\title{
FLUORESZENZCHEMOSENSOREN AUF BASIS DES ANTHRACEN-FLUOROPHORS
}

\author{
Dissertation zur Erlangung des \\ mathematisch-naturwissenschaftlichen Doktorgrades \\ "Doctor rerum naturalium" \\ der Georg-August-Universität Göttingen
}

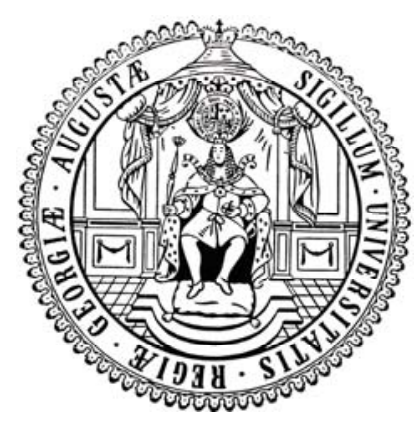

vorgelegt von

Dipl.-Chem. Daniel Stern

aus Hameln 
D7

Referent: Prof. Dr. Dietmar Stalke

Korreferent: Prof. Dr. Georg M. Sheldrick

Tag der Mündlichen Prüfung: 26.01.2010 
Die vorliegende Arbeit wurde in der Zeit von Januar 2007 bis Dezember 2009 am Institut für Anorganische Chemie der Georg-August-Universität zu Göttingen unter der Leitung von Prof. Dr. Dietmar Stalke angefertigt. 
"Ein kluger Mensch macht nicht alle Fehler selber.

Er gibt auch anderen eine Chance." (Winston Churchill) 


\section{Inhaltsverzeichnis}

1 EINLEITUNG 1

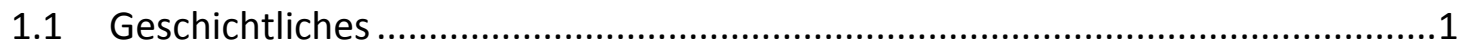

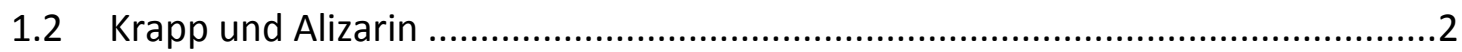

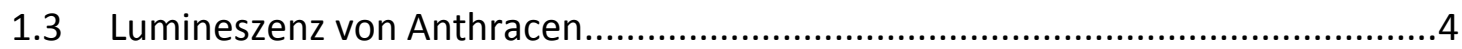

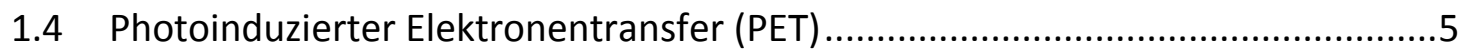

1.5 Ausgewählte Beispiele von Fluoreszenzchemosensoren auf Basis des

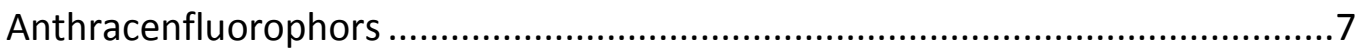

1.6 Vorarbeiten im Arbeitskrei Stalke auf dem Gebiet der

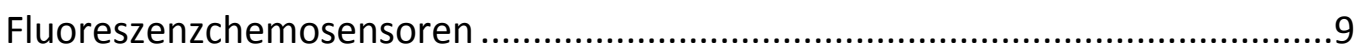

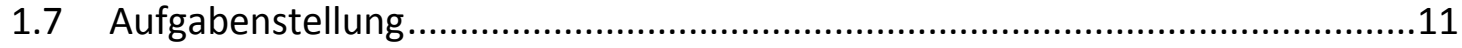

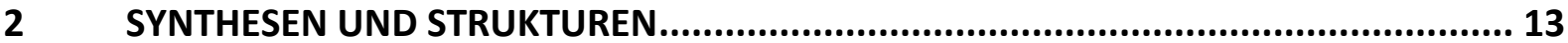

2.1 Bromierte Anthracene als Ausgangsstoffe weiterer Synthesen ........................14

2.1.1 Bromierung des Anthracens.............................................................15

2.1.2 Bromierung der Anthracenderivate ..................................................18

2.2 Funktionalisierung bromierter Anthracene durch Lithiierung ..........................23

2.2.1 Selektivität in der Lithiierung von Dibromanthracen............................24

2.2.2 Reaktivitätssteigerung der Lithiumanthracene durch Substitution von Donoren und durch Deaggregation ............................................26

2.2.3 Der LiX-Effekt in lithiierten Anthracenen ............................................36

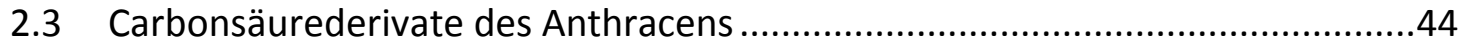

2.4 Addition lithiierter Anthracene an Carbonylgruppen ....................................49

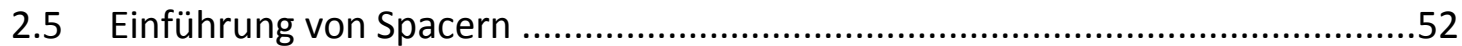

2.6 Aufbau der Rezeptoreinheit durch Substitutionsreaktionen .............................54

2.7 Einführung von Phosphanen in substituierte Anthracene ...............................59

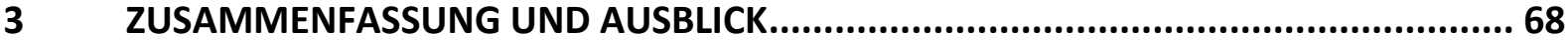

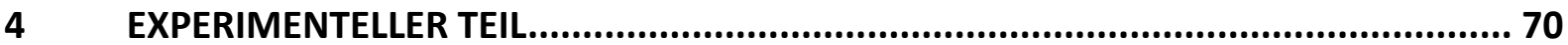

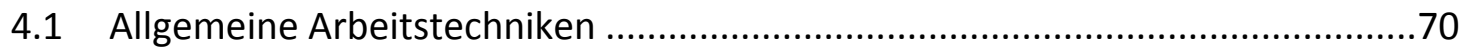

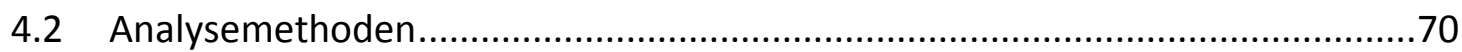

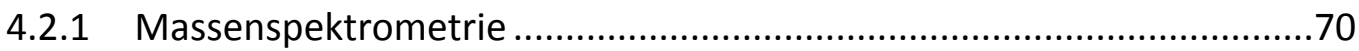

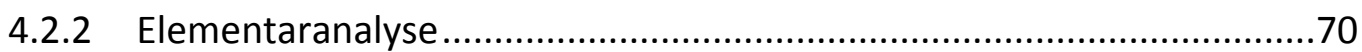

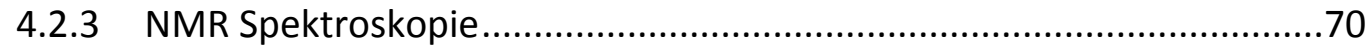




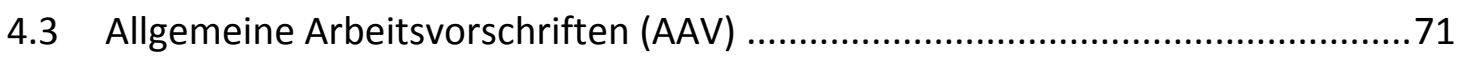

4.3.1 AAV 1 Bromierung von Anthracen(derivaten) .....................................71

4.3.2 AAV2 Lithiierung von Bromanthracenen ohne Abtrennen von

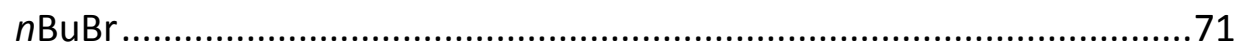

4.3.3 AAV 3 Lithiierung von Bromanthracenen mit Abtrennen von $n \mathrm{BuBr}$.....71

4.3.4 AAV 4 Einführung von Phosphanen in Anthracene ..............................71

4.3.5 AAV 5 Oxidaton von Phosphanylanthracenen mit $\mathrm{H}_{2} \mathrm{O}_{2} \ldots \ldots \ldots \ldots \ldots \ldots . . . . . . . . .72$

4.3.6 AAV 6 Oxidation von Phosphanylanthracenen mit Schwefel oder

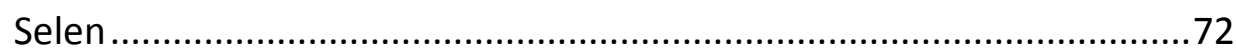

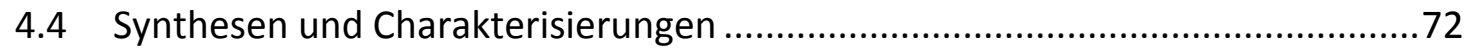

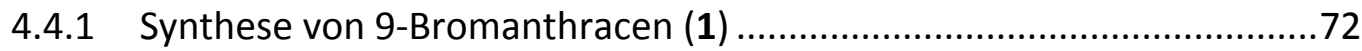

4.4.2 Synthese von 9,10-Dibromanthracen (2) ...........................................73

4.4.3 Synthese von 9-Brom-10-methylanthracen (3) ...................................73

4.4.4 Synthese von 9-Brom-10-brommethylanthracen (4) ............................74

4.4.5 Synthese von 9,10-Di(brommethyl)anthracen (5) ...............................74

4.4.6 Synthese von 9-Brommethylanthracen (6) ..........................................75

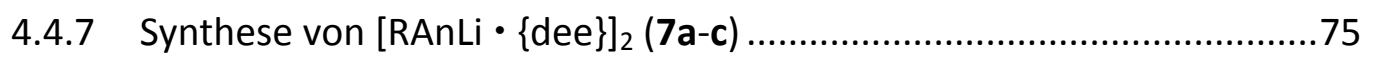

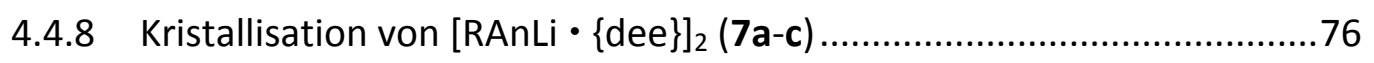

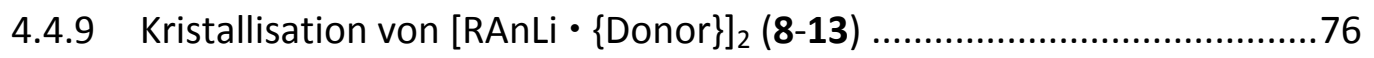

4.4.10 Kristallisation von $\left[\mathrm{RAnLi} \cdot \mathrm{LiBr} \cdot\{\text { Donor }]_{2}(\mathbf{1 4 a}-\mathbf{b}\right.$ und 15a-b) ...............76

4.4.11 Kristallisation von $[n B u A n L i \cdot\{$ pmdeta\}] (16) .........................................77

4.4.12 Synthese von 9,10-Dibuthylanthracen (17) ..........................................77

4.4.13 Synthese von 9-Bromanthracen-10-carbonsäure (18) ..........................77

4.4.14 Synthese von 9-Bromanthracen-10-carbonsäurechlorid (19) ................78

4.4.15 9-Brom-10-carbonsäure-tert-butanesteranthracen (21)......................78

4.4.16 9-Brom-10-anthracen(2-hydroxy-1,1dimethylethyl)carbonsäureamid (22) ..............................................79

4.4.17 9-Brom-10-(4,4-dimethyl-2-oxazyl)-anthracen (23) .............................79

4.4.18 9-Brom-10-(1-hydroxy-1-pyridyl)methylanthracen (25)........................80

4.4.19 9-((2-Methoxyethyl)aminomethyl)anthracen (26) .............................80

4.4.20 9-((2-tetrahydrofuranylethyl)aminomethyl)anthracen (27)..................81

4.4.21 Synthese von 9-Brom-10-(2-hydroxyethyl)anthracen (28)....................82

4.4.22 Synthese von 9-Brom-10-(2-tosyloxyethyl)anthracen (29) .....................82

4.4.23 Synthese von 9-Brom-10-((trimethyethylendiamino)methyl)-

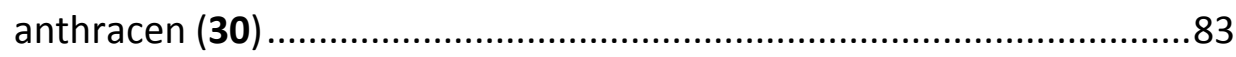

4.4.24 9-Brom-10-(dipyridylaminomethyl)anthracen (31) .............................84

4.4.25 Synthese von 9-Diisopropylphosphanylanthracen (35) .........................84

4.4.26 Synthese von 9-Diisopropyloxophosphanylanthracen (36) ...................84 
4.4.27 Synthese von 9-Diisopropylthiophosphanylanthracen (37) .................85

4.4.28 Synthese von 9-Diisopropylselenophosphanylanthracen (38) ...............86

4.4.29 Synthese von 9-Bis(diisopropylamino)phosphanylanthracen (39) .........86

4.4.30 Synthese von 9-Bis(diisopropylamino)oxophosphanylanthracen (40) ...87

4.4.31 Synthese von 9-Bis(diisopropylamino)thiophosphanylanthracen (41)

4.4.32 Synthese von 9-Bis(N,N-diisopropylamino)selenophosphanylanthracen (42).

4.4.33 Synthese von 9-Brom-10-bis(dimethylamino)phosphanylanthracen (44)

4.4.34 Synthese von 9-Brom-10-bis(dimethylamino)thiophosphoranylanthracen (46).

4.4.35 Synthese von 9-Brom-10-bis(dimethylamino)selenophosphoranylanthracen (47)

4.4.36 Synthese von 9,10-Bis(dimethylamino)phosphanylanthracen (48)........90

4.4.37 Synthese von 9,10-Bis(dimethylamino)oxophosphoranylanthracen (49) .90

4.4.38 Synthese von 9,10-Bis(dimethylamino)thiophosphoranylanthracen (50)

4.4.39 Synthese von 9,10-Bis(dimethylamino)selenophosphoranylanthracen (51) .

4.4.40 Synthese von 9-Bis(dimethylamino)phosphanyl-10-((trimethylethylendiamino)methyl)anthracen (52).....

4.4.41 Synthese von 9-Diphenylphosphanyl-10-((trimethylethylendiamino)methyl)anthracen (53).

4.4.42 Synthese von 9-((trimethylethylendiamino)methyl)anthracen (54) ......92

5 KRISTALLOGRAPHISCHER TEIL

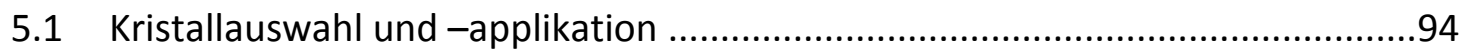

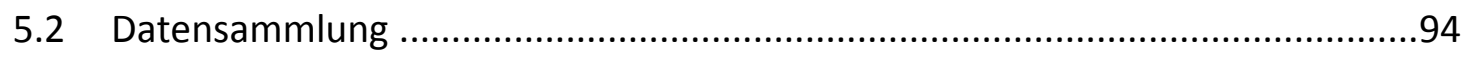

5.3 Strukturlösung und Verfeinerung................................................................95

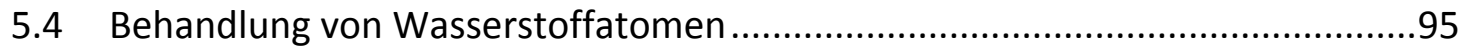

5.5 Berechnung von Wasserstoffpositionen in Methylgruppen .............................97

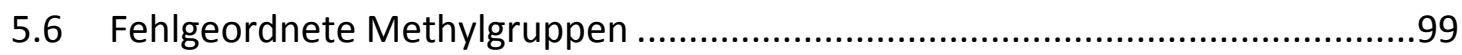

5.6.1 Beschreibung einer fehlgeordneten Methylgruppe mit dem HFIX 123/127 Befehl.

5.6.2 Möglichkeiten zur Verbesserung des mit dem HFIX 123/127 beschriebenen Modells einer fehlgeordneten Methylgruppe 


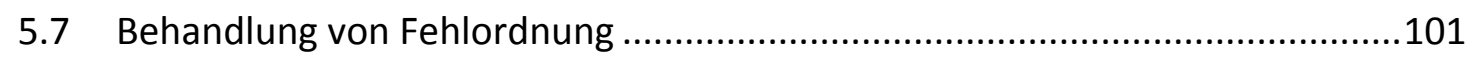

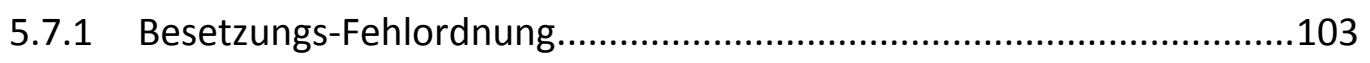

5.7.2 Lage-Fehlordnung .....................................................................103

5.7.3 Fehlordnung auf spezieller Lage ....................................................104

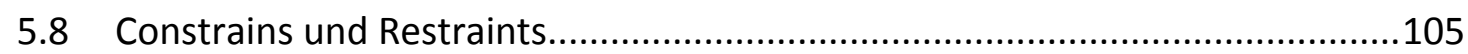

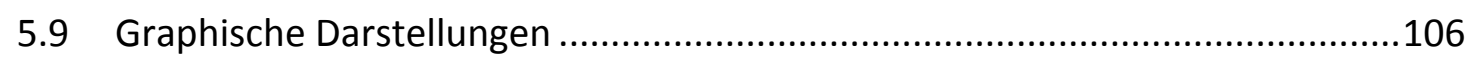

5.10 Kristallographische Daten gemessener Kristallstrukturen .............................107

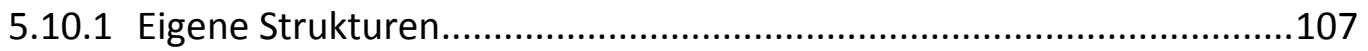

5.10.2 Strukturen des AK Roesky (Universität Göttingen) .............................164

5.10.3 Strukturen des AK Klingebiel (Universität Göttingen) .........................190

5.10.4 Stuktur des AK Beifuß (Universität Hohenstein).................................204

5.10.5 Struktur des AK Breher (Universität Karlsruhe) .................................205

5.10.6 Strukturen des AK Saalfrank (Universität Erlangen) ..........................206

5.10.7 Struktur des AK Beckhaus (Universität Oldenburg) ............................208

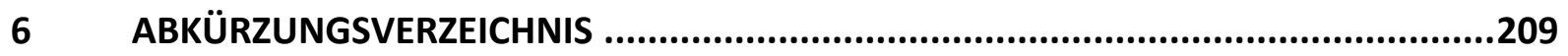

7 VERZEICHNIS DER NUMMERIERTEN VERBINDUNGEN ....................................210

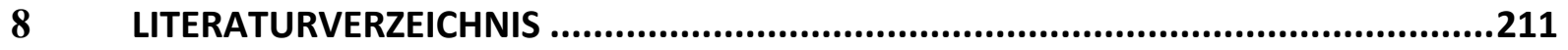




\section{Einleitung}

\subsection{Geschichtliches}

"Man muß die Farbe gesehen haben, ja man muß sie sehen, um sich von der Herrlichkeit dieses kraftvollen Phänomens einen Begriff zu machen." (Johann Wolfgang von Goethe)

In allen Zeitaltern waren Menschen von Farben fasziniert. Belege dafür finden sich in den Höhlenmalereien der Frühzeit, die unter Verwendung von Naturfarben und Erdpigmenten entstanden. Neben den leicht zugänglichen Farben schwarz (Kohle) und weiß (Kalk) kamen auch in den ältesten bisher entdeckten Darstellungen rot (Blut, Manganerde) und ocker (Lehm) zum Einsatz. Abb. 1.1 zeigt das bekannte Motiv 'stehender Bison', dessen Entstehung auf die Jahre 16.000 bis 11.000 v. C. datiert wird und somit zu den ältesten Höhlenmalereien gehört, die bisher entdeckt wurden.

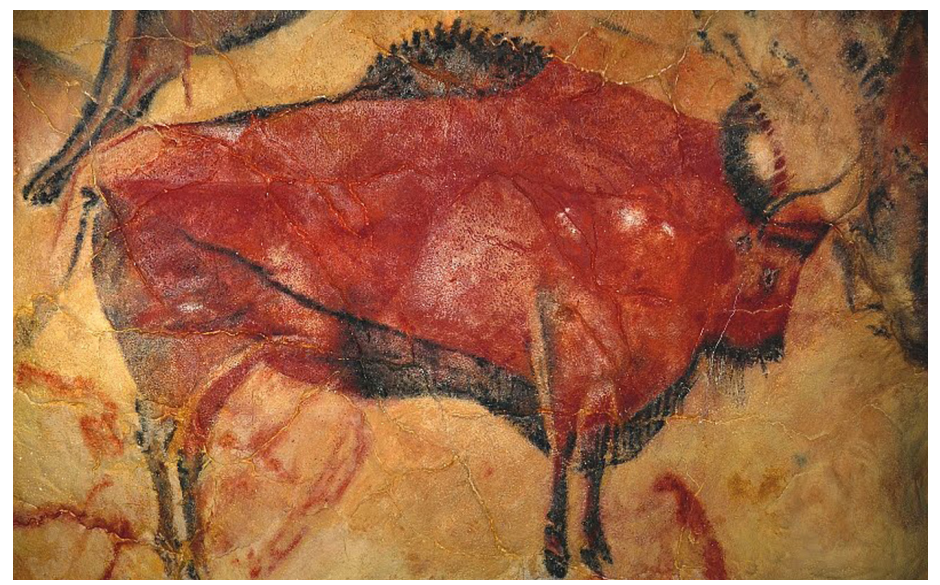

Abb. 1.1: 'Stehender Bison' aus der Höhle bei Altamira (Nordspanien). Entstehung 16.000 bis 11.000 v. C. Die eingesetzten Farbpigmente sind u. a. Holzkohle, Kreide und Manganerde.

Erste Hochkulturen entwickelten die Kunst der Malerei durch immer neue Farbpigmente weiter und waren auch schon früh in der Lage Wolle, Seide und Teppiche zu färben. ${ }^{*}$ Als Färbemittel kamen pflanzliche und tierische Naturfarbstoffe zum Einsatz, die mühevoll gewonnen werden mussten. ${ }^{[1]}$ Neben der Beschaffung der Färbemittel waren die Haltbarkeit und Farbechtheit die größten Probleme. Wie sich aus archäologischen Funden zeigt, waren die frühen Hochkulturen trotz fehlender Kenntnis chemischer Prozesse in der Lage, durch aufwendige Beizverfahren die Farben nicht nur haltbarer zu machen, sondern auch die Farbgebung selbst zu verbessern.

\footnotetext{
*Namentlich Ägypter, Griechen, Römer, Inder, Perser und Türken, wie es z. B. aus der berühmten Enzyklopädie 'Naturalis Historiae Libri' von Gaius Plinius Secundus d. Ä. hervorgeht.
} 


\title{
1.2 Krapp und Alizarin
}

Mit dem stetigen Wachstum der Bevölkerung wurde der Bedarf an Färbepflanzen größer und konnte schon sehr schnell nicht mehr aus den natürlichen Vorkommen gedeckt werden, so dass es zur Kultivierung dieser Pflanzen kam. So ordnete Karl der Große in der Landgüterverordnung Capitulare de villis den Anbau des Krapp ${ }^{\dagger}$ (lat.: Rubia tinctorum) an, dessen roter Farbstoff Alizarin zu ca. $1 \%$ in der Krappwurzel enthalten ist (Abb. 1.2). Die Krapppflanze wurde Ende des 16. Jahrhunderts über Italien nach Europa eingeführt und in Frankreich, in Holland, im Elsaß sowie in anderen Gebieten im großem Maßstab angebaut. ${ }^{[2]}$
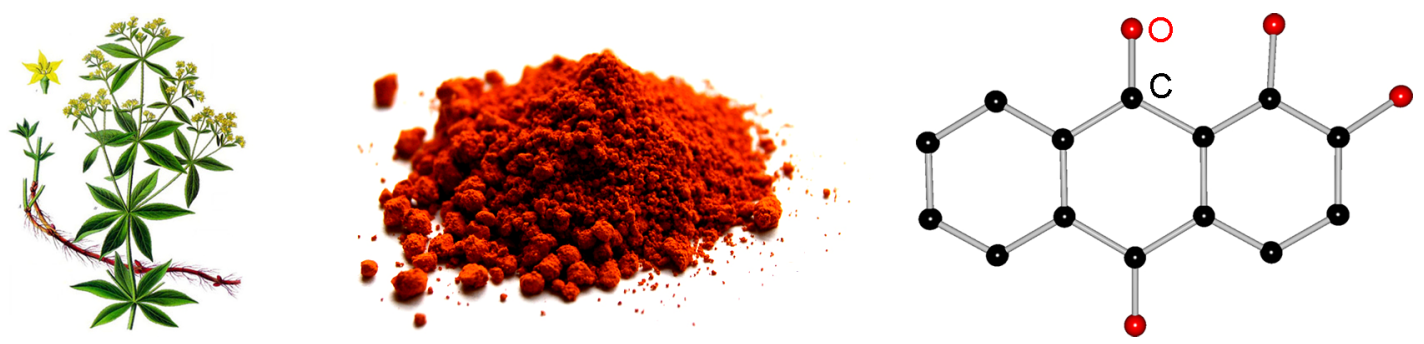

Abb. 1.2: Zeichnung der Krapppflanze (lat.: Rubia tinctorum - links), Alizarinpulver (Mitte) und Molekülstruktur des Alizarins ${ }^{[3]}$ (rechts).

Der Gewinn, der sich aus dem Anbau von Krapp erzielen ließ, überstieg den des Weizens erheblich, so dass Bauern, die sich auf dessen Anbau spezialisierten, schnell zu einem guten Auskommen kamen. Immer größere Flächen wichtigen Ackerlandes ${ }^{\ddagger}$ waren nötig, um den stetig ansteigenden Bedarf an Krapp zu decken.

Ein 'Meilenstein' in der Entwicklung der chemischen Industrie stellte die Aufklärung der Konstitution des im Krapp enthaltenen farbgebenden Stoffes Alizarin dar. ${ }^{[4]}$ Die Erkenntnis, dass Alizarin ein Derivat des Anthracens ist, führte 1868 Carl Lieberman und Carl Gräbe (beide Schüler von Adolf von Baeyer) nicht nur zu der ersten Synthese eines natürlichen Farbstoffes, ${ }^{\S}$ sondern stellte auch gleichzeitig die erste gezielte Synthese eines Naturstoffes dar. $^{[5]}$ Den Forschern war die große Bedeutung ihrer Arbeit durchaus bewusst und so schlossen sie ihre kurze Mitteilung über die gelungene Synthese mit den Worten:

\begin{abstract}
"Von welcher Wichtigkeit unsere Entdeckung für die Krappindustrie sein wird, wenn es gelingt dieselbe technisch verwendbar zu machen, brauchen wir nicht ausführlich hervorzuheben. Der enorme Verbrauch von Krapp in der Kattundruckerei, die großen Strecken fruchtbaren Bodens, die zu dessen Anbau nöthig sind, sprechen hinreichend klar für die Bedeutung, welche ein neuer Industriezweig erlangen würde, der auf der künstlichen Darstellung des Alizarins aus einem Bestandtheil des Steinkohlentheeröls beruht." (Graebe u. Liebermann 1869) ${ }^{[5]}$
\end{abstract}

\footnotetext{
${ }^{\dagger}$ Auch Färberkrapp, Färberröte oder Türkischrot genannt.

${ }^{\ddagger}$ Hier besteht eine Analogie zum Anbau von Raps zur Gewinnung von Bio-Diesel.

${ }^{\S}$ Von 1856 bis 1868 war lediglich die Synthese einiger künstlicher Anilinfarbstoffe bekannt und großtechnisch umgesetzt worden. Diese enttäuschten jedoch durch die ungenügende Farb- und Lichtechtheit.
} 
Nachdem Graebe und Liebermann ihr Verfahren zur Alizarindarstellung patentiert hatten, machten sie ihre Synthese öffentlich und wandten sich an Heinrich Caro (Forschungschef der BASF), um eine großtechnische Umsetzung zu realisieren. ${ }^{[6]}$ Es zeigte sich jedoch, dass ihre erste Synthese über die Bromierung von Anthrachinon für die großtechnische Durchführung ungeeignet war. In der Zusammenarbeit mit Heinrich Caro gelang es innen jedoch noch im selben Jahr ein wirtschaftliches Verfahren zu entwickeln, um diesen Farbstoff im großen Maßstab künstlich herzustellen. ${ }^{[7,8]}$

Der künstliche Farbstoff, der nun wesentlich günstiger gewonnen werden konnte, sorgte schnell für das Einbrechen des Krappanbaus. Auch die Versuche Napoleons, den heimischen Anbau zu fördern, indem er das Rotfärben der Uniformhosen seiner Armee befahl, konnte dies nicht verhindern. Der Preisverfall beim Krapp sorgte jedoch nicht nur dafür, dass sich die Krappbauern umorientieren mussten und auf ihren Feldern nun wieder Weizen anbauten, sondern führte auch zu dem ersten großen Aufschwung der noch jungen chemischen Industrie. So kam es in dieser Zeit mit z. B. der BASF, der AGFA, Bayer und Hoechst zu der Gründung einiger namhafter Chemiefirmen, die zum Teil noch heute bestehen (siehe Tab. 1.1). ${ }^{[2,9]}$

Tab. 1.1: Namen und Gründungsjahre einiger Teerfarbenfabriken. ${ }^{[2,9]}$

\begin{tabular}{lc}
\hline Name & Gründungsjahr \\
\hline Chemische Fabrik Griesheim $^{\text {a }}$ & 1856 \\
Farbwerke Meister, Lucius \& Brüning $^{\text {b }}$ (später Farbwerk Hoechst) & 1862 \\
Farbenfabriken vorm. Friedrich Bayer \& Co (später Bayer) & 1863 \\
Kalle \& Co. Aktiengesellschaft (aufgekauft von Hoechst) & 1863 \\
Badische Anilin- und Sodafabrik (BASF) & 1865 \\
Leopold Cassella \& Co. (jetzt AlessaChemie GmbH) & 1870 \\
Aktiengesellschaft für Anilinfabrikation (AGFA) & 1873 \\
\hline
\end{tabular}

a) später Teilung mit Ausgliederung der heutigen Messer Griesheim GmbH u. Verkauf anderer Teile an verschiedene Unternehmen wie z. B. Hoechst u. Bayer; b) nach Wilhelm Meister, Dr. Eugen Lucius und Dr. Adolf Brüning.

Das Ringen um immer neue Färbemittel, das nicht nur national stattfand, gipfelte in einer Flut von Patenten und führte durch die stete Entwicklung immer neuer Farbstoffe zu einem rasanten Wachstum der Farbstoffindustrie. So konnte zur Weltausstellung 1873 eine vollständige Sammlung künstlicher Farbstoffe mit etwa 400 Exemplaren vorgestellt werden. ${ }^{[9]}$ Mit den heutzutage zur Verfügung stehenden 6000 bis 7000 Farben, von denen 500 in größeren Mengen technisch genutzt werden, ist die Farbigkeit von Stoffen eine Selbstverständlichkeit geworden und weckt keine große Faszination mehr. Lediglich das Aufkommen der Tagesleuchtfarben (umgangssprachlich auch Neonfarben genannt) in den 80er Jahren weckte für kurze Zeit das Interesse der Bevölkerung und beeinflusste sogar die Modewelt, was sich durch die vermehrte Verwendung dieser 'neuen' Farben in skurrilen Farbkombinationen von Alltagskleidung äußerte. 


\subsection{Lumineszenz von Anthracen}

Die Lumineszenz gehört noch heute zu den Farberscheinungen, die seit ihrer Entdeckung nicht an Faszination verloren hat. Nicht nur Schulklassen und Studenten lassen sich immer wieder aufs Neue für Chemolumineszenz und Phosphoreszenz begeistern, auch in den chemischen Laboratorien unserer Zeit gehören Lumineszenzerscheinungen zu den beeindruckensten optischen Phänomenen, die bisweilen zu beobachten sind.

Die vielfältigen Arbeiten auf diesem Gebiet sind Beweis genug für die Faszination, die von lumineszierenden Substanzen ausgeht. Als lumineszierende Stoffe (Fluorophor) kommen ausgedehnte $\pi$-Systeme in Frage, deren HOMO (highest occupied molecule orbital) und LUMO (lowest unoccupied molecule orbital)-Energien die Emission eines Lichtquants im sichtbaren Bereich erlauben.

Anthracen verfügt durch seine drei kondensierten Benzolringe über ein ausgedehntes $\pi$-Elektronensystem und zeigt nicht nur starke Fluoreszenzbanden zwischen 350 und $500 \mathrm{~nm}$, sondern zeichnet sich auch durch seine hohe Fluoreszenzquantenausbeute $\left(\Phi_{\mathrm{F}}\right)$ aus. Das Absorptions- und Emissionsspektrum von Anthracen ist in Abb. 1.3 dargestellt.
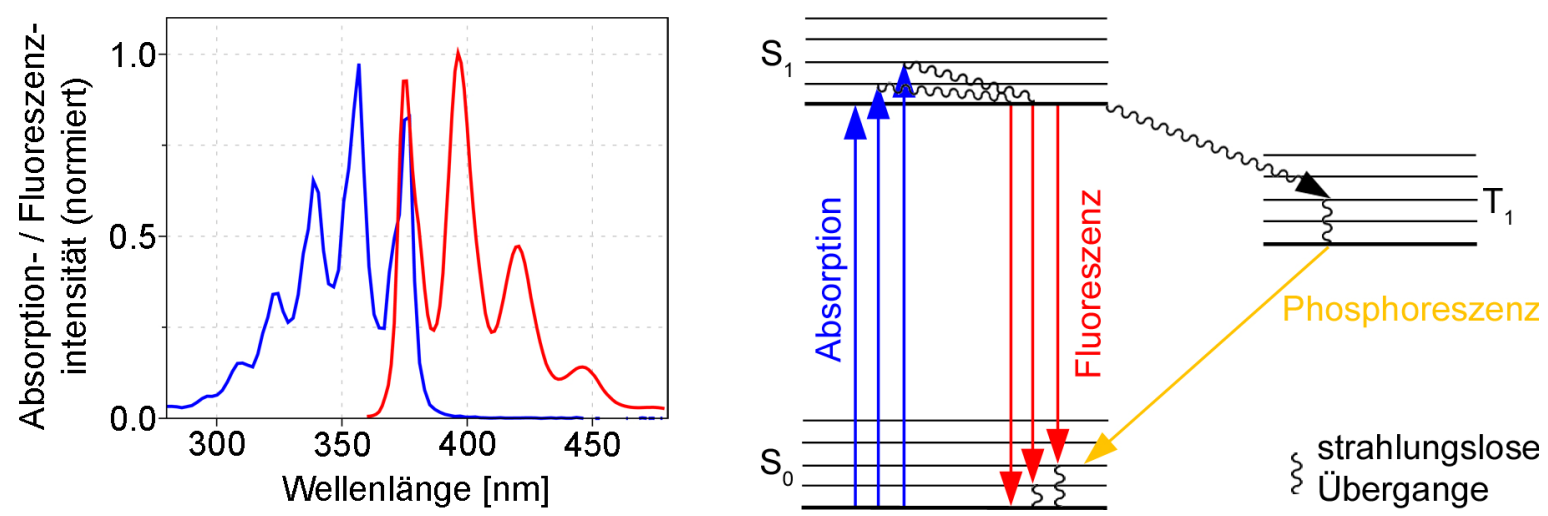

Abb. 1.3: Absorptionsspektrum (blau) und Emmissionsspektrum (rot, $\lambda_{E x}=350 \mathrm{~nm}$ ) von Anthracen in Cyclohexan bei Raumtemperatur ${ }^{[10]}$ (links) und Jablonski Diagramm (rechts).

Die verschiedenen Banden des Absorptions- und Emissionsspektrums lassen sich mit Hilfe des Frank-Condon-Prinzips erklären, nach dem ein elektronischer Übergang immer 'vertikal' erfolgt. Bei Raumtemperatur ist nach der Boltzmann-Statistik praktisch ausschließlich das unterste Schwingungsniveau $(v=0)$ des Grundzustands $S_{0}$ besetzt. Durch Absorption werden verschiedene Schwingungsniveaus $v^{\prime}=0,1,2,3, \ldots$ des ersten angeregten Zustands $S_{1}$ populiert. Durch eine sehr rasche Relaxation nach $v^{\prime}=0$ erfolgt die Emission aus dem Schwingungsgrundzustand des ersten angeregten Zustands $S_{1}$ in verschiedene schwingungsangeregte Zustände des Grundzustandes $S_{0}$. Nach der Kasha-Regel erfolgt die Emission stets aus dem Schwingungsgrundzustand des ersten angeregten Zustandes, wodurch die Emission gegenüber der Absorption rotverschoben ist. ${ }^{[11,12]}$

Die Einführung von Substituenten am Anthracengerüst führt zu einer Veränderung der Orbitalenergien und gegebenenfalls zu einer Vergrößerung des chromophoren Systems, 
woraus eine Verschiebung der Absorptions- und Emissionsbanden resultiert. ${ }^{[13]}$ Die Emissionsbanden einiger mono- und disubstituierter Anthracenderivate sind in Tab. 1.2 angegeben.

Tab. 1.2: Emissionsbanden einiger ausgewählter Anthracenderivate. ${ }^{[14]}$

\begin{tabular}{llll}
\hline Derivat & Messtemperatur [K] & Lösungsmittel & Emissionsbanden [nm] \\
\hline 9-Bromanthracen & 293 & Ethanol & $392.62,415.81,441.51,480.78$ \\
9-Chloranthracen & 293 & Ethanol & $392.16,414.94$ \\
9,10-Dibromanthracen & 293 & Ethanol & $378.79,431.04,457.67,483.10$ \\
9,10-Dichloranthracen & 293 & Ethanol & $404.05,429.19,456.63,480.78$ \\
9-Methylanthracen & 293 & Ethanol & $396.83,469.49,561.81$ \\
9-Brom-10-methylanthracen & 293 & Ethanol & $378.78,429.19,455.59$ \\
9,10-Dimethylanthracen & k. A. & Krist. ${ }^{\text {a }}$ & $436.69,446.43$ \\
9-Cyanoanthracen & 293 & Krist. & $509.95,420.00$ \\
9-Brom-10-ethylanthracen & 293 & Ethanol & $406.51,429.19,455.59,480.78$ \\
9-Ethyl-10-methylanthracen & 293 & Ethanol & $402.42,426.45,432.91,481.93$ \\
9-Brom-10- $n$-propylanthracen & 293 & Ethanol & $409.00,430.11,454.55$ \\
9,10-Diethylanthracen & 293 & Ethanol & $403.23,424.45,453.52,483.10$ \\
9-Brom-10-phenylanthracen & 293 & Ethanol & $408.17,429.19,456.63,485.44$ \\
9,10-Di- $n$-butylanthracen & 293 & Ethanol & $404.05,428.27,455.59$ \\
\hline
\end{tabular}

a) Messungen wurden an kristallinem Material durchgeführt.

Werden komplexere Substituenten verwendet, können die Emissionsbanden deutlich in den sichtbaren Spektralbereich verschoben werden. So verschieben Diphenyloxo- und Diphenylthiophosphoransubstituenten die Fluoreszenzbanden derart, dass eine blaue bzw. gelbe Fluoreszenz beobachtet werden kann. ${ }^{[15-17]}$

\subsection{Photoinduzierter Elektronentransfer (PET)}

Soll die Lumineszenz zur Detektion von Teilchen auf molekularer Ebene genutzt werden, so muss ein Weg gefunden werden, die Lumineszenz eines Fluorophors 'ein-' bzw. 'auszuschalten'. Durch ein solches chemisch induziertes 'Schalten' können chemische Informationen, durch Änderung der Lumineszenzintensität, in leicht detektierbare Signale umgewandelt werden. Dieser Vorgang lässt sich auf einfachem Wege durch den PET-Effekt (Photoinduzierter Elektronentransfer) realisieren. Beim PET-Effekt kommt es zu einem Elektronentransfer von einem Elektronendonator zum HOMO des angeregten Fluorophors, was zu einem effektiven Löschen (Quenchen) der Fluoreszenz führt. Stehen die zur Donation notwendigen Elektronen (meist freie Elektronenpaare) durch kovalente oder donierende Bindungen nicht mehr zur Verfügung, kommt es zum Auftreten der Fluoreszenz. In Abb. 1.4 ist die Lage der Energiniveaus dargestellt, die nötig ist um ein effektives Quenchen durch PET zu ermöglichen. ${ }^{[18]}$ 

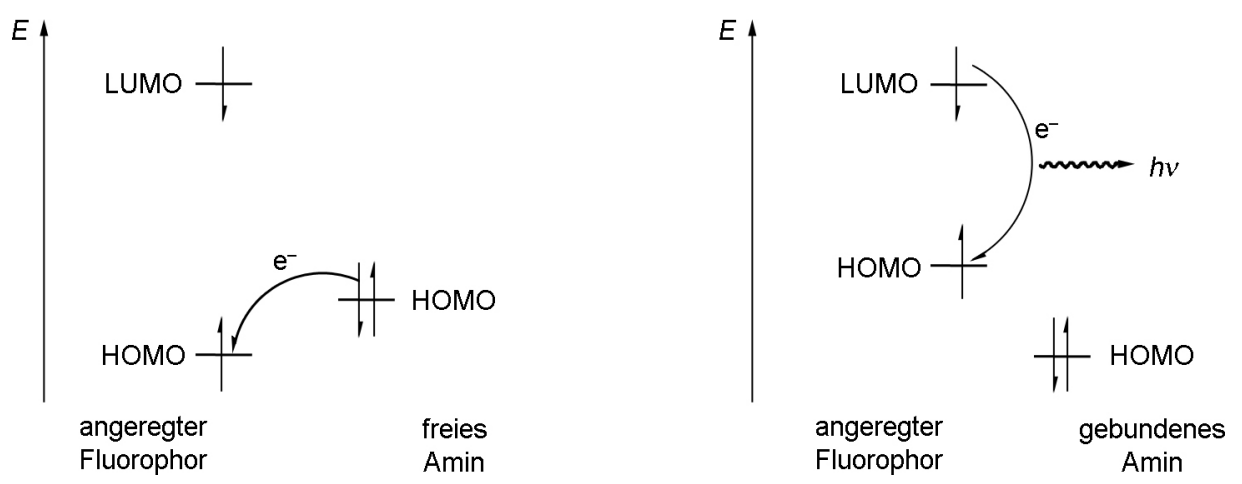

Abb. 1.4: Molekülorbitalenergiediagramme mit einer Lage der Energieniveaus, die einen schnellen photoinduzierten Elektronentransfer ermöglichen (links) oder nicht ermöglichen (rechts).

Die oben beschriebenen Prozesse können anhand eines pH-Fluoreszenzsensors auf Basis des Anthracenfluorophors deutlich gemacht werden. Bei dem Sensormolekül handelt es sich um 9-Diethylaminomethylanthracen. ${ }^{[18,19]}$ Im basischen bis neutralen Bereich liegt der tertiäre Aminsubstituent (Rezeptor) unprotoniert vor und das freie Elektronenpaar führt zu einem effektiven Quenchen der Fluoreszenz des Anthracens (Fluorophor). Wird der pH-Wert ausgehend vom neutralen Bereich abgesenkt, kommt es durch Protonierung zur Quaternisierung der Aminseitenkette. Durch das Einbringen des freien Elektronenpaares in die kovalente Bindung zu einem Proton wird das Oxidationspotential (und damit die energetische Lage des HOMO's) soweit abgesenkt, dass ein Quenchen nicht mehr möglich ist. Als direkte Folge kommt es bei sinkendem $\mathrm{pH}$-Wert zu einem Ansteigen der Fluoreszenzintensität (siehe Abb. 1.5).
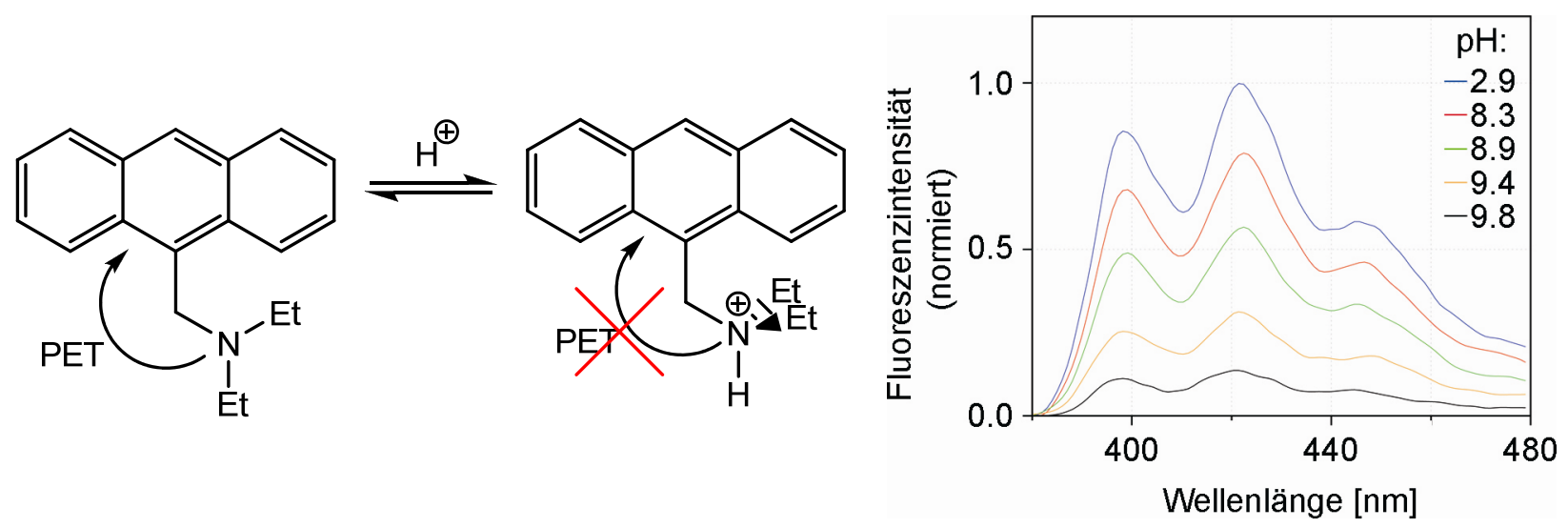

Abb. 1.5: Unterdrücken des PET-Effekts durch Protonierung (links), Abhängigkeit der Fluoreszenzintensität vom pH-Wert (rechts). ${ }^{[18,19]}$

Nicht nur Protonen können das Oxidationspotential des Aminsubstituenten absenken, auch durch Koordination von Metallkationen kann der PET-Effekt unterdrückt werden. Da Stickstoffatome gute Donoren für Metallkationen sind, lässt sich durch Ausnutzen des PET-Effekts eine große Vielfalt an Fluoreszenzchemosensoren für unterschiedliche Analyten (trargets) entwickeln. 


\subsection{Ausgewählte Beispiele von Fluoreszenzchemosensoren auf Basis des Anthracenfluorophors}

Eine der größten Herausforderungen bei der Synthese von Fluoreszenzchemosensoren ist es, hohe Selektivität gegenüber einem gewünschten Analyten zu erreichen. Die Größe und Beschaffenheit der Rezeptoreinheit ist hierfür entscheidend und stellt den schwierigsten Teil bei der Synthese der Sensoren dar. Der Großteil der auf Basis des Anthracenfluorophors publizierten Fluoreszenzchemosensoren wird aus den kommerziell erhältlichen Chlormethylderivaten 9-Chlormethylanthracen und 9,10-Di(chlormethyl)anthracen (siehe Abb. 1.6) dargestellt.<smiles>ClCc1c2ccccc2cc2ccccc12</smiles><smiles>ClCc1c2ccccc2c(CCl)c2ccccc12</smiles>

Abb. 1.6: Strukturformeln der zum Aufbau von Chemosensoren genutzten Ausgangsverbindungen.

Die Rezeptoreinheiten werden stets über $\mathrm{S}_{\mathrm{N}}$-Reaktionen aufgebaut, so dass mono- oder disubstituierte Fluoreszenzchemosensoren nach dem Prinzip Fluorophor-Spacer-Rezeptor oder Rezeptor-Spacer-Fluorophor-Spacer-Rezeptor erhalten werden. Einige ausgewählte Beispiele solcher Systeme sind in Abb. 1.7 dargestellt.

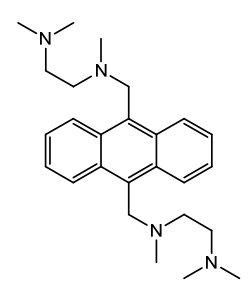

I

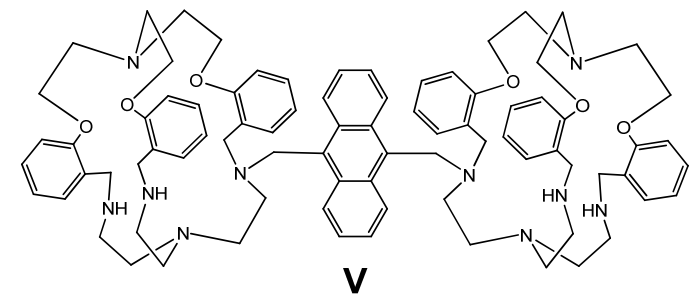

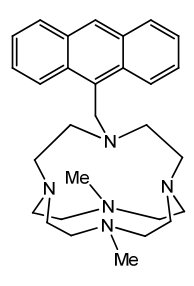

III

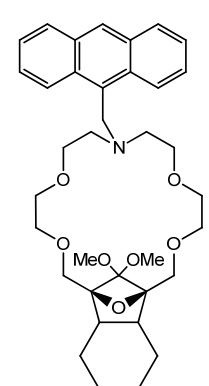

IV

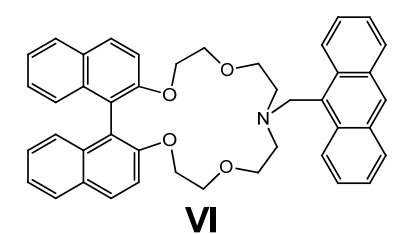

Abb. 1.7: Strukturformeln einiger publizierter Chemosensoren auf Basis des Anthracenfluorophors. Die zugehörigen Publikationen sind: I, ${ }^{[20]} \mathbf{I I},{ }^{[21]} \mathbf{I I I}{ }^{[22]} \mathbf{I V},{ }^{[23]} \mathbf{V}^{[24]}$ und VI. ${ }^{[25]}$

Das Spektrum der in den publizierten Sensoren enthaltenen Rezeptoren reicht von einfachen Chelatliganden (I und II) über Azakronenether (IV und VI) bis hin zu aufwendigen Kryptanden (III und V). 
Während die Sensoren I und II schon durch ihre einfachen Rezeptoren eine geringe Selektivität gegenüber unterschiedlichen Analyten erwarten lassen, haben sie jedoch den Vorteil wasserlöslich zu sein. Dies ermöglicht ihren Einsatz bei der Detektion von Metallkationen in belebten Systemen (in vivo). Es ist bekannt, dass Kronenether und Kryptanden abhängig von ihrer Ringgröße unterschiedliche Selektivitäten gegenüber verschiedenen Metallkationen aufweisen. Bei Fluoreszenzchemosensoren kann diese Selektivität anhand der Fluoreszenzverstärkungen gemessen werden, die durch die Gegenwart unterschiedlicher Metallkationen erreicht werden. In Abb. 1.8 sind Fluoreszenzspektren der Sensoren I bis VI sowie die Fluoreszenzspektren von 9Methylanthracen und 9,10-Dimethylanthracen abgebildet.
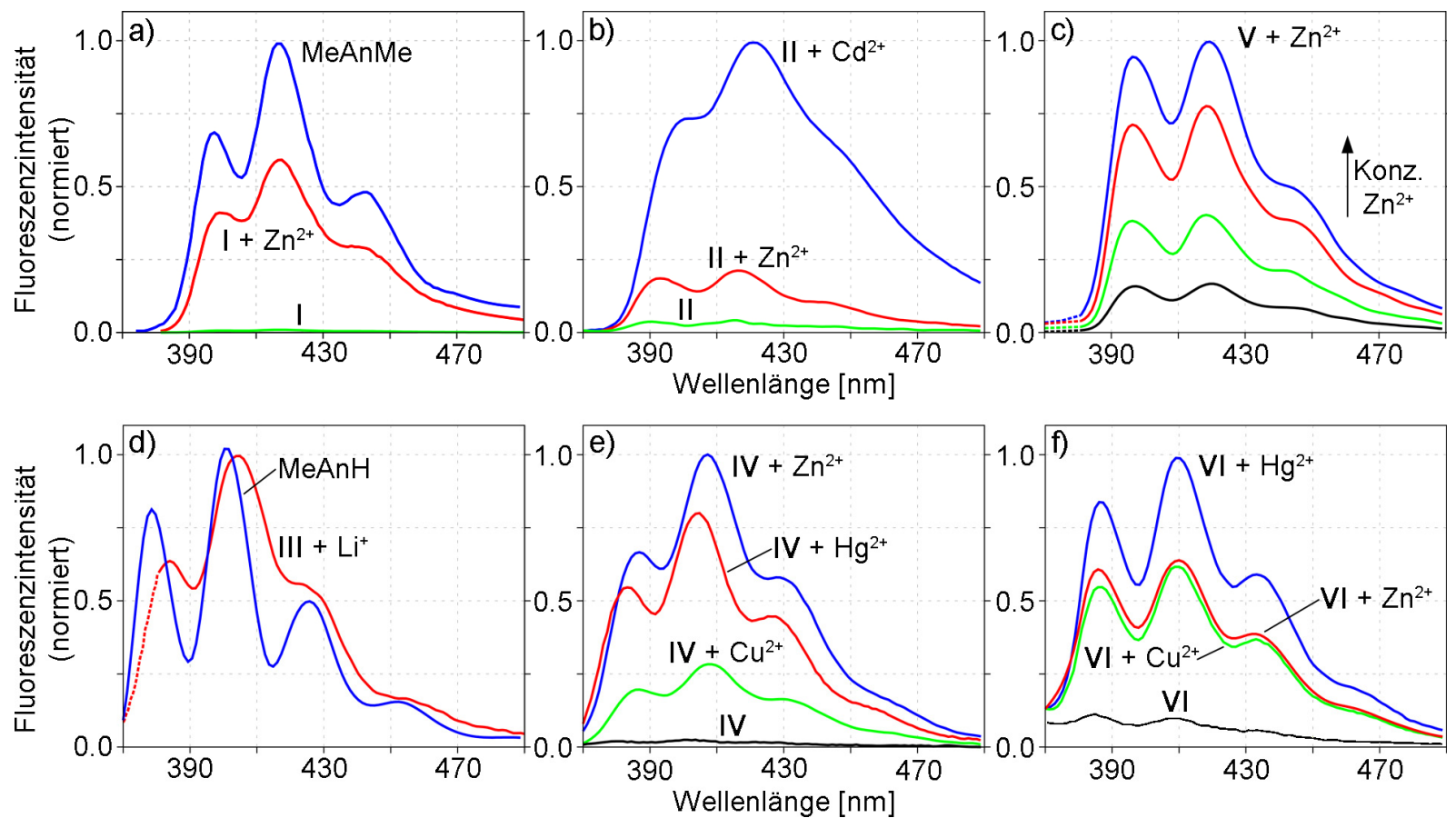

Abb. 1.8: Fluoreszenzspektren von: a) 9,10-Dimethylanthracen (MeAnMe - blau), Sensor I (grün), Sensor I mit $\mathrm{Zn}^{2+}\left(\right.$ rot); b) Sensor II (grün), Sensor II mit $\mathrm{Zn}^{2+}\left(\right.$ rot), Sensor II mit Cd ${ }^{2+}$ (blau); c) Sensor V mit steigender $\mathrm{Zn}^{2+}-$ Konzentration; d) 9-Methylanthracen (MeAnH - blau), Sensor III mit Li (rot); e) Senosor IV (schwarz), Senosor IV mit $\mathrm{Cu}^{2+}$ (grün), Senosor IV mit $\mathrm{Hg}^{2+}$ (rot), Senosor IV mit $\mathrm{Zn}^{2+}$ (blau); f) Sensor VI (schwarz), Sensor VI mit $\mathrm{Cu}^{2+}$ (grün), Sensor VI mit $\mathrm{Zn}^{2+}$ (rot), Sensor VI mit $\mathrm{Hg}^{2+}$ (blau). Die gestrichelten Teile der Kurven in c) und d) stellen interpolierte Werte dar. Sämtliche zur Auftragung nötige Daten wurden den jeweiligen Publikationen entnommen: I und MeAnMe ${ }^{[20]} \mathrm{II}^{[21]}{ }^{[\mathrm{III}}{ }^{[22]} \mathbf{I V},{ }^{[23]} \mathbf{V},{ }^{[24]} \mathbf{V I}^{[25]} \mathrm{MeAnH}^{[26]}$

Für den Sensor I wurden die Fluoreszenzspektren des freien Sensors I und des Sensors I in Gegenwart von $\mathrm{ZnCl}_{2}$ dargestellt um zu zeigen, dass es zu einer Fluoreszenzverstärkung durch Chelatisierung des $\mathrm{Zn}^{2+}$ kommt (Abb. 1.8a). Das Fluoreszenzspektrum von 9,10Dimethylanthracen wurde herangezogen um zu zeigen, dass der Sensor ein nahezu identisches Fluoreszenzspektrum wie 9,10-Dimethylanthracen liefert (Abb. 1.8a). Eine Selektivität gegenüber bestimmten Metallkationen, die auch kaum zu erwarten ist, war in diesem frühen Beispiel (1988) nicht Gegenstand der Untersuchungen. Wie durch die Fluoreszenzspektren der Sensoren IV und VI gezeigt wurde, kann durch die Ringgröße von 
Kronenethern eine gewisse Selektivität gegenüber bestimmten Metallkationen erreicht werden (Abb. 1.8e und f). So zeigt IV eine größere Selektivität gegenüber $\mathrm{Zn}^{2+}$ und weniger gegenüber $\mathrm{Hg}^{2+}$, während bei VI eine umgekehrte Selektivität besteht. Werden wie bei Sensor III Kryptanden eingesetzt, kann die Größenselektivität weiter verbessert werden, was eine eindeutigere Unterscheidung von Metallkationen ermöglicht (Abb. 1.8d). Während die Metallkationen vollständig in die dreidimensionale Koordinationsumgebung der Kryptanden eindringen müssen, reicht eine seitliche Anlagerung an einen Kronenether aus um zu einer Fluoreszenzverstärkung zu führen. Der Sensor III weist selektiv Lithium-Kationen nach und zeigt keine Komplexbildung mit den übrigen Alkalimetallkationen. Der ebenfalls mit Kryptanden versehene Sensor $\mathbf{V}$ arbeitet nach demselben Prinzip, besitzt jedoch pro Kryptand zwei NH-Gruppen. Werden diese zu tertiären Aminen derivatisiert, kann durch den sterischen Anspruch der eingeführten Substituenten der Eintritt von Metallkationen gesteuert und somit die Größenselektivität weiter verbessert werden.

Da allen hier gezeigten Sensoren dasselbe Strukturmotiv zugrunde liegt, weisen diese auch nahezu dieselben Charakteristika in ihren Fluoreszenzspektren bezüglich der Lage und der relativen Intensitäten der Banden auf. Der elektronische Einfluss der Rezeptoreinheit auf die Energieniveaus des Fluorphors ist bereits mit einem $\mathrm{CH}_{2}$-Spacer soweit abgeschwächt, dass die Fluoreszenzspektren der Sensoren sich kaum von denen der jeweiligen Methylderivate unterscheiden. Die Fluoreszenzspektren der disubstituierten Sensoren I, II und V sind in Abb. 1.8 oben, die Fluoreszenzspektren der monosubstituierten Sensoren III, IV und VI unten gegenübergestellt. Für einen direkten Verglich sind die Fluoreszenzspektren von 9,10Dimethylanthracen und 9-Methylanthracen in Abb. 1.8 a) und Abb. 1.8 d) aufgetragen.

Um die Fluoreszenzbanden in den sichtbaren Bereich zu verschieben sind asymmetrisch substituierte Anthracenderivate nötig, die neben dem Rezeptor noch über einen direkt an das Anthracengerüst gebundenen Substituenten verfügen. Dass solche Systeme bisher in der Literatur nicht bekannt sind, liegt sehr wahrscheinlich am fehlenden synthetischen Zugang zu den asymmetrischen Ausgangsmaterialien. Diese sind weder kommerziell erhältlich, noch existieren bislang Synthesevorschriften, nach denen sich diese Ausgangsstoffe in der nötigen Menge und Reinheit herstellen lassen.

\subsection{Vorarbeiten im Arbeitskreis Stalke auf dem Gebiet der Fluoreszenzchemosensoren}

In vorangegangenen Arbeiten des Arbeitskreises Stalke konnte gezeigt werden, dass Phosphor(V)substituenten am Anthracen in der Lage sind, die Emissionsbanden dieses Fluorophors in den sichtbaren Bereich zu verschieben. ${ }^{[15,27,28]}$ Für die beobachtete Emission scheint einerseits der elektronische Einfluss des Phosphorsubstituenten, andererseits die sterische Abschirmung des Fluorophors verantwortlich zu sein. So werden je nach Art des am Phosphoratom gebundenen Chalkogens $(\mathrm{O}, \mathrm{S}$ und $\mathrm{Se})$ unterschiedliche Fluoreszenz- 
eigenschaften beobachtet. Während die Oxophosphane eine starke Tendenz zur Fluoreszenz in Lösung zeigen, tritt bei den mit Schwefel oxidierten Phosphanen bevorzugt Festkörperfluoreszenz auf. Von allen bisher im Arbeitskreis Stalke untersuchten Verbindungen zeigten lediglich sieben Derivate Fluoreszenz im sichtbaren Bereich (vier im Festkörper und drei in Lösung). Erwähnenswert ist, dass es sich bei diesen ausschließlich um Diphenylphosphanylund Diphenylphosphoranylanthracene handelt, weshalb diese zu der Gruppe der bislang am besten untersuchten Derivate zählen. Während lange Zeit davon ausgegangen wurde, dass ausschließlich die mit Schwefel oxidierten Diphenylthiophosphorylanthracene Festkörperfluoreszenz zeigen, konnte in der, dieser Arbeit vorangegangen, Diplomarbeit das erste mit Sauerstoff oxidierte Diphenylphosphoranylanthracen synthetisiert werden, welches ebenfalls im Festkörper fluoresziert (siehe Abb. 1.9). ${ }^{[28]}$

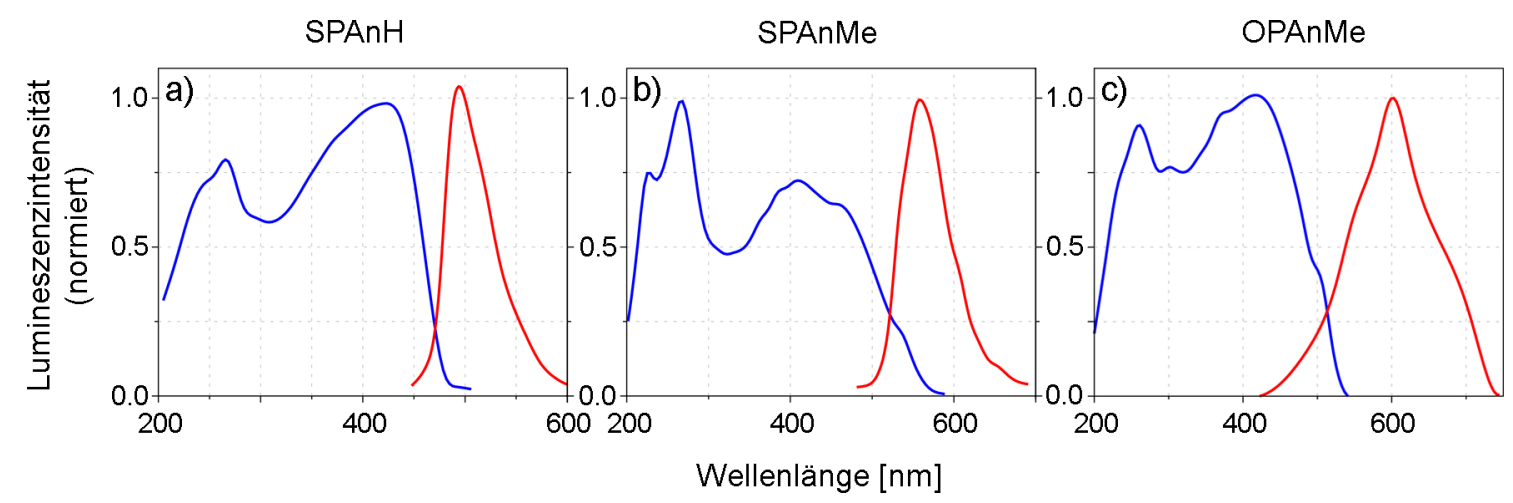

Abb. 1.9: Normierte Absortions- (blau) und Emissionsspekten (rot) von: a) 9-Diphenylthiophosporylanthracen (SPAnH); b) 9-Diphenylthiophosphoranyl-10-methylanthracen (SPAnMe); c) 9-Diphenylphosphoranyl-10methylanthracen (OPAnMe). Aufgenommen bei Raumtemperatur mit einer Anregungswellenlänge von $\lambda_{\mathrm{Ex}}=400 \mathrm{~nm}$. Die Emissionsmaxima liegen bei $\lambda_{\mathrm{Em}}=495 \mathrm{~nm}(\mathrm{SPAnH}), \lambda_{\mathrm{Em}}=524 \mathrm{~nm}$ (SPAnMe) und $\lambda_{\mathrm{Em}}=600 \mathrm{~nm}$ (OPAnMe).

Durch Variation der beiden organischen Reste am Phosphoratom kann ebenfalls Einfluss auf die Lage der Fluoreszenzbanden genommen werden. Dieses Phänomen wurde jedoch bisher nur quantitativ bestimmt.

Dialkyl- und Diarylphosphanylanthracene lassen sich in guten Ausbeuten durch die Umsetzung von lithiierten Anthracenen mit den entsprechenden Chlorphosphanen erhalten. Wird hierbei das 9-Brom-10-anthryllithium eingesetzt, entstehen Anthracene, die neben dem Phosphorsubstituenten ein Bromatom in 9-Position tragen. Da dieses Bromatom bisher nur in geringem Maße über Lithiierung ausgetauscht werden konnte, hat sich diese Substanzklasse zum Aufbau von Fluoreszenzchemosensoren als ungeeignet herausgestellt. Dieses Problem kann umgangen werden, wenn der Phosphorsubstituent erst nach Aufbau des Zweitsubstituenten eingeführt wird, wie es für die 9-Diphenylphosphanyl-10methylanthracene gezeigt werden konnte. ${ }^{[28]}$ 


\subsection{Aufgabenstellung}

In dieser Arbeit soll ein synthetischer Zugang zu einem Fluoreszenzchemosensor (im Weiteren Sensor genannt) auf Basis des Anthracenfluorophors gefunden werden. Wie oben gezeigt sind solche Systeme relativ weit verbreitet, jedoch sind die meisten unter ihnen spiegelsymmetrisch aufgebaut (Rezeptor-Spacer-Fluorophor-Spacer-Rezeptor) ${ }^{[19-22,24,29-32]}$ oder enthalten lediglich monosubstituierte Anthracene (mit einem Wasserstoffatom in 10Position). Das hier angestrebte Sensorsystem soll neben den fluoreszenzsensortypischen Bauteilen Fluorophor, Spacer und Rezeptor noch eine zusätzliche Komponente, den Tuner, enthalten (siehe Abb. 1.10). Über diesen Tuner soll die Möglichkeit erhalten werden, die Fluoreszenzquantenausbeuten $\mathrm{zu}$ steigern und die Lage der Fluoreszenzbanden zu verschieben (tunen).

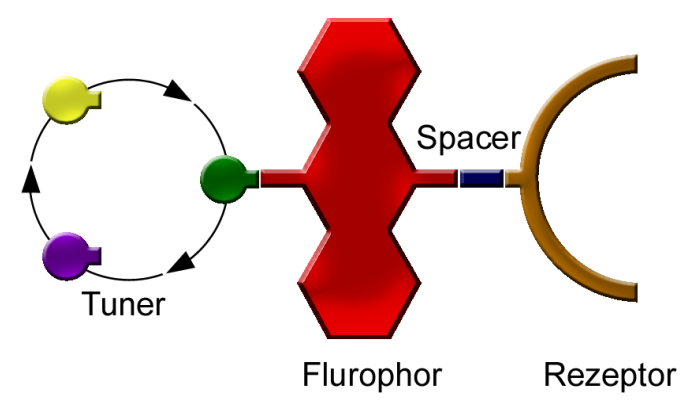

Abb. 1.10: Aufbau eines Sensorsystems mit Fluorophor, Spacer, Rezeptor und variierbarem Tuner.

Da es wenig sinnvoll erscheint, sich a priori auf nur eine konkrete Zielverbindung (target) festzulegen, soll der gesamte Aufbau des Sensors modular geschehen, um das Tauschen verschiedener 'Baugruppen' zu ermöglichen. So soll der Sensor nicht nur durch Variation des Rezeptors auf verschiedene Targets angepasst werden, vielmehr soll ein Baukastenprinzip entwickelt werden, welches das Tauschen aller Bauteile ermöglicht und somit den Zugang zu einem vielseitigen Sensorsystem realisiert (siehe Abb. 1.11).

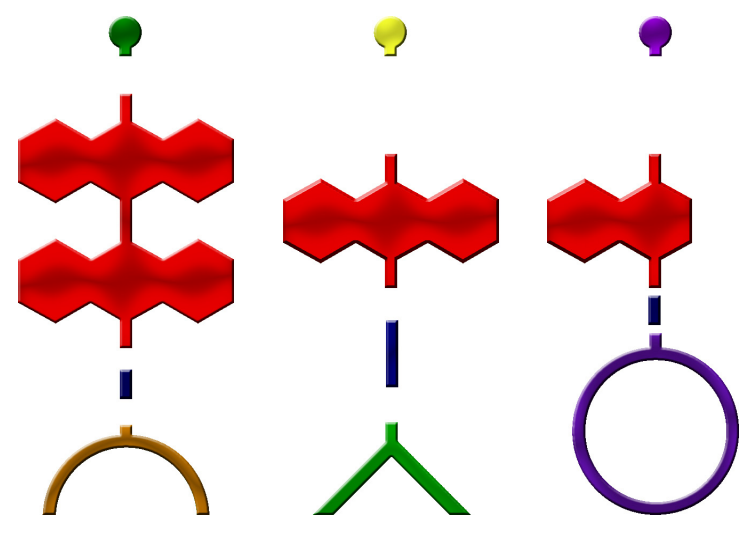

Abb. 1.11: Modularer Aufbau des Sensorsystems. 
Der Anspruch an die zum Aufbau verwendeten Synthesewege ist hoch. Um die oben gesteckten Ziele zu erreichen, dürfen die Synthesen nicht zu spezifisch sein und müssen dennoch hohe Ausbeuten liefern, bei denen die Gewinnung der Reaktionsprodukte möglichst keiner aufwendigen Aufarbeitung bedarf.

Als Basis für die noch zu erschließenden Synthesewegen, soll die schon im Arbeitskreis Stalke bekannte Selektivität bei der Lithiierung von 9,10-Dibromanthracen zur Anwendung kommen. 


\section{Synthesen und Strukturen}

Wie in der Aufgabenstellung erwähnt, soll das angestrebte Sensorsystem über Anthracen als Fluorophor verfügen. Des Weiteren haben sich Phosphane und Phosphorane in Vorarbeiten des Arbeitskreises Stalke durch ihr Vermögen, die Fluoreszenzbanden des Anthracengerüstes in den sichtbaren Bereich des elektromagnetischen Spektrums zu verschieben, hervorgetan und sollen daher als Tuner eingesetzt werden. Phosphanyl- und Phosphoranylanthracene sind aus lithiierten Anthracenen nahezu quantitativ zugänglich und ihre Aufarbeitung beschränkt sich meist auf das Abtrennen von Lithiumsalzen und überschüssigen Oxidationsmitteln (Schwefel und Selen). Der Nachteil dieser Substituenten ist die Desaktivierung des bei asymmetrisch sequentiell geführter Synthese verbleibenden Bromatoms (GI. 2.1).<smiles>Brc1c2ccccc2c(Br)c2ccccc12</smiles><smiles>[R]P([R])c1c2ccccc2c(Br)c2ccc(C(F)(F)Br)cc12</smiles>

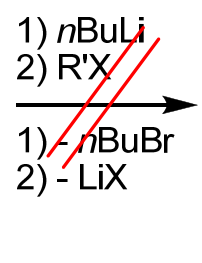<smiles>[R]c1c2ccccc2c(P([R])[R])c2ccccc12</smiles>

GI. 2.1: Desaktivierung des Bromatoms in 9-Brom-10-phosphanylanthracenen.

So hat sich gezeigt, dass dieses nach Einführung von Phosphanen nicht (oder nur in sehr geringem Maße) durch Lithiierung substituiert werden kann. Der Aufbau asymmetrisch substituierter Phosphanylanthracene kann, wie in der, dieser Arbeit vorangegangenen, Diplomarbeit gezeigt wurde, auf dem umgekehrten Weg erfolgen. Hierbei wurden erst die Zweitsubstituenten und danach die Phosphane in das Anthracengerüst eingeführt (GI. 2.2). ${ }^{[28]}$

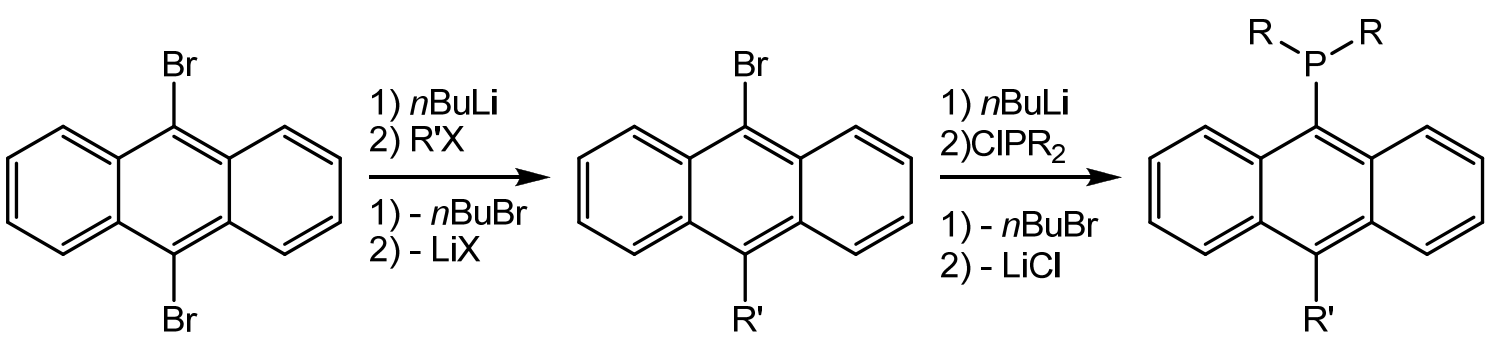

GI. 2.2: Aufbau asymmetrisch substituierter Phosphanylanthracene durch Einführung des Phosphans im letzten Syntheseschritt.

Auf Grund dieser Erkenntnisse soll auch in dieser Arbeit der Aufbau des Rezeptors und damit verbunden auch des Spacers vor der Einführung des Phosphans erfolgen. Um einen PET 
gesteuerten Sensor zu erhalten, werden tertiäre Aminseitenketten als Rezeptoreinheit verwendet, die durch ihr freies Elektronenpaar in der Lage sind, die Fluoreszenz des Anthracengerüstes effektiv zu quenchen. Da PET bis zu Abständen von $10 \AA$ zwischen Rezeptor und Fluorophor beobachtet werden kann, soll der hier verwendete Spacer aus Alkylgruppen mit ein bis zwei Kohlenstoffatomen bestehen. ${ }^{[33]}$ Das sich daraus ergebende Strukturmotiv des gesuchten Sensorsystems ist in Abb. 2.1 dargestellt.

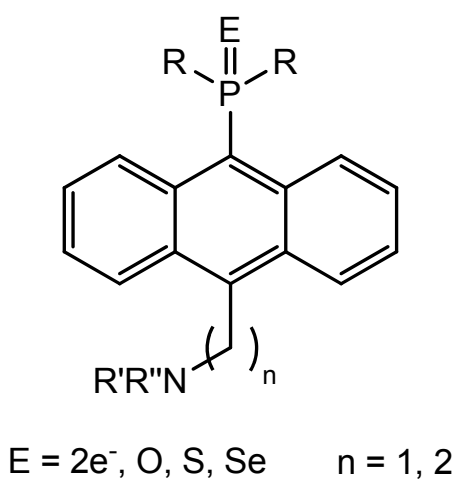

Abb. 2.1: Strukturmotiv des gesuchten Sensors, mit Anthracen als Fluorophor, einem Phosphan als Tuner, $n$ Methylengruppen $(n=1,2)$ als Spacer und eine tertiären Aminseitenkette als Rezeptor.

Um den modularen Aufbau zu gewährleisten sollen klassische organische Synthesewege verfolgt werden, die ein breites Anwendungsspektrum bieten. Hierzu wird in den folgenden Kapiteln die selektive Bromierung von Anthracen und Anthracenderivaten näher untersucht. Auch die Kenntnis über die selektive Lithiierbarkeit von 9,10-Dibromanthracen soll vertieft und um die Möglichkeiten der Reaktivitätssteigerung der lithiierten Zwischenstufen erweitert werden. Die gut untersuchten Reaktionstypen der nukleophilen Substitution, der Additionen von Nukleophilen an Carbonylgruppen sowie die Carbonsäurechemie sollen zum weiteren Aufbau der Rezeptoreinheit zur Anwendung kommen.

\subsection{Bromierte Anthracene als Ausgangsstoffe weiterer Synthesen}

In der modernen Synthesechemie bilden bromierte Alkyl- und Arylverbindungen die Schlüsselreagenzien zur Durchführung von klassischen metallorganischen Reaktionen ( $z$. $B$.

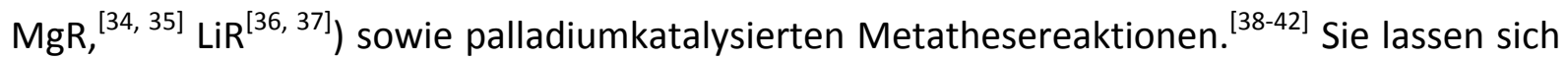
in einer Vielzahl von Reaktionen funktionalisieren und bieten somit den Zugang zum Aufbau komplexer Verbindungen. Viele der gängigen Bromderivate sind kommerziell erhältlich, andere können über klassische Halogenierungsmethoden synthetisiert werden. Anthracen nimmt hierbei wie auch andere kondensierte Aromaten (z. B. Phenanthren und Naphthalin) eine Sonderstellung ein. Zwar ist Anthracen nach klassischen Gesichtspunkten als Aromat anzusehen, das Reaktionsverhalten entspricht jedoch eher dem eines Alkens. So wird elementares Brom an Anthracen schnell unter Bildung des 9,10-Dibrom-9,10dihydroanthracens addiert (GI. 2.3). ${ }^{[43]}$ 
<smiles>c1ccc2cc3ccccc3cc2c1</smiles>

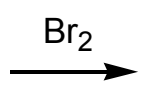<smiles>BrC1c2ccccc2C(Br)c2ccccc21</smiles>

Gl. 2.3: Bildung von 9,10-Dibrom-9,10-dihydroanthracen bei der Reaktion des elementaren Brom mit Anthracen.

Der elektrophile Angriff erfolgt hierbei selektiv unter Verlust der Aromatizität im mittleren Ring an der 9,10-Position. Der Grund für dieses - für Aromaten untypische Reaktionsverhalten liegt in den Resonanzstabilisierungsenergien von Anthracen und den drei möglichen, bei einem elektrophilen Angriff gebildeten, $\sigma$-Komplexen begründet. So weist der durch Angriff der 9,10-Position gebildete $\sigma$-Komplex mit zwei intakten aromatischen Ringen eine um $9 \mathrm{kcal} \mathrm{mol}^{-1}$ größere Stabilisierung auf, als ein Naphthalinsystem, das durch einen Angriff an der 1,4-Position oder der 2,3-Position entstehen würde (siehe GI. 2.4). ${ }^{[43]}$<smiles>Fc1ccc2cc3ccccc3cc2c1</smiles><smiles>Cc1cccc2ccccc12</smiles><smiles>FC1c2ccccc2Cc2ccccc21</smiles>

GI. 2.4: Bildung der beim elektrophilen Angriff auf Anthracen möglichen $\sigma$-Komplexe.

\subsubsection{Bromierung des Anthracens}

Bei der Bromierung des Anthracens wird durch elektrophilen Angriff an die 9-Position ein $\sigma$-Komplex gebildet, an den sich ein Bromid in 10-Position addiert, so dass es zur Bildung des oben genannten 9,10-Dibrom-9,10-dihydroanthracens kommt (siehe GI. 2.5).
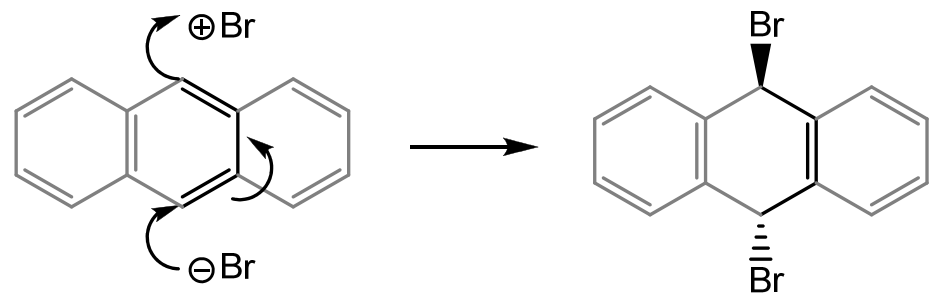

GI. 2.5: Addition von Brom an Anthracen unter Bildung von 9,10-Dibrom-9,10-dihydroanthracen.

Die so gebildete Dibromverbindung ist nicht stabil und spaltet oberhalb von $-78^{\circ} \mathrm{C}$ unter Rearomatisierung Bromwasserstoffsäure $(\mathrm{HBr})$ ab. Das so erhaltene 9-Bromanthracen (1) kann anschließend durch Addition eines weiteren Äquivalents Brom und anschließender Rearomatisierung zu 9,10-Dibromanthracen (2) weiterreagieren. Tatsächlich läuft diese 
Reaktion derart schnell ab, dass es sich als schwierig erweist, die Reaktion selbst bei einem Einsatz von nur einem Äquivalent Brom beim 9-Bromanthracen anzuhalten. Dies spiegelt sich auch in den Preisen beider Derivate wieder (Tab. 2.1).

Tab. 2.1: Preise für die kommerziell erhältlichen Produkte 9-Bromanthracen und 9,10-Dibromanthracen.

\begin{tabular}{lcccc}
\hline Produkt (Reinheit) & Packungsgröße & Packungspreis & Preis/Gramm & Preis/mol \\
\hline \multirow{2}{*}{ 9-Bromanthracen (94 \%) } & $5 \mathrm{~g}$ & $32.80 €$ & $6.56 €$ & $1686.77 €$ \\
& $25 \mathrm{~g}$ & $112.90 €$ & $4.52 €$ & $1242.49 €$ \\
9-Bromanthracen (90\%) & $10 \mathrm{~g}$ & $48.30 €$ & $4.83 €$ & $1241.94 €$ \\
& $50 \mathrm{~g}$ & $214.30 €$ & $4.29 €$ & $1179.21 €$ \\
9,10-Dibromanthracen (98\%) & $10 \mathrm{~g}$ & $39.20 €$ & $3.92 €$ & $1317.20 €$ \\
& $50 \mathrm{~g}$ & $134.70 €$ & $2.69 €$ & $905.24 €$ \\
\hline
\end{tabular}

a) Die übrigen Prozente sind 9,10-Dibromanthracen; b) Die übrigen Prozente sind 9-Bromanthracen. Alle Preise wurden dem Onlinekatalog der Firma Sigma-Aldrich entnommen (abgerufen am 10.12.09).

So kostet 9,10-Dibromanthracen bezogen auf die Molmenge, bei einer Packungsgröße von $50 \mathrm{~g}$, trotz der höheren Reinheit (98\%) gerade einmal das 0.73-fache des Preises für 9-Bromanthracen (Packungsgröße 25 g, 94 \%). Die selektive Synthese von 9-Bromanthracen gelingt erst, wenn die Reaktion so geführt wird, dass die Bildung des 9,10-Dibrom-9,10dihydroanthracen stattfinden kann, die Rearomatisierung zu 9-Bromanthracen jedoch verhindert wird. Ist das gesamte Anthracen auf diese Weise bromiert, kann die $\mathrm{HBr}$ Abspaltung eingeleitet werden. Da auf diese Weise kein freies Brom mehr zur Verfügung steht, kann auch keine Folgereaktion unter Bildung von 9,10-Dibromanthracen eintreten. Diese Bedingungen sind gegeben, wenn eine Lösung von Anthracen in Diethylether auf $-78^{\circ} \mathrm{C}$ gekühlt wird und das Brom in Form einer ebenfalls auf $-78^{\circ} \mathrm{C}$ gekühlten Lösung in Diethylether über längere Zeit zugetropft wird. Die Rearomatisierung durch Abspaltung von $\mathrm{HBr}$ tritt beim Erwärmen automatisch ein und sollte ebenfalls so langsam wie möglich, am besten über Nacht, geschehen. Das so synthetisierte 9-Bromanthracen enthält dennoch geringe Mengen an Anthracen bzw. 9,10-Dibromanthracen, je nachdem ob mit einem leichten Unterschuss oder Überschuss an Brom gearbeitet wurde. Das Abtrennen von 9,10Dibromanthracen kann hierbei durch fraktionierte Kristallisation aus Diethylether erfolgen, bei der 9,10-Dibromanthracen durch seine geringe Löslichkeit als erstes in feinen gelben Nadeln kristallisiert.

Die Dihalogenierung von Anthracen mit Brom bedarf weit weniger Aufwand. So wird die eingesetzte Menge an Brom (typischerweise in einem Überschuss von ca. 2.2 Äq.) bei Raumtemperatur zu einer Suspension von Anthracen in Chloroform gegeben. Das in der Reaktion gebildete 9,10-Dibromanthracen fällt als gelber voluminöser Feststoff aus der Reaktionsmischung aus. Das Ende der Reaktion kann gut an dem Aussetzen der Gasentwicklung erkannt werden. Etwaige Verunreinigungen an Anthracen können ohne größere Schwierigkeiten durch Umkristallisation aus Chloroform abgetrennt werden. 
Obwohl der Additions-Eleminierungsmechanismus zumindest bei der Halogenierung von Anthracen schon früh erkannt wurde, ${ }^{[4]}$ werden immer wieder Halogenierungen von Anthracen (meist innerhalb von Screaning-Experimenten) veröffentlicht, bei denen ungewöhnlich schlechte Ausbeuten als große Erfolge publiziert werden. So liefert z. B. die 'Monobromierung' von Anthracen in Trimethylphosphat, einem "bemerkenswert reaktiven Lösungsmittel für Halogenierungen", erstaunliche Ausbeuten von $42 \%$ an 9,10Dibromanthracen. ${ }^{[45]}$ Einige weitere Beispiele sind in Tab. 2.2 zusammengefasst.

Tab. 2.2: Bromierung von Anthracen zur Synthese von 9,10-Dibromanthracen unter fragwürdigen Bedingungen.

\begin{tabular}{lllcc}
\hline Publikationsjahr & Bromierungsmittel & Lösungsmittel & Reaktionszeit & Ausbeute \\
\hline 2004 & $\mathrm{H}_{2} \mathrm{O}_{2}, \mathrm{HBr}$ & Ethylenchlorid & $30 \mathrm{Min}$. & $61 \%{ }^{[46]}$ \\
2003 & $\mathrm{ZnBr}_{2}, \mathrm{NaBiO}_{3}$ & Essigsäure (konz.) & $3 \mathrm{~h}$ & $52 \%{ }^{[47]}$ \\
1998 & $\mathrm{Bu}_{4} \mathrm{NBr}_{3}$ & Essigsäure & $30 \mathrm{Min}$. & $55 \%{ }^{[48]}$ \\
1989 & $\mathrm{O}_{2} \mathrm{NBr}_{2} \mathrm{CCN}$ & $\mathrm{CH}_{2} \mathrm{Cl}_{2}$ & $3 \mathrm{~h}$ & $65 \%{ }^{[49]}$ \\
\hline
\end{tabular}

Werden zur Bromierung große Überschüsse an Brom verwendet und die Reaktionszeiten deutlich verlängert, kommt es zu einer weiteren Bromierung eines der äußeren Ringe des Anthracengerüsts.

Diese Bildung von sechsfach bromierten Anthracenderivaten wurde schon 1862 bei den ersten Bromierungsversuchen an Anthracen von Anderson beobachtet. ${ }^{[50]}$ Gezielt lassen sich diese Hexabromanthracene durch die Belichtung einer siedenden Suspension von Dibromanthracen und Brom in Tetrachlorkohlenstoff erhalten. Cakmak et al. untersuchten die Mehrfachbromierung von Anthracen und konnten bereits zwei der möglichen Isomere röntgenkristallographisch nachweisen. ${ }^{[51,52]}$ Die Molekülstrukturen dieser beiden Derivate sind in Abb. 2.2 dargestellt.
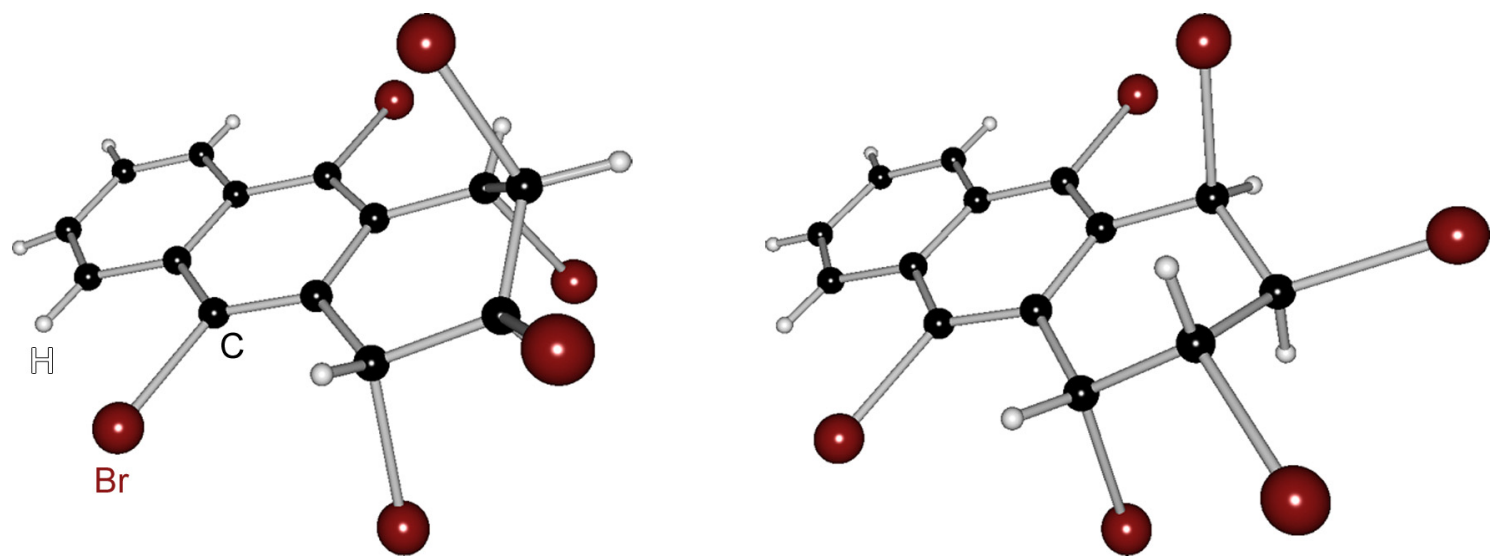

Abb. 2.2: Molekülstrukturen der von Cakmak röntgenkristallographisch nachgewiesene 1,2,3,4,9,10-Hexabrom1,2,3,4-tetrahydroanthracene. ${ }^{[51,52]}$ 


\subsubsection{Bromierung der Anthracenderivate}

Die Bromierung monosubstituierter Anthracenderivate läuft analog zu den oben genannten Mechanismen ab. Das gebildete Produkt ist hierbei jedoch stark von der Art des bereits vorhandenen Substituenten abhängig und folgt nachvollziehbaren Regeln:

A) Bei der Rearomatisierung wird stets die bessere Abgangsgruppe abgespalten. So wird bei der Monobromierung von 9-Chloranthracen selektiv das 9-Brom-10-chloranthracen erhalten. Wird andersherum 9-Bromanthracen mit einem Äquivalent Chlor versetzt, so wird ausschließlich 9,10-Dichloranthracen erhalten.

B) Die Rearomatisierung erfolgt durch Abspaltung von $\mathrm{HX}(\mathrm{X}=\mathrm{Br}, \mathrm{Cl}$ ), was Wasserstoffatome in den 9,10-Positionen erfordert. Sind diese nicht vorhanden, kommt es zur Eliminierung des zuvor addierten Halogens (also der Rückreaktion). Ist der Substituent selbst eine bessere Abgangsgruppe als das addierte Halogen, so wird dieser abgespalten.

C) Sind durch den Substituenten zur 9,10-Position $\beta$-ständige Wasserstoffatome (z. B. an Alkylgruppen) vorhanden, können diese unter Bildung von Doppelbindungen, die ihrerseits wieder schnell $\mathrm{X}_{2}(\mathrm{X}=\mathrm{Br}, \mathrm{Cl})$ addieren, als $\mathrm{HX}(\mathrm{X}=\mathrm{Br}, \mathrm{Cl})$ abgespalten werden. Dieser Reaktion tritt jedoch nur dann auf, wenn nicht gleichzeitig Wasserstoffatome in den 9,10-Positionen vorhanden sind.

Bis auf C) sind diese Konzepte seit langer Zeit bekannt und haben nicht nur Einzug in einschlägige Lehrbücher gefunden, sondern finden auch Anwendung in der aktuellen Forschung. In Gl. 2.6 sind die in dieser Arbeit durchgeführten Monobromierungen zusammengefasst.<smiles>[R]c1c2ccccc2cc2ccccc12</smiles>

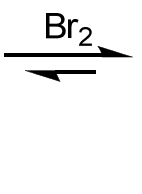<smiles>[R][C@]1(Br)c2ccccc2[C@@H](Br)c2ccccc21</smiles>

I

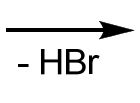<smiles>[R]c1c2ccccc2c(Br)c2ccccc12</smiles>

1: $R=H, 2: R=B r, 3: R=M e$

GI. 2.6: Selektive Bromierung einiger monosubstituierter Anthracenderivate.

Der unter $\mathrm{C}$ ) erläuterte Einfluss von $\beta$-ständigen Wasserstoffatomen wurde bisher weder als Problem bei der Bromierung von z.B. Alkylanthracenen erkannt, noch wurde dessen Potential zum Aufbau seitenkettenbromierter Anthracene ausgelotet. Dies wird insbesondere deutlich, wenn man sich alle bisher publizierten Synthesewege (es sind nur wenige) zu dem auch in dieser Arbeit synthetisierten 9-Brom-10-brommethylanthracen (4) vor Augen führt. Während der Versuch der radikalischen Bromierung von 9Methylanthracen mit dem erklärten Ziel, 9-Brommethylanthracen (6) zu erhalten, noch 
nachvollziehbar scheint, offenbaren andere Darstellungsmethoden das eindeutige Unverständnis der Additions-Eliminierungsmechanismen, die für kondensierte Aromaten im Allgemeinen und Anthracen im Besonderen, typisch sind. Einige publizierte Synthesewege sind in Tab. 2.3 dargestellt.

Tab. 2.3: Einige publizierte Synthesewege zu 9-Brom-10-brommethylanthracen (4).

\begin{tabular}{|c|c|c|c|c|}
\hline Edukt & $\begin{array}{l}\text { Art der } \\
\text { Bromierung }\end{array}$ & Bromierungsmittel & Erhaltenes Produkt & Ausbeute \\
\hline 9-Methylanthracen & radikalisch & NBS & $\begin{array}{l}3 \\
4,4\end{array}$ & $\begin{array}{l}66 \%{ }^{[53]} \\
41 \%,{ }^{[54]} 2 \%{ }^{[55]}\end{array}$ \\
\hline 9-Methanolanthracen & Appel & $\mathrm{PPh}_{3} \cdot x \mathrm{Br}_{2}$ & 4 & k. A. ${ }^{[56,57]}$ \\
\hline 9-Methanolanthracen & $\mathrm{S}_{\mathrm{N}}$ & $\mathrm{HBr}$ & 4 & k. A. ${ }^{[58]}$ \\
\hline 9-Brom-10-methylanthracen & radikalisch & $\mathrm{CuBr}_{2}$ & 4 & $83 \%^{[59]}$ \\
\hline
\end{tabular}

Das in dieser Arbeit zur Synthese des 9-Brom-10-brommethylanthracens als Ausgangssubstanz dienende 9-Brom-10-methylanthracen wurde durch selektive Monolithiierung von 9,10-Dibromanthracen und anschließender Umsetzung mit Methyljodid erhalten. Dieses Edukt kann natürlich, wie oben gezeigt, auch aus 9-Methylanthracen durch selektive Monobromierung synthetisiert werden. Wird die Synthese in beiden Fällen beim Anthracen begonnen, werden sogar rein rechnerisch exakt dieselben Mengen an Reagenzien verbraucht. Da jedoch auf dem zweiten Syntheseweg das aufwendiger zu isolierende 9-Bromanthracen benötigt wird, wird eine deutlich geringere Gesamtausbeute erhalten.

Unter Berücksichtigung der oben genannten Regeln zur Bromierung von Anthracenderivaten mit $\beta$-ständigen Wasserstoffatomen kann leicht der Mechanismus der Bromierung von 9-Brom-10-methylanthracen aufgestellt werden (siehe Gl. 2.7).<smiles>Cc1c2ccccc2c(Br)c2ccccc12</smiles>

3<smiles>C#CCCC</smiles><smiles>CC1(Br)c2ccccc2C(Br)(Br)c2ccccc21</smiles>

II<smiles>CC(C)Br</smiles><smiles>N#CCC1(Br)c2ccccc2C(Br)(Br)c2ccccc21</smiles><smiles>CC=C1c2ccccc2C(Br)(Br)c2ccccc21</smiles>

III<smiles>BrCc1c2ccccc2c(Br)c2ccccc12</smiles>

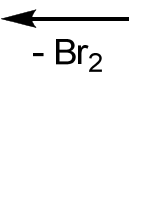

GI. 2.7: Seitenkettenbromierung von 9-Brom-10-methylanthracen (3) unter Bildung von 9-Brom-10brommethylanthracen (4). 
Nach der Addition eines Äquivalents Brom stehen der gebildeten Tribromspezies II zwei Reaktionskanäle offen: Zunächst die Rearomatisierung durch Abspaltung der besten Abgangsgruppe (hier $\mathrm{Br}_{2}$ ), was die Rückbildung des Eduktes (also die Rückreaktion) zur Folge hat und desweiteren die $\mathrm{HBr}$ Eliminierung unter Bildung der in III gezeigten Doppelbindung. III reagiert schnell unter Addition eines weiteren Äquivalents Brom zur Tetrabromspezies IV. Auch hier konkurrieren zwei Reaktionswege miteinander, aus denen letztendlich das stabilere 9-Brom-10-brommethylanthracen hervorgeht. Die Reaktion läuft quantitativ ohne Bildung von Nebenprodukten ab und kann sehr leicht mit ${ }^{1} \mathrm{H}$-NMR-Spektroskopie aus der Reaktionslösung verfolgt werden. Das zur Synthese verwendete und gegen Bromierung inerte Lösungsmittel Tetrachlorkohlenstoff erweist sich hierbei als hilfreich, da durch dessen Unsichtbarkeit im ${ }^{1} \mathrm{H}$-NMR-Spektrum lediglich die Beimischung eines deuterierten Lösungsmittels zu der entnommen Probe aus der Reaktionsmischung erforderlich ist.

Die Signale der Methylgruppe bei $3.07 \mathrm{ppm}$ und der Methylengruppe bei $5.50 \mathrm{ppm}$ erscheinen als separierte Singuletts und deren Integration liefert nach Kalibrierung direkt den Umsatz der Reaktion (siehe Abb. 2.3). Eine Auftragung des Umsatzes über der Zeit zeigte, dass ein $8 \mathrm{~g}$ Ansatz schon in weniger als 30 Min. vollständig umgesetzt wurde. Obwohl meist mit einem leichten Überschuss an Brom gearbeitet wurde, konnte eine Dibromierung der Methylgruppe niemals beobachtet werden.

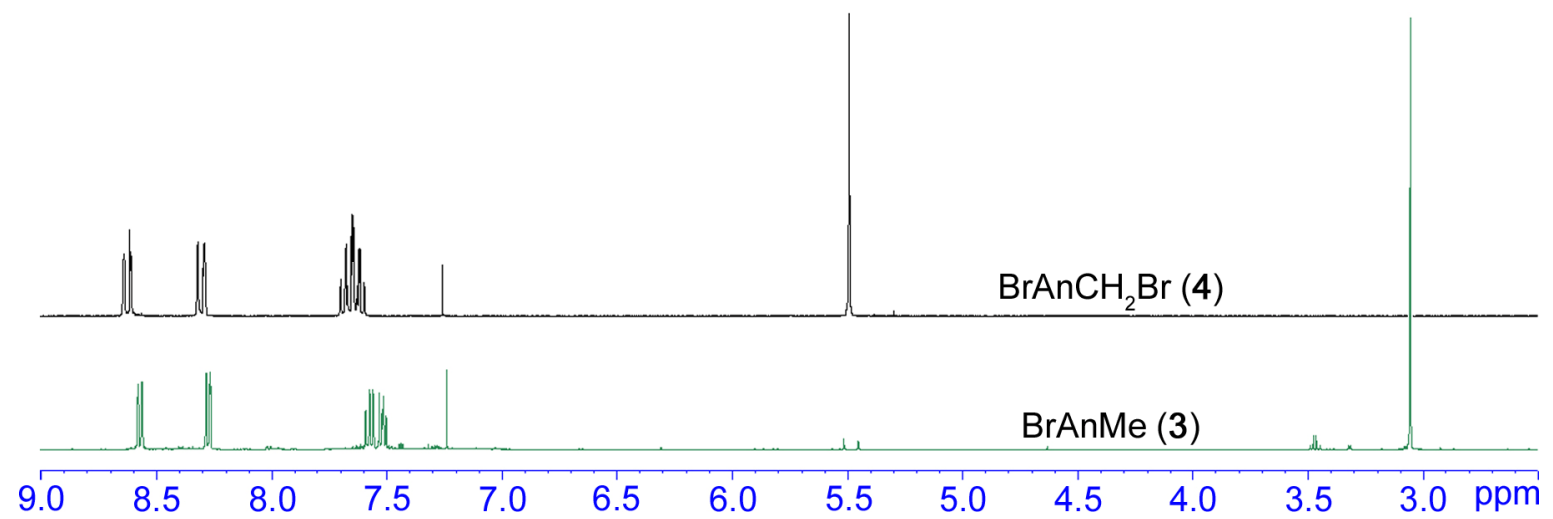

Abb. 2.3: ${ }^{1} \mathrm{H}-\mathrm{NMR}$-Spektren von 9-Brom-10-methylanthracen (3 - unten) und 9-Brom-10-brommethylanthracen (4 - oben).

Die Richtigkeit des oben postulierten Mechanismus wird, unter Betrachtung der in der Reaktion gewählten Reaktionsbedingungen, bestätigt. So wurde die Reaktion mit elementarem Brom in einer abgedunkelten Reaktionsapparatur, also unter nicht radikalischen Reaktionsbedingungen durchgeführt, so dass der vorgeschlagene AdditionsEliminierungsmechanismus der einzig mögliche Reaktionsweg bleibt. Eine zusätzliche Bestätigung des angenommenen Mechanismus sollte die analoge Umsetzung von 9,10Dimethylanthracen bringen, bei der das 9,10-Di(brommethyl)anthracen (5) das erwartete Reaktionsprodukt ist (siehe GI. 2.8). 
<smiles>C=c1c2ccccc2c(=C)c2ccccc12</smiles>

Gl. 2.8: Synthese von 9,10-Di(brommethyl)anthracen (5) unter den zur Synthese von 9-Brom-10methylanthracen (4) analogen Reaktionsbedingungen.

9,10-Di(brommethyl)anthracen (5), das schon früher durch Brommethylierung von Anthracen mit Paraformaldehyd (oder 1,3,5-Trioxan) und $\mathrm{HBr}$ synthetisiert wurde, ${ }^{[31,53]}$ konnte unter den gleichen Reaktionsbedingungen wie schon für $\mathbf{4}$ in vergleichbarer Ausbeute und Reinheit erhalten werden. Zwar wird dieses Reaktionsprodukt für keine weiteren in dieser Arbeit durchgeführten Synthesen benötigt, es besitzt jedoch großes Potential als Ausgangsstoff in der Synthese symmetrischer Anthracenderivate, deren Substituenten durch einen $\mathrm{CH}_{2}$-Spacer vom Anthracengerüst getrennt sind. So könnte es leicht das für diese Zwecke häufig eingesetzte 9,10-Di(chlormethyl)anthracen ersetzen,

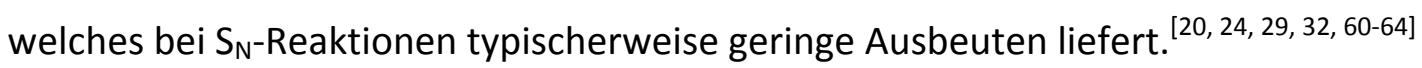

Werden die drei oben genannten Regeln zur Bromierung von Anthracenderivaten als richtig akzeptiert, kann schnell eingesehen werden, dass das Reaktionsprodukt 9-Brommethylanthracen (6) aus keiner Reaktion erhalten werden kann, in der auch nur geringste Spuren an freiem Brom vorhanden sind oder erzeugt werden. Es bleibt dennoch zu erwähnen, dass es in der Literatur nicht an genau diesen Bemühungen fehlt, welche verständlicherweise nicht in dem gewünschten Produkt, sondern in dem schon oben diskutierten 9-Brom-10brommethylanthracen endeten. Auch hier kann durch Zurückgreifen auf andere bekannte Synthesewege in guten Ausbeuten zum gewünschten Produkt gelangt werden. So liefert die Umsetzung von 9-Methanolanthracen mit dem Salz Bromtriphenylphosphoniumbromid das gewünschte Produkt unter Abspaltung von Triphenylphosphoran (siehe GI. 2.9). ${ }^{[53,65]}$

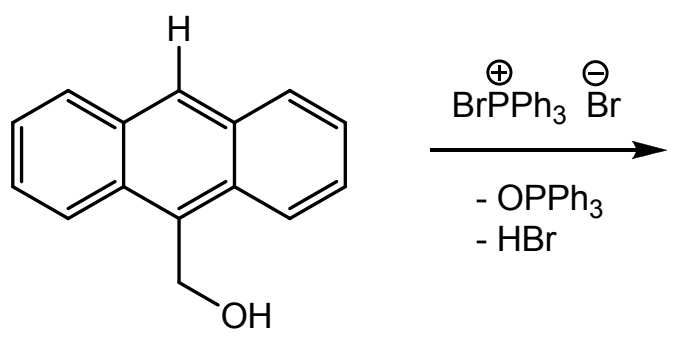<smiles>BrCc1c2ccccc2cc2ccccc12</smiles>

6

GI. 2.9: Synthese des über klassische Bromierung nicht zugänglichen 9-Brommethylanthracens (6) durch die Appel-Reaktion. 
Auch Umsetzungen desselben Eduktes mit Tribromphosphan ${ }^{[53]}$ oder Tribromboran ${ }^{[66]}$ führen zum selben Ergebnis, haben jedoch den Vorteil, dass von einem aufwendigen Abtrennen des Triphenylphosphorans abgesehen werden kann.

Alle in dieser Arbeit synthetisierten bromierten Anthracenderivate lassen sich zur Aufreinigung gut aus chlorierten Lösungsmitteln umkristallisieren. Die auf diesem Wege erhaltenen Kristalle sind stets gelbe Nadeln, deren Größe und Qualität auch ohne große Anstrengungen zur röntgenkristallographischen Analyse ausreichen. Die Kristalle enthalten kein Lösungsmittel und die Atompositionen sind durch das für Anthracen oft beobachtet $\pi$-stacking gut lokalisiert, so dass Belichtungszeiten von einer Sekunde für eine gute atomare Auflösung ausreichen und ein vollständiger Datensatz in wenigen Stunden aufgenommen werden kann. Aus diesen Gründen wurden alle in dieser Arbeit synthetisierten Bromanthracene für eine vollständige Analyse auch kristallographisch untersucht. Die Molekülstrukturen der seitenkettenbromierten Derivate 4 bis 6 sind in Abb. 2.4 dargestellt, Tab. 2.4 enthält ausgewählte Bindungslängen und Torsionswinkel.
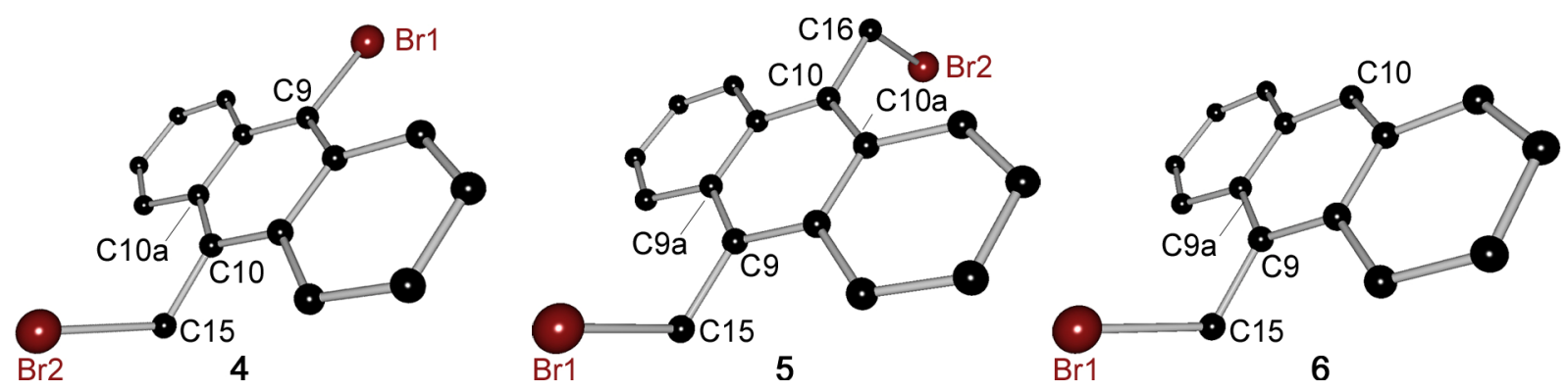

Abb. 2.4: Molekülstrukturen der in dieser Arbeit synthetisierten seitenkettenbromierten Anthracenderivate.

Tab. 2.4: Ausgewählte Bindungslängen $[\AA ̊]$ und Torsionswinkel $\left[^{\circ}\right]$ in 4, 5 und 6.

\begin{tabular}{lccc}
\hline & $\mathbf{4}(298 \mathrm{~K})$ & $\mathbf{5}(100 \mathrm{~K})$ & $\mathbf{6}(100 \mathrm{~K})$ \\
\hline $\operatorname{Br}(1)-\mathrm{C}(9) / \mathrm{Br}(2)-\mathrm{C}(16)$ & $1.889(14)$ & $1.979(3)$ & - \\
$\operatorname{Br}(2)-\mathrm{C}(15) / \operatorname{Br}(1)-\mathrm{C}(15)$ & $1.988(14)$ & $1.979(3)^{\mathrm{a}}$ & $1.996(1)$ \\
$\operatorname{Br}(1) / \mathrm{Br}(2)-\mathrm{C}(15)-\mathrm{C}(10) / \mathrm{C}(9)-\mathrm{C}(10 \mathrm{a}) / \mathrm{C}(9 \mathrm{a})$ & $93.5(6)$ & $92.7(2)$ & $93.7(1)$ \\
$\operatorname{Br}(2)-\mathrm{C}(16)-\mathrm{C}(10)-\mathrm{C}(10 \mathrm{a})$ & - & $92.7(2)^{\mathrm{a}}$ & - \\
\hline
\end{tabular}

a) Gleiche Werte durch Symmetrie.

Mit dem 9-Brom-10-brommethylanthracen konnte schon bei der Bromierung von Anthracenderivaten ein asymmetrisch substituiertes Anthracen erhalten werden, welches bereits einen $\mathrm{CH}_{2}$-Spacer enthält und mit dem benzylisch gebundenen Bromatom auch über eine gute Abgangsgruppe verfügt. Dieser wichtige Synthesebaustein sollte sich in späteren Synthesen leicht durch nukleophile Substitutionsreaktionen des benzylischen Bromides zu den gewünschten Rezeptoren derivatisieren lassen, wohingegen das Bromatom in 9-Position eine Einführung der Tuner durch Lithiierung ermöglichen sollte. Die Derivate 5 und $\mathbf{6}$ bieten analog Zugang zu mono- oder disubstituierten Anthracenen. 


\subsection{Funktionalisierung bromierter Anthracene durch Lithiierung}

Die als Tuner dienenden Phosphane lassen sich nach der Lithiierung der bromierten Derivate an das Anthracengerüst anbringen. Auch der Aufbau der Rezeptoreinheiten kann durch die große Vielfalt an funktionellen Gruppen, die mit lithiumorganischen Reagenzien Reaktionen eingehen, realisiert werden (siehe Abb. 2.5).

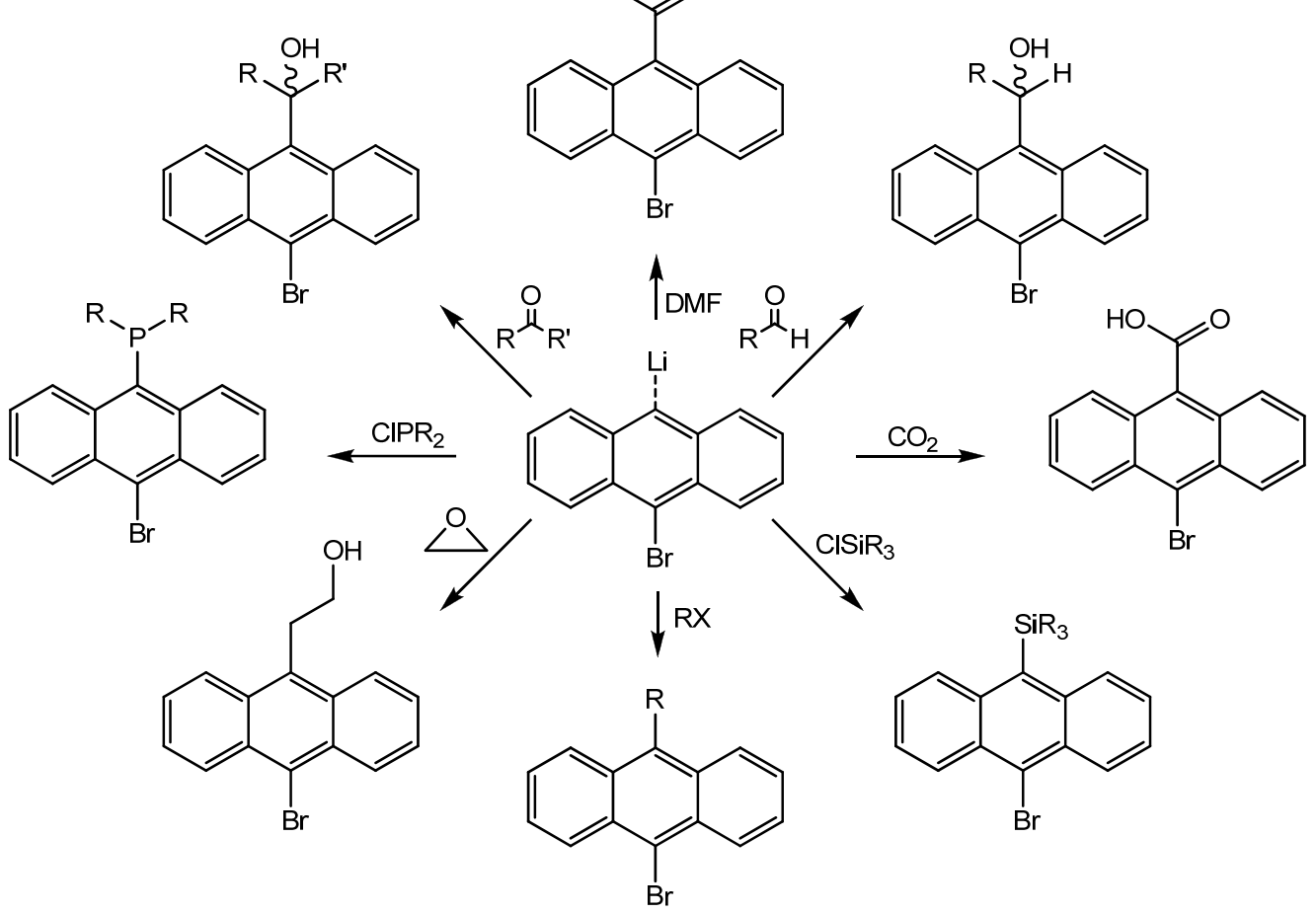

Abb. 2.5: Beispiele zur Umsetzung von 9-Brom-10-anthryllithium mit verschiedenen Elektrophilen. ${ }^{[36,67]}$

In der modernen Synthesechemie sind Lithiumorganyle von größter Bedeutung. Sie lassen sich leicht durch Lithium-Halogenaustausch aus den entsprechenden Halogeniden $(\mathrm{I}>\mathrm{Br}>$ $\mathrm{Cl}$ ) oder durch Deprotonierung $\mathrm{CH}$-azider Substanzen erhalten. Auch eine Direktsynthese aus den jeweiligen bromierten Derivaten und metallischem Lithium ist möglich (siehe GI. 2.10).
a) $\mathrm{R}-\mathrm{H}+\mathrm{R}^{\prime---} \mathrm{Li} \longrightarrow \mathrm{R}^{\prime}-\mathrm{H}+\mathrm{R}---\mathrm{Li}$
b) $\mathrm{R}-\mathrm{X}+\mathrm{R}^{\prime---\mathrm{Li}} \longrightarrow \mathrm{R}^{\prime}-\mathrm{X}+\mathrm{R}---\mathrm{Li}$
c) $\mathrm{R}-\mathrm{X}+2 \mathrm{Li} \longrightarrow \mathrm{R}---\mathrm{Li}+\mathrm{LiX}$

GI. 2.10: Synthese lithiierter Reagenzien durch a) Deprotonierung, b) Lithium-Halogen Austausch oder c) nach der Direktsynthese mit metallischem Lithium.

Neben dem Nachteil mit elementarem Lithium zu arbeiten, liefert Syntheseweg c geringere Ausbeuten und wird durch das Abtrennen nicht umgesetzter Ausgangsstoffe zusätzlich erschwert, so dass diese Darstellung in der Forschung nur noch vereinzelt Anwendung findet. 
Sollen Reaktionen wie hier über eine lithiumorganische Zwischenstufe verlaufen, so müssen Reaktionsbedingungen gefunden werden, die es erlauben das Lithiumorganyl möglichst quantitativ zu erhalten und gleichzeitig dessen Reaktivität so einzustellen, dass die anschließende Umsetzung ebenfalls in hohen Ausbeuten abläuft.

Lithiumorganyle sind hochreaktive, nukleophile Reagenzien und gehen mit einer Vielzahl von funktionellen Gruppen Reaktionen ein. Ihre Nukleophilie ist in der starken Polarisierung der Li-C-Bindung begründet und wird als überwiegend ionisch angesehen. ${ }^{[68]}$ Die Reaktivität des Lithiumorganyls hängt im Wesentlichen vom organischen Rest selbst ab, kann jedoch durch weitere Faktoren wie Konzentration, Lösungsmittel (siehe Abschnitt 2.2.2), Temperatur, Aggregationsgrad und den Salzeffekt (siehe Abschnitt 2.2.3) stark beeinflusst werden. Neben der Reaktivität sind weitere Faktoren wie Chemo-, Regio- und Stereoselektivität für das Gelingen gezielter Umsetzungen von großer Bedeutung. In den nächsten Abschnitten wird auf die Selektivität bei der Lithiierung von bromierten Anthracenen, auf Möglichkeiten zur Reaktivitätssteigerung sowie auf Nebenreaktionen und Desaktivierungsprobleme näher eingegangen.

\subsubsection{Selektivität in der Lithiierung von Dibromanthracen}

Die ersten Lithiierungsversuche an bromierten Anthracenen wurden mit elementarem Lithium in den Jahren $1938^{[69]}$ und $1939^{[70]}$ durchgeführt, blieben jedoch auf Grund mangelhafter Schutzgastechnik ohne nennenswerte Ergebnisse. Diese Arbeiten wurden neun Jahre später von Mikhailov aufgegriffen, welcher ebenfalls durch die Direktsynthese die ersten mono- und dilithiierten Anthracene aus 9-Bromanthracen und 9,10Dibromanthracen synthetisierte und diese durch die Umsetzung mit verschiedenen Nukleophilen nachwies. ${ }^{[71,72]}$ Mikhailov zeigte auch, dass sich die Ausbeuten durch Lithiierung mit $n$-Butyllithium ( $n \mathrm{BuLi}$ ) gegenüber der Direktsynthese deutlich steigern ließen und erkannte die Selektivität bei der Lithiierung von 9,10-Dibromanthracen (siehe Abb. 2.6).

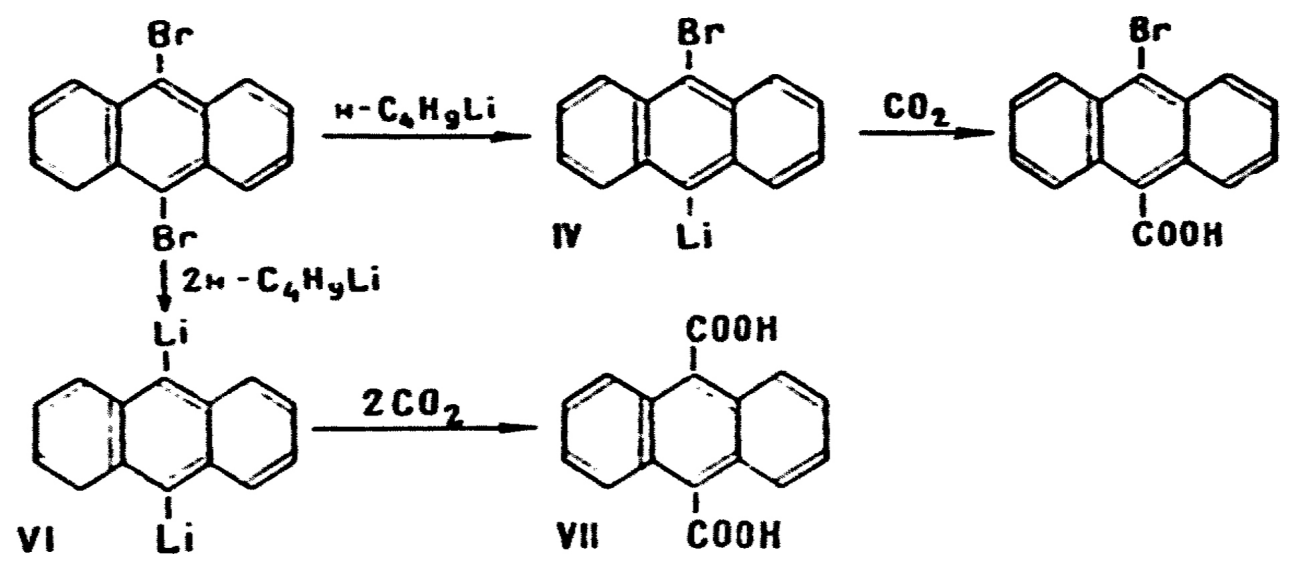

Abb. 2.6: Ausschnitt aus der von Mikhailov im Jahre 1948 veröffentlichten Arbeit zur Lithiierung von 9,10Dibromanthracen. ${ }^{[71]}$ 
Diese revolutionäre Erkenntnis blieb jedoch unbeachtet und fand bis auf einige eigene Arbeiten Mikhailovs, die er bis Mitte der 50er Jahre verfasste, keine weitere Anwendung. ${ }^{\text {[3- }}$ 77] Grund hierfür sind vermutlich die zur Anfangszeit der Lithiumchemie üblichen drastischen Reaktionsbedingungen, bei denen die Reaktionsmischungen vor der weiteren Umsetzung nicht selten über mehrere Stunden zum Rückfluss erhitzt wurden. Das in Lithiierungsreaktionen am häufigsten verwendete Lösungsmittel war zu dieser Zeit Diethylether, welches unter diesen Bedingungen zur Etherspaltung neigt, ${ }^{[78]}$ so dass nicht unerhebliche Mengen an Anthracen aus den entsprechenden Umsetzungen isoliert wurden (siehe GI. 2.11).<smiles>C=C[C@H](C)C(C)OCC</smiles>

GI. 2.11: Etherspaltung von Diethylether mit Anthryllithium unter Bildung von Anthracen.

Die Erkenntnisse Mikhailovs zu der selektiven Lithiierbarkeit standen somit nicht im Einklang mit den von ihm erzielten Ausbeuten, so dass Zweifel an seinen Folgerungen verständlich sind. Auch war das Interesse an asymmetrisch substituierten Anthracenderivaten nicht besonders ausgeprägt, was aus den ersten Anschlussarbeiten an Mikhailovs Veröffentlichungen von Anfang der 60er Jahre ${ }^{[79,80]}$ bis Mitte der 80er Jahre ${ }^{[36,81,82]}$ ersichtlich ist, da diese sich hauptsächlich auf die Dilithiierung von 9,10-Dibromanthracen konzentrierten. Spätere Arbeiten in den 90er Jahren griffen lediglich auf die Literatur der 60 er bis 80er Jahre zurück und mit dem Originalzitat zu Mikhailovs Arbeiten ging auch das Wissen um die selektive Lithiierbarkeit von 9,10-Dibromanthacen verloren. Heutzutage werden mit nur wenigen Ausnahmen monosubstituierte oder symmetrisch disubstituierte Anthracenderivate aus den entsprechenden Anthryllithiumverbindungen synthetisiert. ${ }^{[83,84]}$ Wie so oft in der Wissenschaft war es der Zufall, der die Erkenntnisse Mikhailovs im Arbeitskreis Stalke wieder zutage förderte. So wurden bei der Synthese von 9,10Bis(diphenylthiophosphoranyl)anthracen Kristalle erhalten, die neben dem erwarteten Produkt auch ein unerwartetes Nebenprodukt enthielten (Abb. 2.7).

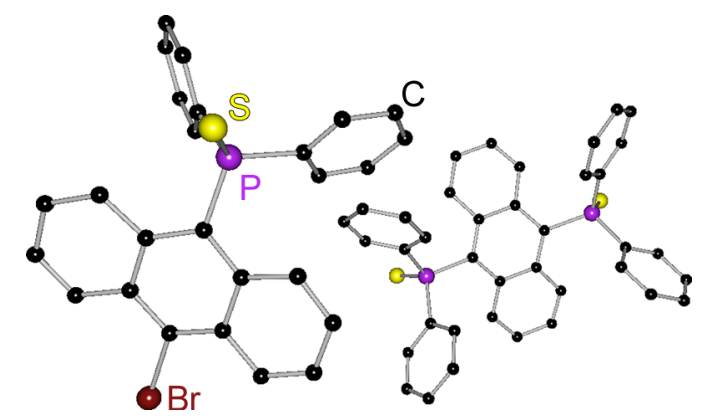

Abb. 2.7: Asymmetrische Einheit der bei der Synthese von 9,10-Bis(diphenylthiophosphoranyl)anthracen erhaltenen Molekülstruktur. 
Bei dem Nebenprodukt handelt es sich um das über Monolithiierung erhaltene 9-Brom-10diphenylthiophosphoranylanthracen, dessen Entstehung auf die Verwendung einer zu gering konzentrierten $n$ BuLi-Lösung zur Lithiierung des 9,10-Dibromanthracen zurückzuführen ist. Durch die nun offensichtliche Tatsache, dass sich bei der Monolithiierung von 9,10Dibromanthracen asymmetrisch substituierte Derivate bilden können, gelang sehr bald die erste gezielte Synthese dieser Verbindung. ${ }^{[27]}$ Durch die Synthese von weiteren 9-Brom-10phosphanylanthracenen konnte das Prinzip der selektiven Lithiierbarkeit von 9,10Dibromanthracen bestätigt werden. ${ }^{[27,85-89]}$ In Tab. 2.9 auf Seite 64 sind alle bisher im Arbeitskreis Stalke synthetisierten und durch Röntgenstrukturanalyse nachgewiesenen Phosphanylanthracene aufgelistet. Diphosphanylverbindungen mit zwei unterschiedlich substituierten Phosphanen am Anthracen, von denen ebenfalls einige röntgenkristallographisch nachgewiesen werden konnten, sind wegen ihrer schlechten Ausbeuten und ihrer fraglichen Reproduzierbarkeit nicht aufgeführt. Die zur Synthese notwendigen Arbeitsschritte sowie die strukturellen und chemischen Eigenschaften einiger in dieser Arbeit synthetisierter Phosphanylanthracene sollen in Abschnitt 2.7 besprochen werden.

Bei den durch selektive Monolithiierung zugänglichen asymmetrisch substituierten Anthracenen befindet sich ein Bromatom in 9-Position, welches Potential zu einer weiteren Derivatisierung besitzen sollte. Versuche der weiteren Derivatisierung dieser Verbindungen über die lithiierten Zwischenstufen, lieferten stets schlechte Ausbeuten und nicht reproduzierbare Ergebnisse. Die im Vergleich zu Kohlenstoff geringere Elektronegativität des Phosphoratoms könnte hier durch positiven induktiven Effekt das bromierte Kohlenstoffatom elektronisch soweit absättigen, dass dessen Elektrophilie zu sehr abgesenkt wird, um mit den eingesetzten Lithiierungsmitteln unter Lithium-Halogenaustausch zu reagieren. Zusätze von Donorbasen zur Reaktivitätssteigerung des Lithiierungsmittels führten ebenfalls nicht zu befriedigenden Ergebnissen. Auch eine Desaktivierung durch den LiX-Effekt könnte dieses Verhalten erklären und soll weiter unten genauer beleuchtet werden.

Da das angestrebte Sensorsystem jedoch ein Phosphan als Tuner am Anthracengerüst tragen soll, wurde neben weiteren Versuchen zur besseren Lithiierbarkeit (siehe Abschnitt 2.2.2 und Abschnitt 2.2.3) nach alternativen Synthesewegen geforscht, bei denen erst die Rezeptoreinheit aufgebaut wird und die Einführung des Phosphans im letzten Syntheseschritt erfolgen sollte (siehe ab Abschnitt 2.3).

\subsubsection{Reaktivitätssteigerung der Lithiumanthracene durch Substitution von Donoren und durch Deaggregation}

Lithiumorganische Reagenzien liegen in Kohlenwasserstoffen als Oligomere vor, deren Oligomerisierungsgrad die Reaktivität dieser Verbindungen maßgeblich beeinflusst. Für die beiden in der Synthese am häufigsten eingesetzten Verbindungen $n B u L i$ und tert- 
Butyllithium (tBuLi) konnte bereits frühzeitig anhand kryoskopischer und spektroskopischer Messungen der Oligomerisierungsgrad ermittelt werden. So liegt $n \mathrm{BuLi}^{[90-93]}$ als Hexamer und $t B u \mathrm{Li}^{[94-97]}$ als Tetramer in Kohlenwasserstoffen vor. Durch Ether wie z. B. Diethylether (DEE), Tetrahydrofuran (THF) oder Dimethoxyethan (DME) insbesondere aber durch tertiäre Amine wie z. B. $N, N, N^{\prime}, N^{\prime}$-Tetramethylethylendiamin (TMEDA) oder $N, N, N^{\prime}, N^{\prime}, N^{\prime \prime}$-Pentamethyldiethylentetraamin (PMDETA), können diese Oligomere aufgespalten werden, was zu einer Reaktivitätssteigerung führt. ${ }^{[98,99]}$ So lässt sich z. B. Toluol mit $n$ BuLi in benzylischer Stellung erst nach Zugabe von TMEDA deprotonieren. ${ }^{[100]}$ In der Praxis werden oft Gemische aus Donorbasen zur Reaktionsführung eingesetzt, die reaktive Spezies ist hierbei meist unbekannt und die eingesetzten Verhältnisse werden häufig empirisch ermittelt. Es wird davon ausgegangen, dass die stärkere Donorbase die gesamte Koordinationssphäre am Lithiumatom auffüllt. Lithiumorganyle, die gleichzeitig von mehreren unterschiedlichen Donorbasen koordiniert werden, sind selten und ihre Reproduzierbarkeit ist meist nicht gegeben. ${ }^{[101,102]}$

Der Oligomerisierungsgrad, welcher hauptsächlich von dem verwendeten Lösungsmittel abhängt, wird zusätzlich durch den Raumbedarf des Alkyl- bzw. Arylrestes beeinflusst und sinkt mit steigender Größe: nBuLi (Hexamer) < tBuLi (Tetramer). Bei Verwendung mehrzähniger Donorbasen lassen sich diese Aggregate weiter bis hin zum Monomer aufspalten, wie es z. B. am Phenyllithium (PhLi) deutlich wird: $[\mathrm{PhLi} \cdot\{\text { dee }\}]_{4}{ }^{[103]}>$ $[\mathrm{PhLi} \cdot\{\mathrm{tmeda}\}]_{2}{ }^{[104]}>[\mathrm{PhLi} \cdot\{\text { pmdeta }\}]_{1}{ }^{[105]}$. Bei der Kombination von schwachen einzähnigen Donorbasen wie DEE und Lithiumorganylen mit großem Raumbedarf lassen sich kleine, meist dimere Aggregate bilden, deren Lithiumatom sterisch so abgeschirmt ist, dass dessen Koordinationssphäre nur zum Teil gefüllt werden kann. Das in Abb. 2.8 dargestellte 2,4,6-Triisopropylphenyllithium, ist eines der wenigen literaturbekannten Beispiele einer solchen Kombination. ${ }^{[106]}$
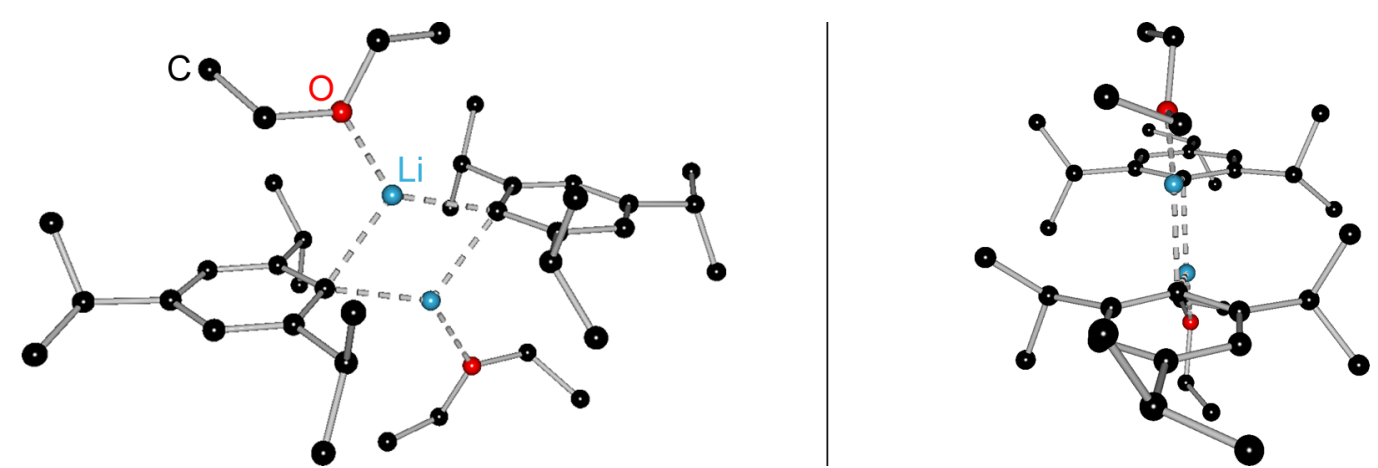

Abb. 2.8: Molekülstruktur des 2,4,6-Triisopropylphenyllithiums mit trivalenten Lithiumatomen. ${ }^{\text {[106] }}$

Durch die beiden Isopropylgruppen in ortho-Position zum lithiierten Kohlenstoffatom besitzt dieses Lithiumorganyl einen dem Anthracen ähnlichen sterischen Anspruch. Somit liegt die Vermutung nahe, dass die (mono)lithiierten Anthracene ähnlich aufgebaute dimere Aggregate bilden. 
Ausgangsmaterialien für die lithiierten Anthracene stellen deren bromierte Derivate dar, welche leicht (wie in Abschnitt 2.1 gezeigt) aus der Reaktion der einfach substituierten Anthracene mit elementarem Brom in inerten Lösungsmitteln erhalten werden können. ${ }^{[107]}$ Unter Verwendung von $n$ BuLi ließen sich alle bisher untersuchten Bromanthracene nahezu quantitativ lithiieren (GI. 2.12). ${ }^{[15,85,89]}$<smiles>[R]c1c2ccccc2c(Br)c2ccccc12</smiles><smiles>[R]c1c2ccccc2c(I)c2cc(CCCCC)ccc12</smiles>

7a: $\mathrm{R}=\mathrm{Br}, \mathbf{7 b}: \mathrm{R}=\mathrm{Me}, \mathbf{7 c}: \mathrm{R}=\mathrm{Cl}$

GI. 2.12: Lithiierung substituierter Anthracenderivate ausgehend von den jeweiligen Bromiden unter Bildung der dimeren Komplexe 7a-c.

Die so erhaltenen Lithiumaryle bilden, wie vermutet, bei Verwendung von ein- oder zweizähnigen Donorbasen stets dimere Aggregate. Wird die Lithiierung unter milden Bedingungen in Diethylether ausgeführt, bilden sich Komplexe der Zusammensetzung $\left[\mathrm{R}\left(\mathrm{C}_{14} \mathrm{H}_{8}\right) \mathrm{Li} \cdot\{\text { dee }\}\right]_{2}(\mathbf{7 a}-\mathrm{c})$. Aus Elementaranalysen sowie NMR-Experimenten zeigt sich, dass die Lithiumatome in diesen Komplexen bemerkenswerter Weise mit einer dreifachen Koordination $(2 \times \mathrm{C}, 1 \times 0)$ von der für Lithium bevorzugten vierfachen Koordination abweichen. Neben dem großen sterischen Anspruch des Anthracengerüstes ist der Raumbedarf eines Diethylethermoleküls für diese - für Lithium ungewöhnliche Koordinationsgeometrie verantwortlich. Röntgenstrukturanalysen dieser Komplexe bestätigen diese Ergebnisse und zeigen eine für dimere Lithiumorganyle ungewöhnliche Orientierung (Abb. 2.9).
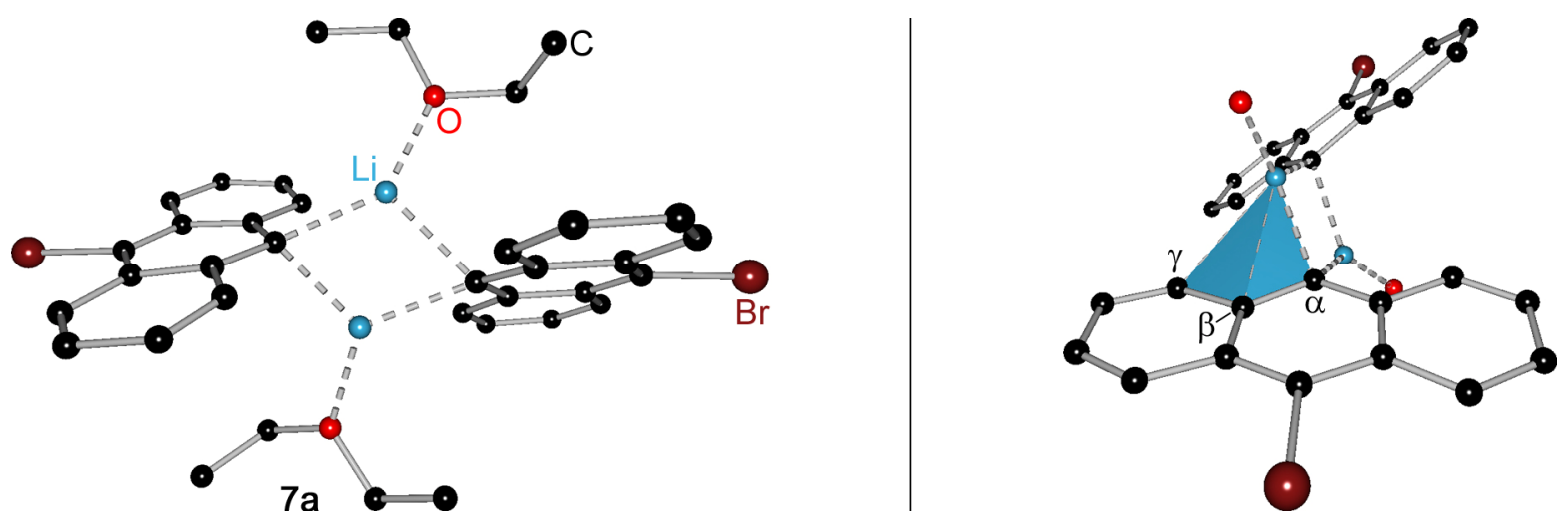

Abb. 2.9: Molekülstruktur des Komplexes 7a (links); Aufsicht auf die allylische $\eta^{3}$-Koordination des Lithiumatoms (rechts). Die Wasserstoffatome sowie die Kohlenstoffatome der Ethermoleküle rechts sind der Übersichtlichkeit halber nicht dargestellt. Ausgewählte Bindungslängen $[\AA]$ : $L i(1)-C(\alpha) 2.211(4), L i(1)-C(\beta)$

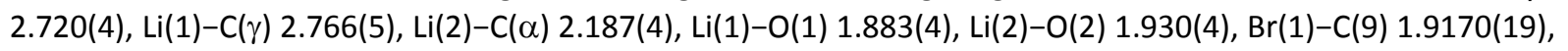
$\operatorname{Br}(2)-C(10) 1.9170(20), \varnothing \mathrm{Li}-\mathrm{C}(\alpha) 2.202, \varnothing \mathrm{Li}-0$ 1.910. 
Alle bisher untersuchten Etherkomplexe kristallisierten in der triklinen Raumgruppe $P \overline{1}$. Verbindung 7a enthält zwei kristallographisch unabhängige Dimere in der asymmetrischen Einheit. Die Anthracengerüste sind nicht wie erwartet parallel zueinander angeordnet, sondern weisen eine deutliche Torsion von 29.9(2) und $17.3(2)^{\circ}$ gegeneinander auf. Daraus resultierend ist auch der zentrale $(\mathrm{Li}-\mathrm{C})_{2}$-viergliedrige Ring nicht orthogonal zur Ebene der Anthracengerüste orientiert $\left(60.50(15)^{\circ}\right.$ und $\left.83.47(13)^{\circ}\right)$. Durch diese Stellung der Anthracengerüste wandert das Lithiumatom von der erwarteten orthogonalen Lage in eine 'Allyltasche' und wird so formal $\eta^{3}$ koordiniert (Abb. 2.9, rechts), wie es z. B. für Benzyllithium ${ }^{[108,109]}$ oder Picolyllithium ${ }^{[110]}$ bekannt ist. Der Abstand des Lithiumatoms zum $\alpha$-Kohlenstoffatom beträgt 2.211(4) $\AA$, der Abstand zum $\beta$-Kohlenstoffatom ist nur um $0.51 \AA$, der zum $\gamma$-Kohlenstoffatom um $0.56 \AA$ größer. Die Koordinationssphäre wird durch ein Diethylethermolekül auf eine Koordinationszahl von formal drei aufgefüllt. Durch den Zusatz stärkerer ein- bis zweizähniger Donorbasen kann zwar keine Deaggregation zum Monomer erwartet werden, eine Reaktivitätssteigerung sollte dennoch erfolgen. Die Koordinationsphäre des Lithiumatoms sollte sich mit kleineren einzähnigen oder zweizähnigen Donorbasen auf die Koordinationszahl vier auffüllen lassen, was zu einer besseren elektronischen Absättigung der Lithiumatome führen würde. Daraus sollten sowohl eine Geometrieänderung unter Aufgabe der $\eta^{3}$ Koordination, zu der für aromatische Lithiumorganyle erwarteten stufenweisen Anordnung, als auch eine längere $\mathrm{Li}-\mathrm{C}_{\alpha}$-Bindung resultieren. Um dieses genauer zu untersuchen, wurden Mischungen mit den Donorbasen THF, DME und TMEDA hergestellt. Bei den Ansätzen mit THF zeigte sich eine weitere, für Lithiumorganyle bisher noch nicht beobachtete Selektivität bei der Substitution von Donorbasen. Der Mechanismus des Ligandenaustausches ist in Gl. 2.13 dargestellt.

2<smiles>Brc1c2ccccc2c(Br)c2ccccc12</smiles>

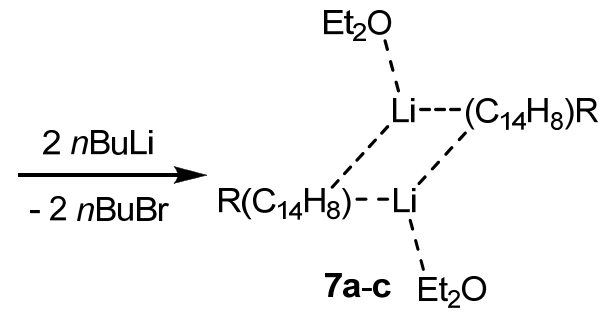

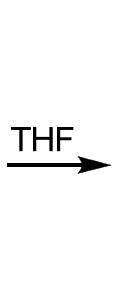

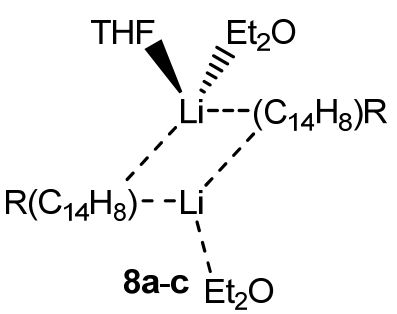

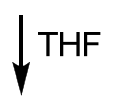

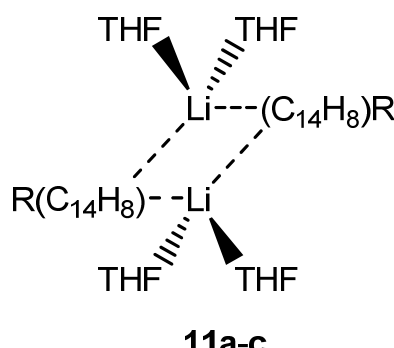
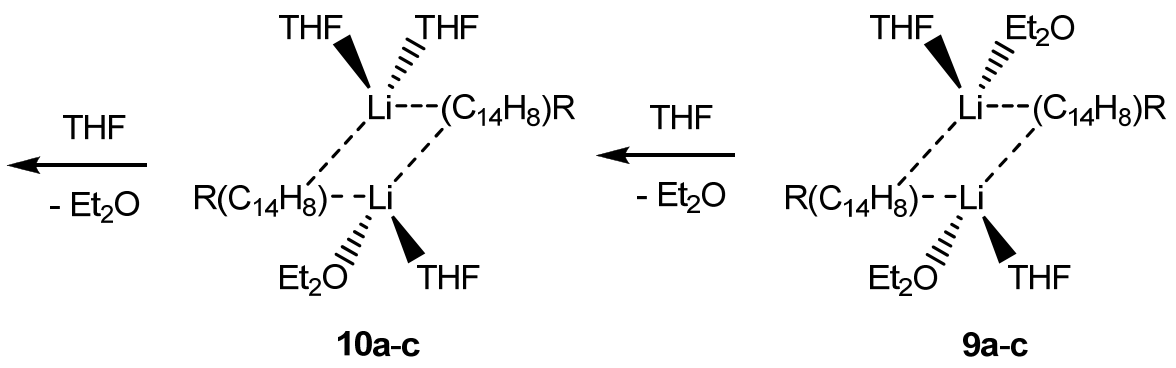

GI. 2.13: Mechanismus des stufenweisen Austausches von DEE gegen THF in den Komplexen 7a-c bis 11a-c. 
Wird genau ein Äquivalent der im Vergleich zu Diethylether stärkeren Donorbase THF zugesetzt, so wird nicht wie erwartet eine Mischung aus verschiedenen Komplexen erhalten, bei denen ein oder zwei Diethylethermoleküle ersetzt wurden, sondern es erfolgt eine selektive Addition an ein Lithiumatom. Da THF weniger Raum als Diethylether beansprucht, ist es in der Lage, die Koordinationssphäre des Lithiumatoms unter Aufgabe der $\eta^{3}$-Koordination aufzufüllen. Die resultierende Verbindung enthält nun ein drei-fach und ein vier-fach koordiniertes Lithiumatom (8). Durch Zugabe eines weiteren Äquivalents THF kann die Koordinationssphäre des zweiten Lithiumatoms unter Ausbildung eines gemischt koordinierten Komplexes (9) vervollständigt werden. Die Addition des zweiten THF Moleküls erfolgt hierbei ausschließlich trans. Erst der Zusatz von mindestens drei Äquivalenten THF führt zu einem Austausch der schwächeren Donorbase DEE und liefert den Komplex 10, in dem nun drei THF und ein Diethylether nebeneinander vorliegen. Ab vier Äquivalenten ist der Austausch komplett und es liegt der THF-Komplex 11 vor. Die ab Komplex $\mathbf{9}$ vollständig abgesättigten Lithiumatome benötigen nun keine $\pi$-Elektronendichte zur Vervollständigung ihrer Koordinationssphäre, so dass die Anthracengerüste nun wie erwartet parallel zueinander orientiert sind. Wie aus den in Abb. 2.10 gezeigten Molekülstrukturen ersichtlich wird, ist diese Umorientierung bereits ab der Addition des ersten THF unter Bildung von $8 \mathrm{zu}$ beobachten. Dieser selektive Ligandenaustausch wurde für alle bisher in diesem Arbeitskreis untersuchten lithiierten Anthracene beobachtet. In Abb. 2.10 ist jeweils ein Exemplar der Verbindungen $\mathbf{8}$ bis $\mathbf{1 1}$ abgebildet.
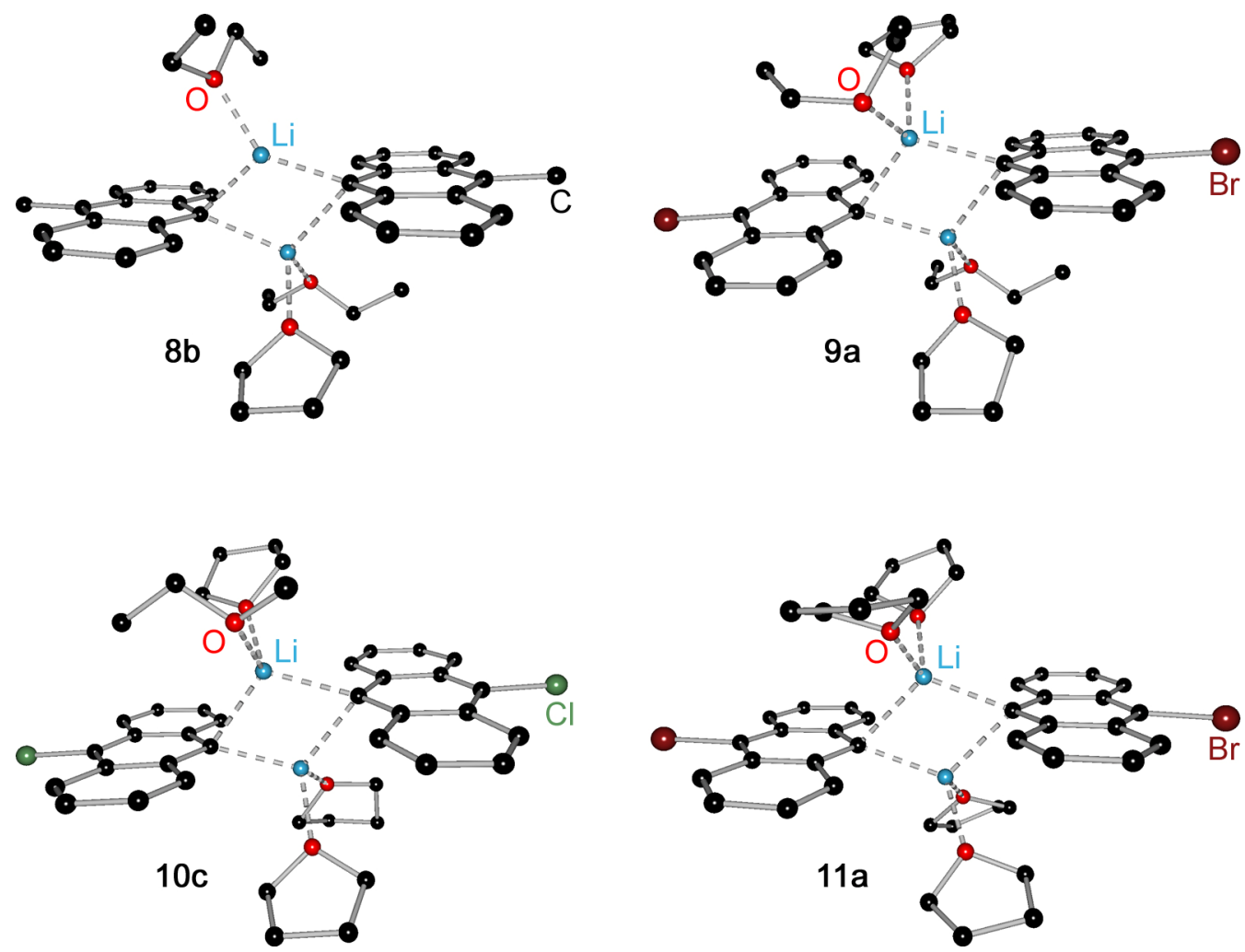

Abb. 2.10: Molekülstrukturen der Komplexe 8b, 9a, 10c und 11a. Die Wasserstoffatome sind der Übersichtlichkeit halber nicht dargestellt. Ausgewählte Bindungslängen $[\AA]$ : $\varnothing \mathrm{Li}-\mathrm{C}(\alpha) 2.276(3)(8 b), 2.318(6)$ (9a), 2.307(6) (10c), 2.305(5) (11a), øLi-O 1.992(9) (8b), 2.035(6) (9a), 2.036(6) (10c), 1.985(5) (11a). 
Der Zusatz der zweizähnigen Donorbasen DME und TMEDA liefert ebenfalls dimere Aggregate (12 und 13), in denen die Lithiumatome jeweils von einem dieser Chelatliganden koordiniert werden. Die Molekülstrukturen dieser beiden Komplexe sind in Abb. 2.11 gegenübergestellt.
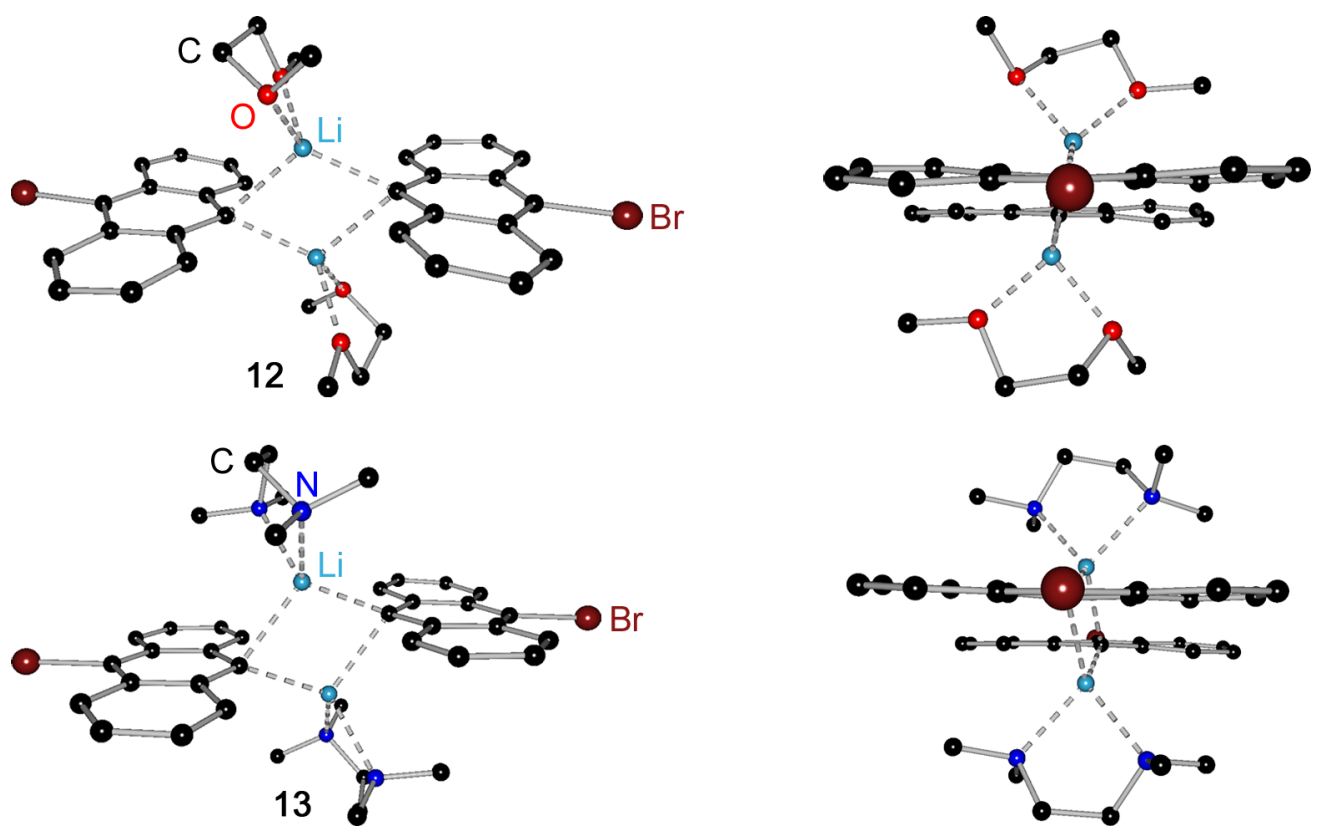

Abb. 2.11: Molekülstrukturen der dimeren Aggregate $[\mathrm{BrAnLi} \cdot\{\mathrm{dme}\}]_{2}(\mathbf{1 2}-$ links $)$ und $[\mathrm{BrAnLi} \cdot\{\mathrm{tmeda}\}]_{2}(\mathbf{1 3}-$ rechts).

Alle bisher untersuchten dimeren Lithiumanthracene enthalten zwei kurze und zwei lange $\mathrm{Li}-\mathrm{C}_{\alpha}$-Bindungsabstände, welche durch die unterschiedliche Koordination der Lithiumatome an das $\alpha$-Kohlenstoffatom zustande kommen und unterschieden werden müssen. Während ein Lithiumatom nahezu in der Ebene eines Anthracengerüstes angeordnet ist, schließt das andere einen Winkel $>50^{\circ}$ mit dieser ein. Der zentrale viergliedrige $(\mathrm{Li}-\mathrm{C})_{2}$-Ring bildet somit ein Parallelogramm. Wird näherungsweise angenommen, dass die Lithiumatome unter Bildung eines Kations ein Elektron auf das Kohlenstoffatom übertragen, sollte sich dieses im Falle von Aromaten in einem $\mathrm{sp}^{2}$-Hybridorbital befinden. Da dieses Hybridorbital orthogonal zur $\pi$-Elekronendichte des aromatischen Systems angeordnet und daher nicht delokalisiert ist, stellt dieses Kohlenstoffatom den stärksten Donor für ein Lithiumatom dar. Diese $\sigma$-Bindung sollte daher sehr stark und damit auch kurz sein, so dass das koordinierte Lithiumatom in die Aromatenebene gezwungen wird. Das zweite Lithiumatom ist oberhalb der Aromatenebene angeordnet und kann so durch das p-Orbital des lithiierten Kohlenstoffatoms koordiniert werden. Da das in diesem p-Orbital befindliche Elektron über den gesamten Aromaten delokalisiert ist, sollte diese $\pi$-Bindung deutlich schwächer und damit auch länger als die $\sigma$-Bindung sein. Die Li-C-Bindungslängen, welche ein Indiz für die Polarität der Bindungen und damit auch für die Reaktivität der gebildeten Lithiumorganyle darstellen, sind für die Komplexe $\mathbf{7 a}, \mathbf{8 b}, \mathbf{9 a}, \mathbf{1 0 c}, \mathbf{1 1 a}, \mathbf{1 2}$, und 13 in Tab. 2.5 gegenübergestellt. 
Tab. 2.5: Ausgewählte Bindungslängen der Komplexe 7a, 8b, 9a, 10c, 11a, 12,und 13.

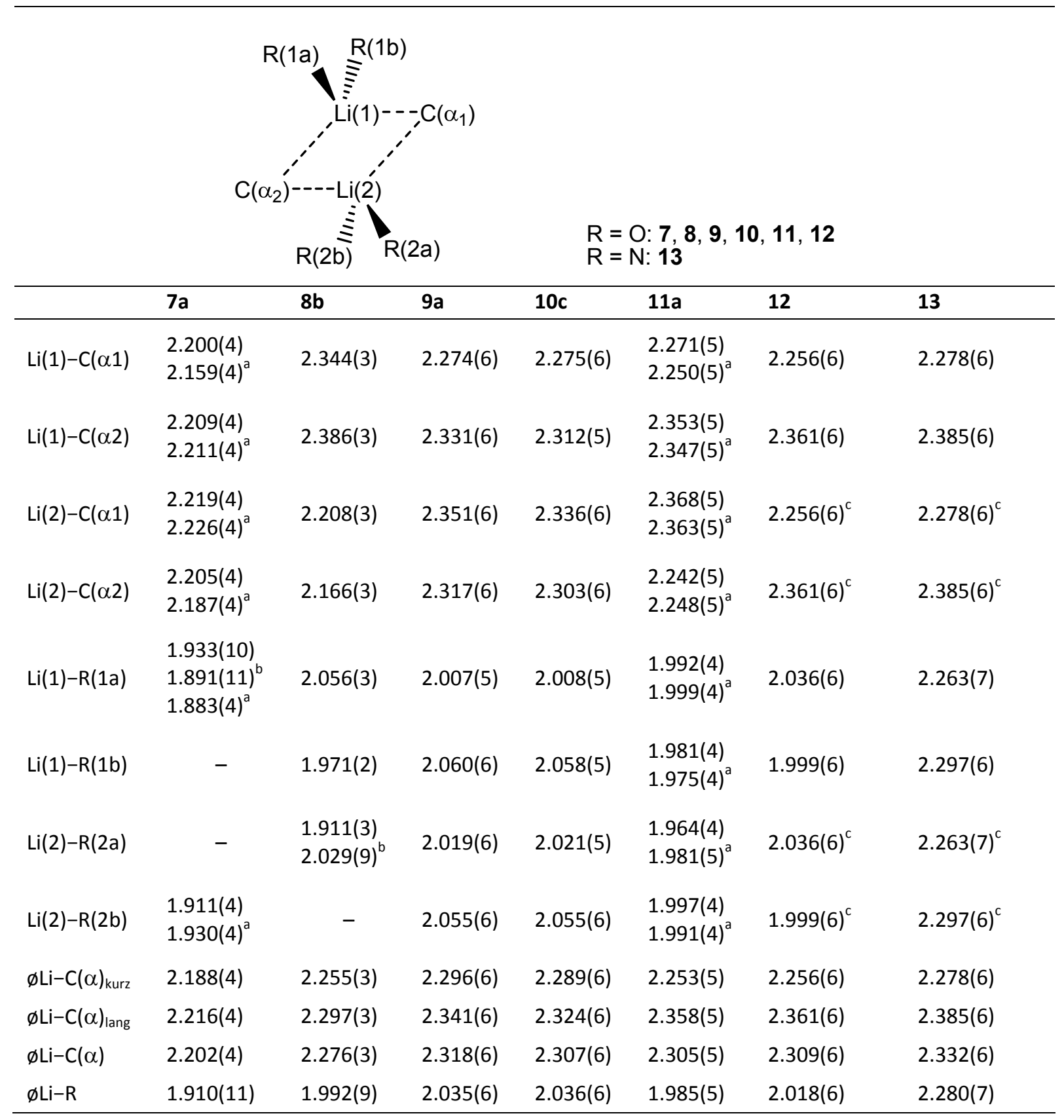

a) Zwei kristallographisch unabhängige Moleküle in der Elementarzelle, b) Fehlordnung, c) Gleiche Werte durch Symmetrie.

In den von einzähnigen Donoren komplexierten Verbindungen $\mathbf{8 b}$ bis 11 a nehmen die $\mathrm{Li}^{-} \mathrm{C}_{\alpha^{-}}$ Bindungslängen im Vergleich zu Komplex 7a zu. Hierbei sind die Bindungslängen von $\mathbf{8 b}$ und 11a sowie die von 9a und 10c innerhalb ihrer Standardabweichungen gleich lang. Überraschend ist jedoch, dass die maximale Bindungslänge bei dem von DEE und THF gemischt koordinierten Komplex 9a und nicht bei dem vollständig mit THF koordinierten Komplex 11a erreicht wird. In Abb. 2.12 sind die $\mathrm{Li}-\mathrm{C}_{\alpha}$-Bindungslängen gegen die Anzahl der im Komplex gebundenen Äquivalente THF aufgetragen. 

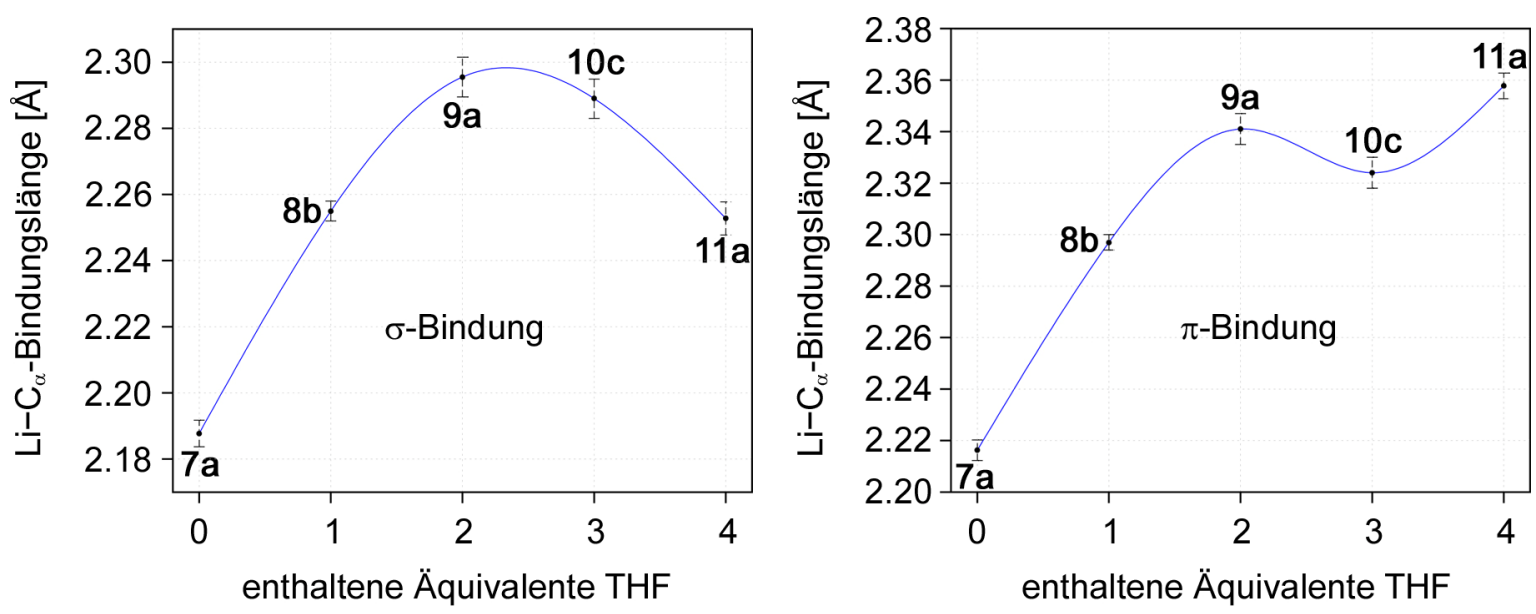

Abb. 2.12: Auftragung der $\mathrm{Li}_{-} \mathrm{C}_{\alpha}$-Bindungslängen der Komplexe 7a bis 11a gegen die im Komplex enthaltenen Äquivalente THF.

Wird - wie oben erörtert - die $\mathrm{Li}-\mathrm{C}_{\alpha}$-Bindungslänge als Indikator für die Reaktivität einer lithiumorganischen Verbindung angenommen, so lässt sich der unerwartete Schluss ziehen, dass die größte Aktivierung bei einem Zusatz von 2 bis maximal 3 Äquivalenten THF zu einer Lösung des lithiierten Anthracens in Diethylether erreicht wird. Da in Reaktionen von lithiumorganischen Reagenzien meist mit einem großen molaren Überschuss an Donorbasen gearbeitet wird, ist dieses Phänomen bisher noch nirgends beobachtet oder beschrieben worden. Es ist aber davon auszugehen, dass dieser Effekt nicht auf Anthryllithiumverbindungen beschränkt ist. So zeigt sich einmal mehr, dass die Strukturaufklärung lithiumorganischer Reagenzien neben dem Oligomerisierungsgrad auch andere wesentliche Beiträge zum Verständnis von deren Aktivierung liefern kann.

Die beiden von Chelatliganden koordinierten Verbindungen $\mathbf{1 2}$ und $\mathbf{1 3}$ verhalten sich wieder wie erwartet. In beiden sind die $\mathrm{Li}-\mathrm{C}_{\alpha}$-Bindungslängen gegenüber Verbindung $7 \mathrm{a}$ deutlich verlängert. Der TMEDA-Komplex 13 zeigt hierbei die längsten Li-C-Bindungen; hier kommt zu dem Chelateffekt noch der für das Lithiumatom bessere (weil härtere) Donor Stickstoff hinzu.

Das durch Zusatz von PMDETA erhaltene monomere Aggregat 16, dessen Molekülstruktur in Abb. 2.15 auf Seite 36 dargestellt ist und dessen Entstehung weiter unten besprochen werden soll, zeigt mit einer gemittelten Bindungslänge von 2.199(4) $\AA$ eine vergleichsweise kurze Li-C-Bindung. Das Lithiumatom dieses Aggregats befindet sich exakt in der Anthracenebene und ist in einer $\sigma$-Bindung (also ausschließlich über das $\mathrm{sp}^{2}$-Hybridorbital) gebunden. Durch diese zu den Dimeren deutlich unterschiedliche Koordination, ist ein direkter Vergleich der Bindungslängen kein aussagekräftiges Indiz für die Reaktivität dieses Komplexes. Kinetische Studien haben gezeigt, dass die Umsetzung lithiumorganischer Reagenzien stets aus monomeren Aggregaten erfolgt, welche sich durch Dissoziation in meist geringen Anteilen in Lösung bilden. Somit ist die Reaktivität monomerer Lithiumkomplexe (abgesehen von solvensseparierten lonenpaaren) als die höchste zu 
erreichende anzusehen. Die untersuchten Anthryllithiumverbindungen lassen sich in drei Reaktivitätsklassen einteilen (siehe Abb. 2.13).

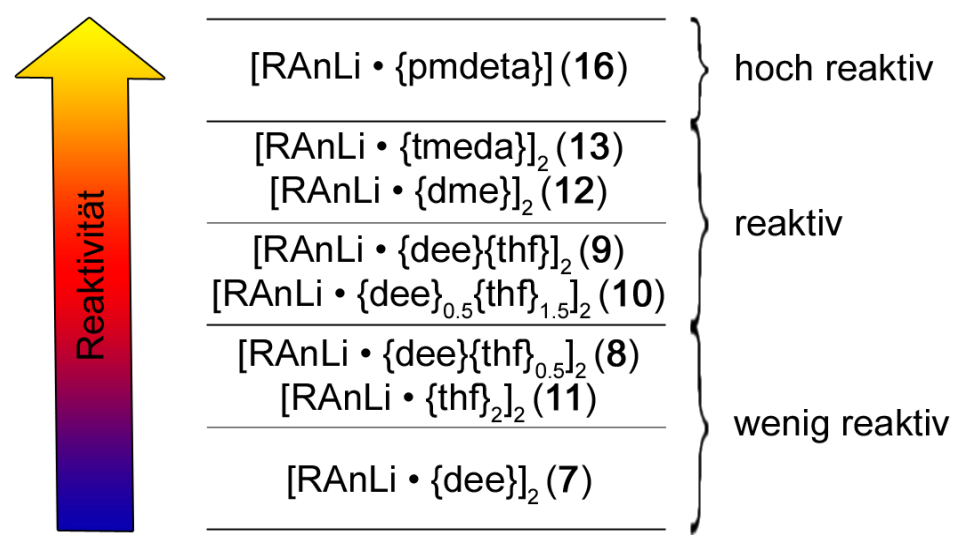

Abb. 2.13: Reaktivitätseinteilung der untersuchten Anthryllithiumverbindungen.

Dass eine Reaktivitätssteigerung durch die Zugabe von Donoren zu den Anthryllithiumlösungen in Diethylether eintritt, zeigte sich anhand von Nebenreaktionen, die zur Bildung von Lithiumbromid führen. So konnten mit Ausnahme der reinen Diethyletherkomplexe aus allen Kristallisationsansätzen Kristalle von Lithiumbromid mit den entsprechenden Donoren erhalten werden. Nach Abtrennen dieser Kristalle wurden oftmals weitere Kristalle erhalten, welche zwar die gewollte lithiumorganische Verbindung beinhalteten, jedoch in Form eines gemischten Aggregates auftraten, in welchem ein Lithiumorganyl mit einem Lithiumbromid ein gemischtes Dimer bildete. Drei dieser Verbindungen sind in Abb. 2.14 dargestellt. Die Bedeutung dieser Komplexe und deren Reaktivitäten im Vergleich zu den reinen lithiumorganischen Verbindungen wird in Abschnitt 2.2.3 besprochen werden.
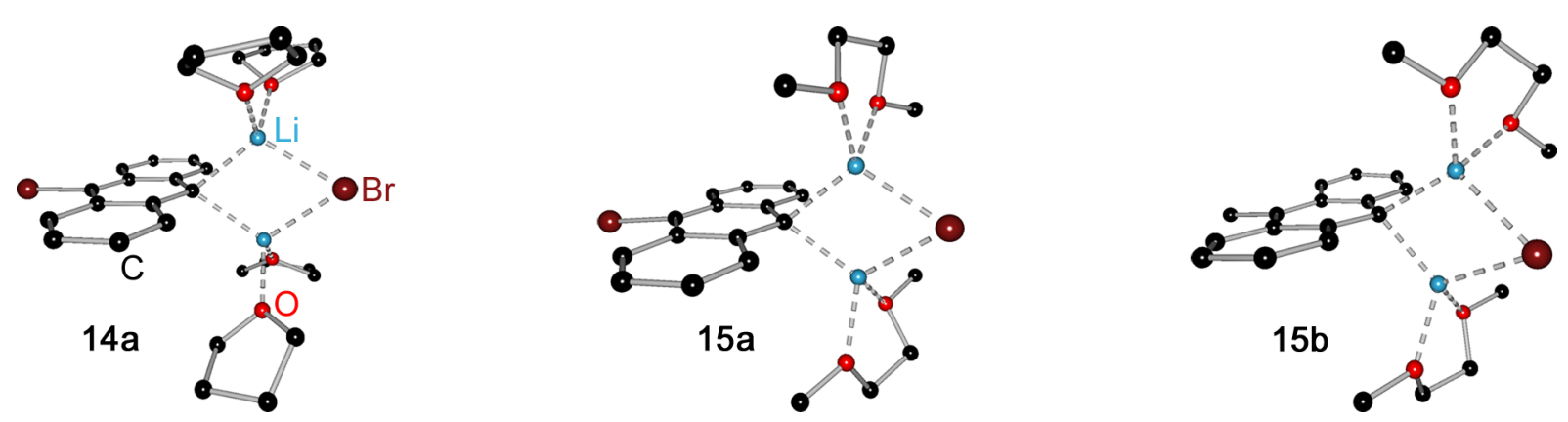

Abb. 2.14: Molekülstrukturen der erhaltenen gemischten Aggregate von Lithiumanthracenen mit Lithiumbromid.

Bei den 9-Brom-10-anthryllithiumverbindungen gibt es zwei mögliche Reaktionswege, die zu einer Bildung von Lithiumbromid führen könnten (Gl. 2.14): Zum Einen könnte Anthryllithium mit noch nicht lithiiertem 9,10-Dibromanthracen oder dem monolithiierten 9-Brom-10-anthryllithium unter C-C-Bindungsknüpfung reagieren, zum Anderen könnte unter Bildung butyllierter Anthracene das 9-Brom-10-anthryllithium mit dem aus der 
Lithiierung mit $n$ BuLi entstehenden $n$-Brombutan $(n \mathrm{BuBr})$ reagieren. Beide Reaktionen würden ein Äquivalent Lithiumbromid erzeugen, welches die Bildung der Salzkomplexe sowie der gemischten Aggregate erklären würde.<smiles>CCCCCc1c2ccccc2c(Br)c2ccccc12</smiles>

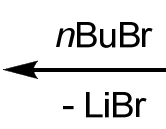<smiles>Clc1c2ccccc2c(Br)c2ccccc12</smiles>

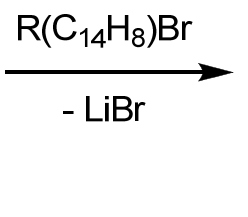<smiles>[R]c1c2ccccc2c(-c2c3ccccc3c(Br)c3ccccc23)c2ccccc12</smiles>

Gl. 2.14: Mögliche Reaktionswege zur Bildung von LiBr aus 9-Brom-10-anthryllithiumverbindungen.

Um die Quelle des Lithiumbromids eindeutig nachzuweisen, wurden mehrere Experimente durchgeführt.

1) Das Bromatom in 9-Position wurde durch eine Methylgruppe substituiert, so dass bei Auftreten von Lithiumbromidkomplexen eine C-C-Bindungsknüpfung zweier Anthracene ausgeschlossen werden konnte.

2) Das bei der Lithiierung entstehende $n$-Brombutan wurde vor der Zugabe der Donoren abgetrennt, so dass nur lithiumbromidfreie Komplexe über Röntgenstrukturanalysen erhalten werden sollten, wenn dieses die Quelle des gebildeten Lithiumbromids ist.

3) Ein Kristallisationsansatz mit PMDETA, bei dem das $n$-Brombutan nicht abgetrennt und welcher länger als vier Wochen bei $-30^{\circ} \mathrm{C}$ gelagert wurde, sollte nach Hydrolyse mittels NMR-Spektroskopie und Massenspektrometrie auf die möglichen Nebenprodukte hin untersucht werden.

Alle drei Experimente führten zum selben Ergebnis und identifizierten das, bei der Lithiierung von Bromanthracenen äquimolar entstehende, $n$-Brombutan als die Quelle des gebildeten Lithiumbromids. So konnte auch bei der Lithiierung von 9-Brom-10methylanthracen die Bildung gemischter Aggregate nachgewiesen werden (siehe Abb. 2.14 rechts). Auch die dimeren Aggregate der chelatisierenden Donoren TMEDA und DME konnten erst nach Abtrennen des $n$-Brombutans isoliert und strukturell nachgewiesen werden. Den letztendlichen Beweis lieferten die angefertigten NMR- und Massenspektren, in denen butylierte Anthracene eindeutig nachgewiesen werden konnten. ${ }^{[111]}$

Das Abtrennen des gebildeten $n$-Brombutans kann lediglich aus den reinen Diethyletherkomplexen erfolgen, da diese Komplexe auch nach mehreren Monaten keine Reaktion mit $n$-Brombutan eingingen. Hierzu wird die Reaktionslösung auf ein Drittel des Volumens eingeengt und anschließend auf $-78^{\circ} \mathrm{C}$ heruntergekühlt. Der ausgefallene Diethyletherkomplex kann nun abfiltriert werden. Der erhaltene Feststoff ist zwar pyrophor, 
lässt sich aber unter Stickstoffatmosphäre auch außerhalb der Argontrockenbox gut handhaben. Nach 30 Min. Trocknen im Vakuum, bei dem kein Verlust des koordinierten Diethylethers beobachtet wurde, ist der hellgelbe Feststoff feinpulverig und lässt sich zur weiteren Reaktionsführung leicht umfüllen. Es empfiehlt sich jedoch den Feststoff vor der Verwendung mit einem unpolaren Lösungsmittel wie Hexan oder Pentan zu waschen, da ansonsten noch geringe Anteile des im Filterkuchen eingeschlossenen $n$-Brombutans mitgeführt werden. So konnten aus dilithiiertem Anthracen, welches nicht gewaschen wurde, das 9-Butyl-10-anthryllithium (16) und 9,10-Dibutylanthracen (17) isoliert und röntgenkristallographisch nachgewiesen werden (Abb. 2.15).
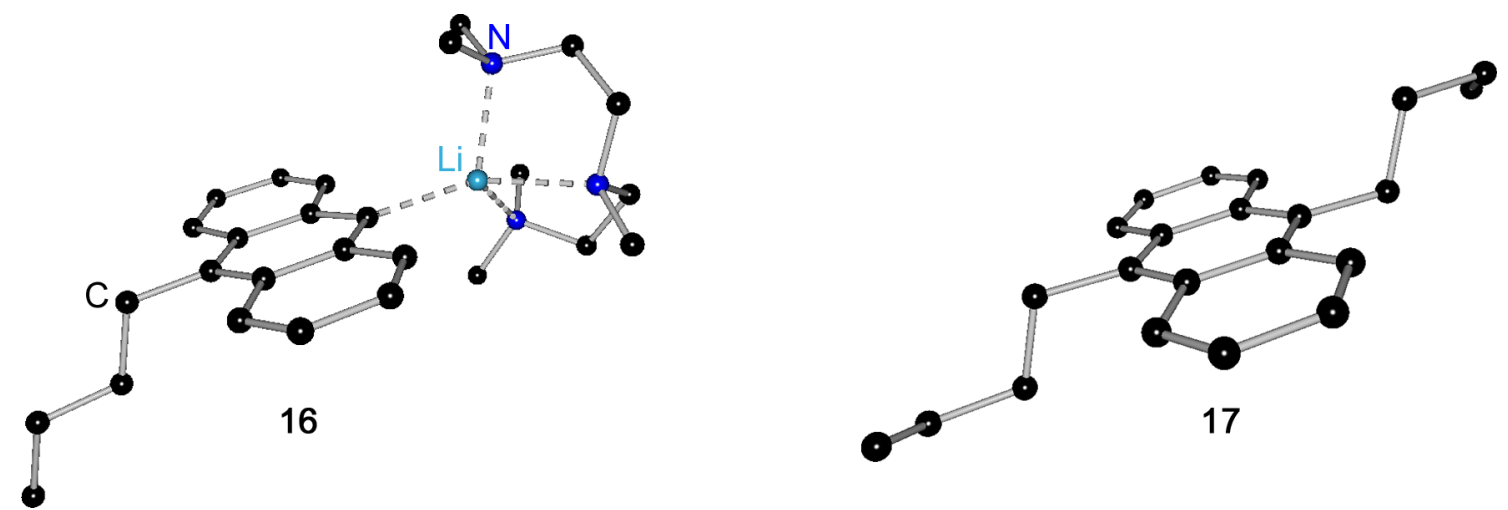

Abb. 2.15: Molekülstrukturen der bei der Dilithiierung von 9,10-Dibromanthracen mit $n$ BuLi möglichen Nebenprodukte 9-Butyl-10-lithioanthracen (16 - links) und 9,10-Dibutylanthracens (17 - rechts).

Zusammenfassend lässt sich sagen, dass die Reaktivität lithiierter Anthracene (wie in Abb. 2.13 dargestellt) in einem breiten Spektrum eingestellt werden kann. Neben der klassischen Methode der Deaggregation (hier lediglich vom Dimer zum Monomer) konnte gezeigt werden, dass auch ein Austausch der Donoren (bzw. deren Addition an den Diethyletherkomplex 7) mit einer Reaktivitätssteigerung einhergeht. Die beobachtete Nebenreaktion der lithiierten Anthracene mit dem $n$-Brombutan kann bei Bedarf durch dessen Abtrennung verhindert werden, sollte jedoch bei in situ Umsetzungen kein Problem darstellen, da die eingesetzten Reaktanden in der Regel die besseren Elektrophile sind.

Die gemischten Aggregate von Anthryllithiumverbindungen mit Lithiumsalzen, die hier nur durch Nebenreaktionen auftraten, sollten sich auch durch gezielte Zugabe von Lithiumsalzlösungen synthetisieren lassen. Die Reaktivität und der Nutzen dieser Verbindungsklasse soll im nächsten Abschnitt besprochen werden.

\subsubsection{Der LiX-Effekt in lithiierten Anthracenen}

Wie im vorherigen Abschnitt gezeigt, kann durch die Wahl des Lithiumorganyls sowie durch die Möglichkeit der Deaggregation, die Reaktivität von Lithiierungsmitteln in einem breiten Bereich eingestellt werden. Mit den optimalen Reaktionsbedingungen lassen sich höhere Ausbeuten auf der einen und bessere Selektivitäten auf der anderen Seite realisieren. 
Bei der Umsetzung der lithiierten Zwischenstufe (wie hier das Anthryllithium), welches meist in situ geschieht, muss sich auf die Deaggregation beschränkt werden. Gerade bei Umsetzungen mit Stoffen, welche mehrere funktionelle Gruppen ähnlicher Reaktivität aufweisen mit denen das Lithiumorganyl umgesetzt weden soll, bietet die Wahl des donierenden Lösungsmittels oftmals nicht genügend Spielraum in der Einstellung der nötigen Selektivität. Wenn keine anderen Synthesewege zur Anwendung kommen, müssen schlechte Ausbeuten und langwierige Aufarbeitungen in Kauf genommen werden.

Gemischte Komplexe von Lithiumorganylen mit Lithiumsalzen, wie sie in Abschnitt 2.2.2 auf Seite 34 gezeigt wurden, könnten hier einen wesentlichen Beitrag zur Verbesserung der Selektivität leisten und sollen in diesem Abschnitt näher untersucht werden.

Die Substanzklassen der Lithiumamide und Lithiumalkoxide zeigen ein zu den Lithiumorganylen analoges Aggregationsverhalten. ${ }^{[112]}$ Während die Oligomerisierung und Deaggregation bei den Lithiumorganylen bisher nur für eine Handvoll Derivate untersucht wurde, ist dieses Feld bei den Lithiumamiden erschöpfend behandelt worden. Der Aufbau der gebildeten Oligomere in verschiedensten Lösungsmitteln sowie deren Deaggregation durch den Zusatz von Donorbasen wurde neben intensiven NMR-Studien auch anhand zahlreicher Festkörperstrukturanalysen und quantenmechanischen Berechnungen aufgezeigt. ${ }^{[113]}$ Als ein weiterer wesentlicher Aspekt bei der Einstellung der Reaktivität und Selektivität der Lithiumamide hat sich der Zusatz von Lithiumsalzen erwiesen. ${ }^{[114,115]}$ So kann aus dem dimer auftretenden Lithiumdiisopropylamin (LDA) durch den Zusatz von Lithiumchlorid ein gemischtes Aggregat erhalten werden (GI. 2.15), dessen Potential bei der enantioselektiven Bildung von Enolaten nicht nur früh erkannt wurde, sondern auch schon seit Generationen Einzug in einschlägige Lehrbücher gehalten hat.

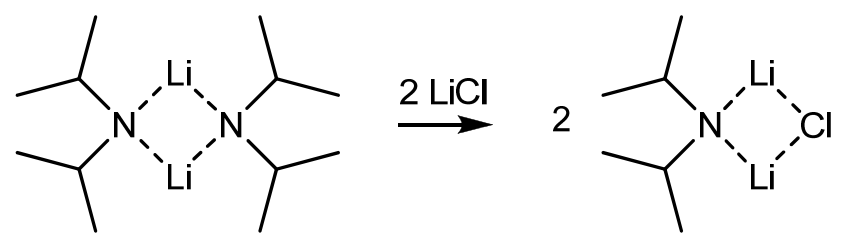

GI. 2.15: Bildung von gemischten Komplexen von Lithiumamiden und Lithiumchlorid am Beispiel LDA.

Auch für Lithiumorganyle wurde schon früh das Auftreten solcher gemischten Aggregate vermutet. ${ }^{[116,117]}$ So wurde das Einbüßen der Reaktivität von $n$ BuLi und PhLi auf die Bildung von Mischverbindungen mit Lithiumhalogeniden zurückgeführt. ${ }^{[116,118]}$ Die damals noch nicht kommerziell erhältlichen Lithiierungsreagenzien wurden stets aus den entsprechenden Halogenalkanen und -arylen (meist $\mathrm{Cl}$ oder $\mathrm{Br}$ ) dargestellt. Das Abtrennen von nicht reagiertem Lithium sowie der ausgefallenen Menge des in der Synthese stöchiometrisch gebildeten Lithiumsalzes stellte lange Zeit den einzigen Aufarbeitungsschritt dar, so dass Lithiumhalogenidkonzentrationen von 5-8 \% keine Seltenheit waren. Auch erste kommerziell erhältliche Produkte wurden als 'halogenhaltig' vertrieben. Nach der Zugänglichkeit 'salzfreier' Lithiumorganyle aus Diorganylquecksilberverbindungen und der Erkenntnis, dass 
diese salzfreien Lithiumorganyle eine gegenüber den salzhaltigen Organylen gesteigerte Reaktivität aufweisen, wurde der Lithiumsalzgehalt als ein Problem angesehen. Schon bald zeigten Untersuchungen, dass die Bildung von Mischverbindungen kein vereinzeltes Phänomen war, so dass davon ausgegangen wurde, dass die Desaktivierung durch Komplexbildung mit Lithiumhalogeniden von allgemeiner Natur sei. ${ }^{[19]}$ Mit der Verbesserung der Kryotechnik konnten mit $\left[\mathrm{Ph}_{3} \mathrm{Li}_{4} \mathrm{Br} \cdot\{\text { dee }\}_{3}\right]^{[103]}$ und $\left[\mathrm{Cy}_{2} \mathrm{Li}_{4} \mathrm{Br}_{2} \cdot\{\text { dee }\}_{4}\right]^{[120]}$ (mit $\mathrm{Cy}=\mathrm{Cyclopropyl)}$ erstmals zwei Mischverbindungen eindeutig strukturell untersucht werden. Durch Verbesserung der industriellen Prozesse zur Darstellung von lithiumorganischen Verbindungen, konnten diese später als 'salzfrei' vertrieben werden und lösten schon bald die 'salzhaltigen' Lithiumorganyle ab, so dass diese heutzutage nicht mehr vertrieben werden. Obwohl das Auftreten von Mischverbindungen seither in Vergessenheit geraten zu sein scheint und dieser Verbindungsklasse keine weitere Bedeutung beigemessen wird, stellt die Bildung von gemischten Aggregaten aus Lithiumorganylen und Lithiumhalogeniden einen wesentlichen Aspekt der lithiumorganischen Chemie dar. Dies wird besonders deutlich, wenn man sich vor Augen führt, dass bei der Umsetzung von Lithiumorganylen mit Halogenalkanen oder -arylen äquimolare Mengen an Lithiumsalzen gebildet werden (GI. 2.16).
a) $\mathrm{R}-\mathrm{Li}+$
$\mathrm{R}^{\prime}-\mathrm{X}$
$R-R^{\prime}$
LiX
$+$
$\mathrm{R}_{3}{ }_{3} \mathrm{Si}-\mathrm{X}$
$\mathrm{R}-\mathrm{SiR}_{3}{ }_{3}+$
LiX
c) $\mathrm{R}-\mathrm{Li}$
$\mathrm{R}_{2} \mathrm{P}-\mathrm{X}$
$\longrightarrow \quad \mathrm{R}-\mathrm{PR}_{2}$
LiX

Gl. 2.16: Bildung von Lithiumhalogeniden bei der Umsetzung von lithiumorganischen Verbindungen mit a) Halogenalkanen / arylen, b) Halogensilanen und c) Halogenphosphanen.

Dieser Effekt ist jedoch nicht auf die C-C-Bindungsknüpfung beschränkt und tritt bei der Umsetzung von Lithiumorganylen (z. B. mit Chlorphosphanen u. Chlorsilanen) in einer Vielzahl von gängigen Reaktionen auf. Die Lithiumsalzkonzentrationen steigen im Reaktionsverlauf linear zum Umsatz an, so dass es zu einer Selbsthemmung der Reaktion durch die Bildung gemischter Aggregate kommt.

In dieser Arbeit sollen neue Erkenntnisse über die Reaktivität gemischter Aggregate durch den Vergleich der Li-C-Bindungslängen der hier erhaltenen lithiumbromidhaltigen Anthryllithiumderivate mit den korrespondierenden lithiumbromidfreien Verbindungen gefunden werden. Unter Einbeziehung der zwei bereits beschriebenen Mischverbindungen, soll eine allgemeingültige Aussage zu der Reaktivität dieser bisher nur ungenügend erforschten Substanzklasse getroffen werden.

1983 wurden von Power et al. $\left(\left[\mathrm{Ph}_{3} \mathrm{Li}_{4} \mathrm{Br} \cdot\{\text { dee }\}_{3}\right]\right)$ und Schmidbaur et al. $\left(\left[\mathrm{Cy}_{2} \mathrm{Li}_{4} \mathrm{Br}_{2} \cdot\{\text { dee }\}_{4}\right]\right)$ unabhängig voneinander die beiden bis heute einzigen, strukturell nachgewiesenen Lithiumorganyle mit Lithiumsalzen ( $\mathrm{LiBr}$ ) beschrieben. Sowohl Power als auch Schmidbaur 
isolierten ihre gemischten Aggregate aus Lösungen, die sie durch die Direktsynthese der Lithiumorganyle erhielten. Die Molekülstrukturen beider Verbindungen sind in Abb. 2.16 dargestellt.
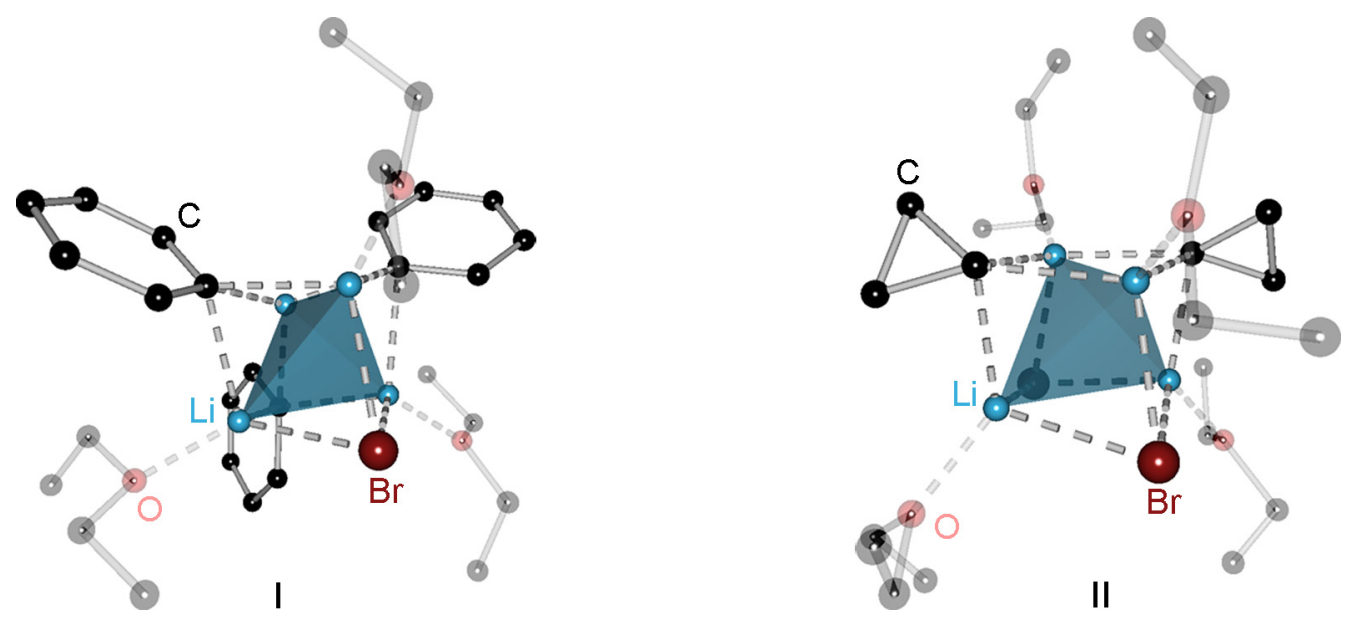

Abb. 2.16: Molekülstrukturen der gemischten Aggregate $\left[\mathrm{Ph}_{3} \mathrm{Li}_{4} \mathrm{Br} \cdot\{\text { dee }\}_{3}\right]$ (I) und $\left[\mathrm{Cy}_{2} \mathrm{Li}_{4} \mathrm{Br}_{2} \cdot\{\text { dee }\}_{4}\right]$ (II). Wasserstoffatome wurden der Übersichtlichkeits halber weggelassen und die Diethylethermoleküle transparent dargestellt.

Beide Strukturen wurden aus Diethylether kristallisiert und bilden daher wie die meisten Lithiumorganyle mit 'kleinen' organischen Resten tetramere Aggregate. ${ }^{[121-125]}$ Das typische Strukturmotiv von zwei sich durchdringenden Tetraedern ist auch in diesen gemischten Derivaten zu beobachten, jedoch wurden ein (I) bzw. zwei (II) organische Reste gegen Bromidionen substituiert, so dass es zu deutlichen geometrischen Verzerrungen kommt, die so in anderen tetraedrischen Lithiumorganylen nicht $\mathrm{zu}$ beobachten sind. Ein direkter Vergleich des gemischten Derivates I mit dem ebenfalls von Power et al. beschriebenen reinen Phenyllithium $\left([\mathrm{PhLi} \cdot\{\text { dee }\}]_{4}-\mathbf{l b}\right)^{[103]}$ zeigt, dass die durch Brom substituierte Ecke des $\mathrm{C}_{3} \mathrm{Br}$-Tetraeders durch den größeren Kovalenzradius herausgezogen wird, was auch eine deutliche Aufweitung der Winkel (die entsprechenden Winkelsummen sind: $\Sigma \mathrm{Li}(1)=341.0$ in I und $\Sigma \mathrm{Li}(1)=324.6$ in $\mathbf{~ l b}$ ) am gegenüberliegenden Lithiumatom zur Folge hat. Wie aus Tab. 2.6 ersichtlich, verkürzen sich die Li-C-Bindungslängen um durchschnittliche $0.09 \AA$, was auf eine geringere Reaktivität schließen lässt. Power macht diesbezüglich zwar keine Angaben, jedoch deckt sich diese Vermutung mit früheren Berichten über gemischte Aggregate des Phenyllithiums mit Lithiumhalogeniden. In II sind zwei Cyclopropylreste gegen Bromidionen substituiert. Von den zwei möglichen Strukturisomeren wurde hier nur das mit $\mathrm{C}_{2}$-Symmetrie, bei dem die Bromidionen auf einer Würfelfläche gegenüberliegend angeordnet sind, beobachtet. Da die Molekülstruktur für reines Cyclopropyllithium nicht bekannt ist, sollten zum Vergleich mit II andere Alkyllithiumverbindungen herangezogen werden. Es zeigte sich jedoch, dass keine geeigneten Daten mit vergleichbaren Bedingungen (Raumtemperatur, ähnlicher sterischer Anspruch des Organylrestes sowie Diethylether als donierendes Lösungsmittel) vorhanden sind. Auch zur Reaktivität des gemischten Aggregates II im Vergleich zum reinen Cyclopropyllithium kann keine Aussage getätigt werden. Zwar 
beschreibt Schmidbaur das von inm synthetisierte gemischte Aggregat als pyrophor, kann jedoch aus Ermangelung an salzfreiem kristallinem Material keine Vergleiche zum reinen Cyclopropyllithium ziehen.

Tab. 2.6: Ausgewählte Bindungslängen und Winkel in I, Ib und II.

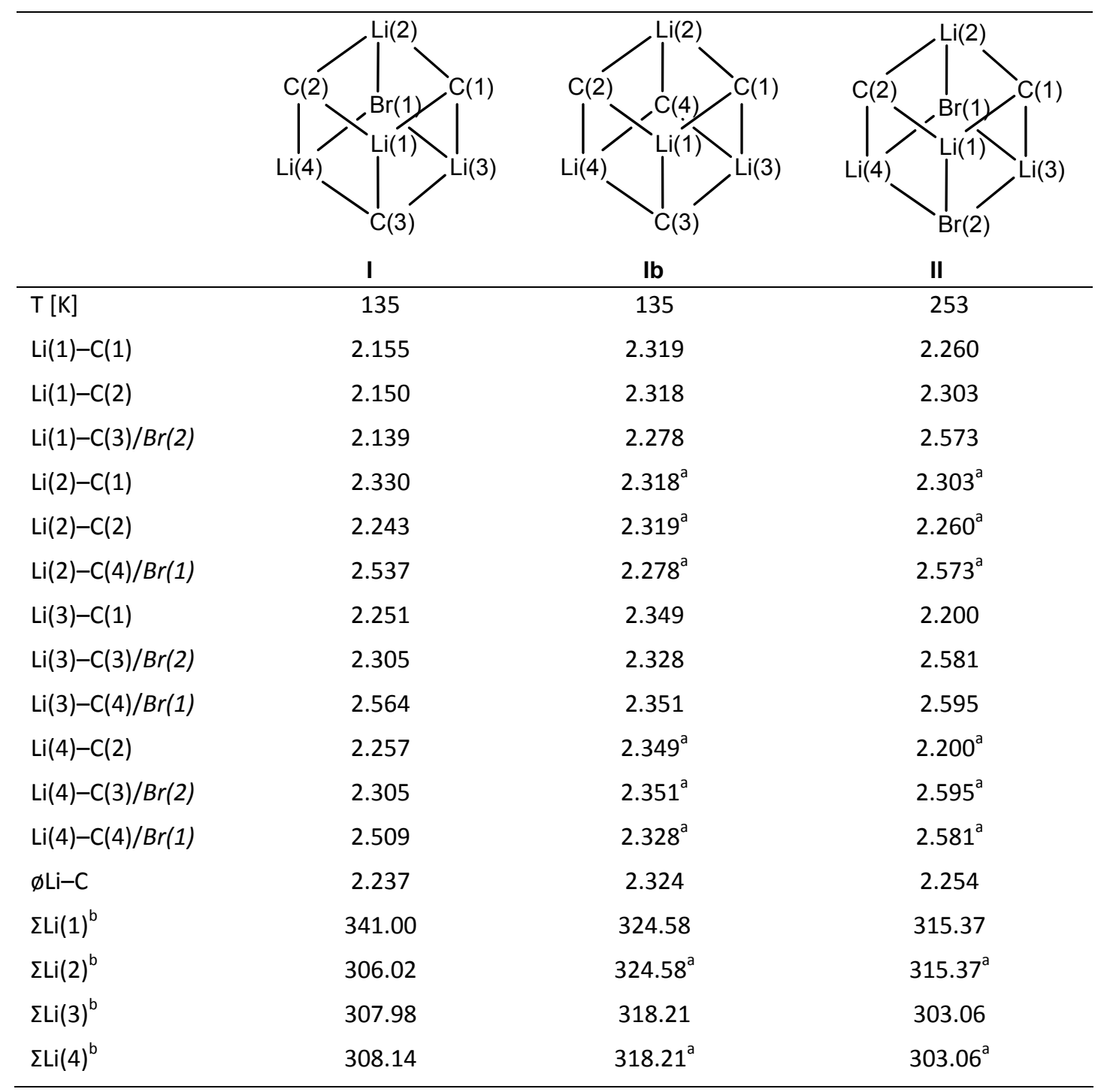

a) identische Werte durch Symmetrie; b) $\Sigma L i=$ Winkelsumme.

Weitere Informationen über den Einfluss von Lithiumsalzen auf die Struktur und Reaktivität von Lithiumorganylen können aus den in dieser Arbeit erhaltenen gemischten Aggregaten gewonnen werden. Um jedoch Vergleiche anstellen zu können, mussten die lithiumhalogenidfreien Komplexe synthetisiert und kristallisiert werden. Wie bereits in früheren Arbeiten des Arbeitkreis Stalke gezeigt wurde, ${ }^{[15,89,126]}$ lassen sich aus bromierten Anthracenen, welche leicht aus der Reaktion der einfach substituierten Anthracene mit elementarem Brom in inerten Lösungsmitteln erhalten werden können, ${ }^{[107]}$ monolithiierte Anthracene erhalten. Unter Verwendung von $n$ BuLi ließen sich bisher alle untersuchten Bromanthracene nahezu quantitativ zu den entsprechenden Lithiumanthracenen umsetzen (GI. 2.17). 


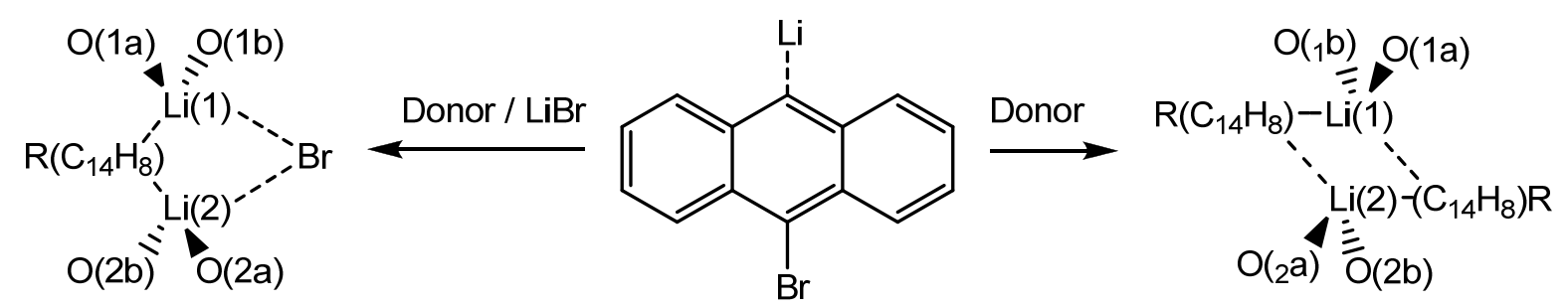

GI. 2.17: Aggregation von lithiierten Anthracenderivaten mit und ohne LiBr, ausgehend von 9-Brom-10-anthryllithium.

Die so erhaltenen Lithiumaryle bilden bei Verwendung von ein- oder zweizähnigen Donorbasen stets dimere Aggregate der Zusammensetzung $\left[R\left(\mathrm{C}_{14} \mathrm{H}_{8}\right) \mathrm{Li} \cdot\{\text { Donor }\}_{n}\right]_{2}$ mit $\mathrm{n}=1$ oder 2 aus. Nach Abtrennen des in der Reaktion gebildeten $n \mathrm{BuBr}$ durch Isolieren und Waschen (Hexan) lassen sich die Etherkomplexe mit der Zusammensetzung $\left[\mathrm{R}\left(\mathrm{C}_{14} \mathrm{H}_{8}\right) \mathrm{Li} \cdot\{\text { dee }\}\right]_{2}$ erhalten.

Werden diese Komplexe wieder in Lösung gebracht und mit den Donorbasen THF oder DME versetzt, werden die dimeren Aggregate $\left[R\left(\mathrm{C}_{14} \mathrm{H}_{8}\right) \mathrm{Li} \cdot\{\text { thf }\}_{2}\right]_{2}(\mathbf{1 1})$ und $\left[\mathrm{R}\left(\mathrm{C}_{14} \mathrm{H}_{8}\right) \mathrm{Li} \cdot\{\mathrm{dme}\}\right]_{2}$ (12) erhalten. Wird das $n$-Brombutan nicht abgetrennt, kommt es wie in Abschnitt 2.2.2 beschrieben zur Bildung von $\mathrm{LiBr}$, so dass die gemischten Aggregate $\left[\mathrm{R}\left(\mathrm{C}_{14} \mathrm{H}_{8}\right) \mathrm{Li} \cdot \mathrm{LiBr} \cdot\{\mathrm{thf}\}_{4}\right]$ (15) und $\left[\mathrm{R}\left(\mathrm{C}_{14} \mathrm{H}_{8}\right) \mathrm{Li} \cdot \mathrm{LiBr} \cdot\{\mathrm{dme}\}_{2}\right](14)$ erhalten werden. Diese Aggregate sollten sich auch durch Zusatz eines Äquivalents Lithiumbromid (in Form einer Maßlösung in THF) erhalten lassen. Die Molekülstrukturen der Verbindungen 11, 12, 14 und 15 sind in Abb. 2.17 dargestellt.
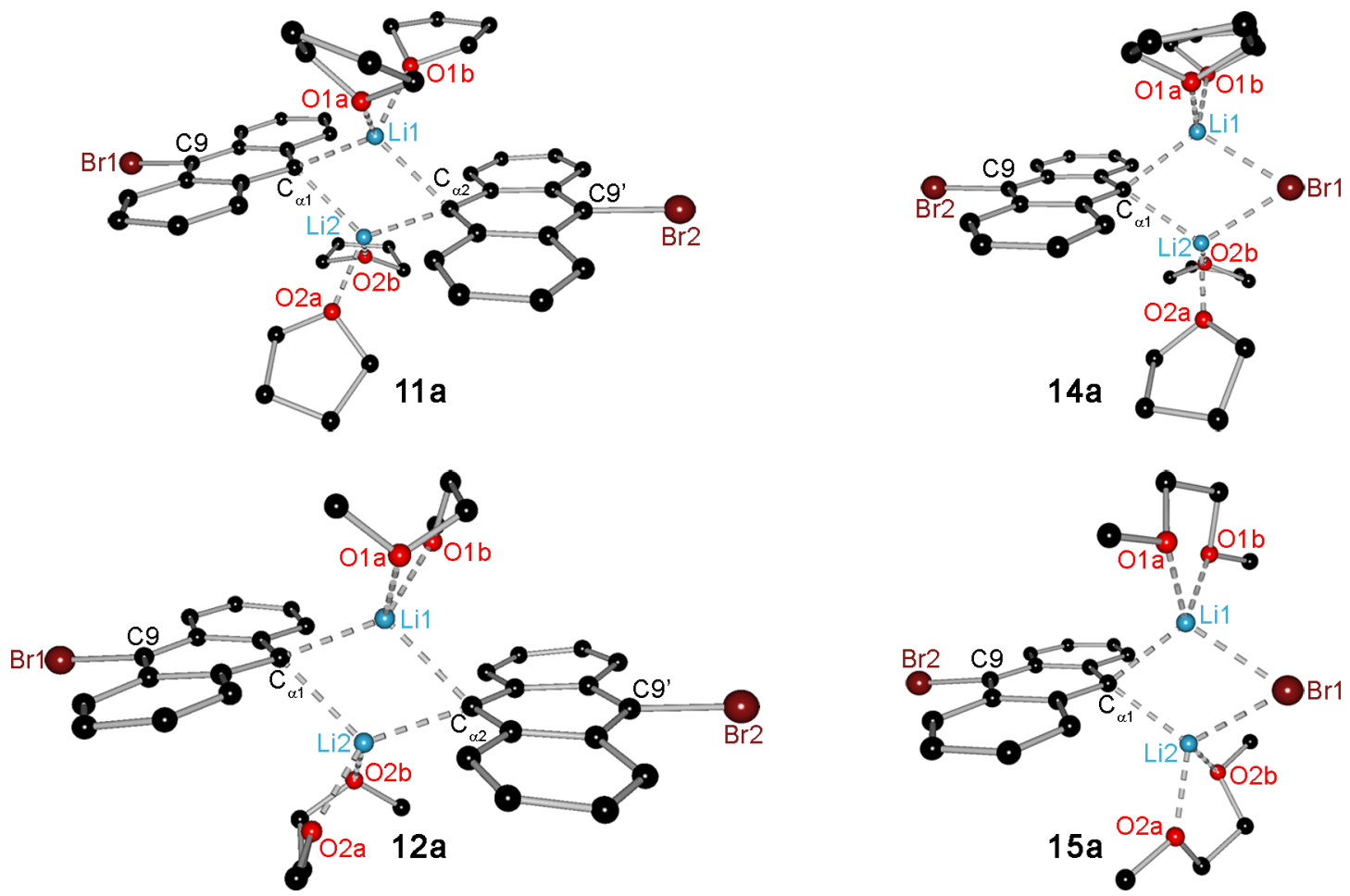

Abb. 2.17: Molekülstrukturen der Komplexe 11a, 12a, 14a und 15a. Die Wasserstoffatome und die Fehlordnung eines DME in 14a wurden der Übersichtlichkeit halber nicht dargestellt. 
Die Dimere 11 und 12 zeigen den für dimere Lithiumaryle typischen Aufbau. Die Anthracengerüste sind mit einer gemittelten Abweichung von 0.036(2) $\AA$ in 11 und 0.079(3) A in 12 nahezu planar und werden über einen, zu ihnen orthogonal orientierten, viergliedrigen $(\mathrm{Li}-\mathrm{C})_{2}$ Ring verbrückt. Die parallele Verschiebung beider Anthracenreste resultiert aus den unterschiedlichen Bindungstypen einer kurzen $\mathrm{Li}-\mathrm{C}-\sigma$ und einer langen $\mathrm{Li}-$ $\mathrm{C}-\pi$ Bindung, welche die $\mathrm{sp}^{2}$-Hybridisierung des lithiierten Kohlenstoffatoms $\mathrm{C}_{\alpha}$ widerspiegeln. Die gemittelten $\mathrm{Li}-\mathrm{C}_{\alpha}$ Bindungslängen (2.305(5) $\AA$ in 11 und 2.309(6) $\AA$ in 12) sind innerhalb ihrer Standardabweichung identisch und lassen auf eine nahezu gleiche Reaktivität schließen. Die Vermutung, dass die gemischten Aggregate 15 und $\mathbf{1 4}$ zu den Verbindungen 11 und 12, mit der Ausnahme, dass ein Anthrylrest gegen ein Bromidion ausgetaucht wurde, identisch aufgebaut sind, bestätigte sich überraschender Weise nicht. Zwar sind auch hier die Anthracengerüste planar und auch der viergliedrige $\mathrm{Li}_{2} \mathrm{CBr}$-Ring ist orthogonal zur Anthracenebene orientiert, die Bindungslängen beider Lithiumatome zum lithiierten Kohlenstoffatom $C_{\alpha}$ sind hier jedoch im Rahmen ihrer Standardabweichung

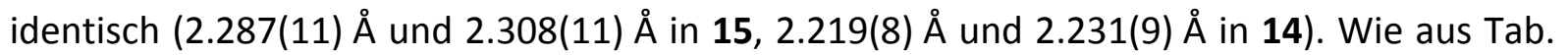
2.7 ersichtlich ist, treten auch hier dieselben Tendenzen bezüglich der Li-C-Bindungslängen wie für das oben diskutierte tetramere Aggregat auf.

Tab. 2.7: Ausgewählte Bindungslängen $[\AA ̊]$ und Winkel $\left[^{\circ}\right]$ in 11, 12, 14 und 15.
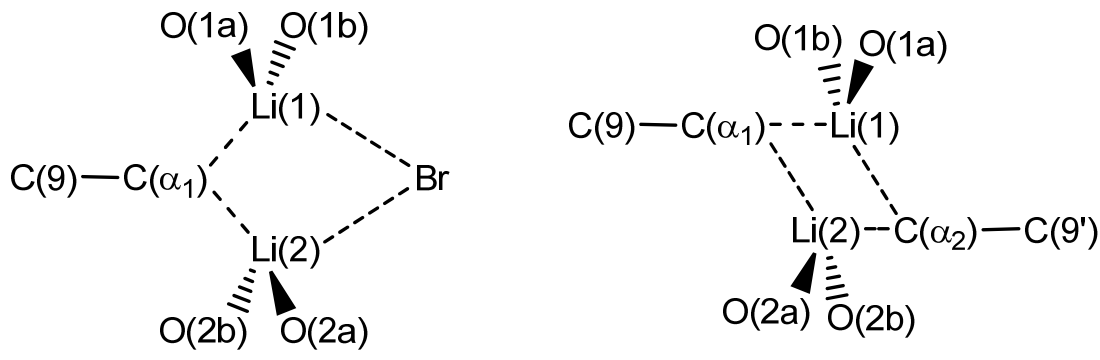

\begin{tabular}{lcccc}
\hline & 15 & 11 & 14 & 12 \\
\hline $\mathrm{C}(\alpha 1)-\mathrm{Li}(1)$ & $2.287(11)$ & $2.250(5) / 2.271(5)^{\mathrm{a}}$ & $2.219(8)$ & $2.256(6)$ \\
$\mathrm{C}(\alpha 1)-\mathrm{Li}(2)$ & $2.308(11)$ & $2.363(5) / 2.368(5)^{\mathrm{a}}$ & $2.231(9)$ & $2.361(6)$ \\
$\mathrm{C}(\alpha 2) / \mathrm{Br}(1)-\mathrm{Li}(1)$ & $2.502(10)$ & $2.347(5) / 2.353(5)^{\mathrm{a}}$ & $2.459(7)$ & $2.361(6) \mathrm{c}$ \\
$\mathrm{C}(\alpha 2) / \mathrm{Br}(1)-\mathrm{Li}(2)$ & $2.514(10)$ & $2.248(5) / 2.242(5)^{\mathrm{a}}$ & $2.494(7)$ & $2.256(6) \mathrm{c}$ \\
$\varnothing \mathrm{Li}-\mathrm{C} \alpha$ & $2.298(11)$ & $2.3053(5)$ & $2.225(9)$ & $2.309(6)$ \\
$\varnothing \mathrm{Li}-\mathrm{Br}(1)$ & $2.51(1)$ & - & $2.3265(70)$ & - \\
$\mathrm{C} 9-\mathrm{C}(\alpha 1)-\mathrm{Li}(1)$ & $141.3(3)$ & $163.6(2) / 160.4(2)^{\mathrm{a}}$ & $141.6(3)$ & $160.3(2)$ \\
$\mathrm{C} 9-\mathrm{C}(\alpha 1)-\mathrm{Li}(2)$ & $145.2(3)$ & $128.6(1) / 132.5(1)^{\mathrm{a}}$ & $143.6(3)$ & $134.6(2)$ \\
$\mathrm{C} 9^{\prime}-\mathrm{C}(\alpha 2)-\mathrm{Li}(1)$ & - & $132.0(1) / 133.1(1)^{\mathrm{a}}$ & - & $134.6(2)^{\mathrm{c}}$ \\
$\mathrm{C} 9^{\prime}-\mathrm{C}(\alpha 2)-\mathrm{Li}(2)$ & - & $159.7(2) / 158.8(2)^{\mathrm{a}}$ & - & $160.3(6)^{\mathrm{c}}$ \\
$\mathrm{Li}(1)-\mathrm{Br}(1)-\mathrm{Li}(2)$ & $66.3(3)$ & - & $66.2(2)$ & - \\
\hline
\end{tabular}

a) Zwei kristallographisch unabhängige Dimere in der Elementarzelle; b) Fehlordnung; c) Identische Werte durch Symmetrie. 
In beiden hier vorgestellten Beispielen sind die gemittelten $\mathrm{Li}-\mathrm{C}_{\alpha}$-Bindungslängen der gemischten Aggregate kürzer als diejenigen der reinen Dimere, was analog zu dem oben diskutierten Beispiel auf eine geringere Reaktivität schließen lässt. Tatsächlich lässt sich eine größere Stabilität der gemischten Aggregate gegenüber Temperatur und Luftsauerstoff beobachten. So ist kristallines Material von $\mathbf{1 5}$ und $\mathbf{1 4}$ über Stunden bei Raumtemperatur stabil und zersetzt sich nur langsam bei Kontakt mit Luftsauerstoff, während sich $\mathbf{1 1}$ und $\mathbf{1 2}$ oberhalb von $-10^{\circ} \mathrm{C}$ und in Gegenwart von Luftsauerstoff schnell zersetzen. Bei Kontakt mit nassen Lösungsmitteln oder Wasser hydrolysieren allen Substanzen jedoch schnell.

Die gemischten Aggregate, welche schon früher als Ruhezustand beschrieben wurden, lassen sich ohne weiteres durch Zusatz von mehrzähnigen Donorbasen wieder reaktivieren. Die Aktivierung erfolgt hierbei nicht durch den bekannten Mechanismus der Deaggregation, sondern durch das Entfernen des Lithiumsalzes aus dem Gleichgewicht. Die Stabilität der gebildeten LiX - Donor-Komplexe ist die Triebkraft der Aktivierung. Die Reaktivierung von gemischten Aggregaten durch Donorbasen sei anhand eines hypothetischen Beispiels dargestellt: Ein Lithiumorganyl RLi, welches in schwach donierenden Donorbasen wie Diethylether als dimeres Aggregat $\left[\mathrm{RLi} \cdot\{\text { dee }]_{2}\right.$ vorliegt, soll mit einem Dialkylchlorphosphan umgesetzt werden. Das während der Reaktion stöchiometrisch gebildete Lithiumchlorid bildet mit dem noch verbliebenen Lithiumorganyl ein gemischtes Aggregat des Typs [RLi $\left.\cdot \mathrm{LiCl} \cdot\{\text { dee }\}_{2}\right]$, dessen Reaktivität nicht ausreicht, um mit dem Chlorphosphan zu reagieren. Nach Beenden der Reaktion wird eine Ausbeute von weniger als $75 \%$ beobachtet. Wird vor Beenden der Reaktion ein Äquivalent PMDETA zugesetzt, so lässt sich die Reaktion auf nahezu quantitative Ausbeuten steigern.

Die Annahme, dass durch Zusatz von PMDETA das Lithiumorganyl in seiner monomeren Form stabilisiert und eine Steigerung der Ausbeute hervorgerufen wird, ist falsch. Die Ausbeute von ca. 75 \% zeigt, dass die Reaktivität des dimeren Lithiumorganyls sehr wohl zur Reaktion mit dem Chlorphosphan ausreicht. Da der starke Donor PMDETA in der Lage ist, Lithiumchlorid stärker zu komplexieren, werden die gemischten Aggregate wieder in ihre dimere Form überführt und stehen so der Reaktion mit dem Chlorphosphan wieder zur Verfügung.

Dieses Beispiel zeigt, dass in Reaktionen bei denen Lithiumsalze gebildet werden weit komplexere Mechanismen ablaufen als weithin angenommen. Zwar führt die Anwendung von akzeptierten Konzepten der Reaktivitätssteigerung durch Deaggregation auch hier in einigen Fällen zum Erfolg, die genaue Kenntnis über die in der Reaktion ablaufenden Prozesse eröffnet jedoch weitere Möglichkeiten. So wird deutlich, dass zur vollständigen Deaggregation zum Monomer mindestens zwei Äquivalente PMDETA eingesetzt werden müssen. 


\subsection{Carbonsäurederivate des Anthracens}

Die Carbonsäurechemie ist eines der am besten untersuchten Gebiete in der organischen Synthese. Neben Mechanismen zur C-C-Bindungsknüpfung lassen sich Carbonsäuren $\mathrm{u}$. a. in Amide, Amine, Ester und Alkohole umwandeln. ${ }^{[127]}$ In diesem Abschnitt sollen Versuche zum Aufbau der gewünschten Rezeptoreinheit durch die Synthese und Reduktion von Carbonsäureamiden erörtert werden. Über diesen Syntheseweg wären tertiäre Amine zugänglich, die über einen $\mathrm{CH}_{2}$-Spacer an das Anthracengerüst gebunden sind (GI. 2.18).<smiles>O=C(O)c1c2ccccc2c(Br)c2ccccc12</smiles><smiles>[R20]C(=O)c1c2ccccc2c(Br)c2ccccc12</smiles><smiles>[R20]Cc1c2ccccc2c(Br)c2ccccc12</smiles>

GI. 2.18: Aufbau der Rezeptoreinheit über Carbonsäureamide.

Die Carbonsäuren von Anthracenderivaten lassen sich in guten Ausbeuten über die lithiierten Stufen darstellen. Je nach verwendeten Ausgangsstoffen sind so die 9-Anthracencarbonsäure, die 9,10-Anthracendicarbonsäure und die 9-Brom-10-anthracencarbonsäure zugänglich. Auch substituierte Anthracenderivate wie das 9-Brom-10-methylanthracen lassen sich in die entsprechende Carbonsäure überführen (GI. 2.19).<smiles>[R]c1c2ccccc2c(Br)c2ccc(Br)cc12</smiles><smiles>[R]c1c2ccccc2c(Cl)c2ccccc12</smiles>

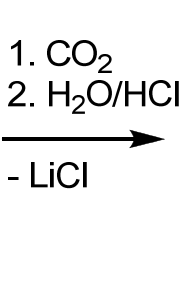<smiles>[R]c1c2ccccc2c(C(=O)O)c2ccccc12</smiles>

GI. 2.19: Synthese von Anthracencarbonsäuren über lithiierte Anthracenderivate.

Da neben der Rezeptoreinheit noch ein Tuner in das Anthracengerüst eingeführt werden soll, kommt für die weitere Derivatisierung nur die 9-Brom-10-anthracencarbonsäure in Betracht. Die Synthese dieser Verbindung erfolgt durch das Zutropfen der Anthryllithiumverbindung auf festes Kohlenstoffdioxid. Der Vorteil dieser Reaktionsführung liegt in der tiefen Temperatur des festen Kohlenstoffdioxids, wodurch es keiner zusätzlichen externen Kühlung der Reaktionsmischung bedarf. Auch auf Schutzgasatmosphäre kann verzichtet werden, da das Eindringen von Luftsauerstoff und Feuchtigkeit durch das, sich beim Auftropfen der ca. $0^{\circ} \mathrm{C}$ kalten Reaktionslösung bildende, gasförmige Kohlenstoffdioxid verhindert wird. Nach saurer Hydrolyse wird die freie Carbonsäure in guten Ausbeuten erhalten. Durch Umkristallisation aus Diethylether wird die Substanz in Form von langen 
gelben Nadeln erhalten. Bei Betrachtung der Molekülpackung (Abb. 2.18) fällt auf, dass je zwei Anthracencarbonsäuren über Wasserstoffbrückenbindungen miteinander verknüpft vorliegen. Eine zusätzliche attraktive Wechselwirkung kommt durch das für Anthracene bekannte $\pi$-stacking zustande. Die monosubstituierte 9-Anthracencarbonsäure wird von verschiedenen Arbeitsgruppen seit Ende der 70er Jahre zur Bildung von Metallkomplexen z. B. der Metalle $\mathrm{Cr}^{2+},{ }^{[128]} \mathrm{Re}^{+},{ }^{[129]} \mathrm{Ru}^{2+},{ }^{[130]} \mathrm{Mn}^{2+},{ }^{[131]} \mathrm{Mo}^{2+},{ }^{[132]} \mathrm{Cd}^{2+},{ }^{[133]} \mathrm{Sn}^{+},{ }^{[134]} \mathrm{Sn}^{2+},[134]$ $\mathrm{Cu}^{2+},{ }^{[135]} \mathrm{Co}^{2+},{ }^{[135]} \mathrm{Ni}^{2+},{ }^{[135]} \mathrm{Mn}^{3+},{ }^{[136]} \mathrm{Zn}^{2+},{ }^{[137]} \mathrm{La}^{3+},{ }^{[138]} \mathrm{Er}^{3+[139]}$ und $\mathrm{Ag}^{+[140]}$ eingesetzt, ${ }^{* *}$ wobei die Zahl an publizierten Metallkomplexen sprunghaft ansteigt, seit die 9-Anthracencarbonsäure kommerziell erhältlich ist.
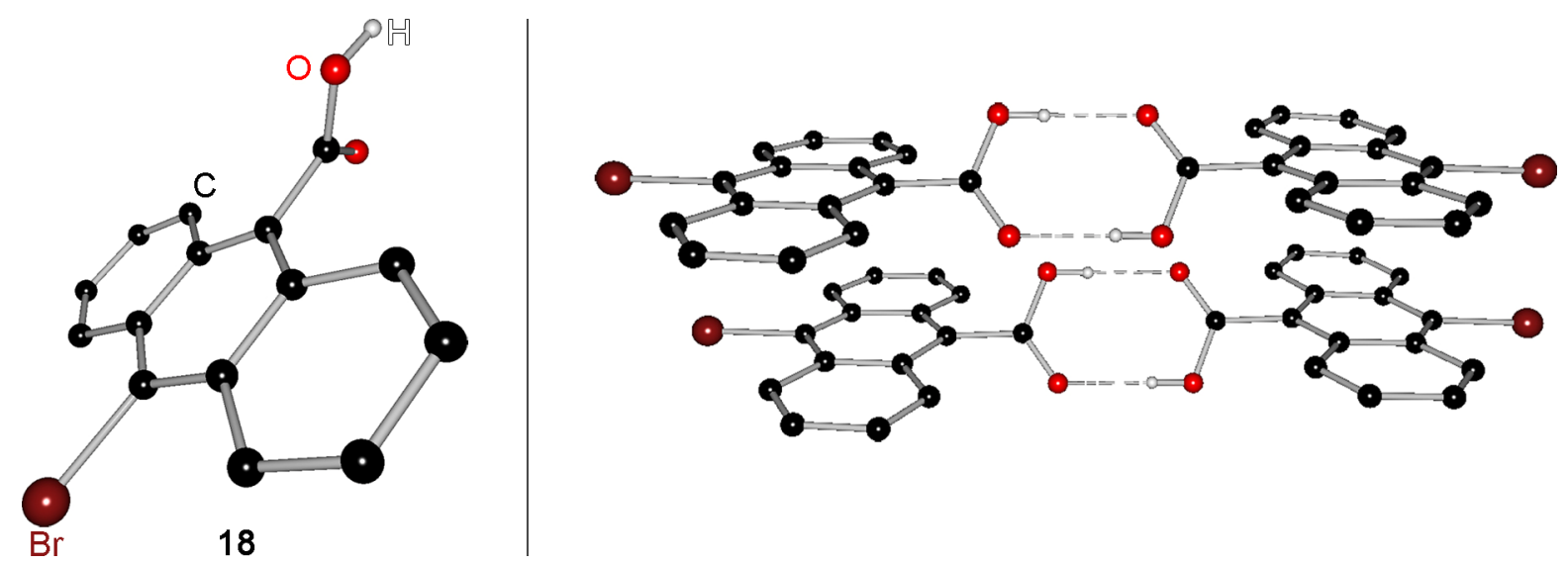

Abb. 2.18: Molekülstruktur der 9-Brom-10-anthrylcarbonsäure (18 - links) und der im Kristall auftretenden intermolekulare Wasserstoffbrückenbindung (rechts).

Die ausnahmslos durch Röntgenstrukturanalyse nachgewiesenen Komplexe zeigen einerseits die vielfältige Einsetzbarkeit dieser Substanzklasse, machen andererseits jedoch deutlich, dass die Carboxylgruppe durch ihre geringe Spezifität für die Funktion als Rezeptor ungeeignet ist. Um die Carbonsäure für eine weitere Derivatisierung zu aktivieren, kann diese nach üblichen Methoden mit Thionylchlorid oder besser Oxalylchlorid zum Carbonsäurechlorid (19) umgewandelt werden (GI. 2.20). ${ }^{[141,142]}$<smiles>O=C(O)c1c2ccccc2c(Br)c2ccccc12</smiles>

18

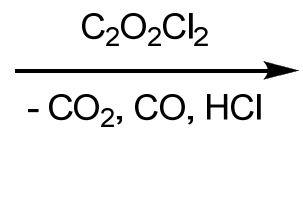

GI. 2.20: Aktivierung der Anthrylcarbonsäure (18) durch Umwandlung zum Anthrylcarbonsäurechlorid (19).<smiles>ClC(Cl)(Cl)c1c2ccccc2c(Br)c2ccccc12</smiles>

19

\footnotetext{
** Die genannten Metalle stellen ausgewählte Beispiele ohne Anspruch auf Vollständigkeit dar.
} 
Das eingesetzte Chlorierungsmittel sollte dabei frisch umkondensiert eingesetzt werden und für spätere Umsetzungen unter Lichtausschluß aufbewahrt werden. Nur so kann die Bildung kleinster Mengen an gelöstem Chlor verhindert werden, welches, wie in Abschnitt 2.1 erwähnt, zu ungewollten Nebenreaktionen führen kann. Die Darstellung auch großer Substanzmengen ist ohne weiteres möglich, die Aufbewahrung des hoch reaktiven Carbonsäurechlorides erweist sich jedoch auch unter Schutzgas als schwierig. Das Anthrylcarbonsäurechlorid reagiert schnell unter Feuchtigkeitseinfluss zur Anthrylcarbonsäure zurück. Das mehrmalige Öffnen des zur Aufbewahrung genutzten Schlenk-Kolbens (selbst im Stickstoffgegenstrom) reicht aus, um signifikante Mengen zur Anthrylcarbonsäure zurückreagieren zu lassen. Die so gebildete Säure (18) kann anschließend mit noch vorhandenem Carbonsäurechlorid (19) zum Anhydrid (20) weiterreagieren (GI. 2.21). ${ }^{[143]}$<smiles>O=C(O)c1c2ccccc2c(Br)c2ccccc12</smiles>

18<smiles></smiles>

19<smiles>O=C(OC(=O)c1c2ccccc2c(Br)c2ccccc12)c1cccc2c(Br)cccc12</smiles>

20

GI. 2.21: Bildung des Anthracencarbonsäureanhydrides 20 aus der Anthrylcarbonsäure 18 und dem Anthrylcarbonsäurechlorid 19. ${ }^{[143]}$

Diese hohe Reaktivität gegenüber Luftfeuchtigkeit beobachteten schon 1995 Schröder et al. für das von ihnen dargestellte 9-Anthrylcarbonsäurechlorid. Auch sie erhielten das Carbonsäureanhydrid als Nebenprodukt und konnten dieses ebenfalls röntgenkristallographisch nachweisen. ${ }^{[144]}$ Eine gezielte Synthese des Anhydrids gelingt, wenn äquimolare Mengen an Säurechlorid und Säure über Nacht in Acetonitril gerührt werden. $^{[143]}$

Eine Aufbewahrung in reinem Oxalylchlorid, welches zwar sämtliche eindringende Feuchtigkeit unter Bildung von $\mathrm{CO}_{2}, \mathrm{CO}$ und $\mathrm{HCl}$ vernichtet, ist ebenfalls nicht zu empfehlen. Beim längeren Lagern des Oxalylchlorides (auch unter Lichtausschluß) entstehen geringe Mengen an Chlorgas, welches bei genügend langer Reaktionszeit im geschlossenen Kolben nicht nur zu einer Substitution des Bromatoms in 9-Position führt, sondern auch, unter Aufhebung der Aromatizität, eine Halogenierung eines der äußeren sechsgliedrigen Ringe induziert. Das so gebildete Pentahalogenanthracencarbonsäurechlorid (19b) ähnelt den Hexabromderivaten die in Abschnitt 2.1.1 vorgestellt wurden. Die Molekülstrukturen der beiden oben beschriebenen Nebenprodukte 19b und $\mathbf{2 0}$ sind in Abb. 2.19 dargestellt. 

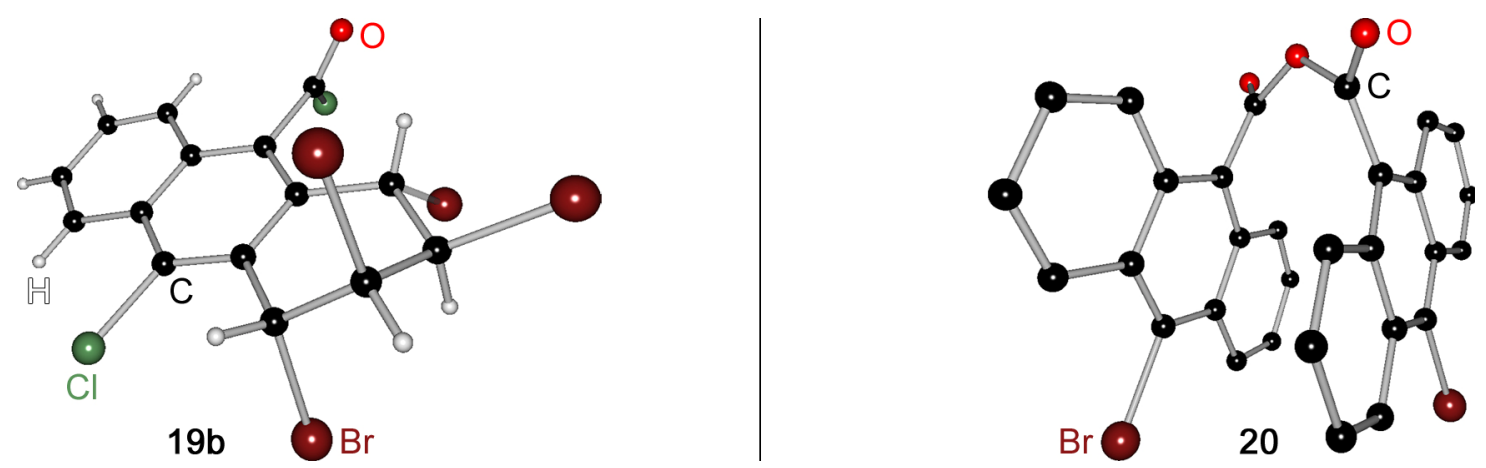

Abb. 2.19: Molekülstrukturen der in dieser Arbeit bei der Lagerung des Anthracencarbonsäurechlorides (19) erhaltenen Nebenprodukte Pentahalogenanthrylcarbonsäurechlorid (19b - links) und Anthracenylcarbonsäureanhydrid (20 - rechts).

Es empfiehlt sich also das Säurechlorid vor der Umsetzung zu den etwaigen Produkten frisch herzustellen, was durch die kurze Reaktionsdauer von ca. einer Stunde kein Problem darstellt. Die so erhaltene hochreaktive Form bietet u. a. Zugang zu Ketonen, Amiden, Alkoholen und Estern, was auch in einer ersten Umsetzung unter Bildung des tertButylesters (21) gezeigt werden konnte. Die Synthese erfolgt durch Umsetzung des Anthracencarbonsäurechlorides bei Raumtemperatur mit Natriumtertbutanolat in THF und liefert den erwarteten Ester (siehe Abb. 2.20). ${ }^{[145]}$
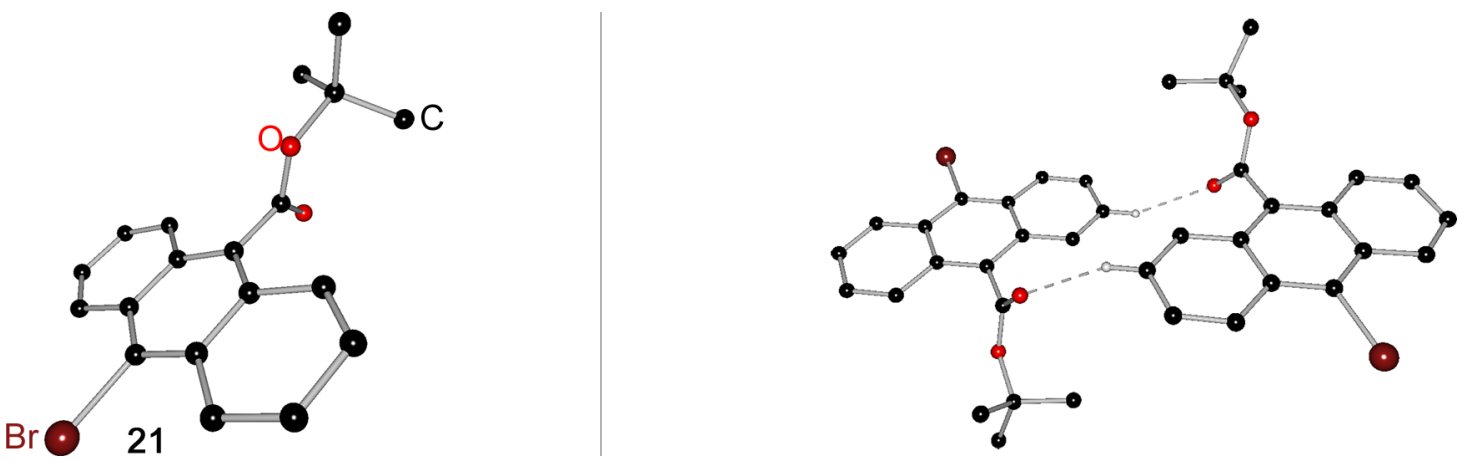

Abb. 2.20: Molekülstruktur des 9-Brom-10-tertbutylanthrylesters (21 - links), intermolekulare Wasserstoffbrückenbindung (rechts).

Auch die Umsetzung von sekundären Aminen zu den entsprechenden Carbonsäureamiden läuft wie erwartet ohne größere Probleme ab. Für die Umsetzung des Carbonsäurechlorides mit sekundären Aminen muss mit einem doppelten Überschuss an Amin gearbeitet werden, da ein Äquivalent Amin durch das entstehende $\mathrm{HCl}$ in das entsprechende Ammoniumsalz überführt wird und somit der Reaktion nicht mehr zur Verfügung steht. Bei teureren oder aufwendig synthetisierten Aminen empfiehlt sich hier der Einsatz einer nicht nukleophilen Hilfsbase um mit stöchiometrischen Mengen Amin arbeiten zu können.

Obwohl die Synthesen der Carbonsäureamide wie erwartet in hohen Ausbeuten verlaufen, konnte im Laufe dieser Arbeit keine praktikable Methode gefunden werden um das Carbonsäureamid zu den gewünschten tertiären Aminen zu reduzieren. 
Die Carbonsäureamide könnten auch ohne Reduktion als Rezeptoreinheit dienen, die Einführung eines Tuners mittels Lithium-Halogenaustausch ist mit ihnen jedoch nicht möglich. Zur Einführung des Phosphan-Tuners als Zweitsubstituent müsste die aufgebaute Rezeptoreinheit, wie in den vorherigen Abschnitten erläutert, inert gegenüber lithiumorganischen Reagenzien sein. Das Schützen der Säurefunktion könnte das Einführen der Phosphane ermöglichen und bei nachfolgender Entschützung die Synthese von Carbonsäureamiden ermöglichen.

$\mathrm{Zu}$ den wenigen Schutzgruppen, die gegenüber lithiumorganischen Reagenzien inert sind, gehört die Substanzklasse der Oxazole. ${ }^{[146]}$ Diese lassen sich bei der Umsetzung von Säurechloriden mit den entsprechenden Aminoethanolen (meist 2-Amino-2,2-dimethylethanol) und anschließender Cyclisierung erhalten (GI. 2.22).<smiles>Brc1ccc2c(Br)c3ccccc3c(Br)c2c1</smiles>

2<smiles>C#C[C@H]1COC(c2c3ccccc3c(Br)c3ccccc23)=N1</smiles>

23<smiles>Clc1c2ccccc2c(Br)c2ccccc12</smiles>

1) $\mathrm{CO}_{2}$

2) $\mathrm{H}_{2} \mathrm{O} / \mathrm{HCl}$

2) $-\mathrm{LiCl}$<smiles>O=C(O)c1c2ccccc2c(Br)c2ccccc12</smiles>

18<smiles>CC(C)(CO)NC(=O)c1c2ccccc2c(Br)c2ccccc12</smiles>

22

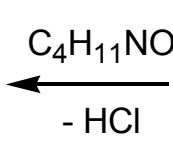<smiles>COC(=O)C(C)(C)C(=O)OCCO</smiles><smiles>ClC(Cl)(Cl)c1c2ccccc2c(Br)c2ccccc12</smiles>

19

GI. 2.22: Syntheseweg zum Aufbau des Oxazolanthracens 23.

Die beim Anthracen begonnene sechsstufige Synthese läuft bis zum Amid $\mathbf{2 2}$ ohne größere Probleme. Alle bis zu diesem Schritt gebildeten Nebenprodukte sind entweder gasförmig oder leicht flüchtig, so dass diese leicht abgetrennt werden können. Nach der wässrigen Aufarbeitung zur Freisetzung der Säure 18 kann sogar im selben Reaktionskolben weitergearbeitet werden, was Aufarbeitungsverluste deutlich minimiert. Das gebildete Amid konnte eindeutig durch NMR- sowie Massenspektren nachgewiesen werden. Die zur Cyclisierung angewendete Chlorierung der Alkoholfunktion des Amids 22, führte zu unerwarteten Problemen. Aus mehreren Ansätzen wurde stets 9,10-Dichloranthracen als Hauptprodukt isoliert. Dieses unerwartete Ergebnis kann nur durch die Bildung von elementarem Chlor während der Chlorierung erklärt werden. Nach Addition von Chlor und 
anschließender Rearomatisierung könnten sich, wie schon in Abschnitt 2.1 besprochen, die zuvor an den 9,10-Positionen befindlichen Substituenten als bessere Abgangsgruppen abgespalten haben. Durch diese Problematik, die auch durch die Verwendung von Oxalylchlorid als Chlorierungsmittel nicht überwunden werden konnte, wurde diese Syntheseroute zugunsten anderer Synthesewege nicht weiter verfolgt.

\subsection{Addition lithiierter Anthracene an Carbonylgruppen}

Lithiumorganyle lassen sich durch Reaktion mit Verbindungen, die über eine Carbonylgruppe verfügen zu Additionsprodukten umsetzen. Durch Umsetzung von z. B. Aldehyden oder Ketonen mit Lithiumanthracenen werden die entsprechenden sekundären und tertiären Alkohole erhalten (GI. 2.23). ${ }^{[147]}$<smiles>[R]C(O)c1c2ccccc2c(Br)c2ccccc12</smiles>
1) $\mathrm{R}_{\mathrm{R}}^{\mathrm{O}}$
2) $\frac{\mathrm{H}_{2} \mathrm{O} / \mathrm{HCl}}{\stackrel{\mathrm{LiCl}}{2}}$<smiles>Clc1c2ccccc2c(Br)c2ccccc12</smiles>

1) ${ }_{R}^{\stackrel{O}{\Perp}} R^{\prime}$

2) $\mathrm{H}_{2} \mathrm{O} / \mathrm{HCl}$

2) $-\mathrm{LiCl}$<smiles>[R]C(O)c1c2ccccc2c(Br)c2ccccc12</smiles>

GI. 2.23: Synthese von sekundären und tertiären Alkoholen durch die Umsetzung von Lithiumanthracenen mit Aldehyden und Ketonen.

Werden komplexere Aldehyde oder Ketone eingesetzt, die in ihrer Peripherie über Donoratome verfügen, lassen sich so in einem Reaktionsschritt maßgeschneiderte Ligandensysteme aufbauen. Diese Addition an die Carboxylgruppe liefert einen Spacer, der für den Aufbau eines Sensorsystems benötigt wird, gleich mit. In der, dieser Arbeit vorangegangenen, Diplomarbeit konnte so ausgehend von 9-Brom-10-anthryllithium und Benzaldehyd der sekundäre Alkohol 9-Bromanthrylphenylmethanol (24) erhalten werden.

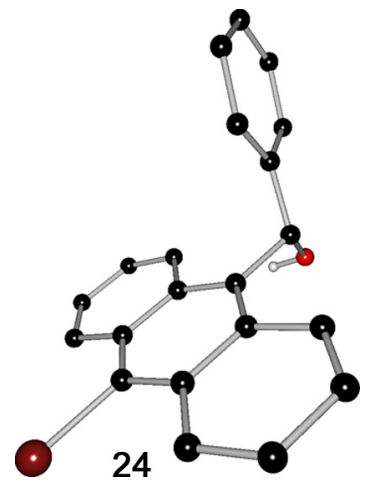<smiles>OC(c1ccccn1)c1c2ccccc2c(Br)c2ccccc12</smiles>

25

Abb. 2.21: Molekülstruktur des durch Addition von 9-Brom-10-anthryllithium an Benzaldehyd erhalten sekundären Alkohols $\mathbf{2 4}$ (I.) und die durch Umsetzung mit 2-Pyridylaldehyd erhaltene Verbindung 25 (r.). 
24 wurde durch die gewählten Reaktionsbedingungen als Racemat erhalten und kristallisiert in der chiralen orthorhombischen Raumgruppe $P 2_{1} 2_{1} 2_{1}$ in Form eines racemischen Zwillings. Die Molekülstruktur, deren Verfeinerung erst im Laufe dieser Arbeit gelang, ist in Abb. 2.21 links dargestellt.

Das für den PET-Effekt nötige Stickstoffatom kann nicht durch die Umsetzung von Anthryllithium mit Carbonsäureamiden eingeführt werden, da diese als schlechte Elektrophile eine zu geringe Reaktivität aufweisen. Das einzuführende Amin muss daher mindestens über eine Ethylenbrücke vom Fluorophor getrennt sein, was die Auswahl an Ausgangsmaterialien limitiert. So wurde in dieser Arbeit auf dem zu $\mathbf{2 4}$ analogem Wege Verbindung $\mathbf{2 5}$ hergestellt. Die Alkoholfunktion, welche auf diesem Reaktionsweg gebildet wird, verhindert, wie auch schon die Carbonylfunktion bei den Carbonsäureamiden, die Einführung der Tuner. Auch hier ist ein Reduktionsschritt erforderlich, welcher bisher nicht gelang.

Aussichtsreicher sind Synthesewege, bei denen ein Anthracencarbaldehyd mit primären Aminen zur Schiffschen-Base umgesetzt wird. Das benötigte 9-Brom-10-anthracencarbaldehyd kann durch die Umsetzung von 9-Brom-10-anthryllithium mit Dimethylformamid (DMF) erhalten werden (GI. 2.24). ${ }^{[67]}$<smiles>Clc1c2ccccc2c(Br)c2ccccc12</smiles>
1) $\mathrm{DMF}$
2) $\mathrm{H}_{2} \mathrm{O}$
2) - $\mathrm{LiOH} /-\mathrm{HNMe}_{2}$<smiles>O=Cc1c2ccccc2c(Br)c2ccccc12</smiles>

GI. 2.24: Synthese von 9-Brom-10-anthracencarbaldehyd durch die Umsetzung von 9-Brom-10-anthryllithium mit DMF. ${ }^{67]}$

Das 9-Brom-10-anthracencarbaldehyd kann je nach Wassergehalt des schwer zu trocknenden DMFs mit Ausbeuten von 65 bis $80 \%$ erhalten werden, wobei 9Bromanthracen durch Hydrolyse als Nebenprodukt entsteht. Anthracencarbaldehyde lassen sich in einer Mischung aus Ethanol und Acetonitril mit primären Aminen bei Raumtemperatur zu den entsprechenden Schiffschen-Basen umsetzen. Eine anschließende Reduktion mit Natriumborhydrid in Ethanol gelingt problemlos, so dass die entsprechenden sekundären Amine in Ausbeuten um $95 \%$ erhalten werden. Auf diesem Weg wurden in dieser Arbeit durch die Umsetzung des kommerziell erhältlichen 9-Anthracencarbaldehyds mit Methoxyethylamin und Furfurylamin zwei sekundäre Anthrylamine (26 und 27) synthetisiert (GI. 2.25). 
<smiles>O=Cc1c2ccccc2cc2ccccc12</smiles><smiles>[R]C/N=C\c1c2ccccc2cc2ccccc12</smiles>

\section{[Red]}<smiles>[R]CNCc1c2ccccc2cc2ccccc12</smiles>

26: $R=1-(2,3,4-$ trihydrofuran)

27: $\mathrm{R}=\mathrm{CH}_{2} \mathrm{OMe}$

GI. 2.25: Synthese von sekundären Anthrylaminen (26 und 27) über die Schiffschen-Basen.

Wird 9-Brom-10-anthracencarbaldehyd für diese Umsetzung verwendet, kann das in 9-Position verbliebene Bromatom augrund der NH-Funktion nicht über Lithium-HalogenAustausch substituiert werden. Einen Ausweg bietet die Derivatisierung zu einem tertiären Amin, welche nicht nur die Einführung des Tuners ermöglicht, sondern auch gleichzeitig den Zugang zu asymmetrisch substituierten Anthracenylaminen eröffnet. ${ }^{[148]}$ Solche asymmetrisch substituierten Systeme sind im Bereich der Fluoreszenzchemosensoren von besonderem Interesse, da durch diese der Zugang zu 'logic-devices' möglich ist. Gegenüber einem einfachen Chemosensor, der ausschließlich über eine 'an-' und 'aus-Funktion' verfügt, können mit 'logic-devices' komplexere Anfragen realisiert werden. Ein Beispiel ist das 'andlogic-device', bei dem zwei Informationen parallel abgefragt werden (Abb. 2.22).
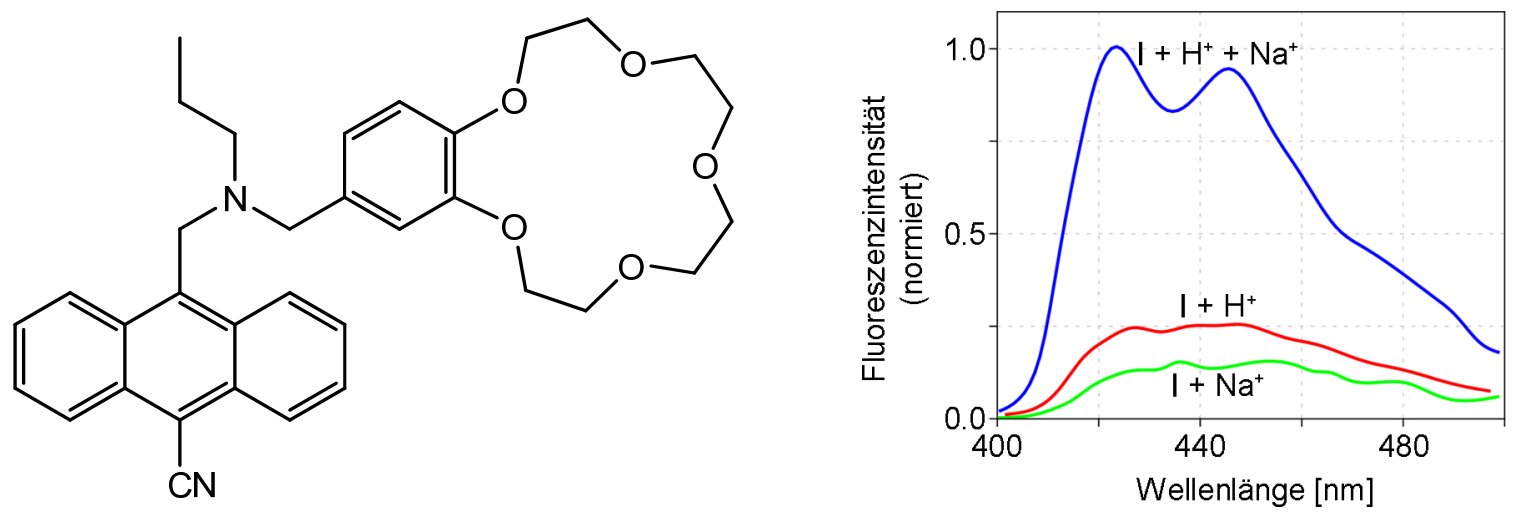

Abb. 2.22: Lewis-Formel eines 'and-logic-device' Fluoreszenzchemosensors (links) und Fluoreszenzspektren des Sensors mit Natriumionen (grün), mit Protonen (rot) und mit sowohl Natriumionen als auch Protonen (blau). ${ }^{[149]}$

So kommt es, sowohl durch das freie Elektronenpaar des Stickstoffatoms, als auch durch den unbesetzten Benzokronenether, zu einem effektiven Quenchen der Fluoreszenz. Um beide PET-Effekte zu unterdrücken ist demnach die Anwesenheit von zwei Analyten (hier Protonen und Natriuminonen) notwendig. Die Anwesenheit von lediglich einem der beiden Analyten führt nur zu einer geringen Verstärkung der Fluoreszenz (siehe Abb. 2.22 rechts). ${ }^{[149]}$ Über die Syntheseroute der Schiffschen Basen lassen sich bevorzugt asymmetrische Amine aufbauen. Die Synthese von Azakronenethern oder Kryptanden, welche hier über eine intramolekulare Cyclisierung erfolgen müsste, kann leichter durch Anwendung der in Abschnitt 2.6 beschriebenen $\mathrm{S}_{\mathrm{N}}$-Chemie erfolgen. 


\subsection{Einführung von Spacern}

Die zur Komplexierung der gewünschten Analyten (Targets) nötige Rezeptoreinheit sollte in einem Fluoreszenzsensorsystem über Alkylgruppen, welche als Abstandshalter (Spacer) dienen, an das Fluorophor gebunden sein. Nicht nur die größere Flexibilität des so gebundenen Rezeptors, sondern auch die Verringerung des elektronischen Einflusses koordinierter Kationen auf die Lage der Fluoreszenzbanden des Fluorophors spielt hier eine entschiedene Rolle.

Mit dem 9-Brom-10-brommethylanthracen (4) konnte in Abschnitt 2.1.2 bereits eine Ausgangsverbindung mit einem $\mathrm{CH}_{2}$-Spacer erhalten werden. Der Aufbau des Fluoreszenzchemosensors kann hier über nukleophile Substitution des Bromatoms an der $\mathrm{CH}_{2}$-Gruppe erfolgen (siehe Abschnitt 2.6). Um eine größere Flexibilität bei der Derivatisierung späterer Fluoreszenzchemosensoren zu haben, sollten auch Ausgangsmaterialien synthetisiert werden, welche über eine Ethylenbrücke als Spacer verfügen (siehe Abb. 2.23).<smiles>[X]Cc1c2ccccc2c(Br)c2ccccc12</smiles>

A

$X=$ Abgangsgruppe<smiles>CCc1c2ccccc2c(Br)c2ccccc12</smiles>

B

Abb. 2.23: Ausgangsstoffe zum Aufbau von Ligandensystemen, die über eine Methylenbrücke bzw. Ethylenbrücke mit dem Fluorophor verbunden sind.

Die Synthese von $\mathbf{A}$ (mit $\mathrm{X}=\mathrm{Br}$ ) erfolgt ausgehend von Anthracen (wie ausführlich in Abschnitt 2.1.2 gezeigt) in einer dreistufigen Synthese (GI. 2.26), welche in allen Schritten mit Ausbeuten $>90 \%$ abläuft. Das Bromatom an der $\mathrm{CH}_{2}$-Brücke ist eine gute Abgangsgruppe und sollte sich leicht substituieren lassen.<smiles>Brc1c2ccccc2c(Br)c2ccccc12</smiles>

2
1) $n \mathrm{BuLi}$

2) Mel

1) $-n \mathrm{BuBr}$

2) - Lil<smiles>Cc1c2ccccc2c(Br)c2ccccc12</smiles>

3

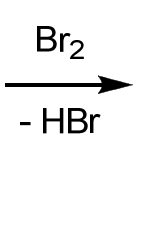

GI. 2.26: Synthese des 9-Brom-10-brommethylanthracens (4) als Ausgangsmaterial zu Sensorsystemen mit einem Methylen-Spacer.

Die Ethylenbrücke in B lässt sich ebenfalls in einer dreistufigen Synthese in das System in Form eines Alkohols einbringen (Gl. 2.27). 
<smiles>Brc1c2ccccc2c(Br)c2ccccc12</smiles>

2
1) $n \mathrm{BuLi}$

2) $\mathrm{C}_{2} \mathrm{H}_{4} \mathrm{O}$

3) $\mathrm{H}_{2} \mathrm{O} / \mathrm{HCl}$

1) $-n \mathrm{BuBr}$

3) - $\mathrm{LiCl}$

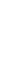<smiles>OCCc1c2ccccc2c(Br)c2ccccc12</smiles>

1) $\mathrm{NaOH}$

2) CITos

1) $-\mathrm{H}_{2} \mathrm{O}$

2) $-\mathrm{NaCl}$<smiles>O[SeH2]CCc1c2ccccc2c(Br)c2ccccc12</smiles>

GI. 2.27: Synthese des Tosylalkohols (29) als Ausgangsmaterial zu Sensorsystemen mit einem Ethylen-Spacer.

Hierzu wird nach einfacher Lithiierung von 9,10-Dibromanthracen in Diethylether Oxiran in die Reaktionslösung eingeleitet. Das nahezu quantitativ gebildete Alkoholat wird durch saure Hydrolyse in den Alkohol 29 umgewandelt.

Um analog zu A die Rezeptoreinheit durch nukleophile Substitution aufbauen zu können, wurde der Alkohol 29 anschließend durch Erhitzen in Pyridin und den Zusatz von Tosylchlorid in den entsprechenden Tosylalkohol 29 überführt. Sowohl $\mathbf{4}$ als auch der Alkohol $\mathbf{2 8}$ und der Tosylalkohol 29 fallen als kristalline Feststoffe an, die gut aus chlorierten Lösungsmitteln umkristallisiert werden können. Die Molekülstrukturen der beiden zuletzt genannten Verbindungen sind in Abb. 2.24 dargestellt.
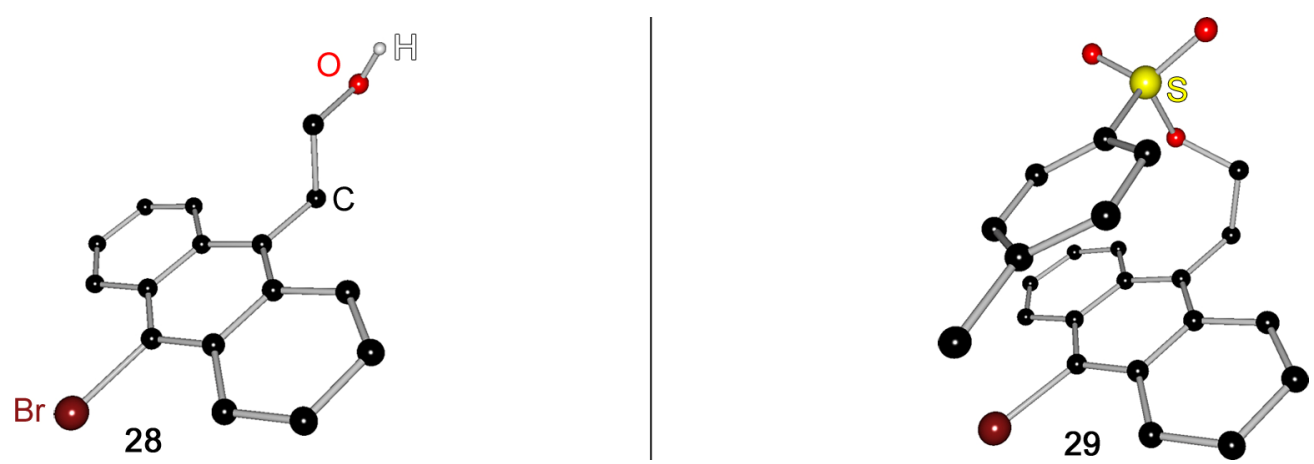

Abb. 2.24: Molekülstrukturen des Alkohols 28 (links) und dem daraus synthetisierten Tosylalkohol 29 (rechts).

In den meisten literaturbekannten Fluoreszenzchemosensoren auf Basis des Anthracenfluorophors werden die Rezeptoreinheiten durch nukleophile Substitution von Chlormethylanthracenen dargestellt (vergleiche Tab. 2.8 in Abschnitt 2.6). Sowohl die Tosylgruppe von 29, als auch das Bromatom in $\mathbf{4}$ sind bessere Abgangsgruppen und sollten sich daher noch leichter substituieren lassen, was zu einer deutlichen Steigerung der Ausbeuten führen sollte.

Für beide Systeme ( $\mathbf{A}$ und $\mathbf{B}$ ) ist die Einführung anderer Abgangsgruppen ohne weiteres möglich. So könnte der Alkohol 28 durch die in Abschnitt 2.1.2 vorgestellte Appel-Reaktion in das entsprechende Bromid überführt werden. Auch der Einsatz anderer Sulfonsäureester wie z. B. Triflat könnte eine Verbesserung für die nachfolgenden Substituitonsreaktionen darstellen. Die Verwendung der Sulfonsäureester als Abgangsgruppe ist nicht auf das System 
B beschränkt. Ausgehend von 9-Brom-10-hydroxymethylanthracen, welches aus der Reduktion des in Abschnitt 2.4 vorgestellten 9-Brom-10-anthracenylcarbaldehyds zugänglich ist, können Sulfonsäureester ebenfalls bei dem System $\mathbf{A}$ als Abgangsgruppe verwendet werden (siehe GI. 2.28).

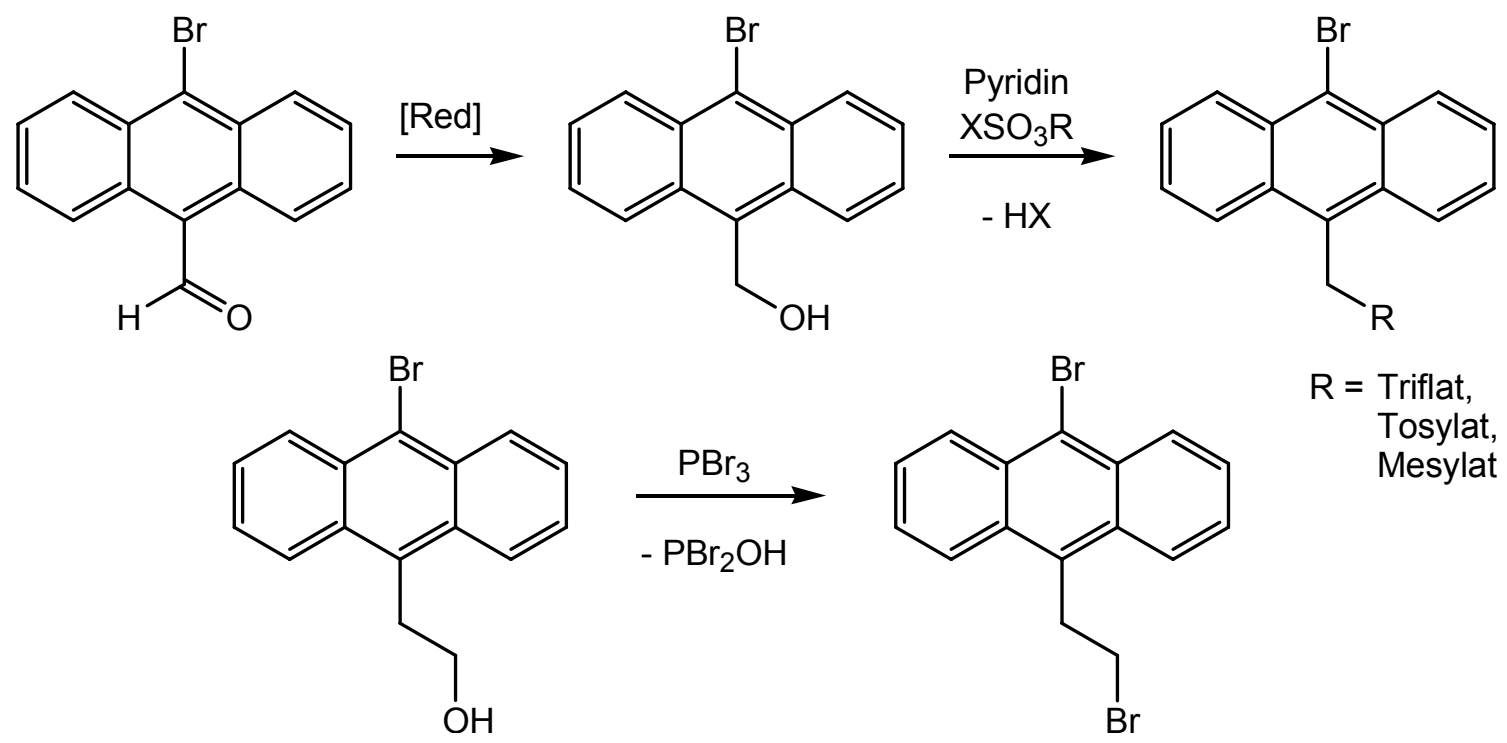

Gl. 2.28: Mögliche Syntheseroute zur Einführung verschiedener Abgangsgruppen in die Systeme $\mathbf{A}$ (oben) und $\mathbf{B}$ (unten).

Beim System B können auf dem in Gl. 2.27 gezeigten Reaktionsweg neben der Tosylgruppe auch andere Sulfonsäureester wie Mesylat oder Triflat mit ähnlichen Ausbeuten eingeführt werden. Auch eine Umwandlung des Alkohols in ein Bromid sollte über die Appel-Reaktion mit guten Ausbeuten möglich sein.

Bei System A lassen sich ebenfalls Abgangsgruppen in Form der Sulfonsäureester synthetisieren. Jedoch ist deren Syntheseweg um eine Stufe länger und müsste von dem 9-Brom-10-hydroxymethylanthracen ausgehen, welches aus der Synthese mit lediglich 65 bis $80 \%$ Ausbeute erhalten wird.

Ob die Einführung anderer Abgangsgruppen für die hier dargestellten Ausgangsstoffe nötig ist, muss anhand der in den jeweiligen Umsetzungen erhaltenen Ausbeuten entschieden werden. Geringere Ausbeuten der Ausgangsstoffe, wie im Fall der Sulfonsäureester des Systems A, könnten sich bei der Umsetzung mit komplexeren Aminen dennoch rentieren, da diese langwieriger Synthesen bedürfen oder sehr teuer sind.

\subsection{Aufbau der Rezeptoreinheit durch Substitutionsreaktionen}

Mit dem brommethylierten Derivaten 4-6 und dem Tosylalkohol 29 wurden in dieser Arbeit Edukte synthetisiert, die sich durch nukleophile Substitution in eine Vielzahl von Rezeptoren umwandeln lassen sollten. Die nukleophile Substitution ist eine der mechanistisch am besten untersuchten organischen Synthesemethoden und findet bei der Synthese von 
Fluoreszenzchemosensoren große Anwendung. Mit der richtigen Wahl des Lösungsmittels, der Abgangsgruppe und dem Nukleophil bietet sich ein großer synthetischer Spielraum zum Aufbau komplexer Systeme. Mit sekundären Aminen als Nukleophile lässt sich ein breites Spektrum substratspezifischer Rezeptoren generieren, das von einfachen Chelatliganden über Azakronenether bis hin zu komplexen Kryptanden reicht, die über nukleophile Substitution an das Fluorophor geknüpft werden können. In Tab. 2.8 sind die Reaktionsbedingungen und Ausbeuten der Synthesen von ausgewählten literaturbekannten Fluoreszenzchemosensoren aufgeführt, die durch nukleophile Substitutionsreaktionen dargestellt wurden.

Tab. 2.8: Ausgewählte literaturbekannte nukleophile Substitutionsreaktionen zum Aufbau von Fluoreszenzchemosensoren.

\begin{tabular}{|c|c|c|c|c|c|}
\hline Edukt & Base & $\operatorname{Amin}\left(N R_{2}\right)$ & Produkt & Ausbeute & Lösungsmittel \\
\hline $\mathrm{BrCH}_{2} \mathrm{AnCH}_{2} \mathrm{Br}$ & Urotropin & Urotropin & $\mathrm{H}_{2} \mathrm{NCH}_{2} \mathrm{AnCH}_{2} \mathrm{NH}_{2}$ & $83 \%^{[31]}$ & $\mathrm{CHCl}_{3}$ \\
\hline $\mathrm{BrCH}_{2} \mathrm{AnCH}_{2} \mathrm{Br}$ & $\mathrm{K}_{2} \mathrm{CO}_{3}$ & & $\mathrm{R}_{2} \mathrm{NCH}_{2} \mathrm{AnCH}_{2} \mathrm{NR}_{2}$ & $87 \%^{[21]}$ & $\mathrm{CHCl}_{3}$ \\
\hline $\begin{array}{l}\mathrm{BrCH}_{2} \mathrm{AnH} \\
\mathrm{BrCH}_{2} \mathrm{AnCN}\end{array}$ & $\mathrm{K}_{2} \mathrm{CO}_{3}$ & & $\begin{array}{l}\mathrm{R}_{2} \mathrm{NCH}_{2} \mathrm{AnH} \\
\mathrm{R}_{2} \mathrm{NCH}_{2} \mathrm{AnCN}\end{array}$ & $\begin{array}{l}90 \%^{[25]} \\
91 \%^{[25]}\end{array}$ & $\mathrm{CH}_{3} \mathrm{CN}$ \\
\hline $\mathrm{BrCH}_{2} \mathrm{AnH}$ & $\mathrm{K}_{2} \mathrm{CO}_{3}$ & & $\mathrm{R}_{2} \mathrm{NCH}_{2} \mathrm{AnH}$ & $79 \%^{[23]}$ & DMF \\
\hline $\begin{array}{l}\mathrm{ClCH}_{2} \mathrm{AnH} \\
\mathrm{ClCH}_{2} \mathrm{AnH} \\
\mathrm{ClCH}_{2} \mathrm{AnCH}_{2} \mathrm{Cl}\end{array}$ & $\begin{array}{l}\mathrm{Na}_{2} \mathrm{CO}_{3} \\
\mathrm{Na}_{2} \mathrm{CO}_{3} / \mathrm{KI} \\
\mathrm{Na}_{2} \mathrm{CO}_{3} / \mathrm{KI}\end{array}$ & & $\begin{array}{l}\mathrm{R}_{2} \mathrm{NCH}_{2} \mathrm{AnH} \\
\mathrm{R}_{2} \mathrm{NCH}_{2} \mathrm{AnH} \\
\mathrm{R}_{2} \mathrm{NCH}_{2} \mathrm{AnCH}_{2} \mathrm{NR}_{2}\end{array}$ & $\begin{array}{l}19 \%^{[32,60,62]} \\
68 \%^{[32,60,62]} \\
61 \%^{[32,60,62]}\end{array}$ & $\begin{array}{l}\mathrm{CH}_{3} \mathrm{CN} \\
\mathrm{CH}_{3} \mathrm{CN} \\
\mathrm{CHCl}_{3} / \\
\mathrm{CH}_{3} \mathrm{CN}\end{array}$ \\
\hline $\mathrm{ClCH}_{2} \mathrm{AnH}$ & $\mathrm{Na}_{2} \mathrm{CO}_{3} / \mathrm{KI}$ & & $\mathrm{R}_{2} \mathrm{NCH}_{2} \mathrm{AnH}$ & $73 \%^{[60,150]}$ & $\mathrm{CH}_{3} \mathrm{CN}$ \\
\hline $\mathrm{ClCH}_{2} \mathrm{AnH}$ & $\overbrace{}^{N H})_{n}$ & & $\mathrm{R}_{2} \mathrm{NCH}_{2} \mathrm{AnH}$ & $\begin{array}{l}n=1: 58 \%^{[30,151]} \\
n=2: 56 \%^{[30,151]} \\
n=4: 40 \%^{[30,151]} \\
n=5: 40 \%^{[151]}\end{array}$ & Toluol \\
\hline $\mathrm{ClCH}_{2} \mathrm{AnH}$ & $\mathrm{K}_{2} \mathrm{CO}_{3}$ & & $\mathrm{R}_{2} \mathrm{NCH}_{2} \mathrm{AnH}$ & $\begin{array}{l}\mathrm{n}=4: 68 \%^{[64,152]} \\
\mathrm{n}=5: 65 \%^{[64,152]}\end{array}$ & Toluol \\
\hline $\mathrm{ClCH}_{2} \mathrm{AnCH}_{2} \mathrm{Cl}$ & $\mathrm{K}_{2} \mathrm{CO}_{3}$ & & $\mathrm{R}_{2} \mathrm{NCH}_{2} \mathrm{AnCH}_{2} \mathrm{NR}_{2}$ & $14 \%^{[20,29]}$ & $\mathrm{DMF}$ \\
\hline $\mathrm{ClCH}_{2} \mathrm{AnH}$ & $\mathrm{NaH}$ & & $\mathrm{R}_{2} \mathrm{NCH}_{2} \mathrm{AnH}$ & $54 \%^{[61,63]}$ & DMF \\
\hline $\begin{array}{l}\mathrm{ClCH}_{2} \mathrm{AnH} \\
\mathrm{ClCH}_{2} \mathrm{AnCH}_{2} \mathrm{Cl}\end{array}$ & $\mathrm{Na}_{2} \mathrm{CO}_{3}$ & & $\begin{array}{l}\mathrm{R}_{2} \mathrm{NCH}_{2} \mathrm{AnH} \\
\mathrm{R}_{2} \mathrm{NCH}_{2} \mathrm{AnCH}_{2} \mathrm{NR}_{2}\end{array}$ & $90 \%$ & $\mathrm{CH}_{3} \mathrm{CN}$ \\
\hline $\mathrm{ClCH}_{2} \mathrm{AnCH}_{2} \mathrm{Cl}$ & $\mathrm{NEt}_{3}$ & & $\mathrm{R}_{2} \mathrm{NCH}_{2} \mathrm{AnCH}_{2} \mathrm{NR}_{2}$ & $70 \%^{[24]}$ & Toluol \\
\hline
\end{tabular}


Es zeigt sich, dass ein Großteil der publizierten Fluoreszenzchemosensoren, die das Anthracengerüst als Fluorophor tragen, über die entsprechenden Chlormethylderivate dargestellt wird. Ein Vergleich der Ausbeuten, die bei der Umsetzung mit Chlormethyl- und Brommethylanthracenen erhalten werden, zeigen, dass die Brommethylderivate durchschnittlich um $30 \%$ bessere Ergebnisse liefern. Dass dennoch hauptsächlich Chlormethylderivate als Ausgangsverbindungen zum Einsatz kommen, liegt zum Einen vermutlich daran, dass diese im Gegensatz zu den Bromderivaten kommerziell erhältlich sind und zum Anderen daran, dass sinnvolle Synthesewege der Bromderivate bisher kaum oder gar nicht bekannt sind.

Für die Umsetzung der Amine mit den Halogenmethylderivaten ist eine Base nötig, um die Amine in bessere Nukleophile umzuwandeln. Die Base selbst darf hierbei nicht nukleophil sein, da ansonsten Konkurrenzreaktionen bei der Substitution des Halogens auftreten würden. Somit kommen tertiäre Amine wie Triethylamin, das zur Umsetzung eingesetzte sekundäre Amin im Überschuss oder Carbonate in Frage. Eine Aktivierung der Amine durch Deprotonierung mit $n$ BuLi unter Bildung von Lithiumamiden, wie sie in dieser Arbeit angewendet wurde, ist ebenfalls möglich (GI. 2.29).
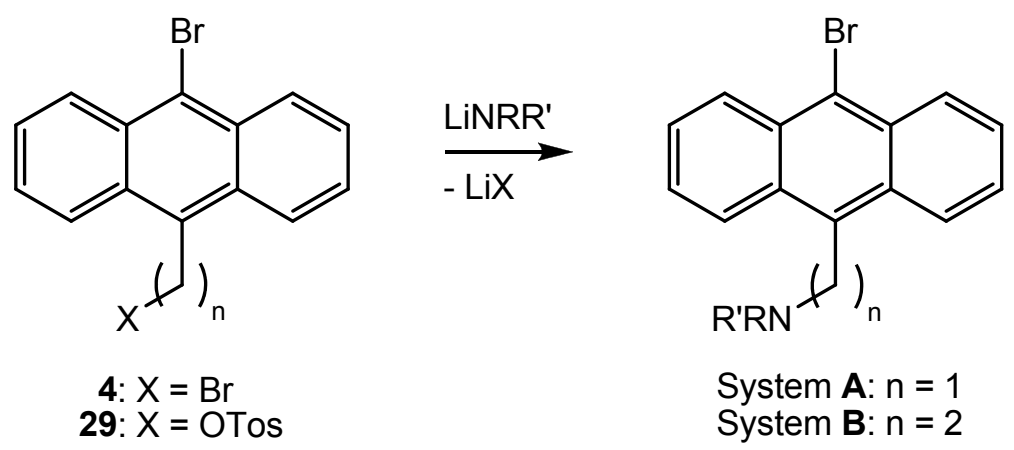

GI. 2.29: Aufbau der Rezeptoreinheit durch nukleophile Substitution bei den Sensorsystemen $\mathbf{A}$ und $\mathbf{B}$.

Die Deprotonierung der Amine wurde in THF bei Raumtemperatur durchgeführt, wobei die

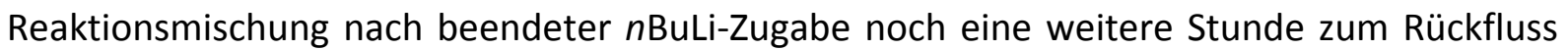
erhitzt wurde.

Da in dieser Arbeit kein spezieller Analyt, sondern der Syntheseweg zu einem modularen Sensorsystem im Vordergrund stand, wurde die Einführung von Rezeptoreinheiten an zwei einfachen sekundären Aminen getestet. Hierzu wurden das aliphatische Amin $\mathrm{N}, \mathrm{N}, \mathrm{N}$-Trimethylethylendiamin (TrMEDA) und das aromatische Amin Dipyridylamin (DPA) verwendet, welche wie oben beschrieben in ihre Lithiumsalze überführt wurden. Die Umsetzung der Lithiumamide wurde bei Raumtemperatur durchgeführt, wobei die Lösung des Lithiumamids in THF mit einer Lösung von 9-Brom-10-brommethylanthracen in THF versetzt wurde. Für eine möglichst vollständige Reaktion wurde die Reaktionsmischung nach beendeter Zugabe eine Stunde zum Rückfluss erhitzt (GI. 2.30). 
<smiles>[Y]Cc1c2ccccc2c(Br)c2ccccc12</smiles>

Gl. 2.30: Aufbau der verschidener Rezeptoreinheiten durch nukleophile Substitution bei den Sensorsystemen A und $\mathbf{B}$ über Lithiumamide.

Auf diese Weise konnten die entsprechenden Amine als Rezeptoreinheiten in das System A in guten Ausbeuten eingeführt werden. Wie auch das literaturbekannte symmetrisch mit TrMEDA substituierte Derivat ist auch die monosubstituierte Verbindung $\mathbf{3 0}$ unter leicht aziden Bedingungen wasserlöslich. Die Aufarbeitung, welche ein Extrahieren der organischen Lösung mit Wasser zur Abtrennung der gebildeten Lithiumsalze beinhaltet, sollte daher unter leicht basischen Bedingungen erfolgen. Auf diese Weise kann $\mathbf{3 0}$ nahezu quantitativ gewonnen werden. Wie aus dem ${ }^{1} \mathrm{H}$-NMR-Spektrum ersichtlich, enthält das so aufgereinigte Produkt keine nennenswerten Verunreinigungen und kann direkt für die weitere Synthese verwendet werden (siehe Abb. 2.25).

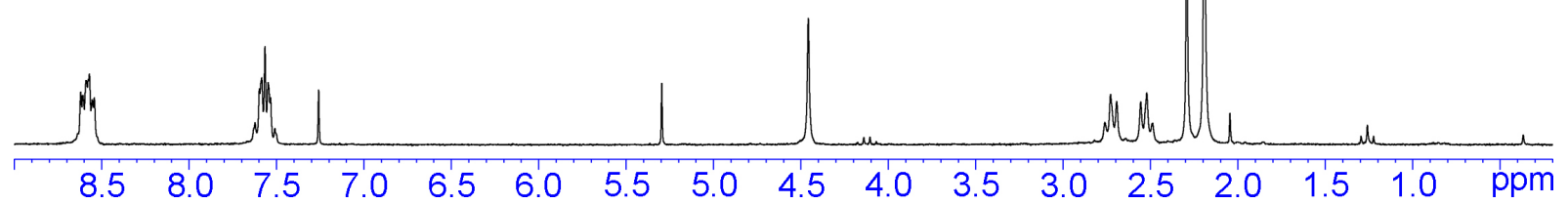

Abb. 2.25: ${ }^{1} \mathrm{H}-\mathrm{NMR}-S p e k t r u m$ der aufgereinigten Verbindung 30. Neben den Singnalen der Verbindung sind noch Spuren von Diethylether, Dichlormethan, Choroform und Aceton enthalten.

Während $\mathbf{3 0}$ als hochviskose Flüssigkeit erhalten wird, bildet das bei der Umsetzung von $\mathbf{4}$ mit LiDPA erhaltene Produkt 31 einen gelben Feststoff, welcher zur Aufreinigung leicht aus chlorierten Lösungsmitteln umkristallisiert werden kann. Die Molekülstruktur von $\mathbf{3 1}$ ist in Abb. 2.26 dargestellt. 

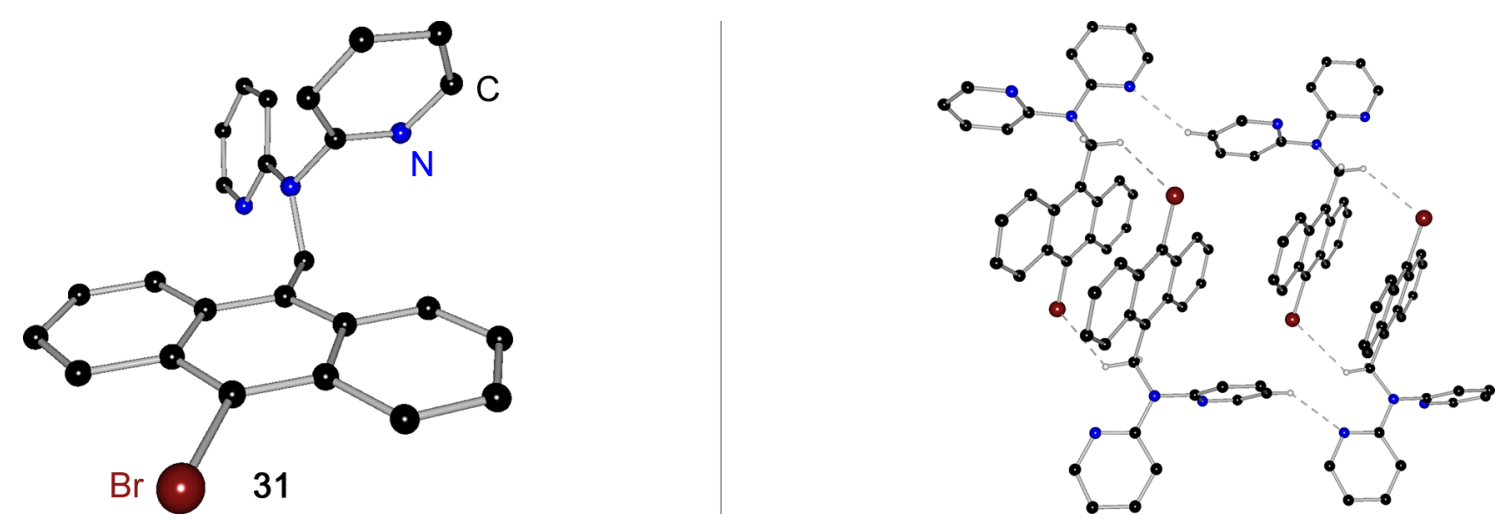

Abb. 2.26: Molekülstruktur von 31 (links) und in der Molekülpackung auftretende intermolekulare Wasserstoffbrückenbindungen (rechts).

Bei den Versuchen zur Umsetzung des Systems B unter analogen Bedingungen traten entgegen der Erwartungen deutliche Probleme auf. Es zeigte sich, dass der Tosylalkohol 29 unter den gewählten Reaktionsbedingungen inert ist, so dass dieser stets aus den Reaktionsansätzen zurückgewonnen wurde. Die Tatsache, dass obwohl mit dem Tosylat eine bessere Abgangsgruppe als im 9-Brom-10-brommethylanthracen vorhanden ist, keine Reaktion stattfindet, kann evtl. in der Orientierung des Tosylrestes zum Anthracengerüst begründet sein. So bilden Protonen am Phenylring des Tosylrestes intramolekulare Wasserstoffbrücken zur $\pi$-Elektronendichte des Anthracengerüstes aus (siehe Abb. 2.27).

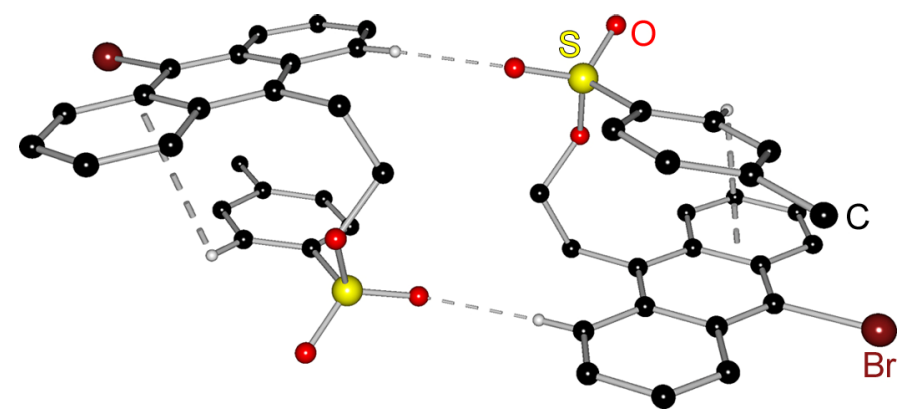

Abb. 2.27: Inter- und Intramolekulare Wasserstoffbrückenbindungen in der Molekülpackung von 29.

Sollte diese Orientierung auch in Lösung auftreten und eine gewisse Stabilität aufweisen, würde diese die $\mathrm{S}_{\mathrm{N}} 2$-Reaktion hemmen. Wie in Gl. 2.31 gezeigt, wird ein fünfbindiges trigonalbipyramidales Kohlenstoffatom bei der $\mathrm{S}_{\mathrm{N}} 2$-Reaktion als Übergangszustand angenommen.

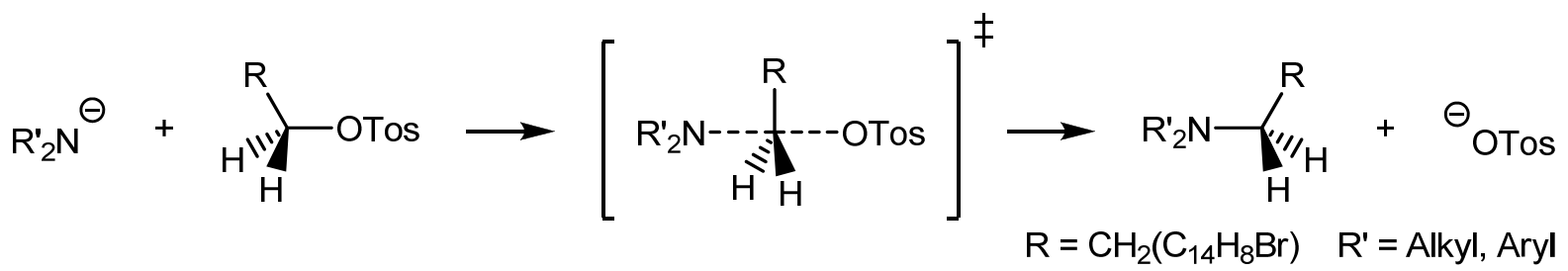

GI. 2.31: Der für $\mathrm{S}_{\mathrm{N}}$ 2-Reaktionen angenommene Mechanismus. 
Abgesehen von der eintretenden Gruppe $\left[\mathrm{NR}_{2}{ }_{2}\right]^{-}$und der abgehenden Gruppe [OTos] müssen die übrigen drei Substituenten dieses Kohlenstoffatoms in einer Ebene angeordnet sein. Diese Geometrieänderung bedingt eine Umorientierung der Tosylgruppe in $\mathbf{2 9}$ und würde zu einem Bruch der Wasserstoffbrücken führen.

Ob die Orientierung des Tosylrestes in Lösung Bestand hat und somit auf die Reaktivität der $\mathrm{S}_{\mathrm{N}}$ 2-Reaktion Einfluss nimmt, konnte im Verlauf dieser Arbeit nicht geklärt werden. Die Verwendung von analogen Derivaten (wie in Abschnitt 2.5 bereits erwähnt) mit anderen Abgangsgruppen wie z. B. Triflat, Mesylat oder Bromid sollte näheren Aufschluss bringen.

\subsection{Einführung von Phosphanen in substituierte Anthracene}

Die Einführung der als Tuner vorgesehenen Phosphorsubstituenten in Anthracene lässt sich leicht über die Umsetzung von Diorganochlorphosphanen mit Anthryllithiumverbindungen (mono- oder dilithiiert) realisieren. Durch die Wahl der organischen Reste am Phosphoratom sowie der Substituenten der 9-Bromanthracenderivate lassen sich eine Vielzahl unterschiedlicher Systeme synthetisieren (GI. 2.32).<smiles>[R]c1c2ccccc2c(Br)c2ccccc12</smiles>

1) $n \mathrm{BuLi}$

2) $\mathrm{CIPR}_{2}$

1) $-n \mathrm{BuBr}$

2) - $\mathrm{LiCl}$<smiles>[R]Cc1c2ccccc2c(P([R])[R])c2ccccc12</smiles>

$\mathrm{E}=\mathrm{O}, \mathrm{S}, \mathrm{Se}$

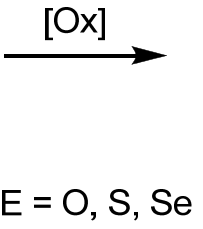<smiles>[R]c1c2ccccc2c([PH]([R])([R])F)c2ccccc12</smiles>

GI. 2.32: Syntheseweg zu Phosphanyl- und Phosphoranylanthracenen.

Dieser Syntheseweg ist nicht auf bestimmte Chlorphosphane beschränkt, was in vorangegangenen Arbeiten eindrucksvoll durch die Synthesen einer Vielzahl von mono- und disubstituierten Phosphanylanthracen gezeigt werden konnte. So wurde auch im Rahmen dieser Arbeit eine Reihe von Phosphanyl- und Phosphoranylanthracenen synthetisiert. Die erhaltenen Molekülstrukturen sind in Abb. 2.28, Abb. 2.30, und Abb. 2.32 dargestellt. Bei den in Abb. 2.28 dargestellten Derivaten handelt es sich um die Diisopropylphosphanyl- und Diisopropylphosphoranylanthracene, welche aus der Reaktion von 9-Anthryllithium mit Diisopropylchlorphosphan und gegebenenfalls anschließender Oxidation erhalten wurden. Anhand dieser Derivate wurde der Einfluss der am Phosphoratom befindlichen Isopropylgruppen auf die Rotationsbarriere des Phosphans und der Einfluss dieser auf die Verbreiterung einiger Signale im ${ }^{1} \mathrm{H}$-NMR-Spektrum untersucht. ${ }^{[89]}$ Da diese Verbindungen in 10-Position lediglich über ein Wasserstoffatom verfügen und es sich bei ihnen bis auf Verbindung 35 um Phosphor(V)-derivate handelt, sind sie für weitere Synthesen uninteressant. Auch Lumineszenz im sichtbaren Bereich konnte für keine dieser 
Verbindungen beobachte werden, so dass diese auch für Versuche zur Photodimerisierung nicht in Betracht gezogen wurden. Aus diesen Dialkylphosphanylanthracenen sind Metallkomplexe, wie sie für einige Diphenylphosphanyl- und Diphenylphosphoranylanthracene bekannt sind, ${ }^{[37,153-156]}$ zugänglich.
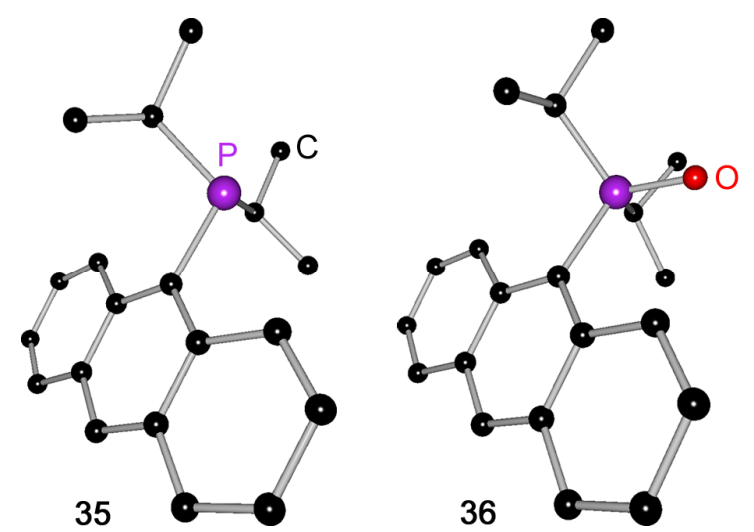
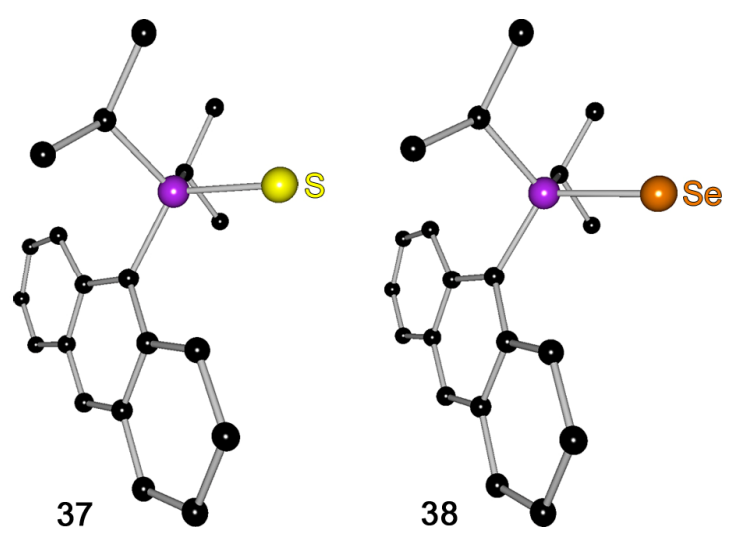

Abb. 2.28: Molekülstrukturen der isopropylsubstituierten Phoshanyl- und Phosphoranylanthracene 35-38.

So stellten Osawa et al. in diesem Jahr einen Europium(III)komplex vor, der neben drei Hexafluroacetylacetonatanionen (HFAC) noch

von

zwei Diisopropylphosphoranylanthracenen (DIOPAn) als Liganden verzerrt quadratischantiprismatisch koordiniert wird. Die beiden Diisopropylphosphoranylanthracene sind transständig angeordnet, so dass die drei Hexafluroacetylacetonatanionen sich zwischen diesen beiden befinden (Abb. 2.29). ${ }^{[157]}$
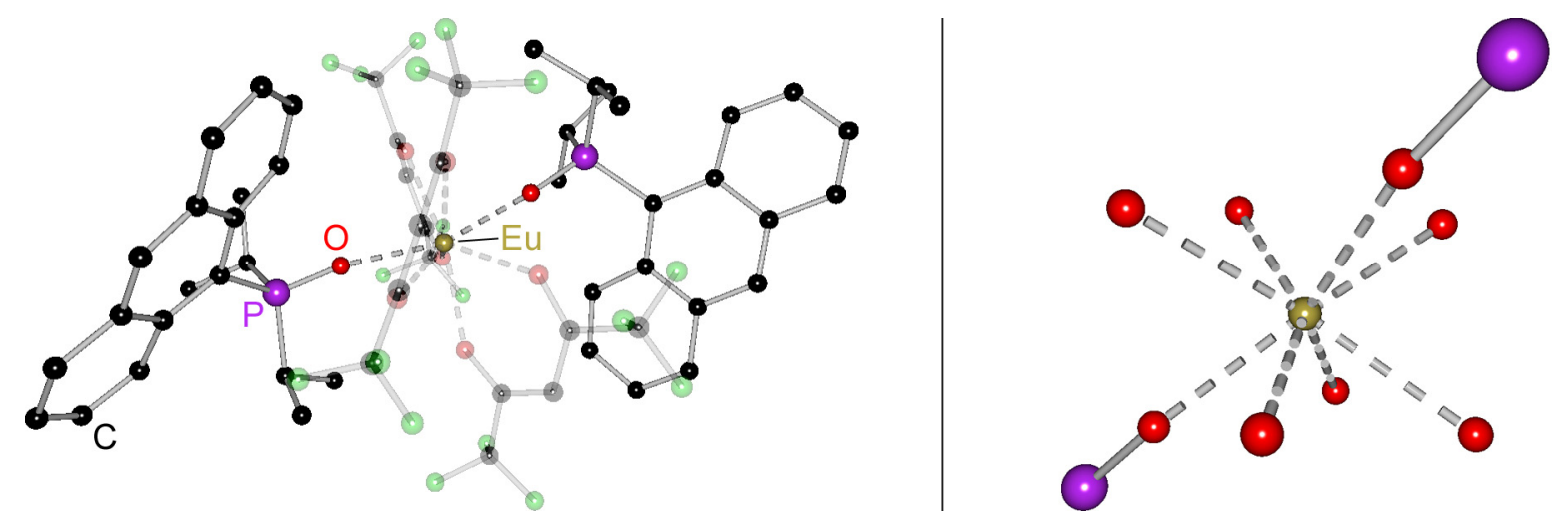

Abb. 2.29: Molekülstruktur des von Osawa et al. synthetisierten Europiumkomplex [Eu\{hfac $\left.\}_{3}\{\text { dipan }\}_{2}\right]$ links; Darstellung des Koordinationspolyeders rechts. ${ }^{[157]}$

Mit insgesamt drei Komplexen wurde der Energietransfer der angeregten Liganden auf das Europiumatom untersucht. Bei dem Komplex [Eu\{hfac $\left.\}_{3}\{\text { dipan }\}_{2}\right]$ wurde festgestellt, dass kein Energietransfer zwischen dem Anthracenliganden und dem Europiumatom stattfindet, so dass die beobachtete Fluoreszenz ausschließlich auf den Liganden zurückgeführt werden konnte. Das Emissionsspektrum des Liganden hat drei Maxima bei 402, 425 und $448 \mathrm{~nm}$ und liegt damit außerhalb des sichtbaren Bereichs. Bemerkenswert ist die mit $\Phi_{f}=0.85(5)$ bestimmte Quantenausbeute des reinen Diisopropylphosphoranylanthracenliganden. 
Ein größerer Nutzen wurde sich von den in Abb. 2.30 und Abb. 2.32 dargestellten Verbindungen erhofft. Bei diesen handelt es sich um Bis(dialkylamino)phosphanyl- und Bis(dialkylamino)phosphoranylanthracene, bei denen die Phosphoratome mit zwei Stickstoffatomen substituiert sind und somit eine Komplexierung von Metallen begünstigen könnten. Die freien Elektronenpaare dieser Stickstoffatome könnten ebenfalls in der Lage sein die Fluoreszenz des Anthracengerüstes zu quenchen, so dass hier ein weiterer Weg zu einem 'logic-device' wie in Abschnitt 2.4 erwähnt, möglich ist.
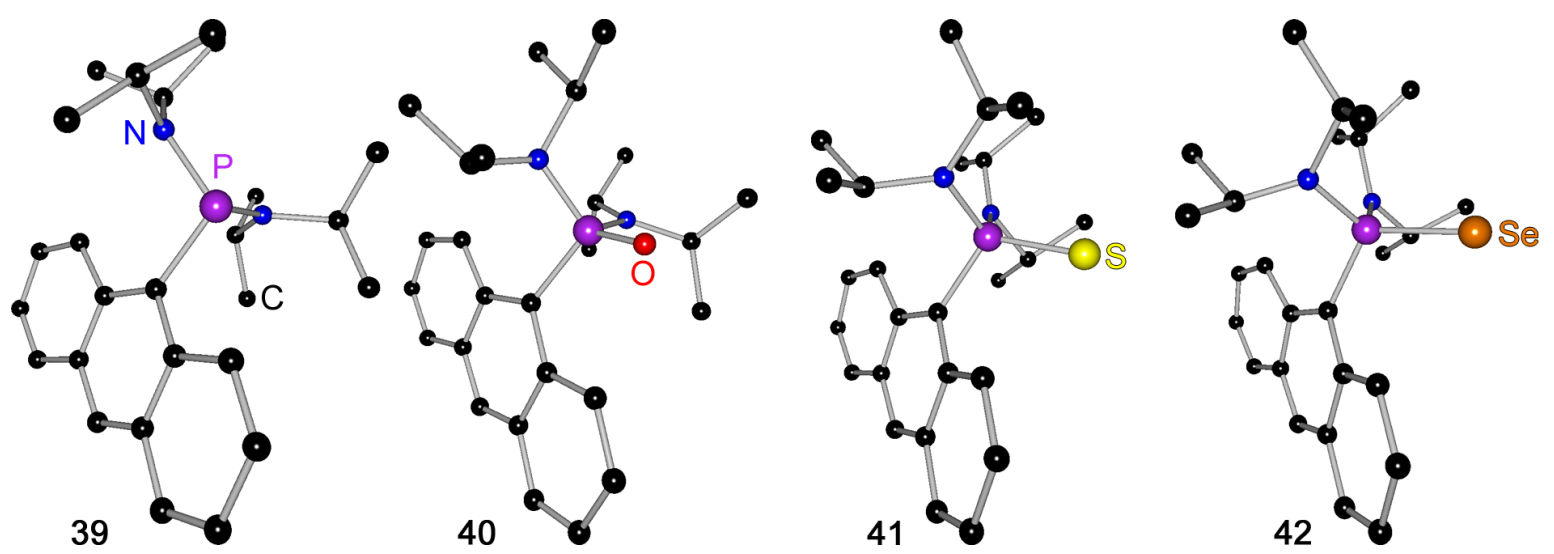

Abb. 2.30: Molekülstrukturen der Bis(diisopropylamino) substituierten Phoshanyl- und Phosphoranylanthracene 39-42.

Wie aus den in Abb. 2.30 dargestellten Molekülstrukturen ersichtlich wird, sind die Stickstoffatome durch die relativ großen Isopropylsubstituenten sterisch stark abgeschirmt, was sich auch in ihrer nahezu planaren Geometrie widerspiegelt. Eine Komplexierung von Metallen als Zweitanalyten über die Stickstoffatome scheint daher wenig wahrscheinlich. Tatsächlich blieben Komplexierungsversuche der Bis(diisopropylamino)phosphanylanthracene mit einigen Metallsalzen, bis auf den in Abb. 2.31 gezeigten Komplex, erfolglos.
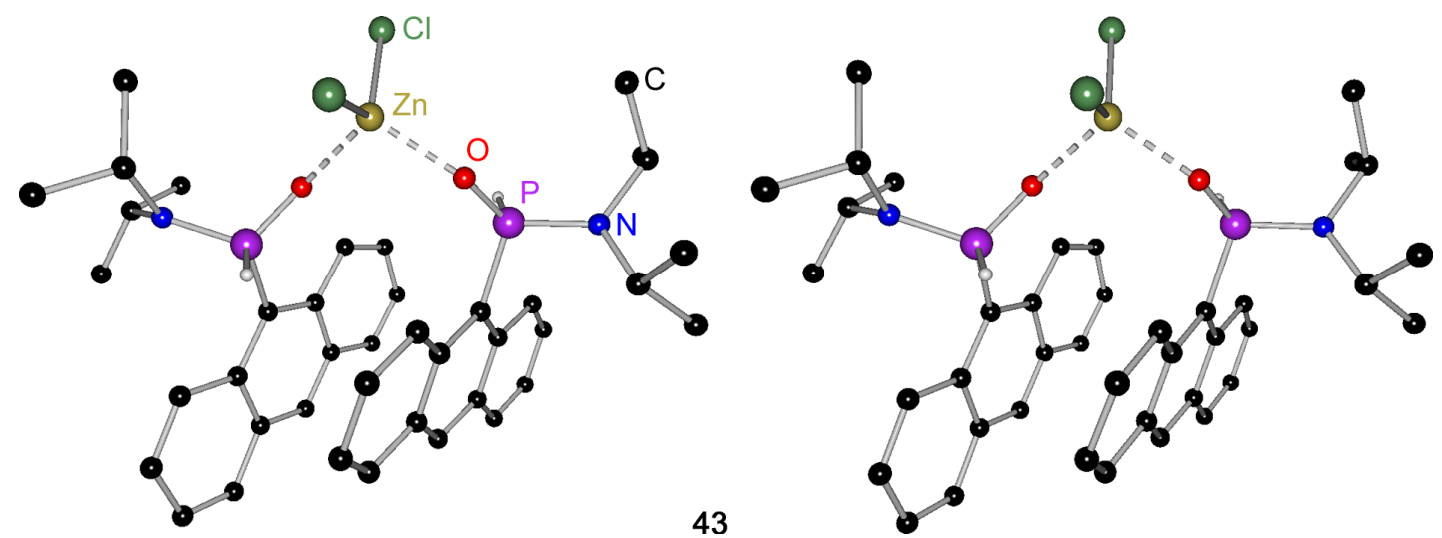

Abb. 2.31: Stereographische Darstellung der Molekülstruktur des aus 39 und Zinkchlorid gebildeten Metallkomplexes 43. 
Der aus Zinkchlorid und Bis(diisopropylamino)phosphanylanthracen gebildete Komplex 43 enthält nicht mehr das eingesetzte Phosphan 39, sondern dessen Reaktionsprodukt mit (vermutlich im Zinkchlorid enthaltenem) Wasser. Eine Diisopropylamingruppe am Phosphoratom wurde gegen eine $\mathrm{OH}-G$ ruppe substituiert, welche sich durch die Oxophilie des Phosphoratoms in das entsprechende Oxophosphoran umwandelt (GI. 2.33).<smiles>CC(C)C(C)N(C(C)C)C(C)C</smiles>

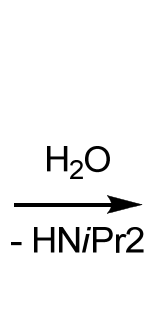<smiles>CC(C)N(C(C)C)C(O)Pc1c2ccccc2cc2ccccc12</smiles><smiles>O=[PH]C(=O)c1c2ccccc2cc2ccccc12</smiles>

GI. 2.33: Entstehung des in Komplex 43 enthaltenen Diisopropylaminooxophosphoranylanthracens.

Das Zinkatom wird tetraedrisch über zwei Chloratome und die Sauerstoffatome zweier Oxophosphorane koordiniert. Die beiden Diisopropylaminogruppen sind auch hier nahezu planar und nehmen wie erwartet nicht an der Koordination des Zinkatoms teil.

Das Problem der sterischen Abschirmung um die Stickstoffatome sollte durch die Synthese analoger Derivate mit kleineren organischen Resten umgangen werden, so dass die Synthese von Bis(dimethylamino)phosphanylanthracen angestrebt wurde. Das für die Synthese benötigte Bis(dimethylamino)chlorphosphan ist kommerziell nicht erhältlich und musste daher synthetisiert werden. Die Darstellung dieses Chlorphosphans kann analog zu der Synthese anderer Bis(dialkylamino)chlorphosphane durchgeführt werden, und erfolgt durch die Umsetzung von $\mathrm{PCl}_{3}$ mit vier Äquivalenten des entsprechenden Amins (GI. 2.34).<smiles>ClP(Cl)Cl</smiles><smiles>[R]N([R])[14CH3]</smiles><smiles>[R]NC[Te]</smiles><smiles>[R]N([R])P(Cl)N([R])[R]</smiles>

GI. 2.34: Synthese von Bis(dialkylamino)chlorphosphanen.

Durch die stöchiometrisch zugesetzten Mengen Amin kann die Anzahl der zu substituierenden Chloratome des $\mathrm{PCl}_{3}$ gesteuert werden; pro Chloratom müssen zwei Äquivalente Amin zugesetzt werden, da ein Amin durch Abfangen des entstehenden $\mathrm{HCls}$ das entsprechende Amoniumsalz bildet. Während die Einhaltung der richtigen Mengen bei den flüssigen Aminen (wie z. B. Diethylamin oder Diisopropylamin) leicht möglich ist, gestaltet sich dies, bei dem für die Synthese des Bis(dimethylamino)chlorphosphan notwendigen gasförmigen Dimethylamin, als schwierig. Die Darstellung lässt sich hier mit einem Umweg über das Tris(dimethylamino)phosphan, welches sich bei Zusatz der richtigen Menge an $\mathrm{PCl}_{3}$ zu dem gewünschten Bis(dimethylamino)chlorphosphan umsetzen lässt, leicht realisieren (GI. 2.35). 


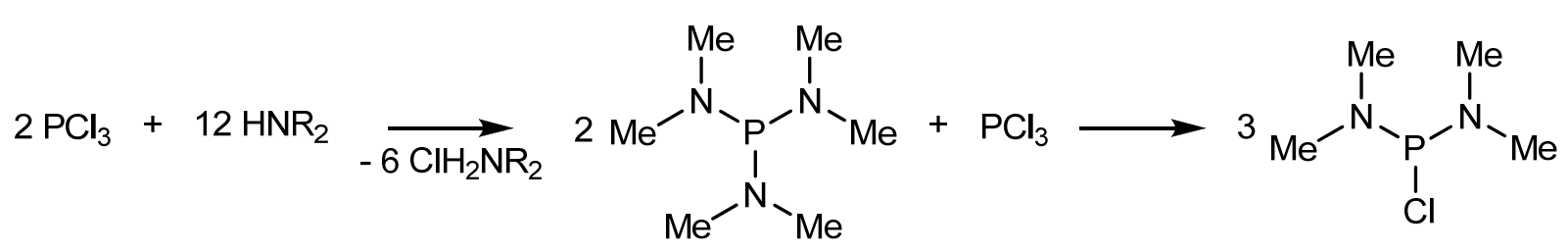

GI. 2.35: Synthese von Bis(dimethylamino)chlorphosphanen über das Tris(dimethylamino)chlorphosphan.

Aus der Synthese der symmetrisch substituierten 9,10-Di(bis(dimethylamino)phosphanyl)anthracene und der asymmetrisch substituierten 9-Brom-10-bis(dimethylamino)phosphanyl)anthracene konnten jeweils die Molekülstrukturen der mit Schwefel und Selen oxidierten Phosphorane (siehe Abb. 2.32) erhalten werden.

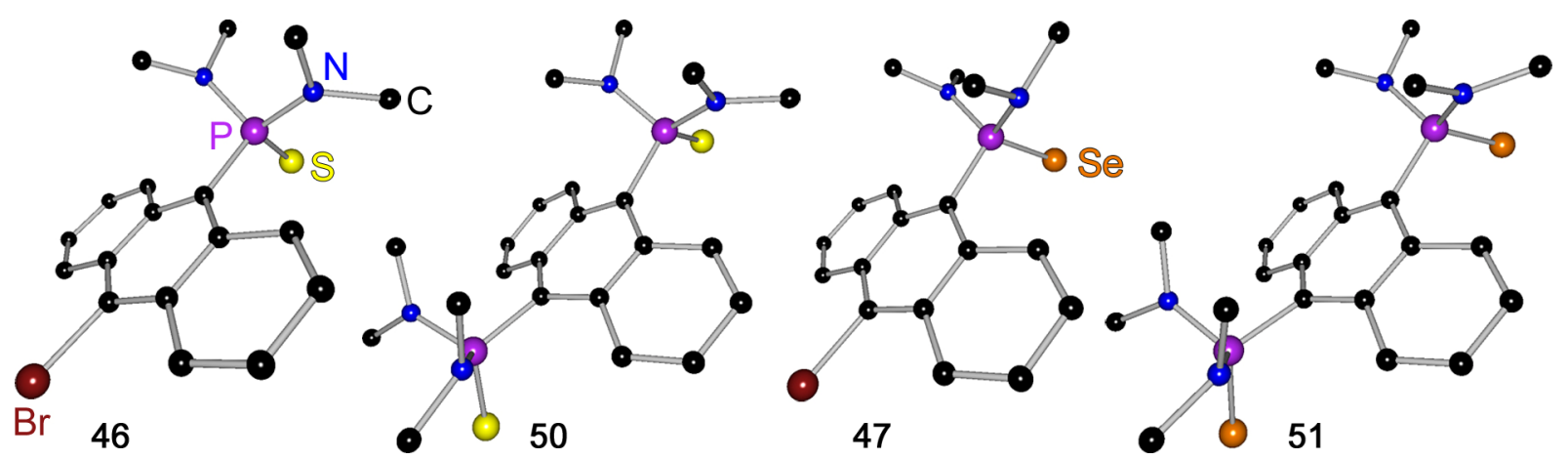

Abb. 2.32: Molekülstrukturen der Bis(dimethylamino)substituierten Phosphoranylanthracene 46, 47, 50 und 51.

Wie die zur Anthracenebene orthogonale Orientierung der Schwefel- und Selenatome zeigt, ist der sterische Anspruch der Dimethylaminogruppen ähnlich gering wie bei den entsprechenden Diphenylderivaten. Von allen bisher im Arbeitskreis Stalke verwendeten Phosphanen scheint dieses, neben dem Diphenylphosphan, welches sich durch den elektronischen Einfluss auf das $\pi$-System des Anthracengerüstes hervorgetan hat, für den Aufbau des gesuchten Chemosensors am Besten geeignet zu sein. Ein weiterer Vorteil der Bis(dialkylamino)phosphane liegt in der Substituierbarkeit der Aminosubstituenten am Phosphoratom, dieses lässt sich durch Einwirkung von $\mathrm{HCl}$ in das entsprechende Dichlorophosphan umwandeln (GI. 2.36). ${ }^{[158]}$<smiles>[R]c1c2ccccc2c(P(N([R])[R])N([R])[R])c2ccccc12</smiles>

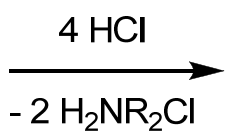<smiles>[R]c1c2ccccc2c(P(Cl)Cl)c2ccccc12</smiles>

Gl. 2.36: Substitution der Diaminosubstituenten gegen Chloratome durch Einwirkung von $\mathrm{HCl}^{[158]}$ 
Das so gebildete Anthracenyldichlorphosphan kann nun mit einer Vielzahl anderer Substituenten zum Aufbau einer zweiten Rezeptoreinheit derivatisiert werden, ${ }^{[159,160]}$ wie es im Arbeitskreis Stalke bereits gezeigt werden konnte. ${ }^{[27]}$

In Tab. 2.9 sind alle bisher im Arbeitskreis Stalke synthetisierten und durch Röntgenstrukturanalyse nachgewiesenen Phosphanylanthracene aufgelistet. Diphosphanylverbindungen mit zwei unterschiedlich substituierten Phosphanen am Anthracen, von denen ebenfalls einige röntgenkristallographisch nachgewiesen werden konnten, sind wegen ihrer schlechten Ausbeuten und ihrer fraglichen Reproduzierbarkeit nicht aufgeführt.

Tab. 2.9: Alle bisher im Arbeitskreis Stalke durch Röngenstrukturanalyse nachgewiesenen Molekülstrukturen von Phosphanyl- und Phosphoranylanthracenen.<smiles>[R]c1c2ccccc2c([PH]([R])(F)F)c2ccccc12</smiles>

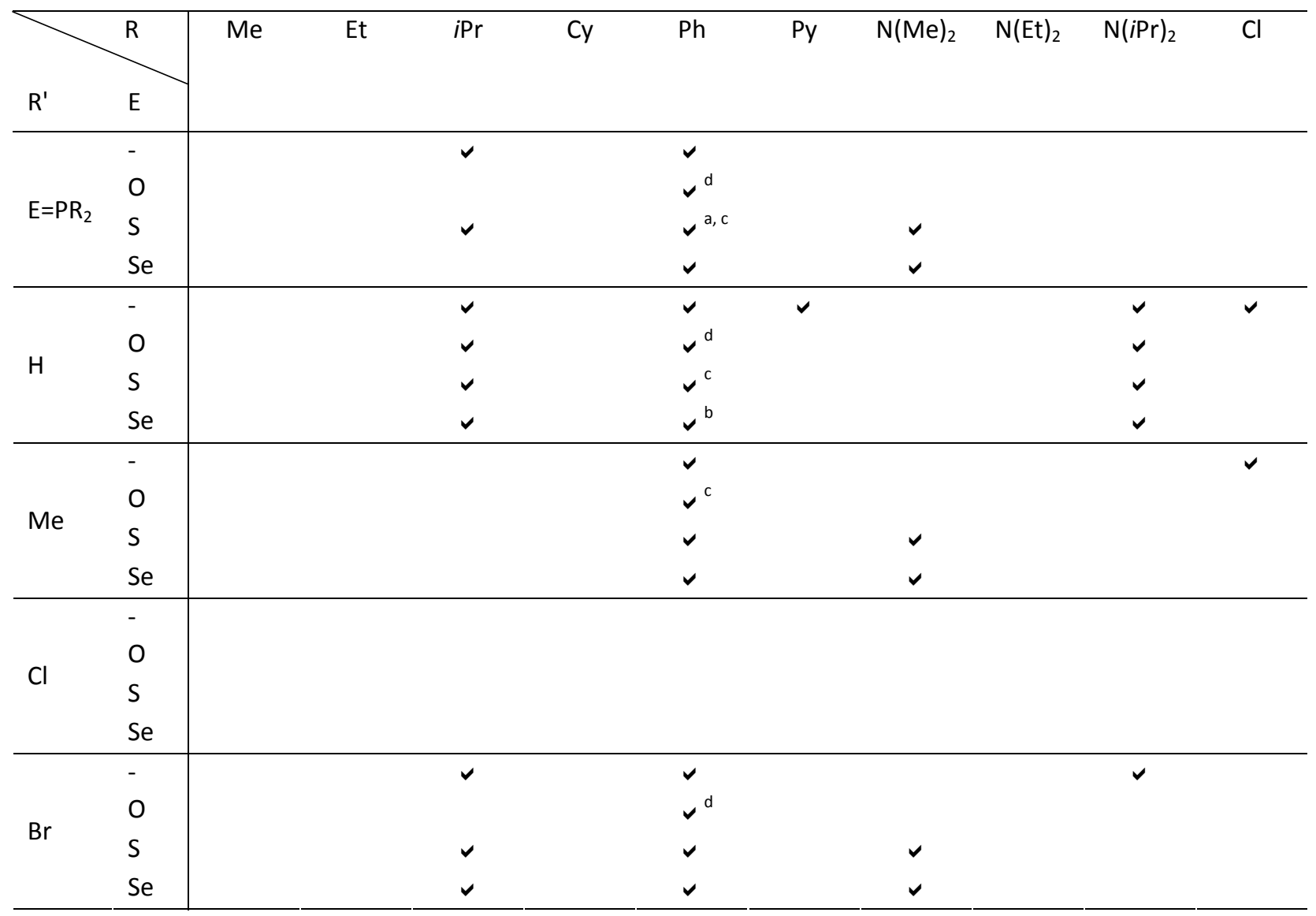

a) es wurden Molekülstrukturen mit Benzol, Toluol, o-Xylol, $m$-Xylol, $p$-Xylol, Styrol, Phenylacetylen, Benzonitril, Anisol, Benzylalkohol, Brombenzol, lodbenzol, $p$-Chlortoluol und Deuterochloroform erhalten; b) es wurden Molekülstrukturen mit Benzol, Toluol, $p$-Xylol und Nitrobenzol erhalten; c) zeigt Festkörperfluoreszenz im sichtbaren Bereich; d) zeigt Fluoreszenz in Lösung. 
Wie aus der obigen Tabelle ersichtlich, lassen sich 9-Bromanthracen und 9,10-Dibromanthracen zu den entsprechenden Phosphanylanthracenen umsetzen. Das bei der Umsetzung von 9-Brom-10-anthryllithium mit Chlorphosphanen zurückbehaltene Bromatom in 9-Positon konnte unter den bisher angewandten Bedingungen nur ungenügend gegen Lithium substituiert werden, so dass eine weitere Derivatisierung zu einem potentiellen Chemosensor ausgeschlossen wurde. Die Synthese von Phosphanylanthracenen aus den asymmetrisch substituierten Anthracenen 9-Brom-10-chloranthracen und 9-Brom-10-methylanthracen geben Anlass zu der Vermutung, dass ein umgekehrter Syntheseweg jedoch möglich sein sollte. Da die Synthese komplex substituierter Anthracene mit einem Bromatom in 9-Position über die in dem vorherigen Abschnitt beschriebenen Synthesen in guten Ausbeuten möglich ist, bleibt die Frage, ob die Einführung der gewünschten Phosphansubstituenten im letzten Schritt der Synthese möglich ist und zu den angestrebten Sensorsystemen führt.

Für einen ersten Versuch wurde das diaminsubstituierte Bromanthracen $\mathbf{3 0}$ auf bewährte Weise mit einem Äquivalent $n \mathrm{BuLi}$ in Diethylether bei $-15^{\circ} \mathrm{C}$ lithiiert und mit dem entsprechenden Chlorphosphan (hier Bis(dimethylamino)chlorphosphan)) versetzt (GI. 2.37).<smiles>CN(C)CCN(C)Cc1c2ccccc2c(Br)c2ccccc12</smiles>

1) $n \mathrm{BuLi}$

2) $\mathrm{CIP}\left(\mathrm{NMe}_{2}\right)_{2}$

1) $-n \mathrm{BuBr}$

2) $-\mathrm{LiCl}$<smiles>CN(C)CCN(C)Cc1c2ccccc2c(P(N(C)C)N(C)C)c2ccccc12</smiles>

GI. 2.37: Synthese des heterosubstituierten Anthracenen $\mathbf{5 2}$ mit Phosphantuner und Rezeptoreinheit.

Durch Massenspektrometrie konnte das gewünschte Produkt eindeutig nachgewiesen werden. NMR-Spektren zeigten jedoch die Bildung eines 1:1-Gemisches zweier Anthracenderivate (Abb. 2.33).

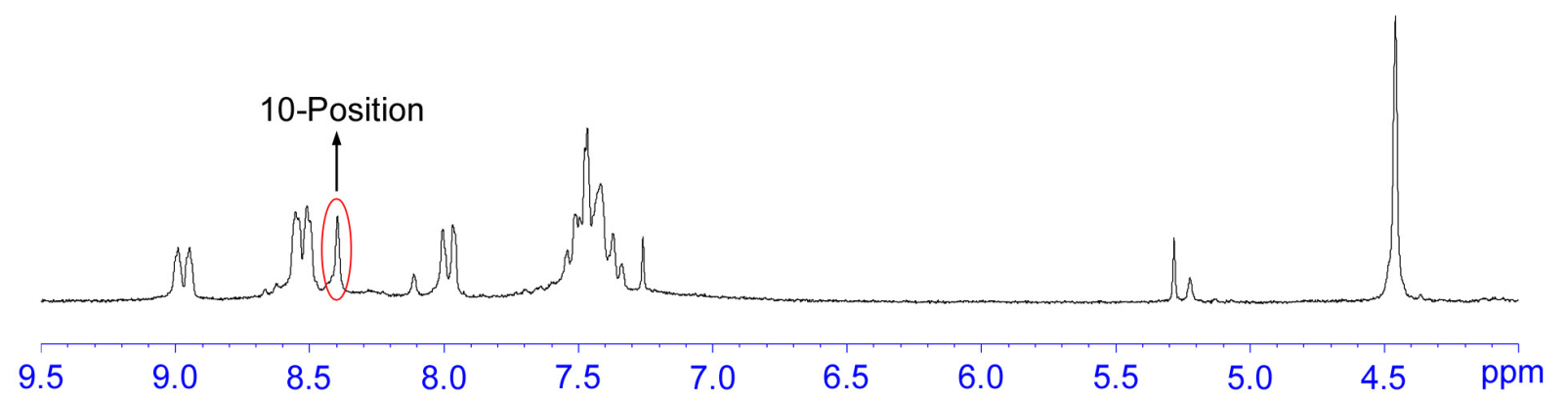

Abb. 2.33: Ausschnitt des ${ }^{1} \mathrm{H}-\mathrm{NMR}$ Spektrum des bei der Synthese von $\mathbf{5 2}$ erhaltenen Produktgemisches. 
Auffällig ist hier das Singulett bei 8.41 ppm, welches (bei Berücksichtigung der eingesetzten Stoffe) nur durch ein Wasserstoffatom in der 9,10-Position des Anthracengerüstes hervorgerufen werden kann. Durch die chemische Verschiebung dieses Signals und die Verschiebung der übrigen Signale konnten mögliche Verunreinigungen an 9-Bromanthracen und/oder Anthracen ausgeschlossen werden. Das Nebenprodukt muss demnach aus $\mathbf{3 0}$ hervorgegangen sein, wobei das Bromatom in 9-Position gegen ein Wasserstoffatom substituiert wurde. Zu einem solchen Reaktionsprodukt kann es jedoch nur kommen, wenn der gesamte Anteil an $\mathbf{3 0}$ lithiiert und im Laufe der Reaktion oder der Aufarbeitung durch Wasser hydrolisiert wurde (GI. 2.38).<smiles>[R]P([R])c1c2ccccc2c(CN(C)CCN(C)C)c2ccccc12</smiles><smiles>CN(C)CCN(C)Cc1c2ccccc2c(Cl)c2ccccc12</smiles><smiles>CN(C)CCN(C)Cc1c2ccccc2cc2ccccc12</smiles>

GI. 2.38: Bildung des Hydrolyseproduktes $\mathbf{5 4}$ bei der Synthese der Sensoren $\mathbf{5 2}$ und $\mathbf{5 3 .}$

Der positive Rückschluss, welcher sich aus dieser Nebenreaktion ziehen lässt ist, dass die Lithiierung von $\mathbf{3 0}$ unter den gewählten Reaktionsbedingungen nahezu quantitativ verläuft. Um das Nebenprodukt und die quantitative Lithiierbarkeit eindeutig nachzuweisen, wurde 30 in einem weiteren Versuch unter den gleichen Reaktionsbedingungen wie zuvor lithiiert und anschließend durch Zugabe einer Lösung von einem Äquivalent Wasser in $10 \mathrm{~mL}$ THF hydrolisiert. Das ${ }^{1} \mathrm{H}$-NMR-Spektrum des erhaltenen Produktes ist in Abb. 2.34 dem ${ }^{1} \mathrm{H}-\mathrm{NMR}-$ Spektrum des oben beschriebenen Reaktionsgemisches gegenübergestellt.

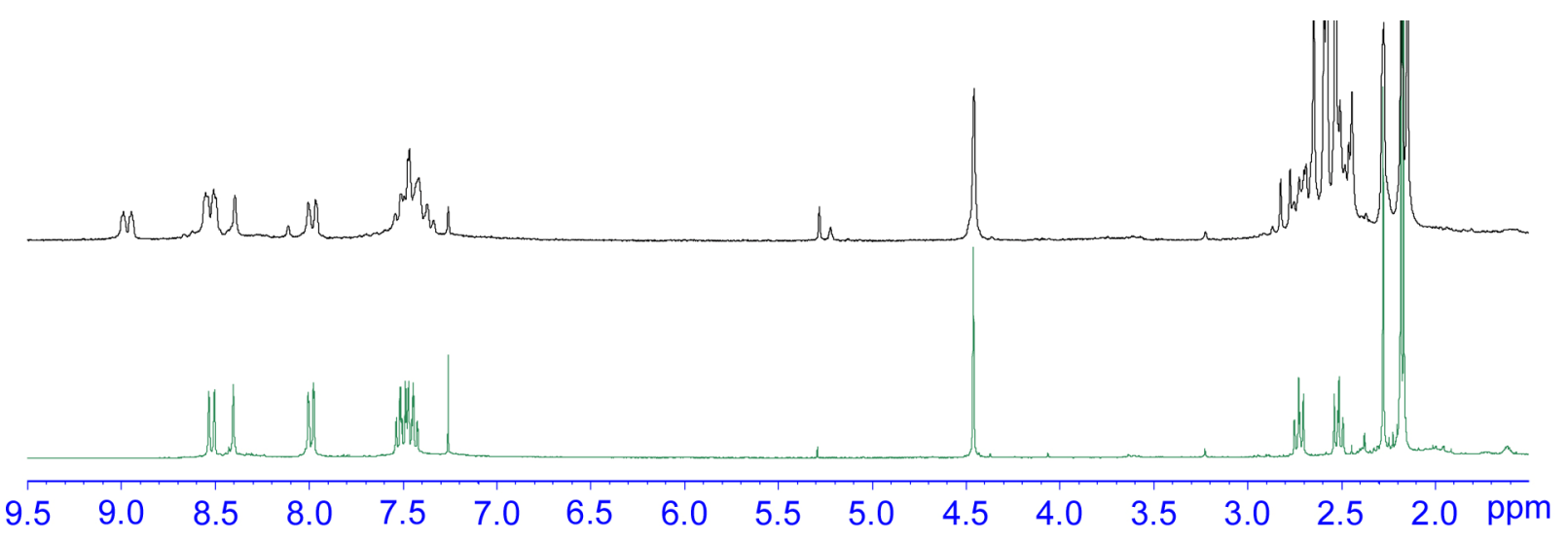

Abb. 2.34: ${ }^{1}$ H-NMR Spektren: des bei der Synthese von 52 erhalten Produktgemisches (oben); des aus der Lithiierung und anschließender Hydrolyse von $\mathbf{3 0}$ erhaltenen Produkts $\mathbf{5 4}$ (unten). 
In dem in Abb. 2.34 unten dargestellten ${ }^{1} \mathrm{H}-\mathrm{NMR}$-Spektrum ist das Hydrolyseprodukt $\mathbf{5 4} \mathrm{zu}$ sehen. Alle Signale lassen sich dem bei der Synthese von $\mathbf{5 2}$ auftretenden Nebenprodukt zuordnen. Die Hydrolyse und damit auch die Annahme, dass die Lithiierung von Verbindung 30 vollständig erfolgt, kann somit bestätigt werden. Da alle Signale der hydrolysierten Verbindung 54 auch in dem Mischspektrum enthalten sind, kann dieses eindeutig als das bei der Umsetzung von $\mathbf{3 0}$ erhaltene Nebenprodukt identifiziert werden. Offen bleibt jedoch die Frage, woher die zur Hydrolyse notwendigen Mengen an Wasser stammen. Eine Wiederholung der Versuche konnte das Lösungsmittel, welches nun frisch destilliert eingesetzt wurde, als Quelle ausschließen. Durch die Verwendung eines anderen Chlorphosphans sollte die einzig verbliebene Quelle überprüft werden. Die Synthese wurde analog zu der von $\mathbf{5 2}$ durchgeführt, jedoch mit frisch destilliertem Diphenylchlorphosphan. Auch dieser Versuch lieferte eine Mischung zweier Produkte wovon das eine das gewünschte Phosphanylanthracen und das andere wieder die Hydrolyseverbindung $\mathbf{5 4}$ war. Da nun alle an der Reaktion beteiligten Stoffe ausgetauscht wurden, bleibt die Aufarbeitung die einzige Quelle des zur Hydrolyse notwendigen Wassers. Demnach liegt vor der Aufarbeitung noch die Hälfte der lithiierten Verbindung $\mathbf{3 0}$ in der Reaktionsmischung vor. In weiteren Versuchen, bei denen die Reaktionszeit deutlich verlängert wurde, konnte die Ausbeute nicht verbessert werden. Auch ein einstündiges Refluxieren der Reaktionsmischung führte nicht zum gewünschten Erfolg. Unter Berücksichtigung des in Abschnitt 2.2.3 beschriebenen Effekts der Selbsthemmung durch Salzbildung können diese Beobachtungen erklärt werden. So muss das in der Reaktion gebildete $\mathrm{LiCl}$ mit den noch verbliebenen Anthryllithiummolekülen einen gemischten Komplex bilden, dessen Reaktivität (selbst bei Temperaturerhöhung) nicht ausreicht um mit dem restlichen Chlorphosphan zu reagieren. Da diese Mischverbindungen jedoch genauso schnell wie Lithiumorganyle mit Wasser reagieren, kommt es beim Abfüllen der NMR-Proben durch das in dem deuterierten Lösungsmittel enthaltenen Wasser zur Bildung des entsprechenden Hydrolyseproduktes. Eine deutliche Umsatzsteigerung sollte sich demnach mit dem Zusatz mindestens eines Äquivaltents PMDETA zur Entfernung des Lithiumsalzes durch Komplexierung ergeben. Versuche die dieses bestätigen, konnten jedoch im Laufe dieser Arbeit nicht mehr durchgeführt werden. 


\section{Zusammenfassung und Ausblick}

In dieser Arbeit konnte ein Syntheseweg $\mathrm{zu}$ einem modular aufgebauten Fluoreszenzchemosensorsystem auf Basis des Anthracen-Fluorophors gefunden werden. Die verwendeten Synthesewege zeichnen sich einerseits durch hohe Ausbeuten und andererseits durch die Flexibilität in den einzusetzenden Bauteilen aus.

Die beim Anthracen begonnene Synthese liefert die jeweiligen Produkte in allen Schritten mit über $80 \%$ Ausbeute. Werden die lithiierten Derivate in situ umgesetzt, verläuft die gesamte Synthese über sechs Stufen, kann jedoch bei Verwendung des kommerziell erhältlichen 9-Brom-10-methylanthracens auf vier Stufen verkürzt werden (siehe Gl. 3.1).<smiles>c1ccc2cc3ccccc3cc2c1</smiles><smiles>BrCC(Br)(Br)Br</smiles><smiles>Brc1c2ccccc2c(Br)c2ccccc12</smiles>

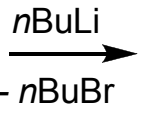<smiles>Clc1c2ccccc2c(Br)c2ccccc12</smiles><smiles>[R]N([R])Cc1c2ccccc2c(Br)c2ccccc12</smiles><smiles>[R]NC(C)(Br)Br</smiles><smiles>BrCc1c2ccccc2c(Br)c2ccccc12</smiles><smiles>BrC(Br)(Br)Br</smiles><smiles>Cc1c2ccccc2c(Br)c2ccccc12</smiles>
3

30: $R=M e, R^{\prime}=E^{\prime N M e} e_{2}$ 31: $R=R^{\prime}=P y$

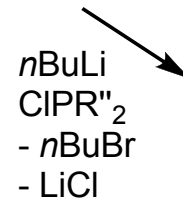<smiles>[R]N([R])Cc1c2ccccc2c(P([R7])[R7])c2ccccc12</smiles><smiles>[R]N([R])Cc1c2ccccc2c([PH]([R7])([R7])F)c2ccccc12</smiles>

52: $\mathrm{R}=\mathrm{Me}, \mathrm{R}^{\prime}=\mathrm{EtNMe}_{2}, \mathrm{R}^{\prime \prime}=\mathrm{NMe}_{2}$

53: $\mathrm{R}=\mathrm{R}^{\prime}=\mathrm{Py}, \mathrm{R}^{\prime \prime}=\mathrm{Ph}$

GI. 3.1: Syntheseweg zu Fluoreszenzchemosensoren mit Fluorophor, Spacer, Rezeptor und Tuner.

Das eingesetzte brommethylierte Anthracenderivat 4, welches sich als herausragendes Edukt zum Aufbau des Rezeptors herausgestellt hat, ist den in der Literatur verwendeten Edukten weit überlegen. In diesem Schlüsselschritt zum Aufbau des Rezeptors konnten nicht nur 
nahezu quantitative Ausbeuten erzielt werden, sondern das Produkt wurde auch in einer solchen Reinheit erhalten, dass zu einer weiteren Umsetzung zur Einführung von Tunern kein zusätzlicher Aufreinigungsschritt notwendig war. Die Substitution des Bromatoms in 9Position konnte ohne Schwierigkeiten durchgeführt werden. Die dadurch ermöglichte Derivatisierung des Fluoreszenzchemosensors ist beispiellos und bietet nicht nur ein enormes Potential zur Einstellung von Emissionsbanden und Steigerung von Quantenausbeuten im Bereich der Fluoreszenzchemosensoren, sondern bietet auch den Zugang zu organischen Farbstoffen wie sie z. B. in OLEDs zum Einsatz kommen.

Die hier angestellten Studien zur Bromierung von Anthracenderivaten, haben maßgeblich zum Verständnis der Mechanismen der Bromierung kondensierter Aromaten beigetragen und die Synthese des 9-Brom-10-brommethylanthracens im großen Maßstab, welches sich als Schlüsselreagenzes dieser Arbeit herausgestellt hat, erst ermöglicht.

Mit der alternativen Syntheseroute über die Anthracencarbaldehyde ist die Synthese komplexer Fluoreszenzchemosensoren in Form von 'logic-devices' möglich. Bei der asymmetrischen Syntheseführung kann auch hier ein Bromatom in 9-Position des Anthracengerüsts zurückbehalten werden, welches anschließend zur Einführung weiterer Gruppen wie z. B. Tunern oder weiteren Rezeptoreinheiten dienen kann.

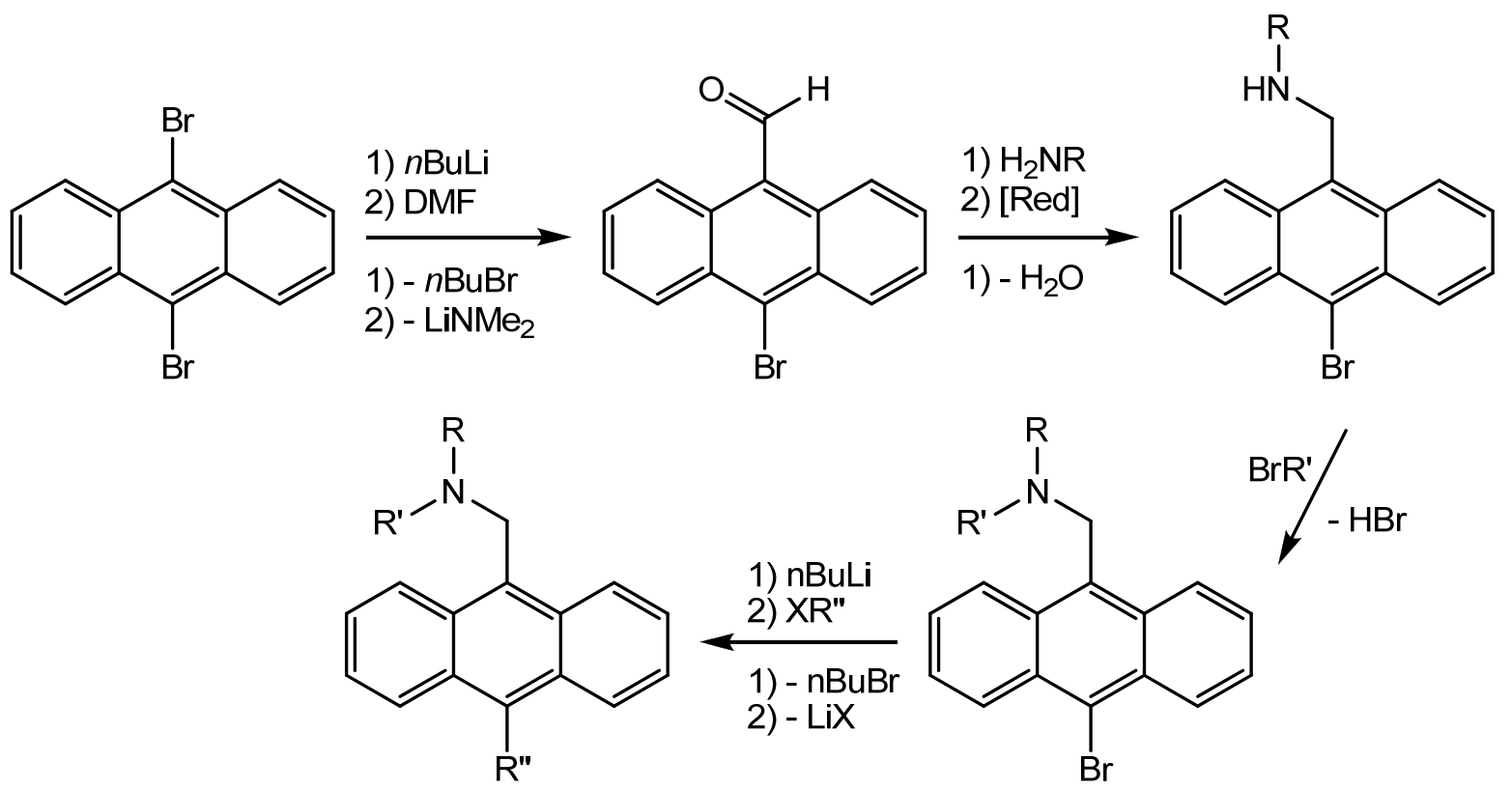

Gl. 3.2: Syntheseweg zu Fluoreszenzchemosensoren mit asymmetrisch substituierten Rezeptoren zum Aufbau von z. B. 'logic-devices'.

Aufgabe zukünftiger Arbeiten muss es sein, substratspeziefische Amine über die hier entwickelten Synthesewege an den Anthracen-Fluorophor zu binden um hochspezifische Fluoreszenzchemosensoren zu entwickeln. Der Nutzen des austauschbaren Tuners muss durch Fluoreszenzanalysen (Lage der Emissionsbanden und Quantenausbeuten) evaluiert werden. 


\section{Experimenteller Teil}

\subsection{Allgemeine Arbeitstechniken}

Alle Reaktionen wurden mit Schlenk-Technik ${ }^{[161]}$ in trockener, nachgereinigter Stickstoffatmosphäre durchgeführt. Die Glasgeräte wurden mehrere Stunden bei $120^{\circ} \mathrm{C}$ ausgeheizt, heiß zusammengesetzt und unter Vakuum abgekühlt. Luft- und feuchtigkeitsempfindliche Substanzen wurden unter Stickstoffatmosphäre oder in einer Argontrockenbox gehandhabt. Alle verwendeten Lösungsmittel wurden nach laborüblichen Methoden getrocknet und vor dem Gebrauch frisch destilliert eingesetzt. Die verwendeten metallorganischen Reagenzien wurden von der Firma Chemmetall, alle übrigen Edukte von der Firma Aldrich bezogen.

\subsection{Analysemethoden}

\subsubsection{Massenspektrometrie}

EI-MS: Gerät MAT 95; Elektronenstoßionisation (EI-MS): $70 \mathrm{eV}$. Den m/z-Werten der Molekülionen und den angegebenen ausgewählten Fragmentionen liegen jeweils die Massenzahlen der Isotope mit den größten natürlichen relativen Häufigkeiten zugrunde $\left({ }^{1} \mathrm{H}\right.$, ${ }^{12} \mathrm{C},{ }^{14} \mathrm{~N},{ }^{31} \mathrm{P},{ }^{32} \mathrm{~S},{ }^{79} \mathrm{Br} /{ }^{81} \mathrm{Br}$ ).

\subsubsection{Elementaranalyse}

Die Bestimmung der prozentualen Anteile von Kohlenstoff und Wasserstoff erfolgte durch Verbrennungsanalyse im Analytischen Labor des Institutes für Anorganische Chemie an der Georg-August-Universität Göttingen.

\subsubsection{NMR Spektroskopie}

Die Aufnahme der Spektren erfolgte mit den Geräten Bruker Avance 500 und Bruker Avance 200. Die Messungen wurden bei Raumtemperatur mit 5-10\%-igen Lösungen durchgeführt. Die chemischen Verschiebungen $\delta$ werden in ppm und die Kopplungskonstanten $J$ in $\mathrm{Hz}$ angegeben. Als externer Standard für ${ }^{1} \mathrm{H}$ - und ${ }^{13} \mathrm{C}$-Spektren diente Tetramethylsilan, für ${ }^{31} \mathrm{P}$ Spektren Orthophosphorsäure und für ${ }^{77}$ Se-Spektren Dimethylselen. Als interner Standard wurden die Restprotonensignale des deuterierten Lösungsmittels verwendet. Die Multiplizitäten werden wie folgt abgekürzt: $b r=$ breites Singal, $s=$ Singulett, $d=$ Dublett, $\mathrm{dd}=$ Dublett von Dubletts, $\mathrm{ddd}=$ Dublett von Dubletts von Dubletts, $\mathrm{m}=$ Multiplett.

Die Zuordnung der ${ }^{1} \mathrm{H}$ - und ${ }^{13} \mathrm{C}$-Signale erfolgte mittels COSY und HSQC Korrelationsspektren. 


\subsection{Allgemeine Arbeitsvorschriften (AAV)}

\subsubsection{AAV 1 Bromierung von Anthracen(derivaten)}

Eine Lösung/Suspension des zu bromierenden Anthracen/-derivates in Tetrachlorkohlenstoff (ca. $20 \mathrm{~mL} / \mathrm{g}$ ) wurde über 30 Min. bei RT mit einer Lösung von Brom in Tetrachlorkohlenstoff (ca. $20 \mathrm{~mL} / \mathrm{mL}$ ) versetzt. Nach weiteren $2 \mathrm{~h}$ Rühren bei RT wurde für ca. $1 \mathrm{~h}$ zum Rückfluss erhitzt. Nach Abziehen des Lösungsmittels und etwaigen Überschuss an Brom wurde der erhaltene gelbe Feststoff aus Dichlormethan umkristallisiert.

\subsubsection{AAV 2 Lithiierung von Bromanthracenen ohne Abtrennen von $n \mathrm{BuBr}$}

Eine Lösung/Suspension des zu lithiierenden Bromanthracens in Diethylether (ca. $20 \mathrm{~mL} / \mathrm{g}$ ) wurde bei $-15^{\circ} \mathrm{C}$ über ca. $30 \mathrm{Min}$. mit einer $n$ BuLi-Lsg. in Hexan (ca. 1.0 bis $2.25 \mathrm{M}$ ) versetzt. Nach beendeter Zugabe wurde zur Vervollständigung der Lithiierung max. 15 Min. bei RT gerührt und die entstandenen Lösung/Suspension vor der weiteren Umsetzung wieder auf $-15^{\circ} \mathrm{C}$ gekühlt.

Anmerkung:

Die so erhaltene Lösung/Suspension enthält $n$-Brombutan das nach AAV 3 abgetrennt werden kann, jedoch bei Lagerung oder Umsetzungen mit Elektrophilen in Diethylether nicht weiter stört. Sollten andere Donorbasen hinzugegeben werden und die Umsetzung nicht sofort erfolgen sollte das Abtrennen des $n \mathrm{BuBr}$ zur Vermeidung von Nebenreaktionen in Betracht gezogen werden.

\subsubsection{AAV 3 Lithiierung von Bromanthracenen mit Abtrennen von $n \mathrm{BuBr}$}

Die nach AAV 2 erhaltene Lösung/Suspension des Lithioanthracens wurde bei $-15^{\circ} \mathrm{C}$ auf ein Drittel des ursprünglichen Volumens eingeengt und anschließend auf $-78^{\circ} \mathrm{C}$ gekühlt. Der entstandene Niederschlag wurde kalt abfiltriert und mit $-78{ }^{\circ} \mathrm{C}$ kaltem Hexan oder Pentan gewaschen (ca. $5 \mathrm{~mL} / \mathrm{g}$ ). Der so erhaltene hellgelbe Feststoff wurde für max. 30 Min. i. Vak. getrocknet und anschließend zur weiteren Umsetzung/Kristallisation wieder in $-15^{\circ} \mathrm{C}$ kaltem Diethylether gelöst/suspendiert.

\subsubsection{AAV 4 Einführung von Phosphanen in Anthracene}

Die nach AVV 2 erhaltene Lösung/Suspension des lithiierten Anthracens wurde bei $-15^{\circ} \mathrm{C}$ über 30 Min. mit dem entsprechenden Chlorphosphan versetzt. Nach beendeter Zugabe wurde weitere $30 \mathrm{Min}$. bei RT gerührt und anschließend das Lösungsmittel i. Vak. entfernt. Zur Abtrennung des entstandenen Lithiumchlorids wurde der erhaltene Feststoff/die erhaltene hochviskose Flüssigkeit in trockenem Dichlormethan gelöst (ca. $10 \mathrm{~mL} / \mathrm{g}$ ) und 
anschließend über Celite ${ }^{\circledR}$ filtriert. Das entsprechende Phosphanylanthracen wurde nach Abziehen des Lösungsmittels als Feststoff/hochviskose Flüssigkeit erhalten.

\subsubsection{AAV 5 Oxidaton von Phosphanylanthracenen mit $\mathrm{H}_{2} \mathrm{O}_{2}$}

Der/die nach AAV 4 erhaltene Feststoff/hochviskose Flüssigkeit des Phosphanylanthracens wurde in einer 1 : 1 Mischung aus Dichlormethan und Methanol gelöst/suspendiert und über $30 \mathrm{Min}$. bei $-15{ }^{\circ} \mathrm{C}$ mit einer $\mathrm{H}_{2} \mathrm{O}_{2}$-Lsg. (35\%ig in Wasser) versetzt. Nach beendeter Zugabe wurde meist über Nacht aber mind. $5 \mathrm{~h}$ bei RT gerührt und anschließend das Lösungsmittelgemsich i. Vak. entfernt. Der erhaltene Feststoff wurde gegebenenfalls zur Reinigung aus Dichlormethan oder Chloroform umkristallisiert.

\subsubsection{AAV 6 Oxidation von Phosphanylanthracenen mit Schwefel oder Selen}

Der/die nach AAV 4 erhaltene Feststoff/hochviskose Flüssigkeit des Phosphanylanthracens wurde mit elementarem Schwefel oder grauem Selen versetzt, in Toluol gelöst/suspendiert (ca. $10 \mathrm{~mL} / \mathrm{g}$ ) und anschließend zur vollständigen Oxidation für mind. $4 \mathrm{~h}$ zum Rückfluss erhitzt. Um überschüssige(n)/s Schwefel/Selen abzutrennen wurde die noch warme Lösung über Celite ${ }^{\circledR}$ filtriert. Nach Abziehen des Lösungsmittels wurde das Phosphoranylanthracen als Feststoff erhalten und gegebenenfalls zur Reinigung aus Toluol umkristallisiert.

\subsection{Synthesen und Charakterisierungen}

\subsubsection{Synthese von 9-Bromanthracen (1)}

Eine Lösung von $2.0 \mathrm{~g}(11.22 \mathrm{mmol})$ Anthracene in $40 \mathrm{~mL} \mathrm{Et}{ }_{2} \mathrm{O}$ wurde bei $-78{ }^{\circ} \mathrm{C}$ über $1 \mathrm{~h}$ mit einer Lösung von $\mathrm{Br}_{2}(0.57 \mathrm{~mL}, 11.22 \mathrm{mmol})$ in $20 \mathrm{~mL} \mathrm{Et}{ }_{2} \mathrm{O}$ versetzt. Nach Erwärmen der Reaktionslösung über Nacht auf RT wurde für $2 \mathrm{~h}$ zum Rückfluss erhitzt. Der nach Abziehen des Lösungsmittels erhaltene gelbe Feststoff wurde säulenchromathographisch (Hexan) gereinigt.

Summenformel: $\quad \mathrm{C}_{14} \mathrm{H}_{9} \mathrm{Br}$.

Molare Masse: $\quad 257.13 \mathrm{~g} / \mathrm{mol}$.

Ausbeute: $\quad 2.77 \mathrm{~g}(10.77 \mathrm{mmol}), 96 \%$.

${ }^{1}$ H-NMR

$\left(500 \mathrm{MHz}^{\mathrm{CDCl}} \mathrm{CDC}_{3}\right): \quad \delta=8.52\left(\mathrm{~d},{ }^{3} \mathrm{~J}_{\mathrm{HH}}=8.8 \mathrm{~Hz},{ }^{4} J_{\mathrm{HH}}=0.7 \mathrm{~Hz}, 2 \mathrm{H}, \mathrm{H}_{1,8}\right), 8.44\left(\mathrm{~s}, 1 \mathrm{H}, \mathrm{H}_{10}\right)$, $8.00\left(\mathrm{~d},{ }^{3} \mathrm{~J}_{\mathrm{HH}}=8.4 \mathrm{~Hz}, 2 \mathrm{H}, \mathrm{H}_{4,5}\right), 7.62-7.59\left(\mathrm{~m}, 2 \mathrm{H}, \mathrm{H}_{2,7}\right), 7.52-7.49$ $\left(\mathrm{m}, 2 \mathrm{H}, \mathrm{H}_{3,6}\right)$. 
${ }^{13} \mathrm{C}\left\{{ }^{1} \mathrm{H}\right\}-\mathrm{NMR}$

$\left(125 \mathrm{MHz}, \mathrm{CDCl}_{3}\right): \quad \delta=132.16\left(\mathrm{~s}, 2 \mathrm{C}, \mathrm{C}_{8 \mathrm{a}, 9 \mathrm{a}}\right), 130.58\left(\mathrm{~s}, 2 \mathrm{C}, \mathrm{C}_{4 \mathrm{a}, 10 \mathrm{a}}\right), 128.59\left(\mathrm{~s}, 2 \mathrm{C}, \mathrm{C}_{4,5}\right)$, 127.62 (s, 2C, $\left.C_{1,8}\right), 127.18\left(\mathrm{~s}, 2 \mathrm{C}, \mathrm{C}_{2,7}\right), 127.09$ (s, 1C, $\left.\mathrm{C}_{10}\right), 125.63$ $\left(\mathrm{s}, 2 \mathrm{C}, \mathrm{C}_{3,6}\right), 122.34\left(\mathrm{~s}, 1 \mathrm{C}, \mathrm{C}_{9}\right)$.

EI-MS: $\mathrm{m} / \mathrm{z}(\%): \quad 256 / 258(100 / 98)[\mathrm{M}]^{+}, 176(36)[\mathrm{M}-\mathrm{Br}]^{+}$.

$\mathrm{CH}$, gef. (ber.) [\%]: $\quad$ C 64.75 (65.40), H 3.40 (3.53).

\subsubsection{Synthese von 9,10-Dibromanthracen (2)}

Die Synthese von 2 erfolgte nach AAV 1 mit $1.0 \mathrm{~g}(5.61 \mathrm{mmol})$ Anthracen und $0.58 \mathrm{~mL}$ (11.32 mmol) $\mathrm{Br}_{2}$.

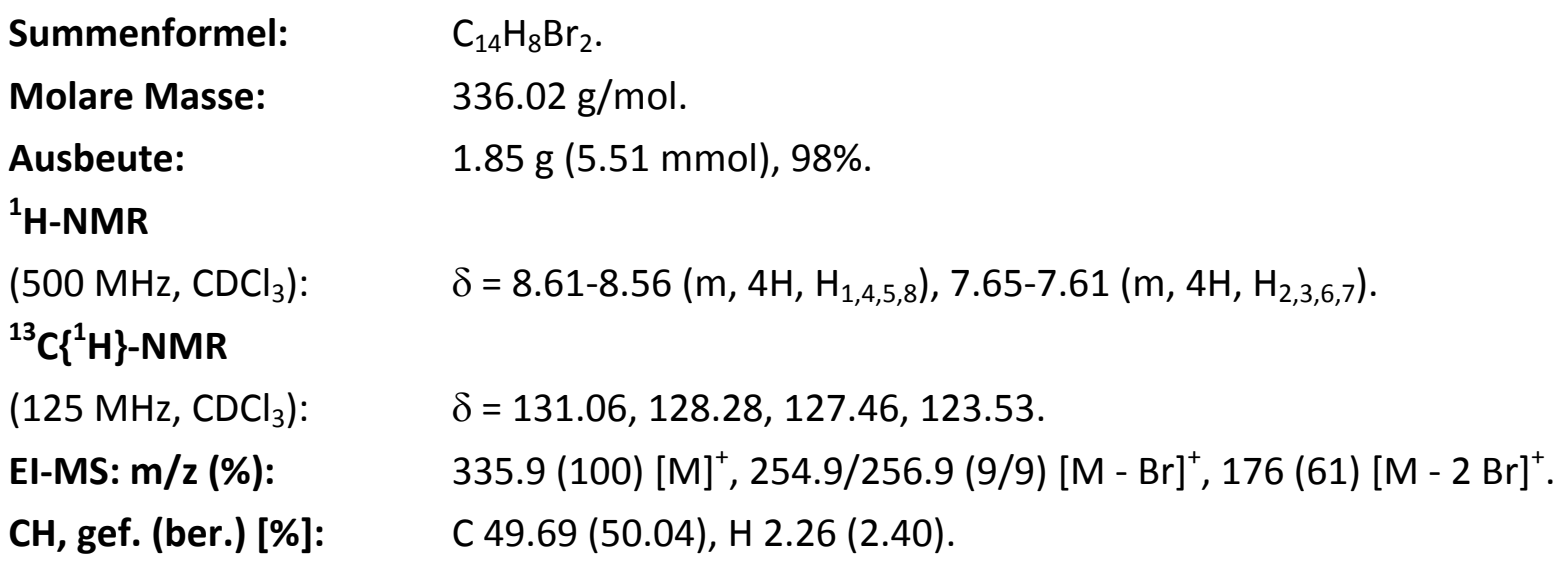

\subsubsection{Synthese von 9-Brom-10-methylanthracen (3)}

Methode A: Die Synthese 3 von erfolgte nach AAV 1 mit $1.0 \mathrm{~g}$ (5.20 mmol) 9Methylanthracen und $0.27 \mathrm{~mL}(5.20 \mathrm{mmol}) \mathrm{Br}_{2}$.

Methode B: Eine Lösung von 9-Brom-10-anthryllithium synthetisiert nach AAV 2, mit $8.00 \mathrm{~g}$ (23.81 mmol) 9,10-Dibromanthracen und $13.22 \mathrm{~mL}$ (23.8 mmol) einer $1.80 \mathrm{M} \mathrm{nBuLi-Lösung} \mathrm{in}$ Hexan wurden bei $-15^{\circ} \mathrm{C}$ über $30 \mathrm{Min}$. mit $1.64 \mathrm{~mL}$ (26.18 mmol) einer lodmethanlösung in $10 \mathrm{~mL}$ Diethyleter versetzt. Nach Entfernen des Lösungsmittels i. Vak. wurde der erhaltene Feststoff in $250 \mathrm{~mL}$ Dichlormethan gelöst, zweimal mit $100 \mathrm{~mL}$ Wasser extrahiert und die organische Phase über $\mathrm{MgSO}_{4}$ getrocknet. Der nach Abziehen des Dichlormethan erhaltene hell gelbe Feststoff wurde aus Dichlormethan umkristallisiert.

Summenformel: $\quad \mathrm{C}_{15} \mathrm{H}_{11} \mathrm{Br}$.

Molare Masse: $\quad 271.15 \mathrm{~g} / \mathrm{mol}$.

Ausbeute: $\quad 6.13 \mathrm{~g}(22.62 \mathrm{mmol}), 95 \%$ (nach Methode $\mathrm{B})$. 
${ }^{1}$ H-NMR

$\left(300 \mathrm{MHz}, \mathrm{CDCl}_{3}\right): \quad \delta=8.59\left(\mathrm{dd},{ }^{3} \mathrm{~J}_{\mathrm{HH}}=7.2 \mathrm{~Hz},{ }^{4} \mathrm{~J}_{\mathrm{HH}}=1.6 \mathrm{~Hz}, 2 \mathrm{H}, \mathrm{H}_{1,8}\right), 8.28\left(\mathrm{dd},{ }^{3} \mathrm{~J}_{\mathrm{HH}}=\right.$ $\left.9.2 \mathrm{~Hz},{ }^{4} J_{\mathrm{HH}}=0.6 \mathrm{~Hz}, 2 \mathrm{H}, \mathrm{H}_{4,5}\right), 7.60$ (ddd, ${ }^{3} J_{\mathrm{HH}}=7.2 \mathrm{~Hz},{ }^{3} J_{\mathrm{HH}}=7.0 \mathrm{~Hz}$, $\left.{ }^{4} J_{\mathrm{HH}}=0.6 \mathrm{~Hz}, 2 \mathrm{H}, \mathrm{H}_{2,7}\right) 7.53\left(\mathrm{ddd},{ }^{3} J_{\mathrm{HH}}=9.2 \mathrm{~Hz},{ }^{3} J_{\mathrm{HH}}=7.0 \mathrm{~Hz},{ }^{4} J_{\mathrm{HH}}=\right.$ $\left.1.6 \mathrm{~Hz}, 2 \mathrm{H}, \mathrm{H}_{3,6}\right), 3.07\left(\mathrm{~s}, 3 \mathrm{H}, \mathrm{CH}_{3}\right)$.

${ }^{13} \mathrm{C}\left\{{ }^{1} \mathrm{H}\right\}-\mathrm{NMR}$

$\left(75 \mathrm{MHz}^{\mathrm{CDCl}}\right)_{3}$ :

$\delta=130.92\left(\mathrm{~s}, 2 \mathrm{C}, \mathrm{C}_{4 \mathrm{a}, 10 \mathrm{a}}\right), 130.22\left(\mathrm{~s}, 2 \mathrm{C}, \mathrm{C}_{8 \mathrm{a}, 9 \mathrm{a}}\right), 128.53\left(\mathrm{~s}, 2 \mathrm{C}, \mathrm{C}_{1,8}\right)$, 126.67 (s, 2C, $\left.C_{2,7}\right), 125.52$ (s, 2C, $\left.C_{3,6}\right), 125.03$ (s, 2C, $\left.C_{4,5}\right), 121.45$ (s, 1C, $\left.\mathrm{C}_{10}\right), 120.35$ (s, 1C, $\left.\mathrm{C}_{9}\right), 14.26\left(\mathrm{~s}, 3 \mathrm{H}, \mathrm{CH}_{3}\right)$.

El-MS: $\mathbf{m} / \mathbf{z}(\%): \quad 270(100)[\mathrm{M}]^{+}, 191(56)[\mathrm{M}-\mathrm{Br}]^{+}, 176(4)[\mathrm{M}-\mathrm{Br}, \mathrm{Me}]^{+}$.

$\mathrm{CH}$, gef. (ber.) [\%]: $\quad$ C 66.18 (66.44), H 4.01 (4.09).

\subsubsection{Synthese von 9-Brom-10-brommethylanthracen (4)}

Die Synthese 4 erfolgte nach AAV 1 mit 4.11 g (15.16 mmol) 9-Brom-10-methylanthracen (3) und $0.81 \mathrm{~mL}$ (15.92 $\mathrm{mmol}) \mathrm{Br}_{2}$.

Summenformel: $\quad \mathrm{C}_{15} \mathrm{H}_{10} \mathrm{Br}_{2}$.

Molare Masse: $\quad 350.05 \mathrm{~g} / \mathrm{mol}$.

Ausbeute: $\quad 5.15 \mathrm{~g}(14.71 \mathrm{mmol}), 97 \%$.

${ }^{1}$ H-NMR

$\left(300 \mathrm{MHz}, \mathrm{CDCl}_{3}\right): \quad \delta=8.63\left(\mathrm{dd},{ }^{3} \mathrm{~J}_{\mathrm{HH}}=8.2 \mathrm{~Hz},{ }^{4} J_{\mathrm{HH}}=1.6 \mathrm{~Hz}, 2 \mathrm{H}, \mathrm{H}_{1,8}\right), 8.31$ (dd, $\left.{ }^{3} J_{\mathrm{HH}}=7.7 \mathrm{~Hz},{ }^{4} J_{\mathrm{HH}}=1.2 \mathrm{~Hz}, 2 \mathrm{H}, \mathrm{H}_{4,5}\right), 7.68$ (ddd, ${ }^{3} J_{\mathrm{HH}}=7.7 \mathrm{~Hz}$, $\left.{ }^{3} J_{\mathrm{HH}}=6.5 \mathrm{~Hz},{ }^{4} J_{\mathrm{HH}}=1.6 \mathrm{~Hz}, 2 \mathrm{H}, \mathrm{H}_{3,6}\right), 7.62\left(\mathrm{ddd},{ }^{3} J_{\mathrm{HH}}=8.2 \mathrm{~Hz}\right.$, $\left.{ }^{3} J_{\mathrm{HH}}=6.5 \mathrm{~Hz},{ }^{4} J_{\mathrm{HH}}=1.2 \mathrm{~Hz}, 2 \mathrm{H}, \mathrm{H}_{2,7}\right), 5.50\left(\mathrm{~s}, 2 \mathrm{H}, \mathrm{CH}_{2} \mathrm{Br}\right)$.

${ }^{13} \mathrm{C}\left\{{ }^{1} \mathrm{H}\right\}-\mathrm{NMR}$

$\left(75 \mathrm{MHz}, \mathrm{CDCl}_{3}\right.$ ): $\quad \delta=130.74\left(\mathrm{~s}, 2 \mathrm{C}, \mathrm{C}_{9 \mathrm{a}, 8 \mathrm{a}}\right), 130.39\left(\mathrm{~s}, 2 \mathrm{C}, \mathrm{C}_{4 \mathrm{a}, 10 \mathrm{a}}\right), 129.00\left(\mathrm{~s}, 2 \mathrm{C}, \mathrm{C}_{1,8}\right)$, 128.69 (s, 1C, $C_{4 a}$ ), 127.32 (s, 2C, $\left.C_{2,7}\right), 127.16$ (s, 2C, $\left.C_{3,6}\right), 125.89$ (s, 1C, $\left.\mathrm{C}_{9}\right), 124.01\left(\mathrm{~s}, 2 \mathrm{C}, \mathrm{C}_{4,5}\right), 26.76\left(\mathrm{~s}, 1 \mathrm{C}, \mathrm{CH}_{2}\right)$.

EI-MS: $\mathbf{m} / \mathbf{z}(\%): \quad 350(5)[\mathrm{M}]^{+}, 269 / 271(100 / 98)[\mathrm{M}-\mathrm{Br}]^{+}$.

CH, gef. (ber.) [\%]: $\quad$ C: 50.63 (51.47), H: 2.96 (2.88).

\subsubsection{Synthese von 9,10-Di(brommethyl)anthracen (5)}

Die Synthese 5 erfolgte nach AAV 1 mit 3.62 g (17.55 mmol) 9,10-Dimethylanthracen und $1.88 \mathrm{~mL}$ (36.86 mmol) $\mathrm{Br}_{2}$.

Summenformel: $\quad \mathrm{C}_{16} \mathrm{H}_{12} \mathrm{Br}_{2}$. 
Molare Masse:

Ausbeute:

${ }^{1} \mathrm{H}-\mathrm{NMR}$

$\left(500 \mathrm{MHz}, \mathrm{CDCl}_{3}\right)$ :

${ }^{13} \mathrm{C}\left\{{ }^{1} \mathrm{H}\right\}-\mathrm{NMR}$

(125 MHz, $\mathrm{CDCl}_{3}$ ):

EI-MS: $\mathrm{m} / \mathrm{z}(\%)$ :

$\mathrm{CH}$, gef. (ber.) [\%]:
$364.07 \mathrm{~g} / \mathrm{mol}$.

$6.20 \mathrm{~g}(17.03 \mathrm{mmol}), 97 \%$.

$\delta=7.62-7.75\left(m, 4 \mathrm{H}, \mathrm{H}_{1,4,5,8}\right), 8.31-8.45\left(\mathrm{~m}, 4 \mathrm{H}, \mathrm{H}_{2,3,6,7}\right), 5.80(\mathrm{~s}, 4 \mathrm{H}$, $\mathrm{CH}_{2} \mathrm{Br}$ ).

$\delta=26.6,124.4,126.7$.

$363(1)[\mathrm{M}]^{+}, 203 / 205(6)[\mathrm{M}-\mathrm{Br}]^{+}, 204(100)[\mathrm{M}-2 \mathrm{Br}]^{+}$.

C: 52.25 (52.78), H: 3.18 (3.32).

\subsubsection{Synthese von 9-Brommethylanthracen (6)}

Eine Lösung von $2.07 \mathrm{~g}$ (10.17 mmol) 9-Methanolanthracen in $20 \mathrm{~mL}$ Acetonitril wurde über 30 Min. zu einer Lösung von Bromtriphenylphosphoniumbromid, hergestellt aus $2.80 \mathrm{~g}$ (10.68 mmol) Triphenylphosphane in $20 \mathrm{~mL}$ trockenem Acetonitril mit $1.62 \mathrm{~g}(0.52 \mathrm{~mL}$, $10.17 \mathrm{mmol}$ ) $\mathrm{Br}_{2}$, getropft. Nach Rühren über Nacht bei RT wurde das Lösungsmittel i. Vak. entfernt und der erhaltene Feststoff säulenchromatographisch gereinigt (Hexane/Etyhlacetate, 2:1). 6 wurde als gelbes Pulver erhalten.

Summenformel:

Molare Masse:

Ausbeute:

${ }^{1} \mathrm{H}-\mathrm{NMR}$

(500 MHz, $\mathrm{CDCl}_{3}$ ):

${ }^{13} \mathrm{C}\left\{{ }^{1} \mathrm{H}\right\}-N M R$

(125 MHz, $\mathrm{CDCl}_{3}$ ):

EI-MS: $\mathrm{m} / \mathrm{z}(\%)$ :

$\mathrm{CH}$, gef. (ber.) [\%]:
$\mathrm{C}_{15} \mathrm{H}_{11} \mathrm{Br}$.

$271.15 \mathrm{~g} / \mathrm{mol}$.

$2.04 \mathrm{~g}(7.52 \mathrm{mmol}), 74 \%$.

$\delta=8.50\left(\mathrm{~s}, 1 \mathrm{H}, \mathrm{H}_{10}\right), 8.31\left(\mathrm{~d},{ }^{3} J_{\mathrm{HH}}=9.0 \mathrm{~Hz},{ }^{4} J_{\mathrm{HH}}=1.0 \mathrm{~Hz}, 2 \mathrm{H}, \mathrm{H}_{1,8}\right)$, $8.05\left(\mathrm{~d},{ }^{3} J_{\mathrm{HH}}=8.5 \mathrm{~Hz}, 2 \mathrm{H}, \mathrm{H}_{4,5}\right), 7.67-7.63\left(\mathrm{~m}, 2 \mathrm{H}, \mathrm{H}_{2,7}\right), 7.52-7.49$ $\left(\mathrm{m}, 2 \mathrm{H}, \mathrm{H}_{3,6}\right), 5.55\left(\mathrm{~s}, 2 \mathrm{H}, \mathrm{CH}_{2}\right)$.

$\delta=131.57$ (s, 2C, C $4 a, 10 a), 129.70$ (s, 2C, C $8 a, 9 a), 129.25$ (s, 2C, C4,5), 129.19 (s, 1C, $\left.C_{10}\right), 127.85$ (s, 1C, $\left.C_{9}\right), 126.77$ (s, 2C, $\left.C_{2,7}\right), 125.35$ (s, 2C, $\left.\mathrm{C}_{3,6}\right), 123.49\left(\mathrm{~s}, 2 \mathrm{C}, \mathrm{C}_{1,8}\right), 26.93\left(\mathrm{~s}, 1 \mathrm{C}, \mathrm{CH}_{2}\right.$ ).

270/272 (7/6) [M] $]^{+}, 191(100)[\mathrm{M}-\mathrm{Br}]^{+}$.

C: 66.30 (66.44), H: 4.22 (4.09).

\subsubsection{Synthese von $[R A n L i \cdot\{\text { dee }\}]_{2}(7 a-c)$}

Eine Suspension von $10 \mathrm{mmol}$ des jeweiligen Bromanthracen in $80 \mathrm{~mL}$ Diethylether wurde bei $-15^{\circ} \mathrm{C}$ über $30 \mathrm{~min}$ mit genau $10 \mathrm{mmol}$ einer $2.25 \mathrm{~m} \mathrm{nBuLi-Lösung} \mathrm{in} \mathrm{Hexan} \mathrm{versetzt} \mathrm{und}$ für weitere $30 \mathrm{~min}$ bei $0^{\circ} \mathrm{C}$ gerührt so dass eine klare orange Lösung erhalten wurde. Die 
Reaktionsmischung wurde anschließend auf ein drittel des Volumens eingeengt und auf $-78^{\circ} \mathrm{C}$ gekühlt. Der so erhaltene Feststoff wurde Abfiltriert mit wenig (5-10 mL) kaltem Hexan gewaschen und für ca. 10 Min i. Vak. getrocknet.

\subsubsection{Kristallisation von $[R A n L i \cdot\{\text { dee }\}]_{2}(7 a-c)$}

ca. $0.5 \mathrm{~g}$ des Komplexes 7 wurden in $10 \mathrm{~mL}$ kaltem Diethylether gelöst. Ungelöste Anteile wurden abfiltriert und die Lösung zur Kristallisation bei $-25{ }^{\circ} \mathrm{C}$ aufbewahrt. Nach $24 \mathrm{~h}$ konnten Kristalle erhalten werden die zur Röntgenstrukturanalyse geeignet waren.

$\mathrm{CH}$, gef. (ber.) [\%]: $\quad$ 7a: C: 63.13 (64.12), H: 5.38 (5.38).

\subsubsection{Kristallisation von $[\mathrm{RAnLi} \cdot\{\text { Donor }\}]_{2}(8-13)$}

$0.1 \mathrm{mmol}$ des Komplexes 7 wurden in $10 \mathrm{~mL}$ Diethylether gelöst auf $-15^{\circ} \mathrm{C}$ gekühlt und langsam mit den entsprechenden Äquivalenten an kaltem Donor versetzt. Etwaiger entstandener Feststoff wurde Abfiltriert und die Lösung zur Kristallisation bei $-25^{\circ} \mathrm{C}$ aufbewahrt. In der Regel konnten nach $24 \mathrm{~h}$ bis max. einer Woche Kristalle erhalten werden die zur Röntgenstrukturanalyse geeignet waren.

\section{${ }^{1}$ H-NMR}

(500 MHz, THF-D8): $\quad 11 \mathrm{a}: \delta=8.31\left(\mathrm{~d},{ }^{3} \mathrm{~J}_{\mathrm{HH}}=8.8 \mathrm{~Hz}, 2 \mathrm{H}, \mathrm{H}_{1,8}\right), 8.19\left(\mathrm{~d},{ }^{3} \mathrm{~J}_{\mathrm{HH}}=8.4 \mathrm{~Hz}, 2 \mathrm{H}\right.$, $\left.\mathrm{H}_{4,5}\right), 7.33\left(\mathrm{dd},{ }^{3} \mathrm{~J}_{\mathrm{HH}}=8.8,{ }^{3} \mathrm{~J}_{\mathrm{HH}}=6.8,2 \mathrm{H}, \mathrm{H}_{2,7}\right), 7.16\left(\mathrm{t},{ }^{3} \mathrm{~J}_{\mathrm{HH}}=8.4 \mathrm{~Hz}\right.$, $\left.{ }^{3} J_{H H}=6.8,2 H, H_{3,6}\right), 3.63(m, 4 H, T H F), 1.78(m, 4 H, T H F)$.

${ }^{13} \mathrm{C}\left\{{ }^{1} \mathrm{H}\right\}-\mathrm{NMR}$

(125 MHz, THF-D8): $\quad$ 11a: $\delta=220.70\left(s, C_{10}\right), 145.94,139.86,128.46,126.95,124.99$, $119.47,116.25,65.54$ (THF), 14.90 (THF).

CH, gef. (ber.) [\%]: $\quad$ 11a: C: 62.61 (64.88), H: 5.25 (5.94).

\subsubsection{Kristallisation von $[\mathrm{RAnLi} \cdot \mathrm{LiBr} \cdot\{\text { Donor }\}]_{2}(14 \mathrm{a}-\mathrm{b}$ und $15 \mathrm{a}-\mathrm{b})$}

Eine Lösung von 9-Brom-10-anthryllithium synthetisiert nach AAV 2, mit $2.00 \mathrm{~g}$ (5.95 mmol) 9,10-Dibromanthracen und $2.35 \mathrm{~mL}(5.95 \mathrm{mmol})$ einer $2.53 \mathrm{M} \mathrm{nBuLi-Lösung} \mathrm{in} \mathrm{Hexan} \mathrm{wurde}$ auf $-15^{\circ} \mathrm{C}$ gekühlt und langsam mit einem 2.5 fachem Überschuss des entsprechenden vorgekühlten Donors versetzt. Etwaiger entstandener Feststoff wurde Abfiltriert und die Lösung zur Kristallisation bei $-25^{\circ} \mathrm{C}$ aufbewahrt. In der Regel konnten nach einer Woche bis 
max. sechs Wochen Kristalle erhalten werden die zur Röntgenstrukturanalyse geeignet waren.

\subsubsection{Kristallisation von $[n B u A n L i \cdot\{p m d e t a\}](16)$}

Eine Suspension von 9,10-Dilihioanthracen synthetisiert nach AAV 2, mit $2.00 \mathrm{~g}$ (5.95 mmol) 9,10-Dibromanthracen und $5.00 \mathrm{~mL}(12.50 \mathrm{mmol})$ einer $2.50 \mathrm{M} \mathrm{nBuLi}$-Lösung in Hexan wurde auf $-15^{\circ} \mathrm{C}$ gekühlt und langsam mit zwei Äquivalenten PMDETA versetzt. Der entstandene Feststoff wurde Abfiltriert und die Lösung zur Kristallisation bei $-25{ }^{\circ} \mathrm{C}$ aufbewahrt. Nach ca. einer Woche konten Kristalle erhalten werden die zur Röntgenstrukturanalyse geeignet waren.

\subsubsection{Synthese von 9,10-Dibuthylanthracen (17)}

Eine Suspension von 9,10-Dilihioanthracen synthetisiert nach AAV 2, mit $2.00 \mathrm{~g}$ (5.95 mmol) 9,10-Dibromanthracen und $5.00 \mathrm{~mL}(12.50 \mathrm{mmol})$ einer $2.50 \mathrm{M} \mathrm{nBuLi-Lösung} \mathrm{in} \mathrm{Hexan} \mathrm{wurde}$ auf $-15^{\circ} \mathrm{C}$ gekühlt und langsam mit einem 5 fachem Überschuss PMDETA versetzt. Nach weitern $64 \mathrm{~h}$ Rühren bei RT wurde das Reaktiongemisch mit $10 \mathrm{~mL}$ Eiswasser hydrolysiert. Nach Abtrennen der organischen Phase und Trocknen über $\mathrm{MgSO}_{4}$ wurde das Reaktionsprodukt als gelber Feststoff erhalten.

\subsubsection{Synthese von 9-Bromanthracen-10-carbonsäure (18)}

Eine Lösung von 9-Brom-10-anthryllithium synthetisiert nach AAV 2, mit $2.00 \mathrm{~g}$ (5.95 mmol) 9,10-Dibromanthracen und $2.35 \mathrm{~mL}(5.95 \mathrm{mmol})$ einer $2.53 \mathrm{M} \mathrm{nBuLi-Lösung} \mathrm{in} \mathrm{Hexan} \mathrm{wurde}$ über 30 Min. zu ca. 20 gemörsertem Trockeneis zugetropft. Nach 2 h Rühren, währen denen sich das Reaktionsgemisch auf RT erwärmte, wurde die Reaktionsmischung auf das halbe Volumen eingeengt der entstandene Feststoff abfiltriert und mit $10 \mathrm{~mL}$ Diethylether gewaschen. Der Rückstand wurde anschließend in $20 \mathrm{~mL}$ demin. Wasser gelöst und die Carbonsäure mit wenig konz. $\mathrm{HCl}$ ausgefällt. Nach Filtration und Trocknen i. Vak. wurde 9Brom-10-anthracencarbonsäure als gelber Feststoff erhalten.

Summenformel: $\quad \mathrm{C}_{15} \mathrm{H}_{9} \mathrm{BrO}_{2}$

Molekulargewicht: $\quad 301.13 \mathrm{~g} / \mathrm{mol}$.

Ausbeute: $\quad 1.67 \mathrm{~g}(5.55 \mathrm{mmol}), 93 \%$. 
${ }^{1}$ H-NMR

(200 MHz, DMSO-D6): $\quad \delta=8.49\left(\mathrm{~m}, 2 \mathrm{H}, \mathrm{H}_{1,8}\right), 8.06\left(\mathrm{~m}, 2 \mathrm{H}, \mathrm{H}_{4,5}\right), 7.77\left(\mathrm{~m}, 2 \mathrm{H}, \mathrm{H}_{2,7}\right), 7.70(\mathrm{~m}$, $\left.2 \mathrm{H}, \mathrm{H}_{3,6}\right), 3.60(\mathrm{~s}, \mathrm{br}, 1 \mathrm{H}, \mathrm{OH})$.

${ }^{13} \mathrm{C}\left\{{ }^{1} \mathrm{H}\right\}-\mathrm{NMR}$

(75 MHz, DMSO-D6): $\quad \delta=169.78(\mathrm{C}=0), 131.29,129.31,128.26,127.56\left(C_{1,8}\right), 127.44$ $\left(C_{2,7}\right), 127.25\left(C_{3,6}\right), 125.63\left(C_{4,5}\right), 123.15$.

EI-MS; m/z (\%): $\quad 300 / 302(100)\left[\mathrm{M}^{+}, 283 / 285\right.$ (24) $[\mathrm{M}-\mathrm{OH}]^{+}, 255 / 257$ (10) [M$\mathrm{COOH}]^{+}, 176(50)[\mathrm{M}-\mathrm{BrCOOH}]^{+}$.

\subsubsection{Synthese von 9-Bromanthracen-10-carbonsäurechlorid (19)}

Eine Suspension von $0.50 \mathrm{~g}(1.66 \mathrm{mmol})$ 9-Bromanthracen-10-carbonsäure in $20 \mathrm{~mL}$ Oxalylchlorid wurde für $5 \mathrm{~h}$ zum Rückfluss erhitzt. Nach abziehen des überschüssigen Oxalylchlorids wurde das Reaktionsprodukt als hell gelber Feststoff erhalten.

Summenformel: $\quad \mathrm{C}_{15} \mathrm{H}_{8} \mathrm{BrClO}$.

Molare Masse: $\quad 319.58 \mathrm{~g} / \mathrm{mol}$.

Ausbeute: $\quad 0.52(1.63 \mathrm{mmol}), 89 \%$.

${ }^{1} \mathrm{H}-\mathrm{NMR}$

(200 MHz, THF-D8): $\quad \delta=8.63-8.54\left(\mathrm{~m}, 2 \mathrm{H}, \mathrm{H}_{1,8}\right), 8.15-8.05\left(\mathrm{~m}, 2 \mathrm{H}, \mathrm{H}_{4,5}\right), 7.75-7.64(\mathrm{~m}$, $\left.4 \mathrm{H}, \mathrm{H}_{2,3,6,7}\right)$.

${ }^{13} \mathrm{C}\left\{{ }^{1} \mathrm{H}\right\}-\mathrm{NMR}$

(125 MHz, THF-D8): $\quad \delta=170.53$ (C=O), 133.48, 130.79, 129.34, 129.08, 128.90, 127.37, 127.07, 125.13.

EI-MS; m/z (\%): $\quad 320(24)[\mathrm{M}]^{+}, 283 / 285(100)[\mathrm{M}-\mathrm{Cl}]^{+}, 255 / 257(17)[\mathrm{M}-\mathrm{COCl}]^{+}, 204$ (2) $[\mathrm{M}-\mathrm{BrCl}]^{+}, 176(76)[\mathrm{M}-\mathrm{BrCOCl}]^{+}$.

\subsubsection{9-Brom-10-carbonsäure-tert-butanesteranthracen (21)}

Eine Lösung von $0.80 \mathrm{~g}(2.50 \mathrm{mmol})$ 9-Bromanthracen-10-carbonsäurechlorid in $30 \mathrm{~mL}$ Dichlormethan wurde bei $-15{ }^{\circ} \mathrm{C}$ mit $0.26 \mathrm{~g}(2.75 \mathrm{mmol})$ Natriumtertbutanolat in $8 \mathrm{~mL}$ Dichlormethan versetzt. Nach $18 \mathrm{~h}$ Rühren bei RT wurde der entstandene Rückstand eingeengt und über Celite filtriert. Nach Abziehen des restlichen Lösungsmittels wurde ein gelber Feststoff erhalten.

Summenformel: $\quad \mathrm{C}_{19} \mathrm{H}_{17} \mathrm{BrO}_{2}$.

Molare Masse $\quad 357.24 \mathrm{~g} / \mathrm{mol}$. 
Ausbeute:

$0.75 \mathrm{~g}(2.10 \mathrm{mmol}), 84 \%$.

${ }^{1} \mathrm{H}-\mathrm{NMR}$

(200 MHz, $\left.\mathrm{CDCl}_{3}\right): \quad \delta=8.61-8.55\left(\mathrm{~m}, 2 \mathrm{H}, \mathrm{H}_{1,8}\right), 8.08-8.01\left(\mathrm{~m}, 2 \mathrm{H}, \mathrm{H}_{4,5}\right), 7.67-7.55(\mathrm{~m}$, $\left.4 \mathrm{H}, \mathrm{H}_{2,3,6,7}\right), 1.79\left(\mathrm{~s}, 3 \mathrm{H}, \mathrm{CH}_{3}\right)$.

EI-MS; m/z (\%): $\quad 356 / 358(12)[\mathrm{M}]^{+}, 285(16)[\mathrm{M}-\mathrm{O} \quad 3 \mathrm{Bu}]^{+}, 257(6)[\mathrm{M}-\mathrm{COOtBu}]^{+}$.

\subsubsection{9-Brom-10-anthracen(2-hydroxy-1,1-dimethylethyl)carbonsäureamid (22)}

Eine Lösung von $2.00 \mathrm{~g}(6.26 \mathrm{mmol})$ 9-Bromanthracen-10-carbonsäurechlorid in $20 \mathrm{~mL}$ Dichlormethan wurde für bei $-15^{\circ} \mathrm{C}$ mit $1.3 \mathrm{~mL}$ (15.6 mmol) 2-Amino-2-methyl-1-propanol in $8 \mathrm{~mL}$ Dichlormethan versetzt. Nach $12 \mathrm{~h}$ Rühren bei RT wurde der enstandene Rückstand abfiltriert und mit Wasser gewaschen. Das Filtrat wurde eingeengt und mit dem gewaschenen Rückstand vereinigt. Nach Umkristallisation aus Dichlormethan wurde das Reaktionsprodukt als beiger Feststoff erhalten.

Summenformel: $\quad \mathrm{C}_{19} \mathrm{H}_{18} \mathrm{BrNO}_{2}$.

Molekulargewicht: $\quad 372.26 \mathrm{~g} / \mathrm{mol}$.

Ausbeute: $\quad 2.07 \mathrm{~g}(5.57 \mathrm{mmol}), 89 \%$.

${ }^{1} \mathrm{H}-\mathrm{NMR}$

(200 MHz, DMSO-D6): $\delta=8.46\left(\mathrm{~m}, 2 \mathrm{H}, \mathrm{H}_{1,8}\right), 8.14\left(\mathrm{~m}, 2 \mathrm{H}, \mathrm{H}_{4,5}\right), 7.73\left(\mathrm{~m}, 2 \mathrm{H}, \mathrm{H}_{2,7}\right), 7.66(\mathrm{~m}$, $\left.2 \mathrm{H}, \mathrm{H}_{3,6}\right), 4.98(\mathrm{~s}, \mathrm{br}, 1 \mathrm{H}, \mathrm{NH}), 3.73\left(\mathrm{~s}, 2 \mathrm{H}, \mathrm{CH}_{2}\right), 3.38(\mathrm{~s}, \mathrm{br}, 1 \mathrm{H}, \mathrm{OH})$, $1.44\left(\mathrm{~s}, 6 \mathrm{H}, 2 \mathrm{xCH}_{3}\right)$.

${ }^{13} \mathrm{C}\left\{{ }^{1} \mathrm{H}\right\}-\mathrm{NMR}$

(125 MHz, DMSO-D6): $\quad \delta=167.39$ (s, 1C, CO), 135.18, 129.42, 128.01, 127.87, 127.06, $126.72,126.25,121.56,66.81\left(\mathrm{~s}, 1 \mathrm{C}, \mathrm{CMe}_{2}\right), 55.71\left(\mathrm{~s}, 1 \mathrm{C}, \mathrm{CH}_{2}\right)$, $23.80\left(\mathrm{~s}, 6 \mathrm{C}, 2 \times \mathrm{CH}_{3}\right)$.

EI-MS; m/z (\%): $\quad 371 / 373$ (27) $[\mathrm{M}]^{+}, 326 / 328$ (1) $\left[\mathrm{M}-\mathrm{C}_{2} \mathrm{H}_{6} \mathrm{O}\right]^{+}, 299 / 301$ (19) [M$\left.\mathrm{C}_{4} \mathrm{H}_{9} \mathrm{O}\right]^{+}, \quad 283 / 285$ (100) $\left[\mathrm{M}-\mathrm{C}_{4} \mathrm{H}_{10} \mathrm{NO}\right]^{+}, 255 / 257$ (15) [M$\left.\mathrm{C}_{5} \mathrm{H}_{10} \mathrm{NO}_{2}\right]^{+}, 220$ (4) $\left[\mathrm{M}-\mathrm{C}_{4} \mathrm{H}_{9} \mathrm{BrO}\right]^{+}, 176(50)\left[\mathrm{M}-\mathrm{BrC}_{5} \mathrm{H}_{10} \mathrm{NO}_{2}\right]^{+}$.

\subsubsection{9-Brom-10-(4,4-dimethyl-2-oxazyl)-anthracen (23)}

$1 \mathrm{~g}(2.69 \mathrm{mmol}) 22$ wurde in $20 \mathrm{~mL}$ Thionylchlorid gelöst und anschließend für $2 \mathrm{~h}$ zum Rückfluss erhitzt. Nach Abziehen des überschüssigen Thionylchlorids wurde der Rückstand in $20 \mathrm{~mL}$ Diethylether gelöst und mit $1 \mathrm{~m} \mathrm{NaOH}$-Lsg extrahiert. Die organische Phase wurde abgetrennt und vom Lösungsmittel befreit wobei das Reaktionsprodukt als gelber Feststoff 
erhalten wurde. Das als Hauptprodukt entstandene 9,10-Dichloranthracen wurde durch fraktionierte Kristallisation abgetrennt.

Summenformel: $\quad \mathrm{C}_{19} \mathrm{H}_{16} \mathrm{BrNO}$.

Molekulargewicht: $\quad 354.24 \mathrm{~g} / \mathrm{mol}$.

Ausbeute: $\quad 0.24 \mathrm{~g}(0.68 \mathrm{mmol}), 25 \%$.

${ }^{1}$ H-NMR

$\left(200 \mathrm{MHz}, \mathrm{CDCl}_{3}\right): \quad \delta=8.46-8.56\left(\mathrm{~m}, 2 \mathrm{H}, \mathrm{H}_{1,8}\right), 8.09-8.18\left(\mathrm{~m}, 2 \mathrm{H}, \mathrm{H}_{4,5}\right), 7.65-7.85(\mathrm{~m}$, $4 \mathrm{H}, \mathrm{H}_{2,3,6,7)}, 4.37\left(\mathrm{~s}, 2 \mathrm{H}, \mathrm{CH}_{2}\right), 1.53\left(\mathrm{~s}, 6 \mathrm{H}, 2 \mathrm{xCH}_{3}\right)$.

EI-MS; m/z (\%): $\quad 353 / 355(23)[\mathrm{M}]^{+}, 309(18)\left[\mathrm{M}-\mathrm{C}_{2} \mathrm{H}_{5} \mathrm{O}\right]^{+}, 283(18)\left[\mathrm{M}-\mathrm{C}_{4} \mathrm{H}_{8} \mathrm{O}\right], 246$

(100) $\left[\mathrm{M}-\mathrm{BrC}_{2} \mathrm{H}_{5}\right]^{+}, 255 / 257$ (3) $\left[\mathrm{M}-\mathrm{C}_{5} \mathrm{H}_{8} \mathrm{NO}\right]^{+}, 176$ (48) [M$\left.\mathrm{BrC}_{5} \mathrm{H}_{8} \mathrm{NO}\right]^{+}$.

\subsubsection{9-Brom-10-(1-hydroxy-1-pyridyl)methylanthracen (25)}

$\mathrm{Zu}$ einer Lösung von 9-Brom-10-anthryllithium synthetisiert nach AAV 2, mit $2.00 \mathrm{~g}$ (5.95 mmol) 9,10-Dibromanthracen, $2.70 \mathrm{~mL}$ (5.97 mmol) einer $2.21 \mathrm{M} \mathrm{nBuLi-Lösung} \mathrm{in}$ Hexan wurde mit einer Lösung von $1.25 \mathrm{~mL}$ (13.07 mmol) Pyridyl-2-carbaldehyd in $5 \mathrm{~mL}$ Diethylether versetzt und für weitere $12 \mathrm{~h}$ bei RT gerührt. Nach Abziehen des Lösungsmittels wurde der entstandene Feststoff in $20 \mathrm{~mL}$ Dichlormethan gelöst und die Reaktionsmischung anschließend mit $150 \mathrm{~mL}$ demin. Wasser hydrolisiert. Die organische Phase wurde abgetrennt und die wässrige Phase mit Diethylether $(2 \times 20 \mathrm{~mL})$ extrahiert. Die organischen Phasen wurden vereinigt und das Lösungsmittel i. Vak entfernt. Es wurden $2.02 \mathrm{~g}$ (5.56 mmol) 9-Brom-10-(1-hydroxy-1-pyridyl)methylanthracen als beiger Feststoff erhalten.

Summenformel: $\quad \mathrm{C}_{21} \mathrm{H}_{15} \mathrm{BrO}$.

Molare Masse: $\quad 363.25 \mathrm{~g} / \mathrm{mol}$.

Ausbeute: $\quad 2.02 \mathrm{~g}(5.56 \mathrm{mmol}), 93 \%$.

${ }^{1}$ H NMR

$\left(\mathrm{CDCl}_{3}, 200 \mathrm{MHz}\right): \quad \delta=8.68-8.60\left(\mathrm{~m}, 2 \mathrm{H}, \mathrm{H}_{1,8}\right), 8.39-8.29\left(\mathrm{~m}, 2 \mathrm{H}, \mathrm{H}_{4,5}\right), 7.67-7.14$ (m, 4H, Py), $7.62-7.51\left(m, 2 \mathrm{H}, \mathrm{H}_{3,6}\right), 7.48-7.37\left(\mathrm{~m}, 2 \mathrm{H}, \mathrm{H}_{2,7}\right)$, $7.289(\mathrm{~s}, 1 \mathrm{H}, \mathrm{CH}), 6.80-6.65(\mathrm{~m}, 1 \mathrm{H}, \mathrm{OH})$.

\subsubsection{9-((2-Methoxyethyl)aminomethyl)anthracen (26)}

$1 \mathrm{~g}(4.85 \mathrm{mmol})$ Anthracen-9-carbaldehyd und $1.25 \mathrm{~mL}$ (14.55 mmol) 2-Methoxyethylamin wurden in $73 \mathrm{~mL}$ einer $1: 1$ Mischung aus Ethanol und Acetonitiril suspendiert und für $48 \mathrm{~h}$ bei RT gerührt. Nach abziehen des Lösungsmittels i. Vak. wurde der Rückstand in Ethanol 
gelöst und mit $1.70 \mathrm{~g}$ Natriumborhydrid versetzt. Die Reaktionsmischung wurde anschließend $4 \mathrm{~h}$ zum Rückfluss erhitzt und nach Abkühlen auf RT mit Wasser hydrolisiert. Nach Ausschütteln mit Dichlormethan $(3 \times 30 \mathrm{~mL})$ wurden die vereinigten organischen Phasen über $\mathrm{MgSO}_{4}$ getrocknet. Nach entfernen des Lösungsmittels wurde das Reaktionsprodkt als hell gelber Feststoff erhalten.

Summenformel:

Molare Masse:

Ausbeute:

${ }^{1}$ H NMR

(300 MHz, DMSO-D6):

${ }^{13} \mathrm{C}\left\{{ }^{1} \mathrm{H}\right\}-N M R$

(75 MHz,DMSO-D6): $\quad \delta=132.27\left(\mathrm{C}_{9}\right), 131.04\left(\mathrm{C}_{8 \mathrm{a}, 9 \mathrm{a}}\right), 129.88\left(\mathrm{C}_{4 \mathrm{a}, 10 \mathrm{a}}\right), 128.79\left(\mathrm{C}_{1,8}\right)$, $126.58\left(C_{10}\right), 125.88\left(C_{2,7}\right), 125.04\left(C_{3,6}\right), 124.65\left(C_{4,5}\right), 71.67$ (O$\left.\mathrm{CH}_{2}\right), 57.97\left(\mathrm{OCH}_{3}\right), 48.77\left(\mathrm{NCH}_{2}\right), 44.88\left(\mathrm{An}-\mathrm{CH}_{2}\right)$.

ESI-MS; $m / z$ (\%):
$\mathrm{C}_{18} \mathrm{H}_{19} \mathrm{NO}$.

$265.35 \mathrm{~g} / \mathrm{mol}$.

$1.22 \mathrm{~g}(4.61 \mathrm{mmol}), 95 \%$.

$\delta=8.55\left(\mathrm{~s}, 1 \mathrm{H}, \mathrm{H}_{10}\right), 8.40\left(\mathrm{dd},{ }^{3} \mathrm{~J}_{\mathrm{HH}}=7.8 \mathrm{~Hz},{ }^{4} \mathrm{~J}_{\mathrm{HH}}=1.1 \mathrm{~Hz}, 2 \mathrm{H}, \mathrm{H}_{4,5}\right)$, $8.06\left(\mathrm{dd},{ }^{3} \mathrm{~J}_{\mathrm{HH}}=6.8 \mathrm{~Hz},{ }^{4} J_{\mathrm{HH}}=1.6 \mathrm{~Hz}, 2 \mathrm{H}, \mathrm{H}_{1,8}\right), 7.52\left(\mathrm{~m}, 4 \mathrm{H}, \mathrm{H}_{2,3,6,7}\right)$, $4.64\left(\mathrm{~s}, 2 \mathrm{H}, \mathrm{An}-\mathrm{CH}_{2}\right), 3.44\left(\mathrm{t},{ }^{3} \mathrm{~J}_{\mathrm{HH}}=5.6 \mathrm{~Hz}, 2 \mathrm{H}, \mathrm{MeOCH}_{2}\right), 3.35(\mathrm{~s}$, $1 \mathrm{H}, \mathrm{NH}), 3.22\left(\mathrm{~s}, 3 \mathrm{H}, \mathrm{OCH}_{3}\right), 2.89\left(\mathrm{t},{ }^{3} \mathrm{~J}_{\mathrm{HH}}=5.6 \mathrm{~Hz}, \mathrm{~N}-\mathrm{CH}_{2}\right)$.

\subsubsection{9-((2-tetrahydrofuranylethyl)aminomethyl)anthracen (27)}

Die Synthese 27 erfolgte analog zu 26 mit $1 \mathrm{~g}$ (4.85 mmol) Anthracen-9-carbaldehyd, $1.50 \mathrm{~mL}$ (14.55 mmol) 2-Methoxyethylamin und $1.70 \mathrm{~g}$ Natriumborhydrid. Durch das eingesetzte Amin wurde das Produkt als Racemat erhalten.

Summenformel: $\quad \mathrm{C}_{20} \mathrm{H}_{21} \mathrm{NO}$.

Molare Masse: $\quad 291.39 \mathrm{~g} / \mathrm{mol}$.

Ausbeute: $\quad 1.34 \mathrm{~g}(4.61 \mathrm{mmol}), 95 \%$.

${ }^{1}$ H NMR

(300 MHz, DMSO-D6): $\quad \delta=8.50\left(\mathrm{~s}, 1 \mathrm{H}, \mathrm{H}_{10}\right), 8.41\left(\mathrm{dd},{ }^{3} J_{\mathrm{HH}}=7.9 \mathrm{~Hz},{ }^{4} J_{\mathrm{HH}}=1.0 \mathrm{~Hz}, 2 \mathrm{H}, \mathrm{H}_{4,5}\right.$ ), $8.05\left(\mathrm{dd},{ }^{3} J_{\mathrm{HH}}=7.4 \mathrm{~Hz},{ }^{4} \mathrm{~J}_{\mathrm{HH}}=1.6 \mathrm{~Hz}, 2 \mathrm{H}, \mathrm{H}_{1,8}\right), 7.51\left(\mathrm{~m}, 4 \mathrm{H}, \mathrm{H}_{2,3,6,7}\right)$, $4.64\left(\mathrm{sm} 2 \mathrm{H}, \mathrm{An}-\mathrm{CH}_{2}\right), 3.90\left(\mathrm{~m},{ }^{3} \mathrm{~J}_{\mathrm{HH}}=6.0 \mathrm{~Hz}, 1 \mathrm{H}, \mathrm{H}_{\mathrm{Fu}-2}\right), 3.70(\mathrm{~m}$, $\left.2 \mathrm{H}, \mathrm{H}_{\text {Fu-5a }}\right), 3.56\left(\mathrm{~m}, 2 \mathrm{H}, \mathrm{H}_{\text {Fu-5b }}\right), 2.77\left(\mathrm{~m}, 2 \mathrm{H}, \mathrm{NHCH}_{2} \mathrm{a}\right), 2.51(\mathrm{~m}, 2 \mathrm{H}$, $\left.\mathrm{NHCH}_{2} \mathrm{~b}\right), 1.78\left(\mathrm{~m}, 6 \mathrm{H}, \mathrm{H}_{\mathrm{Fu}-3 \mathrm{a}, 4 \mathrm{a}, 4 \mathrm{~b}}\right), 1.48\left(\mathrm{~m}, 2 \mathrm{H}, \mathrm{H}_{\mathrm{Fu}-3 \mathrm{~b}}\right)$.

${ }^{13} \mathrm{C}\left\{{ }^{1} \mathrm{H}\right\}-\mathrm{NMR}$

(75 MHz,DMSO-D6): $\quad \delta=132.32\left(C_{9}\right), 131.05\left(C_{8 a}, 9 a\right), 129.90\left(C_{4 a, 10 a}\right), 128.77\left(C_{1,8}\right)$, $126.56\left(C_{10}\right), 125.83\left(C_{2,7}\right), 125.00\left(C_{3,6}\right), 124.64\left(C_{4,5}\right), 80.21\left(C_{F u-5 a}\right)$, 
$78.13\left(\mathrm{C}_{\mathrm{Fu}-2}\right), 67.03$ ( $\left.\mathrm{C}_{\mathrm{Fu}-5 \mathrm{~b}}\right), 53.86\left(\mathrm{NCH}_{2} \mathrm{Furyl} \mathrm{a}\right), 45.99\left(\mathrm{NCH}_{2}\right.$ Furyl

b) $45.12\left(\mathrm{An}-\mathrm{CH}_{2}\right), 28.92\left(\mathrm{C}_{\mathrm{Fu}-3 \mathrm{a}, 3 \mathrm{~b}}\right), 25.31\left(\mathrm{C}_{\mathrm{Fu}-4 \mathrm{a}, 4 \mathrm{~b}}\right)$.

ESI-MS; m/z (\%): $292(12)\left[\mathrm{M}^{+}, 191(100)\left[\mathrm{M}-\mathrm{NHCH}_{2} \text { Furyl }\right]^{+}\right.$.

\subsubsection{Synthese von 9-Brom-10-(2-hydroxyethyl)anthracen (28)}

In eine Lösung von 9-Brom-10-anthryllithium synthetisiert nach AAV 2 mit $4.00 \mathrm{~g}$ (11.9 mmol) 9,10-Dibromanthracen und $6.61 \mathrm{~mL}$ (11.9 mmol) einer $1.8 \mathrm{M} n \mathrm{BuLi}$-Lsg. in Hexan wurde bei $-15{ }^{\circ} \mathrm{C}$ für 20 Min. Ethylenoxid eingeleitet. Danach wurden $20 \mathrm{~mL}$ Eiswasser hinzugegeben, die Lösung mit Salzsäure angesäuert und mit Diethylether $(2 \times 50 \mathrm{~mL})$ extrahiert. Die organischen Phasen wurden vereint, über Magnesiumsulfat getrocknet und abfiltriert. Nach Abziehen des Lösungsmittels und Umkristallisation aus Diethylether (etwa $20 \mathrm{~mL}$ für $700 \mathrm{mg}$ 9-Brom-10-(2-hydroxyethyl)anthracen) wurde das Reaktionsprodukt als orangegelber kristalliner Feststoff erhalten.

Summenformel: $\quad \mathrm{C}_{16} \mathrm{H}_{13} \mathrm{BrO}$.

Molare Masse: $\quad 301.18 \mathrm{~g} / \mathrm{mol}$.

Ausbeute: $\quad 3.13 \mathrm{~g}(10.39 \mathrm{mmol}), 87 \%$.

${ }^{1}$ H-NMR

$\left(200 \mathrm{MHz}, \mathrm{CDCl}_{3}\right): \quad \delta=8.59\left(\mathrm{dd},{ }^{3} \mathrm{~J}_{\mathrm{HH}}=7.6 \mathrm{~Hz},{ }^{4} \mathrm{~J}_{\mathrm{HH}}=1.8 \mathrm{~Hz}, 2 \mathrm{H}, \mathrm{H}_{1,8}\right), 8.33\left(\mathrm{dd},{ }^{3} \mathrm{~J}_{\mathrm{HH}}=\right.$ $\left.8.0 \mathrm{~Hz},{ }^{4} J_{\mathrm{HH}}=2.4 \mathrm{~Hz}, 2 \mathrm{H}, \mathrm{H}_{4,5}\right), 7.56\left(\mathrm{~m}, 4 \mathrm{H}, \mathrm{H}_{2,3,6,7}\right), 4.07\left(\mathrm{~d},{ }^{3} J_{\mathrm{HH}}=\right.$ $7.0 \mathrm{~Hz}, 2 \mathrm{H}, \mathrm{CH}_{2} \mathrm{CH}_{2} \mathrm{OH}$ ), 3.91 (d, ${ }^{3} \mathrm{~J}_{\mathrm{HH}}=7.0 \mathrm{~Hz}, 2 \mathrm{H}, \mathrm{CH}_{2} \mathrm{CH}_{2} \mathrm{OH}$ ).

El-MS: $\mathbf{m} / \mathbf{z}(\%): \quad 300 / 302$ (34) $\left[\mathrm{M}^{+}, 269 / 271\right.$ (100) $\quad[\mathrm{M}-\mathrm{CH} 2 \mathrm{OH}]^{+}, 189$ (63) $[\mathrm{M}-\mathrm{BrCH} 3 \mathrm{OH}]+$.

CH, gef. (ber.) [\%]: $\quad$ C: 64.06 (63.81), H: 4.29 (4.35).

\subsubsection{Synthese von 9-Brom-10-(2-tosyloxyethyl)anthracen (29)}

Zu einer Lösung von $1.50 \mathrm{~g}$ (4.98 mmol) 9-Brom-10-(2-hydroxyethyl)anthracen in $25 \mathrm{~mL}$ Pyridin wurde über $30 \mathrm{Min}$. bei $0^{\circ} \mathrm{C}$ eine Lösung von $8.85 \mathrm{~g}$ (14.94 mmol)paraSulfonsäurechlorid in $15 \mathrm{~mL}$ Pyridin zugetropft. Nach $16 \mathrm{~h}$ Rühren bei RT wurde das Pyridin i. Vak. entfernt. Der Rückstand wurde in $300 \mathrm{~mL}$ Dichlormethan gelöst und mit $1 \mathrm{M}$ Salzsäure gewaschen $(3 \times 150 \mathrm{~mL})$. Die organische Phase wurde über Natriumsulfat getrocknet, abfiltriert und unter vermindertem Druck entfernt. Das 9-Brom-10-(2-tosyloxyethyl)anthracen blieb als gelbbraunes Öl zurück.

Summenformel: $\quad \mathrm{C}_{23} \mathrm{H}_{19} \mathrm{BrO}_{3} \mathrm{~S}$.

Molare Masse: $\quad 455.36 \mathrm{~g} / \mathrm{mol}$. 
Ausbeute:

${ }^{1}$ H-NMR

$\left(200 \mathrm{MHz}, \mathrm{CDCl}_{3}\right)$ :

EI-MS: $m / z(\%):$
$1.95 \mathrm{~g}(4.28 \mathrm{mmol}), 85 \%$.

$\delta=8.55\left(\mathrm{dd},{ }^{3} J_{\mathrm{HH}}=7.8 \mathrm{~Hz},{ }^{4} J_{\mathrm{HH}}=1.4 \mathrm{~Hz}, 2 \mathrm{H}, \mathrm{H}_{1,8}\right), 8.08\left(\mathrm{dd},{ }^{3} \mathrm{~J}_{\mathrm{HH}}=\right.$ $\left.7.6 \mathrm{~Hz},{ }^{4} J_{\mathrm{HH}}=1.6 \mathrm{~Hz}, 2 \mathrm{H}, \mathrm{H}_{4,5}\right), 7.55\left(\mathrm{~m}, 4 \mathrm{H}, \mathrm{H}_{2,3,6,7}\right), 7.30\left(\mathrm{~d},{ }^{3} J_{\mathrm{HH}}=\right.$ $\left.7.0 \mathrm{~Hz}, 2 \mathrm{H}, \mathrm{CH}_{2}\right), 3.96\left(\mathrm{~d},{ }^{3} \mathrm{~J}_{\mathrm{HH}}=7.0 \mathrm{~Hz}, 2 \mathrm{H}, \mathrm{CH}_{2}\right), 2.30\left(\mathrm{~s}, 3 \mathrm{H}, \mathrm{CH}_{3}\right)$ 454/456 (46) [M] $]^{+}, 282 / 284$ (39) [M-TosOH] $]^{+}, 269 / 271$ (65) $\left[\mathrm{M}-\mathrm{TosOCH}_{2}\right]^{+}, \quad 203 \quad(100) \quad[\mathrm{M}-\mathrm{TosOHBr}]^{+}, \quad 189$

\subsubsection{Synthese von 9-Brom-10-((trimethyethylendiamino)methyl)anthracen (30)}

Eine Lösung von $1.48 \mathrm{~g}$ (14.46 mmol) (2-(Dimethylamino)ethyl)methylamin wurde in $100 \mathrm{~mL}$ THF gerührt und anschließend bei $-15^{\circ} \mathrm{C}$ über $30 \mathrm{Min}$. mit $8.00 \mathrm{~mL}$ (14.31 mmol) einer $1.80 \mathrm{M}$ $n$ BuLi-Lösung in Hexan versetzt. Die Reaktionslösung wurde eine Stunde refluxiert. Anschließend wurde $5.09 \mathrm{~g}$ (14.56 mmol) 9-Brom-10-brommethyanthracen in $170 \mathrm{~mL}$ THF bei $-15{ }^{\circ} \mathrm{C}$ über $30 \mathrm{Min}$. zugetropft. Hierbei wechselte die Farbe von farblos über schwarzgrün und gelb-orange zu rot. Die Lösung wurde über Nacht gerührt und anschließend eine Stunde refluxiert. Das Lösungsmittel wurde i. Vak. entfernt, der erhaltene Feststoff in $250 \mathrm{~mL}$ Dichlormethan gelöst. Die Lösung wurde zweimal mit $100 \mathrm{~mL}$ einer Lösung von 8 Tropfen $\mathrm{NEt}_{3}$ zu $100 \mathrm{~mL}$ Wasser extrahiert. Nach Entfernen des Lösungsmittels i. Vak. konnte ein gelb-oranger Feststoff erhalten werden.

Summenformel:

Molare Masse:

Ausbeute:

${ }^{1}$ H-NMR

$\left(200 \mathrm{MHz} \mathrm{CDCl}_{3}\right)$ :

ESI-MS: $\mathrm{m} / \mathrm{z}(\%)$ :
$\mathrm{C}_{20} \mathrm{H}_{23} \mathrm{BrN}_{2}$.

$371.31 \mathrm{~g} / \mathrm{mol}$.

$4.91 \mathrm{~g}(14.38 \mathrm{mmol}), 94 \%$.

$\delta=8.53-8.63\left(\mathrm{~m}, 4 \mathrm{H}, \mathrm{H}_{1,4,5,8}\right), 7.50-7.63\left(\mathrm{~m}, 4 \mathrm{H}, \mathrm{H}_{2,3,6,7}\right), 4.46(\mathrm{~s}, 2 \mathrm{H}$, $\left.\mathrm{H}_{11}\right), 2.68-2.78\left(\mathrm{~m}, 2 \mathrm{H}, \mathrm{H}_{12}\right), 2.47-2.57\left(\mathrm{~m}, 2 \mathrm{H}, \mathrm{H}_{13}\right), 2.29(\mathrm{~s}, 3 \mathrm{H}$, $\left.\mathrm{H}_{14}\right), 2.19\left(\mathrm{~s}, 6 \mathrm{H}, \mathrm{H}_{15}\right)$.

$371.2 / 373.2(100 / 98)[\mathrm{M}+\mathrm{H}]^{+}$.

\subsubsection{9-Brom-10-(dipyridylaminomethyl)anthracen (31)}

$\mathrm{Zu}$ einer Lösung von $0.25 \mathrm{~g}$ (1.48 mmol) 2,2-Dipyridylamin und $0.16 \mathrm{~g}$ (1,61 $\mathrm{mmol})$ Triethylamin in $20 \mathrm{~mL}$ THF wurde bei $-15{ }^{\circ} \mathrm{C}$ über 30 min eine Lösung von $0.42 \mathrm{~g}$ (1.2 mmol) 9-Brom-10-brommethylanthracen (4) in 20 mL THF zugetropft. Das Gemisch wurde $4 \mathrm{~h}$ bei 
RT gerührt. Die Lösung wurde eingeengt und der Feststoff abfiltriert. Trocknen i. Vak. ergab das Produkt als gelben Feststoff.

Summenformel: $\quad \mathrm{C}_{25} \mathrm{H}_{18} \mathrm{BrN}_{3}$.

Molare Masse: $\quad 440.33 \mathrm{~g} / \mathrm{mol}$.

Ausbeute: $\quad 0.48 \mathrm{~g}(1.1 \mathrm{mmol}), 91 \%$.

${ }^{1} \mathrm{H}-\mathrm{NMR}$

$\left(200 \mathrm{MHz} \mathrm{CDCl}_{3}\right): \quad \delta=8.50-8.43\left(\mathrm{~m}, 4 \mathrm{H}, \mathrm{H}_{1,4,5,8}\right), 8.37-8.32\left(\mathrm{~m}, 2 \mathrm{H}, \mathrm{H}_{\mathrm{Py}-3}\right), 7.55-7.37$ $\left(m, 4 \mathrm{H}, \mathrm{H}_{2,3,6,7}\right), 7.28-7.18\left(\mathrm{~m}, 2 \mathrm{H}, \mathrm{H}_{\mathrm{Py}-5}\right), 6.85-6.75\left(\mathrm{~m}, 2 \mathrm{H}, \mathrm{H}_{\mathrm{Py}-6}\right)$, 6.33-6.24 (m, 2H, $\left.\mathrm{H}_{\mathrm{Py}-4}\right), 5.27\left(\mathrm{~s}, 2 \mathrm{H}, \mathrm{CH}_{2}\right)$.

EI-MS: $\mathbf{m} / \mathbf{z}(\%): \quad 441[\mathrm{M}]^{+}, 269\left[\mathrm{M}-\mathrm{NPy}_{2}\right]^{+}, 189[\mathrm{M}-\mathrm{Br}, \mathrm{NPy}]_{2}^{+}$.

\subsubsection{Synthese von 9-Diisopropylphosphanylanthracen (3536)}

Die Synthese von 35 erfolgte nach AAV 4 mit $4.00 \mathrm{~g}$ (15.56 mmol) 9-Bromanthracen, $7.74 \mathrm{~mL}$ (15.56 mmol) einer $2.01 \mathrm{M} \mathrm{nBuLi}$ Lösung in Hexan und $2.28 \mathrm{~mL}$ (15.56 mmol) Diispropylchlorphosphan Das Reaktionsprodukt wurde als hell gelber Feststoff erhalten.

Summenformel: $\quad \mathrm{C}_{20} \mathrm{H}_{23} \mathrm{P}$.

Molare Masse: $\quad 294.15 \mathrm{~g} / \mathrm{mol}$.

Ausbeute: $\quad 3.93 \mathrm{~g}(13.35 \mathrm{mmol}), 86 \%$.

${ }^{1} \mathrm{H}-\mathrm{NMR}$

$\left(300 \mathrm{MHz} \mathrm{CDCl}_{3}\right): \quad \delta=9.20\left(\mathrm{~s}, 2 \mathrm{H}, \mathrm{H}_{1,8}\right), 8.50\left(\mathrm{~s}, 1 \mathrm{H}, \mathrm{H}_{10}\right), 8.00\left(\mathrm{~d},{ }^{3} \mathrm{JHH}_{\mathrm{HH}}=8.6 \mathrm{~Hz}, 2 \mathrm{H}\right.$, $\left.\mathrm{H}_{4,5}\right), 7.53\left(\mathrm{~m}, 4 \mathrm{H}, \mathrm{H}_{2,7}, \mathrm{H}_{3,6}\right), 2.91\left(\mathrm{~m}, 2 \mathrm{H}, 2 \times \mathrm{CH}\left(\mathrm{CH}_{3}\right)_{2}\right), 1.50$ (d, $\left.{ }^{3} J_{\mathrm{HH}}=6.5 \mathrm{~Hz}, \quad 3 \mathrm{H}, \quad i \mathrm{Pr}-\mathrm{CH}_{3}\right), 1.41\left(\mathrm{~d},{ }^{3} J_{\mathrm{HH}}=6.5 \mathrm{~Hz}, 3 \mathrm{H}, \quad i \mathrm{Pr}-\mathrm{CH}_{3}\right)$, $0.69\left(\mathrm{~m}, 6 \mathrm{H}, 2 \times \mathrm{Pr}-\mathrm{CH}_{3}\right)$.

${ }^{31} \mathrm{P}\left\{{ }^{1} \mathrm{H}\right\}-\mathrm{NMR}$

$\left(121 \mathrm{MHz}, \mathrm{CDCl}_{3}\right): \quad \delta=0.10(\mathrm{~s})$.

EI-MS: m/z (\%): $\quad 294(53)[\mathrm{M}]^{+}, 252(14)[\mathrm{M}-i \mathrm{Pr}]^{+}, 209(100)\left[\mathrm{M}-i \mathrm{Pr}_{2}\right]^{+}, 178(22)[\mathrm{M}-$ $\left.\mathrm{PiPr}_{2}\right]^{+}$.

\subsubsection{Synthese von 9-Diisopropyloxophosphanylanthracen (36)}

Die Synthese von 36 erfolgte nach AAV 5 mit $1.21 \mathrm{~g} \quad$ (4.11 mmol) 9Diisopropylphosphanylanthracen und $2 \mathrm{~mL} \quad(23.26 \mathrm{mmol})$ einer $35 \%$ igen Wasserstoffperoxid-Lösung in $\mathrm{H}_{2} \mathrm{O}$. Das Reaktionsprodukt wurde als hellgelber Feststoff erhalten.

Summenformel: $\quad \mathrm{C}_{20} \mathrm{H}_{23} \mathrm{PO}$. 
Molare Masse:

Ausbeute:

${ }^{1} \mathrm{H}-\mathrm{NMR}$

(500 MHz, $\mathrm{CDCl}_{3}$ ):

${ }^{13} \mathrm{C}\left\{{ }^{1} \mathrm{H}\right\}-\mathrm{NMR}$

$\left(125 \mathrm{MHz}, \mathrm{CDCl}_{3}\right)$ :

${ }^{31} \mathrm{P}\left\{{ }^{1} \mathrm{H}\right\}-\mathrm{NMR}$

(121 MHz, $\left.\mathrm{CDCl}_{3}\right)$ :

El-MS: $\mathrm{m} / \mathrm{z}(\%)$ :
$310.15 \mathrm{~g} / \mathrm{mol}$.

$0.91 \mathrm{~g}(2.94 \mathrm{mmol}), 71 \%$.

$\delta=9.19\left(\mathrm{~s}, \mathrm{br}, 2 \mathrm{H}, \mathrm{H}_{1,8}\right), 8.60\left(\mathrm{~s}, 1 \mathrm{H}, \mathrm{H}_{10}\right), 8.02\left(\mathrm{~d},{ }^{3} \mathrm{~J}_{\mathrm{HH}}=8.33 \mathrm{~Hz}\right.$, $\left.2 \mathrm{H}, \mathrm{H}_{4,5}\right), 7.55$ (ddd, ${ }^{3} \mathrm{~J}_{\mathrm{HH}}=9.12 \mathrm{~Hz},{ }^{3} J_{\mathrm{HH}}=6.48 \mathrm{~Hz},{ }^{4} J_{\mathrm{HH}}=6.48 \mathrm{~Hz}$, $\left.2 \mathrm{H}, \mathrm{H}_{4,5}\right), 7.48\left(\mathrm{ddd},{ }^{3} \mathrm{~J}_{\mathrm{HH}}=8.33 \mathrm{~Hz},{ }^{3} J_{\mathrm{HH}}=6.48 \mathrm{~Hz},{ }^{4} J_{\mathrm{HH}}=0.94 \mathrm{~Hz}\right.$, $\left.2 \mathrm{H}, \mathrm{H}_{3,6}\right), 2.81$ (sep, $2 \mathrm{H},{ }^{3} \mathrm{~J}_{\mathrm{HH}}=7.06 \mathrm{~Hz}, \mathrm{CHMe}_{2}$ ), 1.52 (dd, ${ }^{3} \mathrm{~J}_{\mathrm{HH}}=$ $\left.7.02 \mathrm{~Hz},{ }^{3} J_{\mathrm{HP}}=14.86 \mathrm{~Hz}, 6 \mathrm{H}, 2 \times \mathrm{CH}_{3}\right), 1.52\left(\mathrm{dd},{ }^{3} \mathrm{~J}_{\mathrm{HH}}=7.02 \mathrm{~Hz},{ }^{3} J_{\mathrm{HP}}=\right.$ $\left.16.23 \mathrm{~Hz}, 6 \mathrm{H}, 2 \times \mathrm{CH}_{3}\right)$.

$\delta=133.70,133.40\left(C_{10}\right), 131.10\left(C_{4,5}\right), 129.50,126.03,126.74$ $\left(C_{2,7}\right), 124,98\left(C_{3,6}\right), 122.80,30.98\left(d,{ }^{1} J_{C P}=63.84 \mathrm{~Hz}, \mathrm{CHMe}_{2}\right)$, $17.22\left(d,{ }^{2} J_{C P}=3.17 \mathrm{~Hz}, 2 \times \mathrm{CH}_{3}\right), 17.02\left(d,{ }^{2} J_{C P}=3.39 \mathrm{~Hz}, 2 \times \mathrm{CH}_{3}\right)$.

$\delta=59.14(\mathrm{~s})$.

$310(42)[\mathrm{M}]^{+}, 267(39)[\mathrm{M}-i \mathrm{Pr}]^{+}, 225(100)\left[\mathrm{M}-i \mathrm{Pr}_{2}\right]^{+}, 178(22)[\mathrm{M}-$ $\left.\mathrm{PoiPr}_{2}\right]^{+}$.

\subsubsection{Synthese von 9-Diisopropylthiophosphanylanthracen (37)}

Die Synthese von 37 erfolgte nach AAV 6 mit $1.09 \mathrm{~g} \quad(3.70 \mathrm{mmol})$ 9Diisopropylphosphanylanthracen und $0.130 \mathrm{~g}(4.07 \mathrm{mmol})$ Schwefel. Das Reaktionsprodukt wurde als gelber Feststoff erhalten.

Summenformel: $\quad \mathrm{C}_{20} \mathrm{H}_{23} \mathrm{PS}$.

Molare Masse:

$326.13 \mathrm{~g} / \mathrm{mol}$.

Ausbeute:

$0.87 \mathrm{~g}(2.57 \mathrm{mmol}), 72 \%$.

${ }^{1}$ H-NMR

$\left(200 \mathrm{MHz}, \mathrm{CDCl}_{3}\right): \quad \delta=0.83\left(\mathrm{~d},{ }^{3} \mathrm{~J}_{\mathrm{HH}}=7.00 \mathrm{~Hz}, 3 \mathrm{H}, \mathrm{CH}_{3}\right), 0.92\left(\mathrm{~d},{ }^{3} \mathrm{~J}_{\mathrm{HH}}=6.80 \mathrm{~Hz}, 3 \mathrm{H}\right.$, $\left.\mathrm{CH}_{3}\right), 1.40\left(\mathrm{~d},{ }^{3} \mathrm{~J}_{\mathrm{HH}}=6.68 \mathrm{~Hz}, 3 \mathrm{H}, \mathrm{CH}_{3}\right), 1.50\left(\mathrm{~d},{ }^{3} \mathrm{JHH}_{\mathrm{HH}}=6.80 \mathrm{~Hz}, 3 \mathrm{H}\right.$, $\left.\mathrm{CH}_{3}\right), 3.27\left(\mathrm{~m}, 2 \mathrm{H}, 2 \times \mathrm{CH}\left(\mathrm{CH}_{3}\right)_{2}\right), 7.50\left(\mathrm{~m}, 4 \mathrm{H}, \mathrm{H}_{2,7}, \mathrm{H}_{3,6}\right), 7.98(\mathrm{~m}$, $\left.2 \mathrm{H}, \mathrm{H}_{4,5}\right), 8.55\left(\mathrm{~s}, 1 \mathrm{H}, \mathrm{H}_{10}\right), 9.38\left(\mathrm{~d},{ }^{3} J_{\mathrm{HH}}=8.38 \mathrm{~Hz}, \mathrm{H}_{1,8}\right)$.

\section{${ }^{31} \mathrm{P}\left\{{ }^{1} \mathrm{H}\right\}-\mathrm{NMR}$}

(81 $\left.\mathrm{MHz}, \mathrm{CDCl}_{3}\right): \quad \delta=71.21(\mathrm{~s})$.

EI-MS: m/z (\%): $\quad 326(100)\left[\mathrm{M}^{+}, 284(29)[\mathrm{M}-i \mathrm{Pr}]^{+}, 241\right.$ (85) $\left[\mathrm{M}-i \mathrm{Pr}_{2}\right]^{+}, 209$ (17) [M$\left.\mathrm{SiPr}_{2}\right]^{+}, 178(68)\left[\mathrm{M}-\mathrm{PSiPr}_{2}\right]^{+}$. 


\subsubsection{Synthese von 9-Diisopropylselenophosphanylanthracen (38)}

Die Synthese von 38 erfolgte nach AAV 6 mit $1.03 \mathrm{~g} \quad(3.50 \mathrm{mmol})$ 9Diisopropylphosphanylanthracen und $0.41 \mathrm{~g}(5.25 \mathrm{mmol})$ Selen. Das Reaktionsprodukt wurde als gelber Feststoff erhalten.

Summenformel: $\quad \mathrm{C}_{20} \mathrm{H}_{23}$ PSe.

Molare Masse: $\quad 374.07 \mathrm{~g} / \mathrm{mol}$.

Ausbeute: $\quad 0.98 \mathrm{~g}(2.62 \mathrm{mmol}), 75 \%$.

${ }^{1}$ H-NMR

$\left(200 \mathrm{MHz}, \mathrm{CDCl}_{3}\right): \quad \delta=0.83\left(\mathrm{~d}, 3^{3} \mathrm{~J}_{\mathrm{HH}}=7.00 \mathrm{~Hz}, 3 \mathrm{H}, \mathrm{CH}_{3}\right), 0.93\left(\mathrm{~d},{ }^{3} \mathrm{~J}_{\mathrm{HH}}=6.80 \mathrm{~Hz}, 3 \mathrm{H}\right.$, $\left.\mathrm{CH}_{3}\right), 1.40\left(\mathrm{~d},{ }^{3} \mathrm{~J}_{\mathrm{HH}}=6.68 \mathrm{~Hz}, 3 \mathrm{H}, \mathrm{CH}_{3}\right), 1.49\left(\mathrm{~d},{ }^{3} \mathrm{~J}_{\mathrm{HH}}=6.80 \mathrm{~Hz}, 3 \mathrm{H}\right.$, $\left.\mathrm{CH}_{3}\right), 3.34\left(\mathrm{~m}, 2 \mathrm{H}, 2 \times \mathrm{CH}\left(\mathrm{CH}_{3}\right)_{2}\right), 7.50\left(\mathrm{~m}, 4 \mathrm{H}, \mathrm{H}_{2,7}, \mathrm{H}_{3,6}\right), 7.98(\mathrm{~m}$, $\left.2 \mathrm{H}, \mathrm{H}_{4,5}\right), 8.56\left(\mathrm{~s}, 1 \mathrm{H}, \mathrm{H}_{10}\right), 9.40\left(\mathrm{~d},{ }^{3} \mathrm{~J}_{\mathrm{HH}}=8.38 \mathrm{~Hz}, \mathrm{H}_{1,8}\right)$.

${ }^{31} \mathrm{P}\left\{{ }^{1} \mathrm{H}\right\}-\mathrm{NMR}$

( $\left.81 \mathrm{MHz}, \mathrm{CDCl}_{3}\right)$ : $\quad \delta=64.60\left(\mathrm{t},{ }^{1} \mathrm{~J}_{\mathrm{SeP}}=874 \mathrm{~Hz}\right)$.

EI-MS: $\mathbf{m} / \mathbf{z}(\%): \quad 374(48)[\mathrm{M}]^{+}, 331(16)[\mathrm{M}-i \mathrm{Pr}]^{+}, 294(31)\left[\mathrm{M}-i \mathrm{Pr}_{2}\right]^{+}, 209$ (74) $[\mathrm{M}-$ SeiPr $]^{+}, 178(100)\left[\mathrm{M}-\mathrm{SePiPr}_{2}\right]^{+}$.

\subsubsection{Synthese von 9-Bis(diisopropylamino)phosphanylanthracen (39)}

Die Synthese von 39 erfolgte nach AAV 4 mit $3.50 \mathrm{~g}$ (13.61 mmol) 9-Bromanthracen, $6.65 \mathrm{~mL}$ (14.97 mmol) einer $2.25 \mathrm{M} \mathrm{nBuLi} \mathrm{Lösung} \mathrm{in} \mathrm{Hexan} \mathrm{und} \mathrm{einer} \mathrm{Lösung} \mathrm{von} 5.45 \mathrm{~g}$ (13.61 mmol) Bis(diisopropylamino)chlorphosphan in $30 \mathrm{~mL}$ THF.

Summenformel: $\quad \mathrm{C}_{26} \mathrm{H}_{37} \mathrm{~N}_{2} \mathrm{P}$.

Molare Masse: $\quad 408.27 \mathrm{~g} / \mathrm{mol}$.

Ausbeute: $\quad 3.92 \mathrm{~g}(9.61 \mathrm{mmol}), 70 \%$.

${ }^{1}$ H-NMR

(300 MHz, THF-D8): $\quad \delta=0.71\left(\mathrm{~d},{ }^{3} \mathrm{~J}_{\mathrm{HH}}=6.70 \mathrm{~Hz}, 12 \mathrm{H}, 4 \times \mathrm{CH}_{3}\right), 1.35\left(\mathrm{~d},{ }^{3} \mathrm{~J}_{\mathrm{HH}}=6.68 \mathrm{~Hz}\right.$, $\left.12 \mathrm{H}, 4 \times \mathrm{CH}_{3}\right), 3.64-3.75\left(\mathrm{~m}, 4 \mathrm{H}, 4 \times \mathrm{CH}\left(\mathrm{CH}_{3}\right)_{2}\right), 7.38\left(\mathrm{~m}, 4 \mathrm{H}, \mathrm{H}_{2,8}\right.$, $\left.H_{3,6}\right), 7.90\left(m, 2 H, H_{4,5}\right), 8.38\left(s, 1 H, H_{10}\right), 9.61\left(m, 2 H, H_{1,8}\right)$.

\section{${ }^{15} \mathbf{N}\left\{{ }^{1} \mathrm{H}\right\}-\mathrm{NMR}$}

(50 MHz, THF-D8):

${ }^{31} \mathrm{P}\left\{{ }^{1} \mathrm{H}\right\}-\mathrm{NMR}$

$\left(81 \mathrm{MHz}, \mathrm{CDCl}_{3}\right)$ :

$\delta=72.21(\mathrm{~s})$.

El-MS: $\mathrm{m} / \mathrm{z}(\%)$ :

$\delta=-322.8\left(\mathrm{~d},{ }^{1} J_{\mathrm{NP}}=62.3 \mathrm{~Hz}\right)$.

$408(16)[\mathrm{M}]^{+}, 365(2)[\mathrm{M}-i \mathrm{Pr}]^{+}, 308(60)\left[\mathrm{M}-\mathrm{NiPr}_{2}\right]^{+}, 220$ (13) [M$\left.\mathrm{NiPr}_{4}\right]^{+}, 209$ (100) [M-2 NiPr 2$]^{+}, 178$ (10) [M-P(NiPr$\left.)_{2}\right]^{+}$. 


\subsubsection{Synthese von 9-Bis(diisopropylamino)oxophosphanyl-anthracen (40)}

Die Synthese von 40 erfolgte nach AVV 5 mit $0.79 \mathrm{~g}$ (1.94 mmol) 9-Bis(diisopropylamino)phosphanylanthracen und $1 \mathrm{~mL}$ (11.63 mmol) einer 35 \%igen Wasserstoffperoxid-Lösung in $\mathrm{H}_{2} \mathrm{O}$. Das Reaktionsprodukt wurde als roter Feststoff erhalten.

Summenformel:

Molare Masse:

Ausbeute:

${ }^{1} \mathrm{H}-\mathrm{NMR}$

(200 MHz, $\left.\mathrm{CDCl}_{3}\right)$ :

${ }^{31} \mathrm{P}\left\{{ }^{1} \mathrm{H}\right\}-N M R$

$\left(81 \mathrm{MHz}, \mathrm{CDCl}_{3}\right)$ :

EI-MS: $\mathrm{m} / \mathrm{z}(\%)$ :
$\mathrm{C}_{26} \mathrm{H}_{37} \mathrm{~N}_{2} \mathrm{OP}$.

$424.26 \mathrm{~g} / \mathrm{mol}$.

$0.52 \mathrm{~g}(1.23 \mathrm{mmol}), 63 \%$.

$\delta=9.80\left(\mathrm{~s}, \mathrm{br}, 2 \mathrm{H}, \mathrm{H}_{1,8}\right), 8.50\left(\mathrm{~s}, 1 \mathrm{H}, \mathrm{H}_{10}\right), 7.98-7.90\left(\mathrm{~m}, 2 \mathrm{H}, \mathrm{H}_{4,5}\right)$, 7.54-7.37 (m, $\left.4 \mathrm{H}, \mathrm{H}_{2,3,6,7}\right), 4.08-3.85(\mathrm{~m}, 4 \mathrm{H}, \mathrm{CHiPr}), 1.39\left(\mathrm{~d},{ }^{3} J_{\mathrm{HH}}=\right.$ $6.8 \mathrm{~Hz}, 12 \mathrm{H}, i \mathrm{Pr}), 1.00\left(\mathrm{~d},{ }^{3} \mathrm{~J}_{\mathrm{HH}}=6.8 \mathrm{~Hz}, 12 \mathrm{H}, i \mathrm{Pr}\right)$.

$\delta=30.88(\mathrm{~s})$.

$424(15)[\mathrm{M}]^{+}, 324(100)\left[\mathrm{M}-\mathrm{N}(i \mathrm{Pr})_{2}\right]^{+}$.

\subsubsection{Synthese von 9-Bis(diisopropylamino)thiophosphanyl-anthracen (41)}

Die Synthese von 41 erfolgte nach AAV 6 mit $0.63 \mathrm{~g} \quad$ (1.54 mmol) 9Bis(diisopropylamino)phosphanylanthracen und $0.054 \mathrm{~g} \quad(1.69 \mathrm{mmol}) \quad$ Schwefel. Das Reaktionsprodukt wurde als dunkelroter Feststoff erhalten.

Summenformel:

$\mathrm{C}_{26} \mathrm{H}_{37} \mathrm{~N}_{2} \mathrm{SP}$.

Molare Masse: $440.24 \mathrm{~g} / \mathrm{mol}$.

Ausbeute:

$0.50 \mathrm{~g}(1.14 \mathrm{mmol}), 74 \%$.

${ }^{1} \mathrm{H}-\mathrm{NMR}$

$\left(200 \mathrm{MHz}, \mathrm{CDCl}_{3}\right): \quad \delta=9.38-930\left(\mathrm{~m}, 2 \mathrm{H}, \mathrm{H}_{1,8}\right), 8.51\left(\mathrm{~s}, 1 \mathrm{H}, \mathrm{H}_{10}\right), 7.99-7.91\left(\mathrm{~m}, 2 \mathrm{H}, \mathrm{H}_{4,5}\right)$, 7.50-7.38 (m, $\left.4 \mathrm{H}, \mathrm{H}_{2,3,6,7}\right), 4.48-4.28(\mathrm{~m}, 4 \mathrm{H}, \mathrm{CHiPr}), 1.38\left(\mathrm{~d},{ }^{3} J_{\mathrm{HH}}=\right.$ $7.0 \mathrm{~Hz}, 12 \mathrm{H}, i \mathrm{Pr}), 1.36\left(\mathrm{~d},{ }^{3} \mathrm{~J}_{\mathrm{HH}}=6.8 \mathrm{~Hz}, 12 \mathrm{H}, i \mathrm{Pr}\right)$.

EI-MS: $m / z(\%)$ : $440[\mathrm{M}]^{+}, 340[\mathrm{M}-\mathrm{NiPr} 2]^{+}, 240\left[\mathrm{M}-2 \mathrm{NiPr}_{2}\right]^{+}, 209\left[\mathrm{M}-\mathrm{S}, 2 \mathrm{NiPr}_{2}\right]^{+}$, $178\left[\mathrm{M}-\mathrm{PS}\left(\mathrm{NiPr}_{2}\right)_{2}\right]^{+}$.

\subsubsection{Synthese von 9-Bis(N,N-diisopropylamino)selenophosphanyl-anthracen (42)}

Die Synthese von $\mathbf{4 2}$ erfolgte mit $0.70 \mathrm{~g} \quad(1.71 \mathrm{mmol})$ 9-Bisdiisopropylaminophosphanylanthracen und $0.203 \mathrm{~g}(2.57 \mathrm{mmol})$ Selen. Das Reaktionsprodukt wurde als dunkelroter Feststoff erhalten.

Summenformel:

$\mathrm{C}_{26} \mathrm{H}_{37} \mathrm{~N}_{2} \mathrm{SeP}$. 
Molare Masse:

Ausbeute:

EI-MS: $\mathrm{m} / \mathrm{z}(\%)$ :
$488.19 \mathrm{~g} / \mathrm{mol}$.

$0.65 \mathrm{~g}(1.33 \mathrm{mmol}), 76 \%$.

$488[\mathrm{M}]^{+}, 408[\mathrm{M}-\mathrm{Se}]^{+}, 388[\mathrm{M}-\mathrm{NiPr}]^{+}, 287\left[\mathrm{M}-2 \mathrm{NiPr}_{2}\right]^{+}, 209[\mathrm{M}-$ Se, $\left.2 \mathrm{NiPr}_{2}\right]^{+}, 178\left[\mathrm{M}-\mathrm{PSe}\left(\mathrm{NiPr}_{2}\right)_{2}\right]^{+}$.

\subsubsection{Synthese von 9-Brom-10-bis(dimethylamino)phosphanylanthracen (44)}

Die Synthese von 44 erfolgte nach AAV 4 mit $4.00 \mathrm{~g}$ (11.91 mmol) 9,10-Dibromanthracen, $6.62 \mathrm{~mL}$ (11.91 mmol) einer $1.80 \mathrm{M} \mathrm{nBuLi-Lösung} \mathrm{in} \mathrm{Hexan} \mathrm{und} 1.84 \mathrm{~g}$ (11.91 mmol) Bis(dimethylamino)chlorphosphan. Das Produkt wurde als rotes Öl erhalten werden.

Summenformel:

Molare Masse:

Ausbeute:

${ }^{1} \mathrm{H}-\mathrm{NMR}$

$\left(200 \mathrm{MHz} \mathrm{CDCl}_{3}\right)$ :

${ }^{31} \mathrm{P}\left\{{ }^{1} \mathrm{H}\right\}-\mathrm{NMR}$

(81 $\left.\mathrm{MHz}, \mathrm{CDCl}_{3}\right)$ :

EI-MS: $m / z(\%)$ :
$\mathrm{C}_{18} \mathrm{H}_{20} \mathrm{BrN}{ }_{2} \mathrm{P}$.

$375.24 \mathrm{~g} / \mathrm{mol}$.

$4.24 \mathrm{~g}(11.29 \mathrm{mmol}), 95 \%$.

$\delta=8.95-9.04\left(\mathrm{~m}, 2 \mathrm{H}, \mathrm{H}_{1,8}\right), 8.54-8.63\left(\mathrm{~m}, 2 \mathrm{H}, \mathrm{H}_{4,5}\right), 7.50-7.60(\mathrm{~m}$, $\left.2 \mathrm{H}, \mathrm{H}_{3,6}\right), 7.38-7.50\left(\mathrm{~m}, 2 \mathrm{H}, \mathrm{H}_{2,7}\right), 2.58\left(\mathrm{~d},{ }^{3} J_{\mathrm{HP}}=9 \mathrm{~Hz}, \mathrm{CH}_{3}\right)$.

$\delta=108.26(\mathrm{~s})$.

$374 / 376(40 / 39)[\mathrm{M}]^{+}, 330 / 332(99 / 100)\left[\mathrm{M}-\mathrm{Me}_{2} \mathrm{~N}\right]^{+}, 296(21)[\mathrm{M}-$

$\mathrm{Br}]^{+}, \quad 287 / 289(39 / 36) \quad\left[\mathrm{M}-2\left(\mathrm{Me}_{2} \mathrm{~N}\right)\right]^{+}, 256 / 258 \quad(36 / 35) \quad[\mathrm{M}-$ $\left.\mathrm{P}\left(\mathrm{Me}_{2} \mathrm{~N}\right)_{2}\right]^{+}, 252(44)\left[\mathrm{M}-\mathrm{Me}_{2} \mathrm{NBr}\right]^{+}, 207$ (51) [M-(Me $\left.\left.{ }_{2} \mathrm{~N}\right)_{2} \mathrm{Br}\right]^{+}, 176$ (29) $\left[\mathrm{M}-\mathrm{P}\left(\mathrm{Me}_{2} \mathrm{~N}\right)_{2} \mathrm{Br}\right]^{+}, 119(32)[\mathrm{M}-\mathrm{AnBr}]^{+}$.

\subsubsection{Synthese von 9-Brom-10-bis(dimethylamino)thiophosphoranylanthracen (46)}

Die Synthese von 46 erfolgte nach AAV 6 mit $1.32 \mathrm{~g}$ (3.52 mmol) 9-Brom-10-bis(dimethylamino)phosphanylanthracen und $124 \mathrm{mg}$ (3.87 mmol) Schwefel.

Summenformel:

Molare Masse:

Ausbeute:

${ }^{1} \mathrm{H}-\mathrm{NMR}$

(300 $\mathrm{MHz}_{2} \mathrm{CDCl}_{3}$ ):

${ }^{13} \mathrm{C}\left\{{ }^{1} \mathrm{H}\right\}-\mathrm{NMR}$
$\mathrm{C}_{18} \mathrm{H}_{20} \mathrm{BrN}_{2} \mathrm{PS}$.

$407.31 \mathrm{~g} / \mathrm{mol}$.

$1.08 \mathrm{~g}(2.65 \mathrm{mmol}), 75 \%$.

$\delta=8.85-8.89(\mathrm{~m}, 2 \mathrm{H}, \mathrm{H} 1,8), 8.57-8.62\left(\mathrm{~m}, 2 \mathrm{H}, \mathrm{H}_{4,5}\right), 7.53-7.56(\mathrm{~m}$, $\left.2 \mathrm{H}, \mathrm{H}_{3,6}\right), 7.50-7.53\left(\mathrm{~m}, 2 \mathrm{H}, \mathrm{H}_{2,7}\right), 2.70\left(\mathrm{~d},{ }^{3} J_{\mathrm{HP}}=10.8 \mathrm{~Hz}, 12 \mathrm{H}, \mathrm{CH}_{3}\right)$. 
(75.5 MHz, $\left.\mathrm{CDCl}_{3}\right): \quad \delta=133.80\left(\mathrm{~d},{ }^{2} \mathrm{~J}_{\mathrm{CP}}=9.3 \mathrm{~Hz}, 2 \mathrm{C}, \mathrm{C}_{4 \mathrm{a}, 10 \mathrm{a}}\right), 130.48\left(\mathrm{~d},{ }^{3} J_{\mathrm{CP}}=12.5 \mathrm{~Hz}, 2 \mathrm{C}\right.$, $\left.\mathrm{C}_{8 \mathrm{a}, 9 \mathrm{a}}\right), 129.87\left(\mathrm{~d},{ }^{4} \mathrm{~J}_{\mathrm{CP}}=5.1 \mathrm{~Hz}, \mathrm{C}_{10}\right), 128.48\left(\mathrm{~s}, 2 \mathrm{C}, \mathrm{C}_{4,5}\right), 126.71(\mathrm{~s}$, $\left.2 \mathrm{C}, \mathrm{C}_{3,6}\right), 126.57\left(\mathrm{~s}, 2 \mathrm{C}, \mathrm{C}_{2,7}\right), 126.25\left(\mathrm{~d},{ }^{4} \mathrm{~J}_{\mathrm{CP}}=7.6 \mathrm{~Hz}, 2 \mathrm{C}, \mathrm{C}_{1,8}\right)$, $125.42\left(d,{ }^{1} J_{C P}=127.67 \mathrm{~Hz}\right), 37.60\left(d,{ }^{2} J_{C P}=4.2 \mathrm{~Hz}, 4 \mathrm{C}, \mathrm{CH}_{3}\right)$.

${ }^{31} \mathrm{P}\left\{{ }^{1} \mathrm{H}\right\}-\mathrm{NMR}$

(121.5 MHz, $\left.\mathrm{CDCl}_{3}\right): \quad \delta=72.70(\mathrm{~s})$.

EI-MS: $\mathbf{m} / \mathbf{z}(\%): \quad 406 / 408(3 / 3)[\mathrm{M}]^{+}, 317 / 319$ (8/9) $\left[\mathrm{M}-2 \cdot \mathrm{Me}_{2} \mathrm{~N}\right]^{+}, 239$ (9) [M-Br, $\left.2 \cdot \mathrm{NMe}_{2}\right]^{+}, 176(6)\left[\mathrm{M}-\mathrm{Br}, \mathrm{SP}\left(\mathrm{Me}_{2} \mathrm{~N}\right)_{2}\right]^{+}, 119(100)\left[\mathrm{M}-\mathrm{S}, \mathrm{C}_{14} \mathrm{H}_{8} \mathrm{Br}\right]^{+}, 76$ (10) $\left[\mathrm{M}-\mathrm{S}, \mathrm{C}_{14} \mathrm{H}_{8} \mathrm{Br}, \mathrm{NMe}_{2}\right]^{+}$.

CHNS, gef. (ber.) [\%]: $\quad$ C: 53.75 (53.08), H: 5.37 (4.95), N: 6.54 (6.88), S: 8.70 (7.87).

\subsubsection{Synthese von 9-Brom-10-bis(dimethylamino)selenophosphoranylanthracen (47)}

Die Synthese von 47 erfolgte nach AAV 6, mit $1.32 \mathrm{~g}$ (3.52 mmol) 9-Brom-10-Bis(dimethylamino)phosphanylanthracen und $306 \mathrm{mg}(3.88 \mathrm{mmol})$ grauem Selen.

Summenformel: $\quad \mathrm{C}_{18} \mathrm{H}_{20} \mathrm{BrN}_{2}$ PSe.

Molare Masse: $\quad 454.20 \mathrm{~g} / \mathrm{mol}$.

Ausbeute: $\quad 1.52 \mathrm{~g}(3.34 \mathrm{mmol}), 95 \%$.

${ }^{1} \mathrm{H}-\mathrm{NMR}$

$\left(500 \mathrm{MHz} \mathrm{CDCl}_{3}\right): \quad \delta=8.86-8.90\left(\mathrm{~m}, 2 \mathrm{H}, \mathrm{H}_{1,8}\right), 8.56-8.63\left(\mathrm{~m}, 2 \mathrm{H}, \mathrm{H}_{4,5}\right), 7.55-7.57(\mathrm{~m}$, $\left.2 \mathrm{H}, \mathrm{H}_{3,6}\right), 7.53-7.55\left(\mathrm{~m}, 2 \mathrm{H}, \mathrm{H}_{2,7}\right), 2.74\left(\mathrm{~d},{ }^{3} J_{\mathrm{HP}}=11.0 \mathrm{~Hz}, 12 \mathrm{H}, \mathrm{CH}_{3}\right)$.

${ }^{13} \mathrm{C}\left\{{ }^{1} \mathrm{H}\right\}-\mathrm{NMR}$

$\left(125.8 \mathrm{MHz}, \mathrm{CDCl}_{3}\right): \quad \delta=133.33\left(\mathrm{~d},{ }^{2} \mathrm{~J}_{\mathrm{CP}}=9.5 \mathrm{~Hz}, 2 \mathrm{C}, \mathrm{C}_{4 \mathrm{a}, 10 \mathrm{a}}\right), 130.59\left(\mathrm{~d},{ }^{3} J_{\mathrm{CP}}=12.5 \mathrm{~Hz}, 2 \mathrm{C}\right.$, $\left.C_{8 a, 9 a}\right), 129.61\left(d,{ }^{4} J_{C P}=5.2 \mathrm{~Hz}, 1 C, C_{9}\right), 128.28\left(d,{ }^{2} J_{C P}=9.5 \mathrm{~Hz}, 2 \mathrm{C}\right.$, $\left.\mathrm{C}_{4,5}\right), 126.83\left(\mathrm{~s}, 2 \mathrm{C}, \mathrm{C}_{3,6}\right), 126.34\left(\mathrm{~s}, 2 \mathrm{C}, \mathrm{C}_{2,7}\right), 126.25\left(\mathrm{~d},{ }^{4} \mathrm{~J}_{\mathrm{CP}}=\right.$ $\left.7.7 \mathrm{~Hz}, 2 \mathrm{C}, \mathrm{C}_{1,8}\right), 124.81\left(\mathrm{~d},{ }^{1} \mathrm{~J}_{\mathrm{CP}}=115.6 \mathrm{~Hz}, 1 \mathrm{C}, \mathrm{C}_{10}\right), 38.21\left(\mathrm{~d},{ }^{2} \mathrm{~J}_{\mathrm{CP}}=\right.$ $\left.3.89 \mathrm{~Hz}, 4 \mathrm{C}, \mathrm{CH}_{3}\right)$.

\section{${ }^{31} \mathrm{P}\left\{{ }^{1} \mathrm{H}\right\}-\mathrm{NMR}$}

(202.5 MHz, $\mathrm{CDCl}_{3}$ ): $\quad \delta=67.7$ (s).

${ }^{77} \mathrm{Se}\left\{{ }^{1} \mathrm{H}\right\}-\mathrm{NMR}$

(95 $\mathrm{MHz} \mathrm{CDCl}_{3}$ ):

$\delta=-143.75\left(\mathrm{~d},{ }^{1} \mathrm{~J}_{\mathrm{SeP}}=768.9 \mathrm{~Hz}\right)$.

EI-MS: $m / z(\%)$ :

$454 / 456(5 / 4)[\mathrm{M}]^{+}, 365 / 367(8 / 9)\left[\mathrm{M}-2 \cdot \mathrm{Me}_{2} \mathrm{~N}\right]^{+},(330 / 332)(5 / 6)$ $\left[\mathrm{M}-\mathrm{Se}, \mathrm{NMe}_{2}\right]^{+}, 207$ (10) $\left[\mathrm{M}-\mathrm{Br}, \mathrm{Se}, 2 \cdot \mathrm{Me}_{2} \mathrm{~N}\right]^{+}, 176$ (7) [M$\left.\mathrm{Br}, \mathrm{PSe}\left(\mathrm{Me}_{2} \mathrm{~N}\right)_{2}\right]^{+}, \quad 119$ (100) $[\mathrm{M}-\mathrm{Se}, \mathrm{AnBr}]^{+}, \quad 76 \quad$ (17) $[\mathrm{M}-$ $\left.\mathrm{Se}, \mathrm{AnBr}, \mathrm{NMe}_{2}\right]^{+}$.

CHN, gef. (ber.) [\%]: $\quad$ C: 47.92 (47.60), H: 4.49 (4.44), N: 6.21 (6.17). 


\subsubsection{Synthese von 9,10-Bis(dimethylamino)phosphanylanthracen (48)}

Die Synthese von 48 erfolgte nach AAV 4 mit $4.00 \mathrm{~g}$ (11.91 mmol) 9,10-Dibromanthracen, $13.56 \mathrm{~mL}(24.40 \mathrm{mmol})$ einer $1.80 \mathrm{M} \mathrm{nBuLi-Lösung} \mathrm{in} \mathrm{Hexan} \mathrm{und} 3.68 \mathrm{~g}(23.80 \mathrm{mmol})$ Bis(dimethylamino)chlorphosphan. 48 wurde als rote hochviskose Flüssigkeit erhalten.

Summenformel:

Molare Masse:

Ausbeute:

${ }^{1}$ H-NMR

(200 MHz, $\left.\mathrm{CDCl}_{3}\right)$ :

${ }^{31} \mathrm{P}\left\{{ }^{1} \mathrm{H}\right\}-\mathrm{NMR}$

$\left(81 \mathrm{MHz}, \mathrm{CDCl}_{3}\right)$ :

$$
\mathrm{C}_{22} \mathrm{H}_{32} \mathrm{~N}_{4} \mathrm{P}_{2} \text {. }
$$$$
414.46 \mathrm{~g} / \mathrm{mol} \text {. }
$$

$4.74 \mathrm{~g}(11.44 \mathrm{mmol}), 96 \%$.

$\delta=8.54-8.61\left(\mathrm{~m}, 4 \mathrm{H}, \mathrm{H}_{1,4,5,8}\right), 7.24-7.30\left(\mathrm{~m}, 4 \mathrm{H}, \mathrm{H}_{2,3,6,7}\right), 2.50(\mathrm{~d}$, $\left.{ }^{3} J_{\mathrm{HP}}=9.2 \mathrm{~Hz}, 24 \mathrm{H}, \mathrm{CH}_{3}\right)$.

$\delta=105.8(s)$

\subsubsection{Synthese von 9,10-Bis(dimethylamino)oxophosphoranylanthracen (49)}

Die Synthese von 49 erfolgte nach AAV 5 mit $1.23 \mathrm{~g} \quad(2.97 \mathrm{mmol}) \quad 9,10-$ Bis(dimethylamino)phosphanylanthracen und $0.26 \mathrm{~mL}$ (2.97 mmol) einer $35 \%$-igen $\mathrm{H}_{2} \mathrm{O}_{2^{-}}$ Lösung in Wasser. Das Produkt als rot-oranger Feststoff erhalten.

Summenformel: $\quad \mathrm{C}_{22} \mathrm{H}_{32} \mathrm{~N}_{4} \mathrm{O}_{2} \mathrm{P}_{2}$.

Molare Masse: $\quad 446.46 \mathrm{~g} / \mathrm{mol}$.

49 konnte spektroskopisch nicht eindeutig nachgewiesen werden.

\subsubsection{Synthese von 9,10-Bis(dimethylamino)thiophosphoranylanthracen (50)}

Die Synthese von 50 erfolgte nach AAV 6 mit $1.29 \mathrm{~g}$ (3.11 mmol) 9,10-Bis(dimethylamino)phosphanylanthracen und $110 \mathrm{mg}(3.42 \mathrm{mmol})$ Schwefel.

Summenformel:

Molare Masse:

Ausbeute:

${ }^{1} \mathrm{H}-\mathrm{NMR}$

$\left(400 \mathrm{MHz}, \mathrm{CDCl}_{3}\right)$ :
$\mathrm{C}_{22} \mathrm{H}_{32} \mathrm{~N}_{4} \mathrm{~S}_{2} \mathrm{P}_{2}$.

$478.59 \mathrm{~g} / \mathrm{mol}$.

$1.40 \mathrm{~g}(2.93 \mathrm{mmol}), 94 \%$.

$\delta=8.77-8.82\left(m, 4 H, H_{1,4,5,8}\right), 7.40-7.45\left(m, 4 H, H_{2,3,6,7}\right), 2.66(d$, $\left.{ }^{3} J_{\mathrm{HP}}=10.5 \mathrm{~Hz}, 24 \mathrm{H}, \mathrm{CH}_{3}\right)$. 
${ }^{13} \mathrm{C}\left\{{ }^{1} \mathrm{H}\right\}-N M R$

$\left(100.6 \mathrm{MHz}, \mathrm{CDCl}_{3}\right): \quad \delta=133.15\left(\mathrm{dd},{ }^{2} \mathrm{~J}_{\mathrm{CP}}=10.0 \mathrm{~Hz},{ }^{3} J_{\mathrm{CP}}=10 \mathrm{~Hz}, 4 \mathrm{C}, \mathrm{C} 4 \mathrm{a}, 8 \mathrm{a}, 9 \mathrm{a}, 10 \mathrm{a}\right), 129.44$ $\left(d d,{ }^{1} J_{C P}=127.5 \mathrm{~Hz},{ }^{4} J_{C P}=4.9 \mathrm{~Hz} 2 \mathrm{C}, \mathrm{C}_{9,10}\right), 125.54-125.70(\mathrm{~m}, 8 \mathrm{C}$,

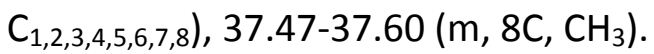

${ }^{31} \mathrm{P}\left\{{ }^{1} \mathrm{H}\right\}-\mathrm{NMR}$

$\left(121.5 \mathrm{MHz} \mathrm{CDCl}_{3}\right)$ : $\quad \delta=72.7(\mathrm{~s})$.

EI-MS: $m / z(\%):$

478.2 (4) $[\mathrm{M}]^{+}, 239.0$ (6) $\left[\mathrm{M}-2 \cdot \mathrm{S}, 4 \cdot \mathrm{Me}_{2} \mathrm{~N}\right]^{+}, 119.1$ (100) [M-S, $\left.\mathrm{C}_{14} \mathrm{H}_{8} \mathrm{PS}\left(\mathrm{NMe}_{2}\right)_{2}\right]^{+}, 76(14)\left[\mathrm{M}-2 \cdot \mathrm{S}, \mathrm{C}_{14} \mathrm{H}_{8} \mathrm{P}, 3 \cdot \mathrm{Me}_{2} \mathrm{~N}\right]^{+}$.

\subsubsection{Synthese von 9,10-Bis(dimethylamino)selenophosphoranylanthracen (51)}

Die Synthese von 51 erfolgte nach AAV 6 mit $1.30 \mathrm{~g}$ (3.14 mmol) 9,10-Bis(dimethylamino)phosphanylanthracen und $272 \mathrm{mg}$ (3.45 mmol) grauem Selen.

Summenformel: $\quad \mathrm{C}_{22} \mathrm{H}_{32} \mathrm{~N}_{4} \mathrm{Se}_{2} \mathrm{P}_{2}$.

Molare Masse: $\quad 572.38 \mathrm{~g} / \mathrm{mol}$.

Ausbeute: $\quad 1.75 \mathrm{~g}(0.05 \mathrm{mmol}), 97 \%$.

${ }^{1} \mathrm{H}-\mathrm{NMR}$

$\left(200 \mathrm{MHz}_{\mathrm{CDCl}}\right): \quad \delta=8.79-8.88\left(\mathrm{~m}, 4 \mathrm{H}, \mathrm{H}_{1,4,5,8}\right), 7.42-7.50\left(\mathrm{~m}, 4 \mathrm{H}, \mathrm{H}_{2,3,6,7}\right), 2.71(\mathrm{~d}$, $\left.{ }^{3} \mathrm{~J}_{\mathrm{HP}}=11.2 \mathrm{~Hz}, 24 \mathrm{H}, \mathrm{CH}_{3}\right)$.

EI-MS: $\mathbf{m} / \mathbf{z}(\%): \quad 574.1 \quad(2) \quad[M]^{+}, 494.2$ (4) $[\mathrm{M}-\mathrm{Se}]^{+}, 119.1$ (100) [M-Se, $\left.\mathrm{C}_{14} \mathrm{H}_{8} \mathrm{PSe}\left(\mathrm{NMe}_{2}\right)_{2}\right]^{+}, 76(12)\left[\mathrm{M}-2 \cdot \mathrm{Se}, \mathrm{C}_{14} \mathrm{H}_{8} \mathrm{P}, 3 \cdot \mathrm{Me}_{2} \mathrm{~N}\right]^{+}$.

\subsubsection{Synthese von 9-Bis(dimethylamino)phosphanyl-10-((trimethylethylen- diamino)methyl)anthracen (52)}

Die Synthese von $\mathbf{5 2}$ erfolgte nach AAV 4 mit $0.45 \mathrm{~g} \quad(1.21 \mathrm{mmol})$ 9-Brom-10((trimethyethylendiamino)methyl)anthracen, $0.67 \mathrm{~mL}(1.21 \mathrm{mmol})$ einer $1.80 \mathrm{M} n \mathrm{nBuLi}$ Lösung in Hexan und $187 \mathrm{mg}$ (1.21 mmol) Bis(dimethylamino)chlorphosphan zugetropft. Das Produkt wurde als roter Feststoff erhalten.

Summenformel:

$\mathrm{C}_{24} \mathrm{H}_{35} \mathrm{~N}_{4} \mathrm{P}$.

Molare Masse: $410.54 \mathrm{~g} / \mathrm{mol}$.

Ausbeute:

$0.20 \mathrm{~g}(0.49 \mathrm{mmol}), 40 \%$. 
${ }^{1}$ H-NMR

$\left(200 \mathrm{MHz}, \mathrm{CDCl}_{3}\right): \quad \delta=8.97\left(\mathrm{~d},{ }^{3} \mathrm{~J}_{\mathrm{HH}}=8.6 \mathrm{~Hz}, 2 \mathrm{H}, \mathrm{H}_{4,5}\right), 8.48-8.58\left(\mathrm{~m}, 2 \mathrm{H}, \mathrm{H}_{1,8}\right), 7.36-7.56$ $\left(\mathrm{m}, 4 \mathrm{H}, \mathrm{H}_{2,3,6,7}\right), 4.45\left(\mathrm{~s}, 2 \mathrm{H}, \mathrm{H}_{11}\right), 2.43-2.84\left(\mathrm{~m}, 4 \mathrm{H}, \mathrm{H}_{12,13}\right), 2.56$ (d, $\left.12 \mathrm{H}, \mathrm{CH}_{3}\right), 2.28\left(\mathrm{~s}, 3 \mathrm{H}, \mathrm{H}_{14}\right), 2.15\left(\mathrm{~s}, 6 \mathrm{H}, \mathrm{H}_{15}\right)$.

ESI-MS: $m / z(\%)$ : $449.2(14)[\mathrm{M}+\mathrm{O}+\mathrm{Na}]^{+}, 427.3(34)[\mathrm{M}+\mathrm{O}+\mathrm{H}]^{+}$.

\subsubsection{Synthese von 9-Diphenylphosphanyl-10-((trimethylethylendiamino)- methyl)anthracen (53)}

Die Synthese von 53 erfolgte nach AAV 4 mit $0.38 \mathrm{~g}$ (1.02 mmol) 9-Brom-10((trimethyethylendiamino)methyl)anthracen, $0.56 \mathrm{~mL}(1.02 \mathrm{mmol})$ einer $1.80 \mathrm{M} n \mathrm{nBuLi}$ Lösung in Hexan und $225 \mathrm{mg}$ (1.02 mmol) Bis(dimethylamino)chlorphosphan zugetropft. 53 wurde in Forum eines roten Feststoffes erhalten.

Summenformel: $\quad \mathrm{C}_{32} \mathrm{H}_{33} \mathrm{~N}_{2} \mathrm{P}$.

Molare Masse: $\quad 476.24 \mathrm{~g} / \mathrm{mol}$.

Ausbeute: $\quad 0.16 \mathrm{~g}(0.33 \mathrm{mmol}), 32 \%$.

${ }^{1}$ H-NMR

$\left(200 \mathrm{MHz}, \mathrm{CDCl}_{3}\right): \quad \delta=8.62\left(\mathrm{~d},{ }^{3} \mathrm{~J}_{\mathrm{HH}}=9.1 \mathrm{~Hz}, 2 \mathrm{H}, \mathrm{H}_{4,5}\right), 7.19-7.84\left(\mathrm{~m}, 16 \mathrm{H}, \mathrm{H}_{\mathrm{Ph}, 1,2,3,6,7,8}\right)$, $4.56\left(\mathrm{~s}, 2 \mathrm{H}, \mathrm{H}_{11}\right), 2.35\left(\mathrm{~s}, 3 \mathrm{H}, \mathrm{H}_{14}\right), 2.21\left(\mathrm{~s}, 6 \mathrm{H}, \mathrm{H}_{15}\right)$ Die Wasserstoffatome an Position 12 und 13 konnten nicht eindeutig zugewiesen werden.

ESI-MS: $\mathbf{m} / \mathbf{z}(\%): \quad 493.2(21)[\mathrm{M}+\mathrm{O}+\mathrm{H}]^{+}, 477.2(34)[\mathrm{M}+\mathrm{H}]^{+}$.

\subsubsection{Synthese von 9-((trimethylethylendiamino)methyl)anthracen (54)}

Die Lithiierung von 31 erfolgte nach AAV 2, mit $0.77 \mathrm{~g} \quad(2.07 \mathrm{mmol})$ 9-Brom-10((trimethyethylendiamino)methyl)anthracen und $1.15 \mathrm{~mL}(2.07 \mathrm{mmol})$ einer $1.80 \mathrm{M} \mathrm{nBuLi}-$ Lösung in Hexan. Die so erhaltene Reaktionslösung wurde anschließend bei $-15^{\circ} \mathrm{C}$ über 20 Min. mit $37 \mathrm{mg}$ (1.21 mmol) Wasser in $5 \mathrm{~mL}$ Diethylether versetzt. Das Lösungsmittel wurde i. Vak. entfernt, der erhaltene Feststoff in $10 \mathrm{~mL}$ Dichlormethan gelöst und über Celite ${ }^{\circledR}$ abfiltriert. Das Reaktionsprodukt wurde in Forum eines roten Feststoffes erhalten.

Summenformel: $\quad \mathrm{C}_{20} \mathrm{H}_{24} \mathrm{~N}_{2}$.

Molare Masse: $\quad 292.42 \mathrm{~g} / \mathrm{mol}$.

Ausbeute: $\quad 0.59 \mathrm{~g}(2.02 \mathrm{mmol}), 97 \%$. 


\section{${ }^{1}$ H-NMR}

$\left(300 \mathrm{MHz}, \mathrm{CDCl}_{3}\right)$ :

\section{${ }^{13} \mathrm{C}\left\{{ }^{1} \mathrm{H}\right\}-\mathrm{NMR}$}

$\left(75 \mathrm{MHz}, \mathrm{CDCl}_{3}\right)$ :

EI-MS: $\mathrm{m} / \mathrm{z}(\%)$ : $\delta=8.53\left(\mathrm{dd},{ }^{3} J_{\mathrm{HH}}=8.9 \mathrm{~Hz},{ }^{4} J_{\mathrm{HH}}=1.2 \mathrm{~Hz}, 2 \mathrm{H}, \mathrm{H}_{1,8}\right), 8.41\left(\mathrm{~s}, 1 \mathrm{H}, \mathrm{H}_{10}\right)$, $7.99\left(\mathrm{dd},{ }^{3} J_{\mathrm{HH}}=8.2 \mathrm{~Hz},{ }^{4} J_{\mathrm{HH}}=1.1 \mathrm{~Hz}, 2 \mathrm{H}, \mathrm{H}_{4,5}\right), 7.51$ (ddd, ${ }^{3} J_{\mathrm{HH}}=$ $8.2 \mathrm{~Hz},{ }^{3} J_{\mathrm{HH}}=6.5 \mathrm{~Hz},{ }^{4} J_{\mathrm{HH}}=1.2 \mathrm{~Hz}, 2 \mathrm{H}, \mathrm{H}_{3,6}$ ), 7.45 (ddd, ${ }^{3} J_{\mathrm{HH}}=8.9 \mathrm{~Hz}$, $\left.{ }^{3} J_{\mathrm{HH}}=6.5 \mathrm{~Hz},{ }^{4} J_{\mathrm{HH}}=1.1 \mathrm{~Hz}, 2 \mathrm{H}, \mathrm{H}_{2,7}\right), 4.44\left(\mathrm{~s}, 2 \mathrm{H}, \mathrm{H}_{11}\right), 2.73\left(\mathrm{dd},{ }^{3} \mathrm{~J}_{\mathrm{HH}}=\right.$ $\left.8.5 \mathrm{~Hz},{ }^{3} J_{\mathrm{HH}}=7.6 \mathrm{~Hz}, 2 \mathrm{H}, \mathrm{H}_{12}\right), 2.52\left(\mathrm{dd},{ }^{3} \mathrm{~J}_{\mathrm{HH}}=8.5 \mathrm{~Hz},{ }^{3} \mathrm{~J}_{\mathrm{HH}}=7.6 \mathrm{~Hz}\right.$, $\left.2 \mathrm{H}, \mathrm{H}_{13}\right), 2.28\left(\mathrm{~s}, 3 \mathrm{H}, \mathrm{H}_{14}\right), 2.19\left(\mathrm{~s}, \mathrm{H}_{15}\right)$.

$\delta=131.39\left(\mathrm{~s}, 2 \mathrm{C}, \mathrm{C}_{8 \mathrm{a}, 9 \mathrm{a}}\right), 131.32$ (s, 2C, $\left.\mathrm{C}_{4 \mathrm{a}, 10 \mathrm{a}}\right), 130.20$ (s, 1C, $\left.\mathrm{C}_{10}\right)$, 128.94 (s, 2C, C4,5), 127.40 (s, 1C, C 10 ), 125.62 (s, 2C, C 3,6 ), 125.03 (s, 2C, C1,8), 124.77 (s, 2C, C2,7), 57.45 (s, 1C, C 13 ), 56.10 (s, 1C, C 12 ) $54.22\left(\mathrm{~s}, 1 \mathrm{C}, \mathrm{C}_{11}\right), 45.69$ (s, 2C, $\left.\mathrm{C}_{15}\right), 42.40$ (s, 1C, $\left.\mathrm{C}_{14}\right)$.

292.2 (7) [M] ${ }^{+}, 234.2$ (10) [M-CH2N(CH3)2]+, 191.1 (100) [M$\left.\mathrm{NCH}_{3}\left(\mathrm{CH}_{2}\right)_{2} \mathrm{~N}\left(\mathrm{CH}_{3}\right)_{2}\right]^{+}, 58.1$ (8) [M-AnCH $\left.\mathrm{NCH}_{3}\left(\mathrm{CH}_{2}\right)_{2}\right]^{+}$. 


\section{Kristallographischer Teil}

\subsection{Kristallauswahl und -applikation}

Luftempfindliche Kristalle wurden im Argongegenstrom aus Schlenk-Kolben entnommen und auf einem Objektträger in perfluoriertes Polyetheröl gegeben. Dieses Öl hat den Vorteil, dass alle bisher untersuchten Verbindungen darin unlöslich waren. So war gewährleistet, dass die Kristalle sich weder auflösten noch Lösungsmittelmoleküle verloren. Ein geeigneter Kristall wurde mit Hilfe eines Polarisationsmikroskops ausgewählt und so auf der Spitze eines Glasfadens positioniert, dass ein Öltropfen den Kristall vollständig umschloss. Dieser wurde sofort im Kaltgasstrom der Tieftemperaturanlage am Diffraktometer schockgefroren. Der Vorteil dieser Methode liegt darin, dass das Öl glasartig erstarrt und der Kristall während der Datensammlung auf dem Glasfaden fixiert bleibt.

Luft- und feuchtigkeitsempfindliche Kristalle wurden gegebenenfalls unter Schutzgaskühlung ausgewählt und appliziert. Dazu wurde eine Kühlvorrichtung genutzt, die im Arbeitskreis Stalke entwickelt wurde. ${ }^{[162,163]}$

Bei dieser wird flüssiger Stickstoff verdampft und über einen Objektträger mit einem Kristall in Polyetheröl geleitet. Durch Variation der Strömungsgeschwindigkeit und der Temperatur des Stickstoffstromes lassen sich definierte Temperaturen einstellen.

\subsection{Datensammlung}

Alle Daten wurden unter Verwendung monochromatisierter Mo- $\mathrm{K}_{\alpha}$-Strahlung $(\lambda=71.073 \mathrm{pm})$ gemessen. Die Sammlung der Daten erfolgte auf einem Bruker D8 Goniometer mit einem APEX II Flächenzähler. Nach der Applikation wurde der Kristall mit Hilfe einer Videokamera zentriert. Um die Kristallqualität bewerten zu können und zur Bestimmung einer vorläufigen Elementarzelle, wurden 50 Frames im $\omega$-scan-fine-slicingModus (Schrittbreite $0.3^{\circ}$ ) detektiert. Entsprechend der Ergebnisse dieser Prescans wurden für die folgenden Datensammlungen passende Belichtungszeiten (1-45s) gewählt. Die Reflexe wurden im $\omega$-scan-Modus mit einer Schrittbreite von $0.3^{\circ}$ detektiert.

Die Bestimmungen und Verfeinerungen der Elementarzellen wurden mit APEX2 ${ }^{[164]}$ durchgeführt. Die Datenintegration erfolgte mit SAINT. ${ }^{[165]}$ An allen Daten wurde mit dem Programm SADABS ${ }^{[166]}$ oder TWINABS ${ }^{[167]}$ eine empirische Absorptionskorrektur vorgenommen. 


\subsection{Strukturlösung und Verfeinerung}

Alle Strukturen wurden entweder mit direkten Methoden oder durch eine PatersonSynthese gelöst (SHELXS). ${ }^{[168,169]}$ Die Strukturverfeinerungen erfolgten mit SHELXL. ${ }^{[169,170]}$

Aus den gemessenen Daten werden nach der Integration mit SAINT die Quadrate der Strukturfaktoren $F_{o}^{2}$ erhalten. Diese sind direkt proportional zu den gemessenen Intensitäten. Da mit SHELXL die Strukturen gegen $F^{2}$ verfeinert werden, wurde der Residualwert $w R 2$ als Gütekriterium verwendet und ist in den Tabellen angegeben.

$$
w R 2=\sqrt{\frac{\sum\left(\left|F_{o}^{2}\right|-\left|F_{c}^{2}\right|\right)^{2}}{\sum w F_{o}^{4}}} \quad \begin{gathered}
w^{-1}=\sigma^{2} \cdot F_{o}^{2}+(g 1 \cdot P)^{2}+g 2 \cdot P \\
\text { mit } P=\left(\frac{F_{o}^{2}+2 \cdot F_{c}^{2}}{3}\right)
\end{gathered}
$$

GI. 5.1: Definition des Residualwerts $w R 2$

In den Verfeinerungen wurden grundsätzlich alle gemessenen Daten berücksichtigt, ausgenommen einzelner Reflexe mit hohen Messfehlern (z. B. $F^{2}<-10$ ). Um Vergleiche mit alten gegen $F$ verfeinerten Strukturen zu ermöglichen, wurde außerdem $R 1$ für alle $F>2 \sigma(F)$ in den Tabellen angegeben.

$$
R 1=\frac{\Sigma|| F_{o}|-| F_{c} \mid}{\Sigma\left|F_{o}\right|}
$$

GI. 5.2: Definition des Residualwerts $R 1$

\subsection{Behandlung von Wasserstoffatomen}

Röntgenstrahlung wechselwirkt mit den Elektronen eines Atoms, so dass die Atompositionen nur aus der Elektronendichteverteilung ermittelt werden können. Wasserstoff verfügt als erstes Element im Periodensystem der Elemente über lediglich ein Elektron, welches durch die geringe Elektronegativität des Wasserstoffatoms zu einem etwaigen Bindungspater hin verschoben ist. Es können also nur Pseudowasserstoffpositionen aus der Differenzfourierkarte erhalten werden.

Der Beitrag eines Atoms zum Diffrakionsbild wird durch den Streufaktor beschrieben, welcher stark zur Anzahl an Elektronen des Atoms korreliert ist und mit steigendem Beugungswinkel stark abnimmt, was die Bestimmung der Pseudowasserstoffpositionen zusätzlich erschwert. Ist die Qualität der zugänglichen Daten schlecht und / oder sind prozentual viele Schweratome vorhanden, ist die Bestimmung der Pseudowasserstoffpositionen aus der Differenzfourierkarte nahezu unmöglich. In den Fällen, bei denen die 
Wasserstoffatompositionen aus der Differenzfourierkarte entnommen und zur Verfeinerung des Strukturmodels verwendet werden, sind die bestimmten Bindungslängen zu kurz und die Atompositionen variieren stark im Laufe des 'least squares' Strukturverfeinerungsprozesses. Die Beschreibung der Elektronendichte der Wasserstoffatome bewirkt jedoch eine deutliche Verbesserung des Strukturmodells, so dass diese in der Regel nicht vernachlässigt werden sollten. Die Atompositionen von Wasserstoffatomen werden daher unter Berücksichtigung der Messtemperatur sowie des Atomtyps und der Atomgeometrie des wasserstofftragenden Atoms berechnet und nach dem Reitermodell verfeinert.

Tab. 5.1: Liste der häufigsten HFIX Befehle.

\begin{tabular}{|c|c|c|c|c|c|}
\hline $\begin{array}{l}\text { HFIX } \\
\text { Befehl }\end{array}$ & $\begin{array}{l}\text { Bindungs- } \\
\text { partner }\end{array}$ & Hybridisierung & & Lewis Formel & $U_{\text {eq }}$ \\
\hline 13 & C & $\mathrm{sp}^{3}$ & $\mathrm{CH}$ & & -1.2 \\
\hline 23 & C & $s p^{3}$ & $\mathrm{CH}_{2}$ & & -1.2 \\
\hline $33^{a}$ & C & $s p^{3}$ & $\mathrm{CH}_{3}$ & & -1.5 \\
\hline 43 & $C, N$ & $\mathrm{sp}^{2}$ & $\mathrm{CH}, \mathrm{NH}$ & & -1.2 \\
\hline $83^{b}$ & $\mathrm{O}$ & $s p^{3}$ & $\mathrm{OH}$ & & -1.5 \\
\hline 93 & $C, N$ & $\mathrm{sp}^{2}$ & $\mathrm{CH}_{2}, \mathrm{NH}_{2}$ & & -1.2 \\
\hline $123^{c}$ & C & $s p^{3}$ & $\mathrm{CH}_{3,3}$ & & -1.5 \\
\hline 137 & C & $\mathrm{sp}^{3}$ & $\mathrm{CH}_{3}$ & & -1.5 \\
\hline 147 & $\mathrm{O}$ & $s p^{3}$ & $\mathrm{OH}$ & & -1.5 \\
\hline 163 & C & $\mathrm{sp}$ & $\mathrm{CH}$ & $\mathrm{R} \equiv$ & -1.2 \\
\hline
\end{tabular}

${ }^{a}$ bei guten Daten sollte der HFIX 137 verwendet werden; ${ }^{b}$ Bei den drei möglichen staggered Position im Fall von gesättigten Kohlenstoffatomen und den beiden Positionen in der Ebene bei aromatischen Ringen wird diejenige Position mit der kürzesten auffindbaren Wasserstoffbrücke zu einem Donoratom gewählt. Bei guten Daten sollte der HFIX 147 verwendet werden; ' nach Berechnung der Wasserstoffpositionen sollte die Besetzung der beiden Domänen mit einer freien Variable (z. B. 21, -21) erfolgen. Um Bindungen zwischen beiden Wasserstoffgruppen zu vermeiden können dise in verschiedene PART's sortiert werden. 
Die Berechnung der Wasserstoffpositonen erfolgt unter Verwendung der HFIX-Befehle (HFIX mn U[\#] d[\#] Atomnamen) im Laufe der Strukturmodellverfeinerung mit SHELXL. Die am häufigsten verwendeten HFIX Befehle sind in Tab. 5.1 aufgeführt. Die Tatsache, dass in dieser Tabelle alleine drei Befehle für die Berechnung von Wasserstoffatompositionen in Methylgruppen und zwei für die Berechnung von Wasserstoffatompositionen in $\mathrm{OH}-G$ ruppen aufgeführt sind, zeigt dass diesen eine Sonderstellung zukommt.

\subsection{Berechnung von Wasserstoffatompositionen in Methylgruppen}

a) b)

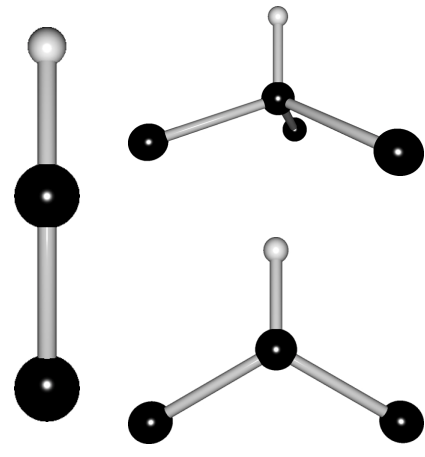

c)

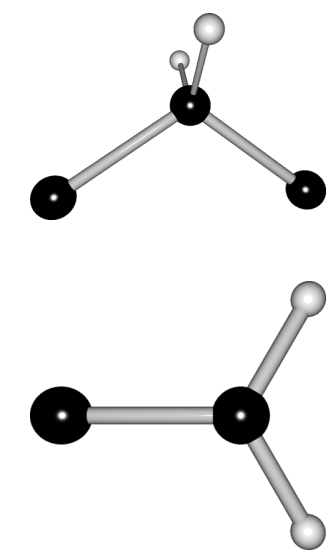

d)

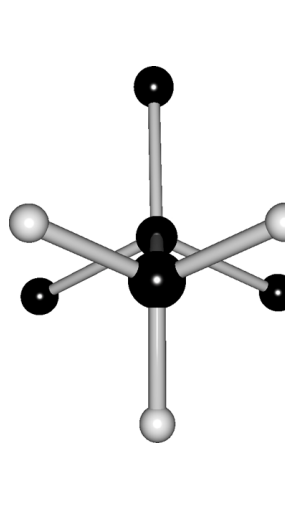

e)
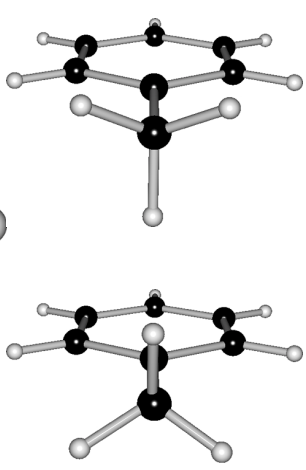

f)

Abb. 5.1: Die verschiedenen Typen von Kohlenstoff-Wasserstoff-Bindungen

Während die Berechnung von Wasserstoffpositionen für $\mathrm{CH}-$ Gruppen (bei $\mathrm{sp}^{3}-\mathrm{sp}^{2}-$ und $\mathrm{sp}$ hybridisierten Kohlenstoffatomen - Abb. 5.1a und b) und $\mathrm{CH}_{2}$-Groupen (bei $\mathrm{sp}^{3}$ - und $\mathrm{sp}^{2}$ hybridisierten Kohlenstoffatomen - Abb. 5.1c) einfach ist, lassen sich die Positionen von Wasserstoffatomen in $\mathrm{CH}_{3}$-Gruppen nur schwer ermitteln (Abb. 5.1d-f). Ist die $\mathrm{CH}_{3}$-Gruppe an ein gesättigtes Kohlenstoffatom gebunden, sollte die thermodynamisch stabilste Form eine gestaffelte Anordnung sein (Abb. 5.1d). Ist die Methylgruppe an einen Aromaten (z. B. Toluol) gebunden gibt es mehrere mögliche Anordnungen mit gleicher Energie (Abb. 5.1e). Für Methylgruppen, die an sp-hybridisierte Kohlenstoffatome (z. B. $\mathrm{CH}_{3}-\mathrm{CN}$ ) gebunden sind, existiert keine bevorzugte Stellung. Zusätzlich führen die relativ geringen Rotationsbarrieren von Methylgruppen (gleiches gilt auch für $\mathrm{OH}$-Gruppen) zu einer Vielzahl möglicher Wasserstoffatompositionen. Diese Problematik wird durch die verschiedenen HFIX-Befehle für Methylgruppen berücksichtigt. 


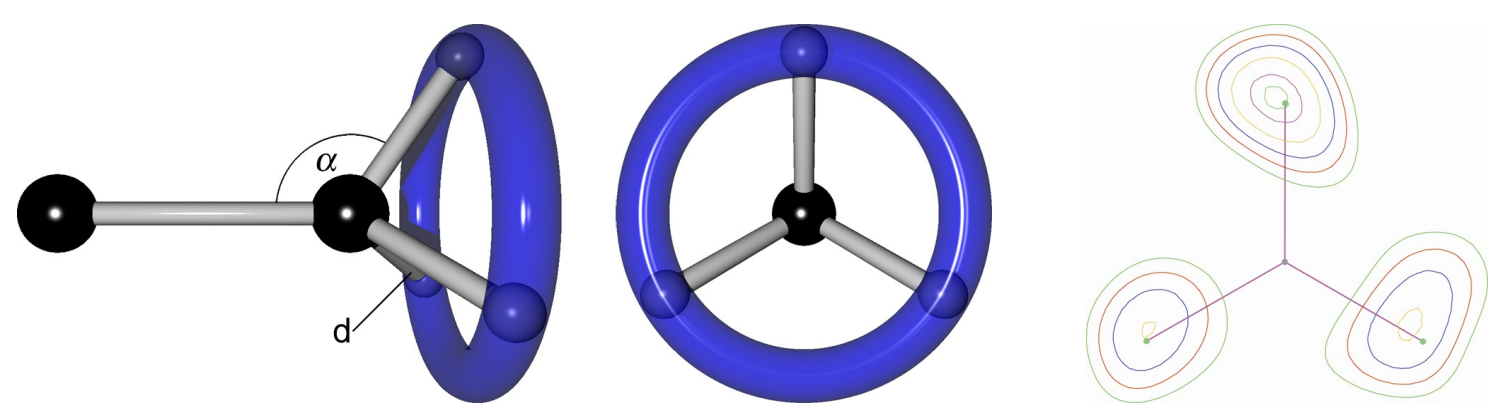

Abb. 5.2: Bestimmung der Wasserstoffpositionen einer Methylgruppe durch den HFIX 137 Befehl in SHELXL unter Verwendung von Bindungslänge $d$ und Winkel $\alpha$ (links). Restelektronendichte einer StandardMethylgruppe berechnet durch eine Wasserstoff-OMIT-map ${ }^{*}$ dargestellt durch einen Konturplot (rechts), erstellt mit dem Programm XP. ${ }^{[171]}$

Um möglichst exakte Atompositionen für Wasserstoffatome in Methylgruppen zu erhalten ist neben der Atomsorte des wasserstofftragenden Atoms auch dessen Hybridisierung notwendig. Werden Wasserstoffatompositionen durch die Verwendung des HFIX 137Befehls berechnet, wird eine Strukturfaktorenberechnung durchgeführt um die maximale Elektronendichte auf einem Kreis der erwarteten Wasserstoffpositionen zu erhalten (Abb. 5.2). Die so erhaltene Elektronendichteverteilung auf einem Kreis, welcher durch die $\mathrm{C}-\mathrm{H}$ Bindungslänge und den $\mathrm{X}-\mathrm{C}-\mathrm{H}$ Bindungswinkel beschrieben wird, sollte drei um $120^{\circ}$ versetzte Maxima mit gleicher integraler Höhe aufweisen. In Abb. 5.3 ist die Verteilung dieser Elektronendichte simuliert.

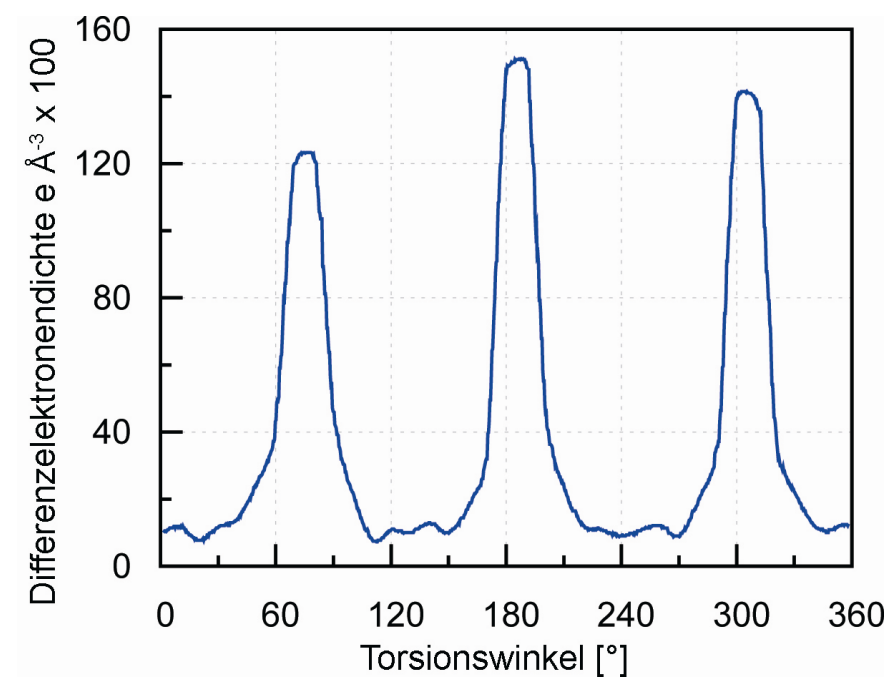

Abb. 5.3: Simulation der Restelektronendichte entlang eines $360^{\circ}$ Kreises, definiert durch die ideale Bindungslänge und den Bindungswinkel einer $\mathrm{CH}_{3}$-Gruppe. Die drei Maxima im Abstand von $120^{\circ}$ mit gleicher integraler Höhe entsprechen den Wasserstoffatompositionen. ${ }^{[172]}$

Die Wasserstoff OMIT-Karte wurde unter Verwendung der Befehle L.S. 0, OMIT \$H und LIST 3 mit SHELXL berechnet. 
Je nach Datenqualität kann die reelle Elektronendichteverteilung weit von den drei erwarteten Maxima abweichen. Aus diesem Grund wird mit SHELXL die Elektronendichteverteilung unter Berücksichtigung der lokalen dreizähligen Symmetrie gemittelt. Auf diese Weise kann eine ausreichend stabile Anordnung auch in nicht eindeutigen Fällen erhalten werden (siehe Abb. 5.4).
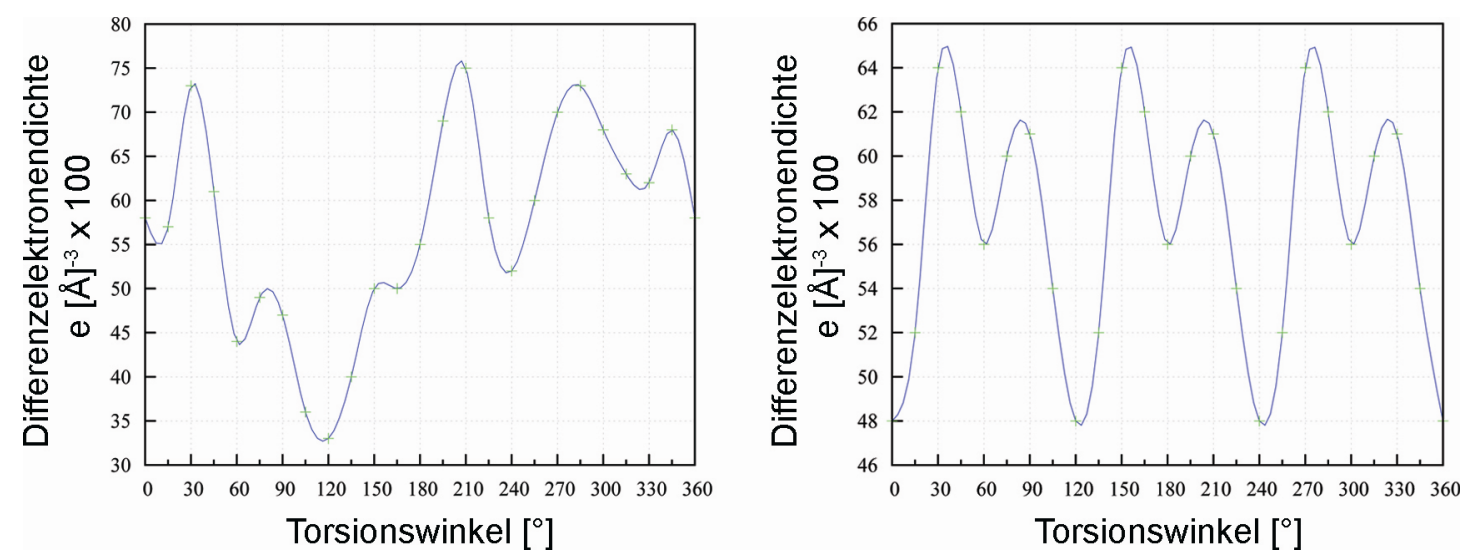

Abb. 5.4: Auftragung der Restelektronendichte am Ort einer $\mathrm{CH}_{3}-\mathrm{Gruppe}$ (links); Auftragung der gemittelten Restelektronendichte (rechts).

Alle diese Berechnungen werden von SHELXL im ersten Verfeinerungsschritt nach setzen des HFIX 137-Befehls durchgeführt. Die Werte der ermittelten Restdichten, die auch zur Auftragung der in Abb. 5.4 dargestellten Graphen verwendet wurden, können dem *.Ist-File entnommen werden.

\subsection{Fehlgeordnete Methylgruppen}

In manchen Fällen führt die Verwendung des HFIX 137-Befehls auch bei sehr guten Daten nicht zu stabilen Wasserstoffpositionen, was an einem kontinuierlichen Shiften des Torsionswinkels der entsprechenden Methylgruppe während der 'least squares'Verfeinerung zu erkennen ist. In einigen anderen Fällen, wie auch in dem in Abb. 5.4 abgebildeten Fall, zeigt sich der bestimmte Torsionswinkel als stabil, es tauchen jedoch Restdichten zwischen den beschriebenen Wasserstoffpositionen auf. Beide Fälle sind ein Anzeichen für Fehlgeordnete Wasserstoffatome der Methylgruppe. Durch die geringe Rotationsbarriere von Methylgruppen tritt diese Art der Fehlordnung sehr häufig auf. Da jedoch nur bei guten Daten, ausgeprägten Fehlordnungen (mit Besetzungen der Beiden Domänen nahe 50\%) und großen Unterschieden im Torsionswinkel (idealer weise $60^{\circ}$ ) die zweite Wasserstoffdomäne durch Restelektronendichtepeaks 'sichtbar' wird, bleibt diese Art der Fehlordnung häufig unbeachtet. Um auch nicht ideal fehlgeordnete Methylgruppen zu erkennen ist die Betrachtung von Restelektronendichtekarten, wie es z. B. mit dem 
Programm $\operatorname{COOT}^{[173,174]}$ möglich ist, von großem Nutzen. Grundsätzlich können drei Typen von fehlgeordneten Methylgruppen unterschieden werden (siehe Abb. 5.5).

a)

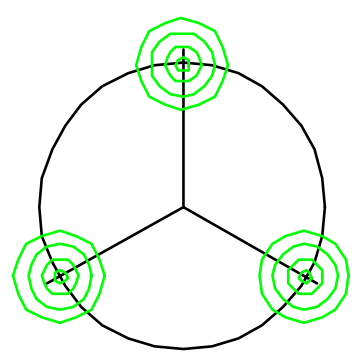

b)

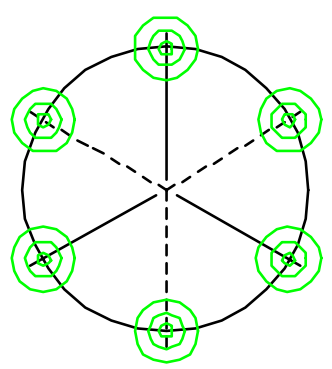

c)

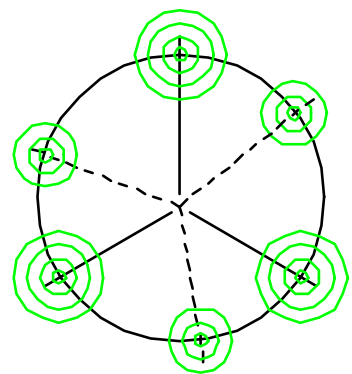

d)

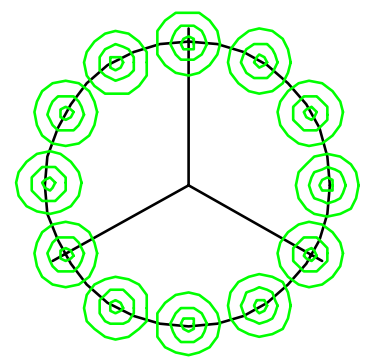

Abb. 5.5: Schematische Darstellung der Restelektronendichte von Methylgruppen: a) nicht fehlgeordnete Methylgruppe; b) Ideal Fehlgeordnete Methylgruppe (Torsionswinkeldifferenz $60^{\circ}$, Besetzung 50\%); c) real Fehlgeordnete Methylgruppe (beliebige Torsionswinkeldifferenz, beliebige Besetzung); d) Worst case, radial verteilte Elektronendichte mit nicht unterscheidbaren Positionen und Besetzungen.

Während in Abb. 5.5a die Restelektronendichte einer noch nicht beschriebenen idealen Methylgruppe schematisch dargestellt ist, zeigen die übrigen Abbildungen $c$ bis $d$ die schematischen Restdichten der drei Arten von fehlgeordnete Methylgruppen:

b) die ideal fehlgeordnete Methylgruppe, mit einer Torsionswinkeldifferenz von $60^{\circ}$ und einer Besetzung von jeweils 50\%. Welche zu finden so wahrscheinlich wie ein 6er im Lotto ist.

c) Die am häufigsten zu findende Fehlordnung mit zwei diskreten Positionen und variierender Besetzung.

d) Der worst case, mit radial verteilter Elektronendichte, bei der keine sinnvollen Wasserstoffpositionen gefunden werden können und die am besten durch einen Donut beschrieben werden könnte. Dies ist jedoch mit SHELXL zurzeit noch nicht möglich. Doch auch hier existiert durch den HFIX 127/123 ein Befehl mit dem Fehlgeordnete Methylgruppen beschrieben werden können.

\subsubsection{Beschreibung einer fehlgeordneten Methylgruppe mit dem HFIX 123/127 Befehl}

Wasserstoffatompositionen einer ideal fehlgeordneten Methylgruppe können mit dem HFIX 123 (bzw. HFIX 127 für $\mathrm{CH}_{3}$-Gruppen die an Aromaten oder Alkine gebunden sind) berechnet werden. Aus der Anwendung dieser HFIX-Befehle resultieren sechs halbbesetzte (sof $=10.50000$ ) Wasserstoffatome welche sich jeweils mit einer Torsionswinkeldifferenz von $60^{\circ}$ voneinander unterscheiden. Im *.res-File erscheinen die berechneten Wasserstoffatome wie auch bei allen anderen HFIX-Befehlen als AFIX-Befehle direkt unterhalb des wasserstoffatomtragenden Atoms: 


$\begin{array}{lcccccc}\text { C1 } & 1 & x & y & z & 11.00000 & U_{\text {eq }} \\ \text { AFIX } & 123 & & & & & \\ \text { H1a } & 2 & x & y & z & 10.50000 & -1.5 \\ \text { H1b } & 2 & x & \text { y } & z & 10.50000 & -1.5 \\ \text { H1C } & 2 & x & \text { y } & z & 10.50000 & -1.5 \\ \text { H1d } & 2 & x & \text { y } & z & 10.50000 & -1.5 \\ \text { H1e } & 2 & x & \text { y } & z & 10.50000 & -1.5 \\ \text { H1f } & 2 & x & \text { y } & \text { z } & 10.50000 & -1.5 \\ \text { AFIX } & 0 & & & & & \end{array}$

Die ersten drei Wasserstoffatome sind der ersten, die übrigen drei der zweiten Domäne zuzuordnen. Da eine solche Beschreibung einer Methylgruppe nur für den sehr selten auftretenden Fall einer ideal fehlgeordneten Methylgruppe gilt, sollte eine weitere Verbesserung des Modells angestrebt werden.

\subsubsection{Möglichkeiten zur Verbesserung des mit dem HFIX 123/127 beschriebenen Modells einer fehlgeordneten Methylgruppe}

Das Modell einer fehlgeordneten Methylgruppe welches durch den HFIX 123/127-Befehl berechnet wurde, gilt streng genommen nur für ideal fehlgeordnete Methylgruppen und kann mit wenig Aufwand der Realität etwas näher gebracht werden. So sollte der side occupation factor (sof) der Wasserstoffatome, welcher durch den HFIX 123/127-Befehl auf 10.5 gesetzt wird, durch eine freie Variable ersetzt werden, die eine unterschiedliche Besetzung der beiden Wasserstoffdomänen ermöglicht. Um eine unterschiedliche Besetzung der beiden Domänen (die jedoch auf eins aufaddieren) durch z. B. der freien Variable FVAR 2 zu realisieren, muss der sof von 10.5 auf 21 für die erste und -21 für die zweite Domäne geändert werden:

\begin{tabular}{|c|c|c|c|c|c|c|}
\hline C1 & 1 & $\mathrm{x}$ & y & Z & 11.00000 & $\mathrm{U}_{\text {eq }}$ \\
\hline AFIX & 123 & & & & & \\
\hline $\mathrm{H} 1 \mathrm{a}$ & 2 & $x$ & $\mathrm{y}$ & $z$ & 21.00000 & -1.5 \\
\hline $\mathrm{H} 1 \mathrm{~b}$ & 2 & $x$ & y & z & 21.00000 & -1.5 \\
\hline $\mathrm{H} 1 \mathrm{C}$ & 2 & $x$ & y & z & 21.00000 & -1.5 \\
\hline $\mathrm{H} 1 \mathrm{~d}$ & 2 & $x$ & $\mathrm{y}$ & z & -21.00000 & -1.5 \\
\hline $\mathrm{H} 1 \mathrm{e}$ & 2 & $x$ & $\mathrm{y}$ & z & -21.00000 & -1.5 \\
\hline $\mathrm{H} 1 \mathrm{f}$ & 2 & $x$ & Y & z & -21.00000 & -1.5 \\
\hline
\end{tabular}

\subsection{Behandlung von Fehlordnung}

Fehlordnung ist das am häufigsten auftretende 'Problem' in der Einkristallröntgendiffraktometrie. Neben vielen anderen Problemen wie Verzwillingung oder Falsche Atomzuordnung hat das Problem der Fehlordnung den deutlichen Vorteil, dass es auch von wenig geübten Kristallographen schnell als solches erkannt, wenn auch oft nicht berücksichtigt wird. Dies ist deshalb so leicht möglich, da direkt zu sehen ist, dass ein Problem vorhanden ist und in vielen Fällen auch um was es sich dabei handelt. 
Doch auch ein so simples Problem wie Fehlordnung kann einige Probleme bei dessen Beschreibung hervorrufen. Dies ist besonders der Fall, wenn es sich um Lösungsmittel auf bzw. in der Nähe von Speziellen Lagen oder um diffuse Lösungsmittelkanäle handelt.

Im Allgemeinen läst sich Fehlordnung in Lage- und Besetzungsfehlordnung einteilen. Während die Besetzungsfehlordnung klar definiert ist, muss die Lagefehlordnung weiter differenziert werden (siehe Abb. 5.6).

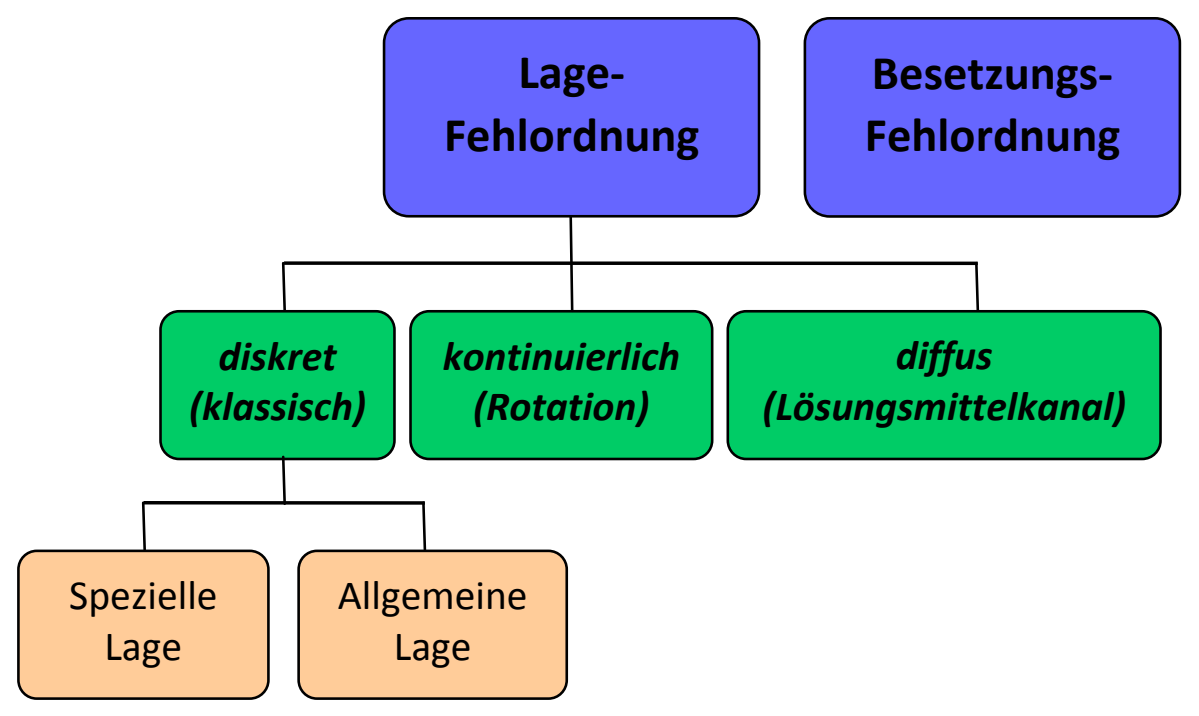

Abb. 5.6: Einteilung von Fehlordnung in verschiedene Klassen.

Unabhängig von der Art der Fehlordnung gibt es generelle Regeln welche die Beschreibung von Fehlordnung ermöglichen bzw. erleichtern:

1. Das Strukturmodell sollte so gut wie möglich beschrieben sein, bevor die Fehlordnung berücksichtigt wird. Dazu sollten alle Atome zugeordnet werden, wobei der Fehlgeordnete Teil der Struktur entweder unberücksichtigt bleibt, oder nur die Hauptdomäne beschrieben wird. Anschließend sollten alle Atome mit Ausnahme der etwaig beschriebenen Fehlgeordneten Atome anisotrop beschrieben und die zugehörigen Wasserstoffatome berechnet werden.

2. Das Wichtungsschema sollte bis zur Fertigstellung des gesamten Strukturmodells auf 0.1 festgehalten werden.

3. Falls nicht bereits der Fall, sollten alle an der Fehlordnung beteiligten Atome mit isotropen Auslenkungsparameter versehen und eventuell gebundene Wasserstoffatome gelöscht werden.

4. Die Fehlordnung sollte nun nach dem splitatom-Modell beschrieben werden. Können die nötigen Atompositionen nicht durch die Restelektronendichtepeaks erhalten werden, können evtl. Startkoordinaten dieser Atome dem *.Ist-File entnommen werden.

Der weitere Gang der Strukturverfeinerung unterscheidet sich nicht von dem einer Standardstruktur. 


\subsubsection{Besetzungs-Fehlordnung ${ }^{\dagger}$}

Die Besetzungs-Fehlordnung ist ein für Mineralien häufig auftretendes Phänomen, ist jedoch nicht auf diese Beschränkt. Das Auftreten dieser Art von Fehlordnung in Kleinmolekülstrukturen soll anhand eines Beispieles verdeutlicht werden.

In 2007 stellten Bolte et al. ein neues Polymorph des Komplexes $\left[\mathrm{MgBr}_{2} \cdot\{\text { thf }\}_{4}\right]$ vor. ${ }^{[175]}$ Die finale Strukturlösung zeigt jedoch einige Ungereimtheiten, welche sich nicht auf die beobachtete Verzwillingung zurückführen lassen. Neben den im Vergleich zu den übrigen Atomen viel zu großen Thermalelipsoiden der Bromatome (e.g. $\left.U_{\text {iso }}(B r 2) / U_{\text {iso }}(M g 1)=2.75\right)$ weisen auch andere untypische Werte, wie eine zu kurze Mg-Br-Bindungslängen (z. B. Mg1$\operatorname{Br} 1=2.562 \AA)$, auf ein Falsches Strukturmodell hin. Diese Annahme spiegelt sich auch in den erhaltenen $R$-Werten ( $\mathrm{w} R 2=0.232, R 1=0.087$ ) und dem GooF-Wert (1.44) wieder.

Wird die Synthese berücksichtigt, welche zu der Bildung der gemessenen Kristalle durchgeführt wurde, wird klar, dass der erhaltenen Komplex eine Mischung der Salze $\mathrm{MgBr}_{2}$ und $\mathrm{MgCl}_{2}$ enthalten muss, bei dem die Atompositionen der Chlorid- und Bromidionen durch Besetzungsfehlordnung nahezu auf die selbe Position fallen.

Eine neue Verfeinerung der von Bolte gemessenen Daten unter Berücksichtigung dieser Fehlordnung verbessert das Strukturmodell erheblich $(w R 2=0.143, \quad R 1=0.054$, GooF = 1.001) ${ }^{[176]}$

\subsubsection{Lage-Fehlordnung}

Die für Kleinmoleküle am Häufigsten auftretende Art der Fehlordnung ist die LageFehlordnung. In Abb. 5.7 sind zwei Beispiele von typischen Lagefehlordnungen abgebildet.
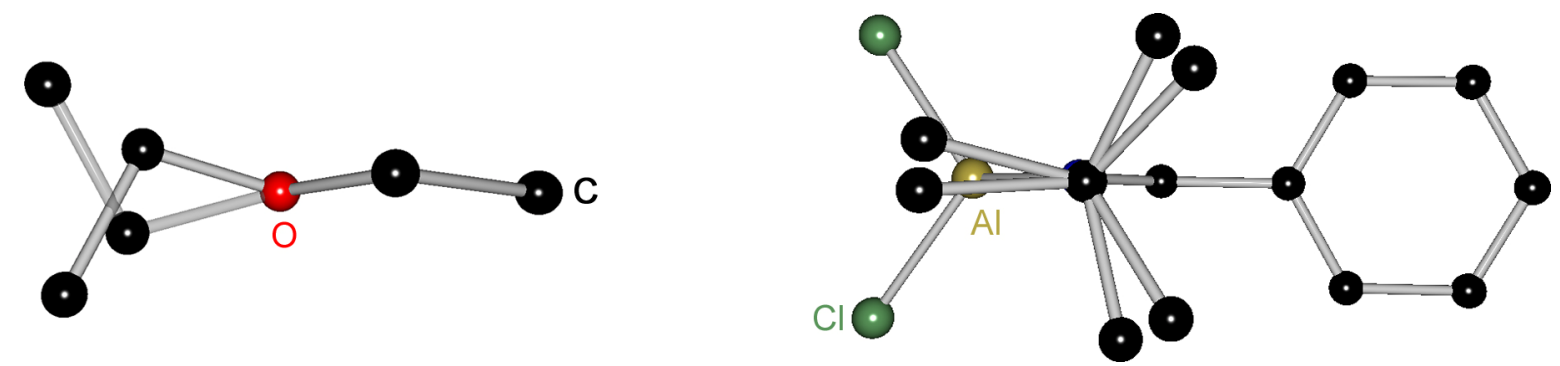

Abb. 5.7: Positionsfehlordnung eines Diethylethermoleküls (links) und einer tert-Buthylgruppe (rechts).

Bei dem linken Beispiel handelt es sich um ein über das Sauerstoffatom Metallkoordinierendes Diethylether Molekül, dessen eine Ethylgruppe zwei der möglichen Orientierungen einnimmt. Das rechte Beispiel zeigt eine fehlgeordnete tert-Buthylgruppe die

\footnotetext{
${ }^{\dagger}$ Diese Art der Fehlordnung ist einer der wenigen Gründe für gebrochene Summenformeln.
} 
zwei equienergetische Positionen einnimmt. Wie auch die meisten Fehlordnungen dieser Art, können auch diese beiden Beispiele durch das splitatom-Modell beschrieben werden. Nachdem die Hauptdomäne zugeordnet wurde können die Atompositionen der zweiten Domäne als Restdichtepeaks gefunden werden. Um Bindungen zwischen den beiden Fehlgeordneten Strukturteilen zu vermeiden, werden diese in Verschiedene PART's sortiert:

$\begin{array}{lllllll}\text { O1 } & 1 & x & y & z & 11.00000 & U_{\text {eq }} \\ \text { PART } & 121 & & & & \\ \text { C1 } & 1 & x & y & z & \mathbf{2 1 . 0 0 0 0 0} \\ \text { C2 } & 1 & x & y & z & \mathbf{2 1 . 0 0 0 0 0} \\ \text { PART } 2-21 & & & & \\ \text { C1 } & 1 & x & y & z & -\mathbf{2 1 . 0 0 0 0 0} \\ \text { C2 } & 1 & x & y & z & -\mathbf{2 1 . 0 0 0 0 0}\end{array}$

Wie auch schon weiter oben für fehlgeordnete Wasserstoffatome diskutiert sollte die Besetzung der beiden Domänen durch eine freie Variable erfolgen. Hierzu kann der sof entweder per Hand angepasst werden oder hinter den jeweiligen PART-Befehl geschrieben werden. Bei der letzteren Methode werden die sof-Werte der Atome des entsprechenden Parts ignoriert und überschrieben. Da den Wasserstoffatomen durch den HFIX-Befehl automatisch der sof-Wert des Wasserstoffatomtragenden Atoms zugewiesen wird, kann die Berechnung der Wasserstoffatompositionen wie gewohnt erfolgen.

\subsubsection{Fehlordnung auf spezieller Lage}

a)

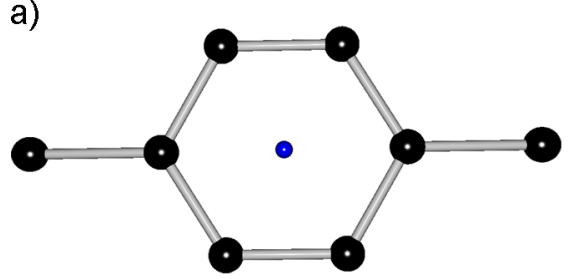

b)

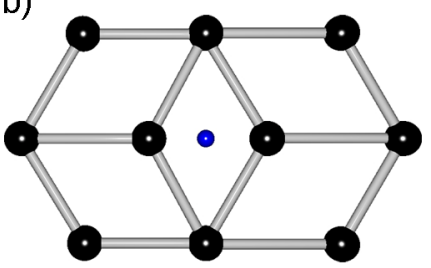

c)

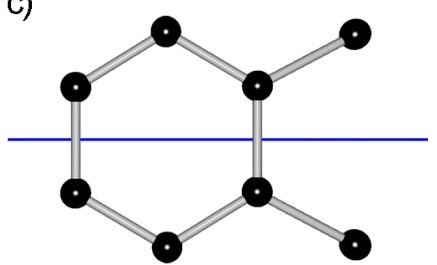

d)

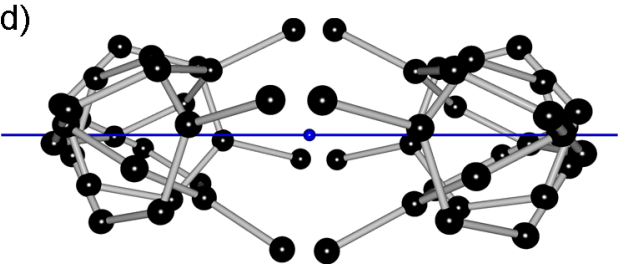

e)

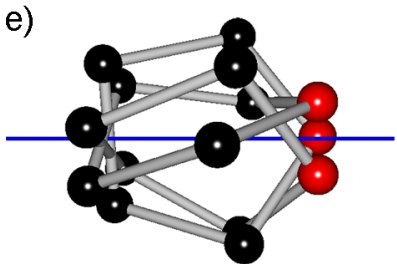

Abb. 5.8: Beispiele für Fehlordnung durch Lösungsmittelmoleküle auf einer speziellen Lage: a) und b) Toluol auf einem Inversionszentrum; c) Toluol auf einem Spiegel oder einer zweizähligen Achse; d) Toluol auf einer $\overline{4}$-Achse; e) THF auf einer dreizähligen Achse.

Weitaus schwieriger lassen sich Fehlordnungen Beschreiben, die z. B. durch Lösungsmittelmoleküle auf speziellen Lagen hervorgerufen werden. Eines der wohl am häufigsten zu findenden Beispiele hierfür ist Toluol. In Abb. 5.8 sind vier Beispiele von Toluol auf speziellen Lagen abgebildet. Der Schwierigkeitsgrad der Beschreibung solcher Fehlordnungen steigt mit der Komplexität des Symmetrieelementes an. So besteht wie bei den beiden letzten Beispielen aus Abb. 5.8 das erste Problem bereits bei der Identifizierung 
des Lösungsmittels selbst. Oftmals ist viel Kreativität und Ausdauer nötig um zu den zur Verfeinerung nötigen Atomkoordinaten zu gelangen. Im Gegensatz zu 'normalen' Positionsfehlordnungen ist die Anwendung von geometriestabilisierenden constranis nicht nur hilfreich sonder essentiell um zu einem stabilen Strukturmodell zu gelangen. Die für ein Toluol auf einem Inversionszentrum nötigen constranis könnten z. B. wie folgt aussehen.

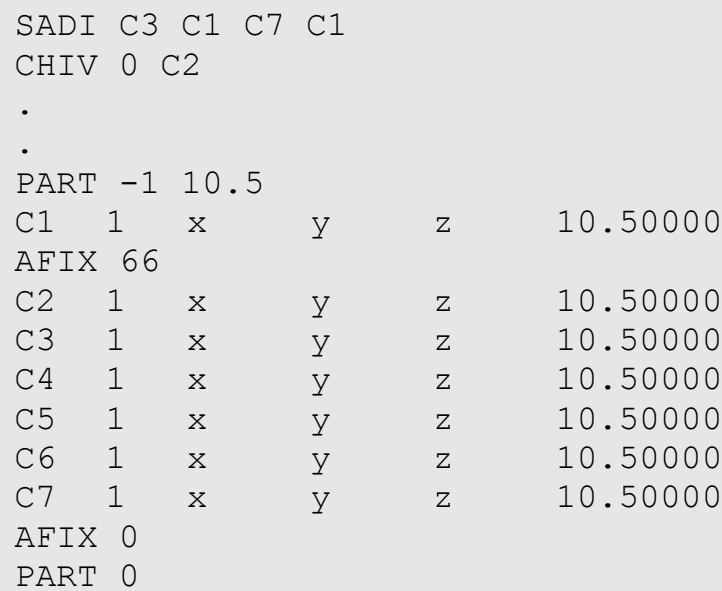<smiles></smiles>

Die Besetzung der Atome muss an das Symmetrieelement angepasst werden und ist für das hier gezeigte Beispiel 0.5. Das sortieren der auf diese weise fehlgeordneten Atome in einen Part -1 sorgt u. a. dafür, dass keine Bindungen zu Symmetrieerzeugten Atomen generiert werden. Mit der Fülle an restraints und constrans stehen auch mehrere Möglichkeiten zur Verfügung um die Geometrie Fehlgeordneter Gruppen zu stabilisieren.

\subsection{Constrains und Restarints}

Die Beschreibung von Fehlordnungen wird unter Zuhilfenahme von constraints und restraints oftmals wesentlich erleichtert.

Ein constraint ist eine mathematische Operation, die Parameter auf einen exakten Wert setzt. So wurden z. B. in den meisten Strukturen Wasserstoffatompositionen nicht frei verfeinert, sondern nach geometrischen Gesichtspunkten berechnet und nach dem Reitermodell in die Verfeinerung mit einbezogen.

Restraints sind zusätzliche chemische oder kristallographische Informationen über die Struktur einer Verbindung, die mit einer Standardabweichung versehen sind. Diese restraints gehen als zusätzliche Daten in die Verfeinerung ein. Die Standardabweichung hat dabei die Funktion einer Gewichtung.

Wenn in einer Struktur chemisch gleiche, aber kristallographisch unabhängige Strukturelemente vorkommen, ist die Anwendung von Abstands-restraints sinnvoll. Hier können vor allem 1,2-Abstände (Bindungslängen) und 1,3-Abstände (Winkel) innerhalb einer Standardabweichung zu einem ähnlichen Wert verfeinert werden. Besonders bei der Verfeinerung fehlgeordneter Positionen kann durch diese restraints eine erhebliche 
Verbesserung des Strukturmodells erfolgen, da über die Mittelung äquivalenter Strukturfragmente chemische Informationen genutzt werden können.

Um auch fehlgeordnete Atompositionen mit Hilfe eines anisotropen Modells verfeinern zu können, wurden restraints, welche die Auslenkungsparameter betreffen (ADP-restraints), verwendet (siehe Abb. 5.9):

- Mittels rigid bond restraints (DELU) werden die Komponenten der anisotropen Auslenkungsparameter entlang der Bindungen innerhalb einer Standardabweichung angeglichen.

- Similarity restraints (SIMU) sind schwache restraints, welche die Auslenkungsparameter benachbarter Atome in einem bestimmten Radius einander annähern.

- ISOR restraints zwingen anisotrope Auslenkungsellipsoide in eine eher sphärische, isotrope Form. Dadurch gelingt häufig, vor allem bei fehlgeordneten Atompositionen mit geringer Besetzung, eine anisotrope Verfeinerung.
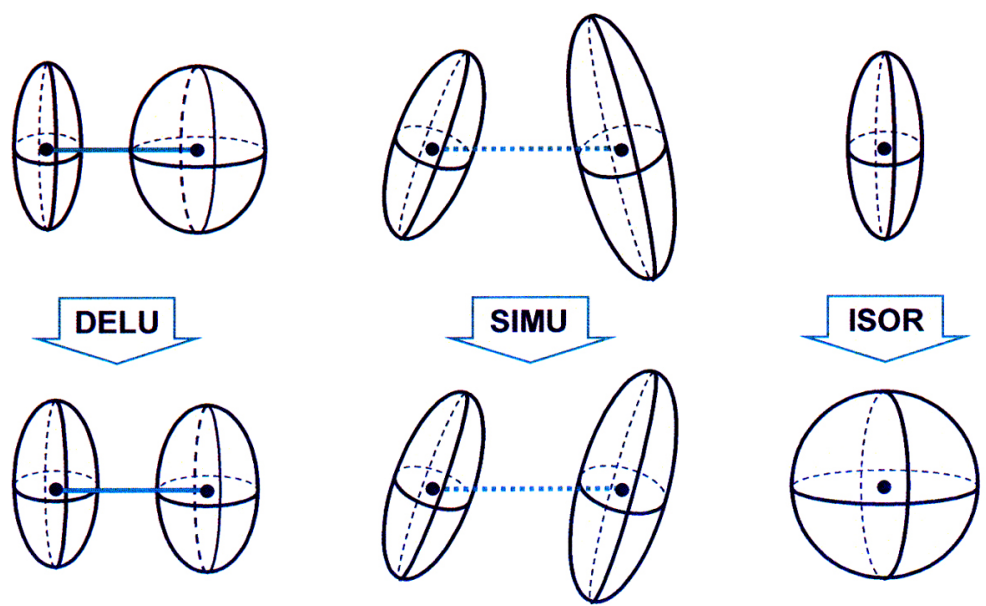

Abb. 5.9: Funktion der ADP-restraints DELU, S IMU und ISOR. ${ }^{[172]}$

\subsection{Graphische Darstellungen}

Die anisotropen Auslenkungsellipsoide der Strukturabbildungen stellen $50 \%$ der Aufenthaltswahrscheinlichkeit der Elektronendichte der Atome dar. Sämtliche im kristallographischen Teil dieser Arbeit dargestellten Strukturbilder wurden mit Hilfe des Programms XSHELL V4.01 erstellt. ${ }^{[177]}$ 


\subsection{Kristallographische Daten gemessener Kristallstrukturen}

\subsubsection{Eigene Strukturen}

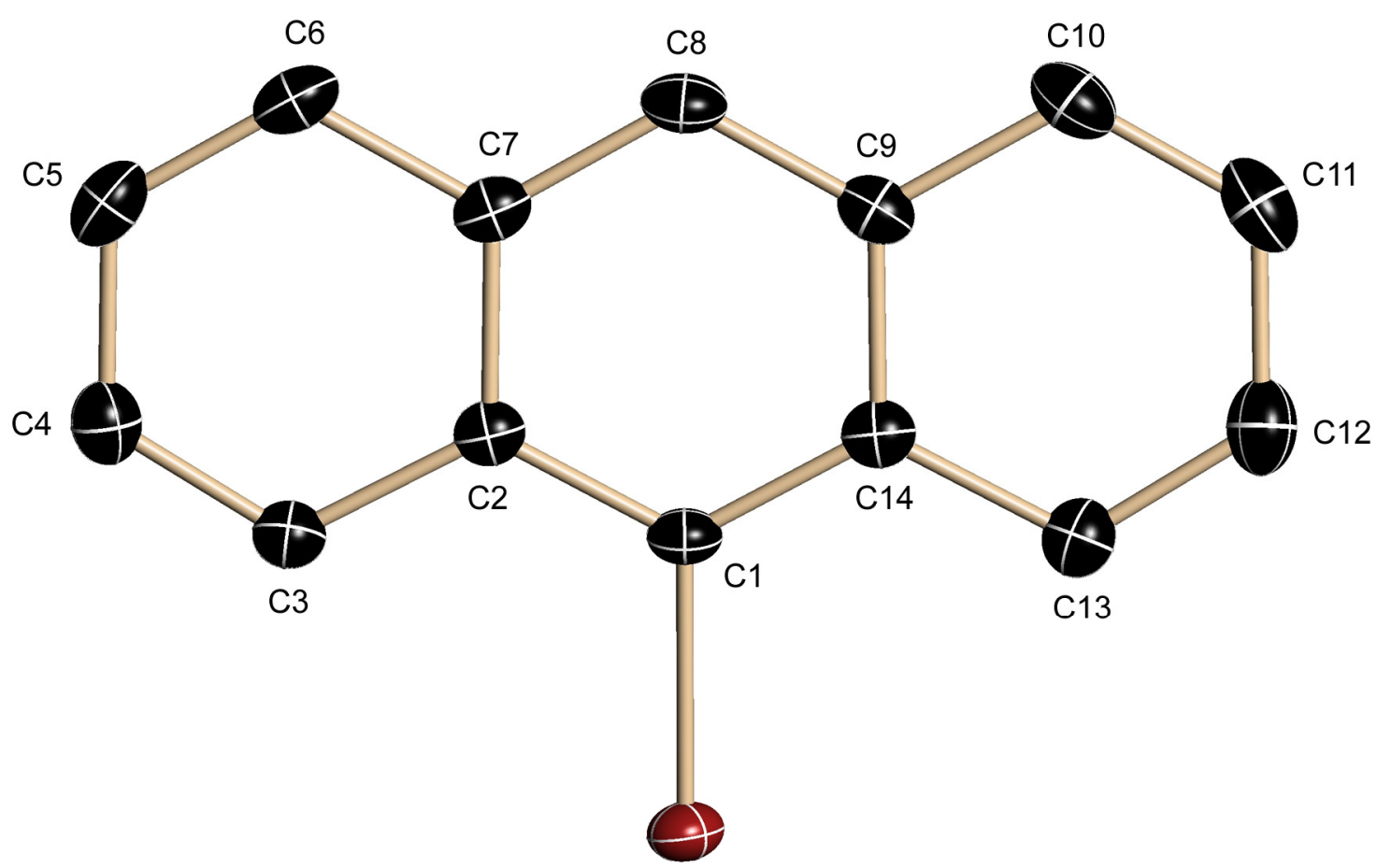

$\mathrm{Br} 1$

Abb. 5.10: Asymmetrische Einheit von 9-Bromanthracen (1); die anisotropen Auslenkungsparameter geben die $50 \%$ ige Aufenthaltswahrscheinlichkeit an.

Tab. 5.2: Kristallographische Daten von 9-Bromanthracen (1).

\begin{tabular}{|c|c|c|c|}
\hline Strukturcode & $\mathrm{BrAnH}$ & $\mu\left[\mathrm{mm}^{-1}\right]$ & 4.050 \\
\hline Summenformel & $\mathrm{C}_{14} \mathrm{H}_{9} \mathrm{Br}$ & $F(000)$ & 512 \\
\hline Molekulare Masse $[\mathrm{g} / \mathrm{mol}]$ & 257.12 & $\theta$-Bereich $\left[{ }^{\circ}\right]$ & $1.84-27.87$ \\
\hline Kristallgröße [mm] & $0.21 \times 0.05 \times 0.03$ & gesammelte Reflexe & 10673 \\
\hline Raumgruppe & $P 2_{1} / c$ & unabhängige Reflexe & 2389 \\
\hline$a[\mathrm{pm}]$ & $394.92(4)$ & Zahl der Restraints & 0 \\
\hline$b[\mathrm{pm}]$ & $1397.52(12)$ & verfeinerte Parameter & 136 \\
\hline$c[\mathrm{pm}]$ & $1829.01(16)$ & $R 1[I>2 \sigma(I)]$ & 0.0254 \\
\hline$\beta\left[^{\circ}\right]$ & $95.9560(10)$ & $w R 2$ [alle Daten] & 0.0676 \\
\hline$V\left[\mathrm{~nm}^{3}\right]$ & $1.00400(16)$ & GooF & 1.056 \\
\hline Z & 4 & Differenzelektronendichte: & \\
\hline Messtemperatur [K] & $100(2)$ & Max. / min. $\left[10^{-6} \mathrm{epm}^{-3}\right]$ & $0.762 /-0.324$ \\
\hline$\rho\left[\mathrm{Mgm}^{-3}\right]$ & 1.701 & Max. / min. Transmission & $0.4322 / 0.3433$ \\
\hline
\end{tabular}




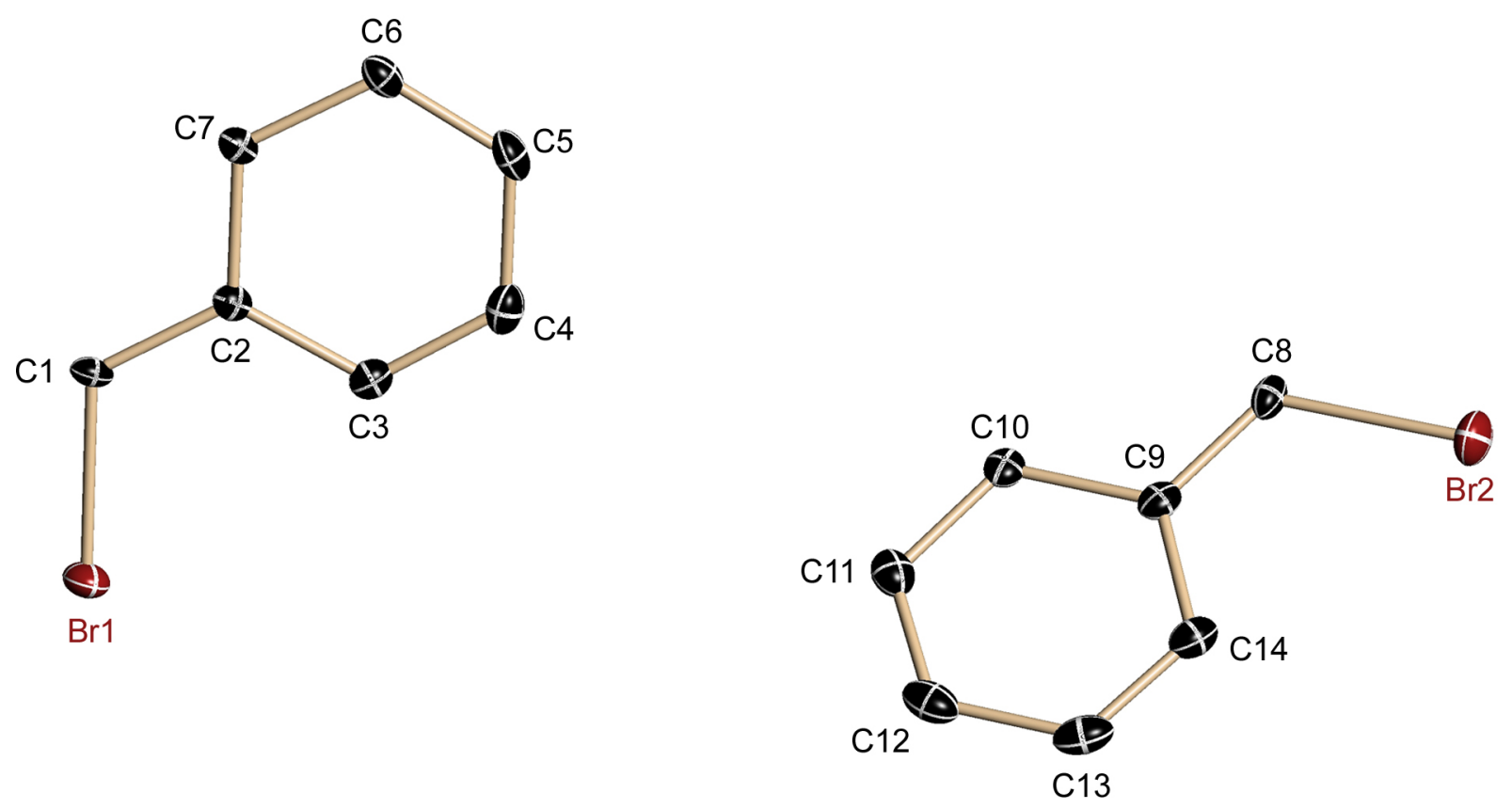

Abb. 5.11: Asymmetrische Einheit von 9,10-Dibromanthracen (2); die anisotropen Auslenkungsparameter geben die $50 \%$ ige Aufenthaltswahrscheinlichkeit an.

Tab. 5.3: Kristallographische Daten von 9,10-Dibromanthracen (2).

\begin{tabular}{|c|c|c|c|}
\hline Strukturcode & $\mathrm{BrAnBr}$ & $\mu\left[\mathrm{mm}^{-1}\right]$ & 7.430 \\
\hline Summenformel & $\mathrm{C}_{14} \mathrm{H}_{8} \mathrm{Br}_{2}$ & $F(000)$ & 324 \\
\hline Molmasse $[\mathrm{g} / \mathrm{mol}]$ & 336.02 & $\theta$-Bereich $\left[{ }^{\circ}\right]$ & $1.29-26.81$ \\
\hline Kristallgröße [mm] & $0.2 \times 0.04 \times 0.03$ & gesammelte Reflexe & 2554 \\
\hline Raumgruppe & $P \overline{1}$ & unabhängige Reflexe & 2308 \\
\hline$a[\mathrm{pm}]$ & $397.29(3)$ & Zahl der Restraints & 0 \\
\hline$b[\mathrm{pm}]$ & $879.70(7)$ & verfeinerte Parameter & 145 \\
\hline$c[\mathrm{pm}]$ & 1608.95(13) & $R 1[\mathrm{I}>2 \sigma(\mathrm{I})]$ & 0.0427 \\
\hline$\alpha\left[^{\circ}\right]$ & $78.8980(10)$ & $w R 2$ [alle Daten] & 0.1208 \\
\hline$\beta\left[^{\circ}\right]$ & $83.8890(10)$ & GooF & 1.125 \\
\hline$\gamma\left[^{\circ}\right]$ & $80.6470(10)$ & & \\
\hline$V\left[\mathrm{~nm}^{3}\right]$ & $0.54282(7)$ & Differenzelektronendichte: & \\
\hline$Z$ & 2 & Max. / min. $\left[10^{-6} \mathrm{epm}^{-3}\right]$ & $1.789 /-0.806$ \\
\hline Messtemperatur [K] & $100(2)$ & Max. / min. Transmission & 0.4299 / 0.1739 \\
\hline$\rho\left[\mathrm{Mgm}^{-3}\right]$ & 2.056 & & \\
\hline
\end{tabular}




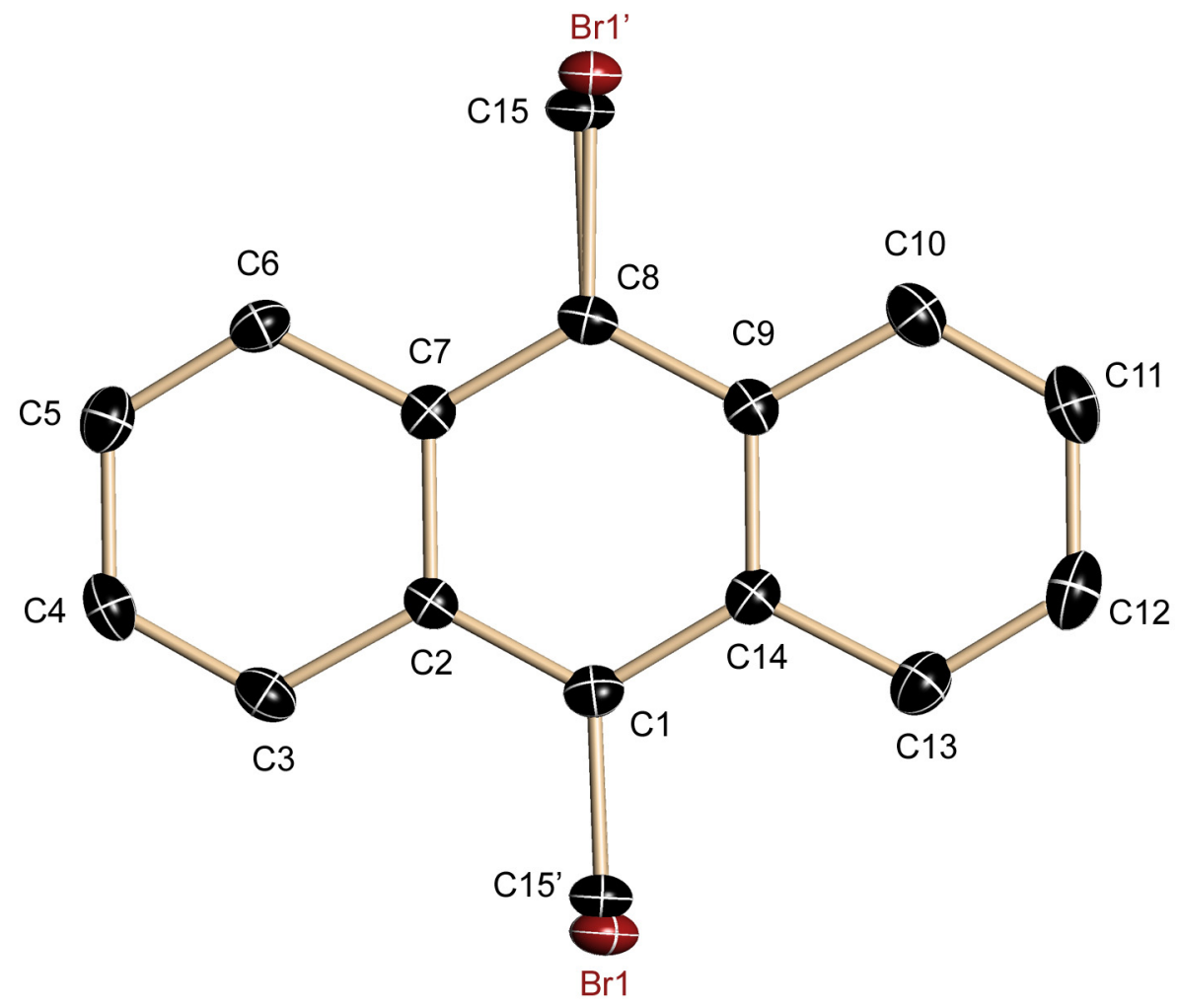

Abb. 5.12: Asymmetrische Einheit von 9-Brom-10-methylanthracen (3); die anisotropen Auslenkungsparameter geben die 50\% ige Aufenthaltswahrscheinlichkeit an.

Das Gesamte Molekül ist fehlgeordnet, was sich jedoch nur auf die Positionen der Methylgruppe und des Bromatoms auswirkt. Hierbei sind die Positionen von Br1 und C15 zu $62 \%$ besetzt.

Tab. 5.4: Kristallographische Daten von 9-Brom-10-methylanthracen (3).

\begin{tabular}{|c|c|c|c|}
\hline Strukturcode & BrAnMe & $\mu\left[\mathrm{mm}^{-1}\right]$ & 3.809 \\
\hline Summenformel & $\mathrm{C}_{15} \mathrm{H}_{11} \mathrm{Br}$ & $F(000)$ & 544 \\
\hline Molmasse $[\mathrm{g} / \mathrm{mol}]$ & 271.15 & $\theta$-Bereich $\left[{ }^{\circ}\right]$ & $2.34-26.76$ \\
\hline Kristallgröße [mm] & $0.2 \times 0.08 \times 0.06$ & gesammelte Reflexe & 26545 \\
\hline Raumgruppe & $P 2_{1} 2_{1} 2_{1}$ & unabhängige Reflexe & 2266 \\
\hline$a[\mathrm{pm}]$ & $387.69(4)$ & Zahl der Restraints & 2 \\
\hline$b[\mathrm{pm}]$ & $1587.34(16)$ & verfeinerte Parameter & 154 \\
\hline$c[\mathrm{pm}]$ & $1737.28(18)$ & $R 1[I>2 \sigma(I)]$ & 0.0187 \\
\hline$\alpha\left[^{\circ}\right]$ & 90 & $w R 2$ [alle Daten] & 0.0519 \\
\hline$\beta\left[^{\circ}\right]$ & 90 & GooF & 1.051 \\
\hline$\gamma\left[^{\circ}\right]$ & 90 & Flack-X & $-0.005(8)$ \\
\hline$V\left[\mathrm{~nm}^{3}\right]$ & $1.06911(19)$ & Differenzelektronendichte: & \\
\hline$Z$ & 4 & Max. / min. $\left[10^{-6} \mathrm{epm}^{-3}\right]$ & $0.252 /-0.258$ \\
\hline Messtemperatur [K] & $100(2)$ & Max. / min. Transmission & $0.4299 / 0.3554$ \\
\hline$\rho\left[\mathrm{Mgm}^{-3}\right]$ & 1.685 & & \\
\hline
\end{tabular}




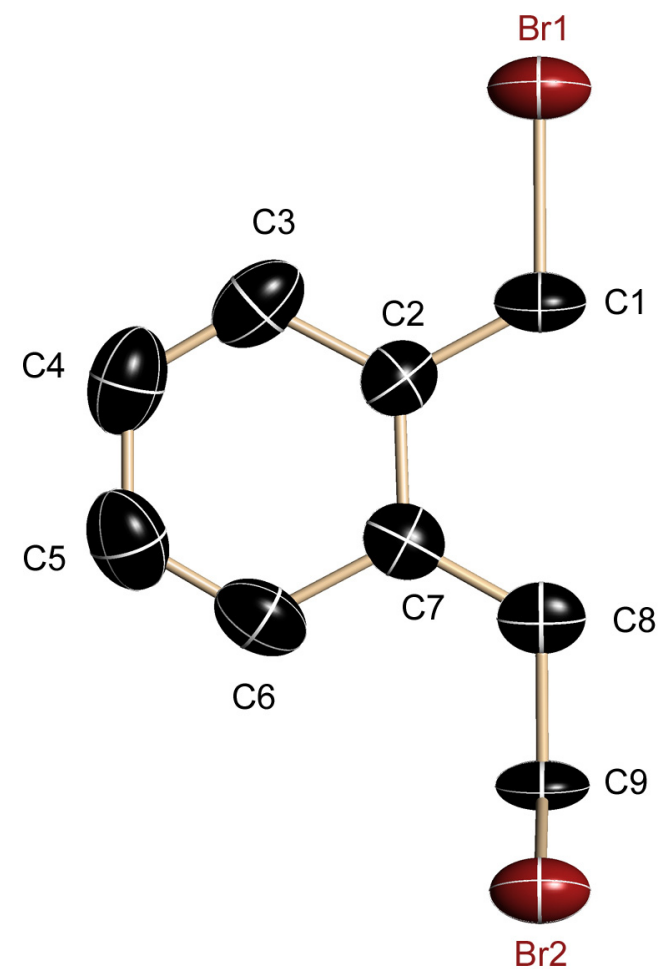

Abb. 5.13: Asymmetrische Einheit von 9-Brom-10-brommethylanthracen (4); die anisotropen Auslenkungsparameter geben die $50 \%$ ige Aufenthaltswahrscheinlichkeit an.

Die Struktur wurde als racemischer Zwilling erhalten dessen Hauptdomäne (63\%) oben dargestellt ist.

Tab. 5.5: Kristallographische Daten von 9-Brom-10-brommethylanthracen (4).

\begin{tabular}{|c|c|c|c|}
\hline Strukturcode & $\mathrm{BrAnBr}$ & $\mu\left[\mathrm{mm}^{-1}\right]$ & 6.615 \\
\hline Summenformel & $\mathrm{C}_{15} \mathrm{H}_{10} \mathrm{Br}_{2}$ & $F(000)$ & 340 \\
\hline Molmasse $[\mathrm{g} / \mathrm{mol}]$ & 350.05 & $\theta$-Bereich $\left[{ }^{\circ}\right]$ & $2.46-25.35$ \\
\hline Kristallgröße [mm] & $0.20 \times 0.20 \times 0.18$ & gesammelte Reflexe & 3586 \\
\hline Raumgruppe & $P m n 2_{1}$ & unabhängige Reflexe & 1070 \\
\hline$a[\mathrm{pm}]$ & $1444.7(6)$ & Zahl der Restraints & 1 \\
\hline$b[\mathrm{pm}]$ & $417.74(18)$ & verfeinerte Parameter & 86 \\
\hline$c[\mathrm{pm}]$ & $1010.9(4)$ & $R 1[I>2 \sigma(I)]$ & 0.444 \\
\hline$\alpha\left[^{\circ}\right]$ & 90 & $w R 2$ [alle Daten] & 0.1063 \\
\hline$\beta\left[^{\circ}\right]$ & 90 & GooF & 1.032 \\
\hline$\gamma\left[^{\circ}\right]$ & 90 & FlackX & s.o. \\
\hline$V\left[\mathrm{~nm}^{3}\right]$ & $0.6101(4)$ & Differenzelektronendichte: & \\
\hline$Z$ & 2 & Max. / min. $\left[10^{-6} \mathrm{epm}^{-3}\right]$ & $0.708 /-0.625$ \\
\hline Messtemperatur [K] & $296(2)$ & Max. / min. Transmission & $0.3823 / 0.3513$ \\
\hline$\rho\left[\mathrm{Mgm}^{-3}\right]$ & 1.906 & & \\
\hline
\end{tabular}




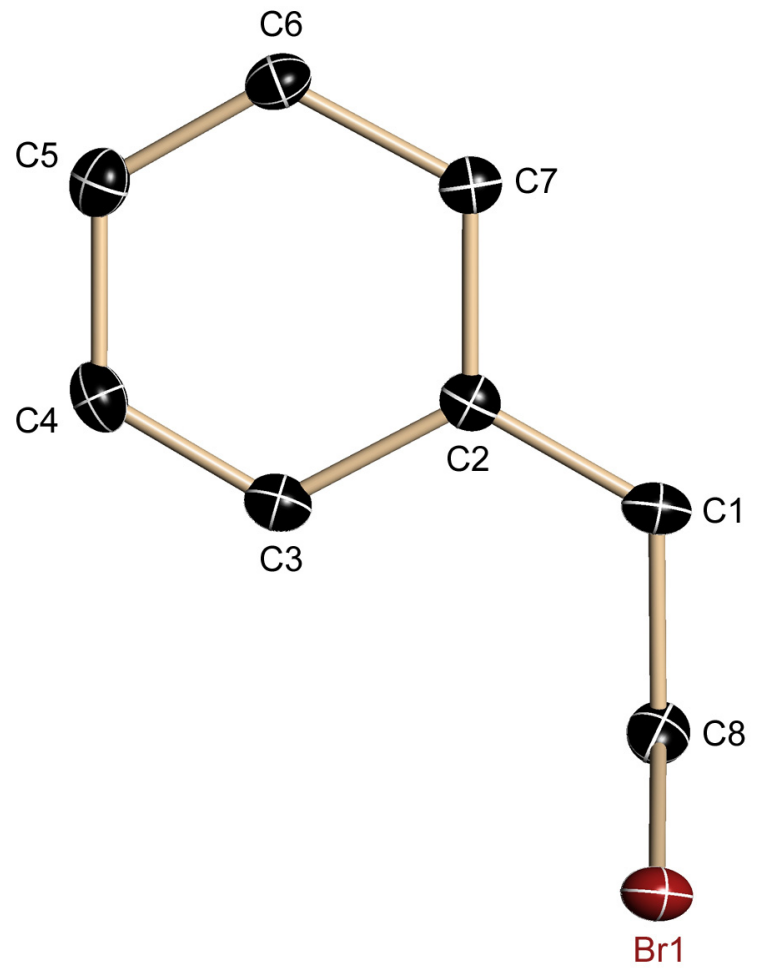

Abb. 5.14: Asymmetrische Einheit von 9,10-Dibrommethylanthracen (5); die anisotropen Auslenkungsparameter geben die 50\% ige Aufenthaltswahrscheinlichkeit an.

Tab. 5.6: Kristallographische Daten von 9,10-Dibrommethylanthracen (5).

\begin{tabular}{|c|c|c|c|}
\hline Strukturcode & $\mathrm{BrAnBr}$ & $\mu\left[\mathrm{mm}^{-1}\right]$ & 6.369 \\
\hline Summenformel & $\mathrm{C}_{16} \mathrm{H}_{12} \mathrm{Br}_{2}$ & $F(000)$ & 356 \\
\hline Molmasse $[\mathrm{g} / \mathrm{mol}]$ & 364.08 & $\theta$-Bereich $\left[^{\circ}\right]$ & $2.63-26.05$ \\
\hline Kristallgröße [mm] & $0.21 \times 0.08 \times 0.05$ & gesammelte Reflexe & 6944 \\
\hline Raumgruppe & $P 2_{1} / n$ & unabhängige Reflexe & 1246 \\
\hline$a[\mathrm{pm}]$ & $850.27(8)$ & Zahl der Restraints & 0 \\
\hline$b[\mathrm{pm}]$ & $482.77(5)$ & verfeinerte Parameter & 82 \\
\hline$c[\mathrm{pm}]$ & $1552.23(15)$ & $R 1[I>2 \sigma(I)]$ & 0.0219 \\
\hline$\alpha\left[^{\circ}\right]$ & 90 & $w R 2$ [alle Daten] & 0.0531 \\
\hline$\beta\left[^{\circ}\right]$ & $95.7040(10)$ & GooF & 1.077 \\
\hline$\gamma\left[^{\circ}\right]$ & 90 & & \\
\hline$V\left[\mathrm{~nm}^{3}\right]$ & $0.63401(11)$ & Differenzelektronendichte: & \\
\hline$Z$ & 2 & Max. / min. $\left[10^{-6} \mathrm{epm}^{-3}\right]$ & $0.704 /-0.311$ \\
\hline Messtemperatur [K] & $100(2)$ & Max. / min. Transmission & $0.4293 / 0.2965$ \\
\hline$\rho\left[\mathrm{Mgm}^{-3}\right]$ & 1.907 & & \\
\hline
\end{tabular}




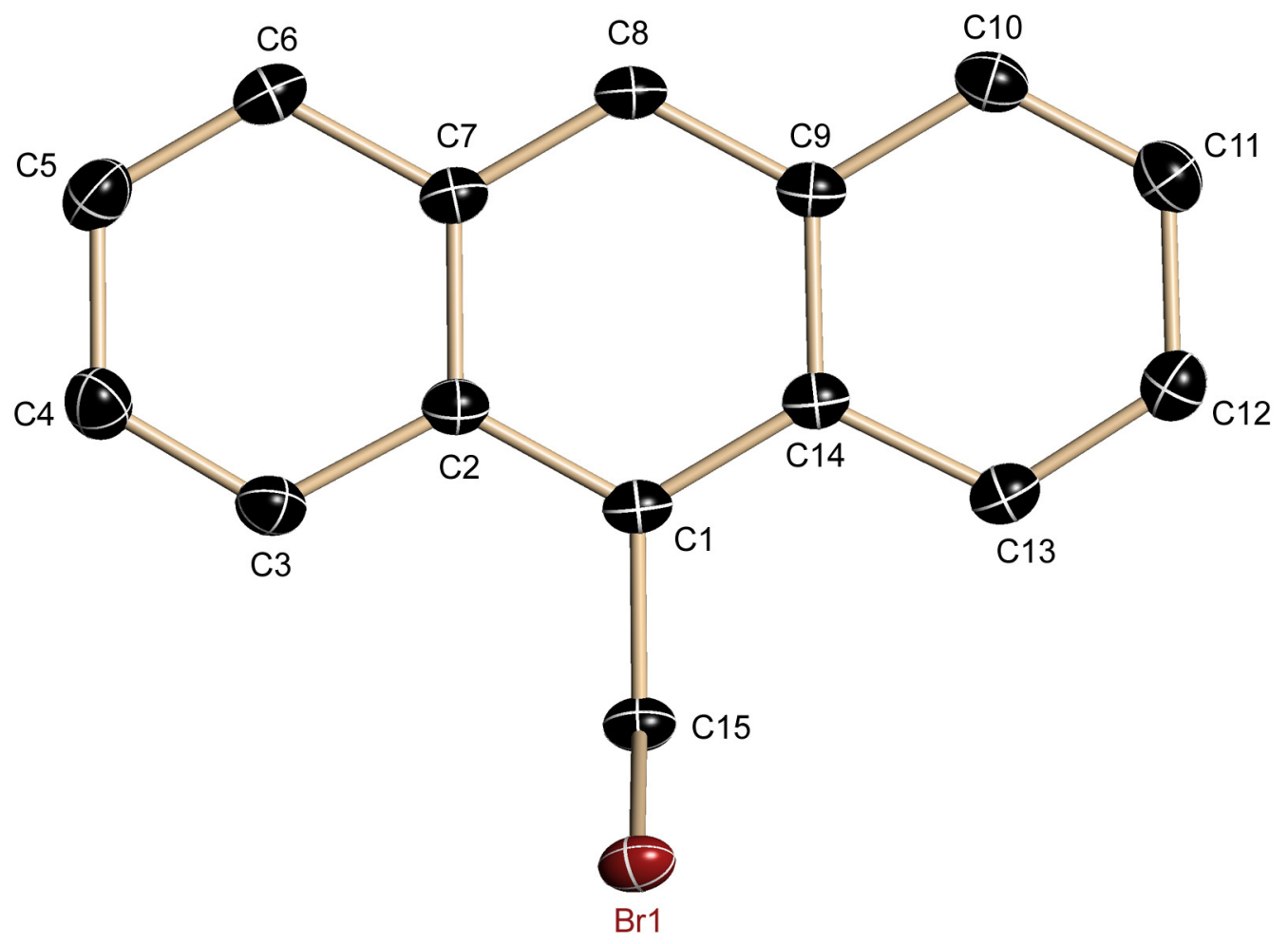

Abb. 5.15: Asymmetrische Einheit von 9-Brommethylanthracen (6); die anisotropen Auslenkungsparameter geben die 50\% ige Aufenthaltswahrscheinlichkeit an.

Tab. 5.7: Kristallographische Daten von 9-Brommethylanthracen (6).

\begin{tabular}{|c|c|c|c|}
\hline Strukturcode & $\mathrm{HAnCH}_{2} \mathrm{Br}$ & $\mu\left[\mathrm{mm}^{-1}\right]$ & 3.674 \\
\hline Summenformel & $\mathrm{C}_{15} \mathrm{H}_{11} \mathrm{Br}$ & $F(000)$ & 544 \\
\hline Molmasse $[\mathrm{g} / \mathrm{mol}]$ & 271.15 & $\theta$-Bereich $\left[{ }^{\circ}\right]$ & $1.83-30.51$ \\
\hline Kristallgröße [mm] & $0.22 \times 0.05 \times 0.04$ & gesammelte Reflexe & 34557 \\
\hline Raumgruppe & $P 2_{1} / c$ & unabhängige Reflexe & 3390 \\
\hline$a[\mathrm{pm}]$ & $444.66(5)$ & Zahl der Restraints & 0 \\
\hline$b[\mathrm{pm}]$ & $1468.52(16)$ & verfeinerte Parameter & 145 \\
\hline$c[\mathrm{pm}]$ & 1697.72(18) & $R 1[I>2 \sigma(I)]$ & 0.0186 \\
\hline$\alpha\left[^{\circ}\right]$ & 90 & $w R 2$ [alle Daten] & 0.0487 \\
\hline$\beta\left[^{\circ}\right]$ & $91.204(2)$ & GooF & 1.056 \\
\hline$\gamma\left[^{\circ}\right]$ & 90 & & \\
\hline$V\left[\mathrm{~nm}^{3}\right]$ & $1.1084(2)$ & Differenzelektronendichte: & \\
\hline$Z$ & 4 & Max. / min. $\left[10^{-6} \mathrm{epm}^{-3}\right]$ & $0.412 /-0.222$ \\
\hline Messtemperatur [K] & $100(2)$ & Max. / min. Transmission & $0.4339 / 0.2891$ \\
\hline$\rho\left[\mathrm{Mgm}^{-3}\right]$ & 1.625 & & \\
\hline
\end{tabular}




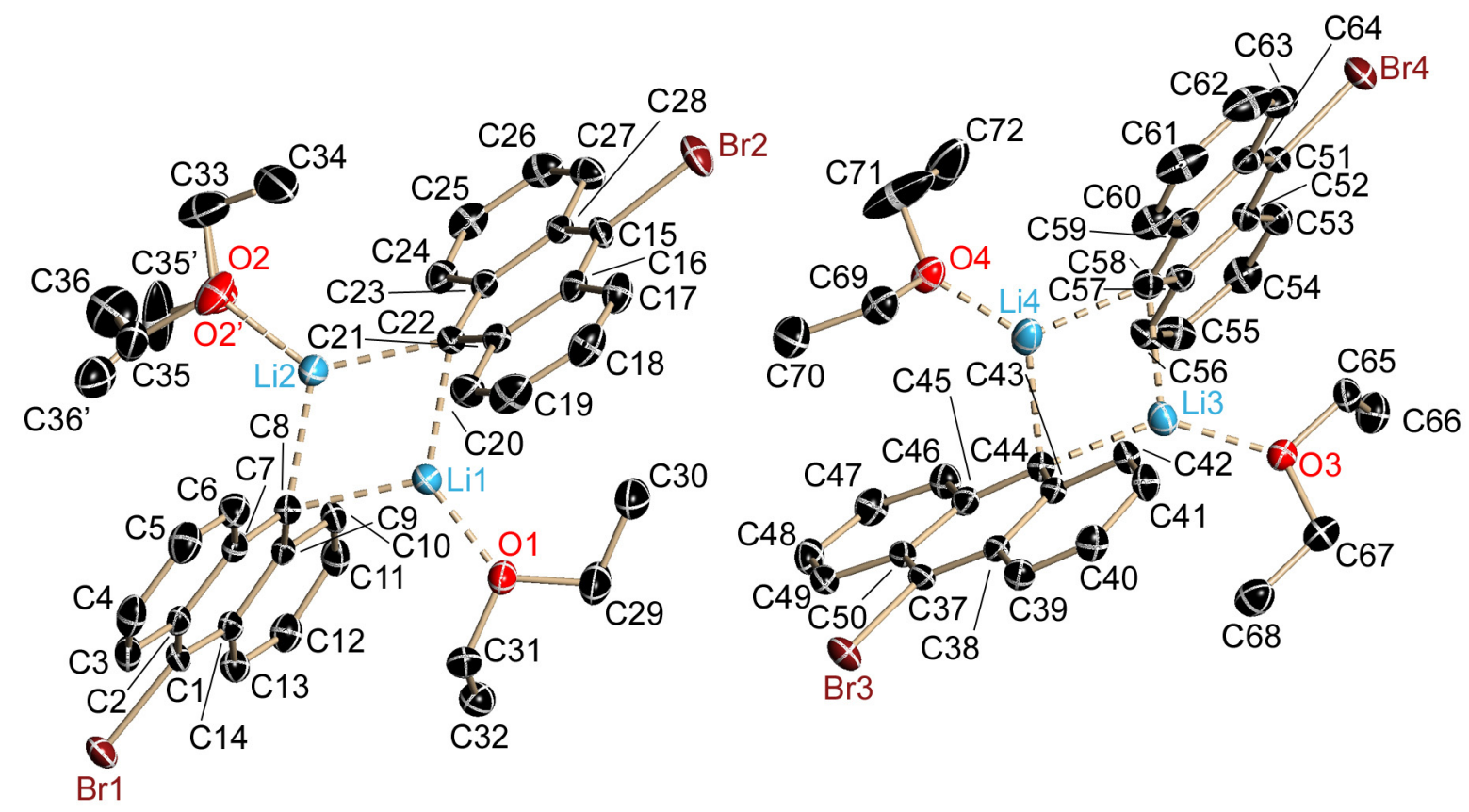

Abb. 5.16: Asymmetrische Einheit von $[\mathrm{BrAnLi} \cdot\{\mathrm{dee}\}]_{2}(7 \mathrm{a})$; die anisotropen Auslenkungsparameter geben die $50 \%$ ige Aufenthaltswahrscheinlichkeit an.

Die Ethylgruppe eines Diethylethers ist fehlgeordnet, der Besetzungsfaktor der Hauptdomäne verfeinerte zu $53 \%$.

Tab. 5.8: Kristallographische Daten von $[\operatorname{BrAnLi} \cdot\{\text { dee }\}]_{2}(7 a)$.

\begin{tabular}{|c|c|c|c|}
\hline Strukturcode & BrAnLi DEE & $\mu\left[\mathrm{mm}^{-1}\right]$ & 2.596 \\
\hline Summenformel & $\mathrm{C}_{36} \mathrm{H}_{36} \mathrm{Br}_{2} \mathrm{Li}_{2} \mathrm{O}_{2}$ & $F(000)$ & 1376 \\
\hline Molmasse $[\mathrm{g} / \mathrm{mol}]$ & 674.35 & $\theta$-Bereich $\left[{ }^{\circ}\right]$ & $1.37-26.81$ \\
\hline Kristallgröße [mm] & $0.21 \times 0.21 \times 0.19$ & gesammelte Reflexe & 60023 \\
\hline Raumgruppe & $P \overline{1}$ & unabhängige Reflexe & 13440 \\
\hline$a[\mathrm{pm}]$ & $1497.82(12)$ & Zahl der Restraints & 1 \\
\hline$b[\mathrm{pm}]$ & $1529.17(12)$ & verfeinerte Parameter & 794 \\
\hline$c[\mathrm{pm}]$ & $1549.14(12)$ & $R 1[I>2 \sigma(I)]$ & 0.0308 \\
\hline$\alpha\left[^{\circ}\right]$ & $63.9660(10)$ & $w R 2$ [alle Daten] & 0.0829 \\
\hline$\beta\left[^{\circ}\right]$ & $87.2590(10)$ & GooF & 1.056 \\
\hline$\gamma\left[^{\circ}\right]$ & $82.3670(10)$ & & \\
\hline$V\left[\mathrm{~nm}^{3}\right]$ & $3.1597(4)$ & Differenzelektronendichte: & \\
\hline$z$ & 4 & Max. / min. $\left[10^{-6} \mathrm{epm}^{-3}\right]$ & $0.998 /-0.687$ \\
\hline Messtemperatur [K] & $100(2)$ & Max. / min. Transmission & $0.7454 / 0.6597$ \\
\hline$\rho\left[\mathrm{Mgm}^{-3}\right]$ & 1.418 & & \\
\hline
\end{tabular}




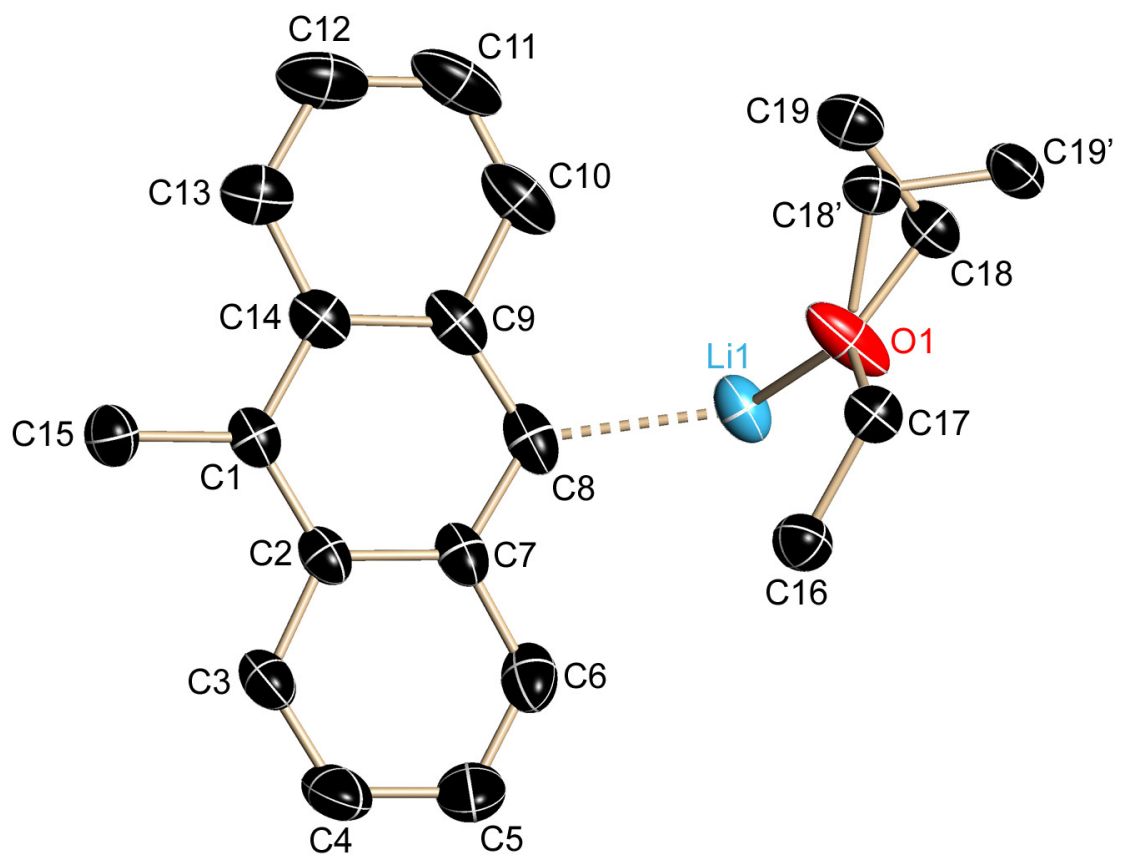

Abb. 5.17: Asymmetrische Einheit von $[\mathrm{MeAnLi} \cdot\{\text { dee }\}]_{2}(7 \mathbf{b})$; die anisotropen Auslenkungsparameter geben die 50\% ige Aufenthaltswahrscheinlichkeit an.

Die Ethylgruppe des Diethylethers ist fehlgeordnet, der Besetzungsfaktor der Hauptdomäne verfeinerte zu $53 \%$.

Tab. 5.9: Kristallographische Daten von $\left[\mathrm{MeAnLi} \cdot\{\text { dee }]_{2}(\mathbf{7 b})\right.$.

\begin{tabular}{|c|c|c|c|}
\hline Strukturcode & BrAnLi DEE & $\mu\left[\mathrm{mm}^{-1}\right]$ & 0.067 \\
\hline Summenformel & $\mathrm{C}_{38} \mathrm{H}_{42} \mathrm{Li}_{2} \mathrm{O}_{2}$ & $F(000)$ & 292 \\
\hline Molmasse [g/mol] & 544.60 & $\theta$-Bereich $\left[{ }^{\circ}\right]$ & $1.98-25.48$ \\
\hline Kristallgröße [mm] & $0.1 \times 0.1 \times 0.1$ & gesammelte Reflexe & 15741 \\
\hline Raumgruppe & $P_{\overline{1}}$ & unabhängige Reflexe & 2921 \\
\hline$a[\mathrm{pm}]$ & $819.65(9)$ & Zahl der Restraints & 0 \\
\hline$b[\mathrm{pm}]$ & $976.61(10)$ & verfeinerte Parameter & 214 \\
\hline$c[\mathrm{pm}]$ & $1041.26(11)$ & $R 1[\mid>2 \sigma(I)]$ & 0.0418 \\
\hline$\alpha\left[^{\circ}\right]$ & $82.3150(10)$ & $w R 2$ [alle Daten] & 0.1172 \\
\hline$\beta\left[^{\circ}\right]$ & $84.1130(10)$ & GooF & 1.084 \\
\hline$\gamma\left[^{\circ}\right]$ & $733630(10)$ & & \\
\hline$V\left[\mathrm{~nm}^{3}\right]$ & $0.78963(14)$ & Differenzelektronendichte: & \\
\hline$z$ & 1 & Max. $/$ min. $\left[10^{-6} \mathrm{epm}^{-3}\right]$ & $0.180 / 0.182$ \\
\hline Messtemperatur [K] & $100(2)$ & Max. / min. Transmission & $0.7452 / 0.6029$ \\
\hline$\rho\left[\mathrm{Mgm}^{-3}\right]$ & 1.145 & & \\
\hline
\end{tabular}




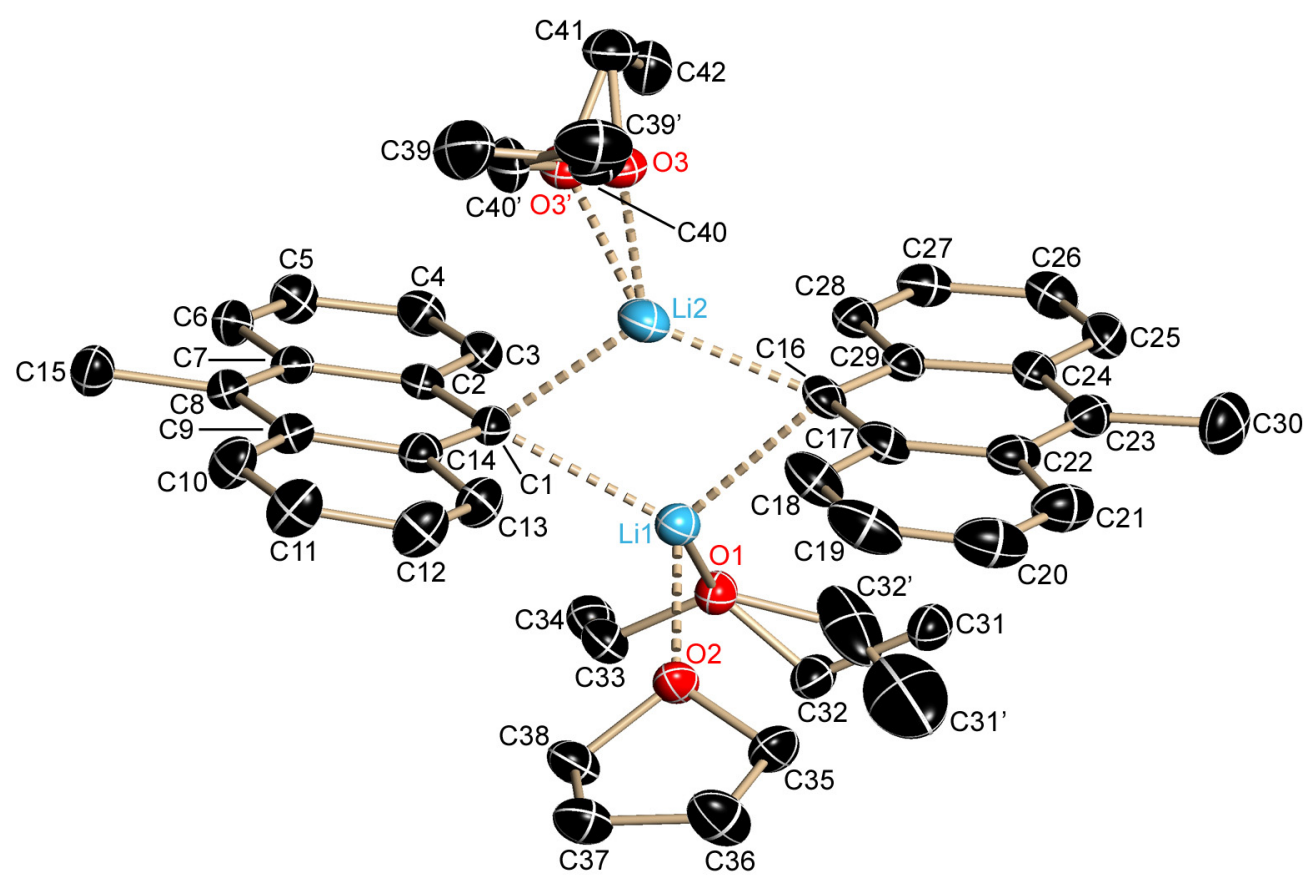

Abb. 5.18: Asymmetrische Einheit von $\left.[\mathrm{BrAnLi} \cdot\{\text { dee }\} \text { thf }\}_{1 / 2}\right]_{2}(\mathbf{8 b})$; die anisotropen Auslenkungsparameter geben die $50 \%$ ige Aufenthaltswahrscheinlichkeit an.

Jeweils ein Ethylrest der beiden Diethylethermoleküle ist fehlgeordnet. Die Besetzungen der Hauptdomänen verfeinerten zu 92 \% (C31, C32) und 75 \% (C39, C40).

Tab. 5.10: Kristallographische Daten von $\left[\mathrm{MeAnLi} \cdot\{\text { dee }\}\{\text { thf }\}_{1 / 2}\right]_{2}(\mathbf{8 b})$.

\begin{tabular}{|c|c|c|c|}
\hline Strukturcode & {$\left[\text { MeAnLi } \bullet\{\text { dee }\}\{\text { thf }\}_{1 / 2}\right]_{2}$} & $\mu\left[\mathrm{mm}^{-1}\right]$ & 0.071 \\
\hline Summenformel & $\mathrm{C}_{42} \mathrm{H}_{50} \mathrm{Li}_{2} \mathrm{O}_{3}$ & $F(000)$ & 664 \\
\hline Molmasse $[\mathrm{g} / \mathrm{mol}]$ & 616.70 & $\theta$-Bereich $\left[{ }^{\circ}\right]$ & $1.69-25.39$ \\
\hline Kristallgröße [mm] & $0.1 \times 0.1 \times 0.05$ & gesammelte Reflexe & 19952 \\
\hline Raumgruppe & $P_{\overline{1}}$ & unabhängige Reflexe & 6364 \\
\hline$a[\mathrm{pm}]$ & $836.9(2)$ & Zahl der Restraints & 0 \\
\hline$b[\mathrm{pm}]$ & $1229.4(3)$ & verfeinerte Parameter & 480 \\
\hline$c[\mathrm{pm}]$ & $1761.3(5)$ & $R 1[I>2 \sigma(I)]$ & 0.419 \\
\hline$\alpha\left[^{\circ}\right]$ & $99.325(3)$ & $w R 2$ [alle Daten] & 0.1123 \\
\hline$\beta\left[^{\circ}\right]$ & $101.564(3)$ & GooF & 1.051 \\
\hline$\gamma\left[^{\circ}\right]$ & $94.111(3)$ & & \\
\hline$V\left[\mathrm{~nm}^{3}\right]$ & $1.7417(8)$ & Differenzelektronendichte: & \\
\hline$z$ & 2 & Max. / min. $\left[10^{-6} \mathrm{epm}^{-3}\right]$ & $0.236 /-0.164$ \\
\hline Messtemperatur [K] & $105(2)$ & Max. / min. Transmission & $0.7452 / 0.6445$ \\
\hline$\rho\left[\mathrm{Mgm}^{-3}\right]$ & 1.176 & & \\
\hline
\end{tabular}




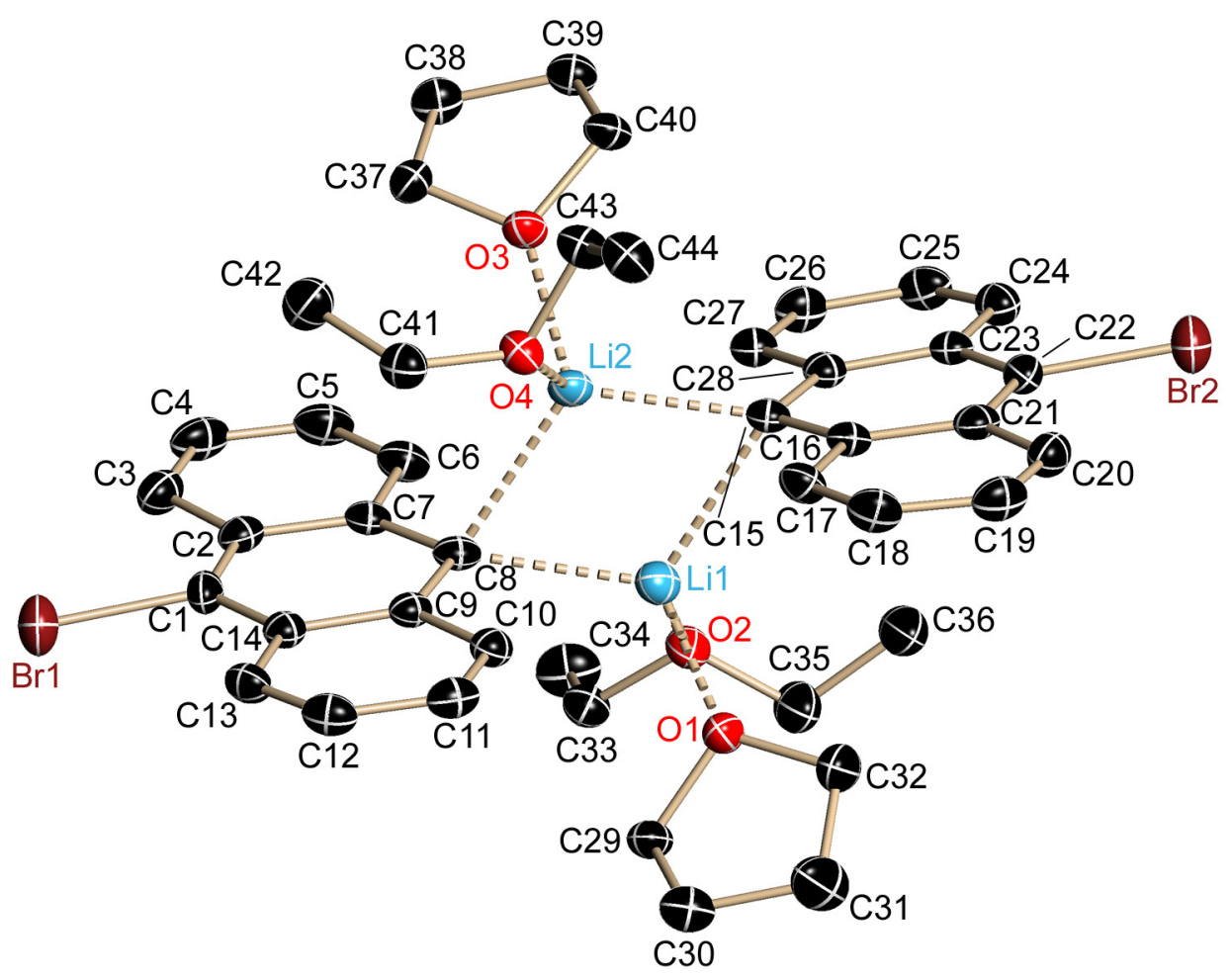

Abb. 5.19: Asymmetrische Einheit von $[\mathrm{BrAnLi} \cdot\{\text { dee }\}\{\text { thf }\}]_{2}$ (9a); die anisotropen Auslenkungsparameter geben die $50 \%$ ige Aufenthaltswahrscheinlichkeit an.

Tab. 5.11: Kristallographische Daten von $[\mathrm{BrAnLi} \cdot\{$ dee $\}$ thf $\}]_{2}(9 \mathrm{9a})$.

\begin{tabular}{|c|c|c|c|}
\hline Strukturcode & BrAnLi DEE THF & $\mu\left[\mathrm{mm}^{-1}\right]$ & 2.113 \\
\hline Summenformel & $\mathrm{C}_{44} \mathrm{H}_{52} \mathrm{Br}_{2} \mathrm{Li}_{2} \mathrm{O}_{4}$ & $F(000)$ & 848 \\
\hline Molmasse [g/mol] & 818.56 & $\theta$-Bereich $\left[^{\circ}\right]$ & $1.70-25.35$ \\
\hline Kristallgröße [mm] & $0.2 \times 0.2 \times 0.03$ & gesammelte Reflexe & 17998 \\
\hline Raumgruppe & $P \overline{1}$ & unabhängige Reflexe & 7128 \\
\hline$a[\mathrm{pm}]$ & $1233.01(18)$ & Zahl der Restraints & 0 \\
\hline$b[\mathrm{pm}]$ & $1237.01(19)$ & verfeinerte Parameter & 473 \\
\hline$c[\mathrm{pm}]$ & $1445.6(2)$ & $R 1[\mid>2 \sigma(I)]$ & 0.0411 \\
\hline$\alpha\left[^{\circ}\right]$ & $82.039(3)$ & $w R 2$ [alle Daten] & 0.1027 \\
\hline$\beta\left[^{\circ}\right]$ & $66.565(3)$ & GooF & 1.073 \\
\hline$\gamma\left[^{\circ}\right]$ & $75.547(2)$ & & \\
\hline$V\left[\mathrm{~nm}^{3}\right]$ & $1.9571(5)$ & Differenzelektronendichte: & \\
\hline$Z$ & 2 & Max. $/ \min .\left[10^{-6} \mathrm{epm}^{-3}\right]$ & 1.014 / -0.724 \\
\hline Messtemperatur [K] & $100(2)$ & Max. / min. Transmission & 0.7452 / 0.6524 \\
\hline$\rho\left[\mathrm{Mgm}^{-3}\right]$ & 1.389 & & \\
\hline
\end{tabular}




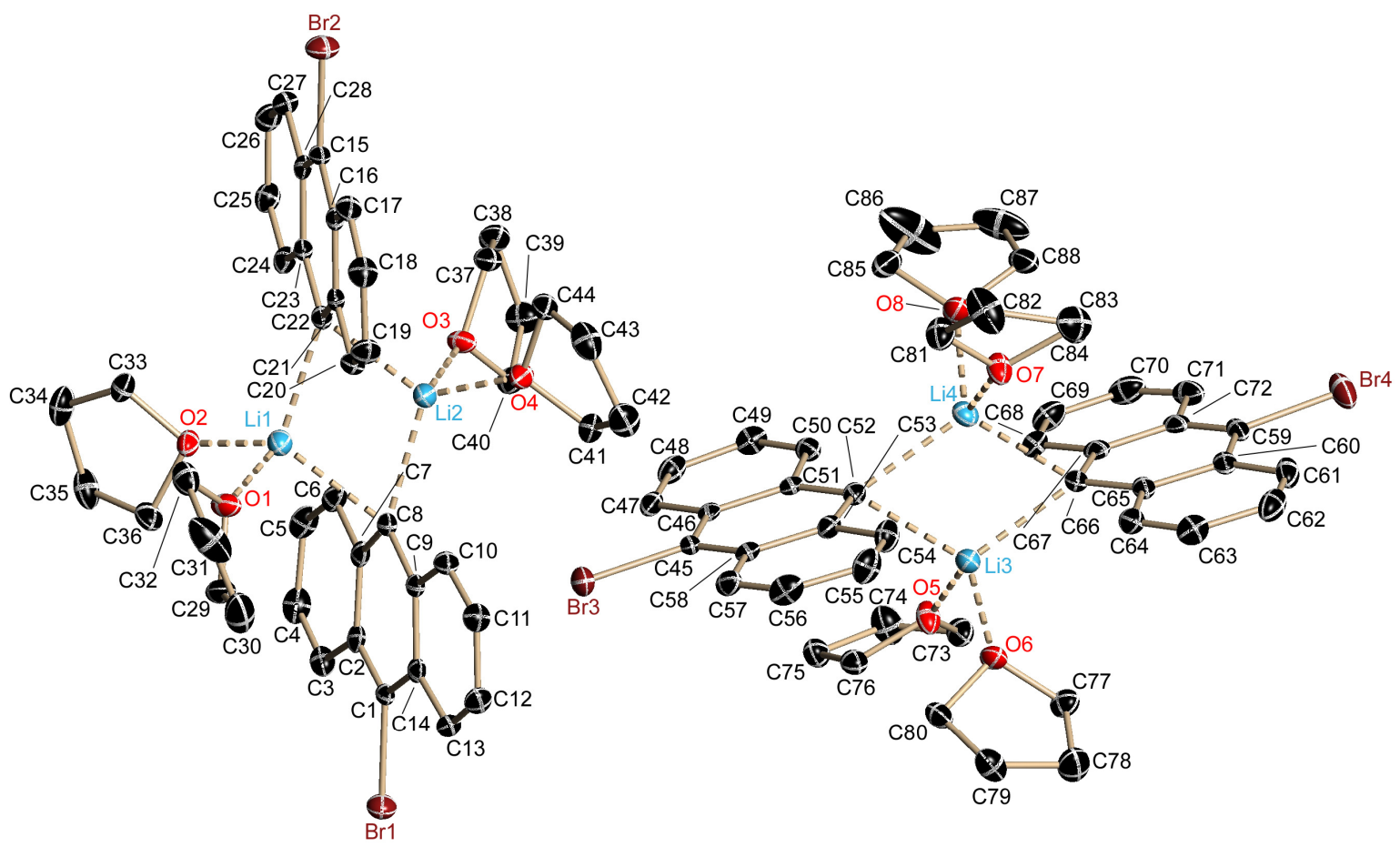

Abb. 5.20: Asymmetrische Einheit von $\left[\mathrm{BrAnLi} \cdot\{\text { thf }\}_{2}\right]_{2}(\mathbf{1 1 a})$; die anisotropen Auslenkungsparameter geben die $50 \%$ ige Aufenthaltswahrscheinlichkeit an.

Tab. 5.12: Kristallographische Daten von $\left[\mathrm{BrAnLi} \cdot\{\text { thf }\}_{2}\right]_{2}(11 \mathrm{a})$.

\begin{tabular}{|c|c|c|c|}
\hline Strukturcode & BrAnLi THF & $\mu\left[\mathrm{mm}^{-1}\right]$ & 2.169 \\
\hline Summenformel & $\mathrm{C}_{44} \mathrm{H}_{48} \mathrm{Br}_{2} \mathrm{Li}_{2} \mathrm{O}_{4}$ & $F(000)$ & 1680 \\
\hline Molmasse $[\mathrm{g} / \mathrm{mol}]$ & 814.52 & $\theta$-Bereich $\left[{ }^{\circ}\right]$ & $1.67-25.68$ \\
\hline Kristallgröße [mm] & $0.2 \times 0.2 \times 0.05$ & gesammelte Reflexe & 77094 \\
\hline Raumgruppe & $P_{\overline{1}}$ & unabhängige Reflexe & 14447 \\
\hline$a$ [pm] & $1247.7(2)$ & Zahl der Restraints & 0 \\
\hline$b[\mathrm{pm}]$ & 1752.9(3) & verfeinerte Parameter & 937 \\
\hline$c[\mathrm{pm}]$ & 1979.2(3) & $R 1[1>2 \sigma(I)]$ & 0.0354 \\
\hline$\alpha\left[^{\circ}\right]$ & $64.145(2)$ & $w R 2$ [alle Daten] & 0.0992 \\
\hline$\beta\left[^{\circ}\right]$ & $78.978(2)$ & GooF & 1.069 \\
\hline$\gamma\left[^{\circ}\right]$ & $89.269(2)$ & & \\
\hline$V\left[\mathrm{~nm}^{3}\right]$ & $3.8114(11)$ & Differenzelektronendichte: & \\
\hline$z$ & 4 & Max. / min. $\left[10^{-6} \mathrm{epm}^{-3}\right]$ & 0.494 / -0.769 \\
\hline Messtemperatur [K] & $100(2)$ & Max. / min. Transmission & $0.7454 / 0.6906$ \\
\hline$\rho\left[\mathrm{Mgm}^{-3}\right]$ & 1.419 & & \\
\hline
\end{tabular}




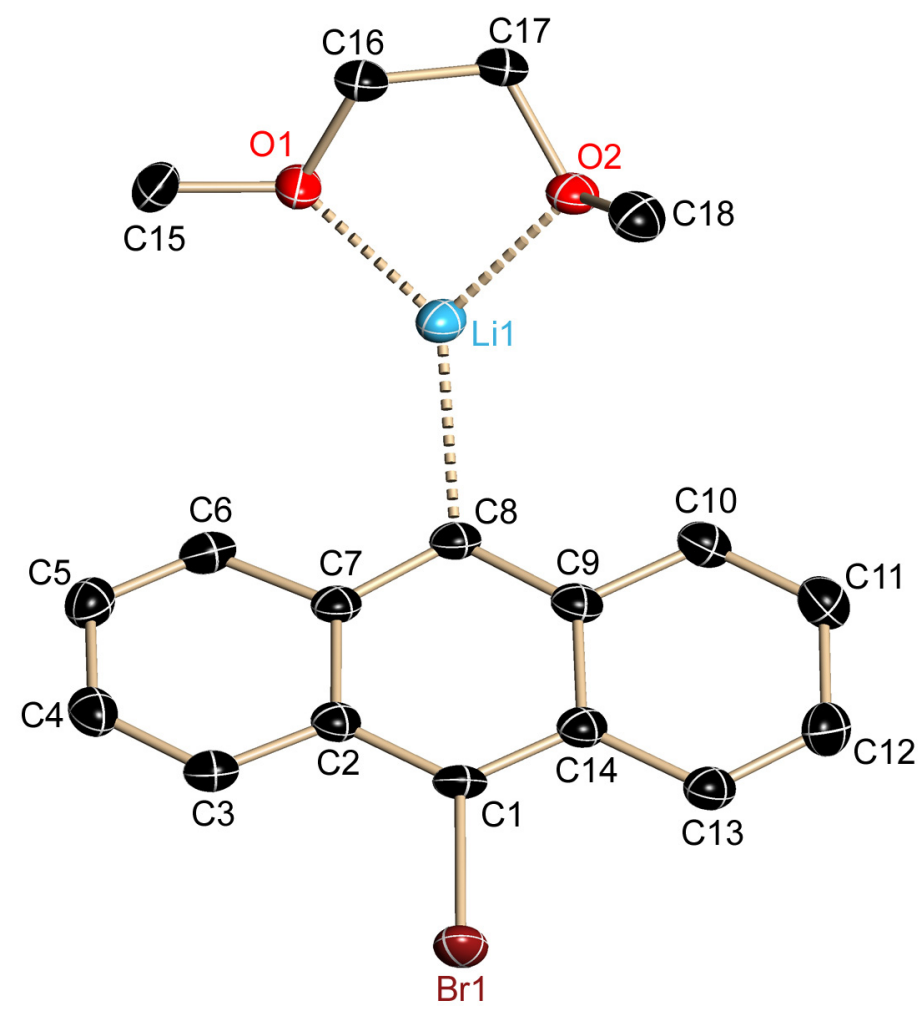

Abb. 5.21: Asymmetrische Einheit von $\left[\mathrm{BrAnLi} \cdot\{\mathrm{dme}\}_{2}\right]_{2}(\mathbf{1 2})$; die anisotropen Auslenkungsparameter geben die $50 \%$ ige Aufenthaltswahrscheinlichkeit an.

Tab. 5.13: Kristallographische Daten von $\left[\mathrm{BrAnLi} \cdot\{\mathrm{dme}\}_{2}\right]_{2}(\mathbf{1 2})$.

\begin{tabular}{|c|c|c|c|}
\hline Strukturcode & BrAnLi DME & $\mu\left[\mathrm{mm}^{-1}\right]$ & 2.664 \\
\hline Summenformel & $\mathrm{C}_{36} \mathrm{H}_{36} \mathrm{Br}_{2} \mathrm{Li}_{2} \mathrm{O}_{2}$ & $F(000)$ & 720 \\
\hline Molmasse $[\mathrm{g} / \mathrm{mol}]$ & 706.34 & $\theta$-Bereich $\left[{ }^{\circ}\right]$ & $2.30-25.45$ \\
\hline Kristallgröße [mm] & $0.1 \times 0.1 \times 0.1$ & gesammelte Reflexe & 15536 \\
\hline Raumgruppe & $P 2_{1} / n$ & unabhängige Reflexe & 3043 \\
\hline$a[\mathrm{pm}]$ & $1057.8(2)$ & Zahl der Restraints & 0 \\
\hline$b[\mathrm{pm}]$ & $915.89(19)$ & verfeinerte Parameter & 201 \\
\hline$c[\mathrm{pm}]$ & 1594.3(3) & $R 1[I>2 \sigma(I)]$ & 0.0425 \\
\hline$\alpha\left[^{\circ}\right]$ & 90 & $w R 2$ [alle Daten] & 0.1173 \\
\hline$\beta\left[^{\circ}\right]$ & $90.795(3)$ & GooF & 1.034 \\
\hline$\gamma\left[^{\circ}\right]$ & 90 & & \\
\hline$V\left[\mathrm{~nm}^{3}\right]$ & $1.5444(6)$ & Differenzelektronendichte: & \\
\hline$Z$ & 2 & Max. / min. $\left[10^{-6} \mathrm{epm}^{-3}\right]$ & $1.831 /-0.536$ \\
\hline Messtemperatur [K] & $100(2)$ & Max. / min. Transmission & $0.7452 / 0.4141$ \\
\hline$\rho\left[\mathrm{Mgm}^{-3}\right]$ & 1.519 & & \\
\hline
\end{tabular}




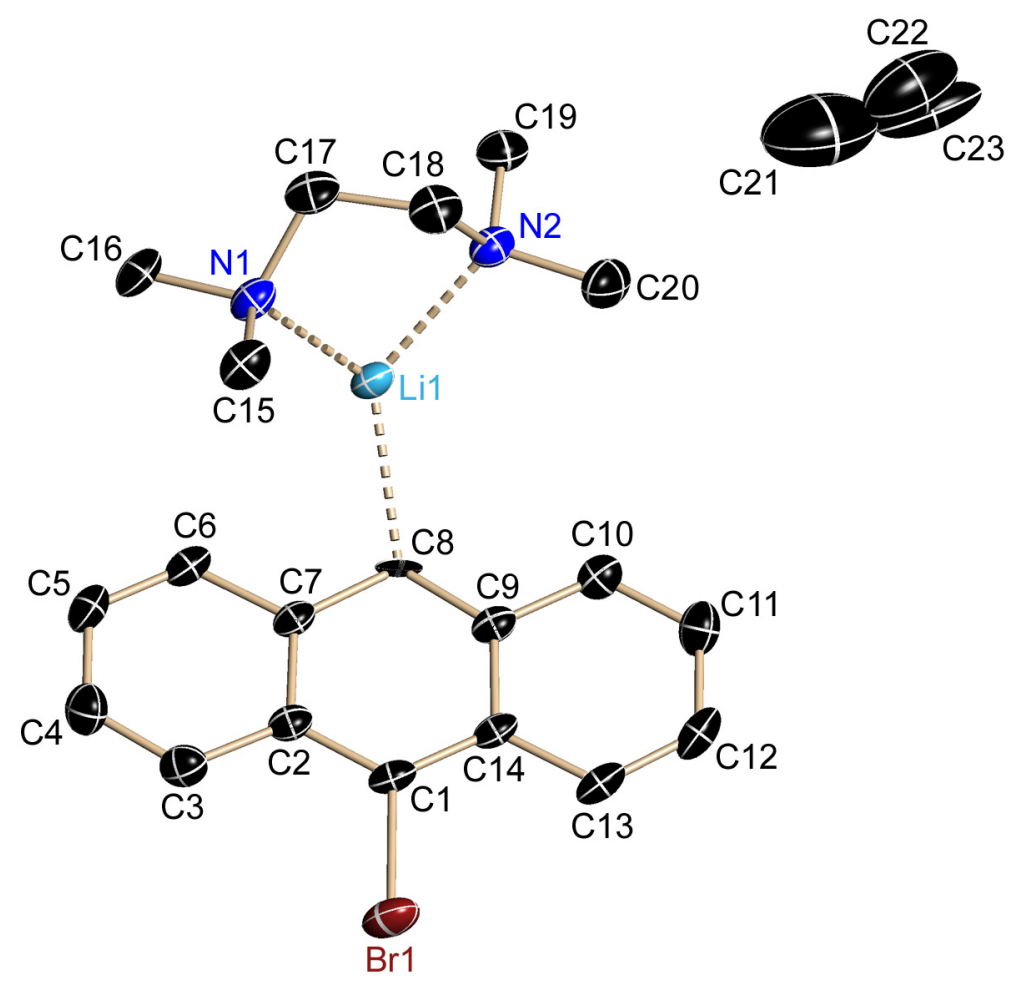

Abb. 5.22: Asymmetrische Einheit von $\left[\mathrm{BrAnLi} \cdot\left\{\mathrm{tmeda}_{2}\right]_{2}\right.$ (13); die anisotropen Auslenkungsparameter geben die $50 \%$ ige Aufenthaltswahrscheinlichkeit an.

Das in der Kristallstruktur enthaltene Hexanmolekül wurde durch die Restraints DFIX und DANG geometrisch optimiert.

Tab. 5.14: Kristallographische Daten von $\left[\mathrm{BrAnLi} \cdot\{\mathrm{tmeda}\}_{2}\right]_{2}(\mathbf{1 3})$.

\begin{tabular}{|c|c|c|c|}
\hline Strukturcode & BrAnLi TMEDA & $\mu\left[\mathrm{mm}^{-1}\right]$ & 1.987 \\
\hline Summenformel & $\mathrm{C}_{46} \mathrm{H}_{62} \mathrm{Br}_{2} \mathrm{Li}_{2} \mathrm{~N}_{4}$ & $F(000)$ & 442 \\
\hline Molmasse $[\mathrm{g} / \mathrm{mol}]$ & 844.70 & $\theta$-Bereich $\left[{ }^{\circ}\right]$ & $1.74-25.33$ \\
\hline Kristallgröße [mm] & $0.1 \times 0.1 \times 0.1$ & gesammelte Reflexe & 12630 \\
\hline Raumgruppe & $P_{\overline{1}}$ & unabhängige Reflexe & 3768 \\
\hline$a[\mathrm{pm}]$ & $904.6(3)$ & Zahl der Restraints & 2 \\
\hline$b[\mathrm{pm}]$ & $1003.7(4)$ & verfeinerte Parameter & 249 \\
\hline$c[\mathrm{pm}]$ & 1207.6(4) & $R 1[\mid>2 \sigma(I)]$ & 0.0481 \\
\hline$\alpha\left[^{\circ}\right]$ & $102.221(4)$ & $w R 2$ [alle Daten] & 0.1495 \\
\hline$\beta\left[^{\circ}\right]$ & $94.897(4)$ & GooF & 1.114 \\
\hline$\gamma\left[^{\circ}\right]$ & $101.776(4)$ & & \\
\hline$V\left[\mathrm{~nm}^{3}\right]$ & $1.0394(6)$ & Differenzelektronendichte: & \\
\hline$Z$ & 1 & Max. / min. $\left[10^{-6} \mathrm{epm}^{-3}\right]$ & $1.376 /-0.654$ \\
\hline Messtemperatur [K] & $100(2)$ & Max. / min. Transmission & $0.7452 / 0.6082$ \\
\hline$\rho\left[\mathrm{Mgm}^{-3}\right]$ & 1.349 & & \\
\hline
\end{tabular}




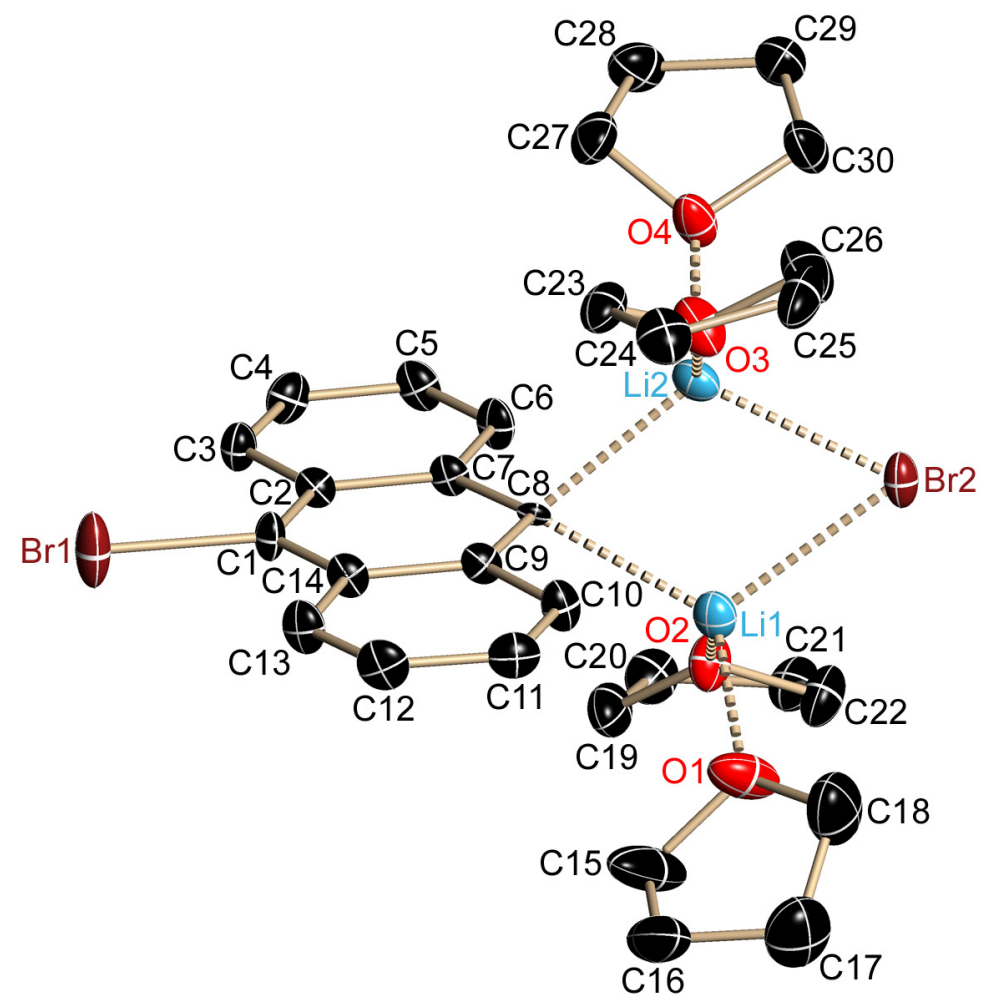

Abb. 5.23: Ausschnitt der asymmetrischen Einheit von $\left[\mathrm{BrAnLi} \cdot \mathrm{LiBr} \cdot\{\mathrm{thf}\}_{4}\right]$ (14a); die anisotropen Auslenkungsparameter geben die 50\% ige Aufenthaltswahrscheinlichkeit an.

Tab. 5.15: Kristallographische Daten von $\left[\mathrm{BrAnLi} \cdot \mathrm{LiBr} \cdot\{\mathrm{thf}\}_{4}\right](\mathbf{1 4 a})$.

\begin{tabular}{|c|c|c|c|}
\hline Strukturcode & {$\left[\mathrm{BrAnLi} \cdot \mathrm{LiBr} \cdot\{\operatorname{thf}\}_{4}\right]$} & $\mu\left[\mathrm{mm}^{-1}\right]$ & 2.657 \\
\hline Summenformel & $\mathrm{C}_{82} \mathrm{H}_{104} \mathrm{Li}_{5} \mathrm{O}_{10} \mathrm{Br}_{5}$ & $F(000)$ & 1732 \\
\hline Molmasse [g/mol] & 1683.90 & $\theta$-Bereich $\left[{ }^{\circ}\right]$ & $1.41-25.14$ \\
\hline Kristallgröße [mm] & $0.1 \times 0.1 \times 0.1$ & gesammelte Reflexe & 57729 \\
\hline Raumgruppe & $P \overline{1}$ & unabhängige Reflexe & 13694 \\
\hline$a[\mathrm{pm}]$ & $938.18(18)$ & Zahl der Restraints & 199 \\
\hline$b[\mathrm{pm}]$ & $1447.0(3)$ & verfeinerte Parameter & 1214 \\
\hline$c[\mathrm{pm}]$ & $2855.4(5)$ & $R 1[\mid>2 \sigma(I)]$ & 0.0752 \\
\hline$\alpha\left[^{\circ}\right]$ & $88.542(3)$ & $w R 2$ [alle Daten] & 0.1989 \\
\hline$\beta\left[^{\circ}\right]$ & $85.980(2)$ & GooF & 1.073 \\
\hline$\gamma\left[^{\circ}\right]$ & $89.426(3)$ & & \\
\hline$V\left[\mathrm{~nm}^{3}\right]$ & $3.8655(13)$ & Differenzelektronendichte: & \\
\hline$Z$ & 2 & Max. / min. $\left[10^{-6} \mathrm{epm}^{-3}\right]$ & 3.947 / -0.837 \\
\hline Messtemperatur [K] & $100(2)$ & Max. / min. Transmission & $0.4286 / 0.3301$ \\
\hline$\rho\left[\mathrm{Mgm}^{-3}\right]$ & 1.447 & & \\
\hline
\end{tabular}



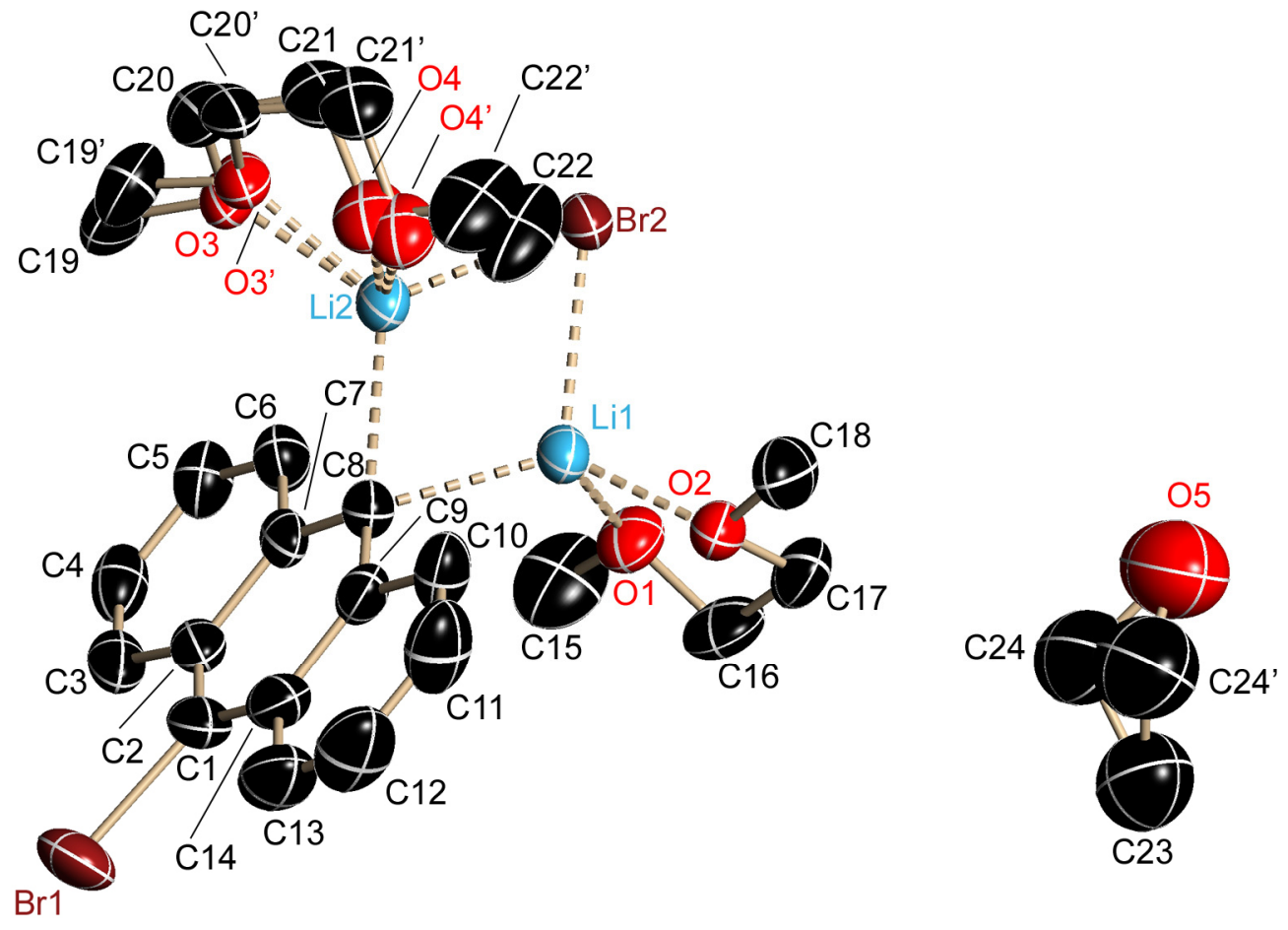

Abb. 5.24: Asymmetrische Einheit von $\left[\mathrm{BrAnLi} \cdot \mathrm{LiBr} \cdot\{\mathrm{dme}\}_{2}\right](\mathbf{1 5 a})$; die anisotropen Auslenkungsparameter geben die $50 \%$ ige Aufenthaltswahrscheinlichkeit an.

Ein DME Molekül und das freie Diethylether sind Fehlgeordnet. Die Besetzung der Hauptdomänen verfeinerten zu $60 \%$ und $59 \%$

Tab. 5.16: Kristallographische Daten von $\left[\mathrm{BrAnLi} \cdot \mathrm{LiBr} \cdot\{\mathrm{dme}\}_{2}\right](\mathbf{1 5 a})$.

\begin{tabular}{|c|c|c|c|}
\hline Strukturcode & BrAnLi LiBr DME & $\mu\left[\mathrm{mm}^{-1}\right]$ & 2.707 \\
\hline Summenformel & $\mathrm{C}_{24} \mathrm{H}_{33} \mathrm{Br}_{2} \mathrm{Li}_{2} \mathrm{O} 4.50$ & $F(000)$ & 2244 \\
\hline Molmasse $[\mathrm{g} / \mathrm{mol}]$ & & $\theta$-Bereich $\left[{ }^{\circ}\right]$ & $2.09-25.39$ \\
\hline Kristallgröße [mm] & $0.2 \times 0.2 \times 0.2$ & gesammelte Reflexe & 30017 \\
\hline Raumgruppe & $C 2 / c$ & unabhängige Reflexe & 5205 \\
\hline$a[\mathrm{pm}]$ & $19.891(3)$ & Zahl der Restraints & 699 \\
\hline$b[\mathrm{pm}]$ & $8.2807(13)$ & verfeinerte Parameter & 368 \\
\hline$c[\mathrm{pm}]$ & $32.847(5)$ & $R 1[I>2 \sigma(I)]$ & 0.0569 \\
\hline$\alpha\left[^{\circ}\right]$ & 90 & $w R 2$ [alle Daten] & 0.1349 \\
\hline$\beta\left[^{\circ}\right]$ & $101.332(2)$ & GooF & 1.118 \\
\hline$\gamma\left[^{\circ}\right]$ & 90 & & \\
\hline$V\left[\mathrm{~nm}^{3}\right]$ & $5304.7(14)$ & Differenzelektronendichte: & \\
\hline$Z$ & 8 & Max. / min. $\left[10^{-6} \mathrm{epm}^{-3}\right]$ & $1.638 /-0.830$ \\
\hline Messtemperatur [K] & $100(2)$ & Max. / min. Transmission & $0.4288 / 0.3363$ \\
\hline$\rho\left[\mathrm{Mgm}^{-3}\right]$ & 1.371 & & \\
\hline
\end{tabular}




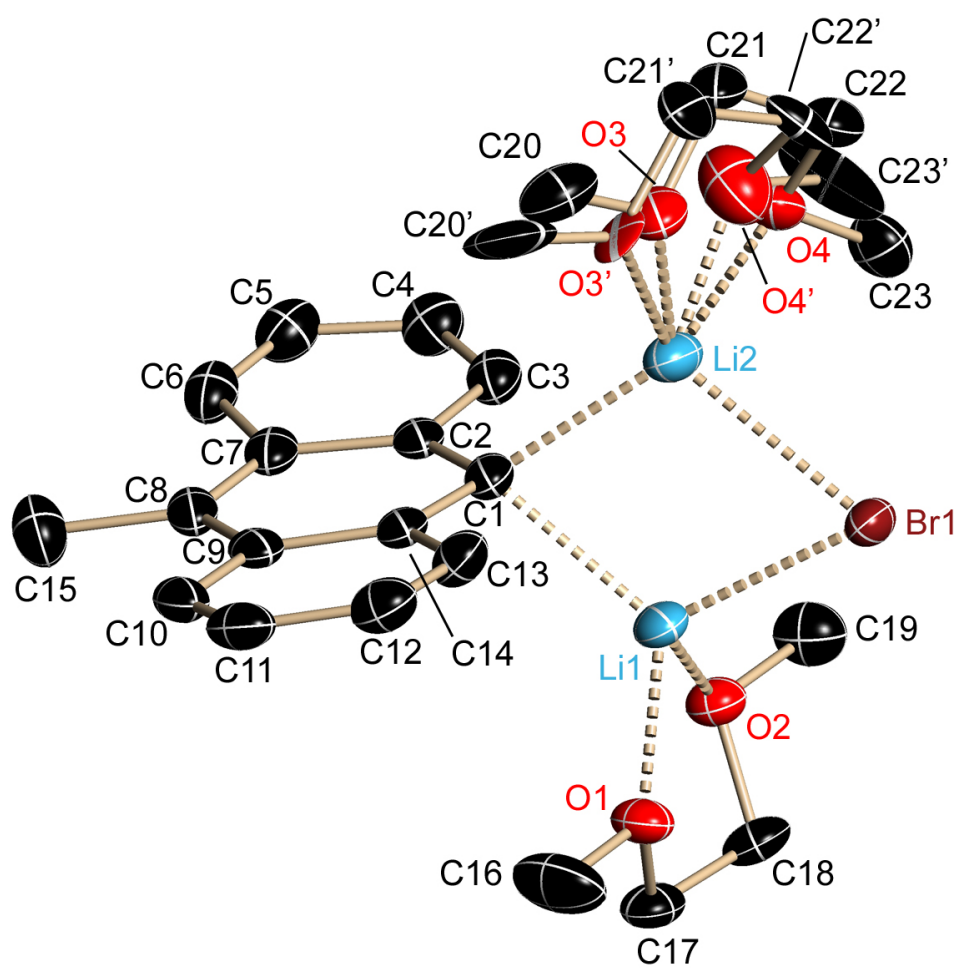

Abb. 5.25: Asymmetrische Einheit von $\left[\mathrm{MeAnLi} \cdot \mathrm{LiBr} \cdot\{\mathrm{dme}\}_{2}\right](\mathbf{1 5 b})$; die anisotropen Auslenkungsparameter geben die $50 \%$ ige Aufenthaltswahrscheinlichkeit an.

Tab. 5.17: Kristallographische Daten von $\left[\mathrm{MeAnLi} \cdot \mathrm{LiBr} \cdot\{\mathrm{dme}\}_{2}\right](\mathbf{1 5 b})$.

\begin{tabular}{|c|c|c|c|}
\hline Strukturcode & BrAnLi LiBr DME & $\mu\left[\mathrm{mm}^{-1}\right]$ & 1.757 \\
\hline Summenformel & $\mathrm{C}_{23} \mathrm{H}_{31} \mathrm{BrLi}_{2} \mathrm{O}_{4}$ & $F(000)$ & 1936 \\
\hline Molmasse $[\mathrm{g} / \mathrm{mol}]$ & 465.27 & $\theta$-Bereich $\left[{ }^{\circ}\right]$ & $2.02-25.47$ \\
\hline Kristallgröße [mm] & $0.18 \times 0.15 \times 0.1$ & gesammelte Reflexe & 85397 \\
\hline Raumgruppe & $C 2 / c$ & unabhängige Reflexe & 4716 \\
\hline$a[\mathrm{pm}]$ & $2014.2(2)$ & Zahl der Restraints & 0 \\
\hline$b[\mathrm{pm}]$ & $827.20(9)$ & verfeinerte Parameter & 333 \\
\hline$c[\mathrm{pm}]$ & $2849.8(3)$ & $R 1[\mid>2 \sigma(I)]$ & 0.403 \\
\hline$\alpha\left[^{\circ}\right]$ & 90 & $w R 2$ [alle Daten] & 0.989 \\
\hline$\beta\left[^{\circ}\right]$ & $92.486(2)$ & GooF & 1.115 \\
\hline$\gamma\left[^{\circ}\right]$ & 90 & & \\
\hline$V\left[\mathrm{~nm}^{3}\right]$ & $4.7437(9)$ & Differenzelektronendichte: & \\
\hline$Z$ & 8 & Max. / min. $\left[10^{-6} \mathrm{epm}^{-3}\right]$ & $1.037 /-0.577$ \\
\hline Messtemperatur [K] & $100(2)$ & Max. / min. Transmission & 0.7452 / 0.6502 \\
\hline$\rho\left[\mathrm{Mgm}^{-3}\right]$ & 1.303 & & \\
\hline
\end{tabular}




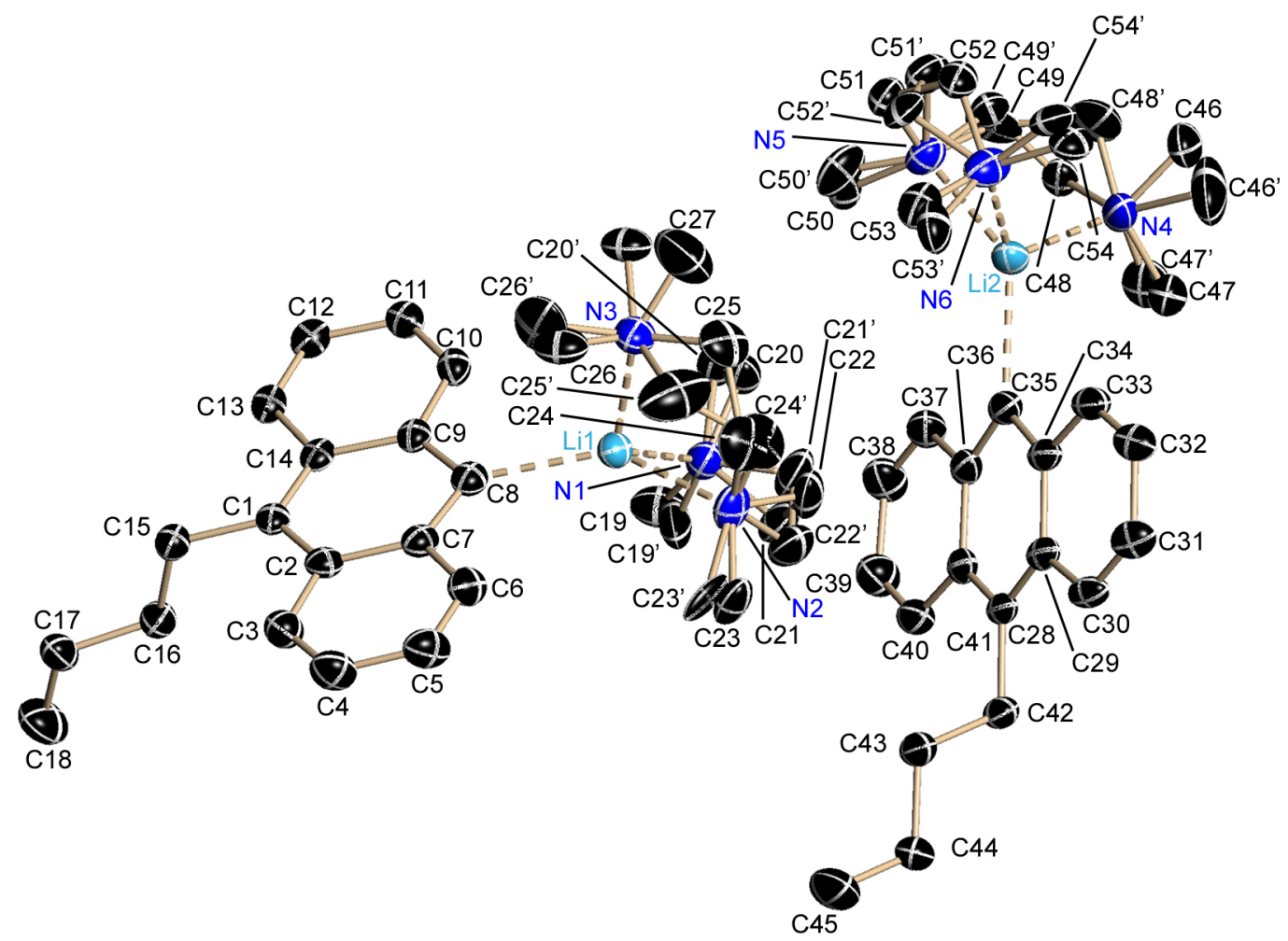

Abb. 5.26: Asymmetrische Einheit von [BuAnLi $-\{$ pmdeta\}] (16); die anisotropen Auslenkungsparameter geben die $50 \%$ ige Aufenthaltswahrscheinlichkeit an.

Tab. 5.18: Kristallographische Daten von [BuAnLi • \{pmdeta\}] (16).

\begin{tabular}{|c|c|c|c|}
\hline Strukturcode & {$[$ BuAnLi $\bullet\{$ pmdeta $\}]$} & $\mu\left[\mathrm{mm}^{-1}\right]$ & 0.061 \\
\hline Summenformel & $\mathrm{C}_{27} \mathrm{H}_{40} \mathrm{LiN}_{3}$ & $F(000)$ & 1808 \\
\hline Molmasse $[\mathrm{g} / \mathrm{mol}]$ & 413.56 & $\theta$-Bereich $\left[{ }^{\circ}\right]$ & $2.61-26.02$ \\
\hline Kristallgröße [mm] & $0.15 \times 0.15 \times 0.15$ & gesammelte Reflexe & 43908 \\
\hline Raumgruppe & $P \overline{4}$ & unabhängige Reflexe & 10166 \\
\hline$a[\mathrm{pm}]$ & $21.123(4)$ & Zahl der Restraints & 474 \\
\hline$b[\mathrm{pm}]$ & $21.123(4)$ & verfeinerte Parameter & 739 \\
\hline$c[\mathrm{pm}]$ & $11.595(5)$ & $R 1[\mid>2 \sigma(I)]$ & 0.435 \\
\hline$\alpha\left[^{\circ}\right]$ & 90 & $w R 2$ [alle Daten] & 0.1133 \\
\hline$\beta\left[^{\circ}\right]$ & 90 & GooF & 1.069 \\
\hline$\gamma\left[^{\circ}\right]$ & 90 & Flack-X & $0.1(17)$ \\
\hline$V\left[\mathrm{~nm}^{3}\right]$ & & Differenzelektronendichte: & \\
\hline$Z$ & 8 & Max. / min. $\left[10^{-6} \mathrm{epm}^{-3}\right]$ & $0.287 /-0.169$ \\
\hline Messtemperatur [K] & $100(2)$ & Max. / min. Transmission & \\
\hline$\rho\left[\mathrm{Mgm}^{-3}\right]$ & 1.062 & & \\
\hline
\end{tabular}



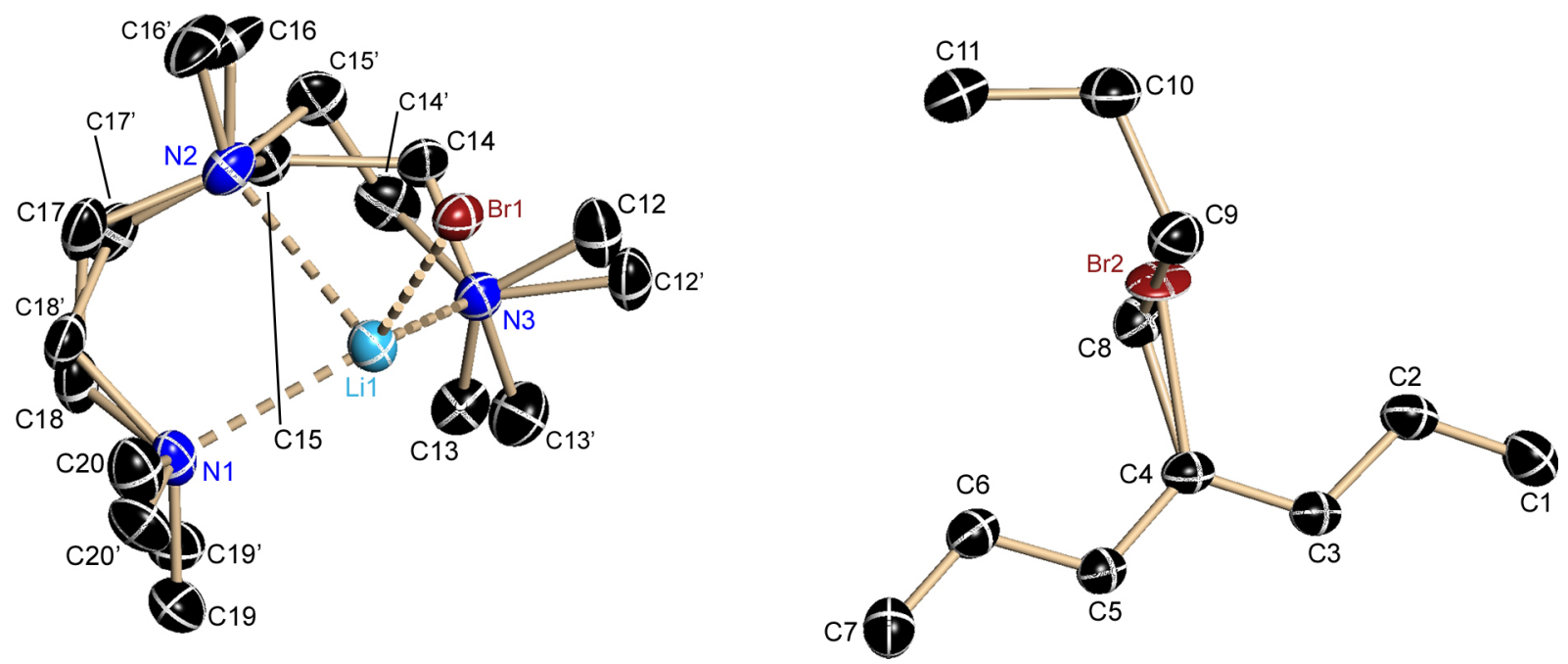

Abb. 5.27: Asymmetrische Einheit von 9,10-Dibutylanthracen (17); die anisotropen Auslenkungsparameter geben die $50 \%$ ige Aufenthaltswahrscheinlichkeit an.

Sowohl das gesammete PMDETA Molekül als auch der Butylrest am Anthracen ist fehlgeordnet. Die Besetzung der Hauptdomäne des PMDETA Moleküls verfeinerte auf $63 \%$, der Buthylrest am Anthracen auf $95 \%$.

Tab. 5.19: Kristallographische Daten von 9,10-Dibutylanthracen (17).

\begin{tabular}{|c|c|c|c|}
\hline Strukturcode & 9,10-Dibutylanthracen & $\mu\left[\mathrm{mm}^{-1}\right]$ & 2.023 \\
\hline Summenformel & $\mathrm{C}_{39.6} \mathrm{H}_{71.1} \mathrm{Br}_{2.1} \mathrm{Li}_{2} \mathrm{~N}_{6}$ & $F(000)$ & 430 \\
\hline Molmasse $[\mathrm{g} / \mathrm{mol}]$ & 813.03 & $\theta$-Bereich $\left[{ }^{\circ}\right]$ & $1.82-25.44$ \\
\hline Kristallgröße [mm] & $0.25 \times 0.03 \times 0.03$ & gesammelte Reflexe & 19524 \\
\hline Raumgruppe & $P \overline{1}$ & unabhängige Reflexe & 3953 \\
\hline$a[\mathrm{pm}]$ & $862.62(13)$ & Zahl der Restraints & 0 \\
\hline$b[\mathrm{pm}]$ & 1127.35(17) & verfeinerte Parameter & 329 \\
\hline$c[\mathrm{pm}]$ & $1180.43(18)$ & $R 1[I>2 \sigma(I)]$ & 0.0261 \\
\hline$\alpha\left[^{\circ}\right]$ & $95.728(2)$ & $w R 2$ [alle Daten] & 0.0674 \\
\hline$\beta\left[^{\circ}\right]$ & $110.504(2)$ & GooF & 1.058 \\
\hline$\gamma\left[^{\circ}\right]$ & $90.270(2)$ & & \\
\hline$V\left[\mathrm{~nm}^{3}\right]$ & $1.0689(3)$ & Differenzelektronendichte: & \\
\hline$Z$ & 1 & Max. / min. $\left[10^{-6} \mathrm{epm}^{-3}\right]$ & $0.494 /-0.0674$ \\
\hline Messtemperatur [K] & $100(2)$ & Max. / min. Transmission & $0.4288 / 0.3106$ \\
\hline$\rho\left[\mathrm{Mgm}^{-3}\right]$ & 1.263 & & \\
\hline
\end{tabular}




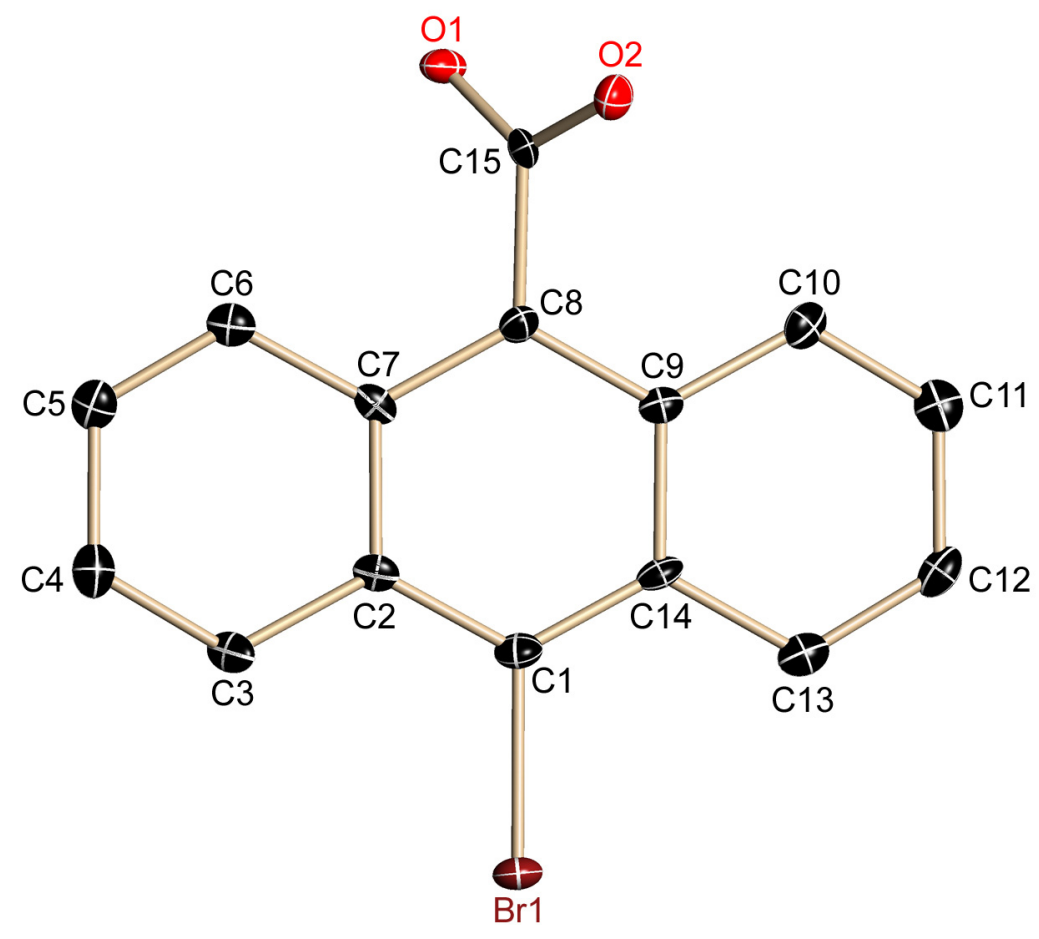

Abb. 5.28: Asymmetrische Einheit von 9-Brom-10-anthrylcarbonsäure (18); die anisotropen Auslenkungsparameter geben die $50 \%$ ige Aufenthaltswahrscheinlichkeit an.

Tab. 5.20: Kristallographische Daten von 9-Brom-10-anthrylcarbonsäure (18).

\begin{tabular}{|c|c|c|c|}
\hline Strukturcode & $\mathrm{BrAnCOOH}$ & $\mu\left[\mathrm{mm}^{-1}\right]$ & 3.671 \\
\hline Summenformel & $\mathrm{C}_{15} \mathrm{H}_{9} \mathrm{O}_{2} \mathrm{Br}$ & $F(000)$ & 600 \\
\hline Molmasse $[\mathrm{g} / \mathrm{mol}]$ & 301.13 & $\theta$-Bereich $\left[{ }^{\circ}\right]$ & $2.23-26.02$ \\
\hline Kristallgröße [mm] & $0.02 \times 0.05 \times 0.05$ & gesammelte Reflexe & 11528 \\
\hline Raumgruppe & $P 2_{1} / c$ & unabhängige Reflexe & 2201 \\
\hline$a[\mathrm{pm}]$ & $920.77(13)$ & Zahl der Restraints & 0 \\
\hline$b[\mathrm{pm}]$ & $386.43(5)$ & verfeinerte Parameter & 164 \\
\hline$c[\mathrm{pm}]$ & $3158.3(4)$ & $R 1[\mathrm{I}>2 \sigma(\mathrm{I})]$ & 0.0533 \\
\hline$\alpha\left[^{\circ}\right]$ & 90 & $w R 2$ [alle Daten] & 0.1260 \\
\hline$\beta\left[^{\circ}\right]$ & $96.748(2)$ & GooF & 1.310 \\
\hline$\gamma\left[^{\circ}\right]$ & 90 & & \\
\hline$V\left[\mathrm{~nm}^{3}\right]$ & $1.1160(3)$ & Differenzelektronendichte: & \\
\hline$Z$ & 4 & Max. / min. $\left[10^{-6} \mathrm{epm}^{-3}\right]$ & $1.287 /-1.415$ \\
\hline Messtemperatur [K] & $100(2)$ & Max. / min. Transmission & $0.6244 / 0.7457$ \\
\hline$\rho\left[\mathrm{Mgm}^{-3}\right]$ & 1.792 & & \\
\hline
\end{tabular}




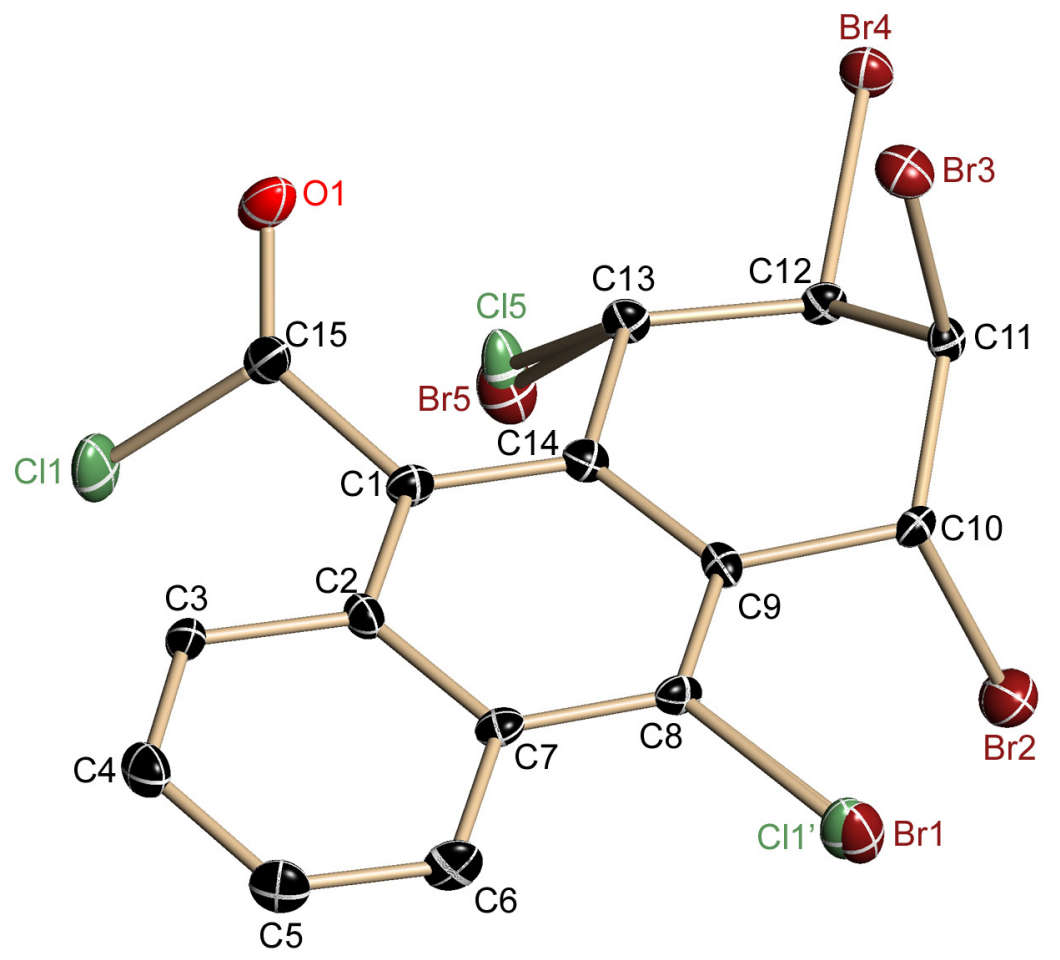

Abb. 5.29: Asymmetrische Einheit von 1,2,3,4,9-Pentachlor-10-anthrachencarbonsäurechlorid (19b); die anisotropen Auslenkungsparameter geben die 50\% ige Aufenthaltswahrscheinlichkeit an.

Während die Bromatome in 1-, 2- und 3-Position voll besetzt sind, ist für die Bromatome in 9- und 4-Position eine Fehlordnung gegen Chloratome zu beobachten. Die Besetzungsfaktoren der Bromatome verfeinerte auf $41 \%(\mathrm{Br} 5)$ und $24 \%(\mathrm{Br} 1)$. Eine anisotope Verfeinerung der fehlgeordneten Atome gelang durch festsetzen der Besetzungsfaktoren und einer BLOC-Verfeinerung, bei der Positionen und Schwingungen getrennt verfeinert wurden.

Tab. 5.21: Kristallographische Daten von 1,2,3,4,9-Pentachlor-10-anthrachencarbonsäurechlorid (19b).

\begin{tabular}{|ll|ll|}
\hline Strukturcode & $\mathrm{Cl}_{1+n} \mathrm{Br}_{4-n} \mathrm{AnCOCl}$ & $\mu\left[\mathrm{mm}^{-1}\right]$ & 9.346 \\
Summenformel & $\mathrm{C}_{15} \mathrm{H}_{8} \mathrm{Br}_{3.67} \mathrm{Cl}_{2.34} \mathrm{O}$ & $\mathrm{F}(000)$ & 1096 \\
Molmasse [g/mol] & 579.86 & $\theta$-Bereich [ $\left.{ }^{\circ}\right]$ & $1.95-26.37$ \\
Kristallgröße [mm] & $0.2 \times 0.2 \times 0.2$ & gesammelte Reflexe & \\
Raumgruppe & $\mathrm{Pna2}_{1}$ & unabhängige Reflexe & 1096 \\
$a[\mathrm{pm}]$ & $752.42(9)$ & Zahl der Restraints & 41 \\
$b[\mathrm{pm}]$ & $1433.78(16)$ & verfeinerte Parameter & 219 \\
$c[\mathrm{pm}]$ & $1525.15(18)$ & $R 1$ [I>2 $\sigma(\mathrm{I})]$ & 0.0218 \\
$\alpha=\beta=\gamma\left[^{\circ}\right]$ & WR2 [alle Daten] & 0.0743 \\
$V\left[\mathrm{~nm}^{3}\right]$ & 90 & GooF & 1.049 \\
$Z$ & $1.6453(3)$ & Flack-X & $0.022(10)$ \\
Messtemperatur $[\mathrm{K}]^{-3}$ & 4 & Differenzelektronendichte: & \\
$\rho\left[\mathrm{Mgm}^{-3}\right]$ & $100(2)$ & Max. / min. [10 & epm $\left.{ }^{-3}\right]$ \\
\hline
\end{tabular}




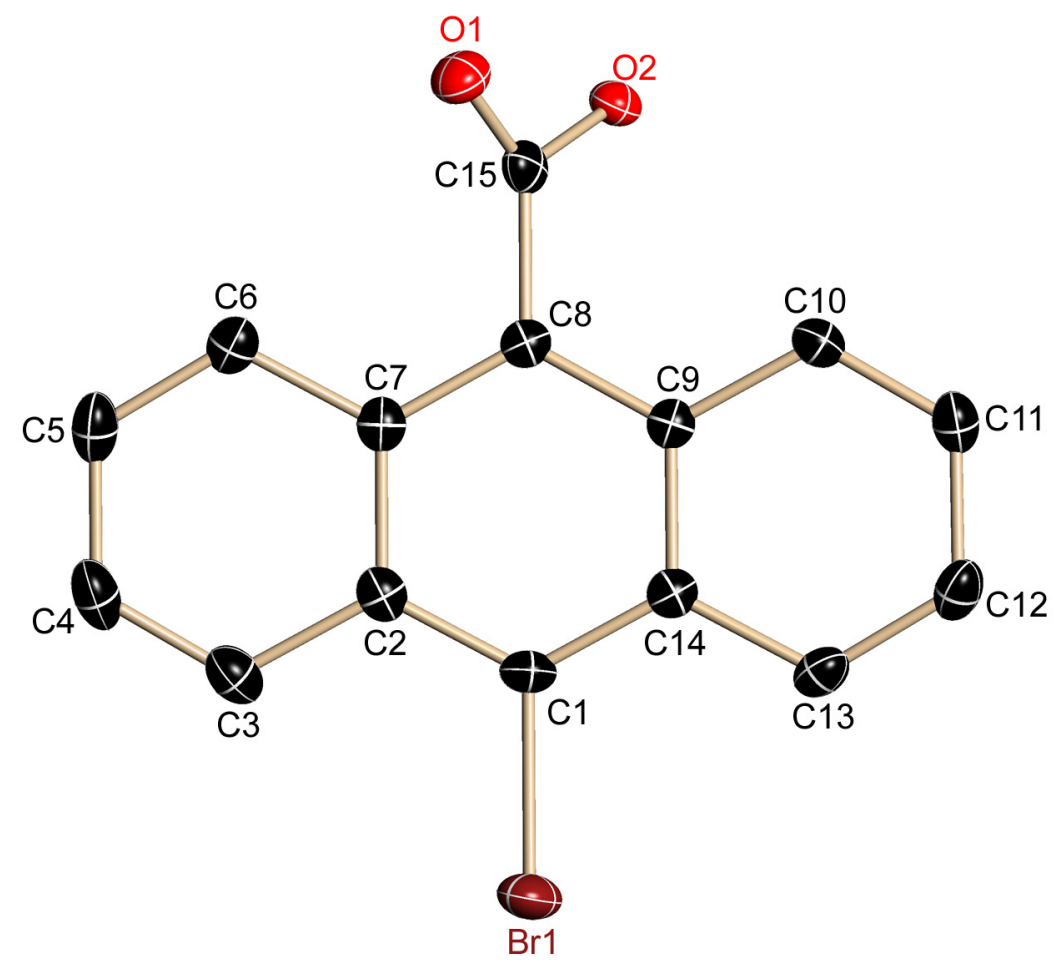

Abb. 5.30: Asymmetrische Einheit von 9-Brom-10-anthrylcarbonsäureanhydrid (20); die anisotropen Auslenkungsparameter geben die 50\% ige Aufenthaltswahrscheinlichkeit an.

Tab. 5.22: Kristallographische Daten von 9-Brom-10-anthrylcarbonsäureanhydrid (20).

\begin{tabular}{|c|c|c|c|}
\hline Strukturcode & BrAnCOOOCAnBr & $\mu\left[\mathrm{mm}^{-1}\right]$ & 3.773 \\
\hline Summenformel & $\mathrm{C}_{30} \mathrm{H}_{16} \mathrm{O}_{3} \mathrm{Br}_{2}$ & $F(000)$ & 1160 \\
\hline Molmasse $[\mathrm{g} / \mathrm{mol}]$ & 584.25 & $\theta$-Bereich $\left[{ }^{\circ}\right]$ & $2.49-25.35$ \\
\hline Kristallgröße [mm] & $0.1 \times 0.1 \times 0.1$ & gesammelte Reflexe & 14206 \\
\hline Raumgruppe & $C 2 / c$ & unabhängige Reflexe & 1979 \\
\hline$a[\mathrm{pm}]$ & $1754.23(15)$ & Zahl der Restraints & 0 \\
\hline$b[\mathrm{pm}]$ & $888.65(8)$ & verfeinerte Parameter & 159 \\
\hline$c[\mathrm{pm}]$ & $1493.00(13)$ & $R 1[I>2 \sigma(I)]$ & 0.0244 \\
\hline$\alpha\left[^{\circ}\right]$ & 90 & $w R 2$ [alle Daten] & 0.0627 \\
\hline$\beta\left[^{\circ}\right]$ & $111.3020(10)$ & GooF & 1.092 \\
\hline$\gamma\left[^{\circ}\right]$ & 90 & & \\
\hline$V\left[\mathrm{~nm}^{3}\right]$ & 2.1684 & Differenzelektronendichte: & \\
\hline$Z$ & 4 & Max. / min. $\left[10^{-6} \mathrm{epm}^{-3}\right]$ & $0.365 /-0.377$ \\
\hline Messtemperatur [K] & $100(2)$ & Max. / min. Transmission & $0.7452 / 0.5537$ \\
\hline$\rho\left[\mathrm{Mgm}^{-3}\right]$ & 1.790 & & \\
\hline
\end{tabular}




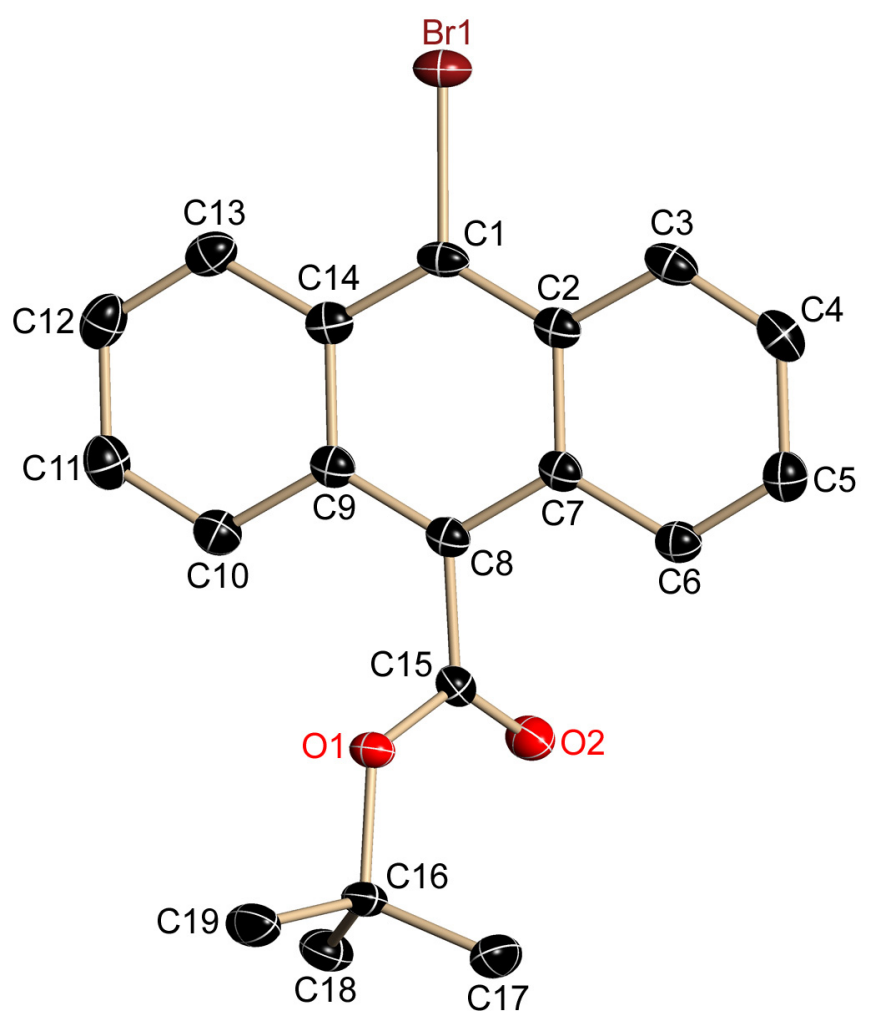

Abb. 5.31: Asymmetrische Einheit von 9-Brom-10-anthracentertbutylcarbonsäureester (21); die anisotropen Auslenkungsparameter geben die 50\% ige Aufenthaltswahrscheinlichkeit an.

Tab. 5.23: Kristallographische Daten von 9-Brom-10-anthracentertbutylcarbonsäureester (21).

\begin{tabular}{|c|c|c|c|}
\hline Strukturcode & BrAnCOOtBu & $\mu\left[\mathrm{mm}^{-1}\right]$ & 2.628 \\
\hline Summenformel & $\mathrm{C}_{19} \mathrm{H}_{17} \mathrm{BrO}_{2}$ & $F(000)$ & 728 \\
\hline Molmasse $[\mathrm{g} / \mathrm{mol}]$ & 357.24 & $\theta$-Bereich $\left[{ }^{\circ}\right]$ & $3.40-26.96$ \\
\hline Kristallgröße [mm] & $0.16 \times 0.15 \times 0.09$ & gesammelte Reflexe & 34102 \\
\hline Raumgruppe & $P 2_{1} / n$ & unabhängige Reflexe & 3541 \\
\hline$a[\mathrm{pm}]$ & 1015.64(7) & Zahl der Restraints & 0 \\
\hline$b[\mathrm{pm}]$ & $1425.54(10)$ & verfeinerte Parameter & 202 \\
\hline$c[\mathrm{pm}]$ & $1177.56(8)$ & $R 1[I>2 \sigma(I)]$ & 0.0260 \\
\hline$\alpha\left[^{\circ}\right]$ & 90 & $w R 2$ [alle Daten] & 0.0667 \\
\hline$\beta\left[^{\circ}\right]$ & $113.2050(10)$ & GooF & 1.104 \\
\hline$\gamma\left[^{\circ}\right]$ & 90 & & \\
\hline$V\left[\mathrm{~nm}^{3}\right]$ & 156699(19) & Differenzelektronendichte: & \\
\hline$Z$ & 4 & Max. / min. $\left[10^{-6} \mathrm{epm}^{-3}\right]$ & $0.365 /-0.283$ \\
\hline Messtemperatur [K] & $100(2)$ & Max. / min. Transmission & $0.7317 / 0.6583$ \\
\hline$\rho\left[\mathrm{Mgm}^{-3}\right]$ & 1.514 & & \\
\hline
\end{tabular}




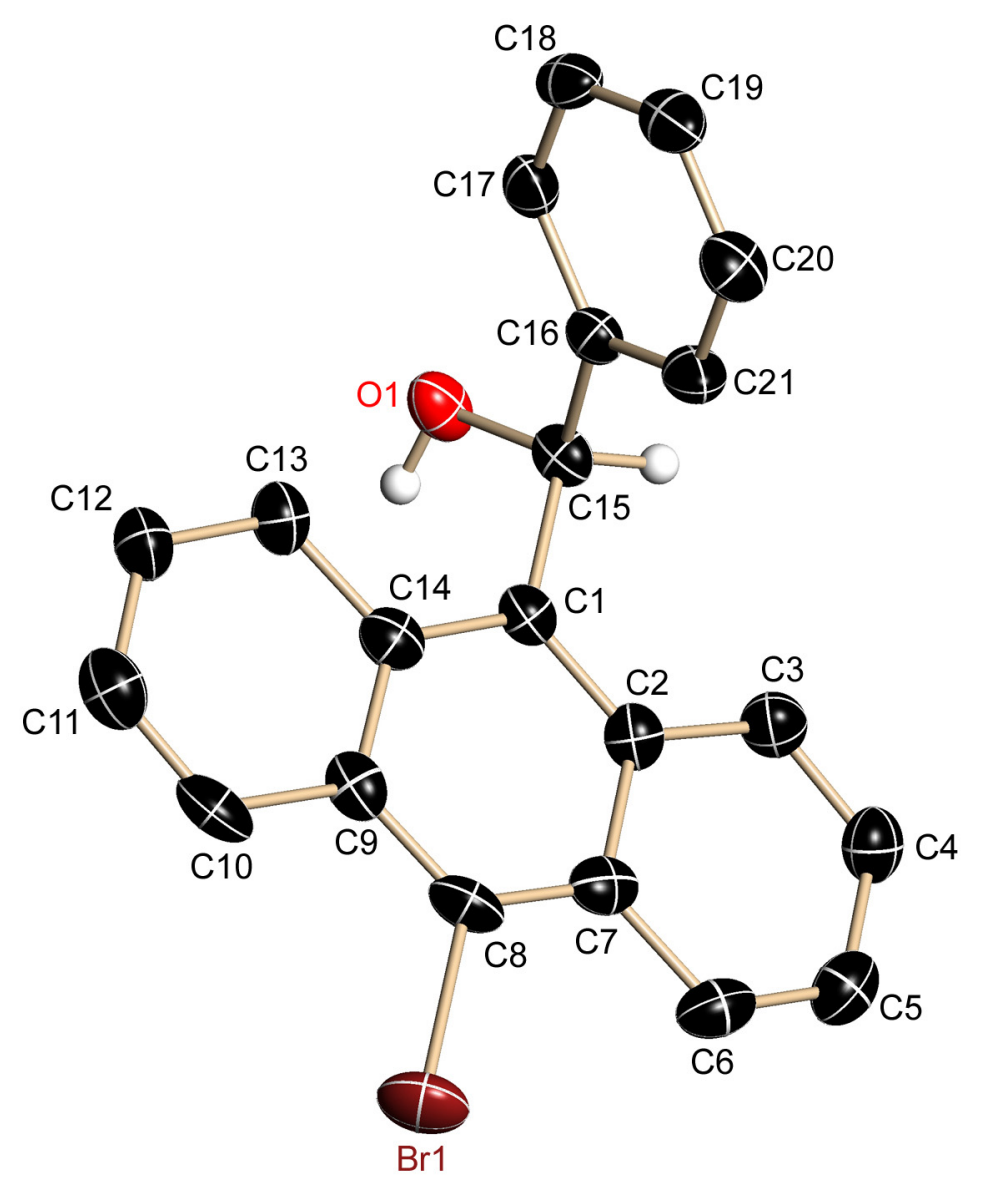

Abb. 5.32: Asymmetrische Einheit von 9-Brom-10-(1-hydroxy-1-phenylmethyl)anthracen (24); die anisotropen Auslenkungsparameter geben die 50\% ige Aufenthaltswahrscheinlichkeit an.

Tab. 5.24: Kristallographische Daten von 9-Brom-10-(1-hydroxy-1-phenylmethyl)anthracen (24).

\begin{tabular}{|c|c|c|c|}
\hline Strukturcode & BrAnCHOHPh & $\mu\left[\mathrm{mm}^{-1}\right]$ & 2.566 \\
\hline Summenformel & $\mathrm{C}_{21} \mathrm{H}_{15} \mathrm{OBr}$ & $F(000)$ & 3095 \\
\hline Molmasse $[\mathrm{g} / \mathrm{mol}]$ & & $\theta$-Bereich $\left[{ }^{\circ}\right]$ & $1.89-25.45$ \\
\hline Kristallgröße [mm] & $0.3 \times 0.2 \times 0.2$ & gesammelte Reflexe & 64272 \\
\hline Raumgruppe & $P 2_{1} 2_{1} 2_{1}$ & unabhängige Reflexe & 1231 \\
\hline$a[\mathrm{pm}]$ & $1944.3(3)$ & Zahl der Restraints & 2 \\
\hline$b[\mathrm{pm}]$ & $1944.3(3)$ & verfeinerte Parameter & 862 \\
\hline$c[\mathrm{pm}]$ & $1746.7(3)$ & $R 1[I>2 \sigma(I)]$ & 0.0415 \\
\hline$\alpha\left[^{\circ}\right]$ & 90 & $w R 2$ [alle Daten] & 0.1067 \\
\hline$\beta\left[^{\circ}\right]$ & 90 & GooF & 1.065 \\
\hline$\gamma\left[^{\circ}\right]$ & 90 & Flack-X & $0.00(4)$ \\
\hline$V\left[\mathrm{~nm}^{3}\right]$ & $6.6029(15)$ & Differenzelektronendichte: & \\
\hline$Z$ & 16 & Max. / min. $\left[10^{-6} \mathrm{epm}^{-3}\right]$ & 1.085 / -0.581 \\
\hline Messtemperatur [K] & $100(2)$ & Max. / min. Transmission & $0.4288 / 0.2957$ \\
\hline$\rho\left[\mathrm{Mgm}^{-3}\right]$ & 1.538 & & \\
\hline
\end{tabular}




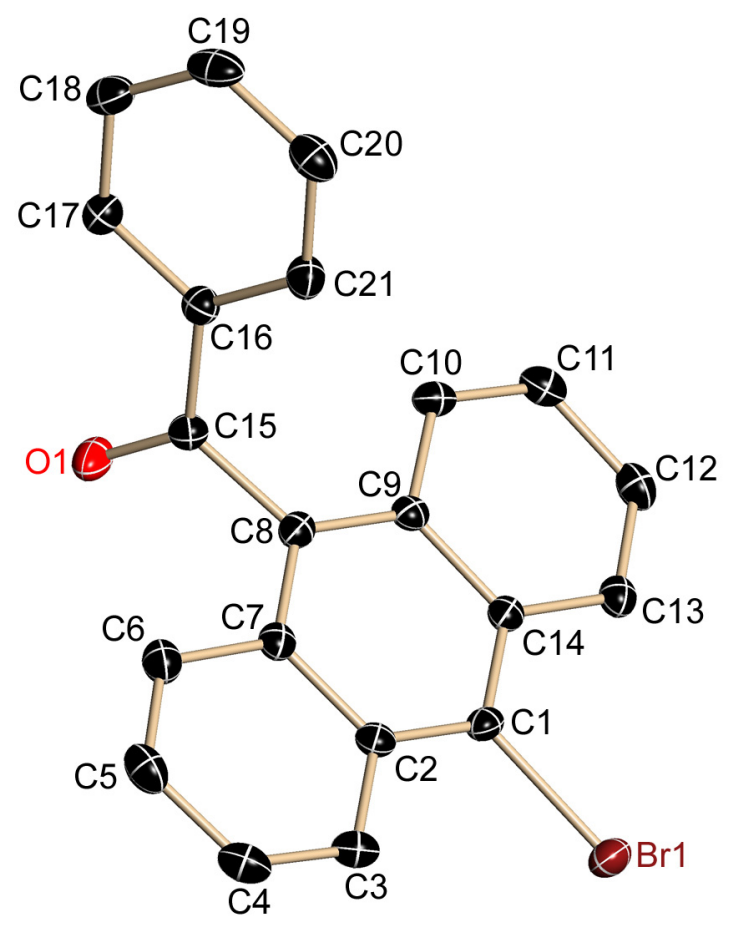

Abb. 5.33: Asymmetrische Einheit von 10-(9-Bromanthracenyl)phenylketon; die anisotropen Auslenkungsparameter geben die $50 \%$ ige Aufenthaltswahrscheinlichkeit an.

Tab. 5.25: Kristallographische Daten von 10-(9-Bromanthracenyl)phenylketon.

\begin{tabular}{|c|c|c|c|}
\hline Strukturcode & BrAnCOPh & $\mu\left[\mathrm{mm}^{-1}\right]$ & 2.685 \\
\hline Summenformel & $\mathrm{C}_{21} \mathrm{H}_{13} \mathrm{OBr}$ & $F(000)$ & 728 \\
\hline Molmasse $[\mathrm{g} / \mathrm{mol}]$ & 361.22 & $\theta$-Bereich $\left[^{\circ}\right]$ & $1.57-27.51$ \\
\hline Kristallgröße [mm] & $0.2 \times 0.25 \times 0.1$ & gesammelte Reflexe & 20909 \\
\hline Raumgruppe & $P 2_{1} / c$ & unabhängige Reflexe & 3517 \\
\hline$a[\mathrm{pm}]$ & $1403.64(13)$ & Zahl der Restraints & 0 \\
\hline$b[\mathrm{pm}]$ & $1043.16(10)$ & verfeinerte Parameter & 208 \\
\hline$c[\mathrm{pm}]$ & $1130.34(11)$ & $R 1[\mid>2 \sigma(I)]$ & 0.0283 \\
\hline$\alpha\left[^{\circ}\right]$ & 90 & $w R 2$ [alle Daten] & 0.0734 \\
\hline$\beta\left[^{\circ}\right]$ & $112.2740(10)$ & GooF & 1.068 \\
\hline$\gamma\left[^{\circ}\right]$ & 90 & & \\
\hline$V\left[\mathrm{~nm}^{3}\right]$ & $1.5316(3)$ & Differenzelektronendichte: & \\
\hline$Z$ & 4 & Max. / min. $\left[10^{-6} \mathrm{epm}^{-3}\right]$ & $0.689 /-0.374$ \\
\hline Messtemperatur [K] & $100(2)$ & Max. / min. Transmission & $0.3854 / 0.4305$ \\
\hline$\rho\left[\mathrm{Mgm}^{-3}\right]$ & 1.567 & & \\
\hline
\end{tabular}




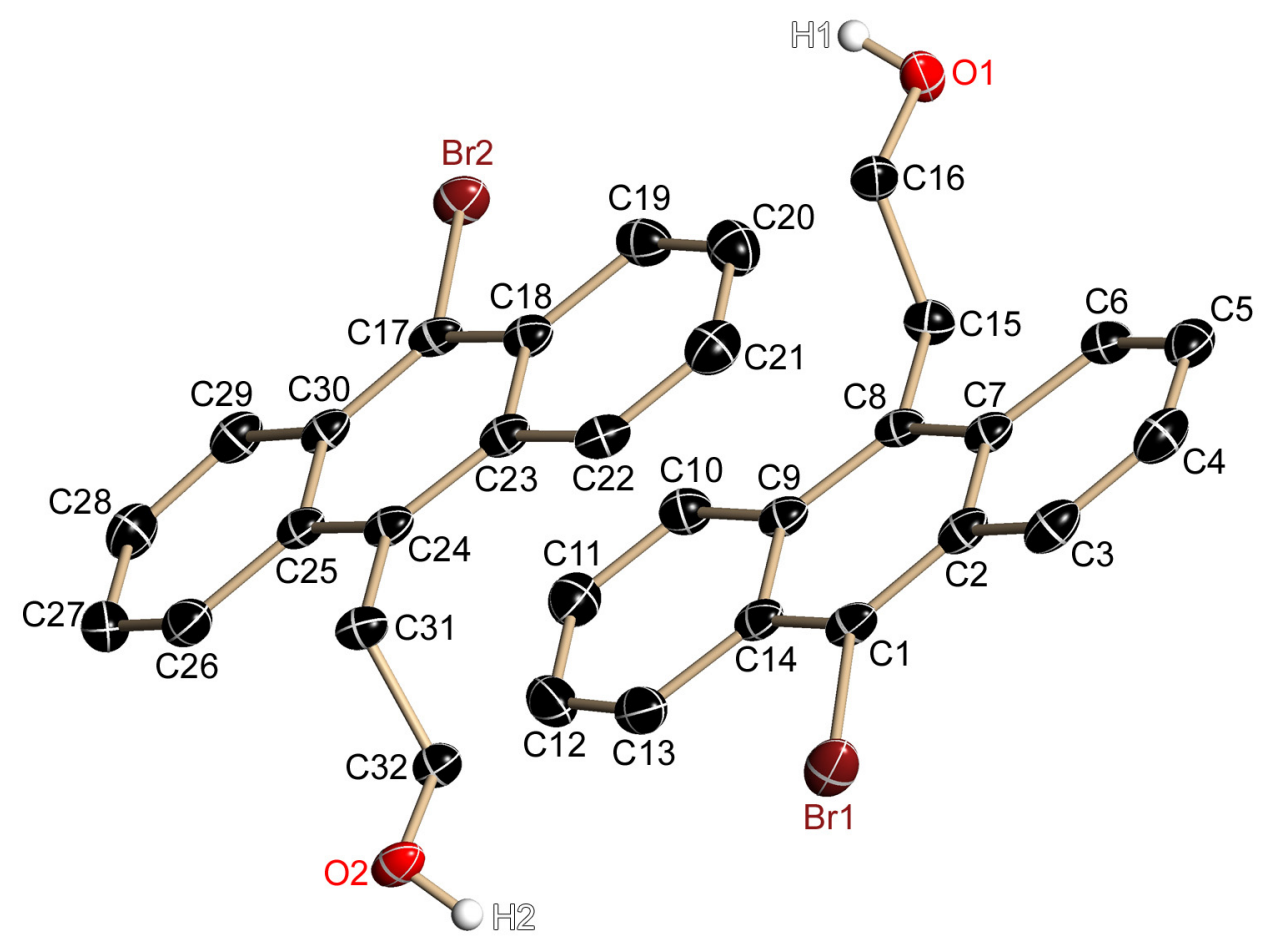

Abb. 5.34: Asymmetrische Einheit von 9-Brom-10-(1-hydroxyethyl)anthracen (27); die anisotropen Auslenkungsparameter geben die 50\% ige Aufenthaltswahrscheinlichkeit an.

Tab. 5.26: Kristallographische Daten von 9-Brom-10-(1-hydroxyethyl)anthracen (27).

\begin{tabular}{|ll|ll|}
\hline Strukturcode & BrAnEtOH & $\mu\left[\mathrm{mm}^{-1}\right]$ & 3.280 \\
Summenformel & $\mathrm{C}_{16} \mathrm{H}_{13} \mathrm{BrO}$ & $\mathrm{F}(000)$ & 1216 \\
Molmasse $[\mathrm{g} / \mathrm{mol}]$ & 301.17 & $\theta$-Bereich [ $\left.{ }^{\circ}\right]$ & $2.59-26.78$ \\
Kristallgröße [mm] & $0.1 \times 0.1 \times 0.1$ & gesammelte Reflexe & 53907 \\
Raumgruppe & $P 2_{1} 2_{1} 2_{1}$ & unabhängige Reflexe & 5326 \\
$a[\mathrm{pm}]$ & $882.68(6)$ & Zahl der Restraints & 0 \\
$b[\mathrm{pm}]$ & $1642.84(11)$ & verfeinerte Parameter & 327 \\
$c[\mathrm{pm}]$ & $1719.93(12)$ & $R 1[\mathrm{I}>2 \sigma(\mathrm{I})]$ & 0.0200 \\
$\alpha\left[^{\circ}\right]$ & 90 & WR2 [alle Daten] & 0.0522 \\
$\beta\left[^{\circ}\right]$ & 90 & GooF & 1.034 \\
$\gamma\left[^{\circ}\right]$ & 90 & FlackX & $0.009(6)$ \\
$V\left[^{\circ} \mathrm{nm}^{3}\right]$ & $2.4941(3)$ & Differenzelektronendichte: & \\
$Z$ & 8 & Max. / min. $\left[10^{-6}\right.$ epm & \\
$\left.\mathrm{Messtemperatur}^{-3}\right]$ & Max. / min. Transmission & $0.428 /-0.250$ \\
$\rho\left[^{-3}\right]$ & $100(2)$ & & $0.7454 / 0.5899$ \\
\hline
\end{tabular}



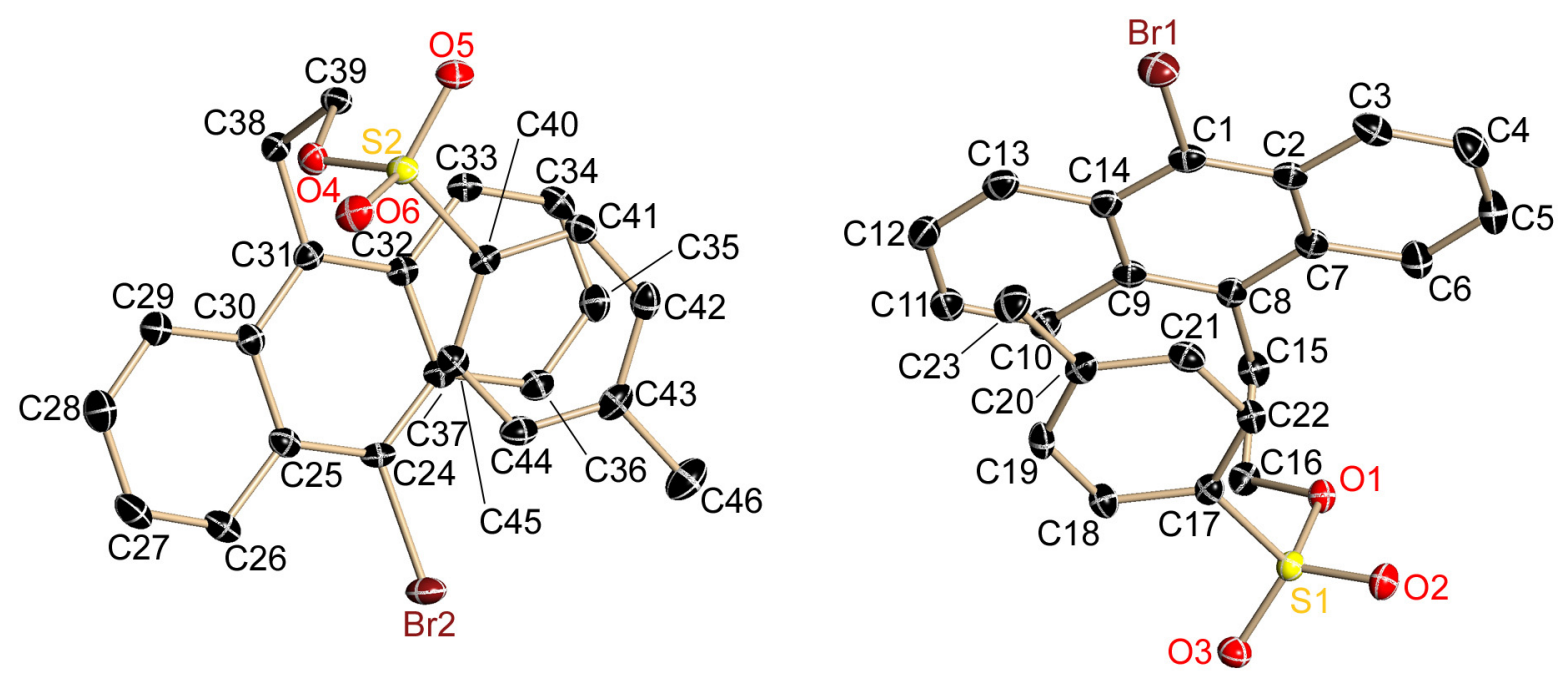

Abb. 5.35: Asymmetrische Einheit von 9-Brom-10-(1-tosylethyl)anthracen (28); die anisotropen Auslenkungsparameter geben die 50\% ige Aufenthaltswahrscheinlichkeit an.

Tab. 5.27: Kristallographische Daten von 9-Brom-10-(1-tosylethyl)anthracen (28).

\begin{tabular}{|c|c|c|c|}
\hline Strukturcode & BrAnEtOTos & $\mu\left[\mathrm{mm}^{-1}\right]$ & 2.280 \\
\hline Summenformel & $\mathrm{C}_{23} \mathrm{H}_{19} \mathrm{BrO}_{3} \mathrm{~S}$ & $F(000)$ & 928 \\
\hline Molmasse $[\mathrm{g} / \mathrm{mol}]$ & 455.35 & $\theta$-Bereich $\left[{ }^{\circ}\right]$ & $1.99-26.02$ \\
\hline Kristallgröße [mm] & $0.18 \times 0.15 \times 0.11$ & gesammelte Reflexe & 17771 \\
\hline Raumgruppe & $P_{\overline{1}}$ & unabhängige Reflexe & 7534 \\
\hline$a[\mathrm{pm}]$ & $775.06(4)$ & Zahl der Restraints & 0 \\
\hline$b[\mathrm{pm}]$ & $1370.23(6)$ & verfeinerte Parameter & 507 \\
\hline$c[\mathrm{pm}]$ & 1848.89(9) & $R 1[1>2 \sigma(I)]$ & 0.0299 \\
\hline$\alpha\left[^{\circ}\right]$ & $83.3910(10)$ & $w R 2$ [alle Daten] & 0.0787 \\
\hline$\beta\left[^{\circ}\right]$ & $89.0400(10)$ & GooF & 1.041 \\
\hline$\gamma\left[^{\circ}\right]$ & $78.7380(10)$ & & \\
\hline$V\left[\mathrm{~nm}^{3}\right]$ & $1.91290(16)$ & Differenzelektronendichte: & \\
\hline$Z$ & 4 & Max. / min. $\left[10^{-6} \mathrm{epm}^{-3}\right]$ & $0.623 /-0.360$ \\
\hline Messtemperatur [K] & $100(2)$ & Max. / min. Transmission & $0.4302 / 0.3802$ \\
\hline$\rho\left[\mathrm{Mgm}^{-3}\right]$ & $1.91290(16)$ & & \\
\hline
\end{tabular}




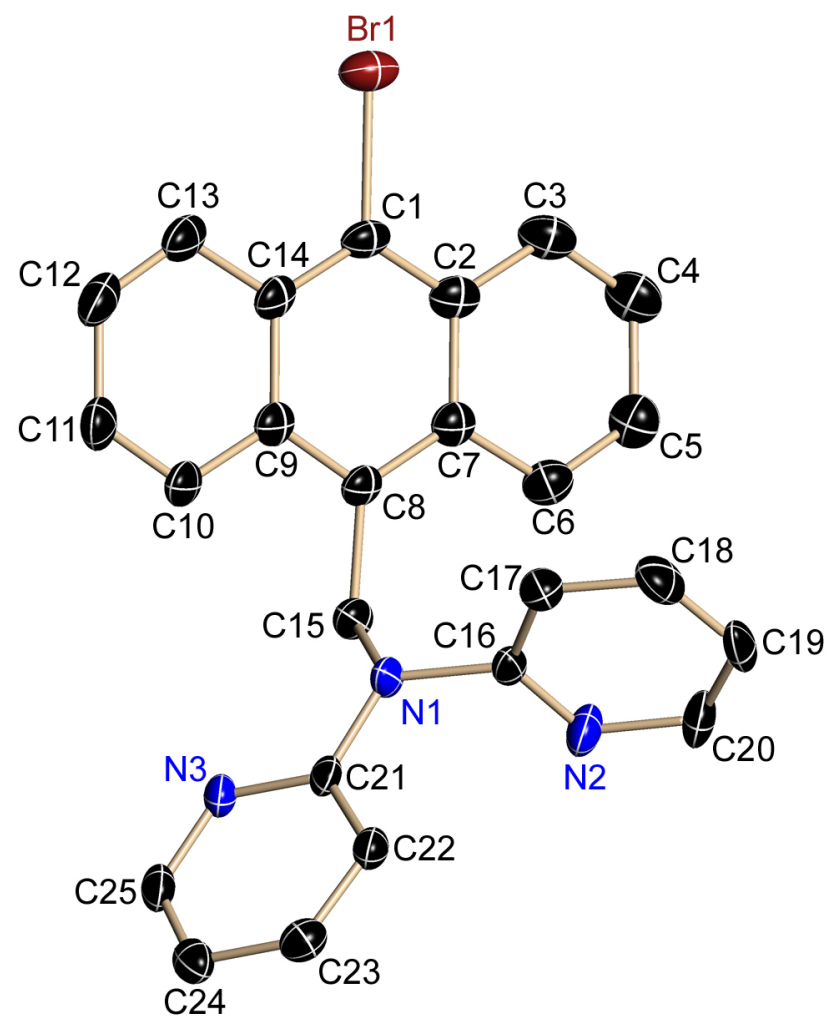

Abb. 5.36: Asymmetrische Einheit von 9-Brom-10-dipyridylaminanthracen (30); die anisotropen Auslenkungsparameter geben die 50\% ige Aufenthaltswahrscheinlichkeit an.

Tab. 5.28: Kristallographische Daten von 9-Brom-10-dipyridylaminanthracen (30).

\begin{tabular}{|ll|ll|}
\hline Strukturcode & $\mathrm{BrAnMeNPy}_{2}$ & $\mu\left[\mathrm{mm}^{-1}\right]$ & 2.186 \\
Summenformel & $\mathrm{C}_{25} \mathrm{H}_{18} \mathrm{BrN}_{3}$ & $\mathrm{~F}(000)$ & 448 \\
Molmasse $[\mathrm{g} / \mathrm{mol}]$ & 440.33 & $\theta$-Bereich [ $\left.{ }^{\circ}\right]$ & $2.73-25.03$ \\
Kristallgröße [mm] & $0.1 \times 0.1 \times 0.2$ & gesammelte Reflexe & 9730 \\
Raumgruppe & $P^{\overline{1}}$ & unabhängige Reflexe & 3341 \\
$a[\mathrm{pm}]$ & $921.6(4)$ & Zahl der Restraints & 0 \\
$b[\mathrm{pm}]$ & $965.1(4)$ & verfeinerte Parameter & 262 \\
$c[\mathrm{pm}]$ & $1249.8(5)$ & $R 1[\mathrm{I}>2 \sigma(\mathrm{I})]$ & 0.0355 \\
$\alpha\left[^{\circ}\right]$ & $76.222(6)$ & WR2 [alle Daten] & 0.0858 \\
$\beta\left[^{\circ}\right]$ & $70.969(6)$ & GooF & 1.068 \\
$\gamma\left[^{\circ}\right]$ & $65.231(6)$ & Differenzelektronendichte: & \\
$V\left[\mathrm{~nm}^{3}\right]$ & $0.9475(7)$ & Max. / min. [10 & \\
$Z$ & 2 & Max. / min. Transmission & $0.606 /-0.417$ \\
$\left.\mathrm{Messtemperatur}[\mathrm{K}]^{-3}\right]$ & $100(2)$ & & $0.7452 / 0.6355$ \\
$\rho$ Mgm $\left.^{-3}\right]$ & 1.543 & & \\
\hline
\end{tabular}




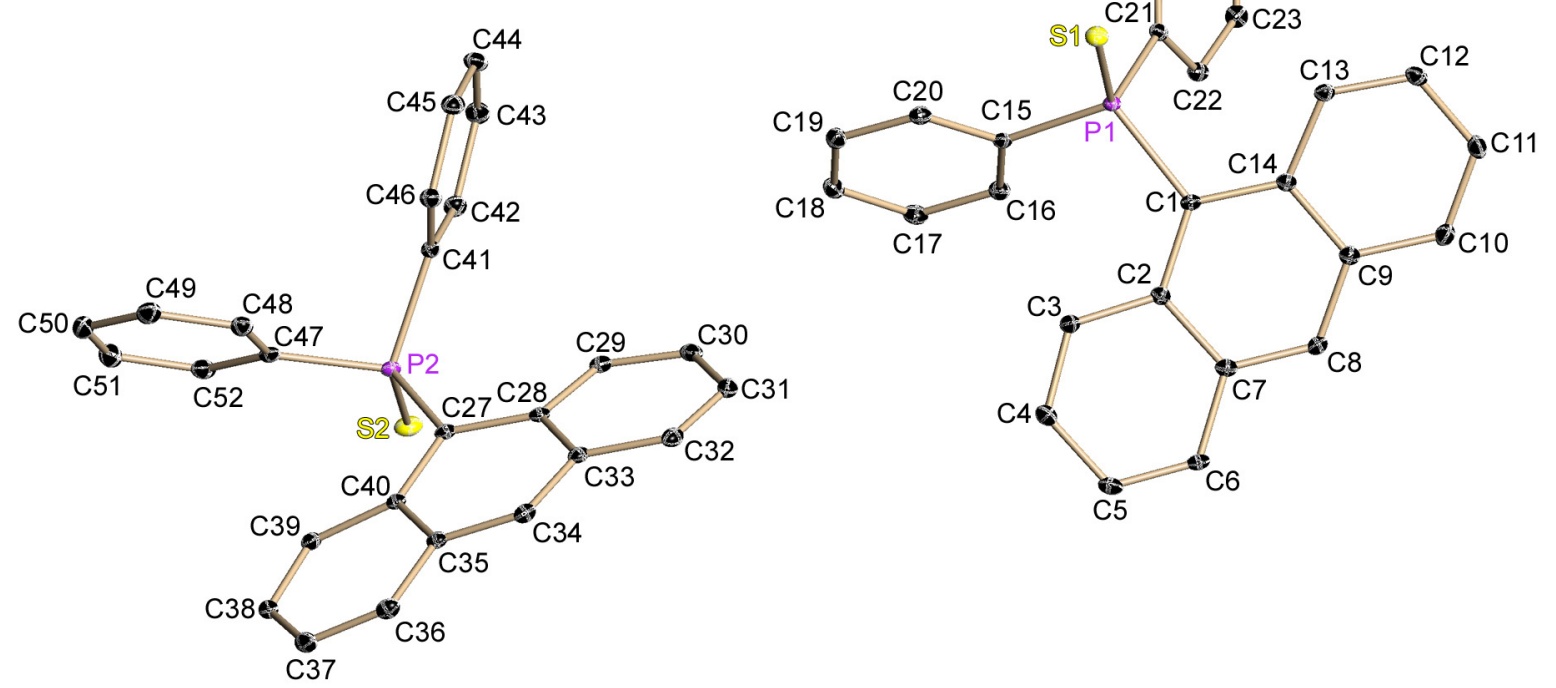

Abb. 5.37: Asymmetrische Einheit von 9-Diphenylthiophosphorylanthracen; die anisotropen Auslenkungsparameter geben die $50 \%$ ige Aufenthaltswahrscheinlichkeit an (He-Temp.).

Tab. 5.29: Kristallographische Daten von 9-Diphenylthiophosphorylanthracen.

\begin{tabular}{|c|c|c|c|}
\hline Strukturcode & SPAnH & $\mu\left[\mathrm{mm}^{-1}\right]$ & 0.256 \\
\hline Summenformel & $\mathrm{C}_{26} \mathrm{H}_{19} \mathrm{PS}$ & $F(000)$ & 824 \\
\hline Molmasse [g/mol] & 394.44 & $\theta$-Bereich $\left[{ }^{\circ}\right]$ & $1.21-31.94$ \\
\hline Kristallgröße [mm] & $0.1 \times 0.1 \times 0.05$ & gesammelte Reflexe & 79502 \\
\hline Raumgruppe & $P \overline{1}$ & unabhängige Reflexe & 12648 \\
\hline$a[\mathrm{pm}]$ & $1020.1(2)$ & Zahl der Restraints & 0 \\
\hline$b[\mathrm{pm}]$ & $1228.6(3)$ & verfeinerte Parameter & 505 \\
\hline$c[\mathrm{pm}]$ & 1733.1(4) & $R 1[\mid>2 \sigma(I)]$ & 0.0347 \\
\hline$\alpha\left[^{\circ}\right]$ & 101.676(3) & $w R 2$ [alle Daten] & 0.0895 \\
\hline$\beta\left[^{\circ}\right]$ & $91.300(4)$ & GooF & 1.035 \\
\hline$\gamma\left[^{\circ}\right]$ & $112.054(3)$ & & \\
\hline$V\left[\mathrm{~nm}^{3}\right]$ & $1.9599(8)$ & Differenzelektronendichte: & \\
\hline$Z$ & 4 & Max. / min. $\left[10^{-6} \mathrm{epm}^{-3}\right]$ & $0.570 /-0.294$ \\
\hline Messtemperatur [K] & $15(2)$ & Max. / min. Transmission & $0.7085 / 0.7464$ \\
\hline$\rho\left[\mathrm{Mgm}^{-3}\right]$ & 1.337 & & \\
\hline
\end{tabular}




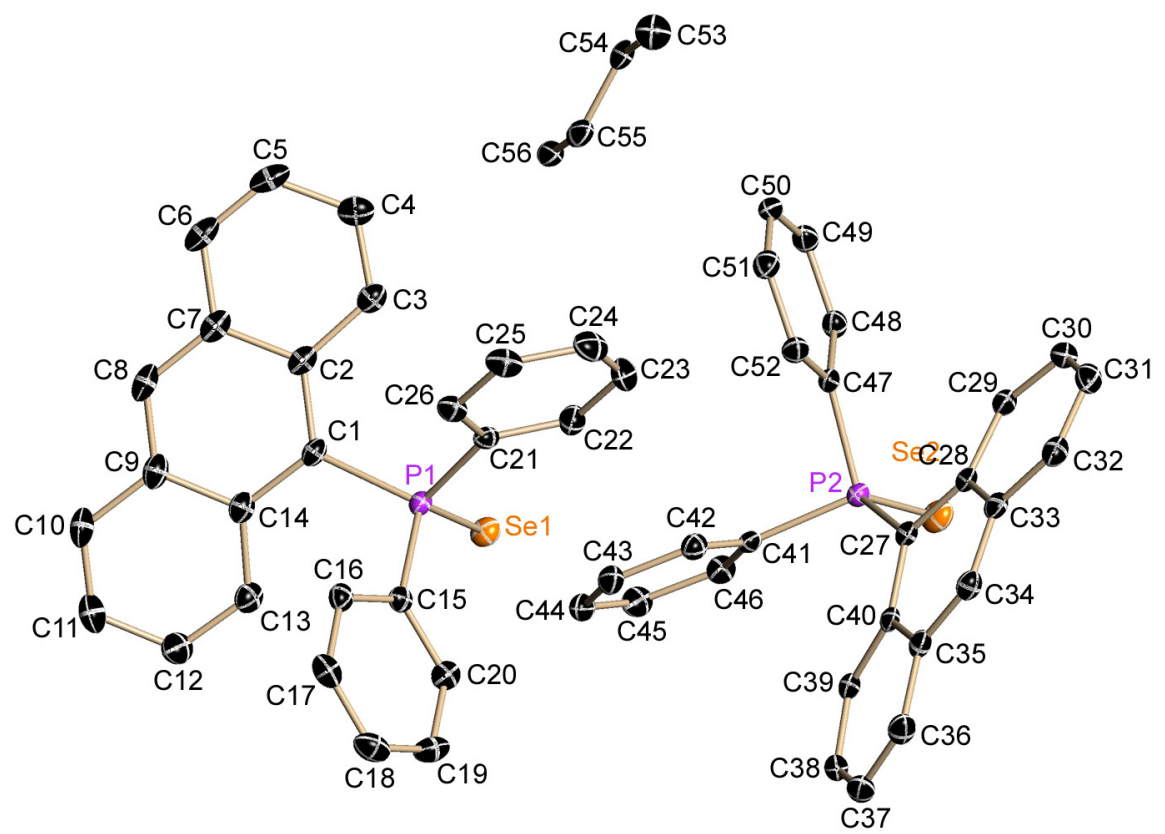

Abb. 5.38: Asymmetrische Einheit von 9-Diphenylselenophosphorylanthracen • $p$ Xylol; die anisotropen Auslenkungsparameter geben die 50\% ige Aufenthaltswahrscheinlichkeit an.

Tab. 5.30: Kristallographische Daten von 9-Diphenylthiophosphorylanthracen $• p$ Xylol.

\begin{tabular}{|c|c|c|c|}
\hline Strukturcode & SePAnH $\cdot p$ Xylol & $\mu\left[\mathrm{mm}^{-1}\right]$ & 1.827 \\
\hline Summenformel & $\mathrm{C}_{26} \mathrm{H}_{19} \mathrm{PSe}$ & $F(000)$ & 954 \\
\hline Molmasse $[\mathrm{g} / \mathrm{mol}]$ & 467.88 & $\theta$-Bereich $\left[{ }^{\circ}\right]$ & $2.13-27.12$ \\
\hline Kristallgröße [mm] & $0.2 \times 0.1 \times 0.1$ & gesammelte Reflexe & 43925 \\
\hline Raumgruppe & $P \overline{1}$ & unabhängige Reflexe & 9440 \\
\hline$a[\mathrm{pm}]$ & $1263.30(6)$ & Zahl der Restraints & 0 \\
\hline$b[\mathrm{pm}]$ & $1275.22(6)$ & verfeinerte Parameter & 542 \\
\hline$c[\mathrm{pm}]$ & $1633.08(8)$ & $R 1[\mid>2 \sigma(I)]$ & 0.0285 \\
\hline$\alpha\left[^{\circ}\right]$ & $92.2060(10)$ & $w R 2$ [alle Daten] & 0.0758 \\
\hline$\beta\left[^{\circ}\right]$ & $112.1950(10)$ & GooF & 1.048 \\
\hline$\gamma\left[^{\circ}\right]$ & $114.4410(10)$ & & \\
\hline$V\left[\mathrm{~nm}^{3}\right]$ & $2.15761(18)$ & Differenzelektronendichte: & \\
\hline$Z$ & 4 & Max. / min. $\left[10^{-6} \mathrm{epm}^{-3}\right]$ & $0.575 /-0.481$ \\
\hline Messtemperatur [K] & $100(2)$ & Max. / min. Transmission & $0.99 / 0.79$ \\
\hline$\rho\left[\mathrm{Mgm}^{-3}\right]$ & 1.440 & & \\
\hline
\end{tabular}




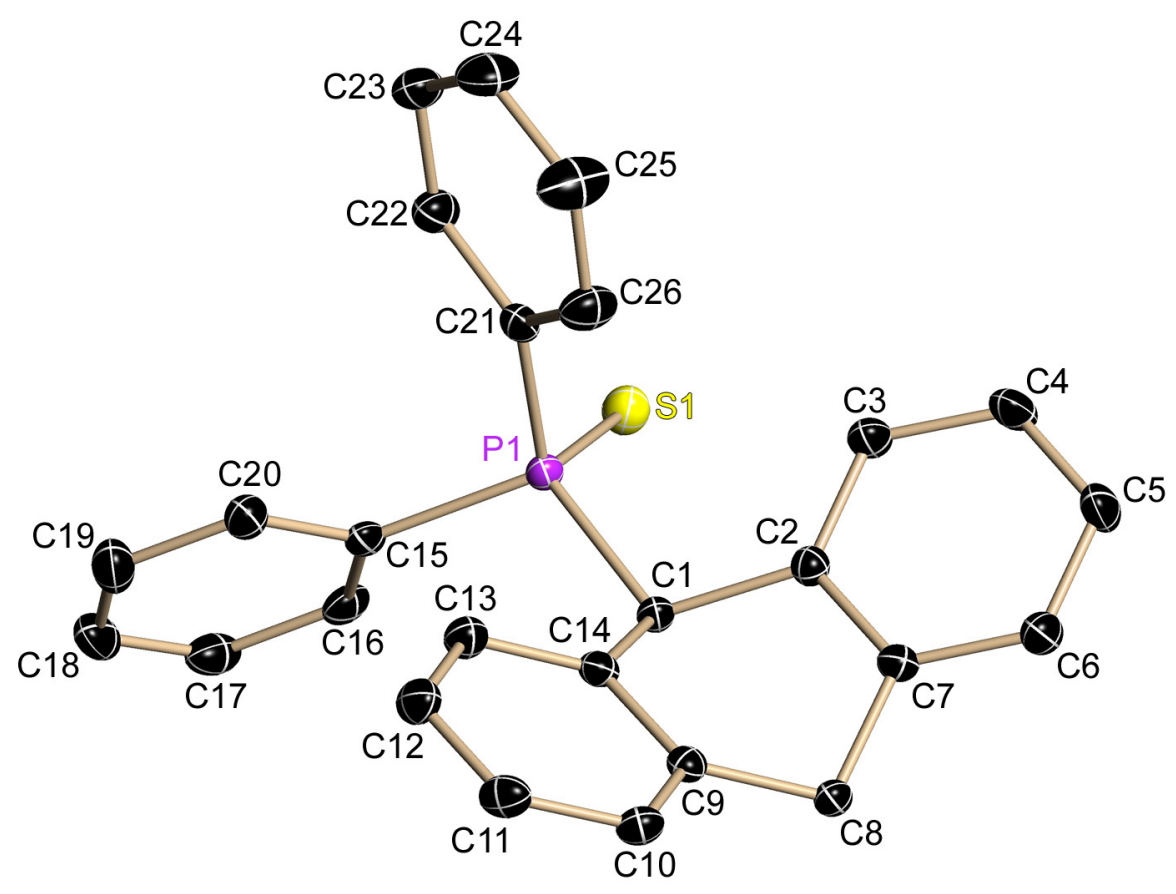

Abb. 5.39: Ausschnitt der asymmetrischen Einheit von 9,9'-Di(Diphenylthiophosphorylanthracen); die anisotropen Auslenkungsparameter geben die 50\% ige Aufenthaltswahrscheinlichkeit an.

Tab. 5.31: Kristallographische Daten von 9,9'-Di(Diphenylthiophosphorylanthracen).

\begin{tabular}{|ll|ll|}
\hline Strukturcode & {$\left[\mathrm{SPh}_{2} \mathrm{PAnH}_{2}\right.$} & $\mu\left[\mathrm{mm}^{-1}\right]$ & 0.192 \\
Summenformel & $\mathrm{C}_{80} \mathrm{H}_{65.1} \mathrm{P}_{2} \mathrm{~S}_{2}$ & $\mathrm{~F}(000)$ & 607 \\
Molmasse $[\mathrm{g} / \mathrm{mol}]$ & 1152.50 & $\theta$-Bereich $\left[^{\circ}\right]$ & $1.70-25.35$ \\
Kristallgröße $[\mathrm{mm}]$ & $0.18 \times 0.16 \times 0.10$ & gesammelte Reflexe & 25442 \\
Raumgruppe & $P \overline{1}$ & unabhängige Reflexe & 5419 \\
$a[\mathrm{pm}]$ & $983.91(11)$ & Zahl der Restraints & 347 \\
$b[\mathrm{pm}]$ & $1292.15(14)$ & verfeinerte Parameter & 522 \\
$c[\mathrm{pm}]$ & $1314.03(14)$ & $R 1[\mathrm{l}>2 \sigma(\mathrm{I})]$ & 0.0382 \\
$\alpha\left[^{\circ}\right]$ & $68.1880(10)$ & WR2 [alle Daten] & 0.1029 \\
$\beta\left[^{\circ}\right]$ & $72.9540(10)$ & GooF & 1.050 \\
$\gamma\left[^{\circ}\right]$ & $85.3760(10)$ & Differenzelektronendichte: & \\
$V\left[\mathrm{~nm}^{3}\right]$ & $1.4821(3)$ & Max. / min. [10 & \\
$Z$ & 1 & Max. / min. Transmission & $0.477 /-0.437$ \\
$\mathrm{Messtemperatur}^{\circ}[\mathrm{K}]$ & $100(2)$ & & $0.7454 / 0.7010$ \\
$\rho\left[\mathrm{Mgm}^{-3}\right]$ & 1.291 & & \\
\hline
\end{tabular}




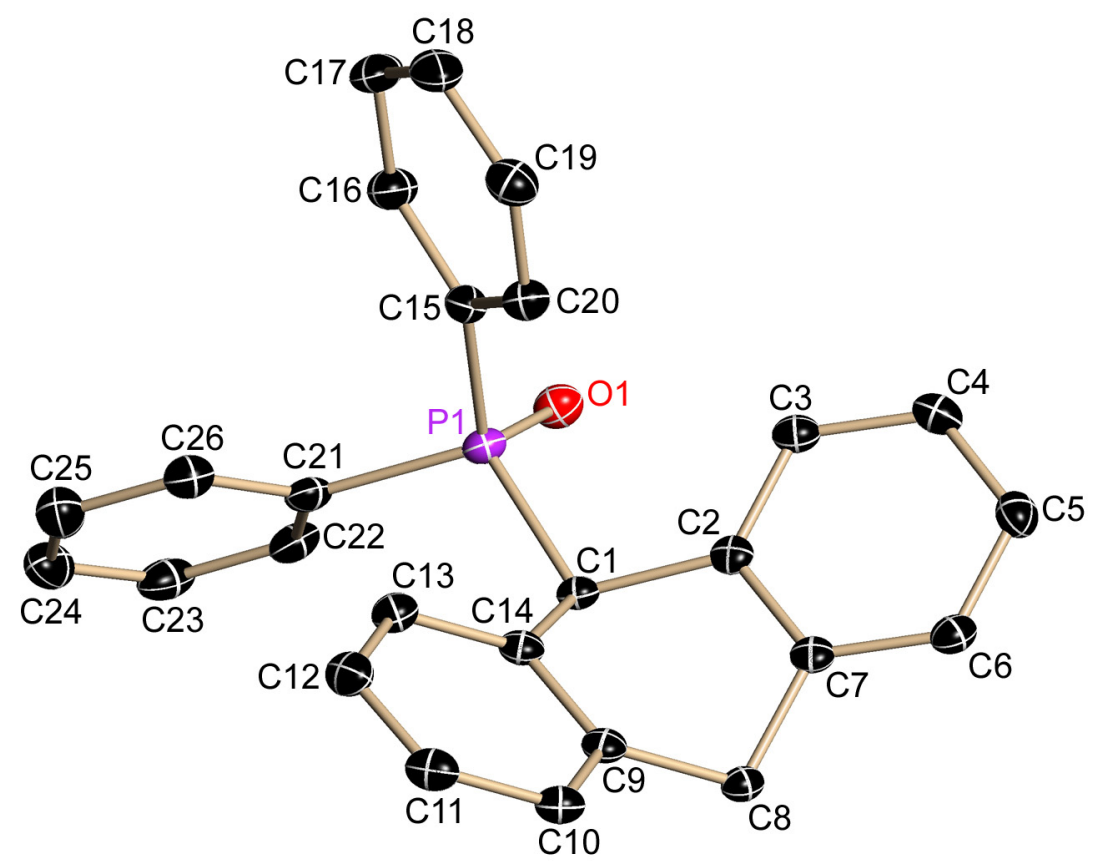

Abb. 5.40: Ausschnitt der asymmetrischen Einheit von 9,9'-Di(Diphenyloxophosphorylanthracen); die anisotropen Auslenkungsparameter geben die 50\% ige Aufenthaltswahrscheinlichkeit an.

Tab. 5.32: Kristallographische Daten von 9,9'-Di(Diphenyloxophosphorylanthracen).

\begin{tabular}{|c|c|c|c|}
\hline Strukturcode & {$\left[\mathrm{OPh}_{2} \mathrm{PAnH}\right]_{2}$} & $\mu\left[\mathrm{mm}^{-1}\right]$ & 0.129 \\
\hline Summenformel & $\mathrm{C}_{76} \mathrm{H}_{62} \mathrm{O}_{2} \mathrm{P}_{2}$ & $F(000)$ & 564 \\
\hline Molmasse $[\mathrm{g} / \mathrm{mol}]$ & 1069.20 & $\theta$-Bereich $\left[{ }^{\circ}\right]$ & $2.87-25.35$ \\
\hline Kristallgröße [mm] & $0.18 \times 0.12 \times 0.10$ & gesammelte Reflexe & 20497 \\
\hline Raumgruppe & $P \overline{1}$ & unabhängige Reflexe & 5123 \\
\hline$a[\mathrm{pm}]$ & $949.61(5)$ & Zahl der Restraints & 273 \\
\hline$b[\mathrm{pm}]$ & $1266.77(7)$ & verfeinerte Parameter & 401 \\
\hline$c[\mathrm{pm}]$ & $1283.95(7)$ & $R 1[\mid>2 \sigma(I)]$ & 0.0397 \\
\hline$\alpha\left[^{\circ}\right]$ & $110.4270(10)$ & $w R 2$ [alle Daten] & 0.1039 \\
\hline$\beta\left[^{\circ}\right]$ & $103.5390(10)$ & GooF & 1.051 \\
\hline$\gamma\left[^{\circ}\right]$ & $91.1340(10)$ & & \\
\hline$V\left[\mathrm{~nm}^{3}\right]$ & $1.39841(13)$ & Differenzelektronendichte: & \\
\hline$Z$ & 1 & Max. / min. $\left[10^{-6} \mathrm{epm}^{-3}\right]$ & $0.395 /-0.380$ \\
\hline Messtemperatur [K] & $100(2)$ & Max. / min. Transmission & $0.9843 / 0.9243$ \\
\hline$\rho\left[\mathrm{Mgm}^{-3}\right]$ & 1.270 & & \\
\hline
\end{tabular}




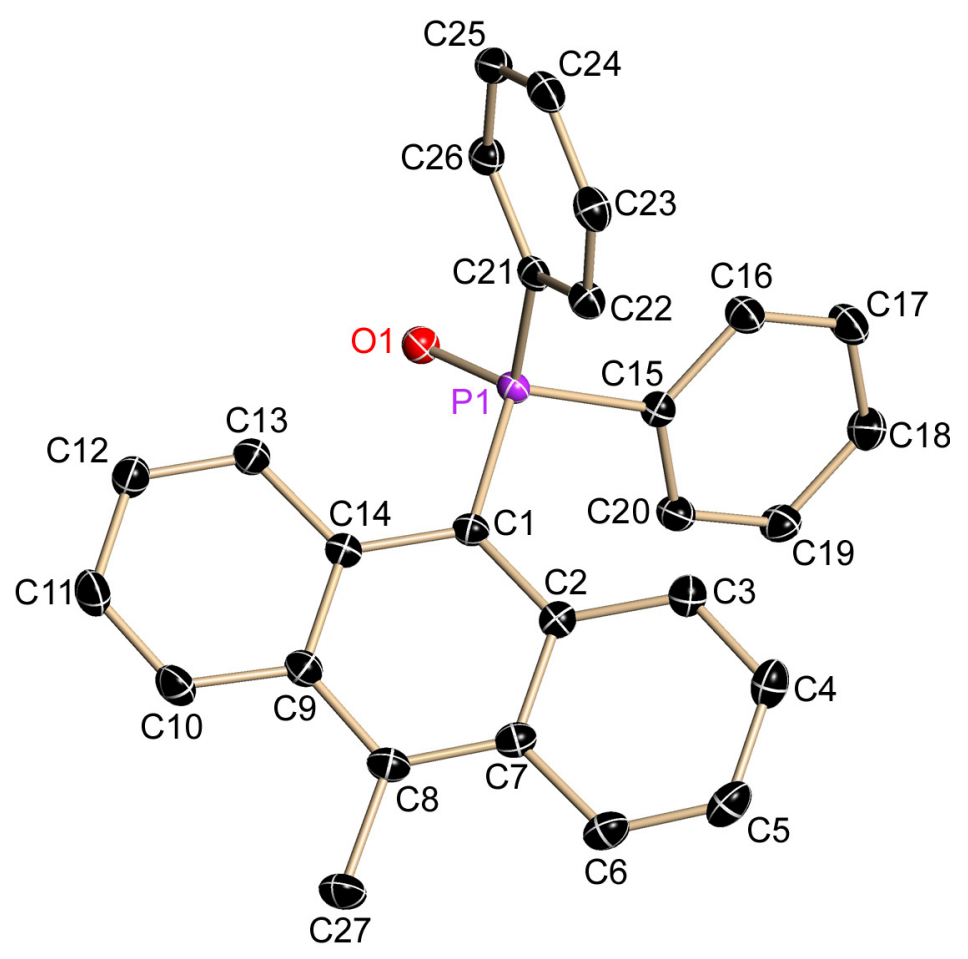

Abb. 5.41: Asymmetrische Einheit von 9-Diphenyloxophosphoryl-10-methylanthracen (33); die anisotropen Auslenkungsparameter geben die 50\% ige Aufenthaltswahrscheinlichkeit an.

Tab. 5.33: Kristallographische Daten von 9-Diphenyloxophosphoryl-10-methylanthracen (33).

\begin{tabular}{|c|c|c|c|}
\hline Strukturcode & OPAnMe & $\mu\left[\mathrm{mm}^{-1}\right]$ & 0.156 \\
\hline Summenformel & $\mathrm{C}_{27} \mathrm{H}_{21} \mathrm{OP}$ & $F(000)$ & 1648 \\
\hline Molmasse $[\mathrm{g} / \mathrm{mol}]$ & 392.41 & $\theta$-Bereich $\left[^{\circ}\right]$ & $1.99-28.29$ \\
\hline Kristallgröße [mm] & $0.4 \times 0.3 \times 0.2$ & gesammelte Reflexe & 43916 \\
\hline Raumgruppe & $C 2 / c$ & unabhängige Reflexe & 4707 \\
\hline$a[\mathrm{pm}]$ & 2962.12(14) & Zahl der Restraints & 0 \\
\hline$b[\mathrm{pm}]$ & $831.20(4)$ & verfeinerte Parameter & 263 \\
\hline$c[\mathrm{pm}]$ & $20.5406(10)$ & $R 1[\mid>2 \sigma(I)]$ & 0.0347 \\
\hline$\alpha\left[^{\circ}\right]$ & 90 & $w R 2$ [alle Daten] & 0.0964 \\
\hline$\beta\left[^{\circ}\right]$ & $129.1240(10)$ & GooF & 1.040 \\
\hline$\gamma\left[^{\circ}\right]$ & 90 & & \\
\hline$V\left[\mathrm{~nm}^{3}\right]$ & $3.9234(3)$ & Differenzelektronendichte: & \\
\hline$z$ & 8 & Max. / min. $\left[10^{-6} \mathrm{epm}^{-3}\right]$ & $0.0451 /-0.331$ \\
\hline Messtemperatur [K] & $100(2)$ & Max. / min. Transmission & \\
\hline$\rho\left[\mathrm{Mgm}^{-3}\right]$ & 1.329 & & \\
\hline
\end{tabular}




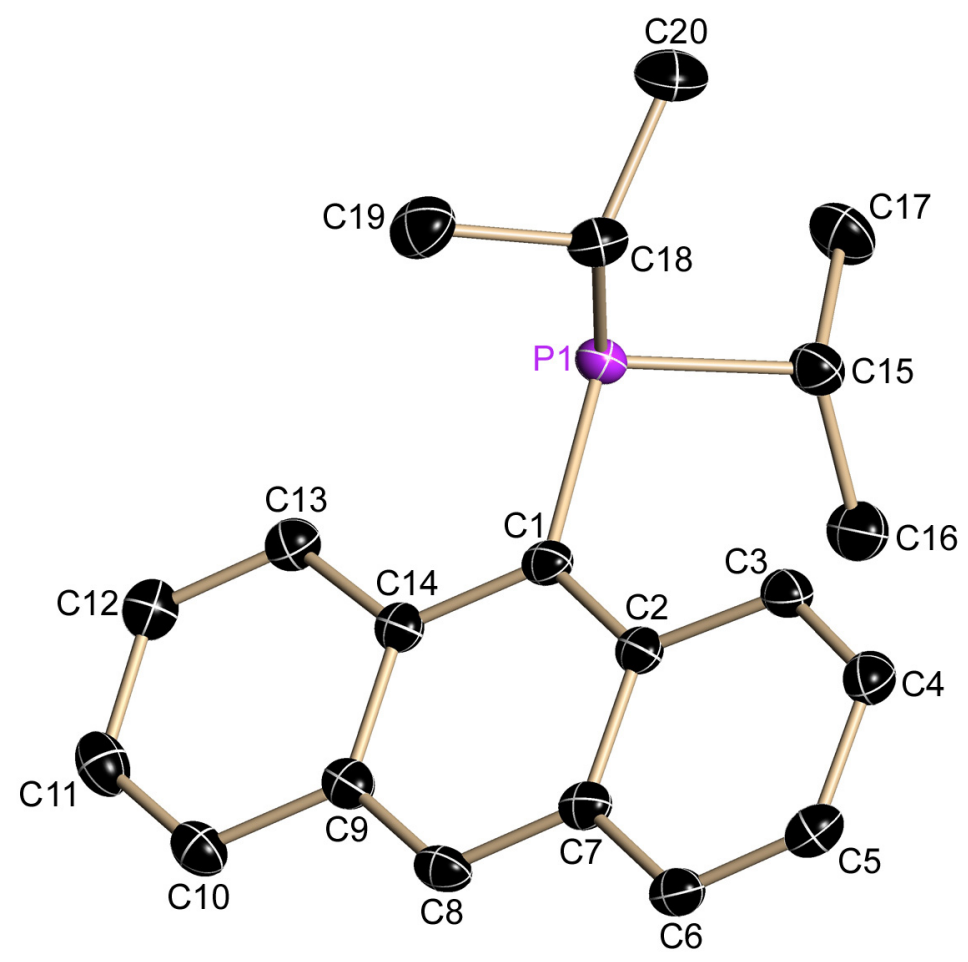

Abb. 5.42: Asymmetrische Einheit von 9-Diisopropylphospanylanthracen (34); die anisotropen Auslenkungsparameter geben die 50\% ige Aufenthaltswahrscheinlichkeit an.

Tab. 5.34: Kristallographische Daten von 9-Diisopropylphospanylanthracen (34).

\begin{tabular}{|ll|ll|}
\hline Strukturcode & $i \mathrm{Pr}_{2} \mathrm{PAnH}$ & $\mu\left[\mathrm{mm}^{-1}\right]$ & 0.284 \\
Summenformel & $\mathrm{C}_{20} \mathrm{H}_{23} \mathrm{PS}$ & $\mathrm{F}(000)$ & 1392 \\
Molmasse $[\mathrm{g} / \mathrm{mol}]$ & 326.41 & $\theta$-Bereich $\left.{ }^{\circ}\right]$ & $2.69-28.33$ \\
Kristallgröße $[\mathrm{mm}]$ & $0.22 \times 0.2 \times 0.1$ & gesammelte Reflexe & 66171 \\
Raumgruppe & $\mathrm{Pbca}$ & unabhängige Reflexe & 4590 \\
$a[\mathrm{pm}]$ & $1091.56(4)$ & Zahl der Restraints & 0 \\
$b[\mathrm{pm}]$ & $1454.13(6)$ & verfeinerte Parameter & 203 \\
$c[\mathrm{pm}]$ & $2106.27(8)$ & $R 1[\mathrm{I}>2 \sigma(\mathrm{I})]$ & 0.0315 \\
$\alpha\left[^{\circ}\right]$ & 90 & WR2 [alle Daten] & 0.0842 \\
$\beta\left[^{\circ}\right]$ & 90 & GooF & 1.059 \\
$\gamma\left[^{\circ}\right]$ & 90 & Differenzelektronendichte: & \\
$V\left[\mathrm{~nm}^{3}\right]$ & $3.3432(2)$ & Max. / min. $\left[10^{-6}\right.$ epm & \\
$Z$ & 8 & Max. / min. Transmission & $0.376 /-0.280$ \\
$\mathrm{Messtemperatur}[\mathrm{K}]^{-3}$ & $100(2)$ & & $0.7457 / 0.7023$ \\
$\rho$ Mgm $\left.^{-3}\right]$ & 1.297 & & \\
\hline
\end{tabular}




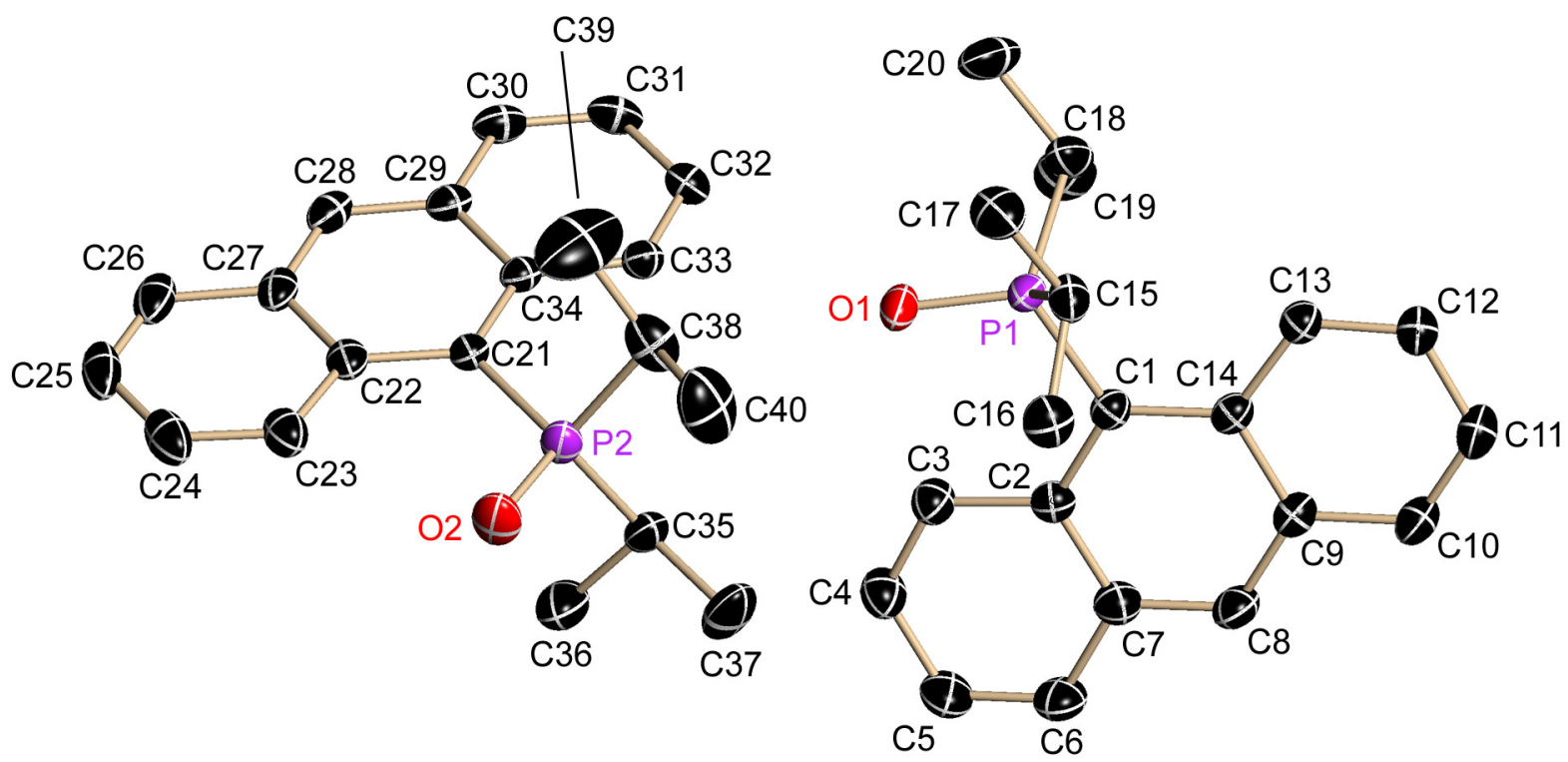

Abb. 5.43: Asymmetrische Einheit von 9-Diisopropyloxophosphorylanthracen (35); die anisotropen Auslenkungsparameter geben die $50 \%$ ige Aufenthaltswahrscheinlichkeit an.

Tab. 5.35: Kristallographische Daten von 9-Diisopropyloxophosphorylanthracen (35).

\begin{tabular}{|c|c|c|c|}
\hline Strukturcode & $\mathrm{OiPr}_{2} \mathrm{PAnH}$ & $\mu\left[\mathrm{mm}^{-1}\right]$ & 0.161 \\
\hline Summenformel & $\mathrm{C}_{20} \mathrm{H}_{23} \mathrm{OP}$ & $F(000)$ & 1328 \\
\hline Molmasse [g/mol] & 310.35 & $\theta$-Bereich $\left[{ }^{\circ}\right]$ & $1.42-25.39$ \\
\hline Kristallgröße [mm] & $0.18 \times 0.15 \times 0.15$ & gesammelte Reflexe & 52184 \\
\hline Raumgruppe & $P 2_{1} / c$ & unabhängige Reflexe & 6247 \\
\hline$a[\mathrm{pm}]$ & $1548.44(15)$ & Zahl der Restraints & 0 \\
\hline$b[\mathrm{pm}]$ & 1429.71(14) & verfeinerte Parameter & 405 \\
\hline$c[\mathrm{pm}]$ & $1660.88(16)$ & $R 1[\mid>2 \sigma(I)]$ & 0.0518 \\
\hline$\alpha\left[^{\circ}\right]$ & 90 & $w R 2$ [alle Daten] & 0.1461 \\
\hline$\beta\left[^{\circ}\right]$ & $112.2170(10)$ & GooF & 1.090 \\
\hline$\gamma\left[^{\circ}\right]$ & 90 & & \\
\hline$V\left[\mathrm{~nm}^{3}\right]$ & 3.4039 & Differenzelektronendichte: & \\
\hline$Z$ & 8 & Max. / min. $\left[10^{-6} \mathrm{epm}^{-3}\right]$ & $0.464 /-0.447$ \\
\hline Messtemperatur [K] & $100(2)$ & Max. / min. Transmission & 0.3279 / 0.3796 \\
\hline$\rho\left[\mathrm{Mgm}^{-3}\right]$ & 1.211 & & \\
\hline
\end{tabular}




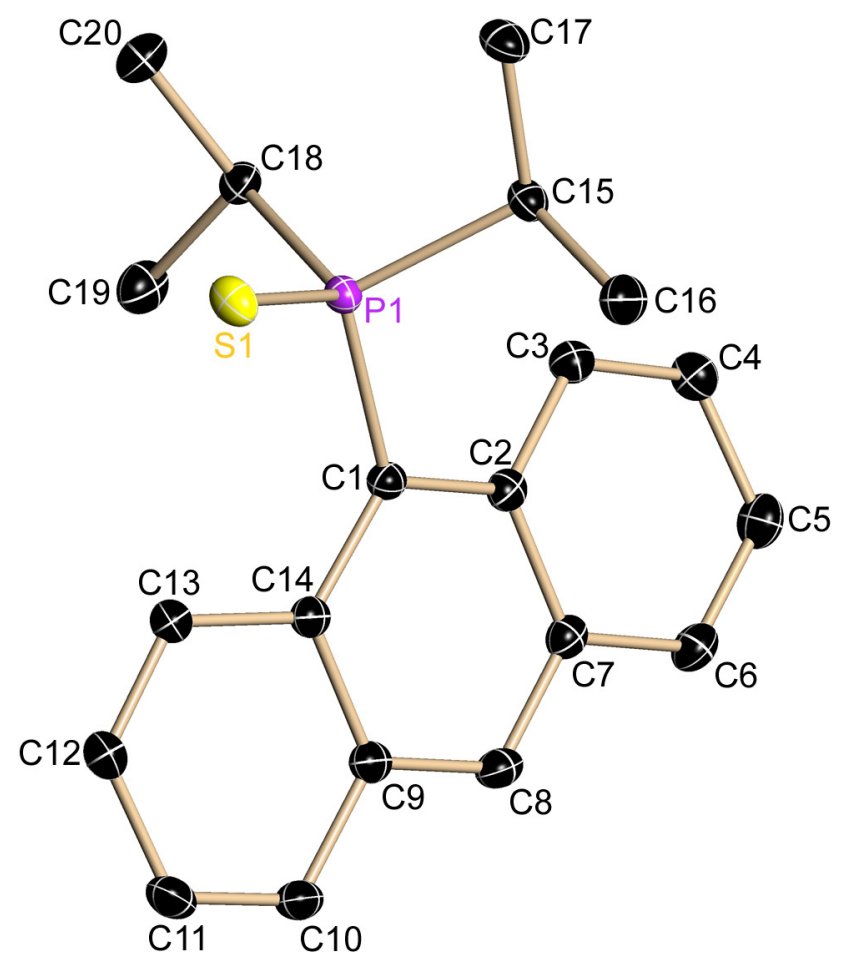

Abb. 5.44: Asymmetrische Einheit von 9-Diisopropylthiophosphorylanthracen (36); die anisotropen Auslenkungsparameter geben die 50\% ige Aufenthaltswahrscheinlichkeit an.

Tab. 5.36: Kristallographische Daten von 9-Diisopropylthiophosphorylanthracen (36).

\begin{tabular}{|c|c|c|c|}
\hline Strukturcode & $\mathrm{SiPr}_{2} \mathrm{PAnH}$ & $\mu\left[\mathrm{mm}^{-1}\right]$ & 0.284 \\
\hline Summenformel & $\mathrm{C}_{20} \mathrm{H}_{23} \mathrm{PS}$ & $F(000)$ & 1392 \\
\hline Molmasse $[\mathrm{g} / \mathrm{mol}]$ & 326.41 & $\theta$-Bereich $\left[{ }^{\circ}\right]$ & $2.69-28.33$ \\
\hline Kristallgröße [mm] & $0.33 \times 0.20 \times 0.20$ & gesammelte Reflexe & 66171 \\
\hline Raumgruppe & Pbca & unabhängige Reflexe & 4590 \\
\hline$a[\mathrm{pm}]$ & $1091.56(4)$ & Zahl der Restraints & 0 \\
\hline$b[\mathrm{pm}]$ & 1454.13(6) & verfeinerte Parameter & 203 \\
\hline$c[\mathrm{pm}]$ & $2106.27(8)$ & $R 1[I>2 \sigma(I)]$ & 0.315 \\
\hline$\alpha\left[^{\circ}\right]$ & 90 & $w R 2$ [alle Daten] & 0.0842 \\
\hline$\beta\left[^{\circ}\right]$ & 90 & GooF & 1.059 \\
\hline$\gamma\left[^{\circ}\right]$ & 90 & & \\
\hline$V\left[\mathrm{~nm}^{3}\right]$ & $3.3432(2)$ & Differenzelektronendichte: & \\
\hline Z & 8 & Max. / min. $\left[10^{-6} \mathrm{epm}^{-3}\right]$ & $0.376 /-0.280$ \\
\hline Messtemperatur [K] & $100(2)$ & Max. / min. Transmission & $0.7457 / 0.7023$ \\
\hline$\rho\left[\mathrm{Mgm}^{-3}\right]$ & 1.297 & & \\
\hline
\end{tabular}




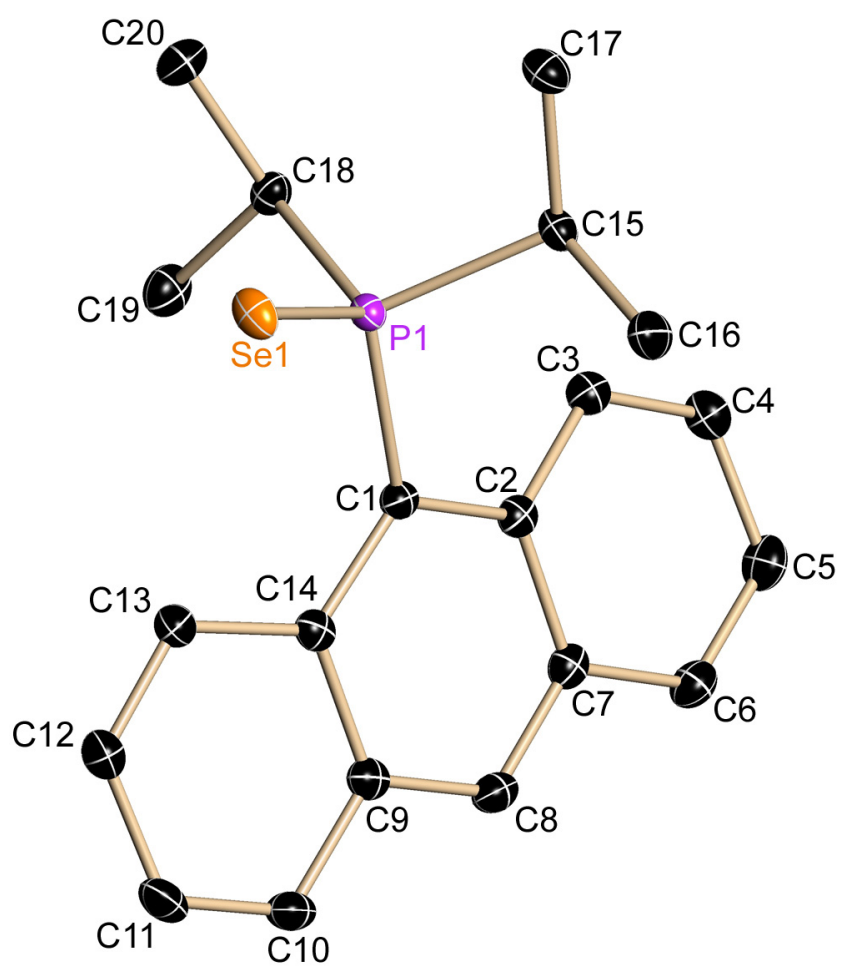

Abb. 5.45: Asymmetrische Einheit von 9-Diisopropylselenophosphorylanthracen (37); die anisotropen Auslenkungsparameter geben die 50\% ige Aufenthaltswahrscheinlichkeit an.

Tab. 5.37: Kristallographische Daten von 9-Diisopropylselenophosphorylanthracen (37).

\begin{tabular}{|c|c|c|c|}
\hline Strukturcode & $\mathrm{SeiPr}_{2} \mathrm{PAnH}$ & $\mu\left[\mathrm{mm}^{-1}\right]$ & 2.291 \\
\hline Summenformel & $\mathrm{C}_{20} \mathrm{H}_{23} \mathrm{PSe}$ & $F(000)$ & 1536 \\
\hline Molmasse $[\mathrm{g} / \mathrm{mol}]$ & 373.31 & $\theta$-Bereich $\left[{ }^{\circ}\right]$ & $2.66 / 26.37$ \\
\hline Kristallgröße [mm] & $0.3 \times 0.27 \times 0.1$ & gesammelte Reflexe & 55146 \\
\hline Raumgruppe & Pbca & unabhängige Reflexe & 3921 \\
\hline$a[\mathrm{pm}]$ & $1102.74(15)$ & Zahl der Restraints & 0 \\
\hline$b[\mathrm{pm}]$ & $1457.7(2)$ & verfeinerte Parameter & 203 \\
\hline$c[\mathrm{pm}]$ & 2121.3(3) & $R 1[I>2 \sigma(I)]$ & 0.0213 \\
\hline$\alpha\left[^{\circ}\right]$ & 90 & $w R 2$ [alle Daten] & 0.0594 \\
\hline$\beta\left[^{\circ}\right]$ & 90 & GooF & 1.085 \\
\hline$\gamma\left[^{\circ}\right]$ & 90 & & \\
\hline$V\left[\mathrm{~nm}^{3}\right]$ & $3.4100(8)$ & Differenzelektronendichte: & \\
\hline$Z$ & 8 & Max. / min. $\left[10^{-6} \mathrm{epm}^{-3}\right]$ & $0.514 /-0.266$ \\
\hline Messtemperatur [K] & $100(2)$ & Max. / min. Transmission & $0.7454 / 0.5472$ \\
\hline$\rho\left[\mathrm{Mgm}^{-3}\right]$ & 1.454 & & \\
\hline
\end{tabular}




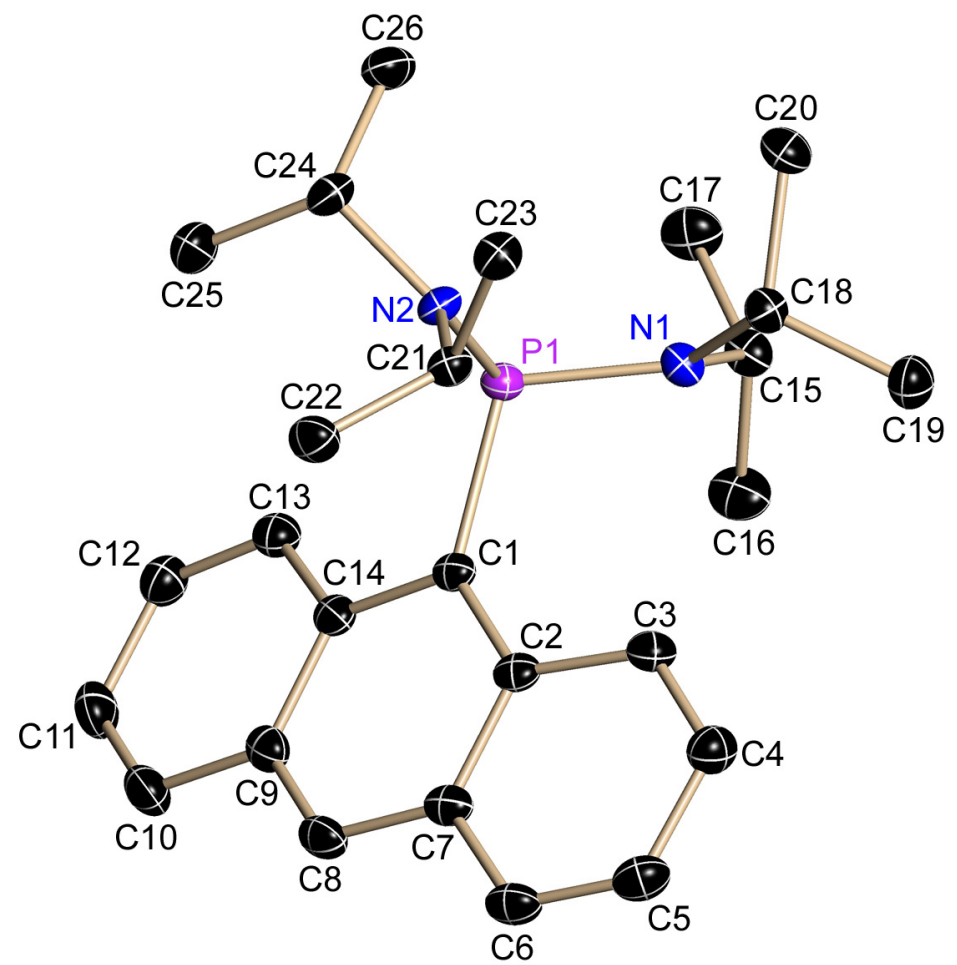

Abb. 5.46: Asymmetrische Einheit von 9-Bis(diisopropylamino)phosphanylanthracen (38); die anisotropen Auslenkungsparameter geben die 50\% ige Aufenthaltswahrscheinlichkeit an.

Tab. 5.38: Kristallographische Daten von 9-Bis(diisopropylamino)phosphanylanthracen (38).

\begin{tabular}{|c|c|}
\hline Strukturcode & $\left(i \mathrm{Pr}_{2} \mathrm{~N}\right)_{2} \mathrm{PAnH}$ \\
\hline Summenformel & $\mathrm{C}_{26} \mathrm{H}_{37} \mathrm{~N}_{2} \mathrm{P}$ \\
\hline Molmasse [g/mol] & 408.55 \\
\hline Kristallgröße [mm] & $0.3 \times 0.3 \times 0.2$ \\
\hline Raumgruppe & $P \overline{1}$ \\
\hline$a[\mathrm{pm}]$ & $810.53(5)$ \\
\hline$b[\mathrm{pm}]$ & $992.27(6)$ \\
\hline$c[\mathrm{pm}]$ & $1550.09(10)$ \\
\hline$\alpha\left[^{\circ}\right]$ & $76.8700(10)$ \\
\hline$\beta\left[^{\circ}\right]$ & $81.6130(10)$ \\
\hline$\gamma\left[^{\circ}\right]$ & $75.8010(10)$ \\
\hline$V\left[\mathrm{~nm}^{3}\right]$ & $1.17162(13)$ \\
\hline$Z$ & 2 \\
\hline Messtemperatur [K] & $100(2)$ \\
\hline$\rho\left[\mathrm{Mgm}^{-3}\right]$ & 1.158 \\
\hline
\end{tabular}

$\begin{array}{ll}\mu\left[\mathrm{mm}^{-1}\right] & 0.132 \\ \mathrm{~F}(000) & 444 \\ \left.\theta \text {-Bereich [ }{ }^{\circ}\right] & 1.36-26.38 \\ \text { gesammelte Reflexe } & 12368 \\ \text { unabhängige Reflexe } & 4746 \\ \text { Zahl der Restraints } & 0 \\ \text { verfeinerte Parameter } & 270 \\ R 1[\text { [l>2 } \sigma(\mathrm{I})] & 0.0377 \\ w R 2 \text { [alle Daten] } & 0.0982 \\ \text { GooF } & 1.078 \\ & \\ \begin{array}{l}\text { Differenzelektronendichte: } \\ \text { Max. / min. [10 }\end{array} & \\ \text { Max. / min. Transmission } & \\ & 0.273 /-0.281 \\ & 0.7454 / 0.6997\end{array}$




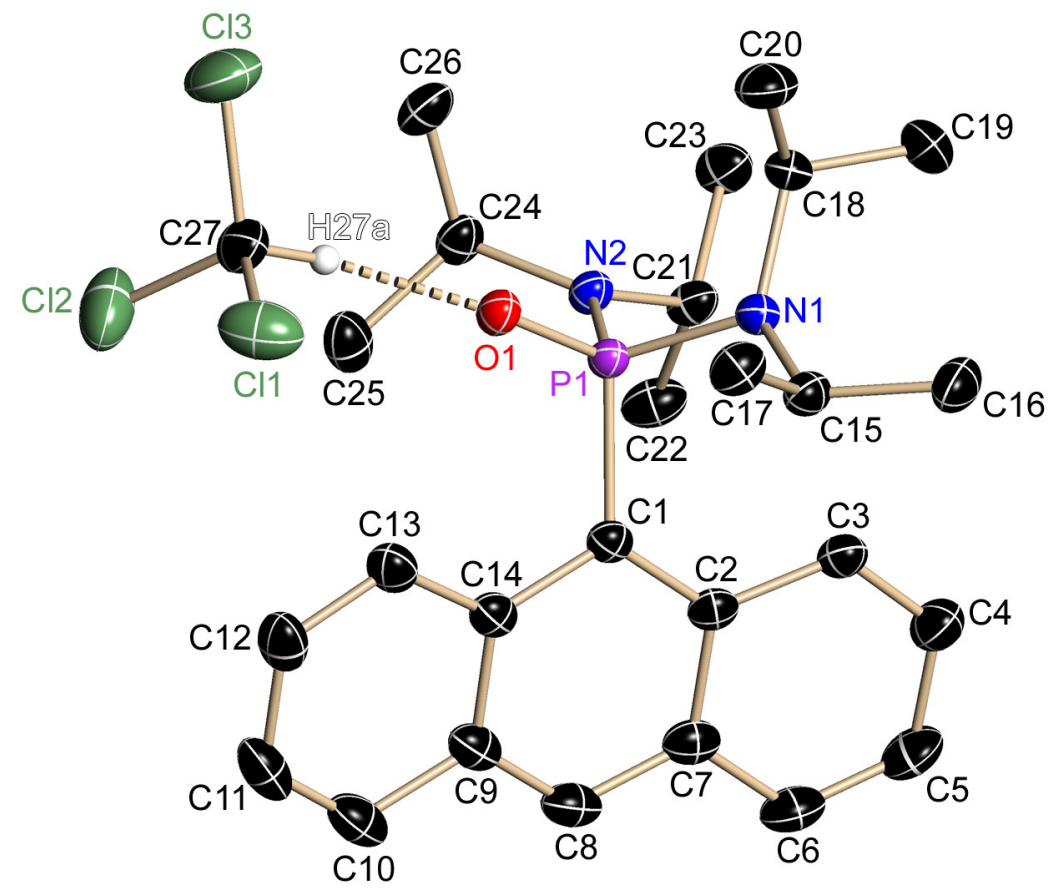

Abb. 5.47: Asymmetrische Einheit von 9-Bis(diisopropylamino)oxophosphorylanthracen (39); die anisotropen Auslenkungsparameter geben die 50\% ige Aufenthaltswahrscheinlichkeit an.

Tab. 5.39: Kristallographische Daten von 9-Bis(diisopropylamino)oxophosphorylanthracen (39).

\begin{tabular}{|c|c|c|c|}
\hline Strukturcode & $\mathrm{O}\left(\mathrm{Prr}_{2} \mathrm{~N}\right)_{2} \mathrm{PAnH}$ & $\mu\left[\mathrm{mm}^{-1}\right]$ & 0.400 \\
\hline Summenformel & $\mathrm{C}_{27} \mathrm{H}_{38} \mathrm{Cl}_{3} \mathrm{~N}_{2} \mathrm{OP}$ & $F(000)$ & 1152 \\
\hline Molmasse $[\mathrm{g} / \mathrm{mol}]$ & 543.91 & $\theta$-Bereich $\left[{ }^{\circ}\right]$ & $1.23-25.38$ \\
\hline Kristallgröße [mm] & $0.1 \times 0.1 \times 0.2$ & gesammelte Reflexe & 45920 \\
\hline Raumgruppe & $P 2_{1} / n$ & unabhängige Reflexe & 5357 \\
\hline$a[\mathrm{pm}]$ & $817.92(6)$ & Zahl der Restraints & 0 \\
\hline$b[\mathrm{pm}]$ & $3321.9(3)$ & verfeinerte Parameter & 315 \\
\hline$c[\mathrm{pm}]$ & $1123.13(10)$ & $R 1[\mid>2 \sigma(I)]$ & 0.0420 \\
\hline$\alpha\left[^{\circ}\right]$ & 90 & $w R 2$ [alle Daten] & 0.0956 \\
\hline$\beta\left[^{\circ}\right]$ & $110.9720(10)$ & GooF & 1.127 \\
\hline$\gamma\left[^{\circ}\right]$ & 90 & & \\
\hline$V\left[\mathrm{~nm}^{3}\right]$ & $2.8494(4)$ & Differenzelektronendichte: & \\
\hline$Z$ & 4 & Max. / min. $\left[10^{-6} \mathrm{epm}^{-3}\right]$ & 0.724 / -0.419 \\
\hline Messtemperatur $[\mathrm{K}]$ & $100(2)$ & Max. / min. Transmission & $0.7452 / 0.6492$ \\
\hline$\rho\left[\mathrm{Mgm}^{-3}\right]$ & 1.268 & & \\
\hline
\end{tabular}




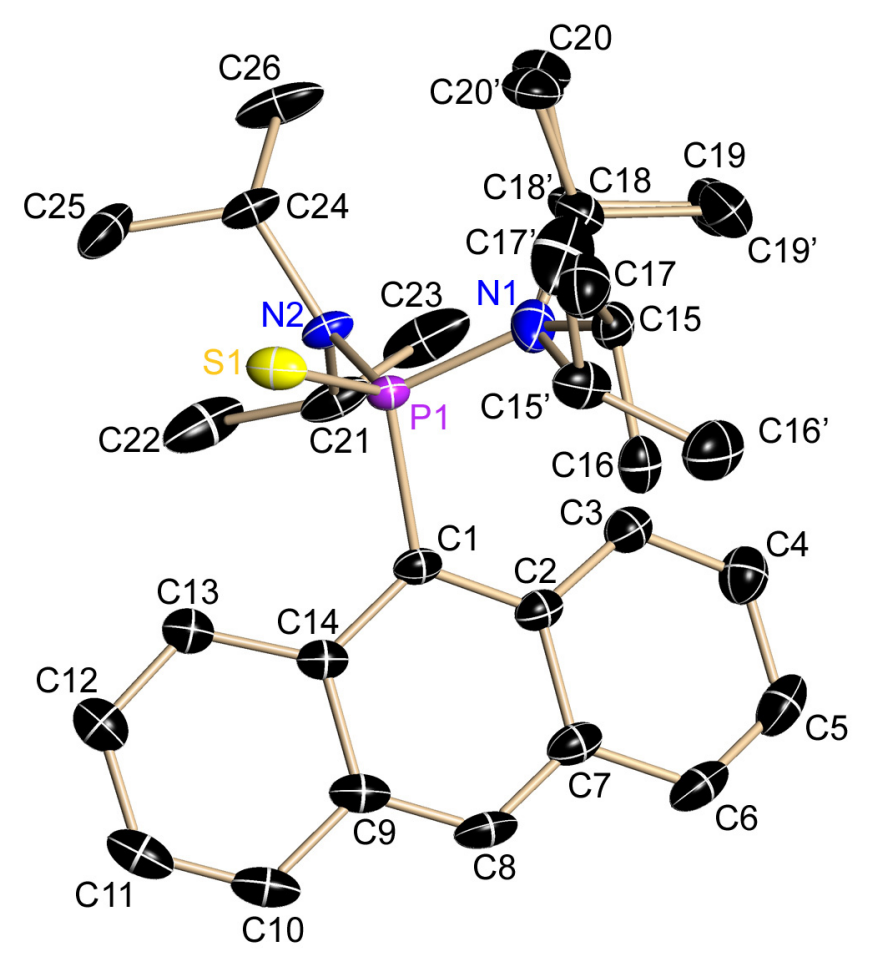

Abb. 5.48: Asymmetrische Einheit von 9-Bis(diisopropylamino)thiophosphorylanthracen (40); die anisotropen Auslenkungsparameter geben die 50\% ige Aufenthaltswahrscheinlichkeit an.

Beide Isopropylgruppen an N1 sind fehlgeordnet, die Besetzung der Hauptdomäne verfeinerte zu $56 \%$.

Tab. 5.40: Kristallographische Daten von 9-Bis(diisopropylamino)thiophosphorylanthracen (40).

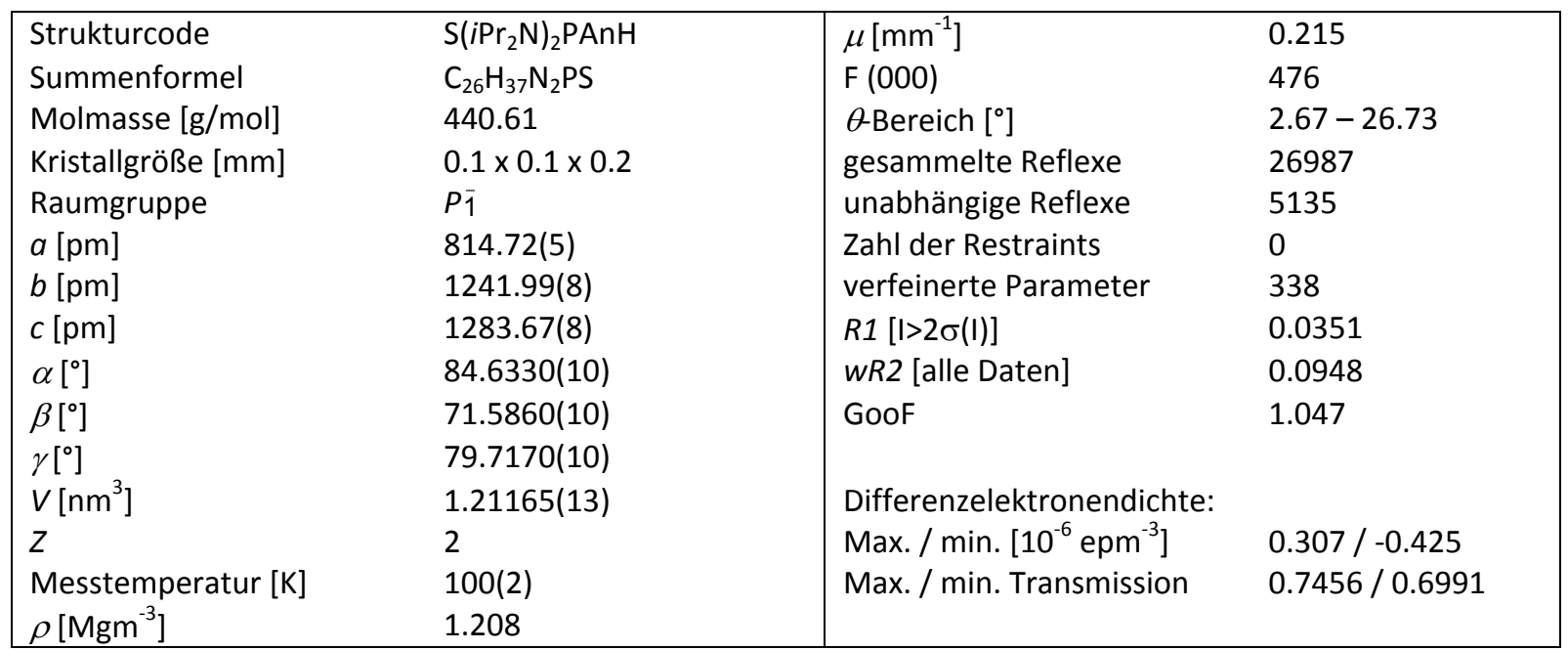




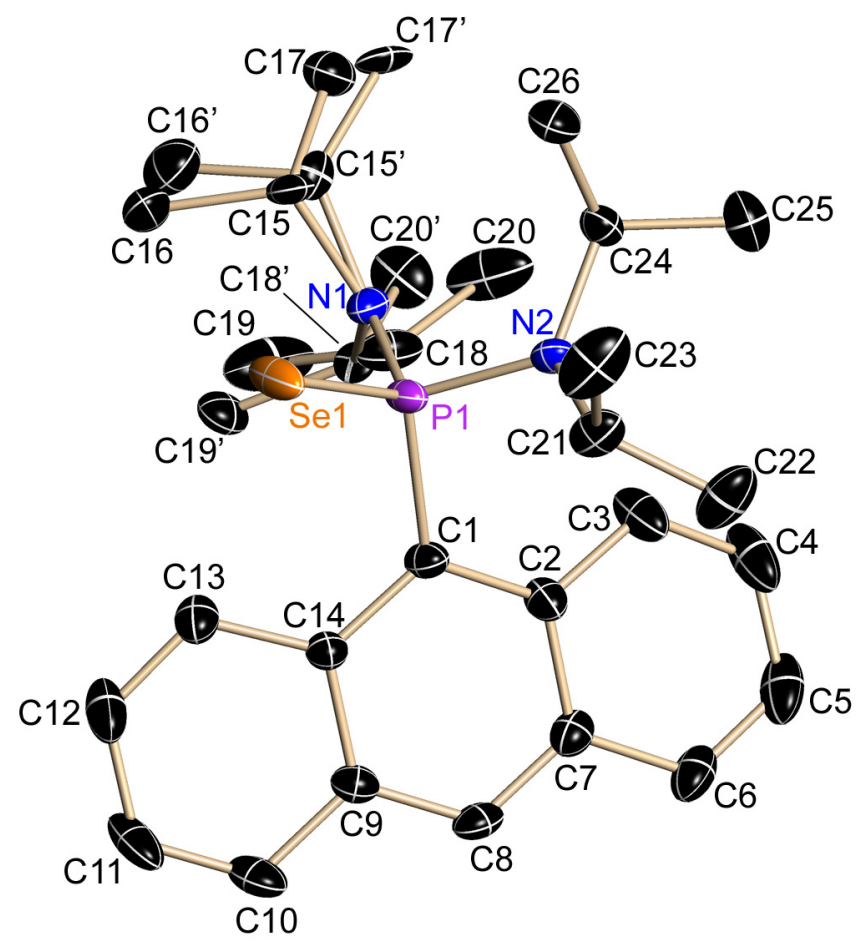

Abb. 5.49: Asymmetrische Einheit von 9-Bis(diisopropylamino)selenophosphorylanthracen (41); die anisotropen Auslenkungsparameter geben die 50\% ige Aufenthaltswahrscheinlichkeit an.

Beide Isopropylgruppen an N1 sind fehlgeordnet, die Besetzung der Hauptdomäne verfeinerte zu $64 \%$.

Tab. 5.41: Kristallographische Daten von 9-Bis(diisopropylamino)selenophosphorylanthracen (41).

\begin{tabular}{|c|c|c|c|}
\hline Strukturcode & $\mathrm{Se}\left(i \mathrm{Pr}_{2} \mathrm{~N}\right)_{2} \mathrm{PAnH}$ & $\mu\left[\mathrm{mm}^{-1}\right]$ & 1.627 \\
\hline Summenformel & $\mathrm{C}_{26} \mathrm{H}_{37} \mathrm{~N}_{2} \mathrm{PSe}$ & $F(000)$ & 2048 \\
\hline Molmasse [g/mol] & 487.51 & $\theta$-Bereich $\left[^{\circ}\right]$ & $2.63-25.34$ \\
\hline Kristallgröße [mm] & $0.1 \times 0.1 \times 0.2$ & gesammelte Reflexe & 28132 \\
\hline Raumgruppe & Pbcn & unabhängige Reflexe & 4993 \\
\hline$a[\mathrm{pm}]$ & $3082.7(17)$ & Zahl der Restraints & 12 \\
\hline$b[\mathrm{pm}]$ & $901.1(5)$ & verfeinerte Parameter & 338 \\
\hline$c[\mathrm{pm}]$ & $1748.6(10)$ & $R 1[\mid>2 \sigma(I)]$ & 0.0375 \\
\hline$\alpha\left[^{\circ}\right]$ & 90 & $w R 2$ [alle Daten] & 0.0888 \\
\hline$\beta\left[^{\circ}\right]$ & 90 & GooF & 1.048 \\
\hline$\gamma\left[^{\circ}\right]$ & 90 & & \\
\hline$V\left[\mathrm{~nm}^{3}\right]$ & $4.86(1)$ & Differenzelektronendichte: & \\
\hline$Z$ & 8 & Max. / min. $\left[10^{-6} \mathrm{epm}^{-3}\right]$ & $0.680 /-1.186$ \\
\hline Messtemperatur [K] & $100(2)$ & Max. / min. Transmission & 0.7452 / 0.5876 \\
\hline$\rho\left[\mathrm{Mgm}^{-3}\right]$ & 1.333 & & \\
\hline
\end{tabular}




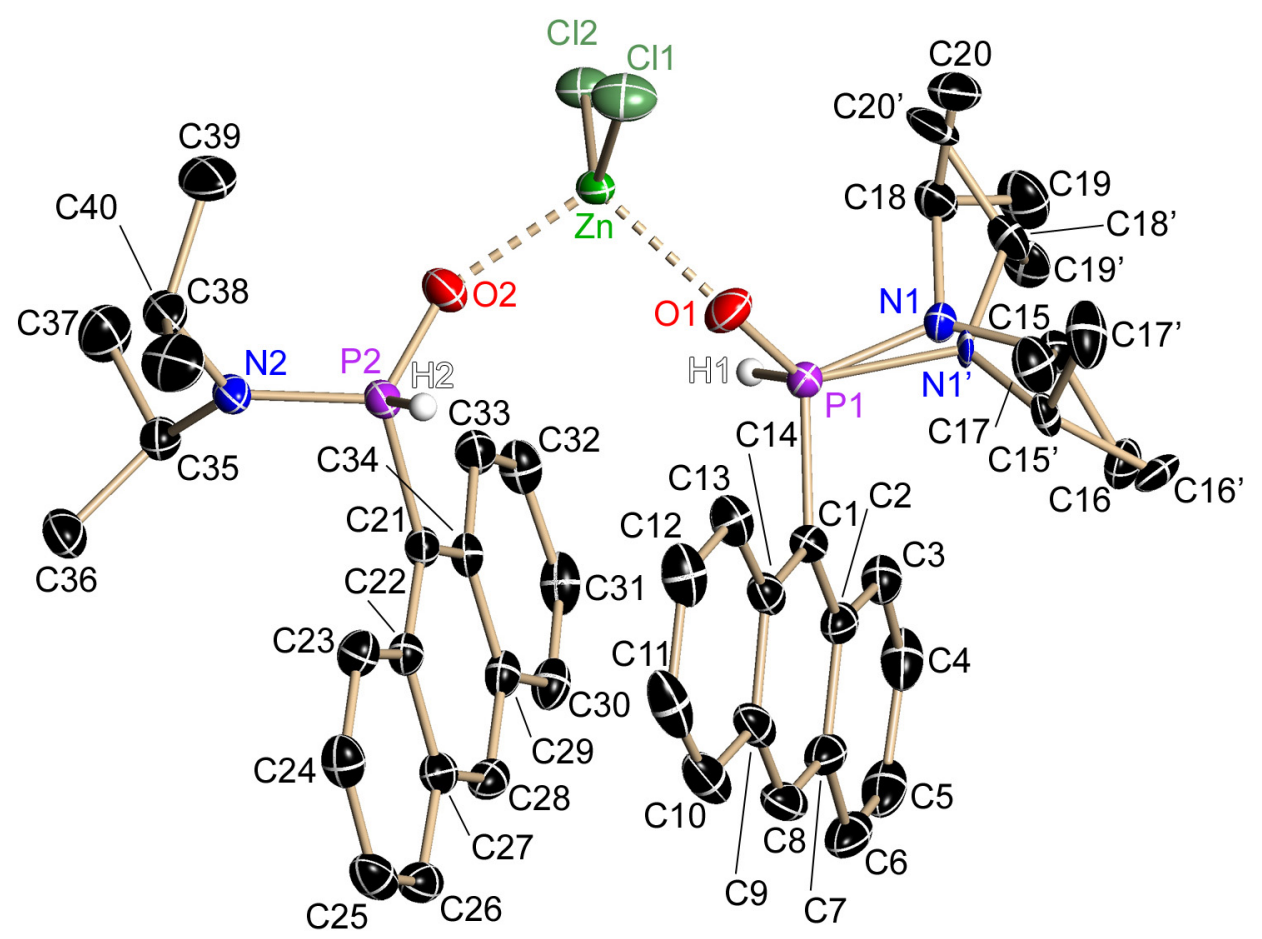

Abb. 5.50: Asymmetrische Einheit von $\left[\mathrm{ZnCl}_{2} \cdot\left\{\mathrm{O}\left(\mathrm{iPr}_{2} \mathrm{~N}\right) \mathrm{HPAnH}\right\}_{2}\right] \quad(42) ;$ die anisotropen Auslenkungsparameter geben die $50 \%$ ige Aufenthaltswahrscheinlichkeit an.

Komplex 42 kristallisiert als nichtmeroerischer Zwilling. Die Bestimmung der Elementarzellen und deren Orientirungsmatizen mit Cell-Now lieferte eine Haupt- und eine Nebendomäne. Nach Integration der beiden Domänen mit Saint wurde eine Absorptionskorrektur mit Twinabs durchgeführt, bei der lediglich die Hauptdomäne verwendet wurde. Eine zufriedenstellende Verfeinerung des Strukturmodells konnte mit dem HKLF5 File durchgeführt werden. Die Diisopropylaminogruppe an P1 ist fehlgeordnet, die Besetzung der Hauptdomäne verfeinerte zu $86 \%$.

Tab. 5.42: Kristallographische Daten von $\left[\mathrm{ZnCl}_{2} \cdot\left\{\mathrm{O}\left(\mathrm{iPr}_{2} \mathrm{~N}\right) \mathrm{HPAnH}\right\}_{2}\right](\mathbf{4 2})$.

\begin{tabular}{|ll|ll|}
\hline Strukturcode & {$\left[\mathrm{ZnCl}_{2} \cdot\left\{\mathrm{O}\left(\mathrm{iPr}_{2} \mathrm{~N}\right) \mathrm{HPAnH}\right\}_{2}\right]$} & $\rho\left[\mathrm{Mgm}^{-3}\right]$ & 1.352 \\
Summenformel & $\mathrm{C}_{40} \mathrm{H}_{48} \mathrm{Cl}_{2} \mathrm{~N}_{2} \mathrm{O}_{2} \mathrm{P}_{2} \mathrm{Zn}$ & $\mu\left[\mathrm{mm}^{-1}\right]$ & 0.893 \\
Molmasse $[\mathrm{g} / \mathrm{mol}]$ & 787.01 & $\mathrm{~F}(000)$ & 824 \\
Kristallgröße [mm] & $0.1 \times 0.1 \times 0.08$ & $\theta$-Bereich [ $\left.{ }^{\circ}\right]$ & $2.84-25.41$ \\
Raumgruppe & $P^{-}$ & gesammelte Reflexe & 11445 \\
$a[\mathrm{pm}]$ & $1097.85(14)$ & unabhängige Reflexe & 7090 \\
$b[\mathrm{pm}]$ & $1401.27(18)$ & Zahl der Restraints & 154 \\
$c[\mathrm{pm}]$ & $1490.40(19)$ & verfeinerte Parameter & 527 \\
$\alpha\left[^{\circ}\right]$ & $93.799(2)$ & $R 1[$ [l>2 $\sigma(\mathrm{I})]$ & 0.0280 \\
$\beta\left[^{\circ}\right]$ & $109.824(2)$ & WR2 [alle Daten] & 0.0775 \\
$\gamma\left[^{\circ}\right]$ & $112.956(2)$ & GooF & 1.049 \\
$V\left[\mathrm{~nm}^{3}\right]$ & $1.9332(4)$ & Differenzelektronendichte: & \\
$Z$ & 2 & Max. / min. [10 & \\
Messtemperatur $\left.^{-3}\right]$ & $0.432 /-0.389$ \\
\hline
\end{tabular}




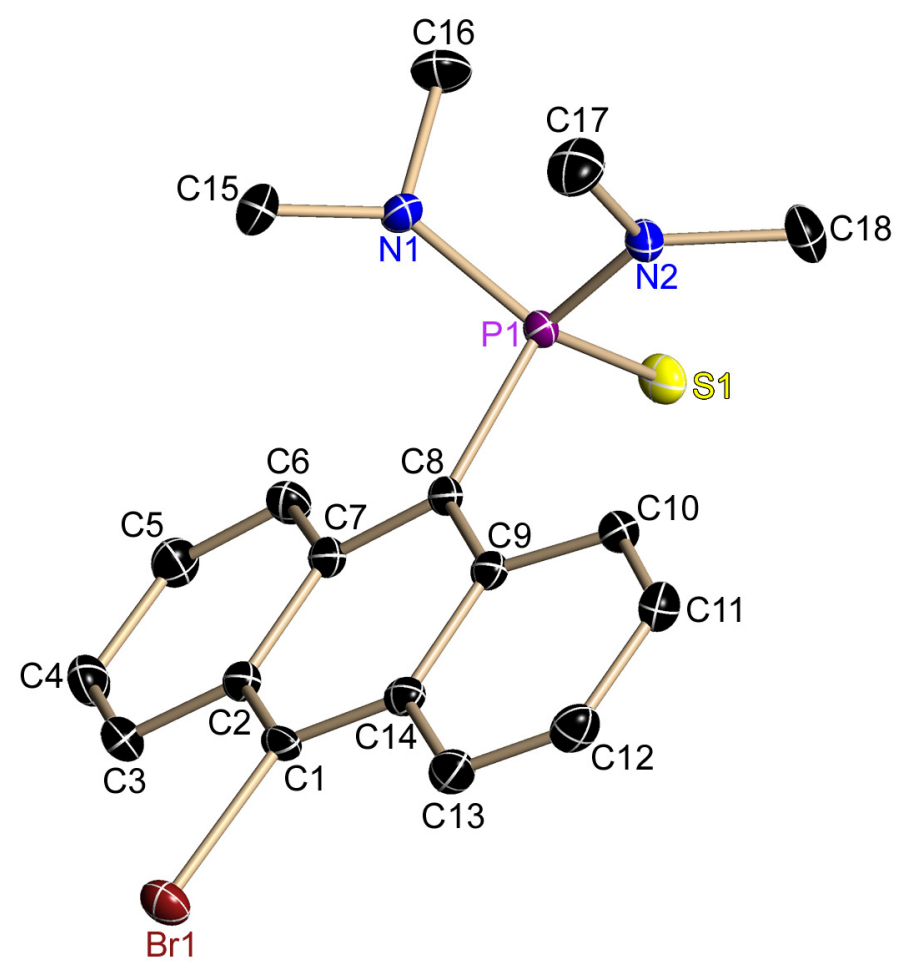

Abb. 5.51: Asymmetrische Einheit von 9-Brom-10-bis(dimethylamino)thiophosphorylanthracen (45); die anisotropen Auslenkungsparameter geben die 50\% ige Aufenthaltswahrscheinlichkeit an.

Tab. 5.43: Kristallographische Daten von 9-Brom-10-bis(dimethylamino)thiophosphorylanthracen (45).

\begin{tabular}{|c|c|c|c|}
\hline Strukturcode & $\mathrm{S}\left(\mathrm{Me}_{2} \mathrm{~N}\right)_{2} \mathrm{PAnBr}$ & $\mu\left[\mathrm{mm}^{-1}\right]$ & 2.555 \\
\hline Summenformel & $\mathrm{C}_{18} \mathrm{H}_{20} \mathrm{~N}_{2} \mathrm{PSBr}$ & $F(000)$ & 832 \\
\hline Molmasse $[\mathrm{g} / \mathrm{mol}]$ & 407.30 & $\theta$-Bereich $\left[{ }^{\circ}\right]$ & $2.14-26.75$ \\
\hline Kristallgröße [mm] & $0.18 \times 0.15 \times 0.12$ & gesammelte Reflexe & 22138 \\
\hline Raumgruppe & $P 2_{1} / n$ & unabhängige Reflexe & 3737 \\
\hline$a[\mathrm{pm}]$ & 1057.15(9) & Zahl der Restraints & 0 \\
\hline$b[\mathrm{pm}]$ & $1338.33(11)$ & verfeinerte Parameter & 212 \\
\hline$c[\mathrm{pm}]$ & $1318.89(11)$ & $R 1[I>2 \sigma(I)]$ & 0.0294 \\
\hline$\alpha\left[^{\circ}\right]$ & 90 & $w R 2$ [alle Daten] & 0.0862 \\
\hline$\beta\left[^{\circ}\right]$ & $110.0050(10)$ & GooF & 1.071 \\
\hline$\gamma\left[^{\circ}\right]$ & 90 & & \\
\hline$V\left[\mathrm{~nm}^{3}\right]$ & $1.7534(3)$ & Differenzelektronendichte: & \\
\hline$Z$ & 4 & Max. / min. $\left[10^{-6} \mathrm{epm}^{-3}\right]$ & 0.449 / -0.914 \\
\hline Messtemperatur [K] & $100(2)$ & Max. / min. Transmission & 0.4299 / 0.3666 \\
\hline$\rho\left[\mathrm{Mgm}^{-3}\right]$ & 1.543 & & \\
\hline
\end{tabular}




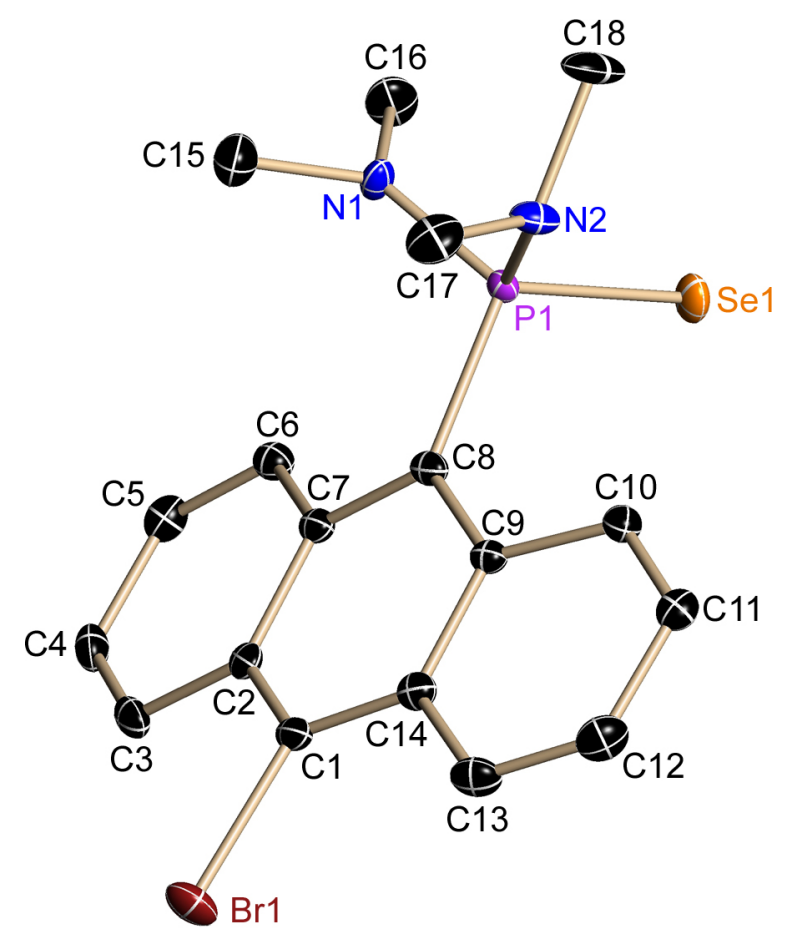

Abb. 5.52: Asymmetrische Einheit von 9-Brom-10-bis(dimethylamino)seleophosphorylanthracen (46); die anisotropen Auslenkungsparameter geben die 50\% ige Aufenthaltswahrscheinlichkeit an.

Komplex 46 kristallisiert als nichtmeroerischer Zwilling. Die Bestimmung der Elementarzellen und deren Orientirungsmatizen mit Cell-Now lieferte zwei Domänen. Nach Integration der beiden Domänen mit Saint wurde eine Absorptionskorrektur mit Twinabs durchgeführt, bei der beide Domänen verwendet wurden. Eine zufriedenstellende Verfeinerung des Strukturmodells konnte mit dem HKLF5 File durchgeführt werden.

Tab. 5.44: Kristallographische Daten von 9-Brom-10-bis(dimethylamino)seleophosphorylanthracen (46).

\begin{tabular}{|c|c|c|c|}
\hline Strukturcode & $\mathrm{BrAnBr}$ & $\mu\left[\mathrm{mm}^{-1}\right]$ & 4.464 \\
\hline Summenformel & $\mathrm{C}_{18} \mathrm{H}_{20} \mathrm{~N}_{2} \mathrm{PBrSe}$ & $F(000)$ & 904 \\
\hline Molmasse $[\mathrm{g} / \mathrm{mol}]$ & 454.20 & $\theta$-Bereich $\left[{ }^{\circ}\right]$ & $2.10-26.06$ \\
\hline Kristallgröße [mm] & $0.16 \times 0.16 \times 0.14$ & gesammelte Reflexe & 5252 \\
\hline Raumgruppe & $P 2_{1} / n$ & unabhängige Reflexe & 3491 \\
\hline$a[\mathrm{pm}]$ & 1059.34(16) & Zahl der Restraints & 0 \\
\hline$b[\mathrm{pm}]$ & 1261.87(19) & verfeinerte Parameter & 213 \\
\hline$c[\mathrm{pm}]$ & $1411.7(2)$ & $R 1[\mid>2 \sigma(I)]$ & 0.0374 \\
\hline$\alpha\left[^{\circ}\right]$ & 90 & $w R 2$ [alle Daten] & 0.1001 \\
\hline$\beta\left[^{\circ}\right]$ & $110.079(3)$ & GooF & 1.075 \\
\hline$\gamma\left[^{\circ}\right]$ & 90 & & \\
\hline$V\left[\mathrm{~nm}^{3}\right]$ & $1.7724(5)$ & Differenzelektronendichte: & \\
\hline$Z$ & 4 & Max. / min. $\left[10^{-6} \mathrm{epm}^{-3}\right]$ & 1.389 / -0.941 \\
\hline Messtemperatur [K] & $100(2)$ & Max. / min. Transmission & 0.42932 / 0.30569 \\
\hline$\rho\left[\mathrm{Mgm}^{-3}\right]$ & 1.702 & & \\
\hline
\end{tabular}




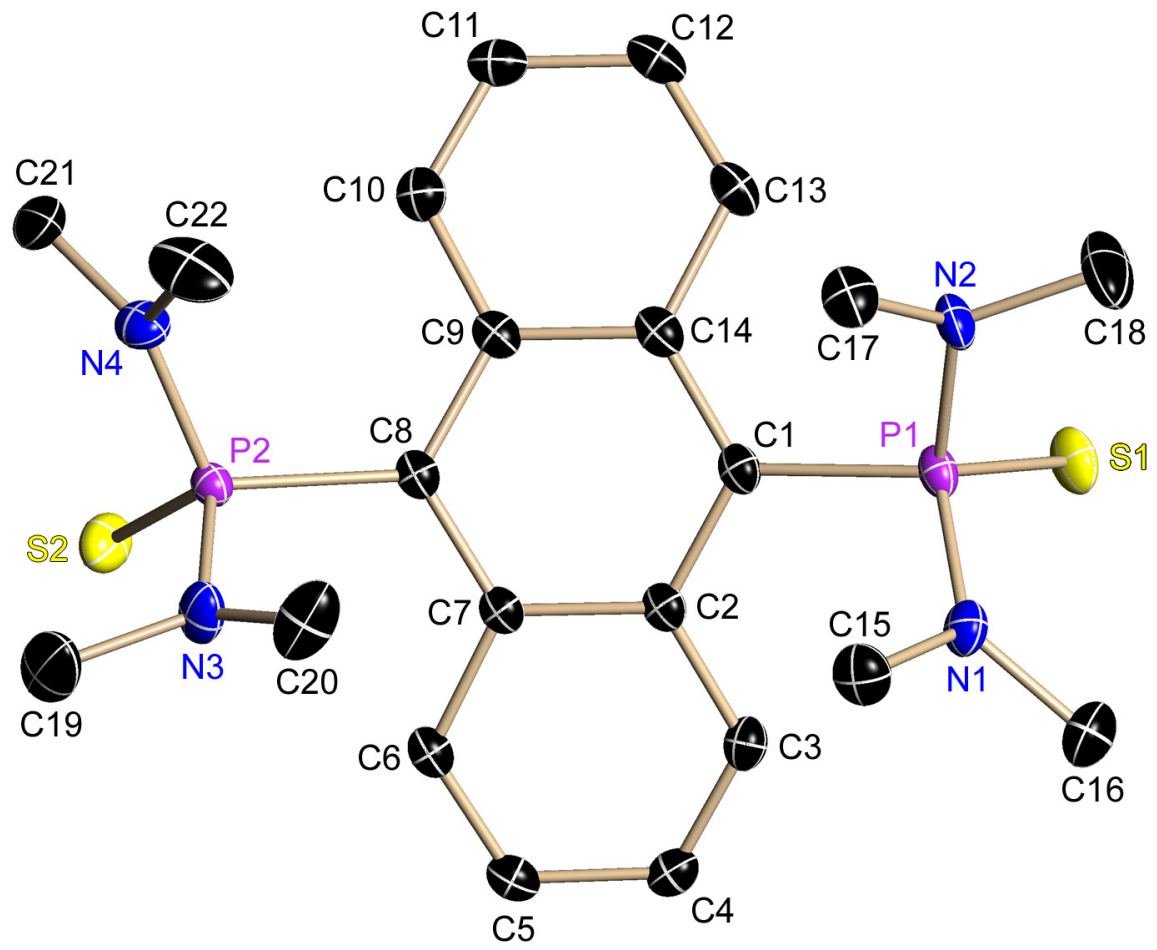

Abb. 5.53: Asymmetrische Einheit von Di(bis(dimethylamino)thiophosphoryl)anthracen (49); die anisotropen Auslenkungsparameter geben die 50\% ige Aufenthaltswahrscheinlichkeit an.

Tab. 5.45: Kristallographische Daten von Di(bis(dimethylamino)thiophosphoryl)anthracen (49).

\begin{tabular}{|c|c|c|c|}
\hline Strukturcode & {$\left[\mathrm{S}\left(\mathrm{Me}_{2} \mathrm{~N}\right)_{2} \mathrm{P}\right]_{2} \mathrm{An}$} & $\mu\left[\mathrm{mm}^{-1}\right]$ & 0.368 \\
\hline Summenformel & $\mathrm{C}_{22} \mathrm{H}_{32} \mathrm{~N}_{4} \mathrm{P}_{2} \mathrm{~S}_{2}$ & $F(000)$ & 1016 \\
\hline Molmasse $[\mathrm{g} / \mathrm{mol}]$ & 478.58 & $\theta$-Bereich $\left[{ }^{\circ}\right]$ & $1.37-26.74$ \\
\hline Kristallgröße [mm] & $0.2 \times 0.18 \times 0.15$ & gesammelte Reflexe & 44397 \\
\hline Raumgruppe & $P 2_{1} 2_{1} 2_{1}$ & unabhängige Reflexe & 5146 \\
\hline$a[\mathrm{pm}]$ & 727.24(4) & Zahl der Restraints & 0 \\
\hline$b[\mathrm{pm}]$ & 1124.95(6) & verfeinerte Parameter & 279 \\
\hline$c[\mathrm{pm}]$ & $2969.22(16)$ & $R 1[I>2 \sigma(I)]$ & 0.0274 \\
\hline$\alpha\left[^{\circ}\right]$ & 90 & $w R 2$ [alle Daten] & 0.0660 \\
\hline$\beta\left[^{\circ}\right]$ & 90 & GooF & 1.095 \\
\hline$\gamma\left[^{\circ}\right]$ & 90 & Flack-X & $0.03(6)$ \\
\hline$V\left[\mathrm{~nm}^{3}\right]$ & $2.4291(2)$ & Differenzelektronendichte: & \\
\hline$Z$ & 4 & Max. / min. $\left[10^{-6} \mathrm{epm}^{-3}\right]$ & $0.286 /-0.201$ \\
\hline Messtemperatur [K] & $100(2)$ & Max. / min. Transmission & $0.7454 / 0.7223$ \\
\hline$\rho\left[\mathrm{Mgm}^{-3}\right]$ & 1.309 & & \\
\hline
\end{tabular}




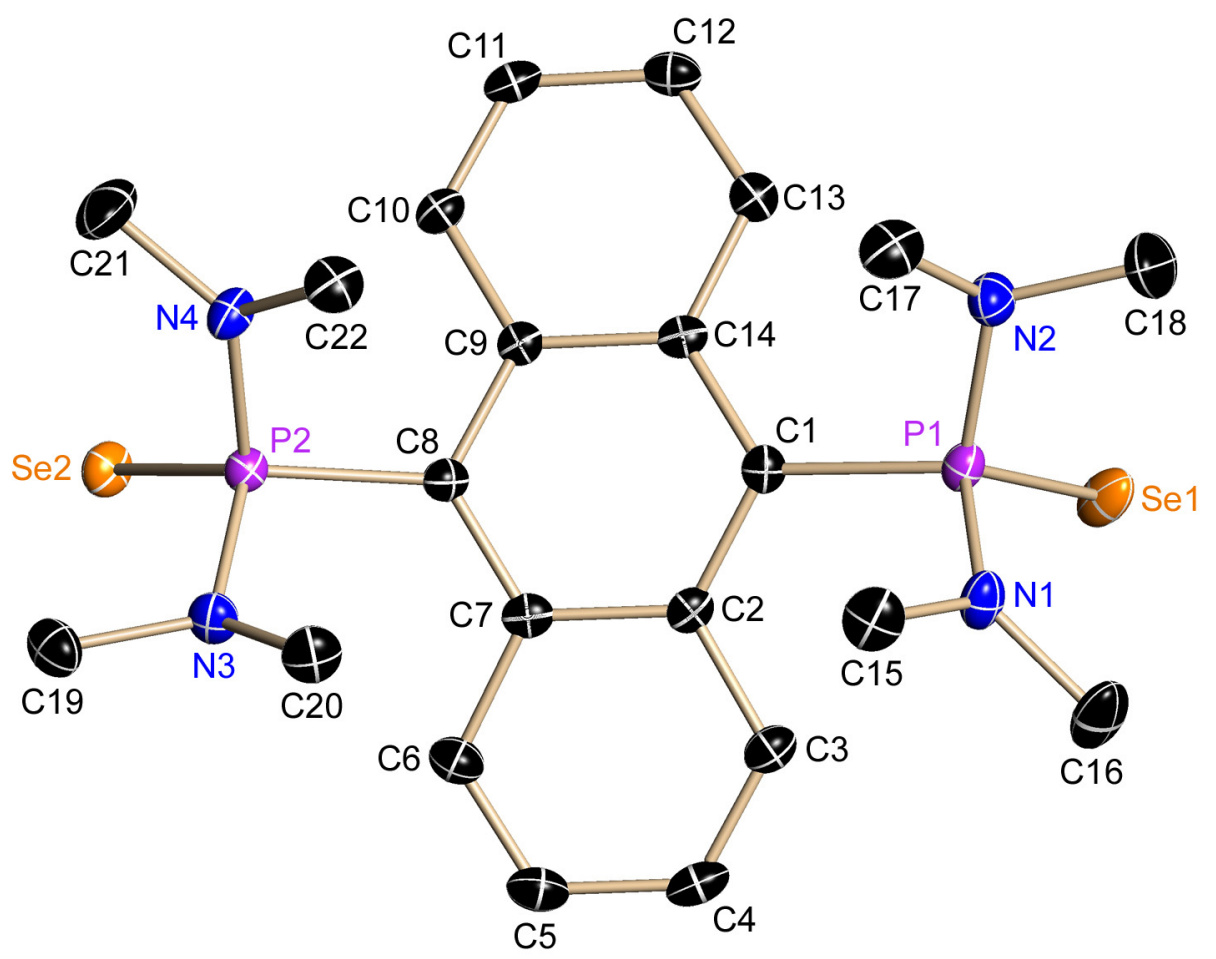

Abb. 5.54: Asymmetrische Einheit von Di(bis(dimethylamino)selenophosphoryl)anthracen (50); die anisotropen Auslenkungsparameter geben die 50\% ige Aufenthaltswahrscheinlichkeit an.

Tab. 5.46: Kristallographische Daten von Di(bis(dimethylamino)selenophosphoryl)anthracen (50).

\begin{tabular}{|ll}
\hline Strukturcode & {$\left[\mathrm{Se}\left(\mathrm{Me}_{2} \mathrm{~N}\right)_{2} \mathrm{P}\right]_{2} \mathrm{An}$} \\
Summenformel & $\mathrm{C}_{22} \mathrm{H}_{32} \mathrm{~N}_{4} \mathrm{P}_{2} \mathrm{Se}_{2}$ \\
Molmasse $[\mathrm{g} / \mathrm{mol}]$ & 572.38 \\
Kristallgröße $[\mathrm{mm}]$ & $0.2 \times 0.18 \times 0.15$ \\
Raumgruppe & $P 2{ }_{1} / n$ \\
$a[\mathrm{pm}]$ & $1287.72(11)$ \\
$b[\mathrm{pm}]$ & $1297.85(11)$ \\
$c[\mathrm{pm}]$ & $1482.19(12)$ \\
$\alpha\left[^{\circ}\right]$ & 90 \\
$\beta\left[^{\circ}\right]$ & $96.5470(10)$ \\
$\left.\gamma{ }^{\circ}\right]$ & 90 \\
$V\left[\mathrm{~nm}^{3}\right]$ & $2.4610(4)$ \\
$Z$ & 4 \\
$\mathrm{Messtemperatur}[\mathrm{K}]^{\circ}$ & $100(2)$ \\
$\rho\left[\mathrm{Mgm}^{-3}\right]$ & 1.545 \\
\hline
\end{tabular}

$\begin{array}{ll}\mu\left[\mathrm{mm}^{-1}\right] & 3.152 \\ \mathrm{~F}(000) & 1160 \\ \left.\theta \text {-Bereich [ }{ }^{\circ}\right] & 1.99-26.04 \\ \text { gesammelte Reflexe } & 24461 \\ \text { unabhängige Reflexe } & 4849 \\ \text { Zahl der Restraints } & 0 \\ \text { verfeinerte Parameter } & 279 \\ R 1 \text { [I>2 } \sigma(\mathrm{I})] & 0.0336 \\ w R 2 \text { [alle Daten] } & 0.0835 \\ \text { GooF } & 1.063 \\ & \\ \text { Differenzelektronendichte: } & \\ \text { Max. / min. [10-6 epm }{ }^{-3} \text { ] } & 1.518 /-0.540 \\ \text { Max. / min. Transmission } & 0.4293 / 0.3565\end{array}$



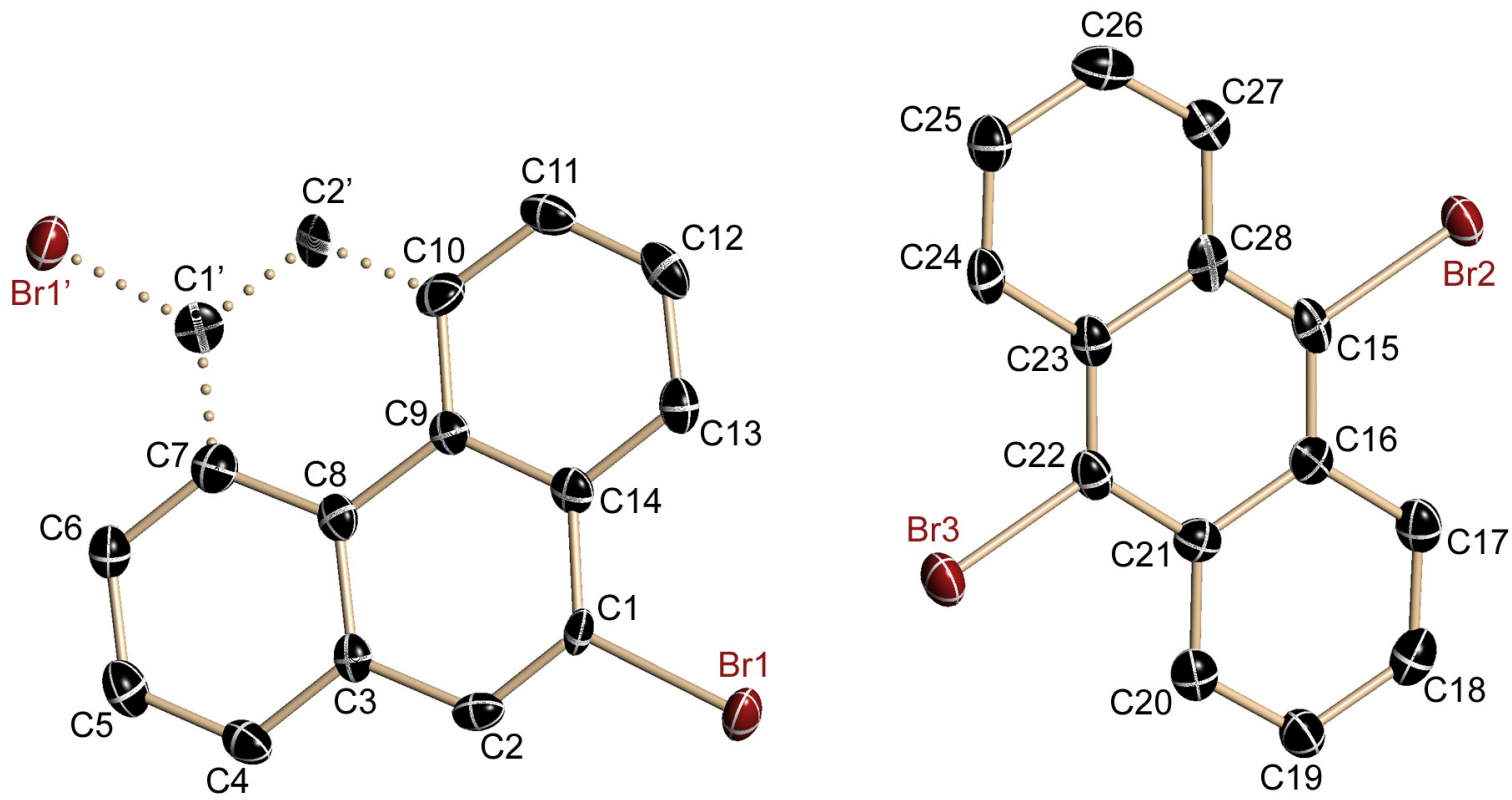

Abb. 5.55: Asymmetrische Einheit von kokristallisiertrn 9-Bromphenanthren und 9,10-Dibromanthracen; die anisotropen Auslenkungsparameter geben die 50\% ige Aufenthaltswahrscheinlichkeit an.

Bei der Aufreinigung von kommerziell erhältlichen 9-Bromphenanthran durch Umkristallisation wurden Kristalle erhalten die neben 9-Bromphenanthran auch 9,10Dibromanthracen enthalten. Die Verbindungen kristalliseren als racemischer Zwilling. Das 9Bromphenathren ist fehlgeordnet, die Besetzung der Hauptdomäne verfeinerte zu 85 \%.

Tab. 5.47: Kristallographische Daten von kokristallisiertrn 9-Bromphenanthren und 9,10-Dibromanthracen.

\begin{tabular}{|c|c|c|c|}
\hline Strukturcode & BrPhen $\mathrm{BrAnBr}$ & $\mu\left[\mathrm{mm}^{-1}\right]$ & 5.767 \\
\hline Summenformel & $\mathrm{C}_{28} \mathrm{H}_{17} \mathrm{Br}_{3}$ & $F(000)$ & 580 \\
\hline Molmasse [g/mol] & 593.15 & $\theta$-Bereich [ $\left.{ }^{\circ}\right]$ & $2.28-26.06$ \\
\hline Kristallgröße [mm] & $0.8 \times 0.06 \times 0.04$ & gesammelte Reflexe & 12242 \\
\hline Raumgruppe & $P n$ & unabhängige Reflexe & 4172 \\
\hline$a[\mathrm{pm}]$ & $1520.18(16)$ & Zahl der Restraints & 50 \\
\hline$b[\mathrm{pm}]$ & $437.38(5)$ & verfeinerte Parameter & 309 \\
\hline$c[\mathrm{pm}]$ & $1710.88(19)$ & $R 1[1>2 \sigma(I)]$ & 0.0270 \\
\hline$\alpha\left[^{\circ}\right]$ & 90 & $w R 2$ [alle Daten] & 0.0713 \\
\hline$\beta\left[^{\circ}\right]$ & $112.3790(10)$ & GooF & 1.021 \\
\hline$\gamma\left[^{\circ}\right]$ & 90 & Flack-X & - \\
\hline$V\left[\mathrm{~nm}^{3}\right]$ & $1.0519(2)$ & Differenzelektronendichte: & \\
\hline$z$ & 2 & Max. / min. $\left[10^{-6} \mathrm{epm}^{-3}\right]$ & $0.503 /-0.476$ \\
\hline Messtemperatur [K] & $100(2)$ & Max. / min. Transmission & $0.4293 / 0.3443$ \\
\hline$\rho\left[\mathrm{Mgm}^{-3}\right]$ & 1.873 & & \\
\hline
\end{tabular}




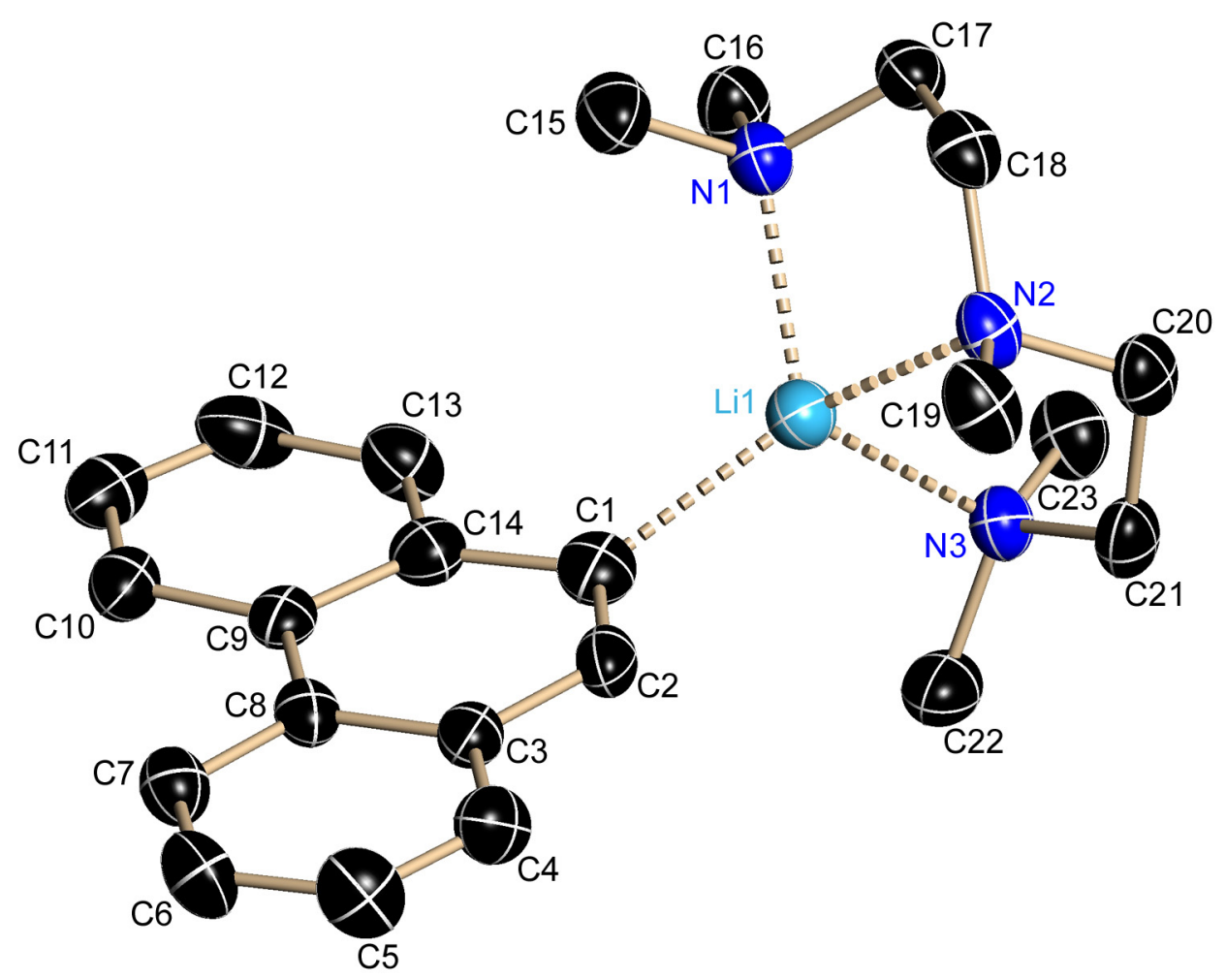

Abb. 5.56: Asymmetrische Einheit des Komplexes 9-Phenanthrenyllithium mit PMDETA; die anisotropen Auslenkungsparameter geben die 50\% ige Aufenthaltswahrscheinlichkeit an.

Tab. 5.48: Kristallographische Daten des Komplexes 9-Phenanthrenyllithium mit PMDETA.

\begin{tabular}{|c|c|c|c|}
\hline Strukturcode & {$[$ PhenLi $\bullet\{$ pmdeta $\}]$} & $\mu\left[\mathrm{mm}^{-1}\right]$ & 0.065 \\
\hline Summenformel & $\mathrm{C}_{23} \mathrm{H}_{32} \mathrm{LiN}_{3}$ & $F(000)$ & 776 \\
\hline Molmasse $[\mathrm{g} / \mathrm{mol}]$ & 357.46 & $\theta$-Bereich $\left[{ }^{\circ}\right]$ & $1.84-20.81$ \\
\hline Kristallgröße [mm] & $0.18 \times 0.16 \times 0.1$ & gesammelte Reflexe & 13996 \\
\hline Raumgruppe & $P 2_{1} / c$ & unabhängige Reflexe & 3371 \\
\hline$a[\mathrm{pm}]$ & $859.7(4)$ & Zahl der Restraints & 352 \\
\hline$b[\mathrm{pm}]$ & $1733.3(7)$ & verfeinerte Parameter & 341 \\
\hline$c[\mathrm{pm}]$ & $1435.0(6)$ & $R 1[\mid>2 \sigma(I)]$ & 0.0804 \\
\hline$\alpha\left[^{\circ}\right]$ & 90 & $w R 2$ [alle Daten] & 0.2023 \\
\hline$\beta\left[^{\circ}\right]$ & $90.122(7)$ & GooF & 1.130 \\
\hline$\gamma\left[^{\circ}\right]$ & 90 & & \\
\hline$V\left[\mathrm{~nm}^{3}\right]$ & 2.1385 & Differenzelektronendichte: & \\
\hline$Z$ & 4 & Max. / min. $\left[10^{-6} \mathrm{epm}^{-3}\right]$ & $0.709 /-0.289$ \\
\hline Messtemperatur [K] & $100(2)$ & Max. / min. Transmission & 0.7454 / 0.4641 \\
\hline$\rho\left[\mathrm{Mgm}^{-3}\right]$ & 1.110 & & \\
\hline
\end{tabular}




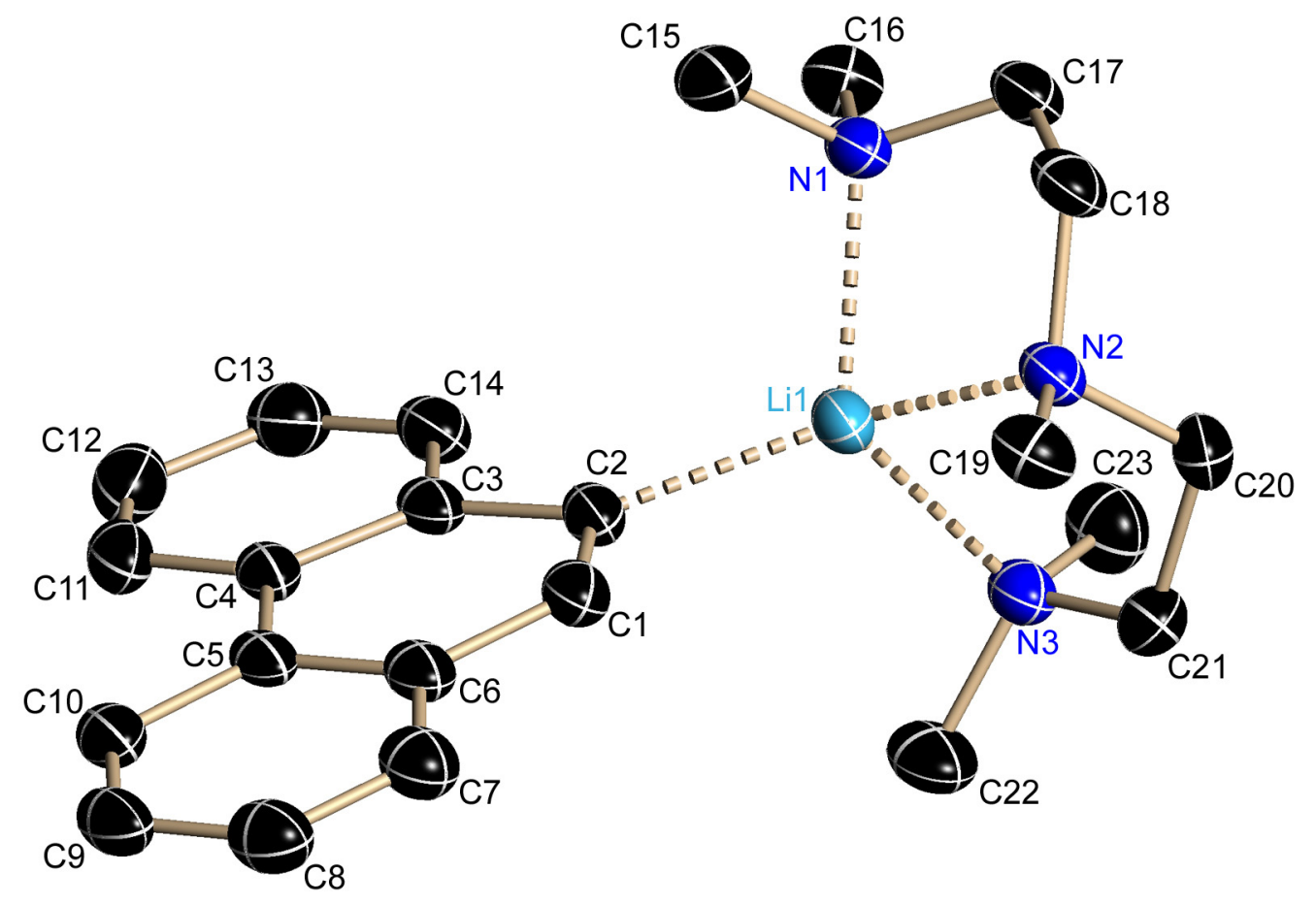

Abb. 5.57: Asymmetrische Einheit von 9-Phenanthrenyllithium mit PMDETA; die anisotropen Auslenkungsparameter geben die 50\% ige Aufenthaltswahrscheinlichkeit an.

Tab. 5.49: Kristallographische Daten von 9-Phenanthrenyllithium mit PMDETA.

\begin{tabular}{|c|c|c|c|}
\hline Strukturcode & $[$ PhenLi • $\{$ pmdeta $\}]]$ & $\mu\left[\mathrm{mm}^{-1}\right]$ & 0.065 \\
\hline Summenformel & $\mathrm{C}_{23} \mathrm{H}_{32} \mathrm{LiN}_{3}$ & $F(000)$ & 780 \\
\hline Molmasse $[\mathrm{g} / \mathrm{mol}]$ & 357.46 & $\theta$-Bereich $\left[{ }^{\circ}\right]$ & $1.41-25.70$ \\
\hline Kristallgröße [mm] & $0.16 \times 0.15 \times 0.15$ & gesammelte Reflexe & 42293 \\
\hline Raumgruppe & $P 2_{1} / c$ & unabhängige Reflexe & 4024 \\
\hline$a[\mathrm{pm}]$ & $1441.51(12)$ & Zahl der Restraints & 0 \\
\hline$b[\mathrm{pm}]$ & $917.59(8)$ & verfeinerte Parameter & 250 \\
\hline$c[\mathrm{pm}]$ & $1603.10(13)$ & $R 1[I>2 \sigma(I)]$ & 0.0599 \\
\hline$\alpha\left[^{\circ}\right]$ & 90 & $w R 2$ [alle Daten] & 0.1506 \\
\hline$\beta\left[^{\circ}\right]$ & $91.0440(10)$ & GooF & 1.054 \\
\hline$\gamma\left[^{\circ}\right]$ & 90 & & \\
\hline$V\left[\mathrm{~nm}^{3}\right]$ & $2.1201(3)$ & Differenzelektronendichte: & \\
\hline$Z$ & 4 & Max. / min. $\left[10^{-6} \mathrm{epm}^{-3}\right]$ & $1.326 /-0.152$ \\
\hline Messtemperatur [K] & $100(2)$ & Max. / min. Transmission & $0.7453 / 0.6953$ \\
\hline$\rho\left[\mathrm{Mgm}^{-3}\right]$ & 1.123 & & \\
\hline
\end{tabular}




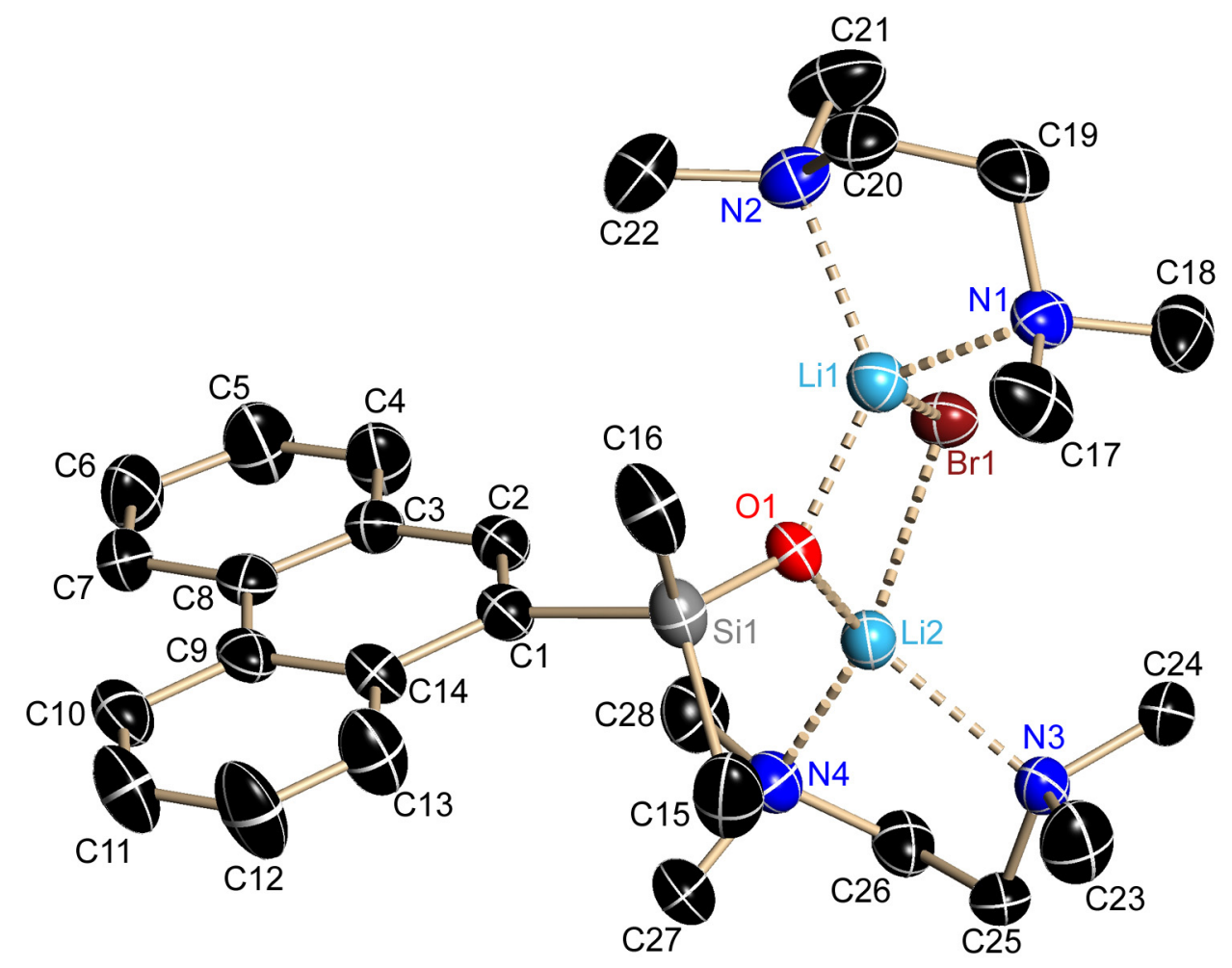

Abb. 5.58: Ausschnitt der asymmetrischen Einheit von $\left[\mathrm{PhenSiMe}_{2} \mathrm{OLi} \cdot \mathrm{LiBr} \cdot\{\mathrm{tmeda}\}_{2}\right]$; die anisotropen Auslenkungsparameter geben die 50\% ige Aufenthaltswahrscheinlichkeit an.

Tab. 5.50: Kristallographische Daten des Komplexes $\left[\mathrm{PhenSiMe}{ }_{2} \mathrm{OLi} \cdot \mathrm{LiBr} \cdot\{\mathrm{tmeda}\}_{2}\right]$.

\begin{tabular}{|ll|ll|}
\hline Strukturcode & & $\mu\left[\mathrm{mm}^{-1}\right]$ & 1.186 \\
Summenformel & $\mathrm{C}_{35} \mathrm{H}_{52} \mathrm{BrLi}_{2} \mathrm{~N}_{4} \mathrm{OSi}$ & $\mathrm{F}(000)$ & 2824 \\
Molmasse $[\mathrm{g} / \mathrm{mol}]$ & 666.69 & $\theta$-Bereich [ $\left.{ }^{\circ}\right]$ & $1.78-24.17$ \\
Kristallgröße $[\mathrm{mm}]$ & $0.18 \times 0.17 \times 0.10$ & gesammelte Reflexe & 17468 \\
Raumgruppe & $\mathrm{C} 2 / \mathrm{c}$ & unabhängige Reflexe & 5828 \\
$a[\mathrm{pm}]$ & $3705.3(2)$ & Zahl der Restraints & 658 \\
$b[\mathrm{pm}]$ & $874.68(6)$ & verfeinerte Parameter & 492 \\
$c[\mathrm{pm}]$ & $2402.06(16)$ & $R 1[\mathrm{I}>2 \sigma(\mathrm{I})]$ & 0.0453 \\
$\alpha\left[^{\circ}\right]$ & 90 & WR2 [alle Daten] & 0.1149 \\
$\beta\left[^{\circ}\right]$ & $109.8150(10)$ & GooF & 1.042 \\
$\gamma\left[^{\circ}\right]$ & 90 & Differenzelektronendichte: & \\
$V\left[\mathrm{~nm}^{3}\right]$ & $7.3240(8)$ & Max. / min. $\left[10^{-6}\right.$ epm $\left.{ }^{-3}\right]$ & $0.504 /-0.389$ \\
$Z$ & 8 & Max. / min. Transmission & $0.4279 / 0.3823$ \\
$\mathrm{Messtemperatur}[\mathrm{K}]^{3}$ & $100(2)$ & & \\
$\rho\left[\mathrm{Mgm}^{-3}\right]$ & 1.209 & & \\
\hline
\end{tabular}




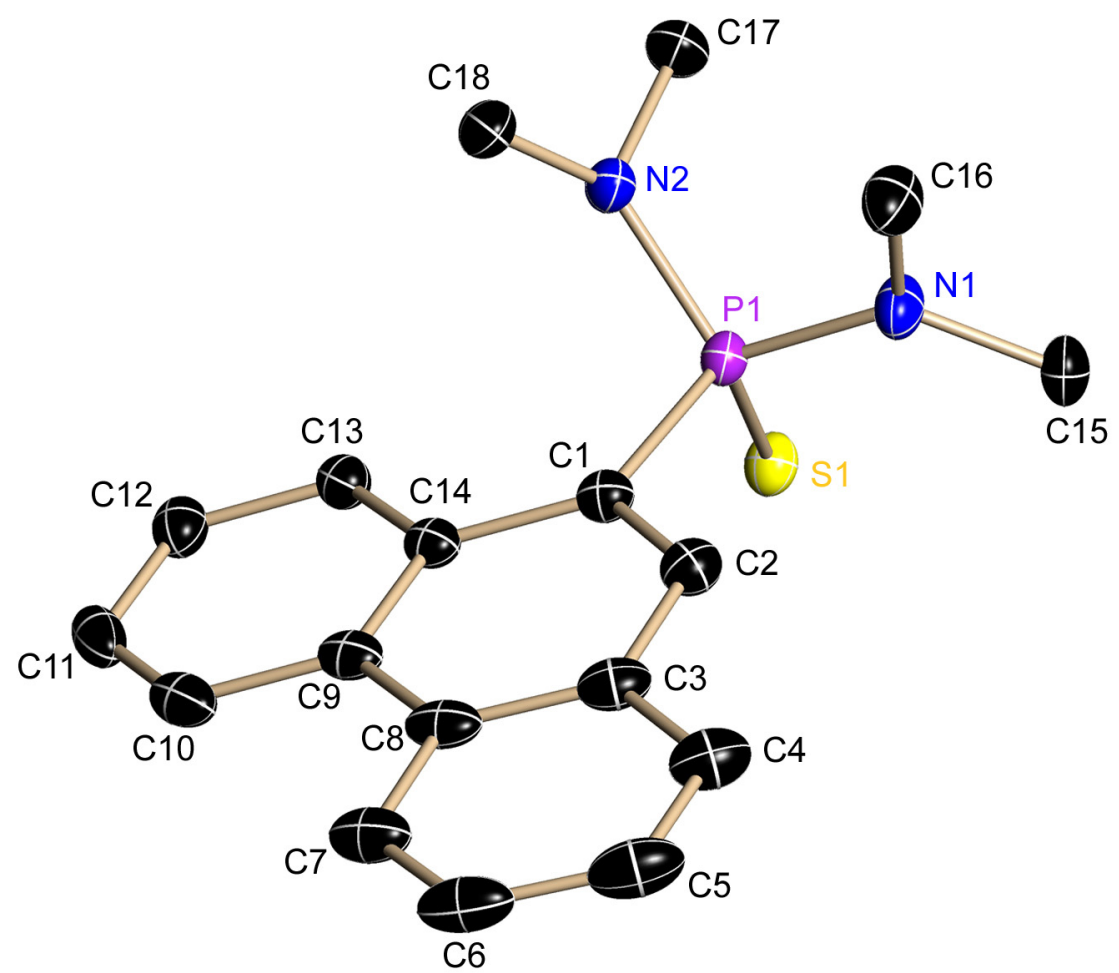

Abb. 5.59: Asymmetrische Einheit von 9-Bis(dimethylamino)thiophosphorylphenanthren; die anisotropen Auslenkungsparameter geben die 50\% ige Aufenthaltswahrscheinlichkeit an.

Tab. 5.51: Kristallographische Daten von 9-Bis(dimethylamino)thiophosphorylphenanthren.

\begin{tabular}{|c|c|c|c|}
\hline Strukturcode & $\mathrm{S}\left(\mathrm{Me}_{2} \mathrm{~N}\right)_{2} \mathrm{PPhen}$ & $\mu\left[\mathrm{mm}^{-1}\right]$ & 0.290 \\
\hline Summenformel & $\mathrm{C}_{18} \mathrm{H}_{21} \mathrm{~N}_{2} \mathrm{PS}$ & $F(000)$ & 768 \\
\hline Molmasse $[\mathrm{g} / \mathrm{mol}]$ & 328.40 & $\theta$-Bereich $\left[{ }^{\circ}\right]$ & $2.24-26.02$ \\
\hline Kristallgröße [mm] & $0.2 \times 0.15 \times 0.12$ & gesammelte Reflexe & 14980 \\
\hline Raumgruppe & $P 2_{1} / n$ & unabhängige Reflexe & 3358 \\
\hline$a[\mathrm{pm}]$ & $1082.55(4)$ & Zahl der Restraints & 0 \\
\hline$b[\mathrm{pm}]$ & 1374.55(6) & verfeinerte Parameter & 203 \\
\hline$c[\mathrm{pm}]$ & $1175.88(5)$ & $R 1[I>2 \sigma(I)]$ & 0.0265 \\
\hline$\alpha\left[^{\circ}\right]$ & 90 & $w R 2$ [alle Daten] & 0.0674 \\
\hline$\beta\left[^{\circ}\right]$ & $108.7690(10)$ & GooF & 1.054 \\
\hline$\gamma\left[^{\circ}\right]$ & 90 & & \\
\hline$V\left[\mathrm{~nm}^{3}\right]$ & $1.65669(12)$ & Differenzelektronendichte: & \\
\hline$Z$ & 4 & Max. / min. $\left[10^{-6} \mathrm{epm}^{-3}\right]$ & $0.897 /-0.393$ \\
\hline Messtemperatur [K] & $100(2)$ & Max. / min. Transmission & $0.4299 / 0.3128$ \\
\hline$\rho\left[\mathrm{Mgm}^{-3}\right]$ & 1.317 & & \\
\hline
\end{tabular}




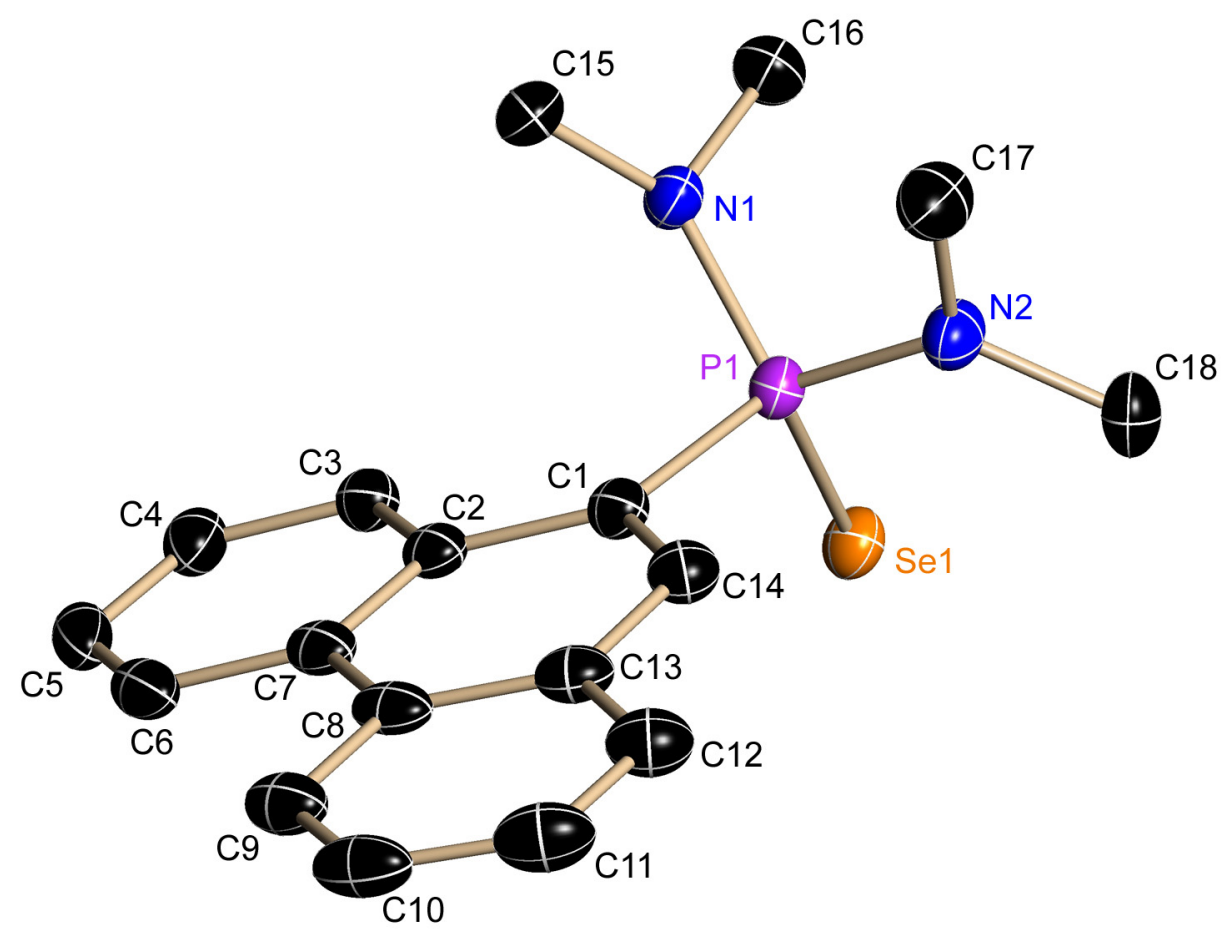

Abb. 5.60: Asymmetrische Einheit von 9-Bis(dimethylamino)selenophosphorylphenanthren; die anisotropen Auslenkungsparameter geben die $50 \%$ ige Aufenthaltswahrscheinlichkeit an.

Tab. 5.52: Kristallographische Daten von 9-Bis(dimethylamino)selenophosphorylphenanthren.

\begin{tabular}{|ll|ll|}
\hline Strukturcode & $\mathrm{Se}\left(\mathrm{Me}_{2} \mathrm{~N}\right)_{2}$ PPhen & $\mu\left[\mathrm{mm}^{-1}\right]$ & 2.298 \\
Summenformel & $\mathrm{C}_{18} \mathrm{H}_{21} \mathrm{~N}_{2} \mathrm{PSe}$ & $\mathrm{F}(000)$ & 768 \\
Molmasse $[\mathrm{g} / \mathrm{mol}]$ & 375.30 & $\theta$-Bereich $\left.{ }^{\circ}\right]$ & $2.24-26.02$ \\
Kristallgröße $[\mathrm{mm}]$ & $0.22 \times 0.18 \times 0.18$ & gesammelte Reflexe & 14980 \\
Raumgruppe & $P 2_{1} / n$ & unabhängige Reflexe & 3358 \\
$a[\mathrm{pm}]$ & $1079.38(8)$ & Zahl der Restraints & 0 \\
$b[\mathrm{pm}]$ & $1401.10(10)$ & verfeinerte Parameter & 203 \\
$c[\mathrm{pm}]$ & $1181.86(9)$ & $R 1[\mathrm{I}>2 \sigma(\mathrm{I})]$ & 0.0265 \\
$\alpha\left[^{\circ}\right]$ & 90 & WR2 [alle Daten] & 0.0674 \\
$\beta\left[^{\circ}\right]$ & $107.7870(10)$ & GooF & 1.054 \\
$\gamma\left[^{\circ}\right]$ & 90 & & \\
$V\left[\mathrm{~nm}^{3}\right]$ & $1.7019(2)$ & Differenzelektronendichte: & \\
$Z$ & 4 & Max. / min. [10 & \\
$\left.\mathrm{Messtemperatur}[\mathrm{K}]^{-3}\right]$ & Max. / min. Transmission & $0.897 /-0.393$ \\
$\rho\left[\mathrm{Mgm}^{-3}\right]$ & $100(2)$ & & $0.4299 / 0.3128$ \\
\hline
\end{tabular}




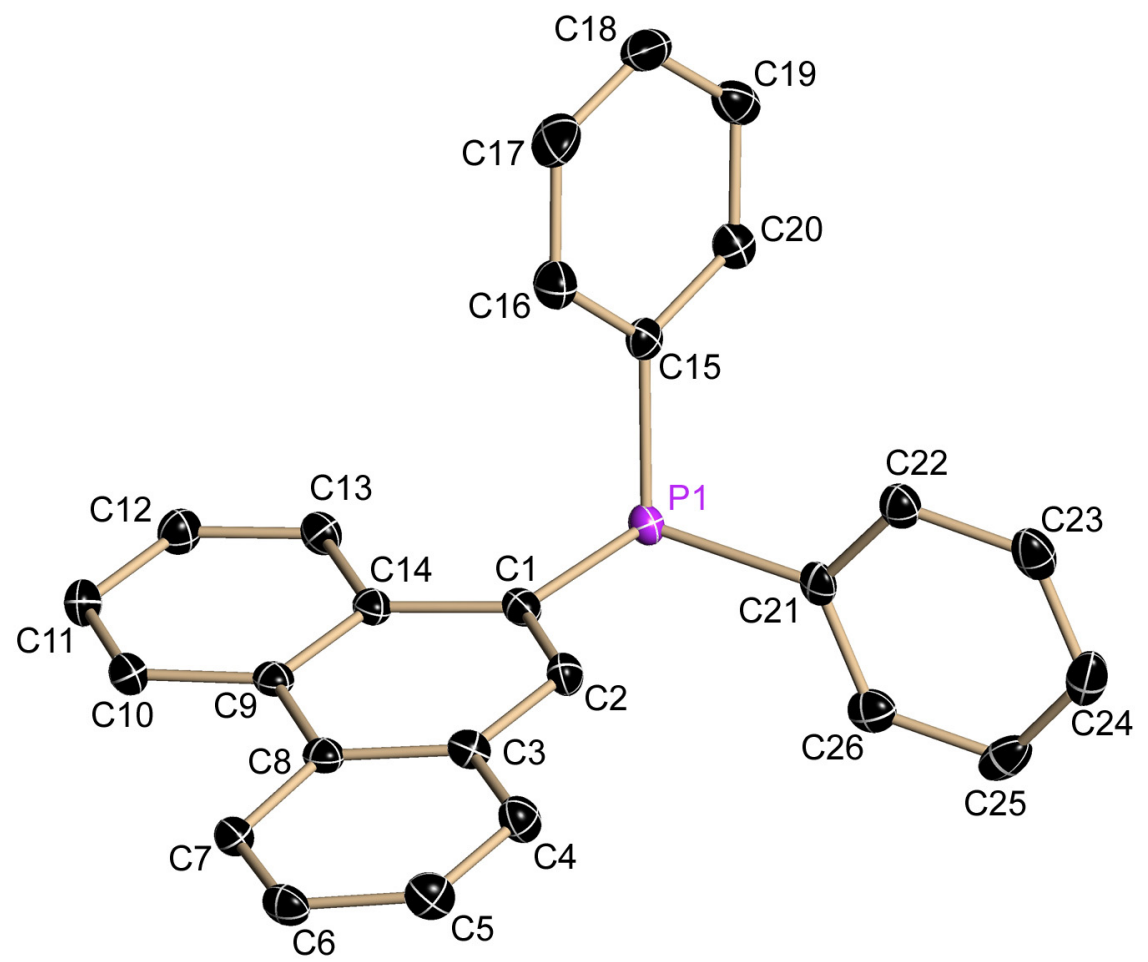

Abb. 5.61: Ausschnitt der asymmetrischen Einheit von 9-Diphenylphosphanylphenanthren; die anisotropen Auslenkungsparameter geben die 50\% ige Aufenthaltswahrscheinlichkeit an.

Tab. 5.53: Kristallographische Daten von 9-Diphenylphosphanylphenanthren.

\begin{tabular}{|c|c|c|c|}
\hline Strukturcode & $\mathrm{Ph}_{2}$ PPhen & $\mu\left[\mathrm{mm}^{-1}\right]$ & 0.157 \\
\hline Summenformel & $\mathrm{C}_{26} \mathrm{H}_{19} \mathrm{P}$ & $F(000)$ & 1520 \\
\hline Molmasse $[\mathrm{g} / \mathrm{mol}]$ & 362.38 & $\theta$-Bereich $\left[{ }^{\circ}\right]$ & $2.19-26.37$ \\
\hline Kristallgröße [mm] & $0.26 \times 0.22 \times 0.20$ & gesammelte Reflexe & 38341 \\
\hline Raumgruppe & $P 2_{1} / n$ & unabhängige Reflexe & 7506 \\
\hline$a[\mathrm{pm}]$ & 1029.37(4) & Zahl der Restraints & 0 \\
\hline$b[\mathrm{pm}]$ & 2241.80(9) & verfeinerte Parameter & 487 \\
\hline$c[\mathrm{pm}]$ & 1604.04(6) & $R 1[I>2 \sigma(I)]$ & 0.0368 \\
\hline$\alpha\left[^{\circ}\right]$ & 90 & $w R 2$ [alle Daten] & 0.1026 \\
\hline$\beta\left[^{\circ}\right]$ & $96.6540(10)$ & GooF & 1.029 \\
\hline$\gamma\left[^{\circ}\right]$ & 90 & & \\
\hline$V\left[\mathrm{~nm}^{3}\right]$ & $3.6766(2)$ & Differenzelektronendichte: & \\
\hline$Z$ & 4 & Max. / min. $\left[10^{-6} \mathrm{epm}^{-3}\right]$ & $0.522 /-0.296$ \\
\hline Messtemperatur [K] & $100(2)$ & Max. / min. Transmission & $0.7454 / 0.6814$ \\
\hline$\rho\left[\mathrm{Mgm}^{-3}\right]$ & 1.309 & & \\
\hline
\end{tabular}




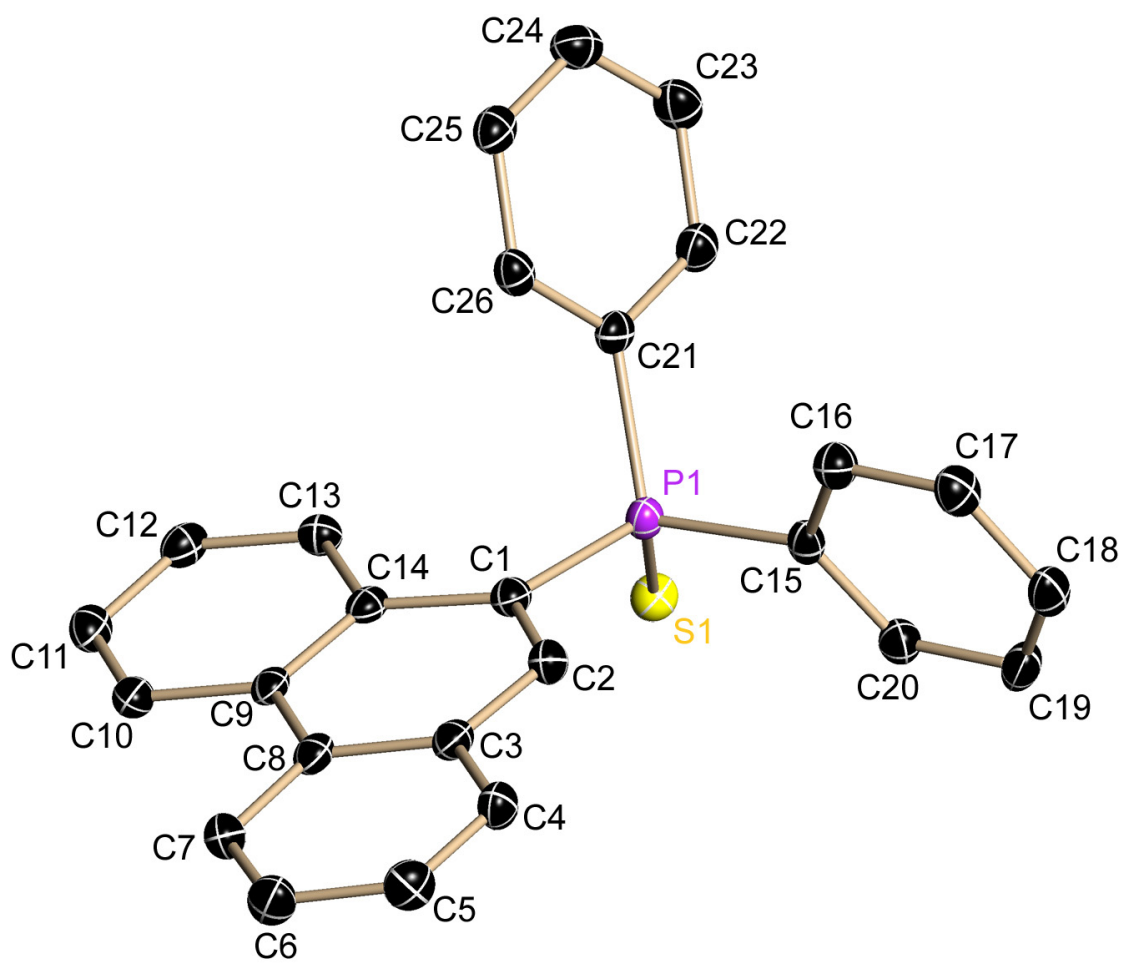

Abb. 5.62: Asymmetrische Einheit von 9-Diphenylthiophosphorylphenanthren; die anisotropen Auslenkungsparameter geben die 50\% ige Aufenthaltswahrscheinlichkeit an.

Tab. 5.54: Kristallographische Daten von 9-Diphenylthiophosphorylphenanthren.

\begin{tabular}{|c|c|c|c|}
\hline Strukturcode & $\mathrm{SPh}_{2}$ PPhen & $\mu\left[\mathrm{mm}^{-1}\right]$ & 0.254 \\
\hline Summenformel & $\mathrm{C}_{26} \mathrm{H}_{19} \mathrm{PS}$ & $F(000)$ & 824 \\
\hline Molmasse [g/mol] & 394.44 & $\theta$-Bereich $\left[^{\circ}\right]$ & $1.97-25.77$ \\
\hline Kristallgröße [mm] & $0.15 \times 0.15 \times 0.05$ & gesammelte Reflexe & 4158 \\
\hline Raumgruppe & $P 2_{1} / n$ & unabhängige Reflexe & 3749 \\
\hline$a[\mathrm{pm}]$ & $1340.3(6)$ & Zahl der Restraints & 0 \\
\hline$b[\mathrm{pm}]$ & $917.9(4)$ & verfeinerte Parameter & 254 \\
\hline$c[\mathrm{pm}]$ & $1601.5(7)$ & $R 1[\mid>2 \sigma(I)]$ & 0.0439 \\
\hline$\alpha\left[^{\circ}\right]$ & 90 & $w R 2$ [alle Daten] & 0.0969 \\
\hline$\beta\left[^{\circ}\right]$ & $90.774(9)$ & GooF & 1.065 \\
\hline$\gamma\left[^{\circ}\right]$ & 90 & & \\
\hline$V\left[\mathrm{~nm}^{3}\right]$ & $1.9701(16)$ & Differenzelektronendichte: & \\
\hline$Z$ & 4 & Max. / min. $\left[10^{-6} \mathrm{epm}^{-3}\right]$ & $0.317 /-0.337$ \\
\hline Messtemperatur [K] & $100(2)$ & Max. / min. Transmission & $0.74524 / 0.55078$ \\
\hline$\rho\left[\mathrm{Mgm}^{-3}\right]$ & 1.330 & & \\
\hline
\end{tabular}




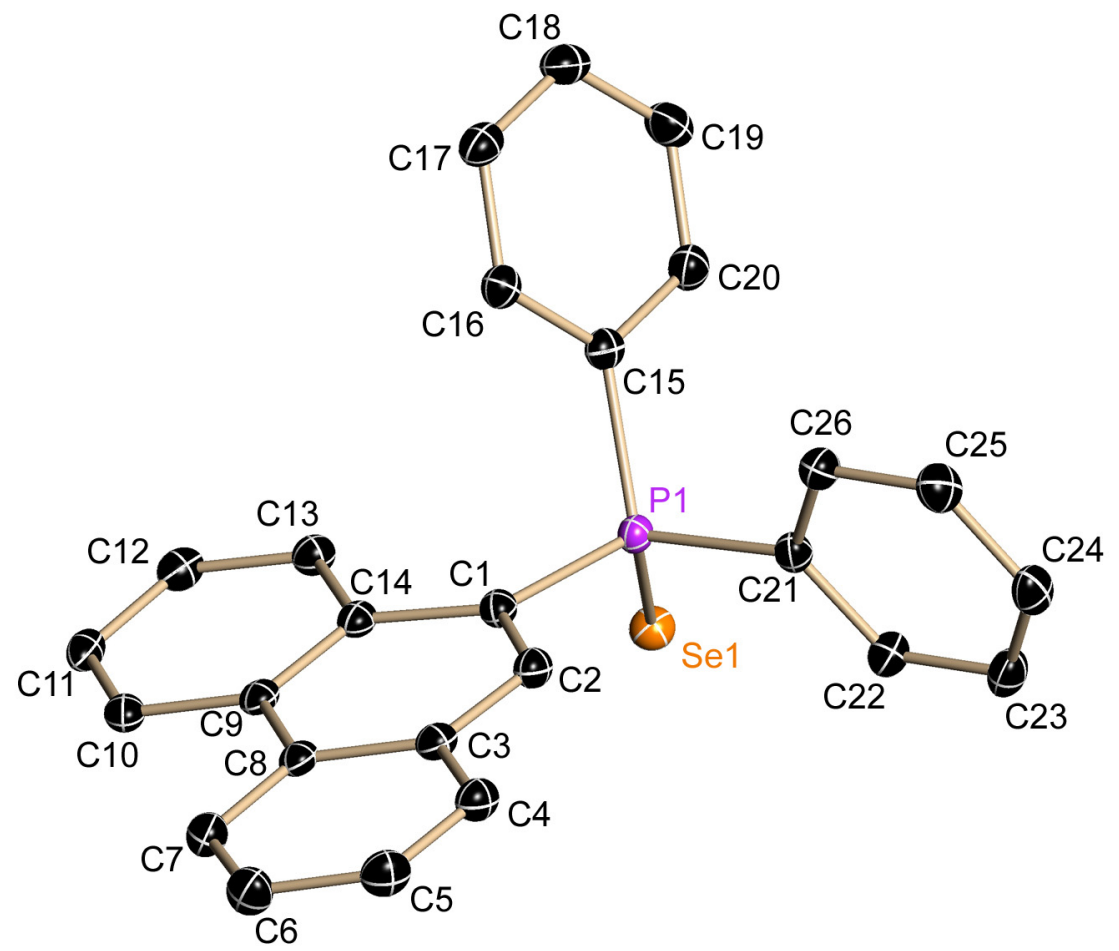

Abb. 5.63: Asymmetrische Einheit von 9-Diphenylselenophosphorylphenanthren; die anisotropen Auslenkungsparameter geben die 50\% ige Aufenthaltswahrscheinlichkeit an.

Tab. 5.55: Kristallographische Daten von 9-Diphenylselenophosphorylphenanthren.

\begin{tabular}{|c|c|c|c|}
\hline Strukturcode & $\mathrm{SePh}_{2} \mathrm{PPhen}$ & $\mu\left[\mathrm{mm}^{-1}\right]$ & 1.967 \\
\hline Summenformel & $\mathrm{C}_{26} \mathrm{H}_{19} \mathrm{PSe}$ & $F(000)$ & 896 \\
\hline Molmasse $[\mathrm{g} / \mathrm{mol}]$ & 441.34 & $\theta$-Bereich $\left[{ }^{\circ}\right]$ & $1.95-27.10$ \\
\hline Kristallgröße [mm] & $0.12 \times 0.11 \times 0.05$ & gesammelte Reflexe & 35738 \\
\hline Raumgruppe & $P 2_{1} / n$ & unabhängige Reflexe & 4417 \\
\hline$a[\mathrm{pm}]$ & 1352.39(6) & Zahl der Restraints & 0 \\
\hline$b[\mathrm{pm}]$ & $916.99(4)$ & verfeinerte Parameter & 253 \\
\hline$c[\mathrm{pm}]$ & $1612.21(8)$ & $R 1[\mid>2 \sigma(I)]$ & 0.0260 \\
\hline$\alpha\left[^{\circ}\right]$ & 90 & $w R 2$ [alle Daten] & 0.0655 \\
\hline$\beta\left[^{\circ}\right]$ & $91.2180(10)$ & GooF & 1.044 \\
\hline$\gamma\left[^{\circ}\right]$ & 90 & & \\
\hline$V\left[\mathrm{~nm}^{3}\right]$ & $1.99890(16)$ & Differenzelektronendichte: & \\
\hline$Z$ & 4 & Max. / min. $\left[10^{-6} \mathrm{epm}^{-3}\right]$ & $0.439 /-0.328$ \\
\hline Messtemperatur [K] & $100(2)$ & Max. / min. Transmission & $0.4305 / 0.3614$ \\
\hline$\rho\left[\mathrm{Mgm}^{-3}\right]$ & 1.467 & & \\
\hline
\end{tabular}




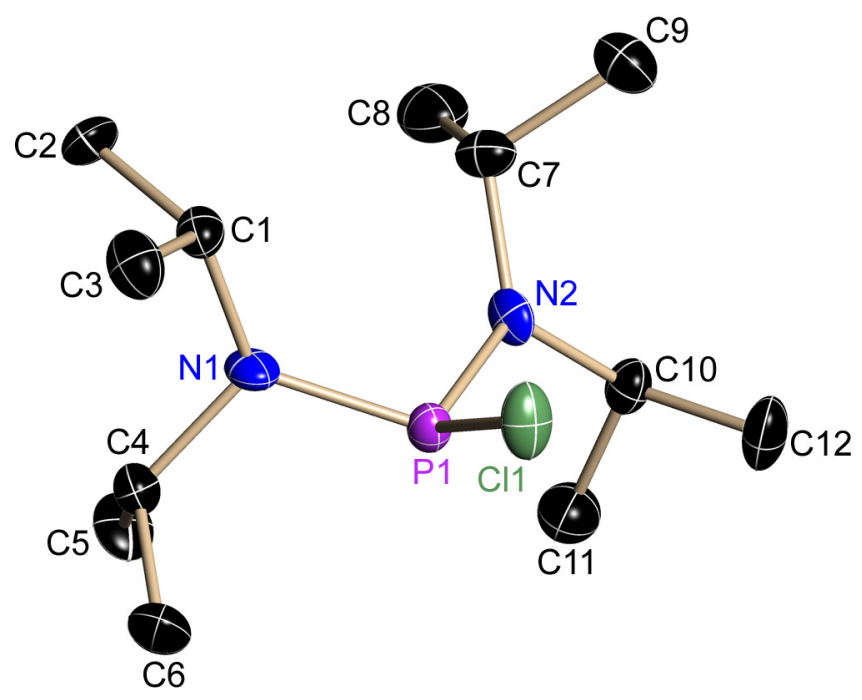

Abb. 5.64: Ausschnitt der asymmetrischen Einheit von Bis(diisopropylamino)chlorphosphan; die anisotropen Auslenkungsparameter geben die 50\% ige Aufenthaltswahrscheinlichkeit an.

Tab. 5.56: Kristallographische Daten von Bis(diisopropylamino)chlorphosphan.

\begin{tabular}{|ll|ll|}
\hline Strukturcode & $\left(i \mathrm{Pr}_{2} \mathrm{~N}\right)_{2} \mathrm{PCl}$ & $\mu\left[\mathrm{mm}^{-1}\right]$ & 0.325 \\
Summenformel & $\mathrm{C}_{12} \mathrm{H}_{28} \mathrm{~N}_{2} \mathrm{PCl}$ & $\mathrm{F}(000)$ & 2336 \\
Molmasse $[\mathrm{g} / \mathrm{mol}]$ & 266.78 & $\theta$-Bereich [ $\left.{ }^{\circ}\right]$ & $1.38-26.78$ \\
Kristallgröße [mm] & $0.3 \times 0.1 \times 0.1$ & gesammelte Reflexe & 51254 \\
Raumgruppe & $C 2 / c$ & unabhängige Reflexe & 6912 \\
$a[\mathrm{pm}]$ & $2178.34(9)$ & Zahl der Restraints & 108 \\
$b[\mathrm{pm}]$ & $2177.07(9)$ & verfeinerte Parameter & 580 \\
$c[\mathrm{pm}]$ & $1437.17(6)$ & $R 1[\mathrm{I}>2 \sigma(\mathrm{I})]$ & 0.0338 \\
$\alpha\left[^{\circ}\right]$ & 90 & WR2 [alle Daten] & 0.0900 \\
$\beta\left[^{\circ}\right]$ & $112.2880(10)$ & GooF & 1.199 \\
$\gamma\left[^{\circ}\right]$ & 90 & Differenzelektronendichte: & \\
$V\left[\mathrm{~nm}^{3}\right]$ & $6.3064(5)$ & Max. / min. $\left[10^{-6}\right.$ epm $\left.{ }^{-3}\right]$ & $0.228 /-0.256$ \\
$Z$ & 18 & Max. / min. Transmission & $0.6965 / 0.7454$ \\
$\mathrm{Messtemperatur}[\mathrm{K}]^{3}$ & $100(2)$ & & \\
$\rho\left[\mathrm{Mgm}^{-3}\right]$ & 1.124 & &
\end{tabular}




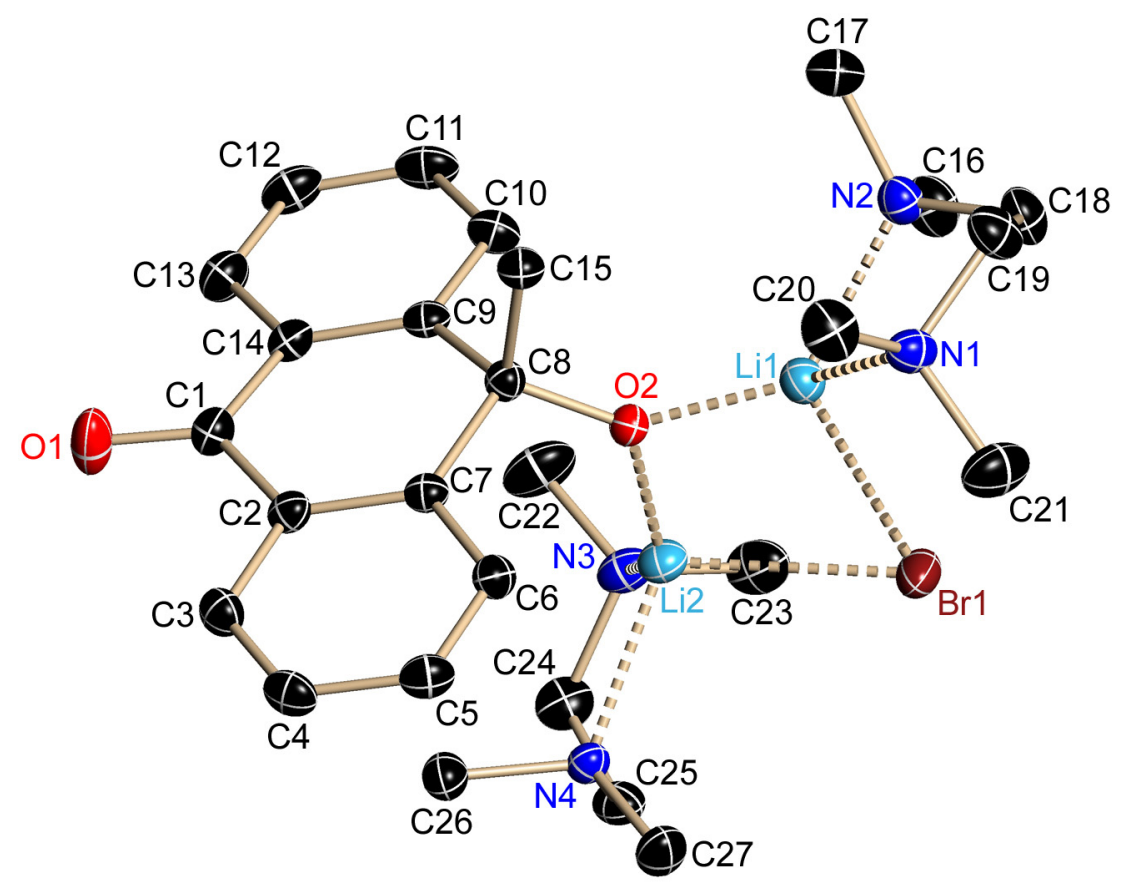

Abb. 5.65: Ausschnitt der asymmetrischen Einheit von [OAnMeOLi $\left.\cdot \mathrm{LiBr} \cdot\{\mathrm{tmeda}\}_{2}\right]$; die anisotropen Auslenkungsparameter geben die $50 \%$ ige Aufenthaltswahrscheinlichkeit an.

Tab. 5.57: Kristallographische Daten von [OAnMeOLi $\left.\cdot \mathrm{LiBr} \cdot\{\text { tmeda }\}_{2}\right]$.

\begin{tabular}{|c|c|c|c|}
\hline Strukturcode & {$\left[\mathrm{OAnMeOLi} \cdot \mathrm{LiBr} \cdot\left\{\right.\right.$ tmeda $\left._{2}\right]$} & $\mu\left[\mathrm{mm}^{-1}\right]$ & 1.417 \\
\hline Summenformel & $\mathrm{C}_{27} \mathrm{H}_{43} \mathrm{Li}_{2} \mathrm{~N}_{4} \mathrm{O}_{2} \mathrm{Br}$ & $F(000)$ & 580 \\
\hline Molmasse $[\mathrm{g} / \mathrm{mol}]$ & 549.44 & $\theta$-Bereich $\left[^{\circ}\right]$ & $2.15-25.42$ \\
\hline Kristallgröße [mm] & $0.17 \times 0.15 \times 0.12$ & gesammelte Reflexe & 21502 \\
\hline Raumgruppe & $P \overline{1}$ & unabhängige Reflexe & 5450 \\
\hline$a[\mathrm{pm}]$ & 1014.66(10) & Zahl der Restraints & 0 \\
\hline$b[\mathrm{pm}]$ & $1213.14(12)$ & verfeinerte Parameter & 334 \\
\hline$c[\mathrm{pm}]$ & $1399.12(14)$ & $R 1[I>2 \sigma(I)]$ & 0.0295 \\
\hline$\alpha\left[^{\circ}\right]$ & $66.9600(10)$ & $w R 2$ [alle Daten] & 0.0757 \\
\hline$\beta\left[^{\circ}\right]$ & $76.171(2)$ & GooF & 1.081 \\
\hline$\gamma\left[^{\circ}\right]$ & $70.229(2)$ & & \\
\hline$V\left[\mathrm{~nm}^{3}\right]$ & $1.4801(3)$ & Differenzelektronendichte: & \\
\hline$Z$ & 2 & Max. / min. $\left[10^{-6} \mathrm{epm}^{-3}\right]$ & $0.679 /-0.602$ \\
\hline Messtemperatur [K] & $100(2)$ & Max. / min. Transmission & $0.3747 / 0.4288$ \\
\hline$\rho\left[\mathrm{Mgm}^{-3}\right]$ & 1.233 & & \\
\hline
\end{tabular}




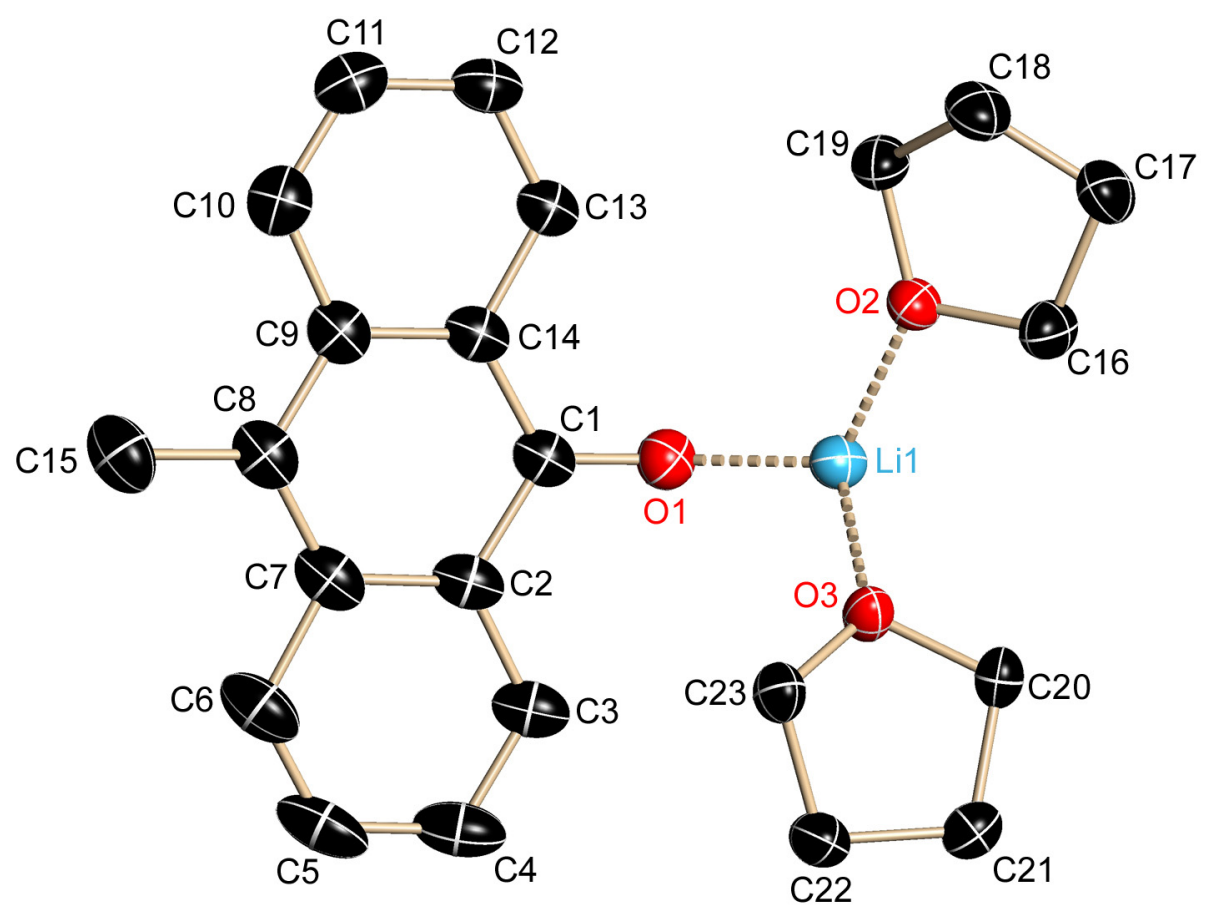

Abb. 5.66: Ausschnitt der asymmetrischen Einheit von $\left[\mathrm{MeAnOLi} \cdot\{\text { thf }\}_{2}\right]_{2}$; die anisotropen Auslenkungsparameter geben die $50 \%$ ige Aufenthaltswahrscheinlichkeit an.

Tab. 5.58: Kristallographische Daten von $\left[\mathrm{MeAnOLi} \cdot\{\text { thf }\}_{2}\right]_{2}$.

\begin{tabular}{|c|c|c|c|}
\hline Strukturcode & {$\left[\mathrm{MeAnOLi} \cdot\{\text { thf }\}_{2}\right]_{2}$} & $\mu\left[\mathrm{mm}^{-1}\right]$ & 0.081 \\
\hline Summenformel & $\mathrm{C}_{46} \mathrm{H}_{54} \mathrm{Li}_{2} \mathrm{O}_{6}$ & $F(000)$ & 768 \\
\hline Molmasse [g/mol] & 716.77 & $\theta$-Bereich $\left[^{\circ}\right]$ & $2.06-26.83$ \\
\hline Kristallgröße [mm] & $0.1 \times 0.1 \times 0.1$ & gesammelte Reflexe & 30168 \\
\hline Raumgruppe & $P 2_{1} / n$ & unabhängige Reflexe & 4036 \\
\hline$a[\mathrm{pm}]$ & $1180.84(7)$ & Zahl der Restraints & 0 \\
\hline$b[\mathrm{pm}]$ & $934.52(6)$ & verfeinerte Parameter & 245 \\
\hline$c[\mathrm{pm}]$ & $1715.30(10)$ & $R 1[\mid>2 \sigma(I)]$ & 0.0553 \\
\hline$\alpha\left[^{\circ}\right]$ & 90 & $w R 2$ [alle Daten] & 0.1461 \\
\hline$\beta\left[^{\circ}\right]$ & $92.3000(10)$ & GooF & 1.055 \\
\hline$\gamma\left[^{\circ}\right]$ & 90 & & \\
\hline$V\left[\mathrm{~nm}^{3}\right]$ & $1.8913(2)$ & Differenzelektronendichte: & \\
\hline$Z$ & 2 & Max. / min. $\left[10^{-6} \mathrm{epm}^{-3}\right]$ & $0.391 /-0.310$ \\
\hline Messtemperatur [K] & $100(2)$ & Max. / min. Transmission & 0.7817 / 0.8620 \\
\hline$\rho\left[\mathrm{Mgm}^{-3}\right]$ & 1.259 & & \\
\hline
\end{tabular}




\subsubsection{Strukturen des AK Roesky (Universität Göttingen)}

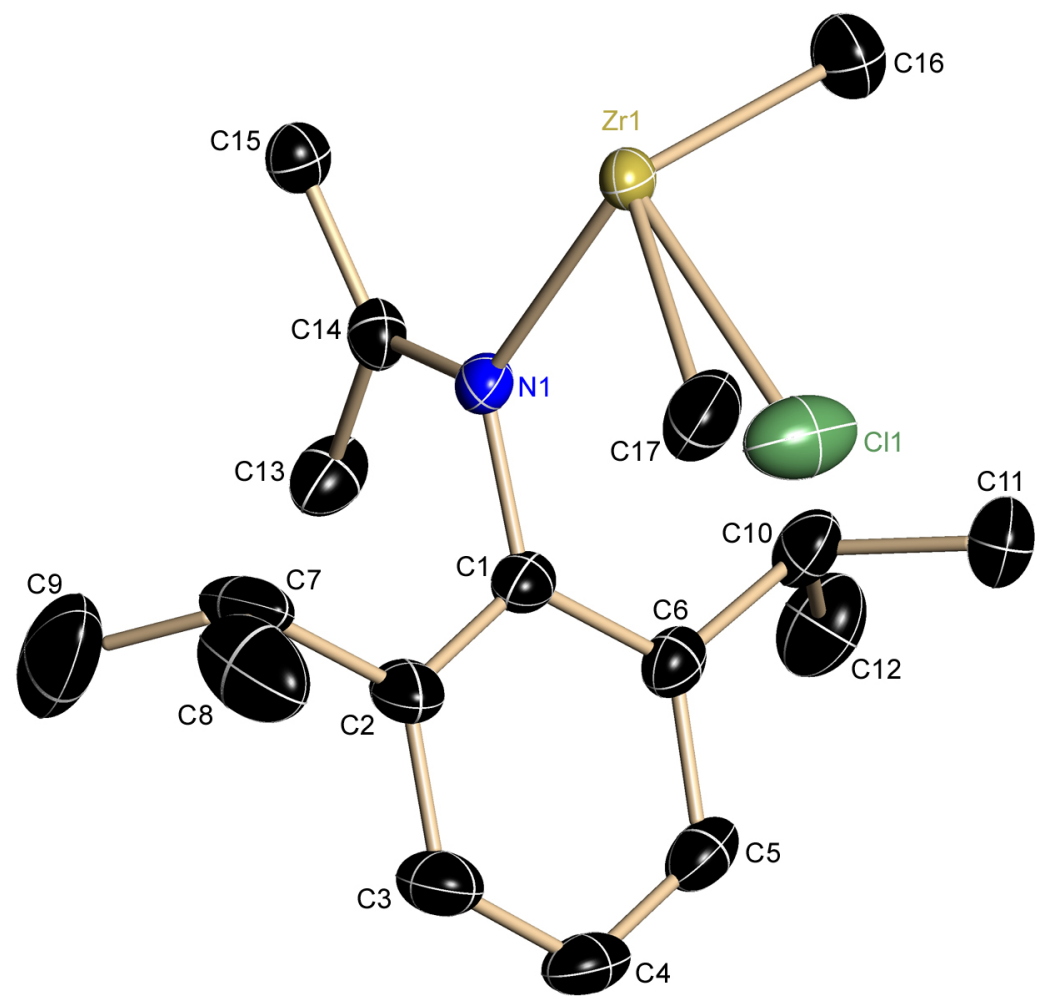

Abb. 5.67: Asymmetrische Einheit von Bj209; die anisotropen Auslenkungsparameter geben die $50 \%$ ige Aufenthaltswahrscheinlichkeit an.

Tab. 5.59: Kristallographische Daten von Bj209.

\begin{tabular}{|ll|ll|}
\hline Strukturcode & $\mathrm{Bj} 209$ & $\rho\left[\mathrm{Mgm}^{-3}\right]$ & 1.263 \\
Summenformel & $\mathrm{C}_{31} \mathrm{H}_{47} \mathrm{ClN} \mathrm{Zr}_{2} \mathrm{Zr}$ & $\mu\left[\mathrm{mm}^{-1}\right]$ & 0.473 \\
Molekulare Masse [g/mol] & 574.38 & $F(000)$ & 1216 \\
Kristallgröße [mm] & $0.2 \times 0.1 \times 0.1$ & $\theta$-Bereich [ $\left.{ }^{\circ}\right]$ & 1.88 to 26.76 \\
Raumgruppe & $P n m a$ & Gesammelte Reflexe & 65230 \\
$a[\mathrm{pm}]$ & $1425.79(14)$ & Unabhängige Reflexe & 3293 \\
$b[\mathrm{pm}]$ & $2166.4(2)$ & Restraints & 7 \\
$c[\mathrm{pm}]$ & $978.10(10)$ & Parameter & 179 \\
$\alpha\left[^{\circ}\right]$ & 90 & $R 1[I>2 \sigma(I)]$ & 0.0286 \\
$\beta\left[^{\circ}\right]$ & 90 & $w R 2[$ alle Daten] & 0.0750 \\
$\gamma\left[^{\circ}\right]$ & 90 & Goof & 1.042 \\
$\mathrm{~V}\left[\mathrm{~nm}^{3}\right]$ & Restdichte: & \\
$Z$ & $3.0212(5)$ & Max. $/$ min. $\left[10^{-6} \mathrm{epm}^{-3}\right]$ & $0.550 /-0.452$ \\
Temperatur $[\mathrm{K}]$ & 4 & Max. $/$ min. Transmission & $0.9458 / 0.8657$ \\
\hline
\end{tabular}




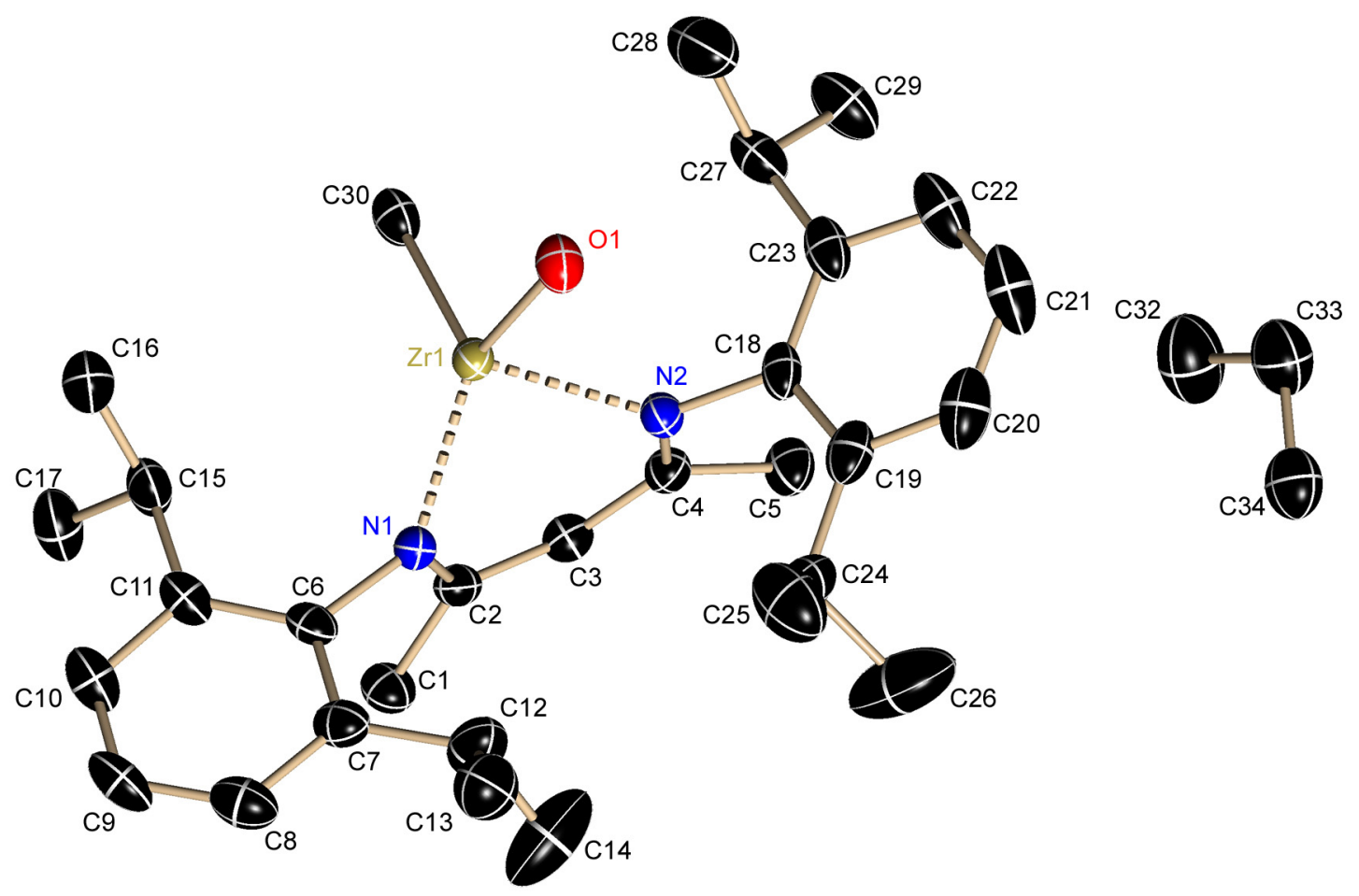

Abb. 5.68: Asymmetrische Einheit von Bj214; die anisotropen Auslenkungsparameter geben die 50\% ige Aufenthaltswahrscheinlichkeit an.

Tab. 5.60: Kristallographische Daten von Bj214.

\begin{tabular}{|c|c|c|c|}
\hline Strukturcode & Bj214 & $\rho\left[\mathrm{Mgm}^{-3}\right]$ & 1.246 \\
\hline Summenformel & $\mathrm{C}_{33} \mathrm{H}_{51} \mathrm{~N}_{2} \mathrm{OZr}$ & $\mu\left[\mathrm{mm}^{-1}\right]$ & 0.380 \\
\hline Molekulare Masse $[\mathrm{g} / \mathrm{mol}]$ & 582.98 & $F(000)$ & 1244 \\
\hline Kristallgröße [mm] & $0.24 \times 0.23 \times 0.21$ & $\theta$-Bereich $\left[^{\circ}\right]$ & $1.55-25.34$ \\
\hline Raumgruppe & $P 2_{1} / c$ & Gesammelte Reflexe & 5693 \\
\hline$a[\mathrm{pm}]$ & $1346.5(2)$ & Unabhängige Reflexe & 5693 \\
\hline$b[\mathrm{pm}]$ & $1356.2(2)$ & Restraints & 36 \\
\hline$c[\mathrm{pm}]$ & $1749.1(3)$ & Parameter & 346 \\
\hline$\alpha\left[^{\circ}\right]$ & 90 & $R 1[I>2 \sigma(I)]$ & 0.0408 \\
\hline$\beta\left[^{\circ}\right]$ & $103.272(2)$ & $w R 2$ [alle Daten] & 0.1030 \\
\hline$\gamma\left[^{\circ}\right]$ & 90 & g1 / g2 & $0.0436 / 5.0433$ \\
\hline $\mathrm{V}\left[\mathrm{nm}^{3}\right]$ & $3.1089(8)$ & Restdichte: & \\
\hline$Z$ & 4 & Max. / min. $\left[10^{-6} \mathrm{epm}^{-3}\right]$ & $0.919 /-0.666$ \\
\hline Temperatur [K] & $100(2)$ & Max. / min. Transmission & $0.4289 / 0.3785$ \\
\hline
\end{tabular}




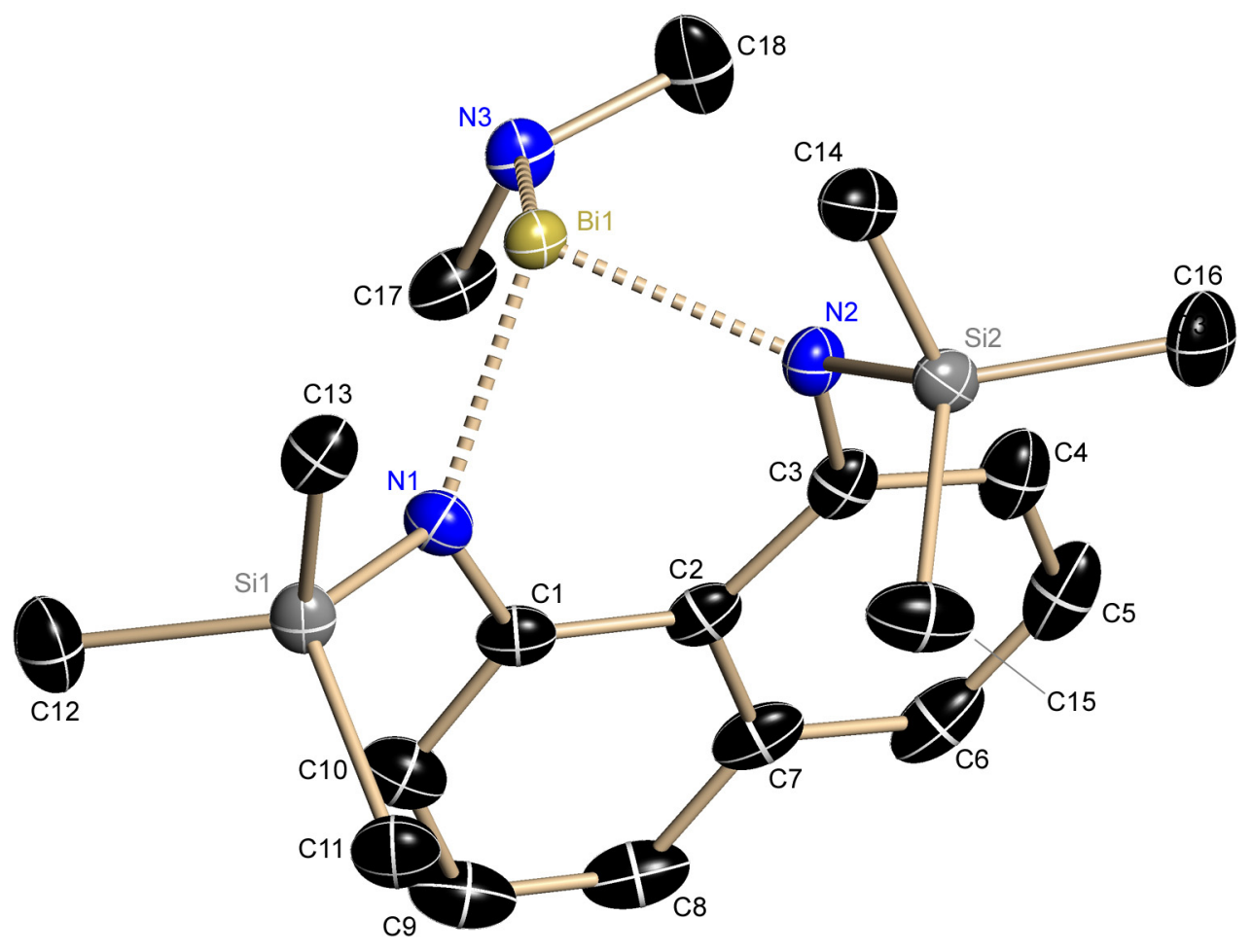

Abb. 5.69: Asymmetrische Einheit von Bj231; die anisotropen Auslenkungsparameter geben die $50 \%$ ige Aufenthaltswahrscheinlichkeit an.

Tab. 5.61: Kristallographische Daten von Bj231.

\begin{tabular}{|ll|ll|}
\hline Strukturcode & $\mathrm{Bj} 231$ & $\rho\left[\mathrm{Mgm}^{-3}\right]$ & 1.728 \\
Summenformel & $\mathrm{C}_{18} \mathrm{H}_{30} \mathrm{BiN}_{3} \mathrm{Si}_{2}$ & $\mu\left[\mathrm{mm}^{-1}\right]$ & 8.405 \\
Molekulare Masse [g/mol] & 553.61 & $F(000)$ & 1080 \\
Kristallgröße [mm] & $0.18 \times 0.15 \times 0.15$ & $\theta$-Bereich [ $\left.{ }^{\circ}\right]$ & $2.02-27.48$ \\
Raumgruppe & $P 21 / n$ & Gesammelte Reflexe & 35183 \\
$a[\mathrm{pm}]$ & $998.70(9)$ & Unabhängige Reflexe & 4871 \\
$b[\mathrm{pm}]$ & $1259.84(12)$ & Restraints & 0 \\
$c[\mathrm{pm}]$ & $1693.83(16)$ & Parameter & 225 \\
$\alpha\left[^{\circ}\right]$ & $R 1[/>2 \sigma(I)]$ & 0.0138 \\
$\beta\left[^{\circ}\right]$ & 90 & $W R 2$ [alle Daten] & 0.0331 \\
$\gamma\left[^{\circ}\right]$ & $93.3040(10)$ & g1 / g2 & 1.081 \\
$\mathrm{~V}\left[\mathrm{~nm}{ }^{3}\right]$ & 90 & Restdichte: & \\
$Z$ & $2.1276(3)$ & Max. / min. [10 $\left.{ }^{-6} \mathrm{epm}^{-3}\right]$ & $0.439 /-0.677$ \\
Temperatur $[\mathrm{K}]$ & 4 & Max. / min. Transmission & $0.4308 / 0.2846$ \\
\hline
\end{tabular}




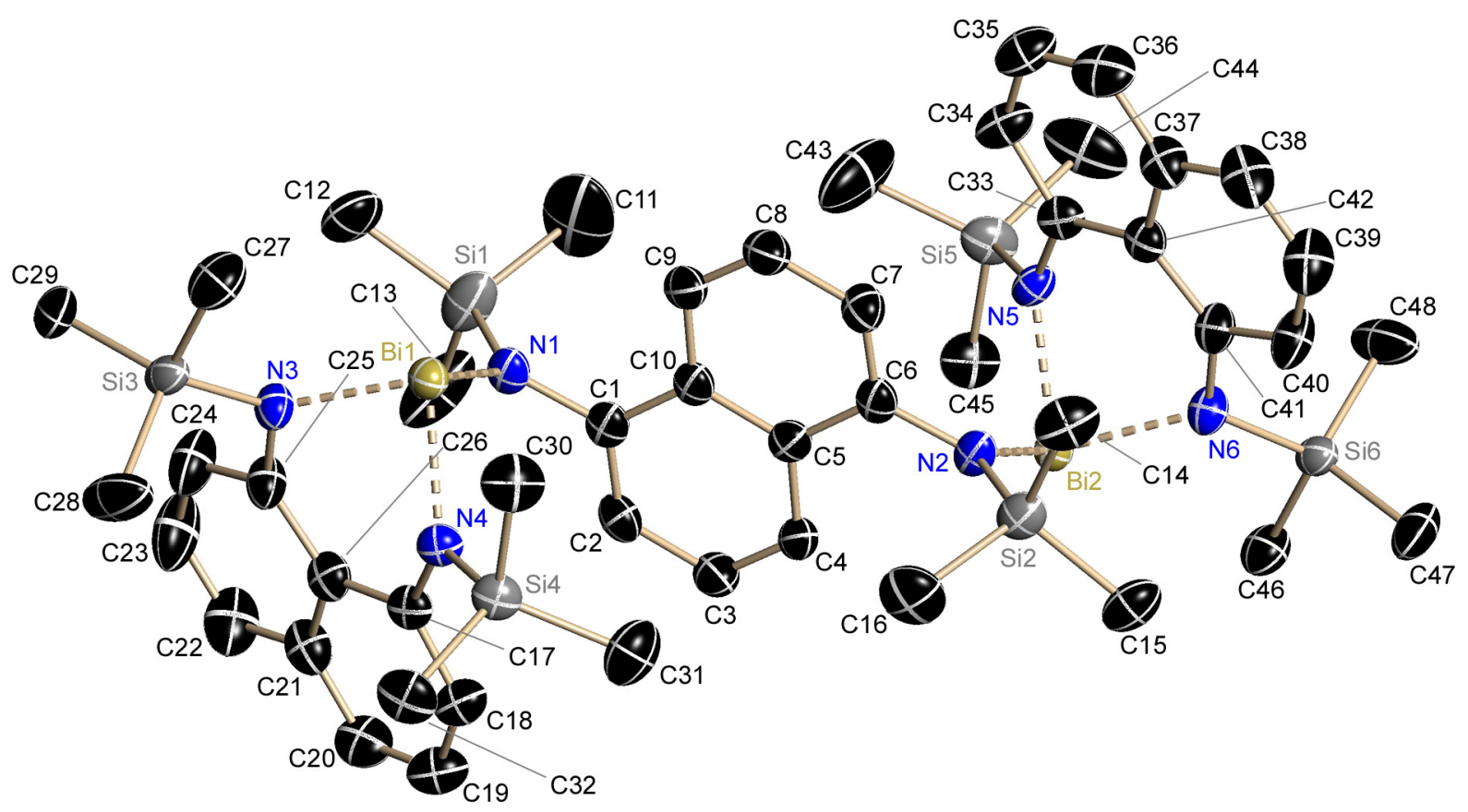

Abb. 5.70: Asymmetrische Einheit von Bj232; die anisotropen Auslenkungsparameter geben die $50 \%$ ige Aufenthaltswahrscheinlichkeit an.

Tab. 5.62: Kristallographische Daten von Bj232.

\begin{tabular}{|ll|ll|}
\hline Strukturcode & $\mathrm{Bj} 232$ & $\rho\left[\mathrm{Mgm}^{-3}\right]$ & 1.597 \\
Summenformel & $\mathrm{C}_{48} \mathrm{H}_{72} \mathrm{Bi}_{2} \mathrm{~N}_{6} \mathrm{Si}_{6}$ & $\mu\left[\mathrm{mm}^{-1}\right]$ & 6.573 \\
Molekulare Masse [g/mol] & 1319.62 & $F(000)$ & 2608 \\
Kristallgröße [mm] & $0.17 \times 0.14 \times 0.10$ & $\theta$-Bereich [ $\left.{ }^{\circ}\right]$ & $1.02-26.73$ \\
Raumgruppe & $P 2{ }_{1} / c$ & Gesammelte Reflexe & 11676 \\
$a[\mathrm{pm}]$ & $2008.1(3)$ & Unabhängige Reflexe & 11676 \\
$b[\mathrm{pm}]$ & $2082.7(3)$ & Restraints & 0 \\
$c[\mathrm{pm}]$ & $1316.5(2)$ & Parameter & 577 \\
$\left.\alpha{ }^{\circ}\right]$ & 90 & $R 1[/>2 \sigma(I)]$ & 0.0405 \\
$\beta\left[^{\circ}\right]$ & wR2 [alle Daten] & 0.1135 \\
$\gamma\left[^{\circ}\right]$ & g1 / g2 & 1.097 \\
$\mathrm{~V}\left[\mathrm{~nm}{ }^{3}\right]$ & $90.712(2)$ & Restdichte: & \\
$Z$ & $5.4873(15)$ & Max. / min. [10 $\left.{ }^{-6} \mathrm{epm}^{-3}\right]$ & $3.697 /-2.754$ \\
Temperatur $[\mathrm{K}]$ & 4 & Max. / min. Transmission & $0.4305 / 0.2509$ \\
\hline
\end{tabular}




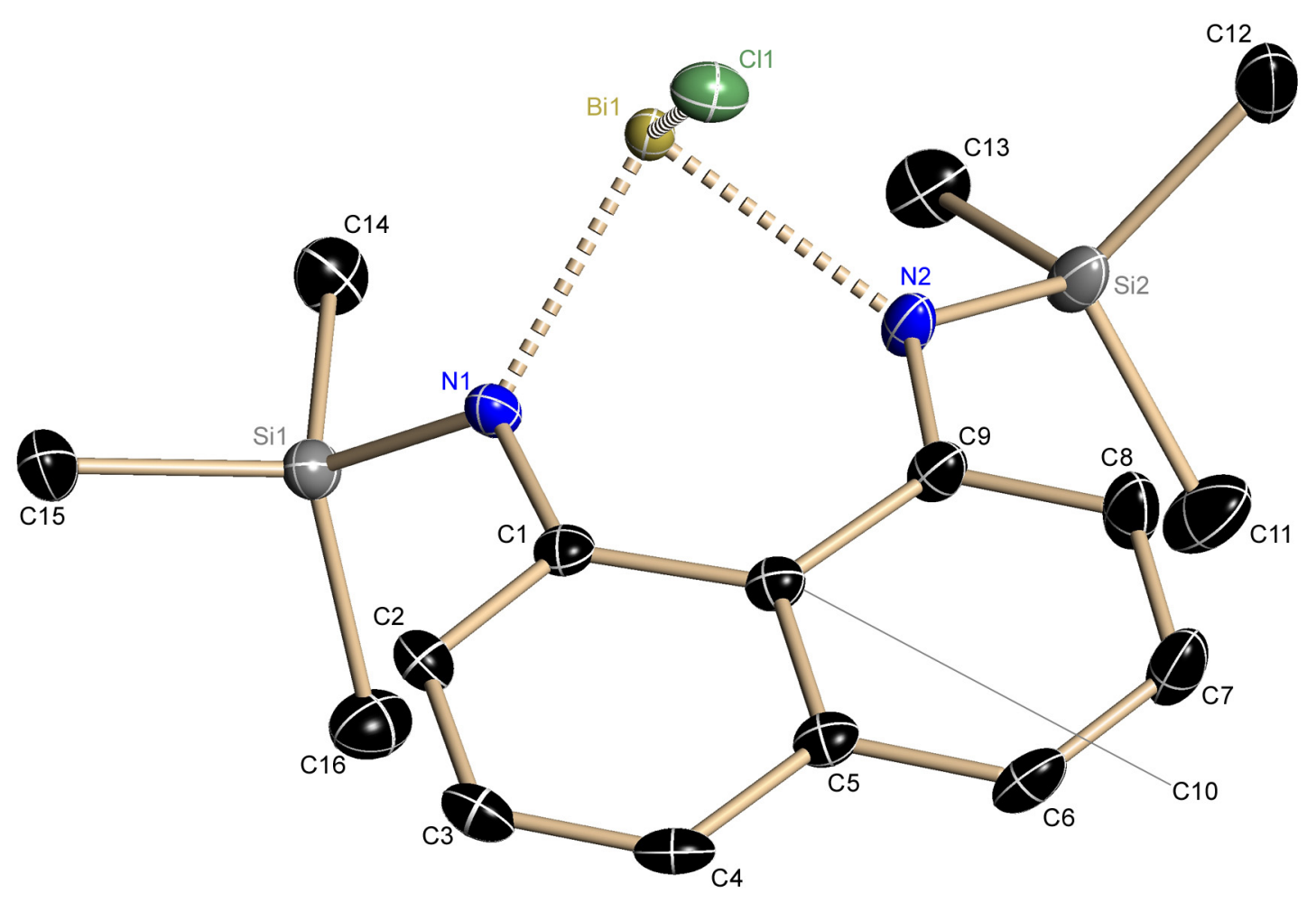

Abb. 5.71: Asymmetrische Einheit von Bj243; die anisotropen Auslenkungsparameter geben die $50 \%$ ige Aufenthaltswahrscheinlichkeit an.

Tab. 5.63: Kristallographische Daten von Bj243.

\begin{tabular}{|c|c|c|c|}
\hline Strukturcode & Bj243 & $\rho\left[\mathrm{Mgm}^{-3}\right]$ & 1.765 \\
\hline Summenformel & $\mathrm{C}_{16} \mathrm{H}_{24} \mathrm{BiClN}_{2} \mathrm{Si}_{2}$ & $\mu\left[\mathrm{mm}^{-1}\right]$ & 8.840 \\
\hline Molekulare Masse $[\mathrm{g} / \mathrm{mol}]$ & 544.98 & $F(000)$ & 2096 \\
\hline Kristallgröße [mm] & $0.14 \times 0.14 \times 0.13$ & $\theta$-Bereich $\left[{ }^{\circ}\right]$ & $1.50-26.75$ \\
\hline Raumgruppe & $1 \overline{4}$ & Gesammelte Reflexe & 33075 \\
\hline$a[\mathrm{pm}]$ & 1924.78(14) & Unabhängige Reflexe & 4367 \\
\hline$b[\mathrm{pm}]$ & 1924.78(14) & Restraints & 0 \\
\hline$c[\mathrm{pm}]$ & 1107.43(17) & Parameter & 205 \\
\hline$\alpha\left[^{\circ}\right]$ & 90 & $R 1[I>2 \sigma(I)]$ & 0.0114 \\
\hline$\beta\left[^{\circ}\right]$ & 90 & $w R 2$ [alle Daten] & 0.0289 \\
\hline$\gamma\left[{ }^{\circ}\right]$ & 90 & g1 / g2 & 1.093 \\
\hline$V\left[\mathrm{~nm}^{3}\right]$ & $4.1028(8)$ & Restdichte: & \\
\hline$z$ & 8 & Max. / min. $\left[10^{-6} \mathrm{epm}^{-3}\right]$ & $0.565 /-0.485$ \\
\hline Temperatur [K] & $100(2)$ & Max. / min. Transmission & 0.2499 / 0.3625 \\
\hline
\end{tabular}




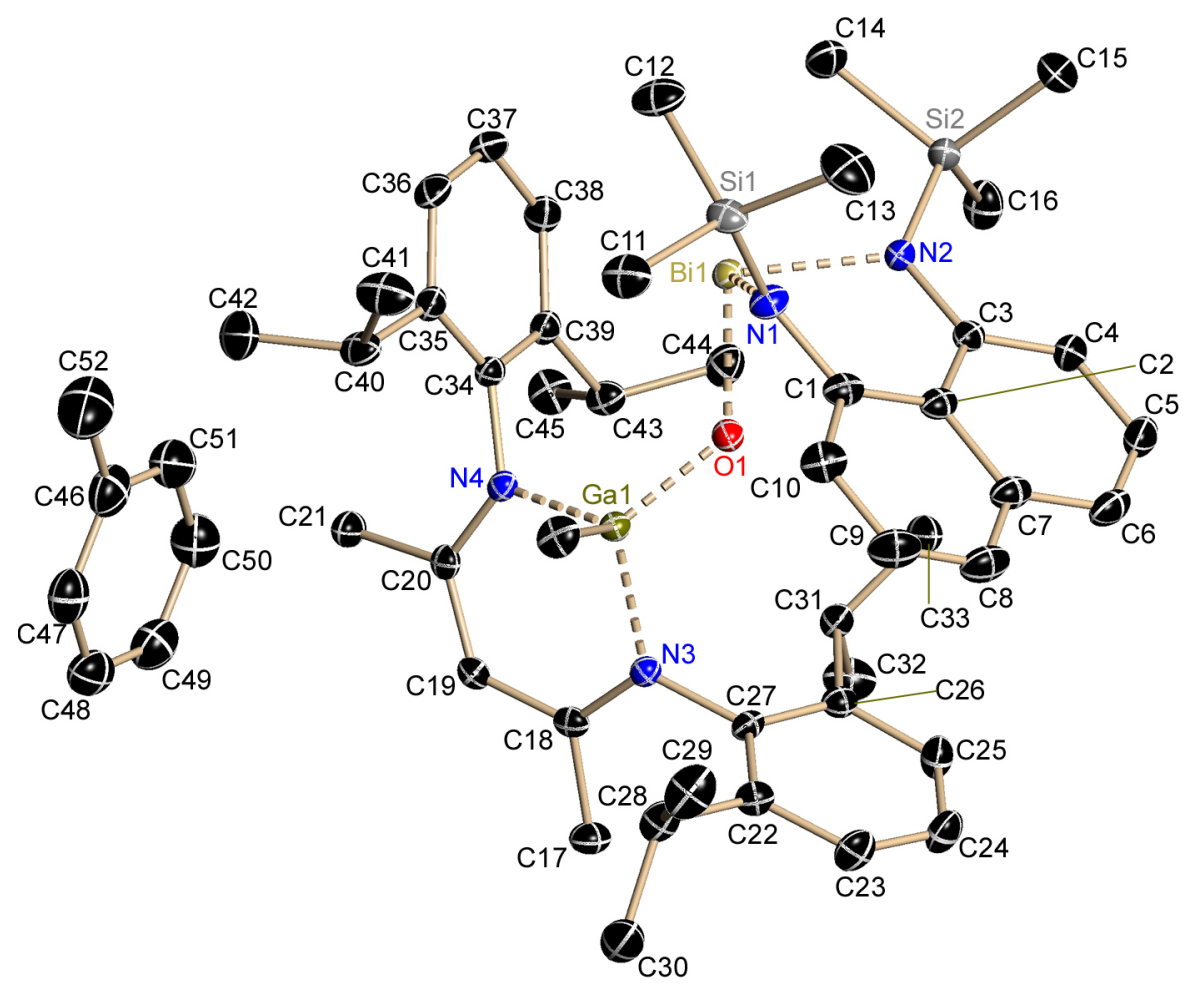

Abb. 5.72: Asymmetrische Einheit von Bj250; die anisotropen Auslenkungsparameter geben die $50 \%$ ige Aufenthaltswahrscheinlichkeit an.

Tab. 5.64: Kristallographische Daten von Bj250.

\begin{tabular}{|ll|ll|}
\hline Strukturcode & $\mathrm{Bj} 250$ & $\rho\left[\mathrm{Mgm}^{-3}\right]$ & 1.399 \\
Summenformel & $\mathrm{C}_{53} \mathrm{H}_{76} \mathrm{BiGaN}_{4} \mathrm{OSi}_{2}$ & $\mu\left[\mathrm{mm}^{-1}\right]$ & 3.894 \\
Molekulare Masse $[\mathrm{g} / \mathrm{mol}]$ & 1120.06 & $F(000)$ & 2288 \\
Kristallgröße [mm] & $0.1 \times 0.1 \times 0.1$ & $\theta$-Bereich [ $\left.{ }^{\circ}\right]$ & $2.05-26.77$ \\
Raumgruppe & $P 2_{1} / c$ & Gesammelte Reflexe & 11321 \\
$a[\mathrm{pm}]$ & $1926.51(17)$ & Unabhängige Reflexe & 11321 \\
$b[\mathrm{pm}]$ & $1071.95(10)$ & Restraints & 0 \\
$c[\mathrm{pm}]$ & $2611.8(2)$ & Parameter & 577 \\
$\alpha\left[^{\circ}\right]$ & 90 & $R 1[/>2 \sigma(I)]$ & 0.0176 \\
$\beta\left[^{\circ}\right]$ & $99.5340(10)$ & wR2 [alle Daten] & 0.0397 \\
$\gamma\left[^{\circ}\right]$ & 90 & g1/g2 & 1.036 \\
$\mathrm{~V}\left[\mathrm{~nm}^{3}\right]$ & $5.3192(8)$ & Restdichte: & \\
$Z$ & 4 & Max. / min. [10 $\left.{ }^{-6} \mathrm{epm}^{-3}\right]$ & $0.418 /-0.403$ \\
Temperatur $[\mathrm{K}]^{2}$ & $100(2)$ & Max. / min. Transmission & $0.4299 / 0.3684$ \\
\hline
\end{tabular}



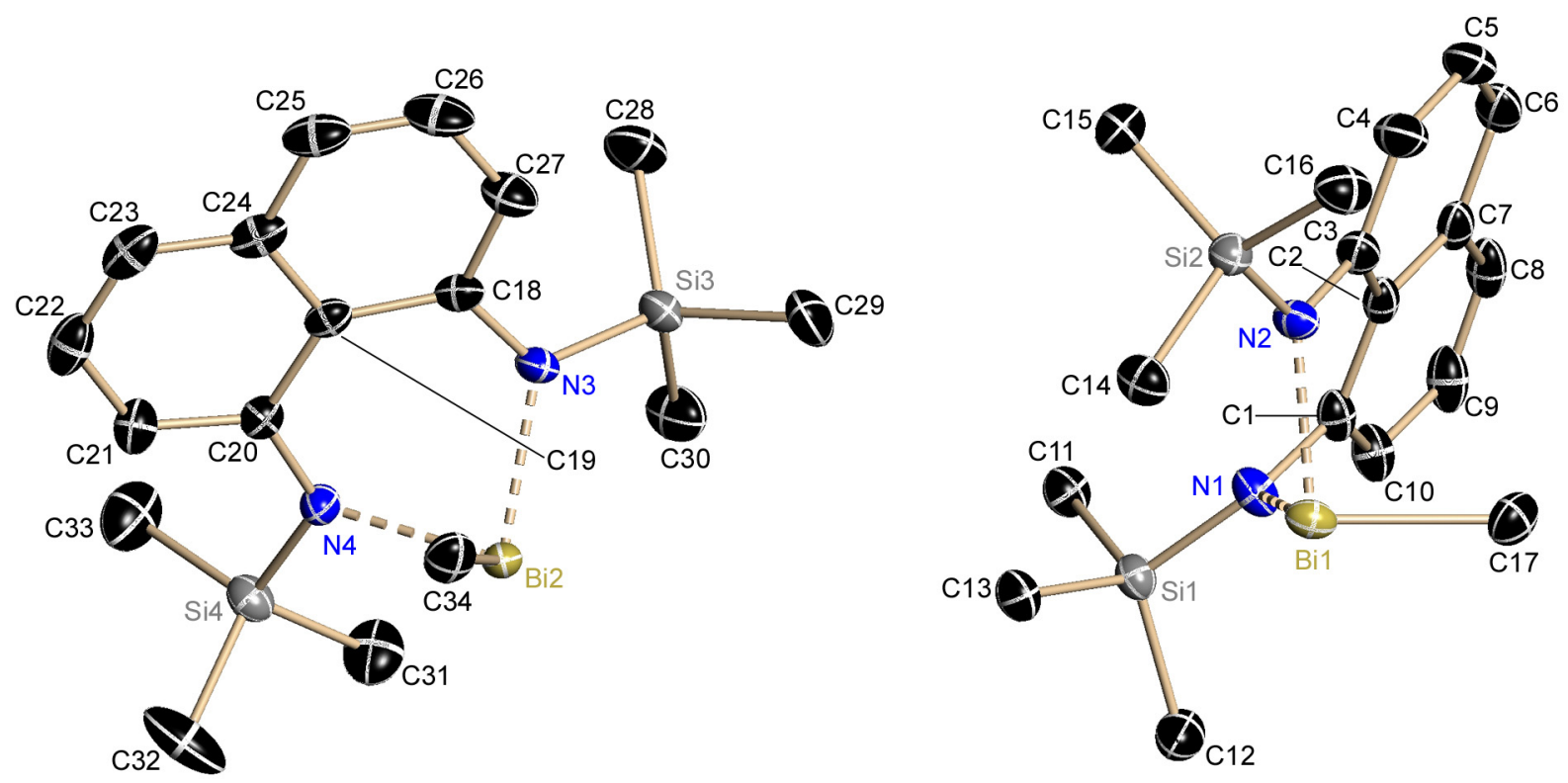

Abb. 5.73: Asymmetrische Einheit von Bj251; die anisotropen Auslenkungsparameter geben die $50 \%$ ige Aufenthaltswahrscheinlichkeit an.

Tab. 5.65: Kristallographische Daten von Bj251.

\begin{tabular}{|ll|ll|}
\hline Strukturcode & $\mathrm{Bj} 251$ & $\rho\left[\mathrm{Mgm}^{-3}\right]$ & 1.691 \\
Summenformel & $\mathrm{C}_{17} \mathrm{H}_{27} \mathrm{BiN}_{2} \mathrm{Si}_{2}$ & $\mu\left[\mathrm{mm}^{-1}\right]$ & 8.671 \\
Molekulare Masse [g/mol] & 524.57 & $F(000)$ & 2032 \\
Kristallgröße [mm] & $0.12 \times 0.11 \times 0.1$ & $\theta$-Bereich [ $\left.{ }^{\circ}\right]$ & $1.25-27.66$ \\
Raumgruppe & $P 2_{1} / n$ & Gesammelte Reflexe & 108907 \\
$a[\mathrm{pm}]$ & $1906.73(9)$ & Unabhängige Reflexe & 9604 \\
$b[\mathrm{pm}]$ & $1178.76(5)$ & Restraints & 0 \\
$c[\mathrm{pm}]$ & $1995.82(9)$ & Parameter & 411 \\
$\alpha\left[^{\circ}\right]$ & 90 & $R 1[I>2 \sigma(I)]$ & 0.0168 \\
$\beta\left[^{\circ}\right]$ & $113.2460(10)$ & wR2 [alle Daten] & 0.0409 \\
$\gamma\left[^{\circ}\right]$ & 90 & g1/g2 & 1.092 \\
$\mathrm{~V}\left[\mathrm{~nm}{ }^{3}\right]$ & $4.1216(3)$ & Restdichte: & \\
$Z$ & 8 & Max. / min. [10 & epm $\left.{ }^{-3}\right]$ \\
Temperatur $[\mathrm{K}]$ & $100(2)$ & Max. / min. Transmission & $1.033 /-1.282$ \\
\hline
\end{tabular}




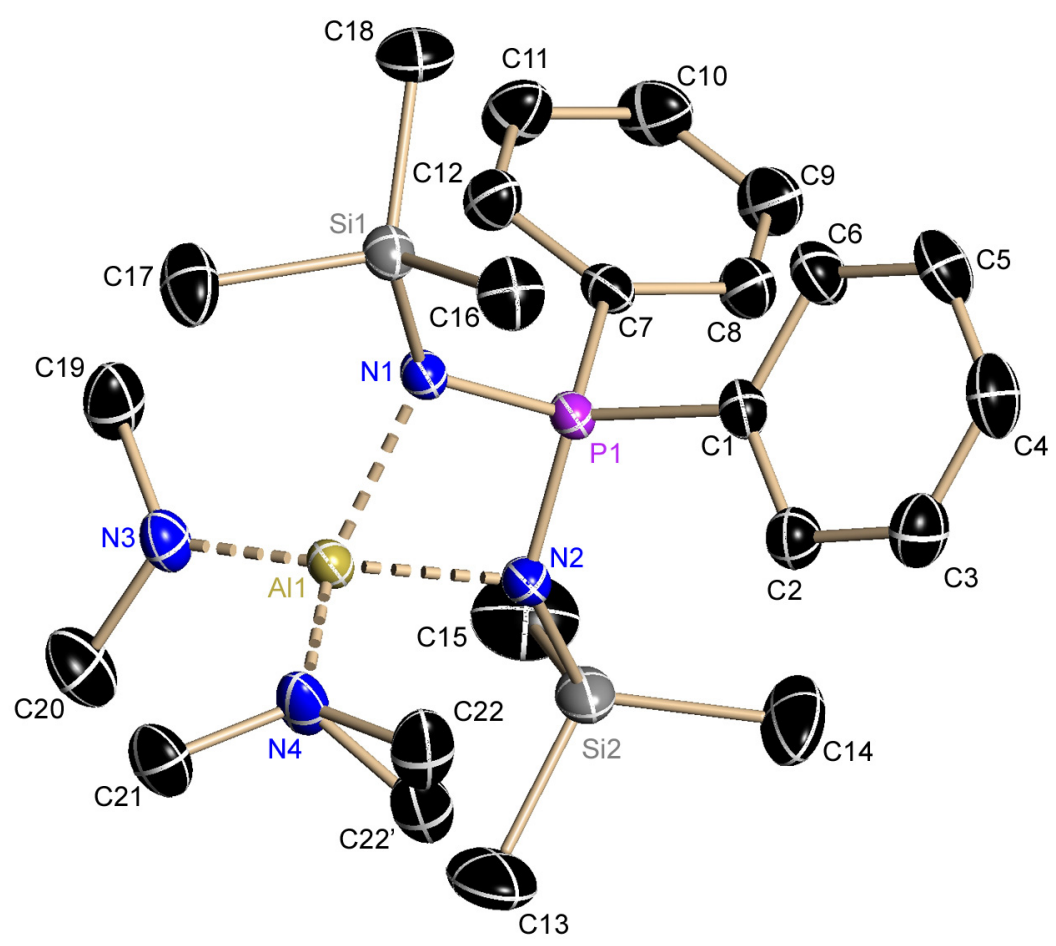

Abb. 5.74: Asymmetrische Einheit von Bj260; die anisotropen Auslenkungsparameter geben die 50\% ige Aufenthaltswahrscheinlichkeit an.

Tab. 5.66: Kristallographische Daten von Bj260.

\begin{tabular}{|c|c|c|c|}
\hline Strukturcode & Bj260 & $\rho\left[\mathrm{Mgm}^{-3}\right]$ & 1.126 \\
\hline Summenformel & $\mathrm{C}_{22} \mathrm{H}_{40} \mathrm{AlN}_{4} \mathrm{PSi}_{2}$ & $\mu\left[\mathrm{mm}^{-1}\right]$ & 0.231 \\
\hline Molekulare Masse $[\mathrm{g} / \mathrm{mol}]$ & 474.71 & $F(000)$ & 1024 \\
\hline Kristallgröße [mm] & $0.21 \times 0.2 \times 0.2$ & $\theta$-Bereich $\left[{ }^{\circ}\right]$ & 1.84 to 27.51 \\
\hline Raumgruppe & $P 2_{1} / n$ & Gesammelte Reflexe & 72209 \\
\hline$a[\mathrm{pm}]$ & 1129.41(8) & Unabhängige Reflexe & 6427 \\
\hline$b[\mathrm{pm}]$ & $1714.85(12)$ & Restraints & 0 \\
\hline$c[\mathrm{pm}]$ & $1446.40(10)$ & Parameter & 292 \\
\hline$\alpha\left[^{\circ}\right]$ & 90 & $R 1[I>2 \sigma(I)]$ & 0.0361 \\
\hline$\beta\left[^{\circ}\right]$ & $91.8650(10)$ & $w R 2$ [alle Daten] & 0.0989 \\
\hline$\gamma\left[^{\circ}\right]$ & 90 & g1 / g2 & 1.077 \\
\hline$V\left[\mathrm{~nm}^{3}\right]$ & $2.7999(3)$ & Restdichte: & \\
\hline$Z$ & 4 & Max. / min. $\left[10^{-6} \mathrm{epm}^{-3}\right]$ & $0.342 /-0.280$ \\
\hline Temperatur $[\mathrm{K}]$ & $100(2)$ & Max. / min. Transmission & $0.4305 / 0.3824$ \\
\hline
\end{tabular}



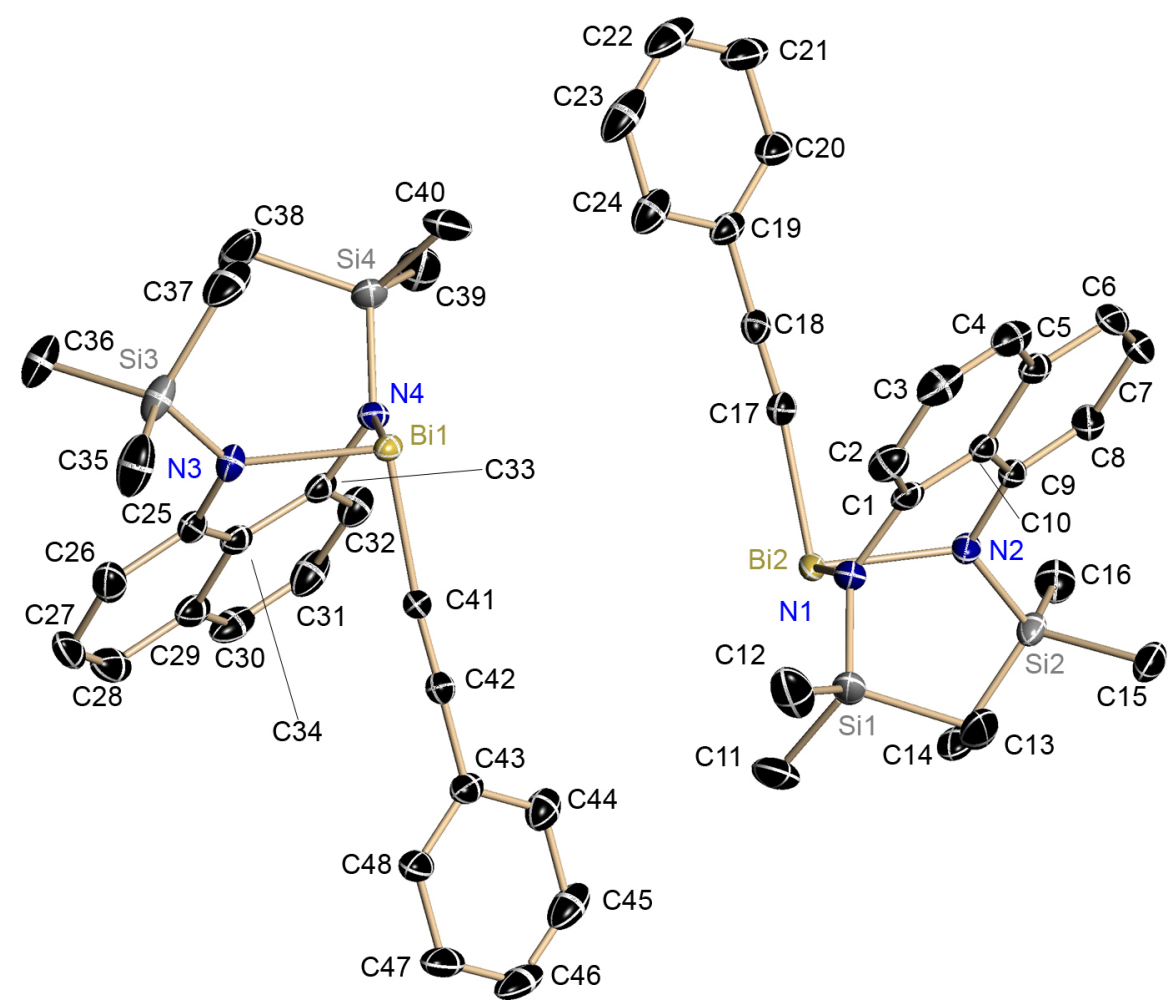

Abb. 5.75: Asymmetrische Einheit von Bj263; die anisotropen Auslenkungsparameter geben die $50 \%$ ige Aufenthaltswahrscheinlichkeit an.

Tab. 5.67: Kristallographische Daten von Bj263.

\begin{tabular}{|c|c|c|c|}
\hline Strukturcode & Bj263 & $\rho\left[\mathrm{Mgm}^{-3}\right]$ & 1.626 \\
\hline Summenformel & $\mathrm{C}_{24} \mathrm{H}_{29} \mathrm{BiN}_{2} \mathrm{Si}_{2}$ & $\mu\left[\mathrm{mm}^{-1}\right]$ & 7.175 \\
\hline Molekulare Masse $[\mathrm{g} / \mathrm{mol}]$ & 610.65 & $F(000)$ & 2384 \\
\hline Kristallgröße [mm] & $0.25 \times 0.2 \times 0.1$ & $\theta$-Bereich $\left[{ }^{\circ}\right]$ & $1.42-27.21$ \\
\hline Raumgruppe & $P 2_{1} / c$ & Gesammelte Reflexe & 82856 \\
\hline$a[\mathrm{pm}]$ & 1475.74(9) & Unabhängige Reflexe & 11106 \\
\hline$b[\mathrm{pm}]$ & $1292.20(8)$ & Restraints & 0 \\
\hline$c[\mathrm{pm}]$ & 2686.96(17) & Parameter & 535 \\
\hline$\alpha\left[^{\circ}\right]$ & 90 & $R 1[I>2 \sigma(I)]$ & 0.0201 \\
\hline$\beta\left[^{\circ}\right]$ & 103.1330(10) & $w R 2$ [alle Daten] & 0.0468 \\
\hline$\gamma\left[^{\circ}\right]$ & 90 & GooF & 1.053 \\
\hline$V\left[\mathrm{~nm}^{3}\right]$ & $4.9899(5)$ & Restdichte: & \\
\hline$Z$ & 8 & Max. / min. $\left[10^{-6} \mathrm{epm}^{-3}\right]$ & $1.544 /-0.975$ \\
\hline Temperatur $[\mathrm{K}]$ & $100(2)$ & Max. / min. Transmission & $0 . .4302 / 0.2544$ \\
\hline
\end{tabular}



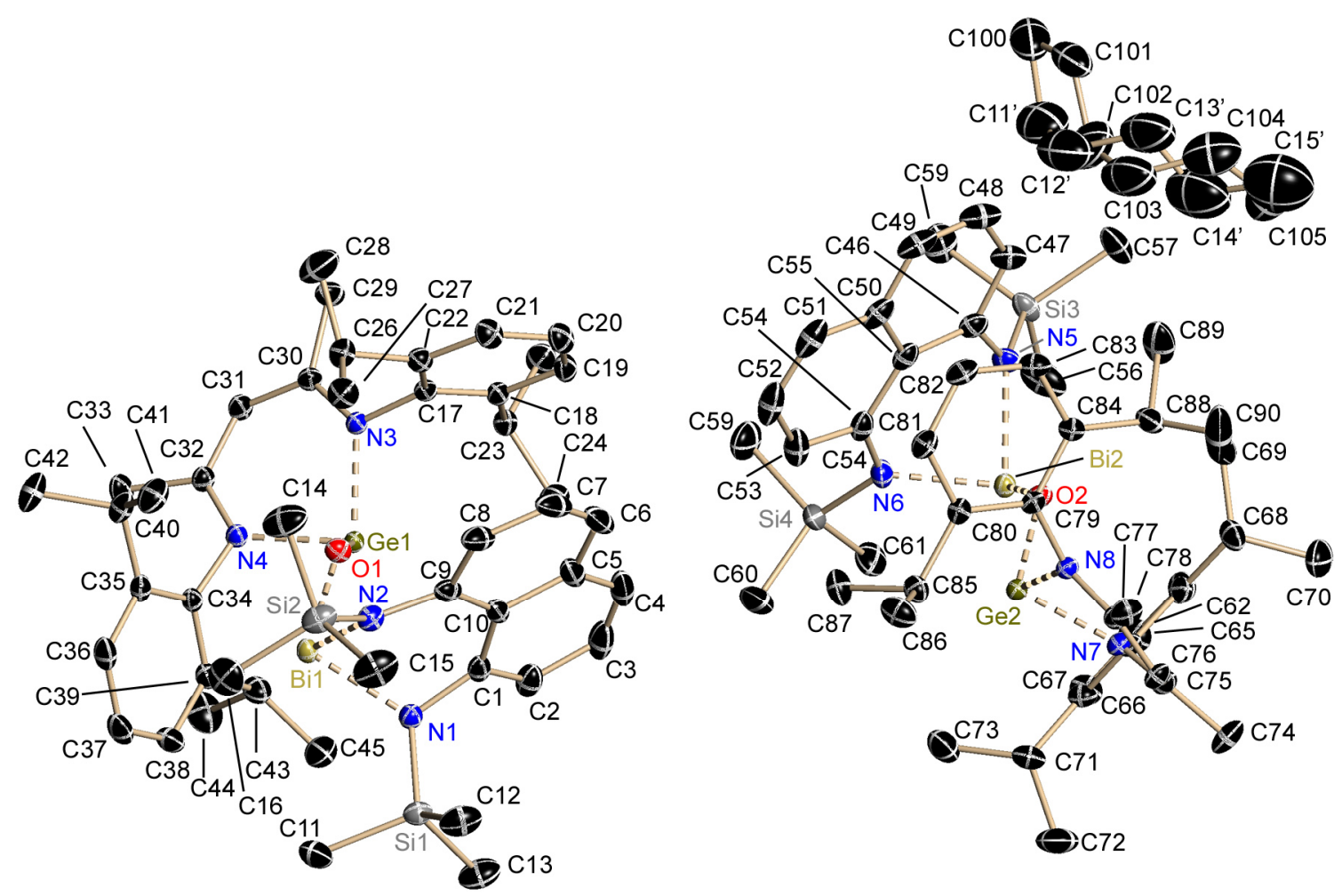

Abb. 5.76: Asymmetrische Einheit von Bj275; die anisotropen Auslenkungsparameter geben die 50\% ige Aufenthaltswahrscheinlichkeit an.

Tab. 5.68: Kristallographische Daten von Bj275.

\begin{tabular}{|ll|ll|}
\hline Strukturcode & $\mathrm{Bj} 275$ & $\rho\left[\mathrm{Mgm}^{-3}\right]$ & 1.408 \\
Summenformel & $\mathrm{C}_{48} \mathrm{H}_{72} \mathrm{BiGeN}_{4} \mathrm{OSi}_{2}$ & $\mu\left[\mathrm{mm}^{-1}\right]$ & 4.206 \\
Molekulare Masse $[\mathrm{g} / \mathrm{mol}]$ & 1058.85 & $F(000)$ & 4312 \\
Kristallgröße [mm] & $0.2 \times 0.1 \times 0.1$ & $\theta$-Bereich [ $\left.{ }^{\circ}\right]$ & 1.05 to 39.39 \\
Raumgruppe & $P 2_{1} / n$ & Gesammelte Reflexe & 461531 \\
$a[\mathrm{pm}]$ & $2259.13(18)$ & Unabhängige Reflexe & 59552 \\
$b[\mathrm{pm}]$ & $2188.04(17)$ & Restraints & 69 \\
$c[\mathrm{pm}]$ & $2279.18(18)$ & Parameter & 1104 \\
$\alpha\left[^{\circ}\right]$ & 90 & $R 1[/>2 \sigma(I)]$ & 0.0339 \\
$\beta\left[^{\circ}\right]$ & $117.5600(10)$ & WR2 [alle Daten] & 0.0637 \\
$\gamma\left[^{\circ}\right]$ & 90 & GooF & 1.041 \\
$\mathrm{~V}\left[\mathrm{~nm}^{3}\right]$ & $9.9877(14)$ & Restdichte: & \\
$Z$ & 8 & Max. / min. [10 $\left.{ }^{-6} \mathrm{epm}^{-3}\right]$ & 1.952 and -2.626 \\
Temperatur $[\mathrm{K}]^{2}$ & $100(2)$ & Max. / min. Transmission & 0.4421 and 0.2690 \\
\hline
\end{tabular}




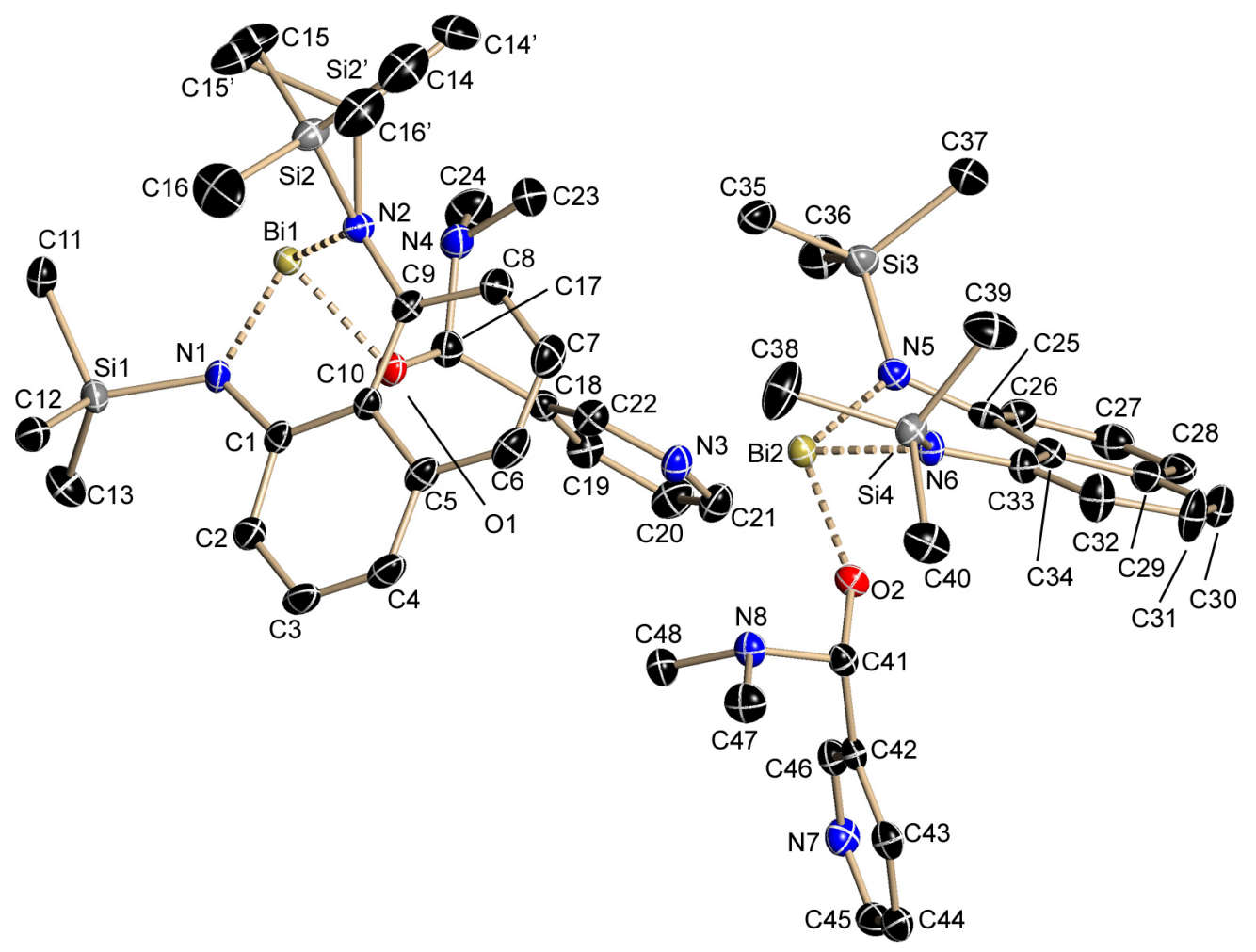

Abb. 5.77: Asymmetrische Einheit von Bj277; die anisotropen Auslenkungsparameter geben die $50 \%$ ige Aufenthaltswahrscheinlichkeit an.

Tab. 5.69: Kristallographische Daten von Bj277.

\begin{tabular}{|ll|ll|}
\hline Strukturcode & $\mathrm{Bj} 277$ & $\rho\left[\mathrm{Mgm}^{-3}\right]$ & 1.598 \\
Summenformel & $\mathrm{C}_{24} \mathrm{H}_{35} \mathrm{BiN}_{4} \mathrm{OSi}_{2}$ & $\mu\left[\mathrm{mm}^{-1}\right]$ & 6.528 \\
Molekulare Masse [g/mol] & 660.72 & $F(000)$ & 1304 \\
Kristallgröße [mm] & $0.2 \times 0.13 \times 0.09$ & $\theta$-Bereich [ $\left.{ }^{\circ}\right]$ & $1.27 / 26.02$ \\
Raumgruppe & $P \overline{1}$ & Gesammelte Reflexe & 40462 \\
$a[\mathrm{pm}]$ & $10.8242(7)$ & Unabhängige Reflexe & 9652 \\
$b[\mathrm{pm}]$ & $15.9185(10)$ & Restraints & 6 \\
$c[\mathrm{pm}]$ & $16.6061(10)$ & Parameter & 621 \\
$\alpha\left[^{\circ}\right]$ & $R 1[/>2 \sigma(I)]$ & 0.0207 \\
$\beta\left[^{\circ}\right]$ & $104.2030(10)$ & GR2 [alle Daten] & 0.0477 \\
$\gamma\left[^{\circ}\right]$ & $92.2510(10)$ & GooF & 1.037 \\
$\mathrm{~V}\left[\mathrm{~nm}{ }^{3}\right]$ & $96.9070(10)$ & Restdichte: & \\
$Z$ & $2746.7(3)$ & Max. / min. [10 $\left.{ }^{-6} \mathrm{epm}^{-3}\right]$ & $0.928 /-0.775$ \\
Temperatur $[\mathrm{K}]$ & 4 & Max. / min. Transmission & $0.3109 / 0.4299$ \\
\hline
\end{tabular}




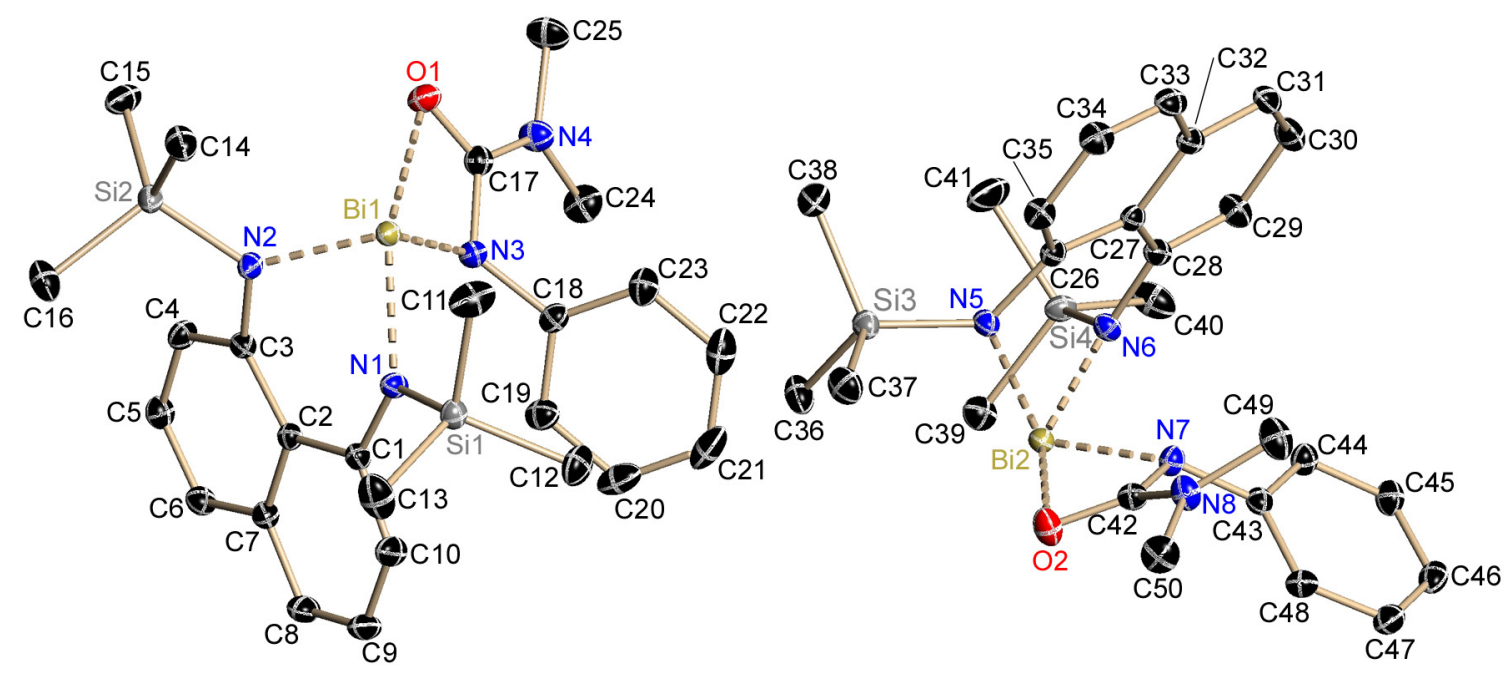

Abb. 5.78: Asymmetrische Einheit von Bj282; die anisotropen Auslenkungsparameter geben die $50 \%$ ige Aufenthaltswahrscheinlichkeit an.

Tab. 5.70: Kristallographische Daten von Bj282.

\begin{tabular}{|ll|ll|}
\hline Strukturcode & $\mathrm{Bj} 282$ & $\rho\left[\mathrm{Mgm}^{-3}\right]$ & 1.630 \\
Summenformel & $\mathrm{C}_{25} \mathrm{H}_{35} \mathrm{BiN}_{4} \mathrm{OSi}_{2}$ & $\mu\left[\mathrm{mm}^{-1}\right]$ & 6.542 \\
Molekulare Masse $[\mathrm{g} / \mathrm{mol}]$ & 672.73 & $F(000)$ & 1328 \\
Kristallgröße [mm] & $0.21 \times 0.21 \times 0.18$ & $\theta$-Bereich [ $\left.{ }^{\circ}\right]$ & $1.20-30.51$ \\
Raumgruppe & $P^{-}$ & Gesammelte Reflexe & 87377 \\
$a[\mathrm{pm}]$ & $1232.88(12)$ & Unabhängige Reflexe & 16706 \\
$b[\mathrm{pm}]$ & $1341.05(13)$ & Restraints & 0 \\
$c[\mathrm{pm}]$ & $1698.98(16)$ & Parameter & 611 \\
$\alpha\left[^{\circ}\right]$ & 88.0950 & $R 1[/>2 \sigma(I)]$ & 0.0164 \\
$\beta\left[^{\circ}\right]$ & $85.964(2)$ & wR2 [alle Daten] & 0.0339 \\
$\gamma\left[^{\circ}\right]$ & $78.1350(10)$ & g1/g2 & 1.024 \\
$\mathrm{~V}\left[\mathrm{~nm}^{3}\right]$ & $2.7417(5)$ & Restdichte: & \\
$Z$ & 4 & Max. / min. [10 & epm \\
Temperatur $\left.[\mathrm{K}]^{-3}\right]$ & Max. / min. Transmission & $0.437 /-0.538$ \\
\hline
\end{tabular}



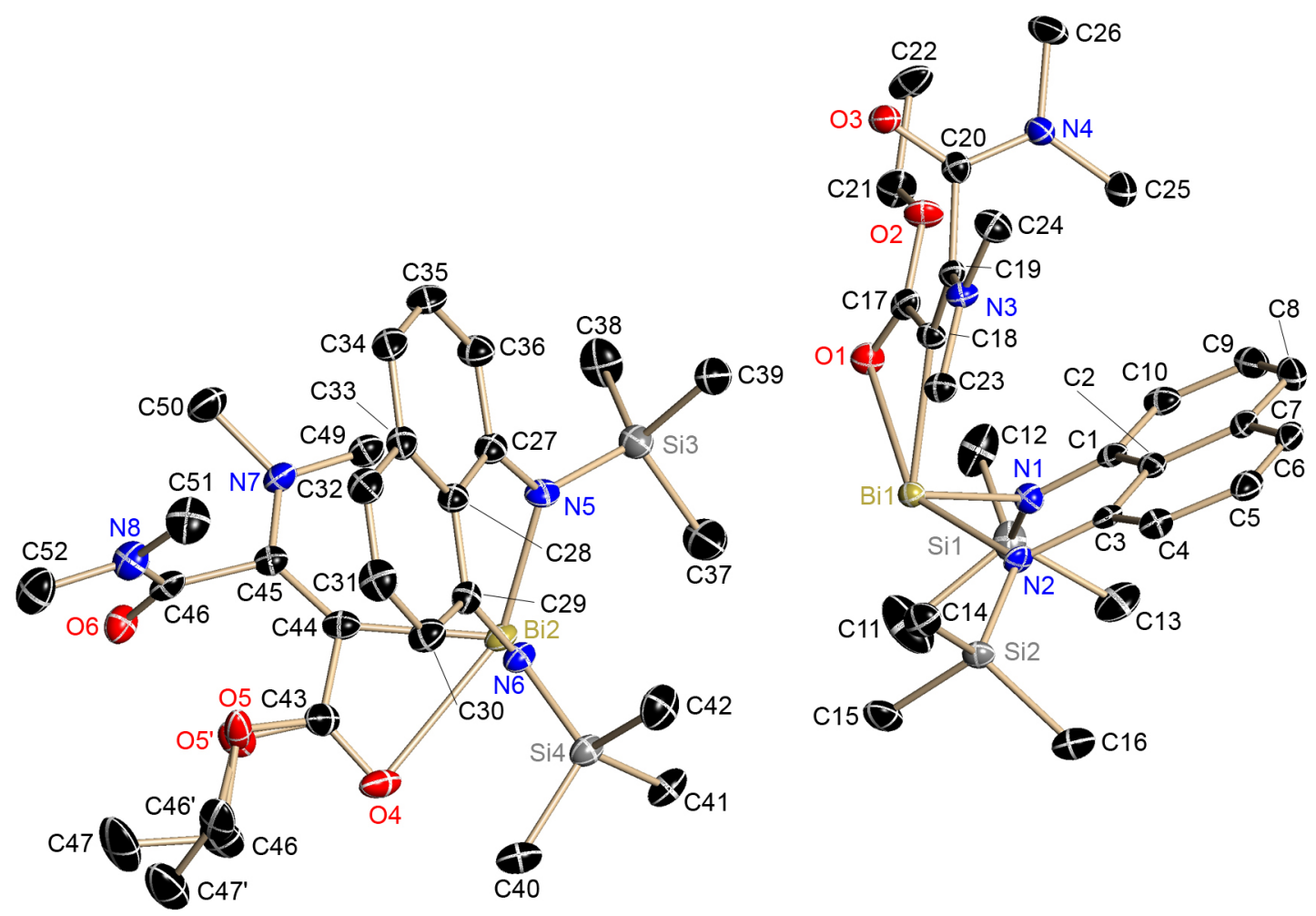

Abb. 5.79: Asymmetrische Einheit von Bj289; die anisotropen Auslenkungsparameter geben die $50 \%$ ige Aufenthaltswahrscheinlichkeit an.

Tab. 5.71: Kristallographische Daten von Bj289.

\begin{tabular}{|ll|ll|}
\hline Strukturcode & $\mathrm{Bj} 289$ & $\rho\left[\mathrm{Mgm}^{-3}\right]$ & 1.571 \\
Summenformel & $\mathrm{C}_{26} \mathrm{H}_{41} \mathrm{BiN}_{4} \mathrm{O}_{3} \mathrm{Si}_{2}$ & $\mu\left[\mathrm{mm}^{-1}\right]$ & 5.880 \\
Molekulare Masse [g/mol] & 722.79 & $F(000)$ & 1440 \\
Kristallgröße [mm] & $0.15 \times 0.15 \times 0.14$ & $\theta$-Bereich [ $\left.{ }^{\circ}\right]$ & $1.12-27.96$ \\
Raumgruppe & $P^{-}$ & Gesammelte Reflexe & 25770 \\
$a[\mathrm{pm}]$ & $1300(200)$ & Unabhängige Reflexe & 14608 \\
$b[\mathrm{pm}]$ & 1337.2 & Restraints & 33 \\
$c[\mathrm{pm}]$ & 1823.6 & Parameter & 701 \\
$\alpha\left[^{\circ}\right]$ & $R 1[I>2 \sigma(I)]$ & 0.0270 \\
$\beta\left[^{\circ}\right]$ & wR2 [alle Daten] & 0.0716 \\
$\gamma\left[^{\circ}\right]$ & GooF & 1.057 \\
$\mathrm{~V}\left[\mathrm{~nm}{ }^{3}\right]$ & 94.06 & Restdichte: & \\
$Z$ & 91.53 & Max. / min. [10-6 epm $\left.{ }^{-3}\right]$ & $1.430 /-2.338$ \\
Temperatur $[\mathrm{K}]$ & 98.82 & Max. / min. Transmission & $0.430757 / 0.288636$ \\
\hline
\end{tabular}




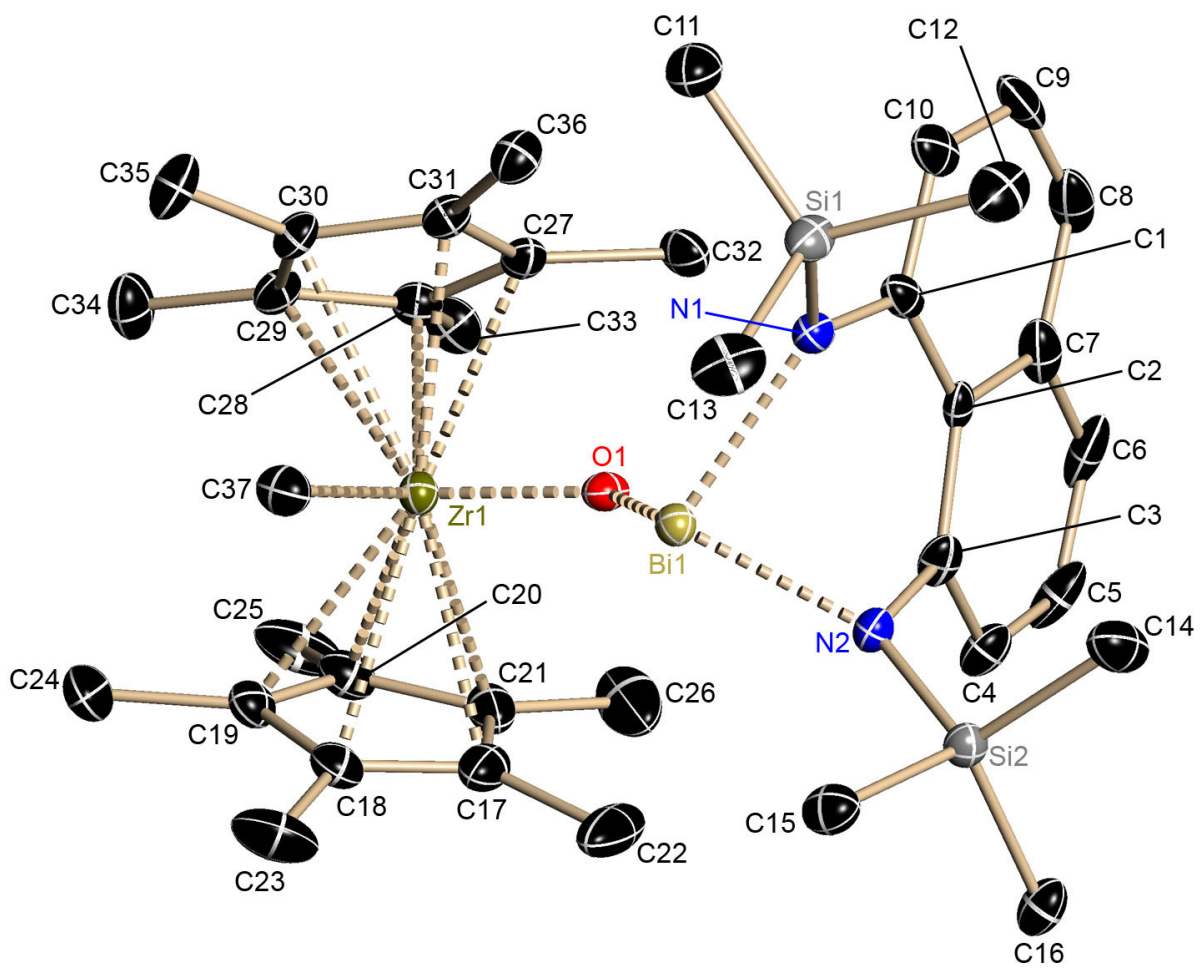

Abb. 5.80: Asymmetrische Einheit von Bj297; die anisotropen Auslenkungsparameter geben die 50\% ige Aufenthaltswahrscheinlichkeit an.

Tab. 5.72: Kristallographische Daten von Bj297.

\begin{tabular}{|c|c|c|c|}
\hline Strukturcode & Bj297 & $\mu\left[\mathrm{mm}^{-1}\right]$ & 4.927 \\
\hline Summenformel & $\mathrm{C}_{37} \mathrm{H}_{57} \mathrm{BiN}_{2} \mathrm{OSi}_{2} \mathrm{Zr}$ & $F(000)$ & 904 \\
\hline Molekulare Masse $[\mathrm{g} / \mathrm{mol}]$ & 902.23 & $\theta$-Bereich $\left[{ }^{\circ}\right]$ & 1.83 to 25.36 \\
\hline Kristallgröße [mm] & $0.15 \times 0.05 \times 0.05$ & Gesammelte Reflexe & 17310 \\
\hline Raumgruppe & $P n$ & Unabhängige Reflexe & 7013 \\
\hline$a[\mathrm{pm}]$ & $1236.4(5)$ & Restraints & 2 \\
\hline$b[\mathrm{pm}]$ & $1037.7(4)$ & Parameter & 414 \\
\hline$c[\mathrm{pm}]$ & $1582.6(6)$ & $R 1[I>2 \sigma(I)]$ & 0.0260 \\
\hline$\alpha=\gamma\left[^{\circ}\right]$ & 90 & $w R 2$ [alle Daten] & 0.0542 \\
\hline$\beta\left[^{\circ}\right]$ & $108.472(5)$ & GooF & 1.061 \\
\hline$V\left[\mathrm{~nm}^{3}\right]$ & $1.9258(12)$ & Flack-X & $-0.027(3)$ \\
\hline$Z$ & 2 & Restdichte: & \\
\hline Temperatur [K] & $100(2)$ & Max. / min. $\left[10^{-6} \mathrm{epm}^{-3}\right]$ & $0.627 /-1.004$ \\
\hline$\rho\left[\mathrm{Mgm}^{-3}\right]$ & 1.556 & Max. / min. Transmission & $0.7804 / .5660$ \\
\hline
\end{tabular}




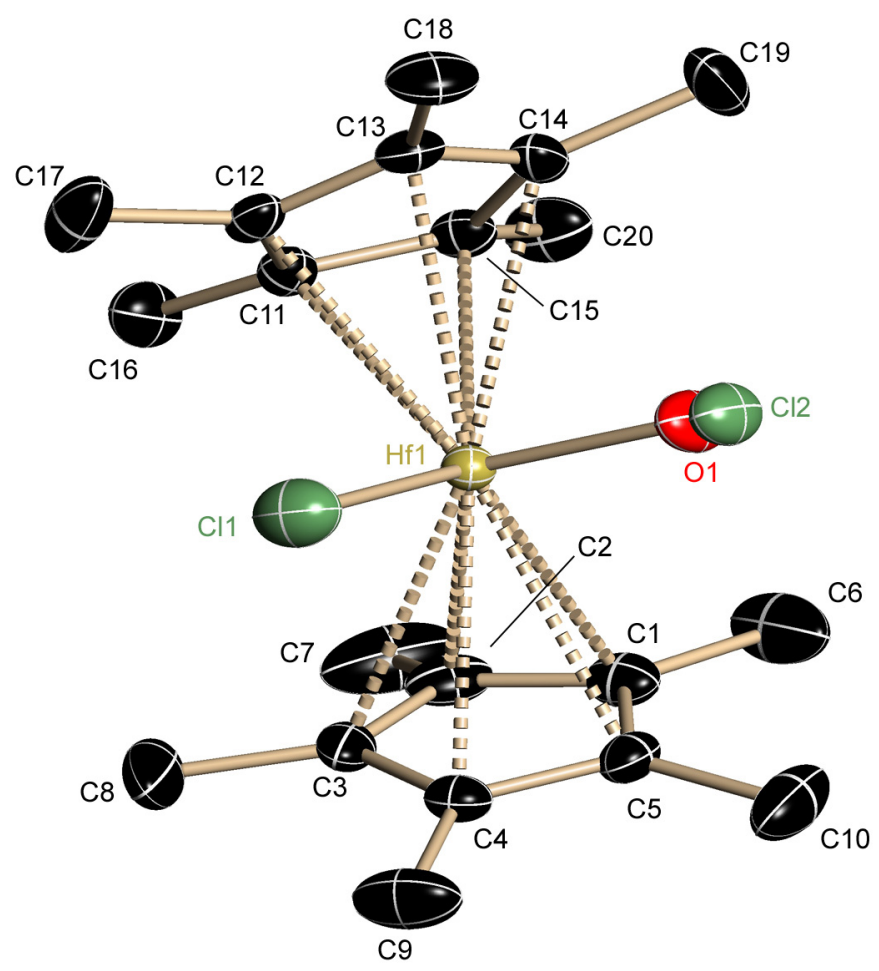

Abb. 5.81: Asymmetrische Einheit von Bj298; die anisotropen Auslenkungsparameter geben die $50 \%$ ige Aufenthaltswahrscheinlichkeit an.

Tab. 5.73: Kristallographische Daten von Bj298.

\begin{tabular}{|ll|ll|}
\hline Strukturcode & $\mathrm{Bj} 298$ & $\rho\left[\mathrm{Mgm}^{-3}\right]$ & 1.754 \\
Summenformel & $\mathrm{C}_{10} \mathrm{H}_{15} \mathrm{Cl}_{0.73} \mathrm{Hf}_{0.50} \mathrm{O}_{0.27}$ & $\mu\left[\mathrm{mm}^{-1}\right]$ & 5.611 \\
Molekulare Masse [g/mol] & 254.59 & $F(000)$ & 1004 \\
Kristallgröße [mm] & $0.2 \times 0.18 \times 0.18$ & $\theta$-Bereich [ $\left.{ }^{\circ}\right]$ & $1.84-27.50$ \\
Raumgruppe & $P 2{ }_{1}{ }_{1} 2_{1}$ & Gesammelte Reflexe & 43559 \\
$a[\mathrm{pm}]$ & $806.41(6)$ & Unabhängige Reflexe & 4421 \\
$b[\mathrm{pm}]$ & $1078.07(8)$ & Restraints & 1 \\
$c[\mathrm{pm}]$ & $2218.19(17)$ & Parameter & 228 \\
$\alpha=\beta=\gamma\left[^{\circ}\right]$ & 90 & $R 1[/>2 \sigma(I)]$ & 0.0123 \\
FlackX & $-0.012(7)$ & GR2 [alle Daten] & 0.0305 \\
$\mathrm{~V}\left[\mathrm{~nm}{ }^{3}\right]$ & $1.9284(3)$ & ReoF & 1.138 \\
$Z$ & 8 & Max. / min. [10 & \\
Temperatur $[\mathrm{K}]$ & $100(2)$ & Max. / min. Transmission & $0.4305 / 0.3371$ \\
\hline
\end{tabular}




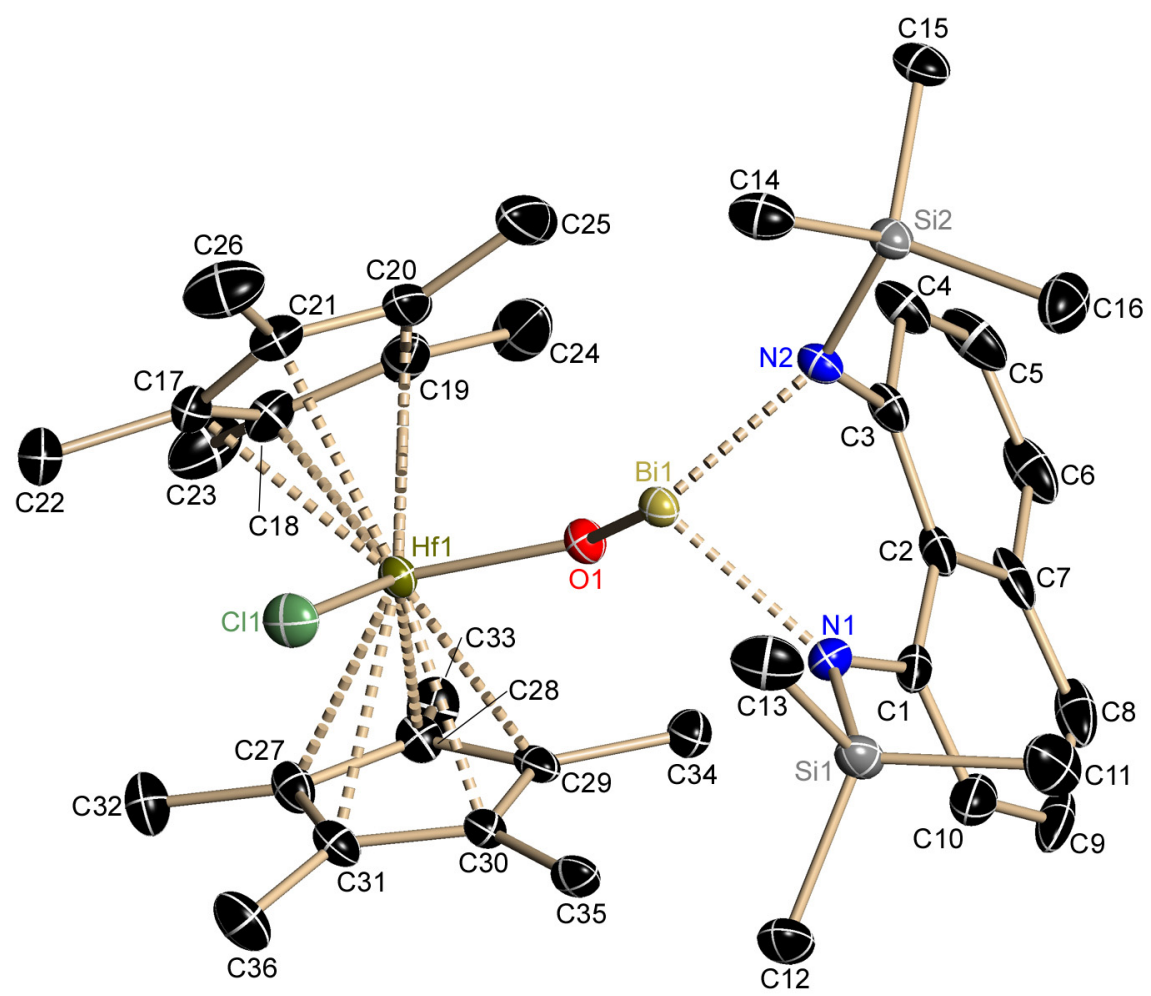

Abb. 5.82: Asymmetrische Einheit von Bj299; die anisotropen Auslenkungsparameter geben die 50\% ige Aufenthaltswahrscheinlichkeit an.

Tab. 5.74: Kristallographische Daten von Bj299.

\begin{tabular}{|c|c|c|c|}
\hline Strukturcode & Bj299 & $\mu\left[\mathrm{mm}^{-1}\right]$ & 7.481 \\
\hline Summenformel & $\mathrm{C}_{36} \mathrm{H}_{54} \mathrm{BiClHfN}{ }_{2} \mathrm{OSi}_{2}$ & $F(000)$ & 984 \\
\hline Molekulare Masse $[\mathrm{g} / \mathrm{mol}]$ & 1009.91 & $\theta$-Bereich $\left[{ }^{\circ}\right]$ & 1.84 / 26.71 \\
\hline Kristallgröße [mm] & $0.1 \times 0.1 \times 0.1$ & Gesammelte Reflexe & 39941 \\
\hline Raumgruppe & $P n$ & Unabhängige Reflexe & 8051 \\
\hline$a[\mathrm{pm}]$ & 1234.04(14) & Restraints & 2 \\
\hline$b[\mathrm{pm}]$ & $1033.40(11)$ & Parameter & 413 \\
\hline$c[\mathrm{pm}]$ & $1575.96(18)$ & $R 1[I>2 \sigma(I)]$ & 0.0245 \\
\hline$\alpha=\gamma\left[^{\circ}\right]$ & 90 & $w R 2$ [alle Daten] & 0.0604 \\
\hline$\beta\left[^{\circ}\right]$ & $108.1890(10)$ & GooF & 1.066 \\
\hline $\mathrm{V}\left[\mathrm{nm}^{3}\right]$ & $1.9093(4)$ & Flack-X & $-0.041(4)$ \\
\hline$Z$ & 2 & Restdichte: & \\
\hline Temperatur [K] & $100(2)$ & Max. / min. $\left[10^{-6} \mathrm{epm}^{-3}\right]$ & 1.122 / 1.251 \\
\hline$\rho\left[\mathrm{Mgm}^{-3}\right]$ & 1.757 & Max. / min. Transmission & 0.6775 / 0.4697 \\
\hline
\end{tabular}




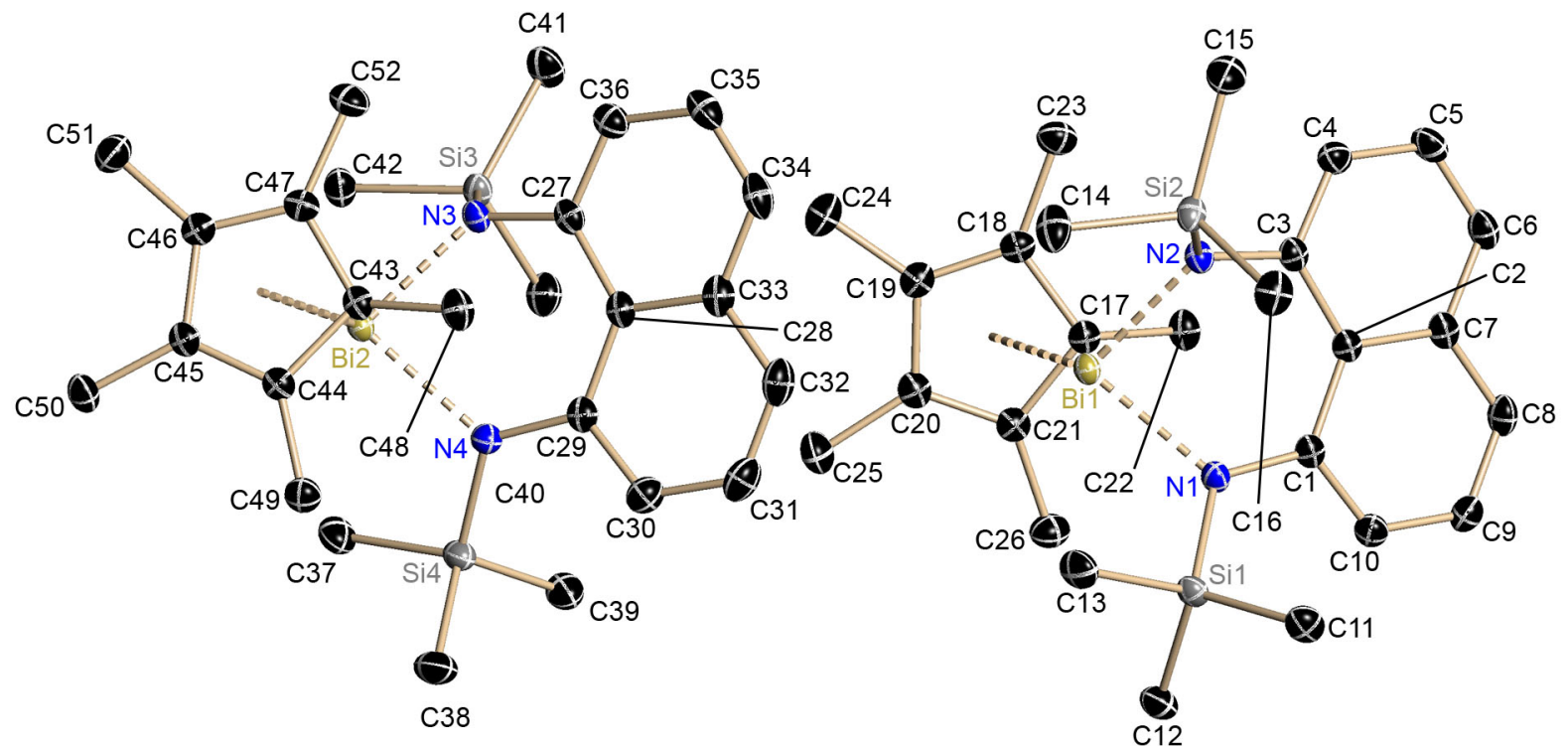

Abb. 5.83: Asymmetrische Einheit von Bj305; die anisotropen Auslenkungsparameter geben die $50 \%$ ige Aufenthaltswahrscheinlichkeit an.

Tab. 5.75: Kristallographische Daten von Bj305.

\begin{tabular}{|ll|ll|}
\hline Strukturcode & $\mathrm{Bj} 305$ & $\rho\left[\mathrm{Mgm}^{-3}\right]$ & 1.578 \\
Summenformel & $\mathrm{C}_{26} \mathrm{H}_{39} \mathrm{BiN}_{2} \mathrm{Si}_{2}$ & $\mu\left[\mathrm{mm}^{-1}\right]$ & 6.600 \\
Molekulare Masse [g/mol] & 644.75 & $F(000)$ & 1280 \\
Kristallgröße [mm] & $0.16 \times 0.16 \times 0.05$ & $\theta$-Bereich [ $\left.{ }^{\circ}\right]$ & $2.06-26.70$ \\
Raumgruppe & $P^{\overline{1}}$ & Gesammelte Reflexe & 66490 \\
$a[\mathrm{pm}]$ & $1025.37(4)$ & Unabhängige Reflexe & 11446 \\
$b[\mathrm{pm}]$ & $1672.84(6)$ & Restraints & 0 \\
$c[\mathrm{pm}]$ & $1740.01(6)$ & Parameter & 581 \\
$\alpha\left[^{\circ}\right]$ & 109.39 & $R 1[/>2 \sigma(I)]$ & 0.0139 \\
$\beta\left[^{\circ}\right]$ & 101.63 & wR2 [alle Daten] & 0.0368 \\
$\gamma\left[^{\circ}\right]$ & $95.5090(10)$ & GooF & 1.049 \\
$V\left[\mathrm{~nm}^{3}\right]$ & $2.71421(17)$ & Restdichte: & \\
$Z$ & 4 & Max. / min. $\left[10^{-6} \mathrm{epm}^{-3}\right]$ & $1.197 /-0.753$ \\
Temperatur $[\mathrm{K}]$ & $100(2)$ & Max. / min. Transmission & $0.4299 / 0.3176$ \\
\hline
\end{tabular}




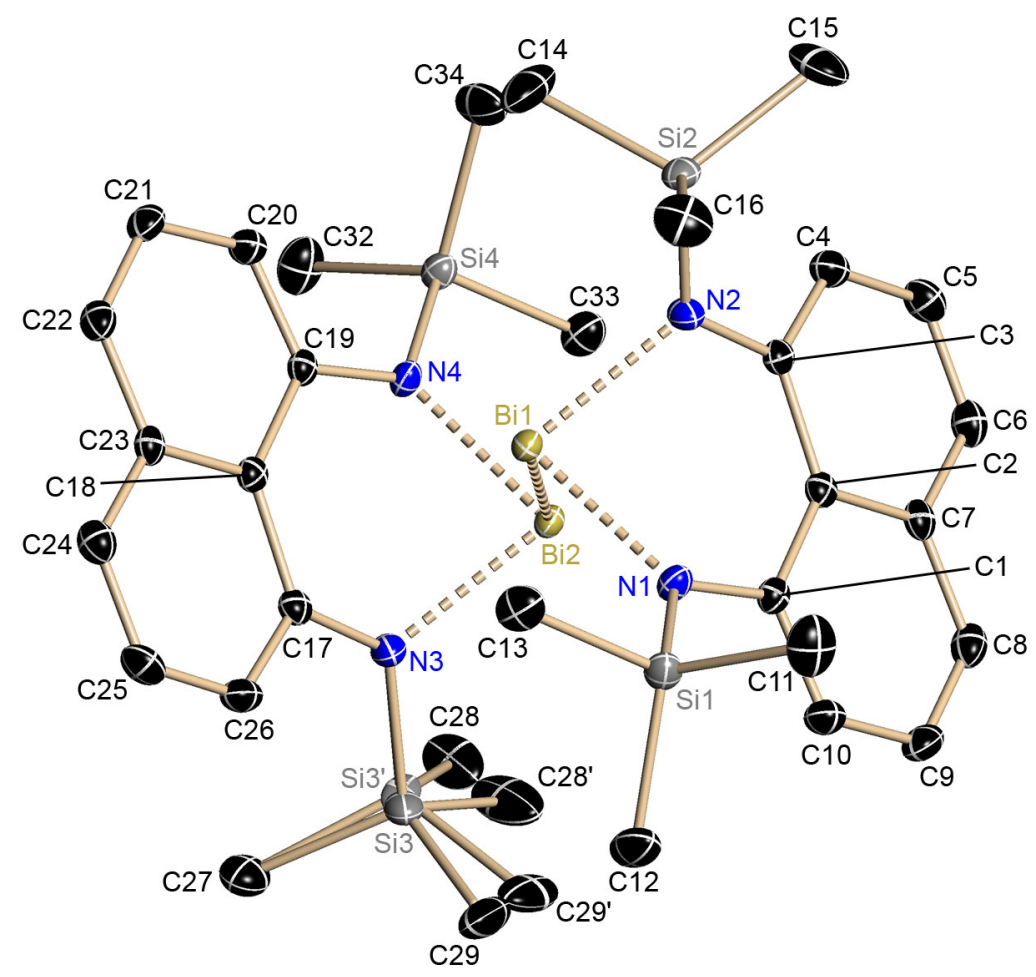

Abb. 5.84: Asymmetrische Einheit von Bj314; die anisotropen Auslenkungsparameter geben die 50\% ige Aufenthaltswahrscheinlichkeit an.

Tab. 5.76: Kristallographische Daten von Bj314.

\begin{tabular}{|ll|ll|}
\hline Strukturcode & $\mathrm{Bj} 314$ & $\rho\left[\mathrm{Mgm}^{-3}\right]$ & 1.789 \\
Summenformel & $\mathrm{C}_{32} \mathrm{H}_{48} \mathrm{Bi}_{2} \mathrm{~N}_{4} \mathrm{Si}_{4}$ & $\mu\left[\mathrm{mm}^{-1}\right]$ & 9.445 \\
Molekulare Masse [g/mol] & 1019.06 & $F(000)$ & 1960 \\
Kristallgröße [mm] & $0.18 \times 0.09 \times 0.08$ & $\theta$-Bereich [ $\left.{ }^{\circ}\right]$ & $1.18-26.89$ \\
Raumgruppe & $P 2_{1} / c$ & Gesammelte Reflexe & 91288 \\
$a[\mathrm{pm}]$ & $1804.62(9)$ & Unabhängige Reflexe & 8157 \\
$b[\mathrm{pm}]$ & $1222.80(6)$ & Restraints & 42 \\
$c[\mathrm{pm}]$ & $1789.30(9)$ & Parameter & 421 \\
$\alpha\left[^{\circ}\right]$ & 90 & $R 1[/>2 \sigma(I)]$ & 0.0151 \\
$\beta\left[^{\circ}\right]$ & $106.6630(10)$ & wR2 [alle Daten] & 0.0322 \\
$\gamma\left[^{\circ}\right]$ & 90 & GooF & 1.054 \\
$\mathrm{~V}\left[\mathrm{~nm}^{3}\right]$ & $3.7826(3)$ & Restdichte: & \\
$Z$ & 4 & Max. / min. [10 & epm $\left.{ }^{-3}\right]$ \\
Temperatur $[\mathrm{K}]$ & $100(2)$ & Max. / min. Transmission & $0.364 /-0.734$ \\
\hline
\end{tabular}




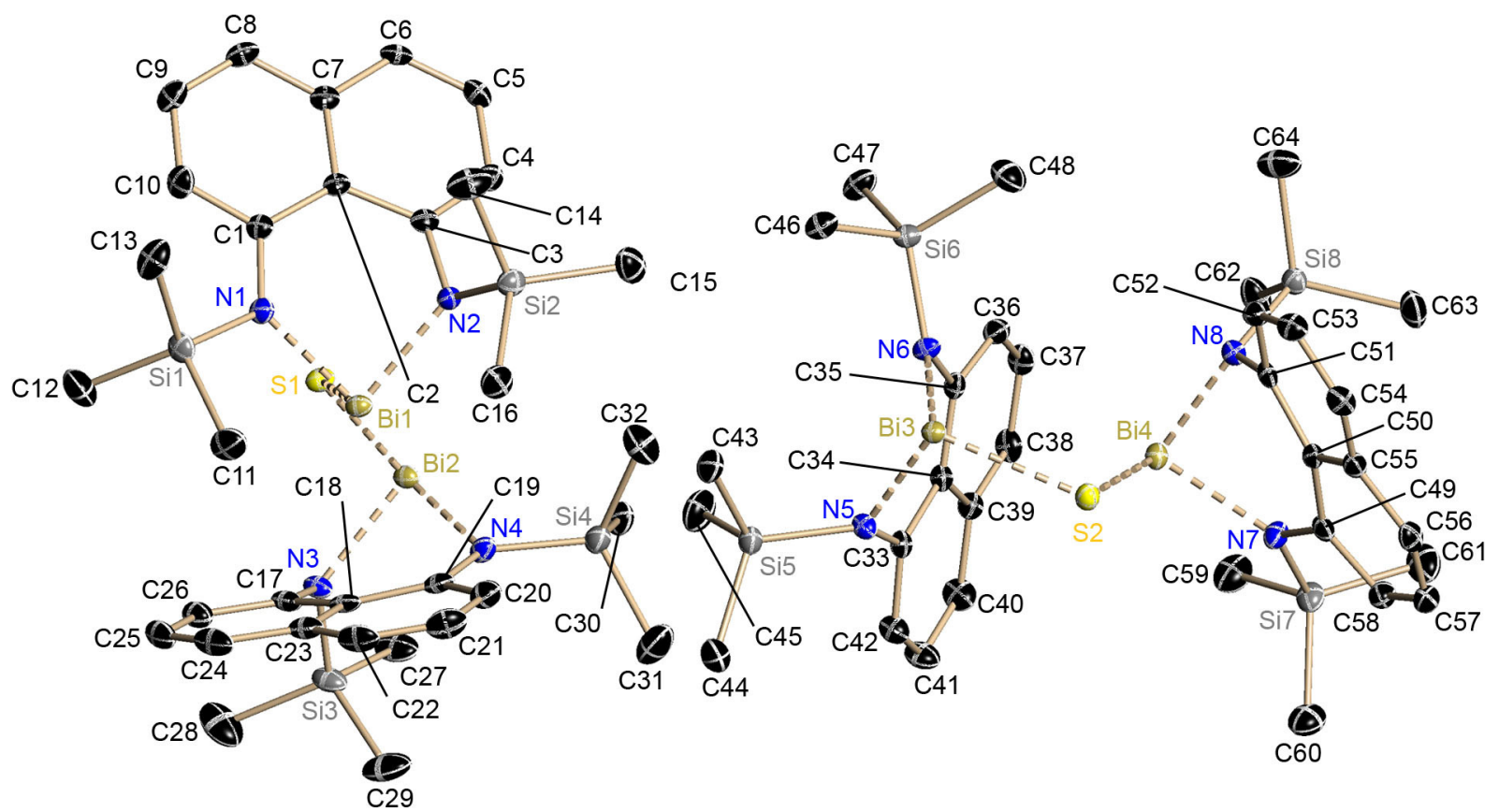

Abb. 5.85: Asymmetrische Einheit von Bj326; die anisotropen Auslenkungsparameter geben die $50 \%$ ige Aufenthaltswahrscheinlichkeit an.

Tab. 5.77: Kristallographische Daten von Bj326.

\begin{tabular}{|ll|ll|}
\hline Strukturcode & $\mathrm{Bj} 326$ & $\rho\left[\mathrm{Mgm}^{-3}\right]$ & 1.795 \\
Summenformel & $\mathrm{C}_{32} \mathrm{H}_{48} \mathrm{Bi}_{2} \mathrm{~N}_{4} \mathrm{SSi}_{4}$ & $\mu\left[\mathrm{mm}^{-1}\right]$ & 9.238 \\
Molekulare Masse [g/mol] & 1051.12 & $F(000)$ & 4048 \\
Kristallgröße [mm] & $0.19 \times 0.08 \times 0.06$ & $\theta$-Bereich [ $\left.{ }^{\circ}\right]$ & $1.65-26.76$ \\
Raumgruppe & $P 2{ }_{1} / n$ & Gesammelte Reflexe & 109915 \\
$a[\mathrm{pm}]$ & $2434.81(14)$ & Unabhängige Reflexe & 16556 \\
$b[\mathrm{pm}]$ & $1293.25(8)$ & Restraints & 0 \\
$c[\mathrm{pm}]$ & $2723.52(16)$ & Parameter & 799 \\
$\alpha\left[^{\circ}\right]$ & 90 & $R 1[/>2 \sigma(I)]$ & 0.0170 \\
$\beta\left[^{\circ}\right]$ & $114.8660(10)$ & wR2 [alle Daten] & 0.0344 \\
$\gamma\left[^{\circ}\right]$ & 90 & GooF & 1.016 \\
$\mathrm{~V}\left[\mathrm{~nm}^{3}\right]$ & $7.7808(8)$ & Restdichte: & \\
$Z$ & 8 & Max. / min. $\left[10^{-6} \mathrm{epm}^{-3}\right]$ & $0.549 /-0.558$ \\
Temperatur $[\mathrm{K}]$ & $100(2)$ & Max. / min. Transmission & $0.5761 / 0.4350$ \\
\hline
\end{tabular}




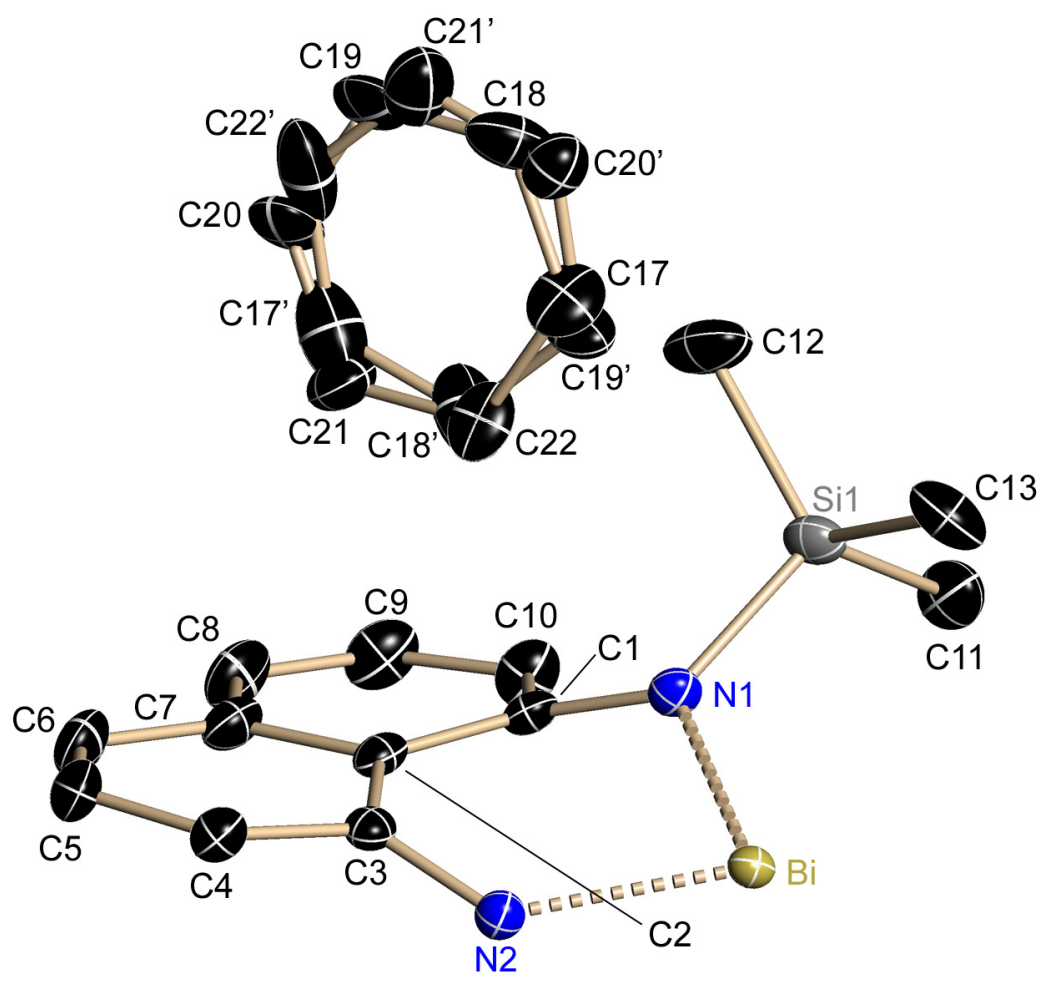

Abb. 5.86: Asymmetrische Einheit von Bj329; die anisotropen Auslenkungsparameter geben die 50\% ige Aufenthaltswahrscheinlichkeit an.

Tab. 5.78: Kristallographische Daten von Bj329.

\begin{tabular}{|ll|ll|}
\hline Strukturcode & $\mathrm{Bj} 329$ & $\rho\left[\mathrm{Mgm}^{-3}\right]$ & 1.853 \\
Summenformel & $\mathrm{C}_{19} \mathrm{H}_{21} \mathrm{BiN}_{2} \mathrm{Si}$ & $\mu\left[\mathrm{mm}^{-1}\right]$ & 9.626 \\
Molekulare Masse [g/mol] & 514.45 & $F(000)$ & 1968 \\
Kristallgröße [mm] & $0.13 \times 0.13 \times 0.13$ & $\theta$-Bereich [ $\left.{ }^{\circ}\right]$ & $1.84-26.01$ \\
Raumgruppe & $I \overline{4}$ & Gesammelte Reflexe & 52237 \\
$a[\mathrm{pm}]$ & $1501.84(3)$ & Unabhängige Reflexe & 3624 \\
$b[\mathrm{pm}]$ & $1501.84(3)$ & Restraints & 141 \\
$c[\mathrm{pm}]$ & $1635.25(3)$ & Parameter & 244 \\
$\alpha=\beta=\gamma\left[^{\circ}\right]$ & $R 1[/>2 \sigma(I)]$ & 0.0089 \\
Flack-X & 90 & WR2 [alle Daten] & 0.0232 \\
& $-0.032(3)$ & GooF & 1.091 \\
$\mathrm{~V}\left[\mathrm{~nm}^{3}\right]$ & & Restdichte: & \\
$Z$ & 1.853 & Max. / min. [10 & epm \\
Temperatur $[\mathrm{K}]$ & 8 & Max. / min. Transmission & $0.907 /-0.204$ \\
\hline
\end{tabular}




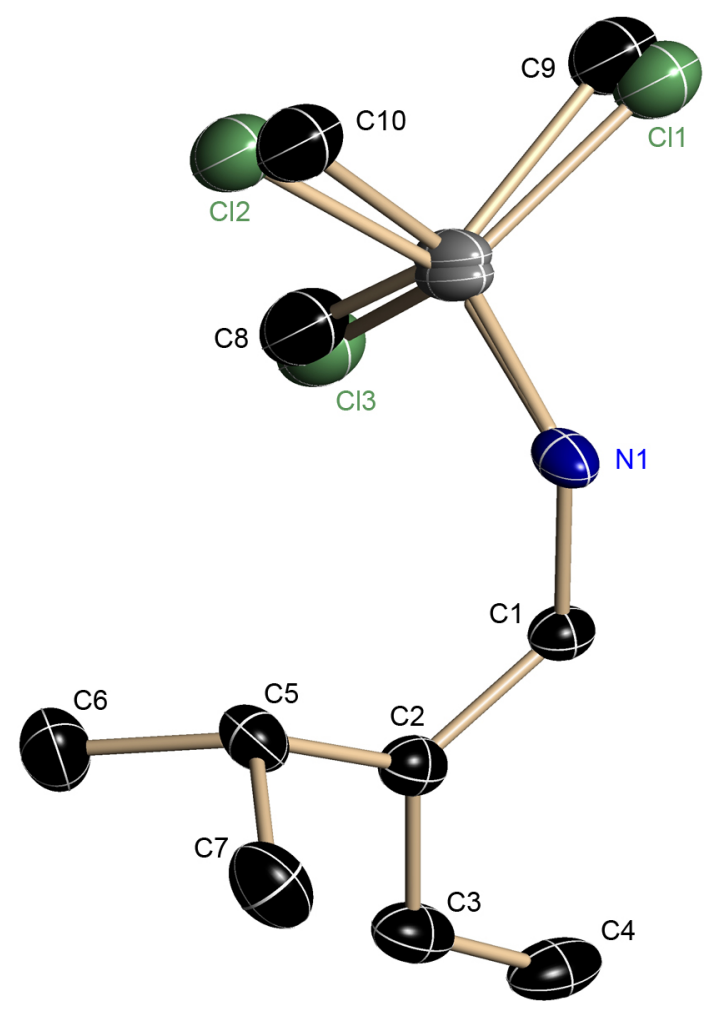

Abb. 5.87: Asymmetrische Einheit von S003; die anisotropen Auslenkungsparameter geben die 50\% ige Aufenthaltswahrscheinlichkeit an.

Tab. 5.79: Kristallographische Daten von S003.

\begin{tabular}{|ll|ll|}
\hline Strukturcode & $\mathrm{S} 003$ & $\rho\left[\mathrm{Mgm}^{-3}\right]$ & 1.071 \\
Summenformel & $\mathrm{C}_{17.43} \mathrm{H}_{33.31} \mathrm{NSi}_{2} \mathrm{Cl}_{0.56}$ & $\mu\left[\mathrm{mm}^{-1}\right]$ & 0.241 \\
Molekulare Masse [g/mol] & 333.19 & $F(000)$ & 730 \\
Kristallgröße [mm] & $0.26 \times 0.24 \times 0.22$ & $\theta$-Bereich [ $\left.{ }^{\circ}\right]$ & $2.89-26.78$ \\
Raumgruppe & $P b c n$ & Gesammelte Reflexe & 16777 \\
$a[\mathrm{pm}]$ & $1220.87(12)$ & Unabhängige Reflexe & 2198 \\
$b[\mathrm{pm}]$ & $1202.85(11)$ & Restraints & 6 \\
$c[\mathrm{pm}]$ & $1407.32(14)$ & Parameter & 103 \\
$\alpha\left[^{\circ}\right]$ & $R 1[/>2 \sigma(I)]$ & 0.0521 \\
$\beta\left[^{\circ}\right]$ & WR2 [alle Daten] & 0.1391 \\
$\gamma\left[^{\circ}\right]$ & GooF & 1.099 \\
$\mathrm{~V}\left[\mathrm{~nm}{ }^{3}\right]$ & 90 & Restdichte: & \\
$Z$ & 90 & Max. / min. [10 $\left.{ }^{-6} \mathrm{epm}^{-3}\right]$ & $0.472 /-0.546$ \\
Temperatur $[\mathrm{K}]$ & 2.0667 & Max. / min. Transmission & $0.7454 / 0.6313$ \\
\hline
\end{tabular}



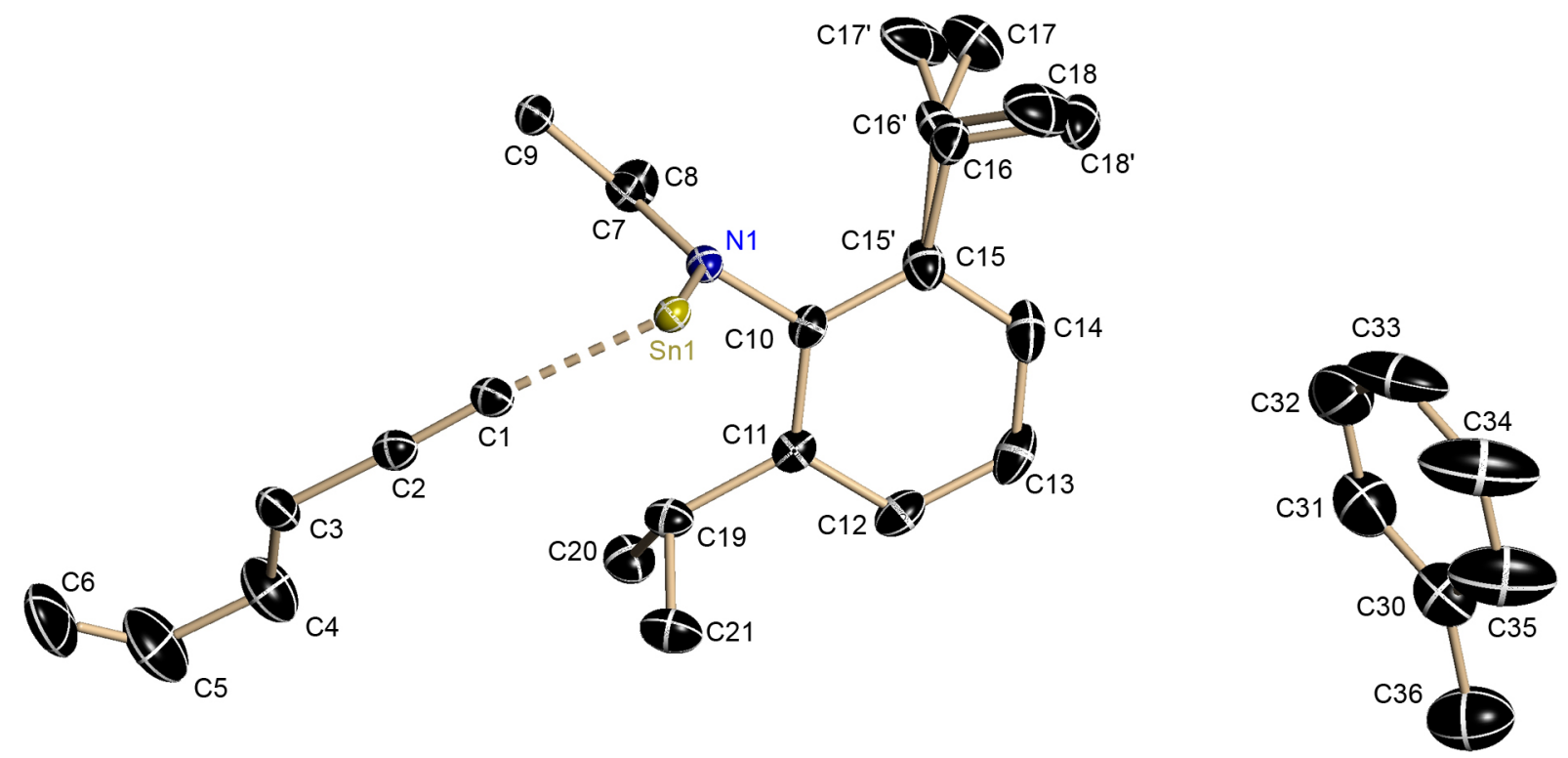

Abb. 5.88: Asymmetrische Einheit von S005; die anisotropen Auslenkungsparameter geben die 50\% ige Aufenthaltswahrscheinlichkeit an.

Tab. 5.80: Kristallographische Daten von S005.

\begin{tabular}{|ll|ll|}
\hline Strukturcode & $\mathrm{S} 005$ & $\rho\left[\mathrm{Mgm}^{-3}\right]$ & 1.274 \\
Summenformel & $\mathrm{C}_{40.5} \mathrm{H}_{50} \mathrm{~N}_{2} \mathrm{Sn}$ & $\mu\left[\mathrm{mm}^{-1}\right]$ & 0.746 \\
Molekulare Masse [g/mol] & 683.52 & $F(000)$ & 2856 \\
Kristallgröße [mm] & $0.14 \times 0.14 \times 0.11$ & $\theta$-Bereich [ $\left.{ }^{\circ}\right]$ & $1.47-26.74$ \\
Raumgruppe & $14 / m$ & Gesammelte Reflexe & 19185 \\
$a[\mathrm{pm}]$ & $18.4182(9)$ & Unabhängige Reflexe & 3910 \\
$b[\mathrm{pm}]$ & $18.4182(9)$ & Restraints & 108 \\
$c[\mathrm{pm}]$ & $21.009(2)$ & Parameter & 277 \\
$\alpha\left[^{\circ}\right]$ & 90.0 & $R 1[/>2 \sigma(I)]$ & 0.0228 \\
$\beta\left[^{\circ}\right]$ & 90.0 & WR2 [alle Daten] & 0.0608 \\
$\gamma\left[^{\circ}\right]$ & 90.0 & GooF & 1.056 \\
$\mathrm{~V}\left[\mathrm{~nm}^{3}\right]$ & $7126.9(8)$ & Restdichte: & \\
$Z$ & 8 & Max. / min. [10 & epm \\
Temperatur $[\mathrm{K}]$ & $100(2)$ & Max. / min. Transmission & $0.848 /-0.365$ \\
\hline
\end{tabular}



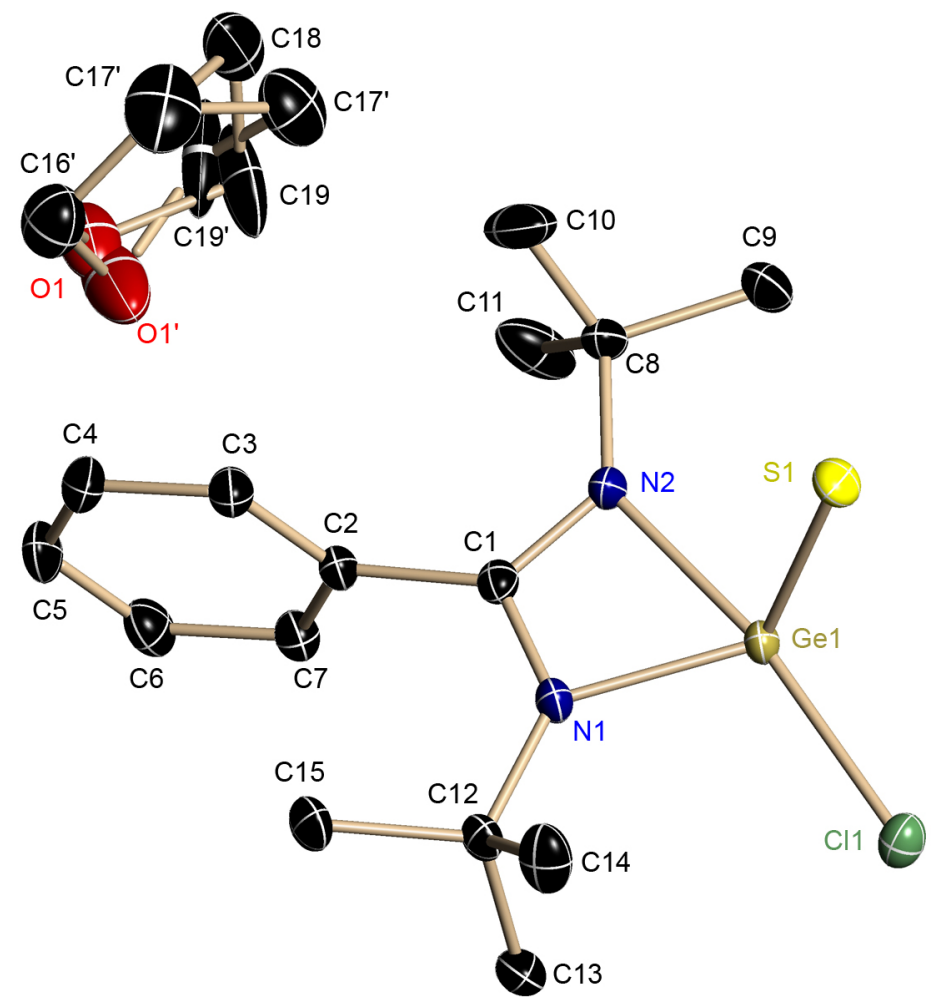

Abb. 5.89: Asymmetrische Einheit von S008; die anisotropen Auslenkungsparameter geben die $50 \%$ ige Aufenthaltswahrscheinlichkeit an.

Tab. 5.81: Kristallographische Daten von S008.

\begin{tabular}{|c|c|c|c|}
\hline Strukturcode & S008 & $\rho\left[\mathrm{Mgm}^{-3}\right]$ & 1.381 \\
\hline Summenformel & $\mathrm{C}_{19} \mathrm{H}_{31} \mathrm{ClGeN}_{2} \mathrm{OS}$ & $\mu\left[\mathrm{mm}^{-1}\right]$ & 1.669 \\
\hline Molekulare Masse $[\mathrm{g} / \mathrm{mol}]$ & 443.56 & $F(000)$ & 928 \\
\hline Kristallgröße [mm] & $0.21 \times 0.19 \times 0.11$ & $\theta$-Bereich $\left[{ }^{\circ}\right]$ & $2.43-26.04$ \\
\hline Raumgruppe & $P 2_{1} / \mathrm{n}$ & Gesammelte Reflexe & 43618 \\
\hline$a[\mathrm{pm}]$ & $8890.39(7)$ & Unabhängige Reflexe & 4199 \\
\hline$b[\mathrm{pm}]$ & $1673.99(14)$ & Restraints & 116 \\
\hline$c[\mathrm{pm}]$ & $1441.41(12)$ & Parameter & 260 \\
\hline$\alpha\left[^{\circ}\right]$ & 90 & $R 1[I>2 \sigma(I)]$ & 0.0224 \\
\hline$\beta\left[^{\circ}\right]$ & $96.3220(10)$ & $w R 2$ [alle Daten] & 0.0657 \\
\hline$\gamma\left[^{\circ}\right]$ & 90 & GooF & 1.082 \\
\hline$V\left[\mathrm{~nm}^{3}\right]$ & $2.1330(3)$ & Restdichte: & \\
\hline$Z$ & 4 & Max. / min. $\left[10^{-6} \mathrm{epm}^{-3}\right]$ & $0.53 /-0.623$ \\
\hline Temperatur [K] & $100(2)$ & Max. / min. Transmission & $0.4293 /$ \\
\hline
\end{tabular}




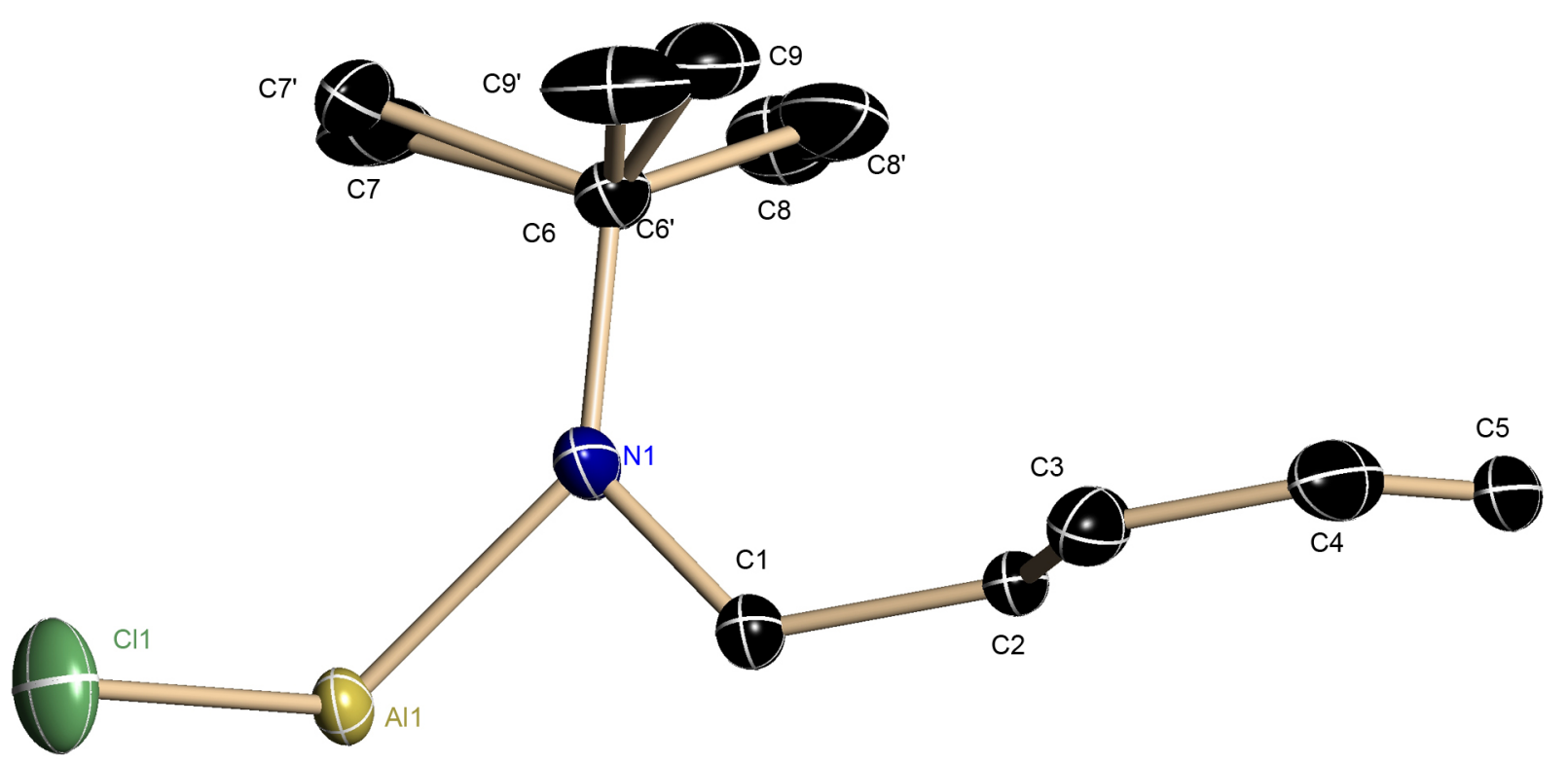

Abb. 5.90: Asymmetrische Einheit von S011; die anisotropen Auslenkungsparameter geben die 50\% ige Aufenthaltswahrscheinlichkeit an.

Tab. 5.82: Kristallographische Daten von S011.

\begin{tabular}{|c|c|c|c|}
\hline Strukturcode & S011 & $\rho\left[\mathrm{Mgm}^{-3}\right]$ & 1.211 \\
\hline Summenformel & $\mathrm{C}_{15} \mathrm{H}_{23} \mathrm{AlCl}_{2} \mathrm{~N}_{2}$ & $\mu\left[\mathrm{mm}^{-1}\right]$ & 0.401 \\
\hline Molekulare Masse $[\mathrm{g} / \mathrm{mol}]$ & 329.23 & $F(000)$ & 696 \\
\hline Kristallgröße [mm] & $0.15 \times 0.15 \times 0.14$ & $\theta$-Bereich $\left[^{\circ}\right]$ & $2.42-27.50$ \\
\hline Raumgruppe & $C 2 / c$ & Gesammelte Reflexe & 8542 \\
\hline$a[\mathrm{pm}]$ & $1453.34(9)$ & Unabhängige Reflexe & 2060 \\
\hline$b[\mathrm{pm}]$ & $1126.86(7)$ & Restraints & 54 \\
\hline$c[\mathrm{pm}]$ & $1261.81(13)$ & Parameter & 127 \\
\hline$\alpha\left[^{\circ}\right]$ & 90 & $R 1[I>2 \sigma(I)]$ & 0.0260 \\
\hline$\beta\left[^{\circ}\right]$ & 119.0760(10) & $w R 2$ [alle Daten] & 0.0702 \\
\hline$\gamma\left[^{\circ}\right]$ & 90 & GooF & 1.060 \\
\hline$V\left[\mathrm{~nm}^{3}\right]$ & $1.8061(2)$ & Restdichte: & \\
\hline$Z$ & 4 & Max. / min. $\left[10^{-6} \mathrm{epm}^{-3}\right]$ & $0.363 /-0.235$ \\
\hline Temperatur $[\mathrm{K}]$ & $100(2)$ & Max. / min. Transmission & $0.7456 / 0.6871$ \\
\hline
\end{tabular}




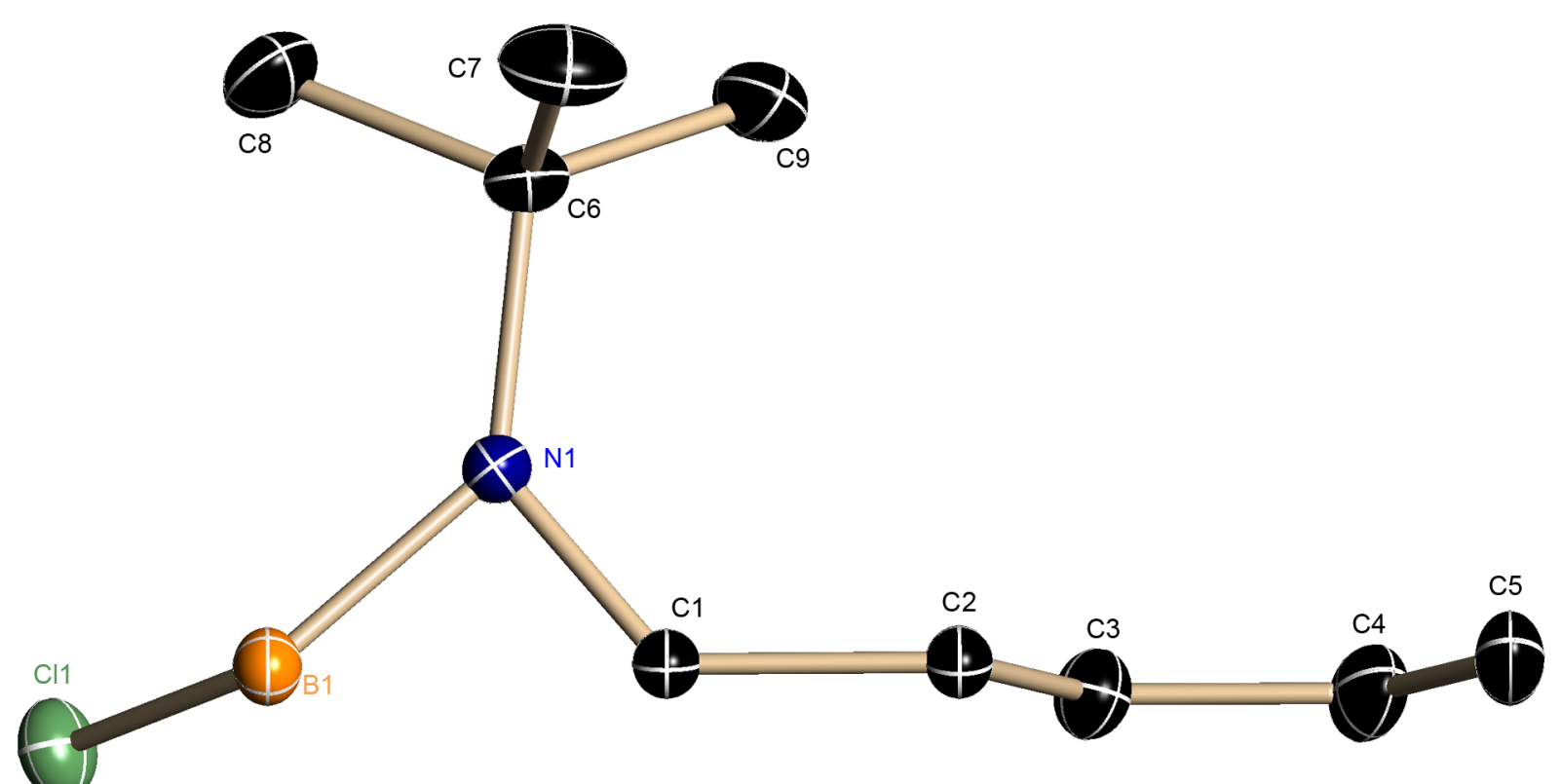

Abb. 5.91: Asymmetrische Einheit von S012; die anisotropen Auslenkungsparameter geben die $50 \%$ ige Aufenthaltswahrscheinlichkeit an.

Tab. 5.83: Kristallographische Daten von S012.

\begin{tabular}{|ll|ll|}
\hline Strukturcode & $\mathrm{S} 012$ & $\rho\left[\mathrm{Mgm}^{-3}\right]$ & 1.237 \\
Summenformel & $\mathrm{C}_{15} \mathrm{H}_{23} \mathrm{BCl}_{2} \mathrm{~N}_{2}$ & $\mu\left[\mathrm{mm}^{-1}\right]$ & 0.378 \\
Molekulare Masse [g/mol] & 313.06 & $F(000)$ & 664 \\
Kristallgröße [mm] & $0.18 \times 0.17 \times 0.16$ & $\theta$-Bereich [ $\left.{ }^{\circ}\right]$ & $2.49-45.44$ \\
Raumgruppe & $C 2 / c$ & Gesammelte Reflexe & 57104 \\
$a[\mathrm{pm}]$ & $1424.87(2)$ & Unabhängige Reflexe & 7056 \\
$b[\mathrm{pm}]$ & $1065.810(10)$ & Restraints & 0 \\
$c[\mathrm{pm}]$ & $1240.19(3)$ & Parameter & 96 \\
$\alpha\left[^{\circ}\right]$ & $R 1[I>2 \sigma(I)]$ & 0.0292 \\
$\beta\left[^{\circ}\right]$ & 116.83 & WR2 [alle Daten] & 0.0900 \\
$\gamma\left[^{\circ}\right]$ & 90 & GooF & 1.074 \\
$\mathrm{~V}\left[\mathrm{~nm}{ }^{3}\right]$ & $1.68071(5)$ & Restdichte: & \\
$Z$ & 4 & Max. / min. [10 $\left.{ }^{-6} \mathrm{epm}^{-3}\right]$ & $0.627 /-0.192$ \\
Temperatur $[\mathrm{K}]$ & $100(2)$ & Max. / min. Transmission & $0.7490 / 0.7152$ \\
\hline
\end{tabular}




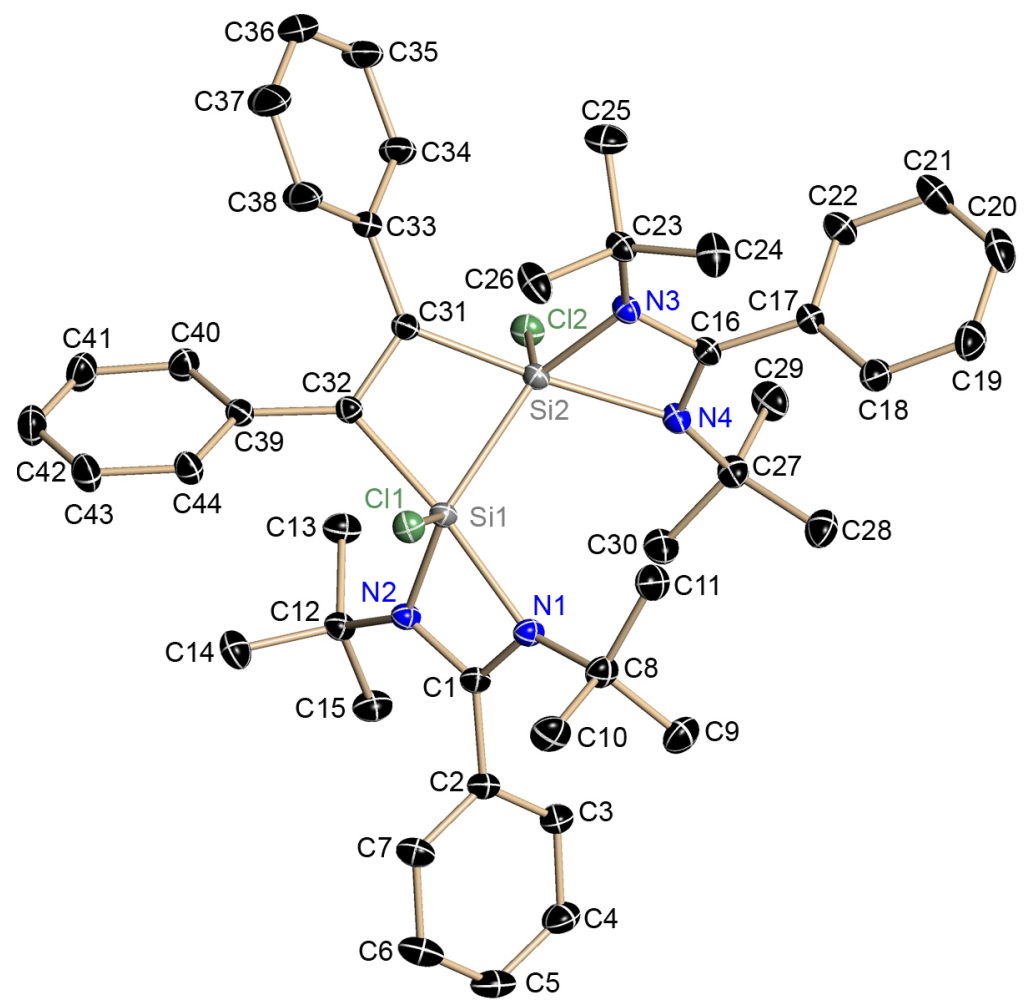

Abb. 5.92: Asymmetrische Einheit von S013; die anisotropen Auslenkungsparameter geben die 50\% ige Aufenthaltswahrscheinlichkeit an.

Tab. 5.84: Kristallographische Daten von S013.

\begin{tabular}{|ll|ll|}
\hline Strukturcode & $\mathrm{S} 013$ & $\rho\left[\mathrm{Mgm}^{-3}\right]$ & 1.200 \\
Summenformel & $\mathrm{C}_{44} \mathrm{H}_{56} \mathrm{Cl}_{2} \mathrm{~N}_{4} \mathrm{Si}_{2}$ & $\mu\left[\mathrm{mm}^{-1}\right]$ & 0.244 \\
Molekulare Masse [g/mol] & 768.01 & $F(000)$ & 1640 \\
Kristallgröße [mm] & $0.2 \times 0.2 \times 0.18$ & $\theta$-Bereich [ $\left.{ }^{\circ}\right]$ & $1.55-39.39$ \\
Raumgruppe & $P 2_{1} / c$ & Gesammelte Reflexe & 287828 \\
$a[\mathrm{pm}]$ & $1318.30(4)$ & Unabhängige Reflexe & 25354 \\
$b[\mathrm{pm}]$ & $1322.75(4)$ & Restraints & 0.0672 \\
$c[\mathrm{pm}]$ & $2445.48(8)$ & Parameter & 481 \\
$\alpha\left[^{\circ}\right]$ & 90 & $R 1[/>2 \sigma(I)]$ & 0.0479 \\
$\beta\left[^{\circ}\right]$ & $94.5010(10)$ & WR2 [alle Daten] & 0.1388 \\
$\gamma\left[^{\circ}\right]$ & 90 & GooF & 1.130 \\
$\mathrm{~V}\left[\mathrm{~nm}^{3}\right]$ & $4.2512(2)$ & Restdichte: & \\
$Z$ & 4 & Max. / min. [10 & epm $\left.{ }^{-3}\right]$ \\
Temperatur $[\mathrm{K}]$ & $100(2)$ & Max. / min. Transmission & $0.755 /-0.351$ \\
\hline
\end{tabular}




\subsubsection{Strukturen des AK Klingebiel (Universität Göttingen)}

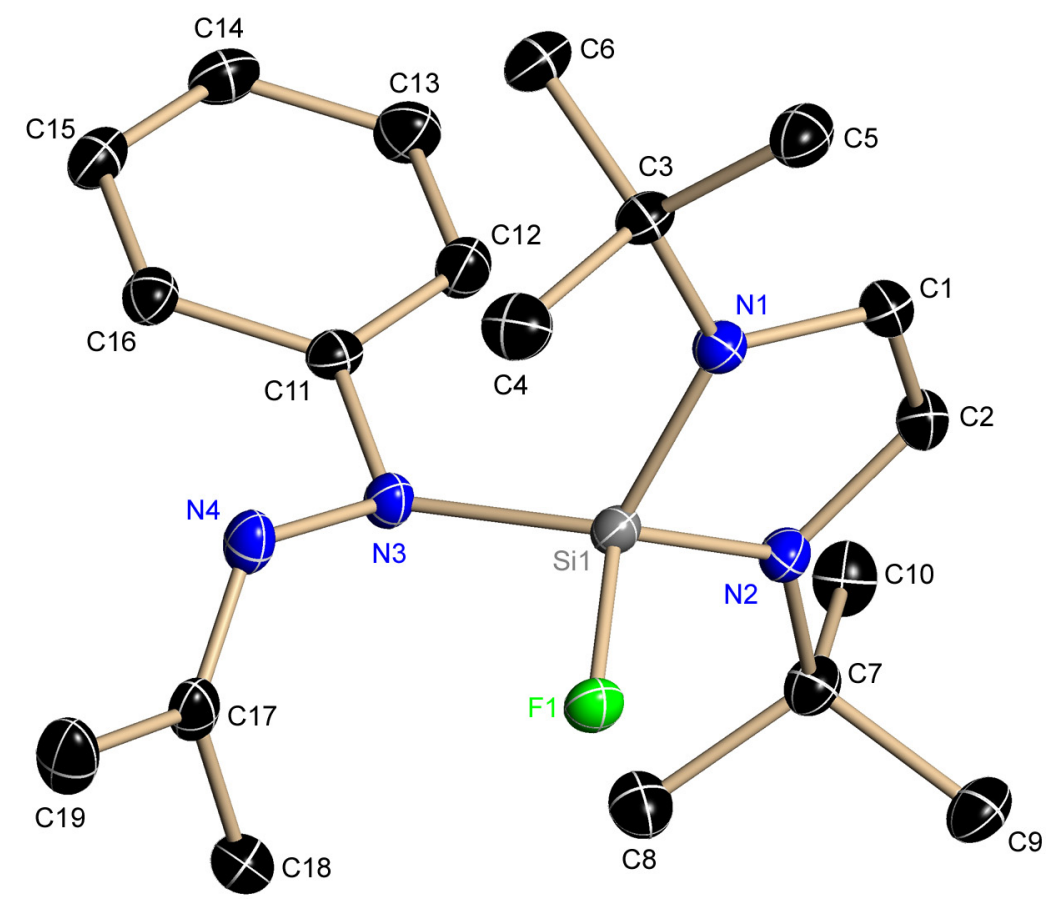

Abb. 5.93: Asymmetrische Einheit von F0407; die anisotropen Auslenkungsparameter geben die 50\% ige Aufenthaltswahrscheinlichkeit an.

Tab. 5.85: Kristallographische Daten von F0407.

\begin{tabular}{|ll|ll|}
\hline Struktur Code & $\mathrm{F} 0407$ & $\rho\left[\mathrm{Mgm}^{-3}\right]$ & 1.222 \\
Summenformel & $\mathrm{C}_{19} \mathrm{H}_{31} \mathrm{FN}_{4} \mathrm{Si}$ & $\mu\left[\mathrm{mm}^{-1}\right]$ & 0.138 \\
Molekulare Masse $[\mathrm{g} / \mathrm{mol}]$ & 362.57 & $F(000)$ & 784 \\
Kristall Größe $[\mathrm{mm}]$ & $0.2 \times 0.2 \times 0.2$ & $\theta$-Bereich [ $\left.{ }^{\circ}\right]$ & 2.82 to 26.98 \\
Raumgruppe & $P 2_{1} / c$ & Gesammelte Reflexe & 43023 \\
$a[\mathrm{pm}]$ & $961.34(12)$ & Unabhängige Reflexe & 4285 \\
$b[\mathrm{pm}]$ & $1101.40(14)$ & Restraints & 0 \\
$c[\mathrm{pm}]$ & $1883.8(2)$ & Parameter & 234 \\
$\alpha\left[^{\circ}\right]$ & 90 & $R 1[/>2 \sigma(I)]$ & 0.0323 \\
$\beta\left[^{\circ}\right]$ & WR2 [alle Daten] & 0.0839 \\
$\gamma\left[^{\circ}\right]$ & GooF & 1.034 \\
$\mathrm{~V}\left[\mathrm{~nm}^{3}\right]$ & $90.9310(10)$ & Rest Dichte: & \\
$Z$ & $1.9704(4)$ & Max. / min. $\left[10^{-6} \mathrm{eÁ}^{-3}\right]$ & $0.300 /-0.294$ \\
Temperatur $[\mathrm{K}]$ & 4 & Max. / min. Transmission & $0.99 / 0.7725$ \\
\hline
\end{tabular}




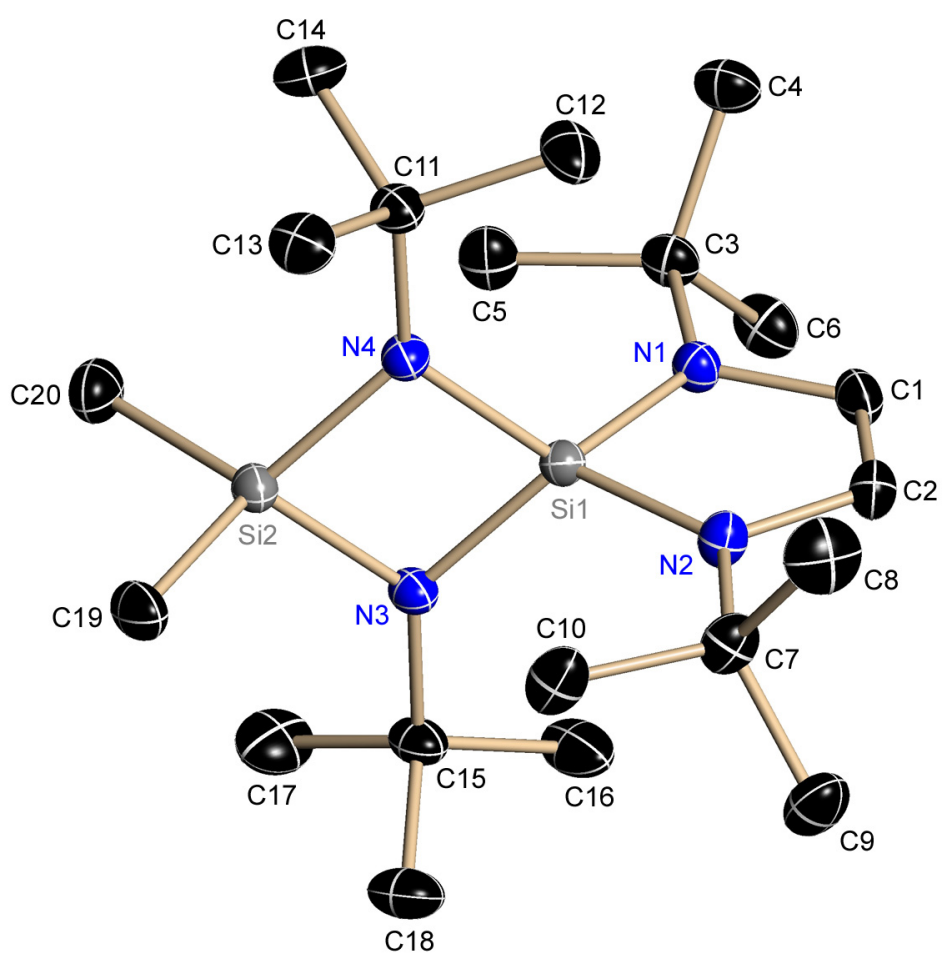

Abb. 5.94: Asymmetrische Einheit von F0907; die anisotropen Auslenkungsparameter geben die 50\% ige Aufenthaltswahrscheinlichkeit an.

Tab. 5.86: Kristallographische Daten von F0907.

\begin{tabular}{|c|c|c|c|}
\hline Struktur Code & F0907 & $\rho\left[\mathrm{Mgm}^{-3}\right]$ & 1.085 \\
\hline Summenformel & $\mathrm{C}_{20} \mathrm{H}_{44} \mathrm{~N}_{4} \mathrm{Si}_{2}$ & $\mu\left[\mathrm{mm}^{-1}\right]$ & 0.157 \\
\hline Molekular Gewicht [g/mol] & 396.77 & $F(000)$ & 880 \\
\hline Kristall Größe [mm] & $0.2 \times 0.1 \times 0.1$ & $\theta$-Bereich $\left[{ }^{\circ}\right]$ & 2.40 to 27.10 \\
\hline Raumgruppe & $P 2_{1} / n$ & Gesammelte Reflexe & 31569 \\
\hline$a[\mathrm{pm}]$ & $927.86(8)$ & Unabhängige Reflexe & 5339 \\
\hline$b[\mathrm{pm}]$ & $1694.89(15)$ & Restraints & 0 \\
\hline$c[\mathrm{pm}]$ & 1569.94(14) & Parameter & 237 \\
\hline$\alpha\left[^{\circ}\right]$ & 90 & $R 1[I>2 \sigma(I)]$ & 0.0366 \\
\hline$\beta\left[^{\circ}\right]$ & $100.3840(10)$ & $w R 2$ [alle Daten] & 0.1032 \\
\hline$\gamma\left[^{\circ}\right]$ & 90 & GooF & 1.028 \\
\hline$V\left[\mathrm{~nm}^{3}\right]$ & $2.4285(4)$ & Rest Dichte: & \\
\hline$Z$ & 4 & Max. / min. $\left[10^{-6} \mathrm{e}^{-3}\right]$ & $0.335 /-0.357$ \\
\hline Temperatur $[\mathrm{K}]$ & $100(2)$ & Max. / min. Transmission & $1 / 0.880785$ \\
\hline
\end{tabular}




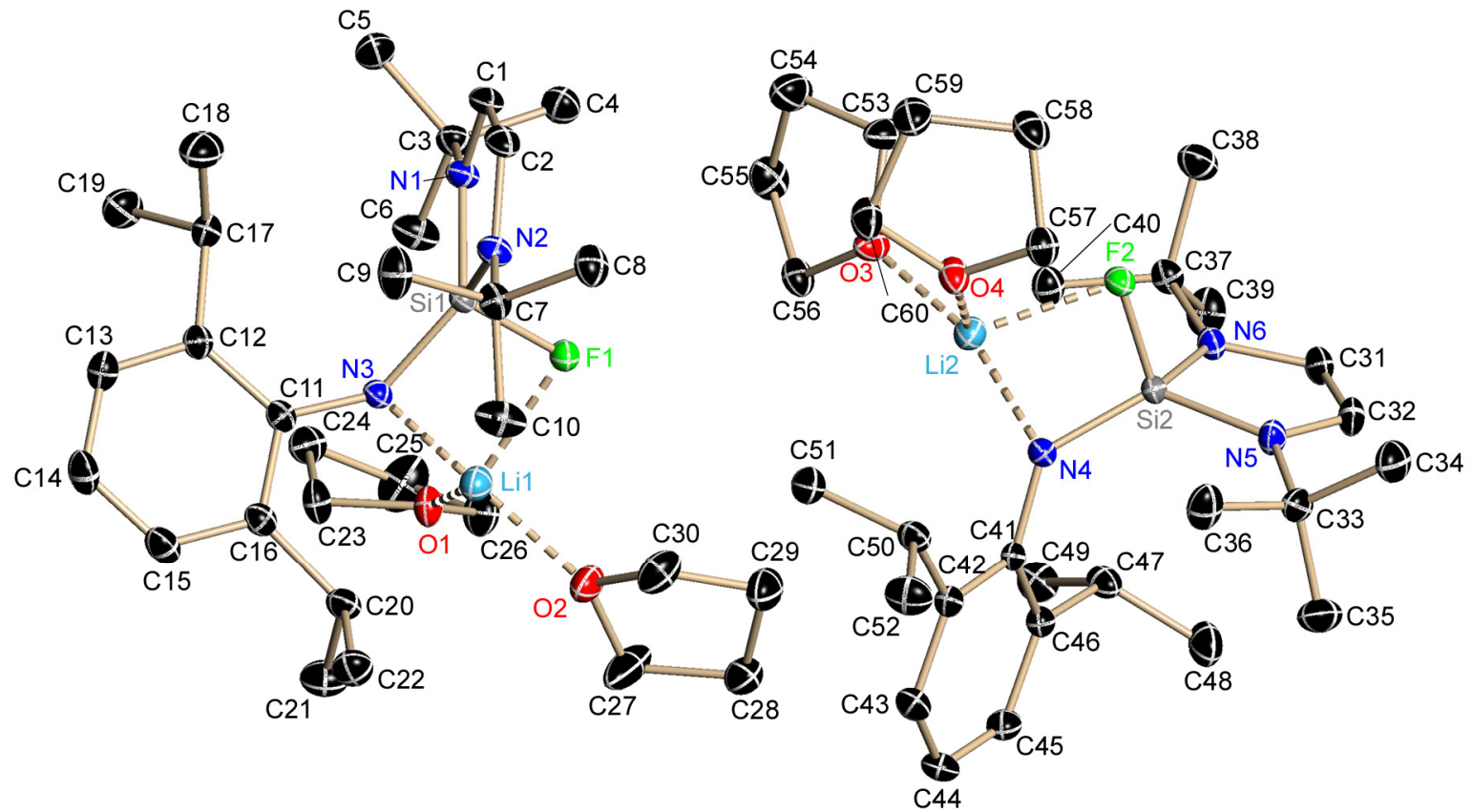

Abb. 5.95: Asymmetrische Einheit von F2007; die anisotropen Auslenkungsparameter geben die $50 \%$ ige Aufenthaltswahrscheinlichkeit an.

Tab. 5.87: Kristallographische Daten von F2007.

\begin{tabular}{|c|c|c|c|}
\hline Struktur Code & F2007 & $\rho\left[\mathrm{Mgm}^{-3}\right]$ & 1.148 \\
\hline Summenformel & $\mathrm{C}_{30} \mathrm{H}_{53} \mathrm{FLiN}_{3} \mathrm{O}_{2} \mathrm{Si}$ & $\mu\left[\mathrm{mm}^{-1}\right]$ & 0.110 \\
\hline Molekular Gewicht [g/mol] & 541.78 & $F(000)$ & 1184 \\
\hline Kristall Größe [mm] & $0.2 \times 0.2 \times 0.18$ & $\theta$-Bereich $\left[{ }^{\circ}\right]$ & 1.62 / 27.10 \\
\hline Raumgruppe & $P_{\overline{1}}$ & Gesammelte Reflexe & 71805 \\
\hline$a[\mathrm{pm}]$ & 1260.94(9) & Unabhängige Reflexe & 13773 \\
\hline$b[\mathrm{pm}]$ & $1545.40(12)$ & Restraints & 0 \\
\hline$c[\mathrm{pm}]$ & 1644.55(12) & Parameter & 13773 \\
\hline$\alpha\left[{ }^{\circ}\right]$ & $101.5360(10)$ & $R 1[I>2 \sigma(I)]$ & 0.0383 \\
\hline$\beta\left[^{\circ}\right]$ & 92.7950(10) & $w R 2$ [alle Daten] & 0.0988 \\
\hline$\gamma\left[{ }^{\circ}\right]$ & $90.5650(10)$ & GooF & 1.108 \\
\hline$V\left[\mathrm{~nm}^{3}\right]$ & $3.1356(4)$ & Rest Dichte: & \\
\hline$Z$ & 4 & Max. / min. $\left[10^{-6} \mathrm{epm}^{-3}\right]$ & 0.562 / -0.315 \\
\hline Temperatur [K] & $100(2)$ & Max. / min. Transmission & $0.99 / 0.859624$ \\
\hline
\end{tabular}




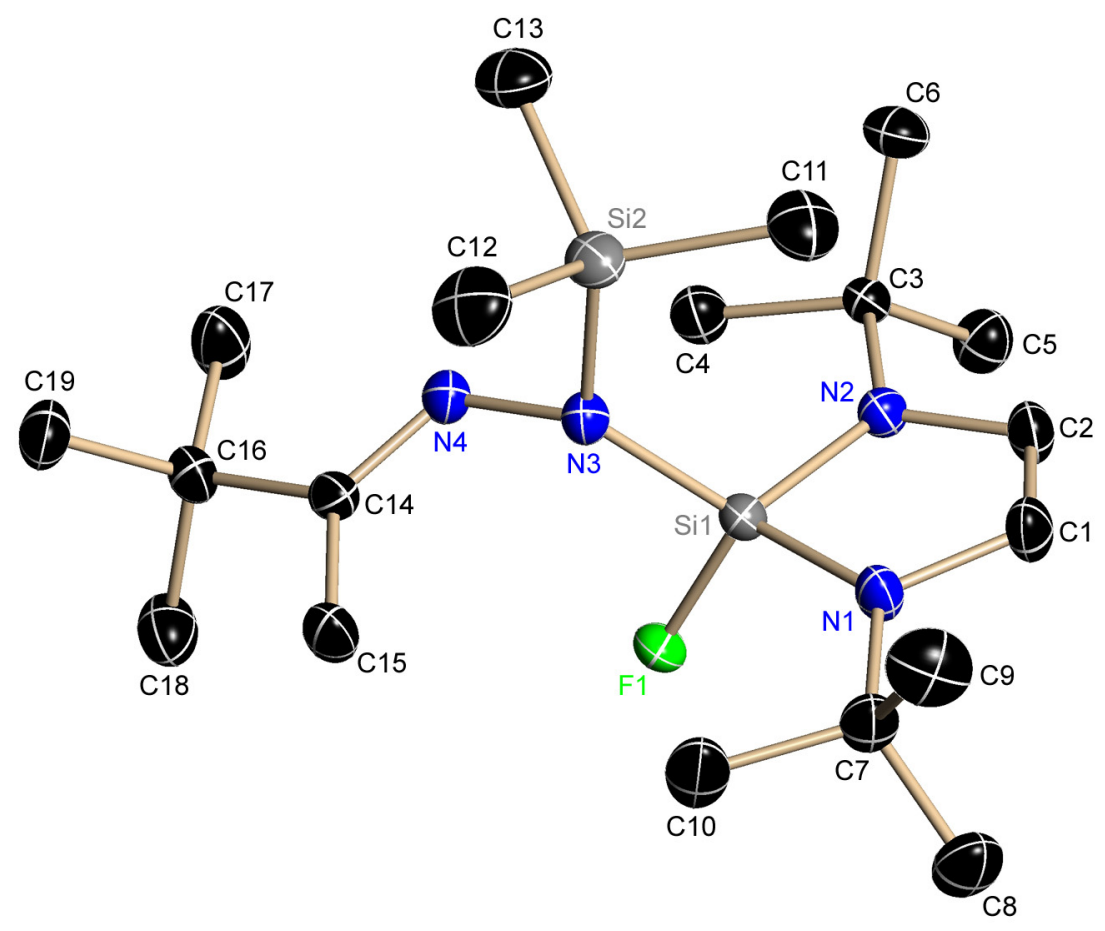

Abb. 5.96: Asymmetrische Einheit von F2407; die anisotropen Auslenkungsparameter geben die 50\% ige Aufenthaltswahrscheinlichkeit an.

Tab. 5.88: Kristallographische Daten von F2407.

\begin{tabular}{|ll|ll|}
\hline Struktur Code & $\mathrm{F} 2407$ & $\rho\left[\mathrm{Mgm}^{-3}\right]$ & 1.104 \\
Summenformel & $\mathrm{C}_{19} \mathrm{H}_{41} \mathrm{FN}_{4} \mathrm{Si}_{2}$ & $\mu\left[\mathrm{mm}^{-1}\right]$ & 0.165 \\
Molekular Gewicht [g/mol] & 400.74 & $F(000)$ & 880 \\
Kristall Größe [mm] & $0.13 \times 0.09 \times 0.06$ & $\theta$-Bereich [ $\left.{ }^{\circ}\right]$ & 2.03 to 26.37 \\
Raumgruppe & $P 2_{1} / n$ & Gesammelte Reflexe & 30603 \\
$a[\mathrm{pm}]$ & $1157.3(4)$ & Unabhängige Reflexe & 4910 \\
$b[\mathrm{pm}]$ & $1631.1(5)$ & Restraints & 0 \\
$c[\mathrm{pm}]$ & $1279.2(4)$ & Parameter & 248 \\
$\alpha\left[^{\circ}\right]$ & 90 & $R 1[/>2 \sigma(I)]$ & 0.0518 \\
$\beta\left[^{\circ}\right]$ & $93.084(5)$ & wR2 [alle Daten] & 0.1208 \\
$\gamma\left[^{\circ}\right]$ & 90 & GooF & 1.051 \\
$\mathrm{~V}\left[\mathrm{~nm}^{3}\right]$ & $2.4112(13)$ & Rest Dichte: & \\
$Z$ & 4 & Max. / min. [10 & epm $\left.{ }^{-3}\right]$ \\
Temperatur $[\mathrm{K}]^{3}$ & $100(2)$ & Max. / min. Transmission & $0.297 /-0.405$ \\
\hline
\end{tabular}




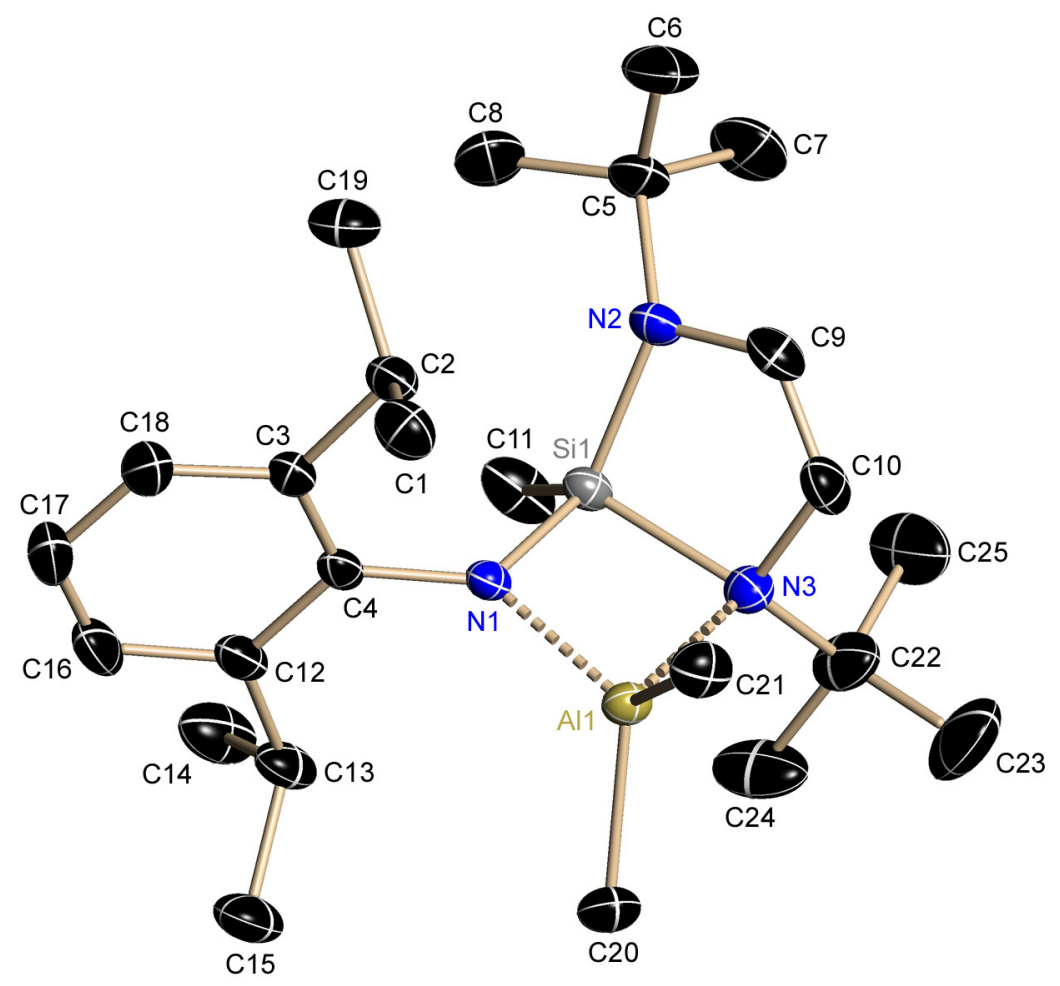

Abb. 5.97: Asymmetrische Einheit von F4107; die anisotropen Auslenkungsparameter geben die 50\% ige Aufenthaltswahrscheinlichkeit an.

Tab. 5.89: Kristallographische Daten von F4107.

\begin{tabular}{|c|c|c|c|}
\hline Struktur Code & F4107 & $\rho\left[\mathrm{Mgm}^{-3}\right]$ & 1.081 \\
\hline Summenformel & $\mathrm{C}_{25} \mathrm{H}_{46} \mathrm{AlN}_{3} \mathrm{Si}$ & $\mu\left[\mathrm{mm}^{-1}\right]$ & 0.134 \\
\hline Molekular Gewicht [g/mol] & 443.72 & $F(000)$ & 976 \\
\hline Kristall Größe [mm] & $0.18 \times 0.18 \times 0.15$ & $\theta$-Bereich $\left[^{\circ}\right]$ & 1.70 to 27.87 \\
\hline Raumgruppe & $P 2_{1} / n$ & Gesammelte Reflexe & 62890 \\
\hline$a[\mathrm{pm}]$ & $944.81(16)$ & Unabhängige Reflexe & 6510 \\
\hline$b[\mathrm{pm}]$ & 1690.1(3) & Restraints & 0 \\
\hline$c[\mathrm{pm}]$ & $1725.3(3)$ & Parameter & 284 \\
\hline$\alpha\left[^{\circ}\right]$ & 90 & $R 1[I>2 \sigma(I)]$ & 0.0359 \\
\hline$\beta\left[^{\circ}\right]$ & $98.228(2)$ & $w R 2$ [alle Daten] & 0.1032 \\
\hline$\gamma\left[{ }^{\circ}\right]$ & 90 & GooF & 1.068 \\
\hline$V\left[\mathrm{~nm}^{3}\right]$ & $2.7267(8)$ & Rest Dichte: & \\
\hline$Z$ & 4 & Max. / min. $\left[10^{-6}\right.$ e $\left.\AA^{-3}\right]$ & $0.318 /-0.226$ \\
\hline Temperatur [K] & $100(2)$ & Max. / min. Transmission & $0.99 / 0.787363$ \\
\hline
\end{tabular}




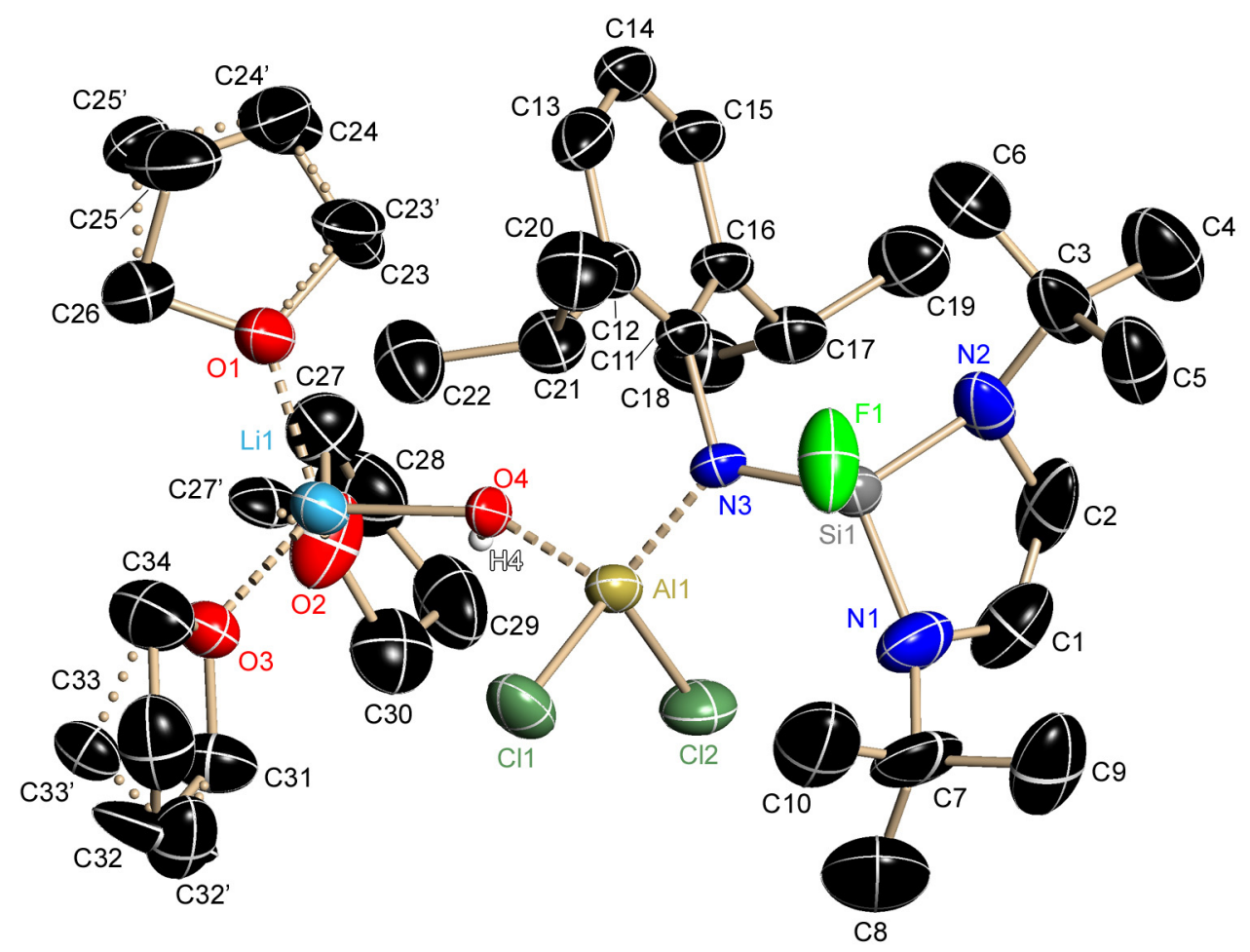

Abb. 5.98: Asymmetrische Einheit von F4507; die anisotropen Auslenkungsparameter geben die 50\% ige Aufenthaltswahrscheinlichkeit an.

Tab. 5.90: Kristallographische Daten von F4507.

\begin{tabular}{|ll|ll|}
\hline Struktur Code & $\mathrm{F} 4507$ & $\mu\left[\mathrm{mm}^{-1}\right]$ & 0.252 \\
Summenformel & $\mathrm{C}_{34} \mathrm{H}_{62} \mathrm{AlCl}_{2} \mathrm{FLiN}_{3} \mathrm{O}_{4} \mathrm{Si}$ & $F(000)$ & 784 \\
Molekular Gewicht [g/mol] & 364.39 & $\theta$-Bereich [ $\left.{ }^{\circ}\right]$ & 2.25 to 25.57 \\
Kristall Größe [mm] & $0.1 \times 0.1 \times 0.2$ & Gesammelte Reflexe & 29985 \\
Raumgruppe & $P n$ & Unabhängige Reflexe & 7944 \\
$a[\mathrm{pm}]$ & $1151.76(10)$ & Restraints & 94 \\
$b[\mathrm{pm}]$ & $1070.73(10)$ & Parameter & 491 \\
$c[\mathrm{pm}]$ & $1655.85(15)$ & $R 1[/>2 \sigma(I)]$ & 0.0642 \\
$\alpha=\gamma\left[^{\circ}\right]$ & 90 & WR2 [alle Daten] & 0.1785 \\
$\beta\left[^{\circ}\right]$ & $94.8820(10)$ & GooF & 1.042 \\
$\mathrm{~V}\left[\mathrm{~nm}^{3}\right]$ & $2.0346(3)$ & Rlack-X & $0.06(9)$ \\
$Z$ & 2 & Max. / min. [10 & \\
Temperatur $\left.[\mathrm{K}]^{-6} \AA^{-3}\right]$ & $0.650 /-0.533$ \\
$\rho\left[\mathrm{Mgm}^{-3}\right]$ & $100(2)$ & Max. / min. Transmission & $0.4288 / 0.3867$ \\
\hline
\end{tabular}




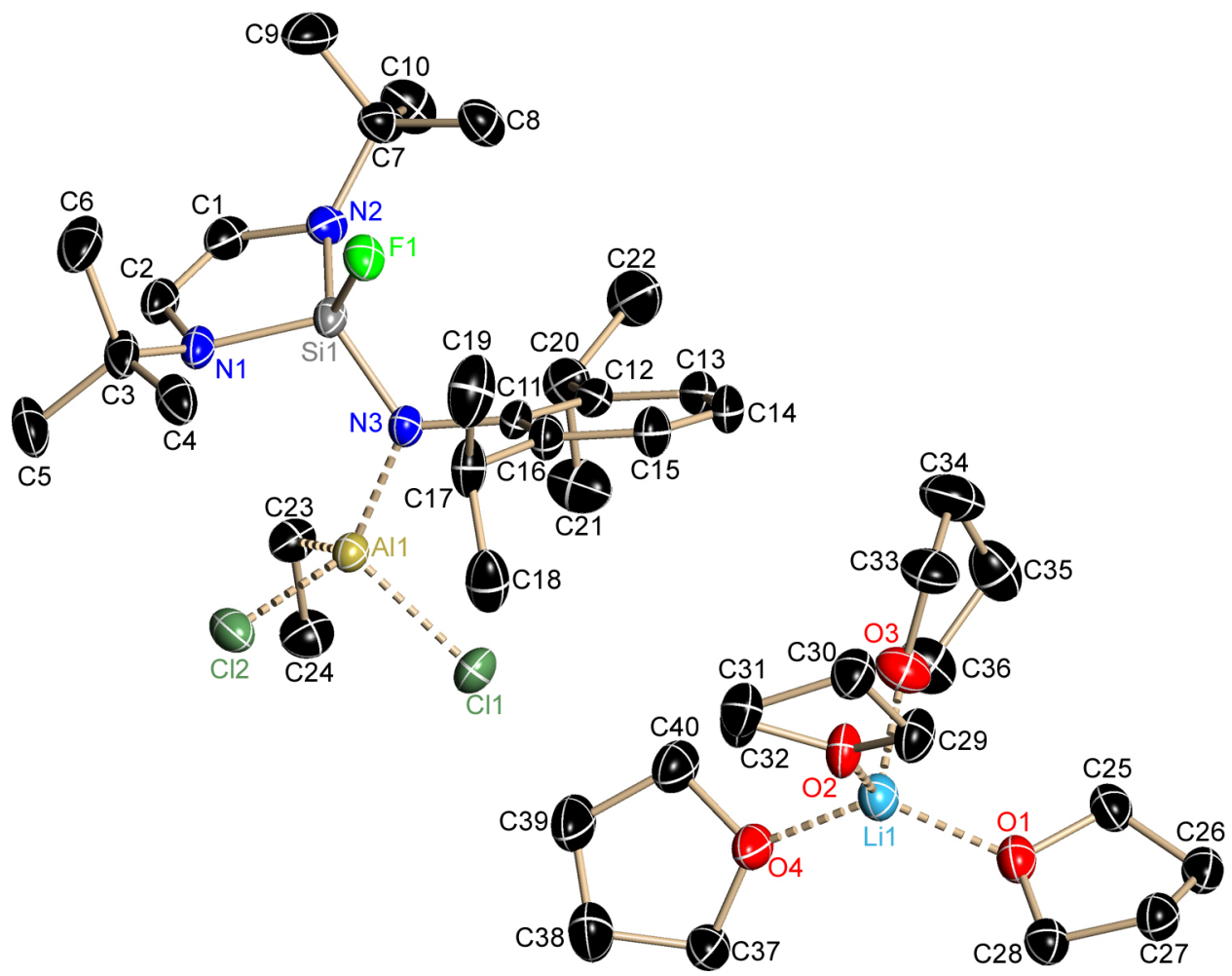

Abb. 5.99: Asymmetrische Einheit von F5607; die anisotropen Auslenkungsparameter geben die $50 \%$ ige Aufenthaltswahrscheinlichkeit an.

Tab. 5.91: Kristallographische Daten von F5607.

\begin{tabular}{|ll|ll|}
\hline Struktur Code & $\mathrm{F} 5607$ & $\rho\left[\mathrm{Mgm}^{-3}\right]$ & 1.176 \\
Summenformel & $\mathrm{C}_{40} \mathrm{H}_{74} \mathrm{AlCl}_{2} \mathrm{FLiN}_{3} \mathrm{O}_{4} \mathrm{Si}$ & $\mu\left[\mathrm{mm}^{-1}\right]$ & 0.230 \\
Molekular Gewicht $[\mathrm{g} / \mathrm{mol}]$ & 812.93 & $F(000)$ & 880 \\
Kristall Größe [mm] & $0.20 \times 0.19 \times 0.10$ & $\theta$-Bereich [ $\left.{ }^{\circ}\right]$ & 2.59 to 25.48 \\
Raumgruppe & $P^{-}$ & Gesammelte Reflexe & 26493 \\
$a[\mathrm{pm}]$ & $1151.8(3)$ & Unabhängige Reflexe & 8305 \\
$b[\mathrm{pm}]$ & $1275.7(3)$ & Restraints & 0 \\
$c[\mathrm{pm}]$ & $1575.9(4)$ & Parameter & 489 \\
$\alpha\left[^{\circ}\right]$ & $92.819(4)$ & $R 1[I>2 \sigma(I)]$ & 0.0522 \\
$\beta\left[^{\circ}\right]$ & $93.379(4)$ & WR2 [alle Daten] & 0.1343 \\
$\gamma\left[^{\circ}\right]$ & $96.130(4)$ & GooF & 1.059 \\
$\mathrm{~V}\left[\mathrm{~nm}{ }^{3}\right]$ & $2.2948(10)$ & Rest Dichte: & \\
$\mathrm{Z}$ & 2 & Max. / min. [10 & \\
Temperatur $[\mathrm{K}]$ & $100(2)$ & Max. / min. Transmission & $0.461 /-0.408$ \\
\hline
\end{tabular}




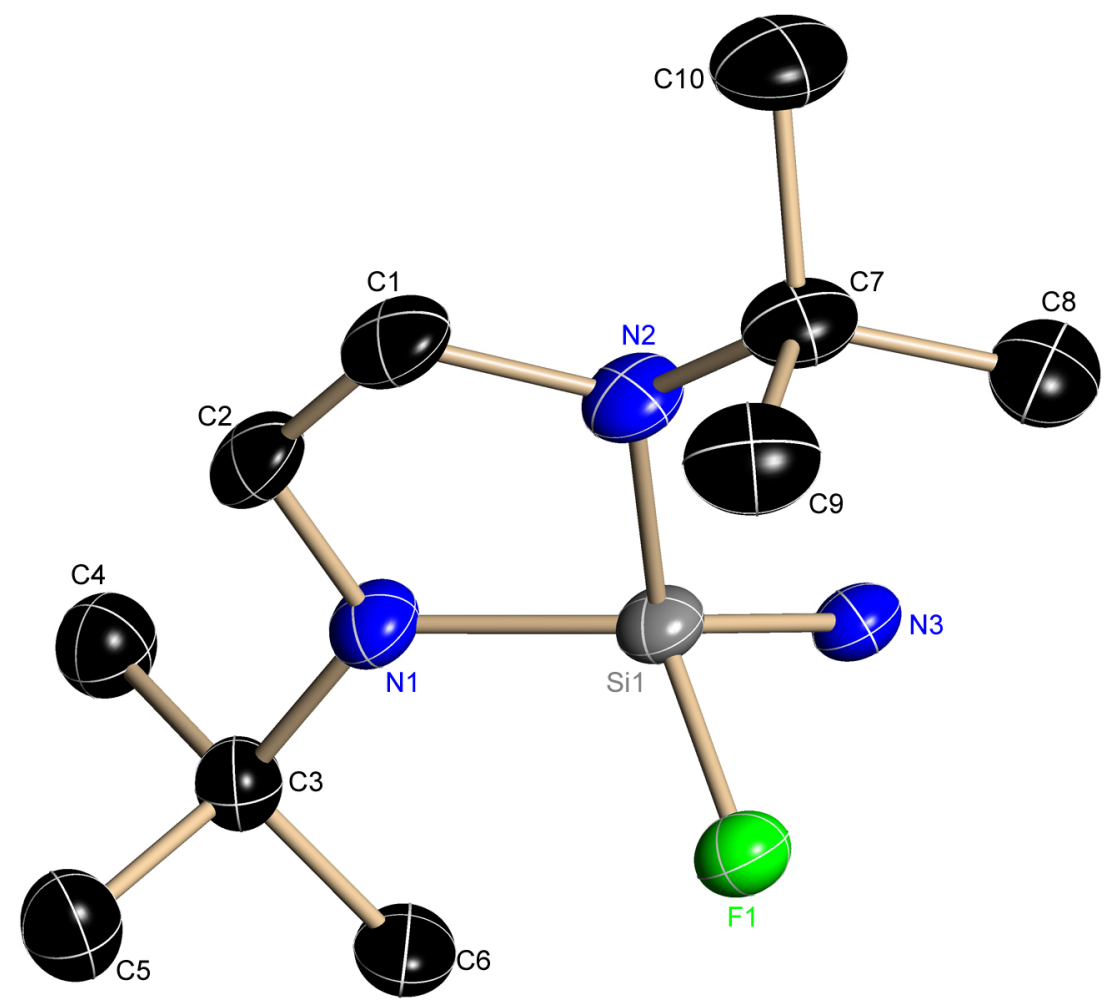

Abb. 5.100: Asymmetrische Einheit von F5707; die anisotropen Auslenkungsparameter geben die 50\% ige Aufenthaltswahrscheinlichkeit an.

Tab. 5.92: Kristallographische Daten von F5707.

\begin{tabular}{|c|c|c|c|}
\hline Struktur Code & F5707 & $\rho\left[\mathrm{Mgm}^{-3}\right]$ & 1.166 \\
\hline Summenformel & $\mathrm{C}_{20} \mathrm{H}_{42} \mathrm{~F}_{2} \mathrm{~N}_{6} \mathrm{Si}_{2}$ & $\mu\left[\mathrm{mm}^{-1}\right]$ & 0.167 \\
\hline Molekular Gewicht [g/mol] & 460.78 & $F(000)$ & 1000 \\
\hline Kristall Größe [mm] & $0.1 \times 0.2 \times 0.2$ & $\theta$-Bereich $\left[^{\circ}\right]$ & 2.61 to 25.44 \\
\hline Raumgruppe & Pbcn & Gesammelte Reflexe & 25670 \\
\hline$a[\mathrm{pm}]$ & $1307.9(4)$ & Unabhängige Reflexe & 2766 \\
\hline$b[\mathrm{pm}]$ & $1676.1(5)$ & Restraints & 0 \\
\hline$c[\mathrm{pm}]$ & $1197.6(4)$ & Parameter & 142 \\
\hline$\alpha\left[^{\circ}\right]$ & 90 & $R 1[I>2 \sigma(I)]$ & 0.0569 \\
\hline$\beta\left[^{\circ}\right]$ & 90 & $w R 2$ [alle Daten] & 0.1556 \\
\hline$\gamma\left[{ }^{\circ}\right]$ & 90 & GooF & 1.058 \\
\hline $\mathrm{V}\left[\mathrm{nm}^{3}\right]$ & $2.6254(15)$ & Rest Dichte: & \\
\hline$Z$ & 4 & Max. / min. $\left[10^{-6} \mathrm{e}^{-3}\right]$ & $0.461 /-0.738$ \\
\hline Temperatur [K] & $100(2)$ & Max. / min. Transmission & $0.7452 / 0.3006$ \\
\hline
\end{tabular}




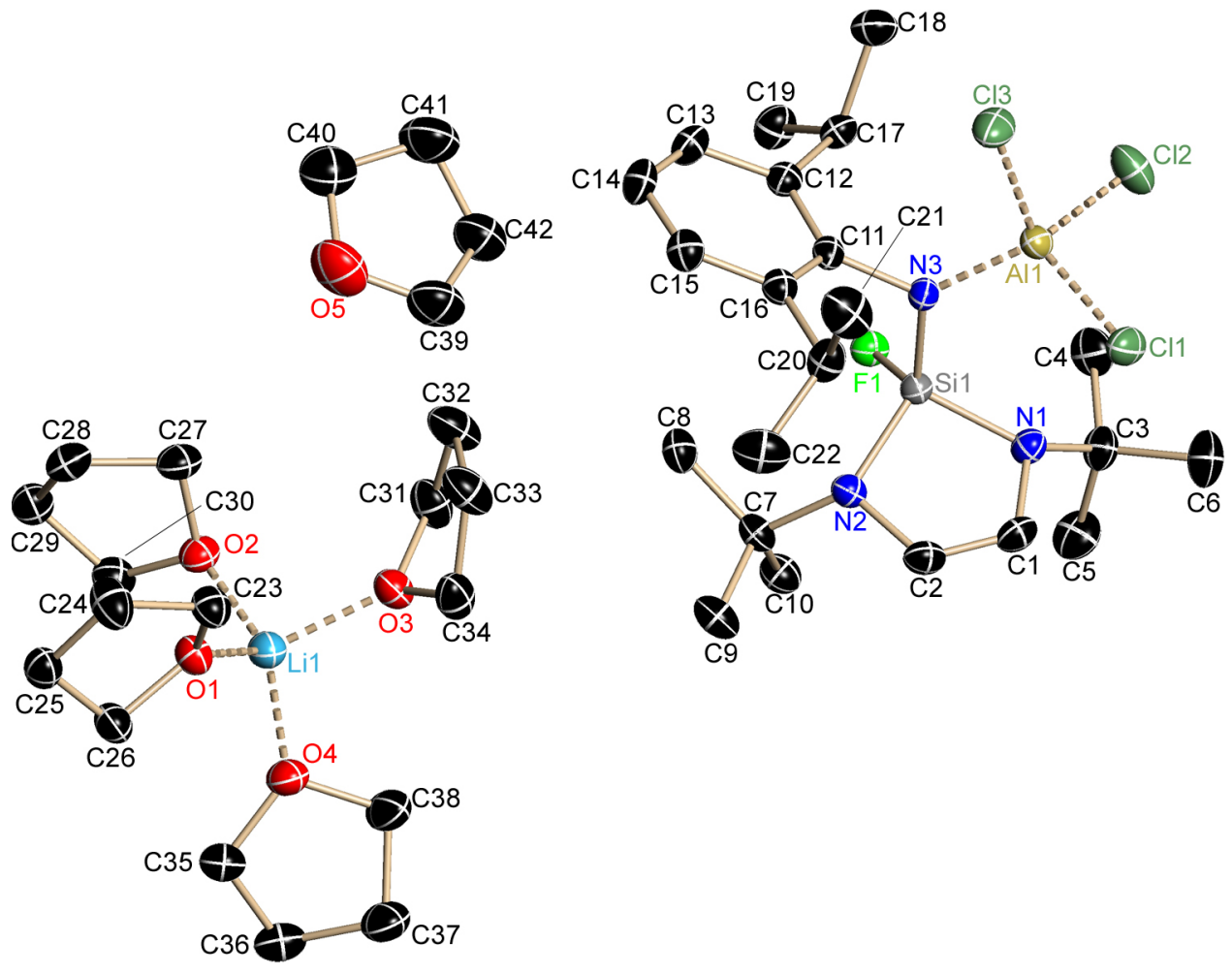

Abb. 5.101: Asymmetrische Einheit von F6107; die anisotropen Auslenkungsparameter geben die 50\% ige Aufenthaltswahrscheinlichkeit an.

Tab. 5.93: Kristallographische Daten von F6107.

\begin{tabular}{|ll|ll|}
\hline Struktur Code & $\mathrm{F} 6107$ & $\rho\left[\mathrm{Mgm}^{-3}\right]$ & 1.215 \\
Summenformel & $\mathrm{C}_{42} \mathrm{H}_{77} \mathrm{AlCl}_{3} \mathrm{FLiN}_{3} \mathrm{O}_{5} \mathrm{Si}$ & $\mu\left[\mathrm{mm}^{-1}\right]$ & 0.277 \\
Molekular Gewicht $[\mathrm{g} / \mathrm{mol}]$ & 891.43 & $F(000)$ & 1920 \\
Kristall Größe $[\mathrm{mm}]$ & $0.2 \times 0.1 \times 0.1$ & $\theta$-Bereich $\left[^{\circ}\right]$ & 2.62 to 25.02 \\
Raumgruppe & $P 22_{1} / c$ & Gesammelte Reflexe & 53841 \\
$a[\mathrm{pm}]$ & $1084.41(15)$ & Unabhängige Reflexe & 8581 \\
$b[\mathrm{pm}]$ & $1829.9(3)$ & Restraints & 17 \\
$c[\mathrm{pm}]$ & $2466.5(3)$ & Parameter & 524 \\
$\alpha\left[^{\circ}\right]$ & 90 & $R 1[/>2 \sigma(I)]$ & 0.0348 \\
$\beta\left[^{\circ}\right]$ & $95.068(2)$ & wR2 [alle Daten] & 0.0935 \\
$\gamma\left[^{\circ}\right]$ & GooF & 1.044 \\
$V\left[\mathrm{~nm}^{3}\right]$ & 40 & Rest Dichte: & \\
$Z$ & $4.8751(12)$ & Max. / min. $\left[10^{-6} \mathrm{eA}^{-3}\right]$ & $0.392 /-0.253$ \\
Temperatur $[\mathrm{K}]$ & 4 & Max. / min. Transmission & $0.4288 / 0.3390$ \\
\hline
\end{tabular}




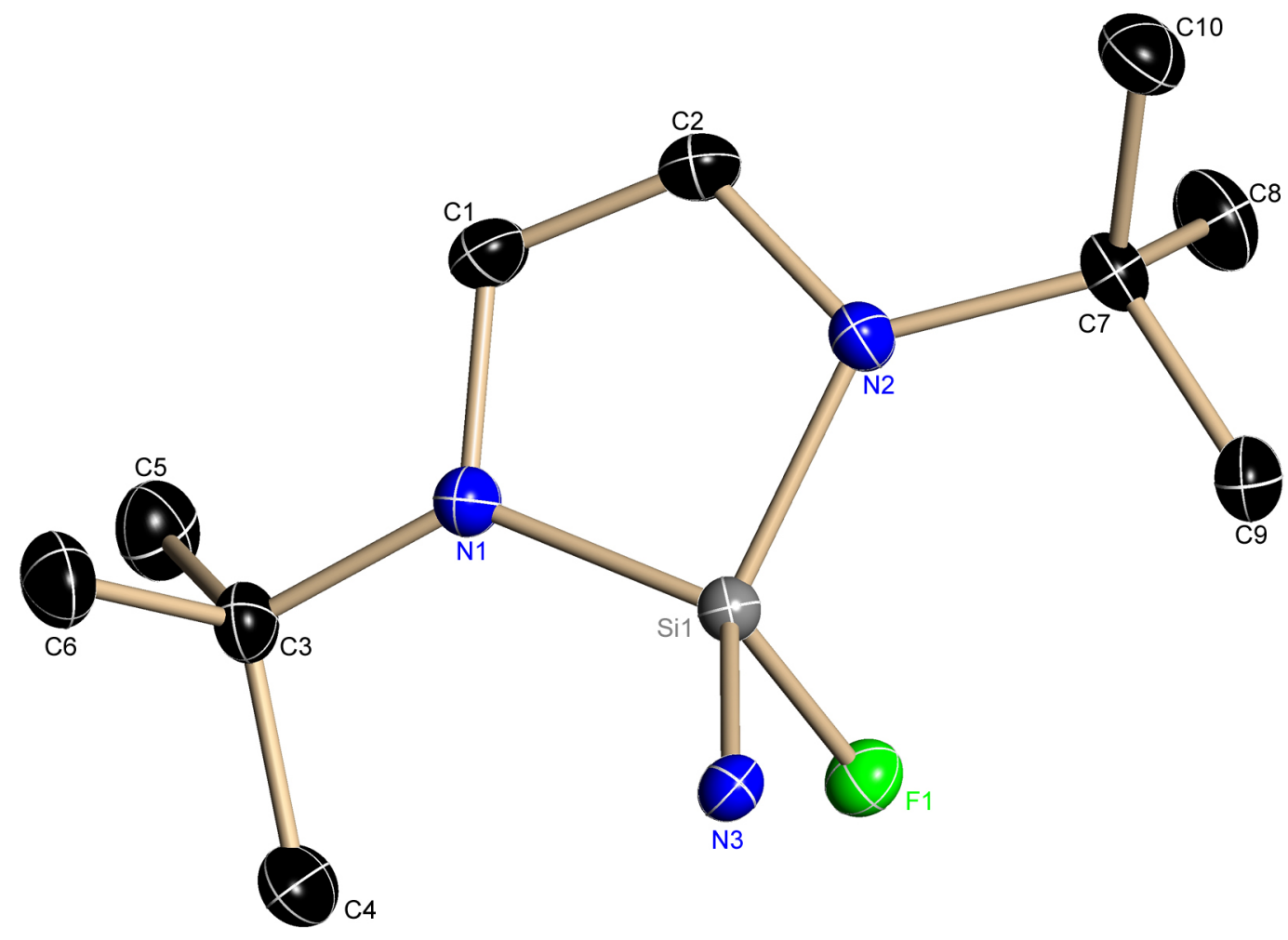

Abb. 5.102: Asymmetrische Einheit von F6307; die anisotropen Auslenkungsparameter geben die $50 \%$ ige Aufenthaltswahrscheinlichkeit an.

$\mathrm{NH}$ auf Spiegelebene

Tab. 5.94: Kristallographische Daten von F6307.

\begin{tabular}{|ll|ll|}
\hline Struktur Code & $\mathrm{F} 6307$ & $\rho\left[\mathrm{Mgm}^{-3}\right]$ & 1.194 \\
Summenformel & $\mathrm{C}_{10} \mathrm{H}_{20.50} \mathrm{FN}_{2.50} \mathrm{Si}$ & $\mu\left[\mathrm{mm}^{-1}\right]$ & 0.174 \\
Molekular Gewicht [g/mol] & 222.88 & $F(000)$ & 484 \\
Kristall Größe $[\mathrm{mm}]$ & $0.4 \times 0.4 \times 0.3$ & $\theta$-Bereich [ $\left.{ }^{\circ}\right]$ & 2.93 to 26.02 \\
Raumgruppe & $P 2 / n$ & Gesammelte Reflexe & 26757 \\
$a[\mathrm{pm}]$ & $1310.34(12)$ & Unabhängige Reflexe & 2665 \\
$b[\mathrm{pm}]$ & $694.22(6)$ & Restraints & 0 \\
$c[\mathrm{pm}]$ & $1365.12(13)$ & Parameter & 138 \\
$\alpha\left[^{\circ}\right]$ & 90 & $R 1[/>2 \sigma(I)]$ & 0.0333 \\
$\beta\left[^{\circ}\right]$ & $93.4330(10)$ & $w R 2[$ alle Daten] & 0.0876 \\
$\gamma\left[^{\circ}\right]$ & 90 & GooF & 1.092 \\
$\mathrm{~V}\left[\mathrm{~nm}^{3}\right]$ & $1.2396(2)$ & Rest Dichte: & \\
$Z$ & 4 & Max. / min. $\left[10^{-6} \mathrm{eA}^{-3}\right]$ & $0.285 /-0.338$ \\
Temperatur $[\mathrm{K}]$ & $100(2)$ & Max. / min. Transmission & $0.7172 / 0.6272$ \\
\hline
\end{tabular}




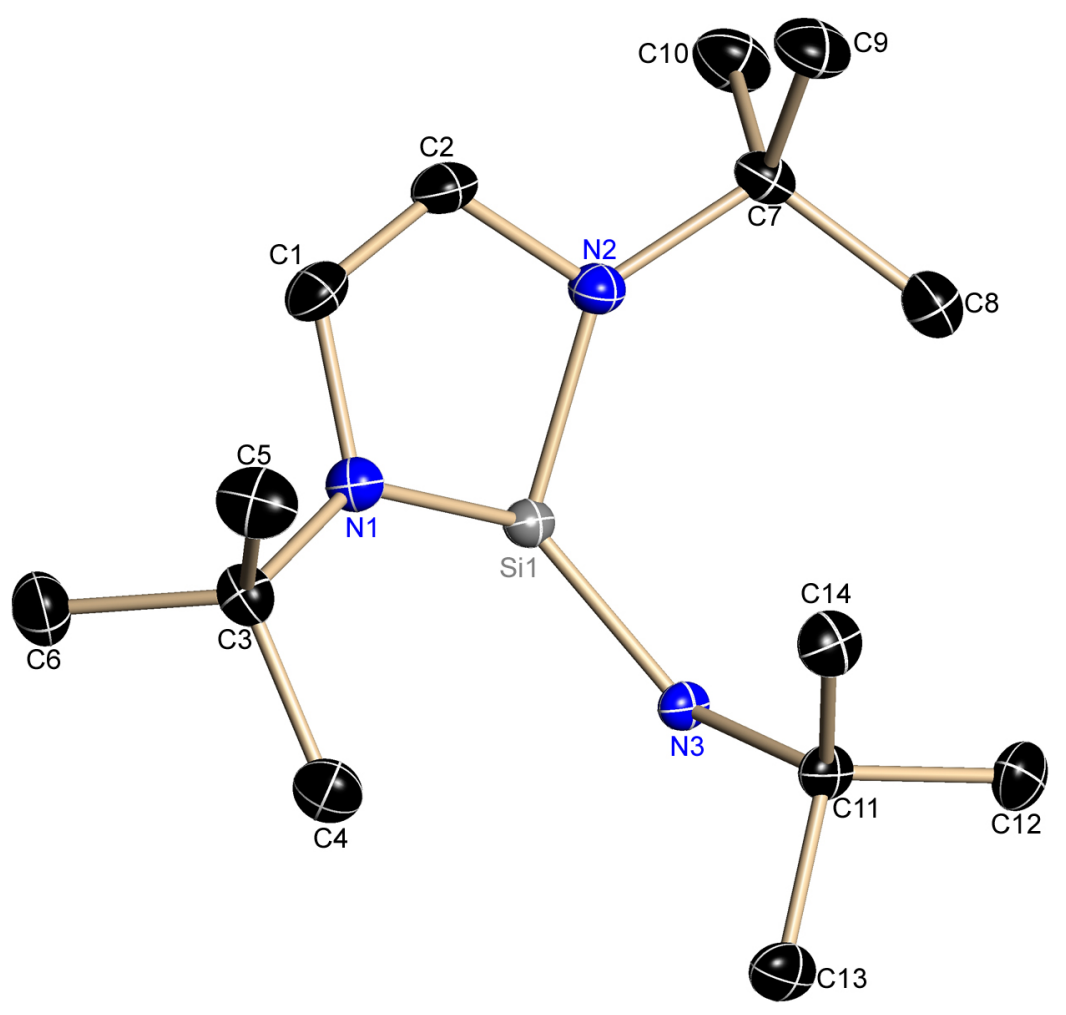

Abb. 5.103: Asymmetrische Einheit von F6407; die anisotropen Auslenkungsparameter geben die $50 \%$ ige Aufenthaltswahrscheinlichkeit an.

Tab. 5.95: Kristallographische Daten von F6407.

\begin{tabular}{|ll|ll|}
\hline Struktur Code & $\mathrm{F} 6407$ & $\rho\left[\mathrm{Mgm}^{-3}\right]$ & 1.118 \\
Summenformel & $\mathrm{C}_{28} \mathrm{H}_{58} \mathrm{~N}_{6} \mathrm{Si}_{2}$ & $\mu\left[\mathrm{mm}^{-1}\right]$ & 0.138 \\
Molekular Gewicht [g/mol] & 534.98 & $F(000)$ & 592 \\
Kristall Größe [mm] & $0.1 \times 0.1 \times 0.1$ & $\theta$-Bereich [ $\left.{ }^{\circ}\right]$ & 2.26 to 26.76 \\
Raumgruppe & $P 2_{1} / n$ & Gesammelte Reflexe & 20330 \\
$a[\mathrm{pm}]$ & $970.46(9)$ & Unabhängige Reflexe & 3599 \\
$b[\mathrm{pm}]$ & $1000.70(9)$ & Restraints & 0 \\
$c[\mathrm{pm}]$ & $1666.66(15)$ & Parameter & 172 \\
$\alpha\left[^{\circ}\right]$ & 90 & $R 1[I>2 \sigma(I)]$ & 0.0397 \\
$\beta\left[^{\circ}\right]$ & $101.0450(10)$ & WR2 [alle Daten] & 0.1051 \\
$\gamma\left[^{\circ}\right]$ & 90 & GooF & 1.045 \\
$\mathrm{~V}\left[\mathrm{~nm}{ }^{3}\right]$ & $1.5886(3)$ & Rest Dichte: & \\
$Z$ & 2 & Max. / min. [10 & \\
Temperatur $[\mathrm{K}]$ & $100(2)$ & Max. / min. Transmission & $0.943 /-0.274$ \\
\hline
\end{tabular}


Structure of F6507
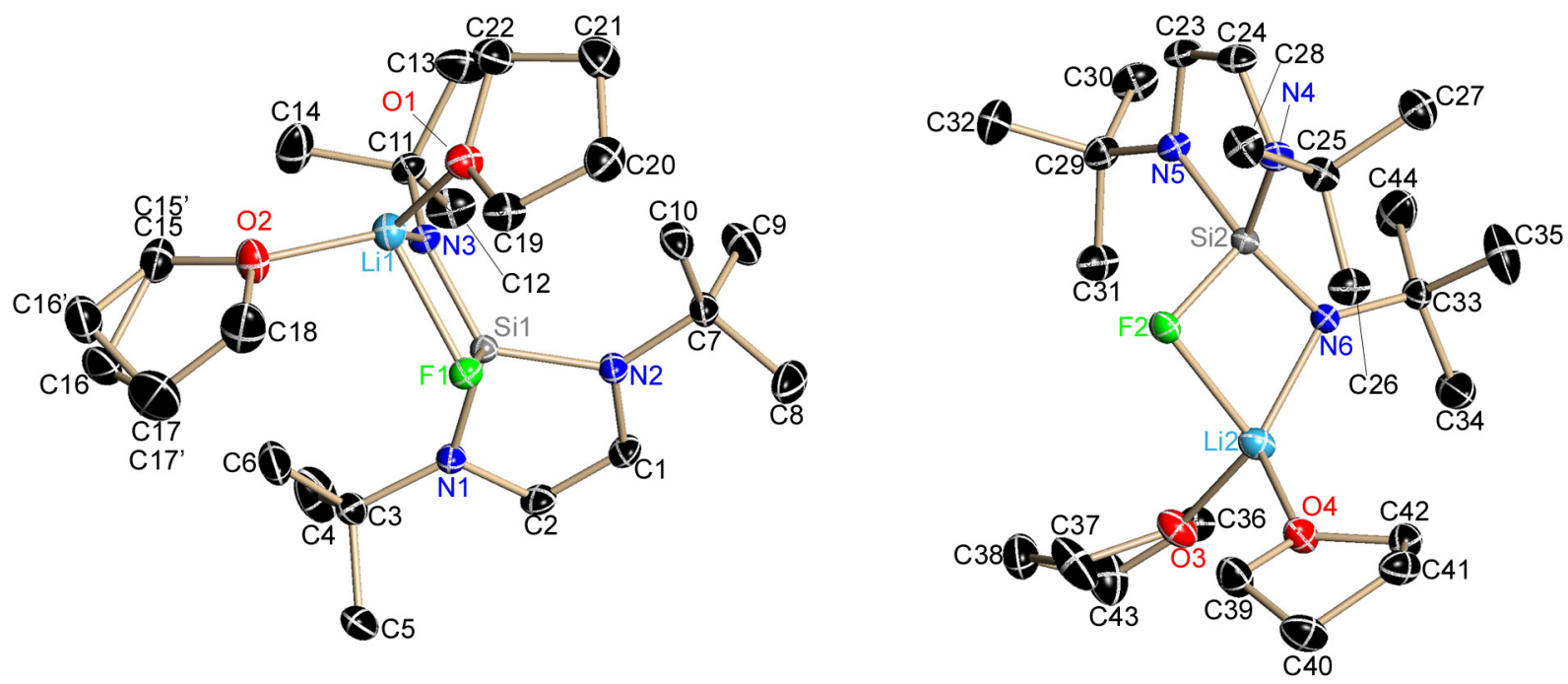

Abb. 5.104: Asymmetrische Einheit von F6507; die anisotropen Auslenkungsparameter geben die 50\% ige Aufenthaltswahrscheinlichkeit an.

Tab. 5.96: Kristallographische Daten von F6507.

\begin{tabular}{|ll|ll|}
\hline Struktur Code & $\mathrm{F} 6507$ & $\rho\left[\mathrm{Mgm}^{-3}\right]$ & 1.129 \\
Summenformel & $\mathrm{C}_{22} \mathrm{H}_{45} \mathrm{FLiN}_{3} \mathrm{O}_{2} \mathrm{Si}$ & $\mu\left[\mathrm{mm}^{-1}\right]$ & 0.119 \\
Molekular Gewicht [g/mol] & 437.64 & $F(000)$ & 960 \\
Kristall Größe [mm] & $0.1 \times 0.1 \times 0.1$ & $\theta$-Bereich [ $\left.{ }^{\circ}\right]$ & 1.39 to 26.37 \\
Raumgruppe & $P^{-}$ & Gesammelte Reflexe & 27110 \\
$a[\mathrm{pm}]$ & $1003.20(8)$ & Unabhängige Reflexe & 10424 \\
$b[\mathrm{pm}]$ & $1511.64(12)$ & Restraints & 0 \\
$c[\mathrm{pm}]$ & $1757.88(14)$ & Parameter & 566 \\
$\alpha\left[^{\circ}\right]$ & $90.3510(10)$ & $R 1[/>2 \sigma(I)]$ & 0.0365 \\
$\beta\left[^{\circ}\right]$ & $93.7730(10)$ & WR2 [alle Daten] & 0.0966 \\
$\gamma\left[^{\circ}\right]$ & $104.4790(10)$ & GooF & 1.065 \\
$\mathrm{~V}\left[\mathrm{~nm}{ }^{3}\right]$ & $2.5748(4)$ & Rest Dichte: & \\
$Z$ & 4 & Max. / min. [10 $\left.{ }^{-6} \mathrm{eA}^{-3}\right]$ & $0.421 /-0.279$ \\
Temperatur $[\mathrm{K}]$ & $100(2)$ & Max. / min. Transmission & $0.7454 / 0.6861$ \\
\hline
\end{tabular}




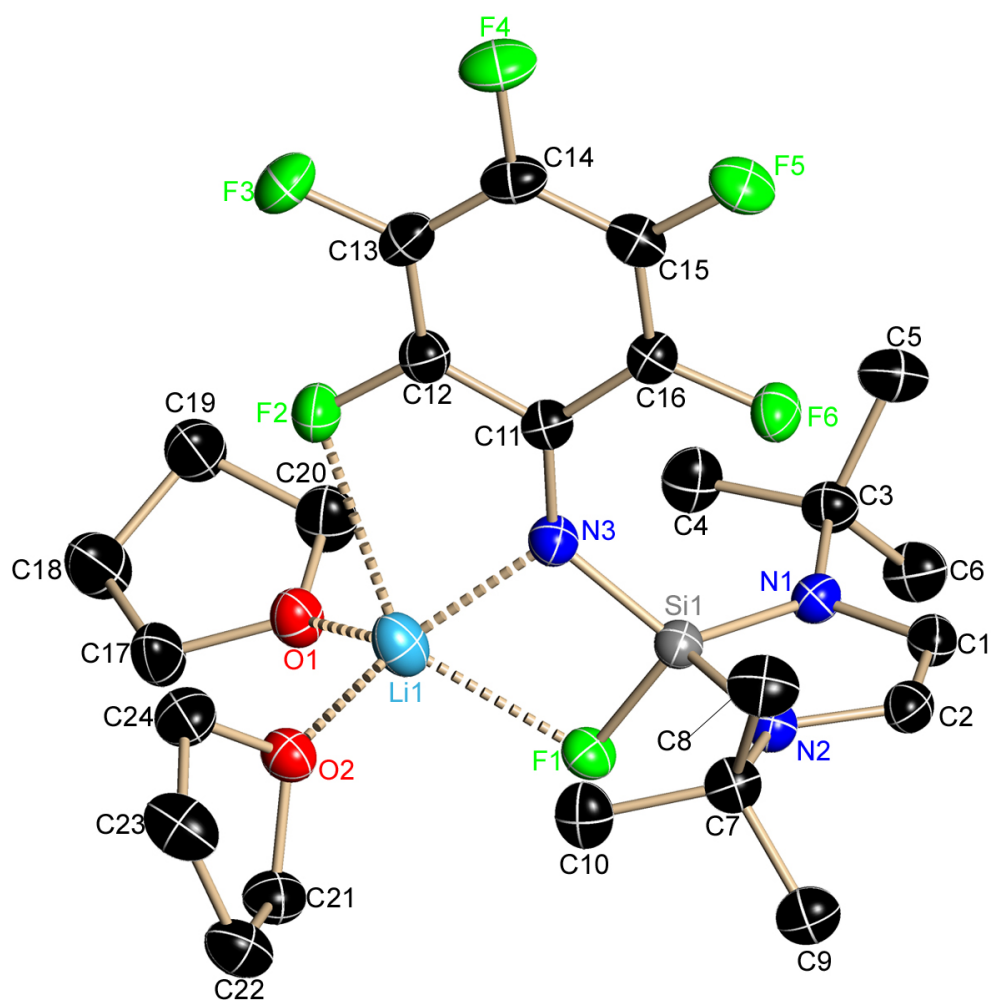

Abb. 5.105: Asymmetrische Einheit von F6707; die anisotropen Auslenkungsparameter geben die 50\% ige Aufenthaltswahrscheinlichkeit an.

Tab. 5.97: Kristallographische Daten von F6707.

\begin{tabular}{|ll|ll|}
\hline Struktur Code & $\mathrm{F} 6707$ & $\rho\left[\mathrm{Mgm}^{-3}\right]$ & 1.354 \\
Summenformel & $\mathrm{C}_{24} \mathrm{H}_{36} \mathrm{~F}_{6} \mathrm{LiN}_{3} \mathrm{O}_{2} \mathrm{Si}$ & $\mu\left[\mathrm{mm}^{-1}\right]$ & 0.155 \\
Molekular Gewicht $[\mathrm{g} / \mathrm{mol}]$ & 547.59 & $F(000)$ & 1152 \\
Kristall Größe [mm] & $0.1 \times 0.1 \times 0.09$ & $\theta$-Bereich [ $\left.{ }^{\circ}\right]$ & 2.39 to 25.40 \\
Raumgruppe & $P 2_{1} / n$ & Gesammelte Reflexe & 23909 \\
$a[\mathrm{pm}]$ & $1068.64(16)$ & Unabhängige Reflexe & 4915 \\
$b[\mathrm{pm}]$ & $1477.7(2)$ & Restraints & 0 \\
$c[\mathrm{pm}]$ & $1722.0(3)$ & Parameter & 340 \\
$\alpha\left[^{\circ}\right]$ & 90 & $R 1[I>2 \sigma(I)]$ & 0.0380 \\
$\beta\left[^{\circ}\right]$ & $98.857(2)$ & WR2 [alle Daten] & 0.1038 \\
$\gamma\left[^{\circ}\right]$ & 90 & GooF & 1.043 \\
$\mathrm{~V}\left[\mathrm{~nm}{ }^{3}\right]$ & $2.6869(7)$ & Rest Dichte: & \\
$\mathrm{Z}$ & 4 & Max. / min. [10 & \\
Temperatur $[\mathrm{K}]$ & $100(2)$ & Max. / min. Transmission & $0.263 /-0.373$ \\
\hline
\end{tabular}




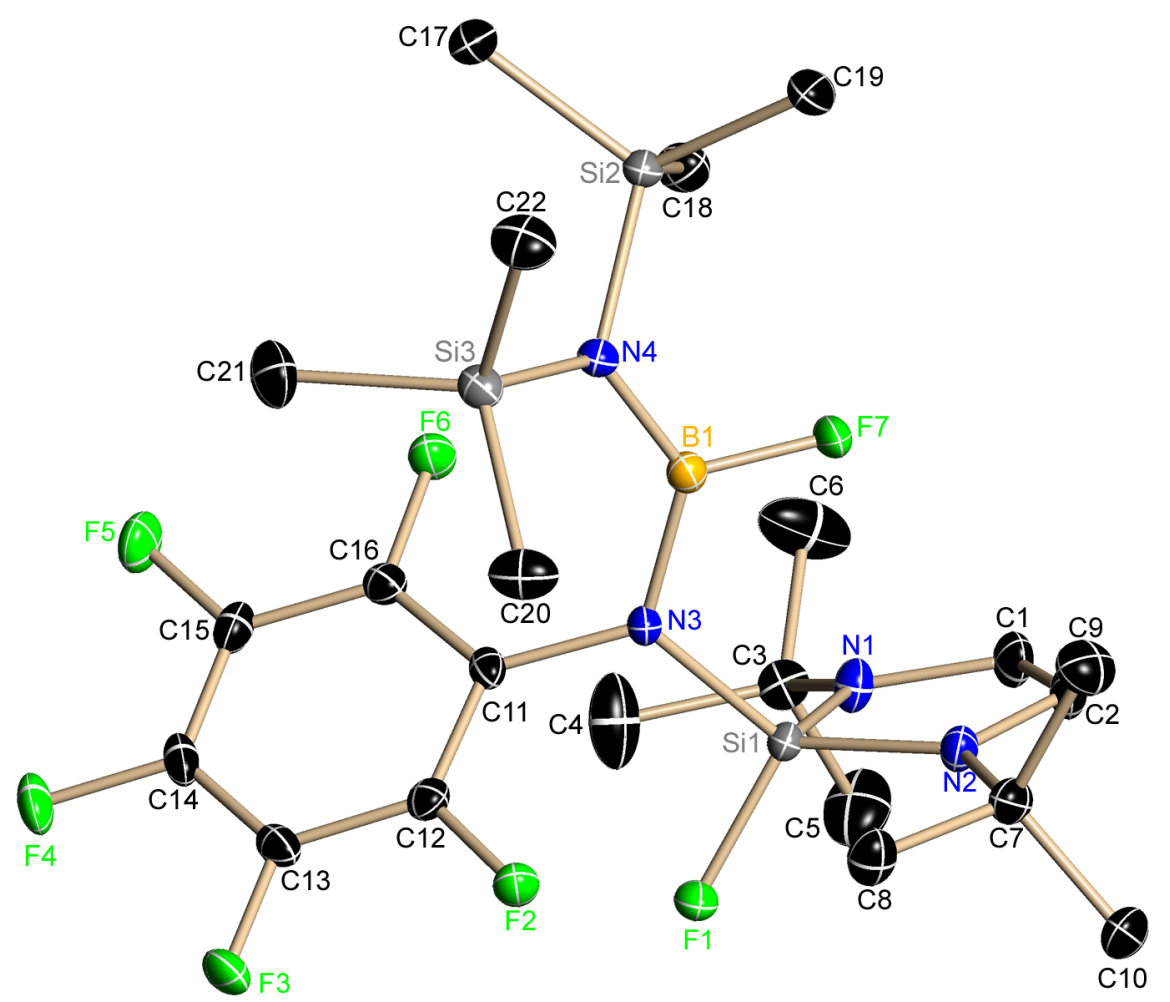

Abb. 5.106: Asymmetrische Einheit von F7007; die anisotropen Auslenkungsparameter geben die $50 \%$ ige Aufenthaltswahrscheinlichkeit an.

Tab. 5.98: Kristallographische Daten von F7007.

\begin{tabular}{|c|c|c|c|}
\hline Struktur Code & F7007 & $\rho\left[\mathrm{Mgm}^{-3}\right]$ & 1.320 \\
\hline Summenformel & $\mathrm{C}_{22} \mathrm{H}_{38} \mathrm{BF}_{7} \mathrm{~N}_{4} \mathrm{Si}_{3}$ & $\mu\left[\mathrm{mm}^{-1}\right]$ & 0.224 \\
\hline Molekular Gewicht [g/mol] & 586.64 & $F(000)$ & 616 \\
\hline Kristall Größe [mm] & $0.4 \times 0.4 \times 0.3$ & $\theta$-Bereich $\left[{ }^{\circ}\right]$ & 1.66 to 25.35 \\
\hline Raumgruppe & $P_{\overline{1}}$ & Gesammelte Reflexe & 19928 \\
\hline$a[\mathrm{pm}]$ & $1028.51(8)$ & Unabhängige Reflexe & 5388 \\
\hline$b[\mathrm{pm}]$ & $1231.49(10)$ & Restraints & 0 \\
\hline$c[\mathrm{pm}]$ & $1253.43(10)$ & Parameter & 347 \\
\hline$\alpha\left[^{\circ}\right]$ & $78.4660(10)$ & $R 1[I>2 \sigma(I)]$ & 0.0290 \\
\hline$\beta\left[^{\circ}\right]$ & $83.5480(10)$ & $w R 2$ [alle Daten] & 0.0772 \\
\hline$\gamma\left[^{\circ}\right]$ & $71.8710(10)$ & GooF & 1.013 \\
\hline$V\left[\mathrm{~nm}^{3}\right]$ & $1.4762(2)$ & Rest Dichte: & \\
\hline$Z$ & 2 & Max. / min. $\left[10^{-6} \mathrm{e}^{-3}\right]$ & $0.357 /-0.259$ \\
\hline Temperatur $[\mathrm{K}]$ & $100(2)$ & Max. / min. Transmission & 0.7454 / 0.7010 \\
\hline
\end{tabular}




\subsubsection{Struktur des AK Beifuß (Universität Hohenstein)}

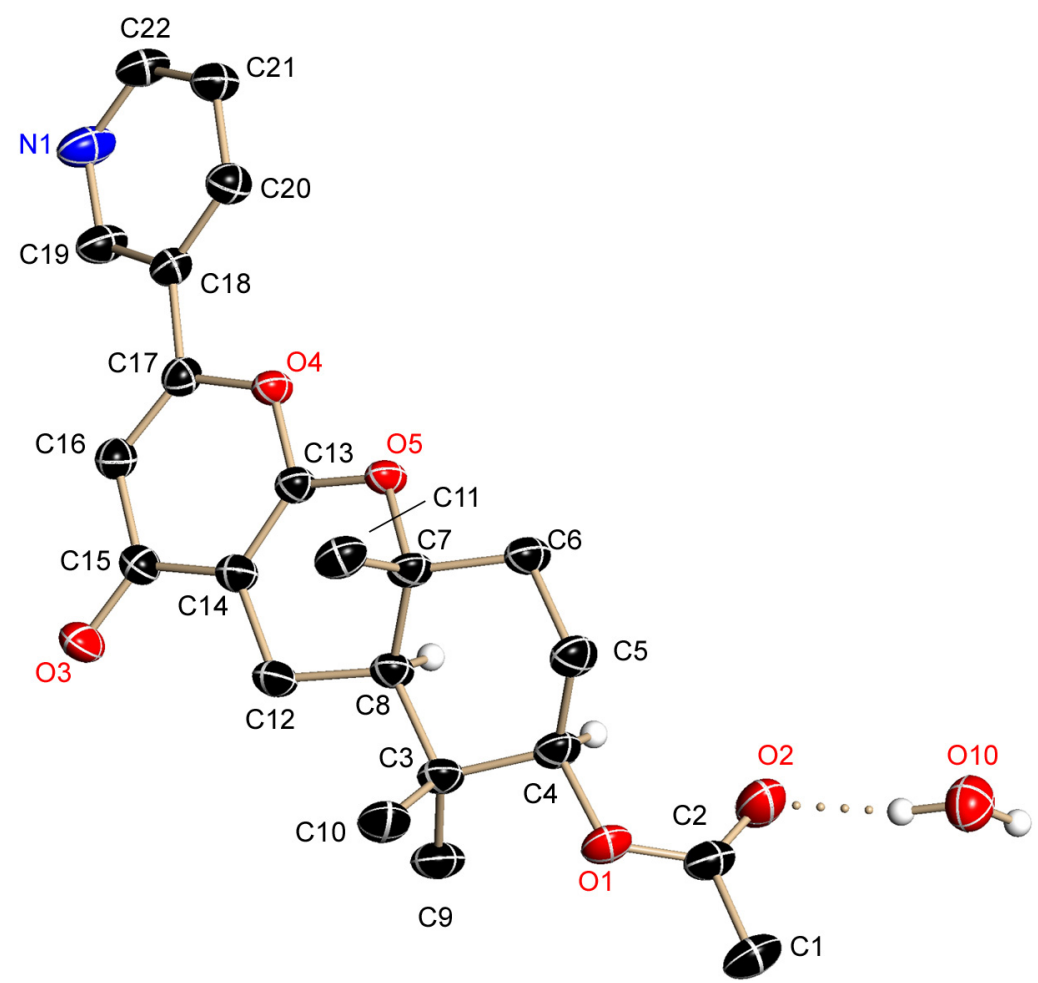

Abb. 5.107: Asymmetrische Einheit von DSR003; die anisotropen Auslenkungsparameter geben die 50\% ige Aufenthaltswahrscheinlichkeit an.

Das Wassermolekül ist nur zu 30 \% besetzt.

Tab. 5.99: Kristallographische Daten von DSR003.

\begin{tabular}{|c|c|c|c|}
\hline Strukturcode & DSR003 & $\rho\left[\mathrm{Mgm}^{-3}\right]$ & 1.285 \\
\hline Summenformel & $\mathrm{C}_{22} \mathrm{H}_{25.6} \mathrm{ONO}_{5.30}$ & $\mu\left[\mathrm{mm}^{-1}\right]$ & 0.092 \\
\hline Molekulare Masse $[\mathrm{g} / \mathrm{mol}]$ & 388.83 & $F(000)$ & 1656 \\
\hline Kristallgröße [mm] & $0.15 \times 0.14 \times 0.14$ & $\theta$-Bereich $\left[{ }^{\circ}\right]$ & $2.62-25.02$ \\
\hline Raumgruppe & $C 2 / c$ & Gesammelte Reflexe & 33895 \\
\hline$a[\mathrm{pm}]$ & $1852.2(4)$ & Unabhängige Reflexe & 3699 \\
\hline$b[\mathrm{pm}]$ & $1556.0(3)$ & Restraints & 3 \\
\hline$c[\mathrm{pm}]$ & $1564.6(3)$ & Parameter & 275 \\
\hline$\alpha\left[^{\circ}\right]$ & 90 & $R 1[I>2 \sigma(I)]$ & 0.0364 \\
\hline$\beta\left[^{\circ}\right]$ & $116.927(2)$ & $w R 2$ [alle Daten] & 0.0906 \\
\hline$\gamma\left[^{\circ}\right]$ & 90 & Goof & 1.048 \\
\hline $\mathrm{V}\left[\mathrm{nm}^{3}\right]$ & $4.0203(13)$ & Restdichte: & \\
\hline$Z$ & 8 & Max. / min. $\left[10^{-6} \mathrm{epm}^{-3}\right]$ & $0.211 /-0.169$ \\
\hline Temperatur [K] & $100(2)$ & Max. / min. Transmission & $0.8620 / 0.7198$ \\
\hline
\end{tabular}




\subsubsection{Struktur des AK Breher (Universität Karlsruhe)}

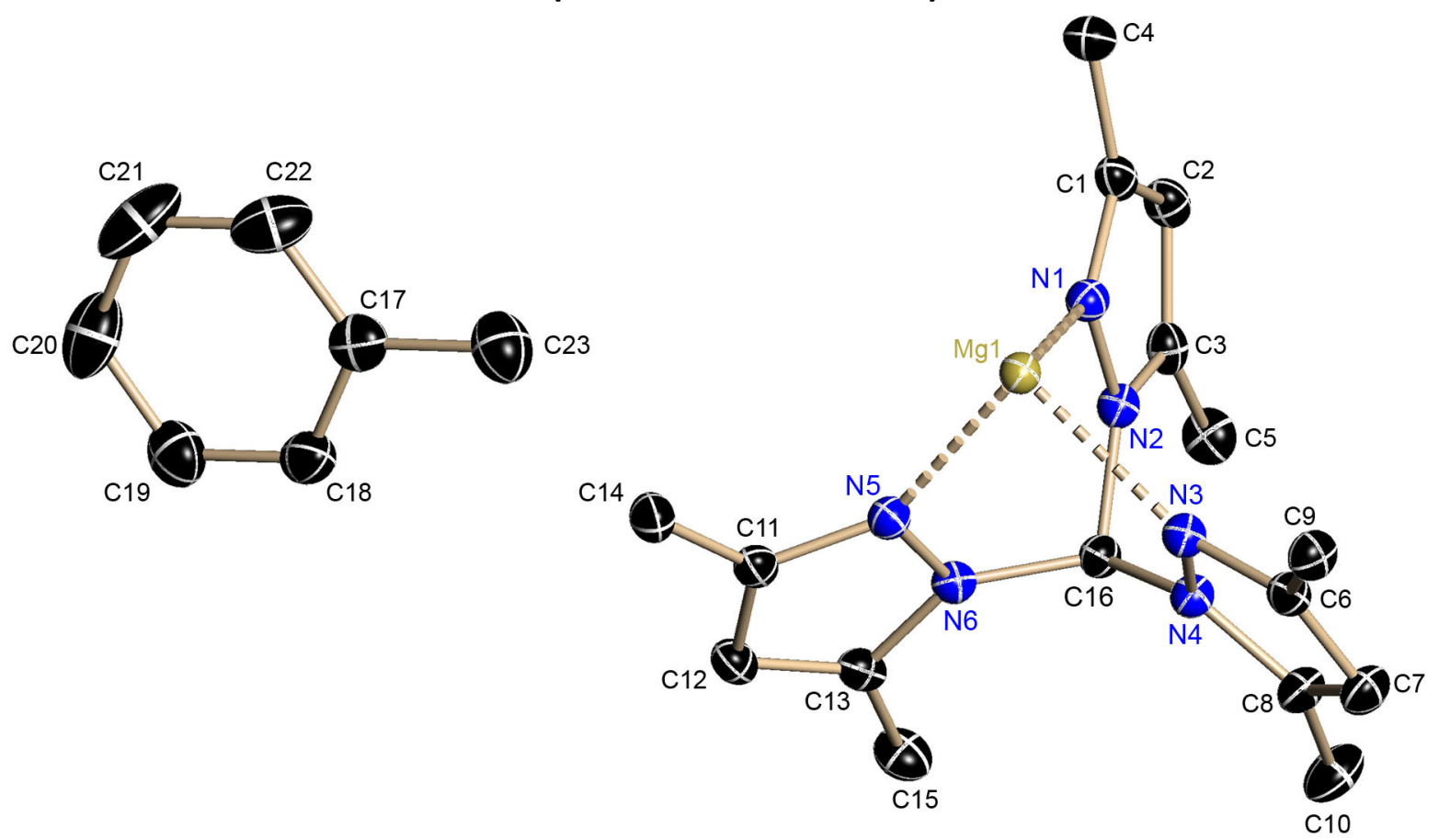

Abb. 5.108: Asymmetrische Einheit von $\left[\mathrm{Mg}\left({ }^{\mathrm{Me}} \mathrm{Tpmd}\right)_{2}\right] \cdot(\text { Toluol })_{2}$; die anisotropen Auslenkungsparameter geben die $50 \%$ ige Aufenthaltswahrscheinlichkeit an.

Tab. 5.100: Kristallographische Daten von $\left[\mathrm{Mg}\left({ }^{\mathrm{Me}} \mathrm{Tpmd}\right)_{2}\right] \cdot(\text { Toluol })_{2}$.

\begin{tabular}{|ll|ll|}
\hline Strukturcode & {$\left[\mathrm{Mg}\left({ }^{\mathrm{Me}} \mathrm{Tpmd}\right)_{2}\right] \cdot\left(\right.$ Toluol $_{2}$} & $\rho\left[\mathrm{Mgm}^{-3}\right]$ & 1.219 \\
Summenformel & $\mathrm{C}_{46} \mathrm{H}_{58} \mathrm{MgN}_{12}$ & $\mu\left[\mathrm{mm}^{-1}\right]$ & 0.088 \\
Molekulare Masse [g/mol] & 803.35 & $F(000)$ & 430 \\
Kristallgröße [mm] & $0.3 \times 0.2 \times 0.08$ & $\theta$-Bereich [ $\left.{ }^{\circ}\right]$ & $1.50-26.82$ \\
Raumgruppe & $P^{\overline{1}}$ & Gesammelte Reflexe & 4662 \\
$a[\mathrm{pm}]$ & $852.76(6)$ & Unabhängige Reflexe & 4662 \\
$b[\mathrm{pm}]$ & $973.36(7)$ & Restraints & 0 \\
$c[\mathrm{pm}]$ & $1396.64(10$ & Parameter & 278 \\
$\alpha\left[^{\circ}\right]$ & $102.7840(10)$ & $R 1[/>2 \sigma(I)]$ & 0.0382 \\
$\beta\left[^{\circ}\right]$ & $92.4480(10)$ & $w R 2[$ alle Daten $]$ & 0.0995 \\
$\gamma\left[^{\circ}\right]$ & $103.4640(10)$ & GooF & 1.050 \\
$\mathrm{~V}\left[\mathrm{~nm}^{3}\right]$ & $1.09402(14)$ & Restdichte: & \\
$Z$ & 1 & Max. / min. $\left[10^{-6} \mathrm{epm}^{-3}\right]$ & $0.289 /-0.223$ \\
Temperatur $[\mathrm{K}]$ & $100(2)$ & Max. / min. Transmission & $0.8620 / 0.7751$ \\
\hline
\end{tabular}




\subsubsection{Strukturen des AK Saalfrank (Universität Erlangen)}

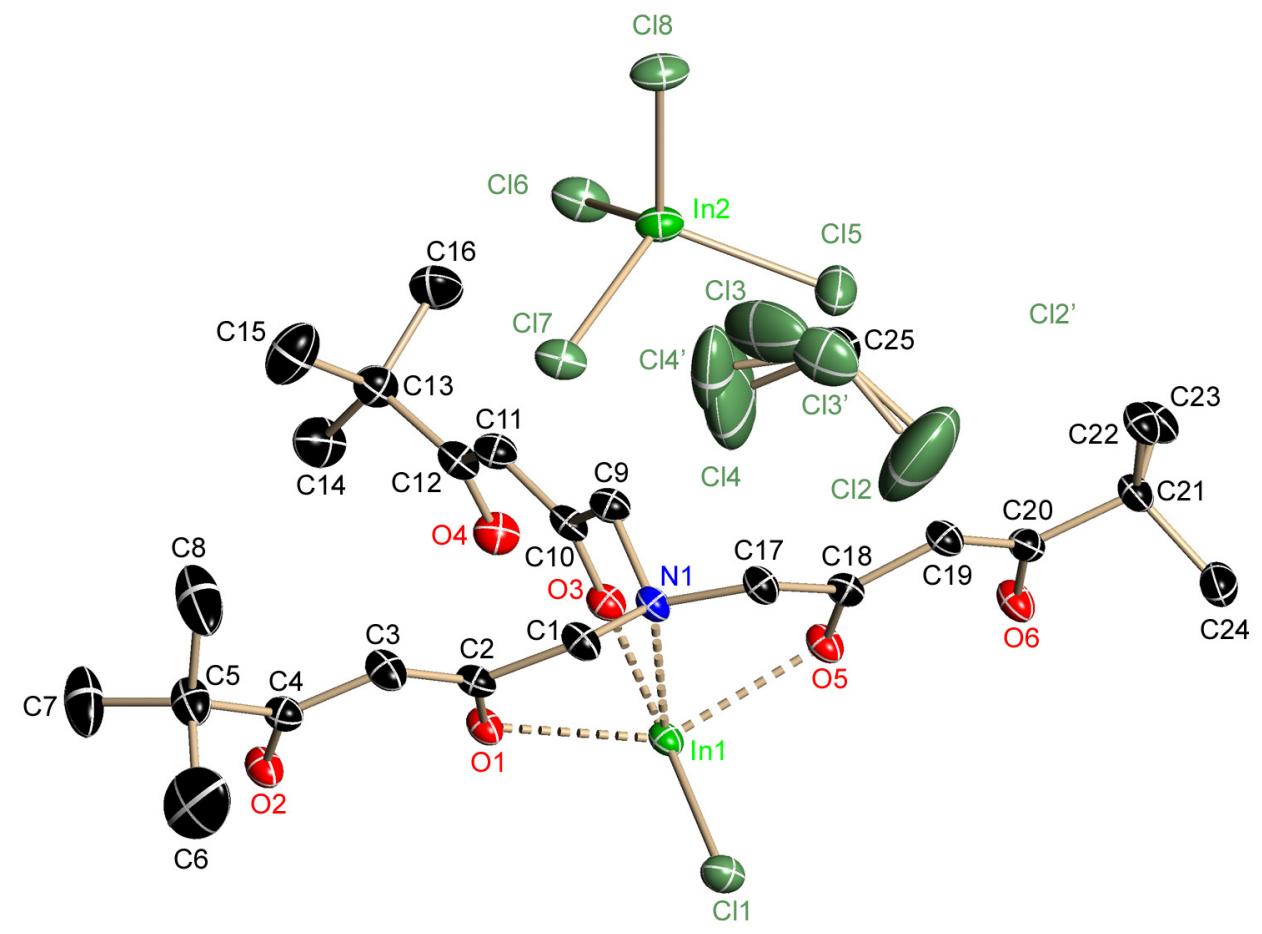

Abb. 5.109: Asymmetrische Einheit von MH488; die anisotropen Auslenkungsparameter geben die 50\% ige Aufenthaltswahrscheinlichkeit an.

Tab. 5.101: Kristallographische Daten von MH488.

\begin{tabular}{|ll|ll|}
\hline Strukturcode & $\mathrm{MH} 488$ & $\rho\left[\mathrm{Mgm}^{-3}\right]$ & 1.643 \\
Summenformel & $\mathrm{C}_{50} \mathrm{H}_{78} \mathrm{O}_{12} \mathrm{~N}_{2} \mathrm{Cl}_{16} \mathrm{In}_{4}$ & $\mu\left[\mathrm{mm}^{-1}\right]$ & 1.769 \\
Molekulare Masse [g/mol] & 1925.62 & $F(000)$ & 3824 \\
Kristallgröße [mm] & $0.2 \times 0.2 \times 0.2$ & $\theta$-Bereich [ $\left.{ }^{\circ}\right]$ & $1.55-26.73$ \\
Raumgruppe & $\mathrm{C} / \mathrm{c}$ & Gesammelte Reflexe & 60157 \\
$a[\mathrm{pm}]$ & $2151.7(2)$ & Unabhängige Reflexe & 8261 \\
$b[\mathrm{pm}]$ & $1672.35(17)$ & Restraints & 24 \\
$c[\mathrm{pm}]$ & $2192.1(2)$ & Parameter & 418 \\
$\alpha\left[^{\circ}\right]$ & 90 & $R 1[/>2 \sigma(I)]$ & 0.0269 \\
$\beta\left[^{\circ}\right]$ & $99.3410(10)$ & $w R 2[$ alle Daten] & 0.0661 \\
$\gamma\left[^{\circ}\right]$ & 90 & GooF & 1.078 \\
$\mathrm{~V}\left[\mathrm{~nm}^{3}\right]$ & $7.7832(13)$ & Restdichte: & \\
$Z$ & 4 & Max. $/$ min. $\left[10^{-6} \mathrm{epm}^{-3}\right]$ & $1.173 /-0.509$ \\
Temperatur $[\mathrm{K}]$ & $100(2)$ & Max. /min. Transmission & $0.99 / 0.8075$ \\
\hline
\end{tabular}




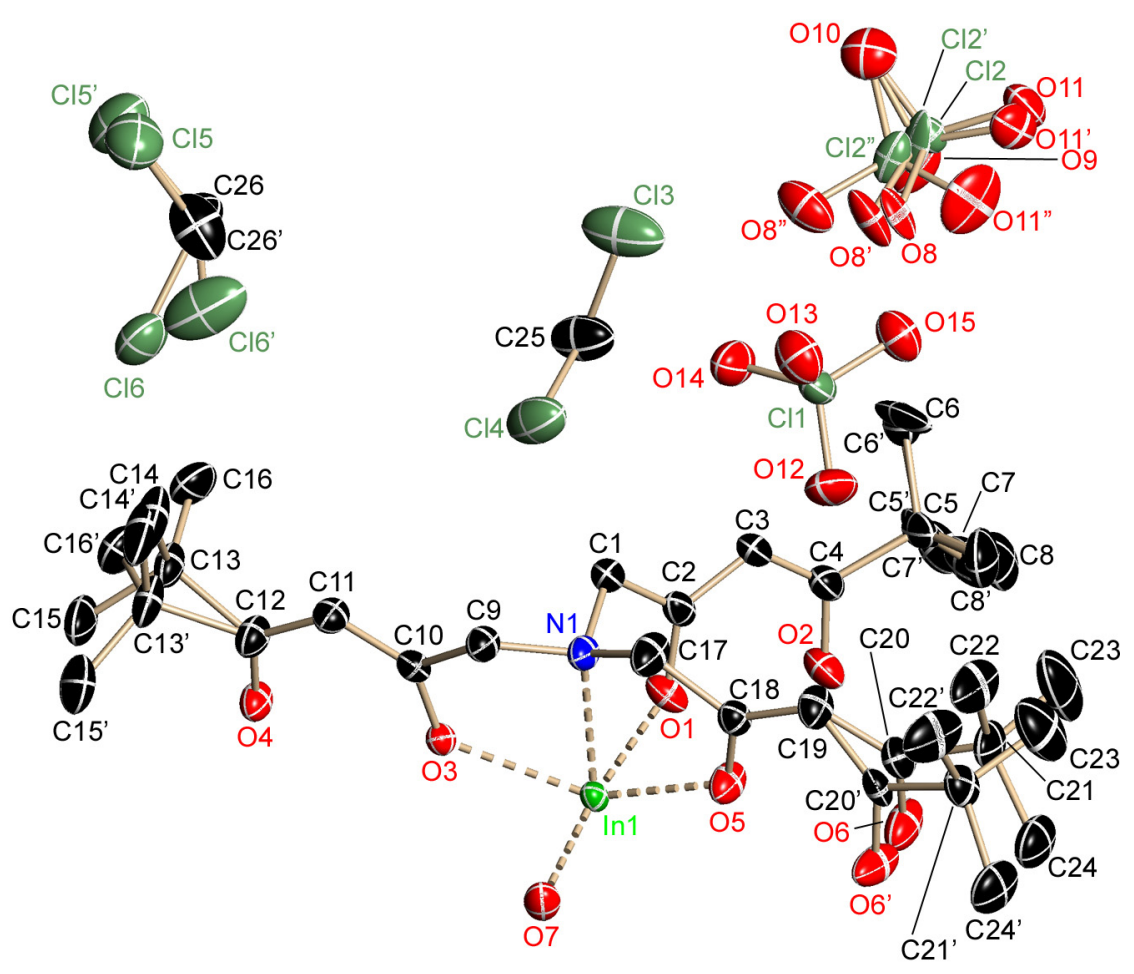

Abb. 5.110: Asymmetrische Einheit von SA420; die anisotropen Auslenkungsparameter geben die 50\% ige Aufenthaltswahrscheinlichkeit an.

Tab. 5.102: Kristallographische Daten von SA420.

\begin{tabular}{|ll|ll|}
\hline Strukturcode & $\mathrm{SA} 420$ & $\rho\left[\mathrm{Mgm}^{-3}\right]$ & 1.586 \\
Summenformel & $\mathrm{C}_{52} \mathrm{H}_{88} \mathrm{O}_{30} \mathrm{~N}_{2} \mathrm{Cl}_{12} \mathrm{In}_{2}$ & $\mu\left[\mathrm{mm}^{-1}\right]$ & 1.072 \\
Molekulare Masse [g/mol] & 1876.23 & $F(000)$ & 1912 \\
Kristallgröße [mm] & $0.2 \times 0.2 \times 0.1$ & $\theta$-Bereich [ $\left.{ }^{\circ}\right]$ & $1.91-26.75$ \\
Raumgruppe & $P 2_{1} / c$ & Gesammelte Reflexe & 60369 \\
$a[\mathrm{pm}]$ & $1760.5(2)$ & Unabhängige Reflexe & 8325 \\
$b[\mathrm{pm}]$ & $1472.94(18)$ & Restraints & 91 \\
$c[\mathrm{pm}]$ & $1731.4(2)$ & Parameter & 676 \\
$\alpha\left[^{\circ}\right]$ & 90 & $R 1[/>2 \sigma(I)]$ & 0.0431 \\
$\beta\left[^{\circ}\right]$ & $118.9210(10)$ & WR2 [alle Daten] & 0.1001 \\
$\gamma\left[^{\circ}\right]$ & 90 & GooF & \\
$\mathrm{V}\left[\mathrm{nm}^{3}\right]$ & $3.9297(8)$ & Restdichte: & \\
$Z$ & 2 & Max. / min. [10 & epm $\left.{ }^{-3}\right]$ \\
Temperatur $[\mathrm{K}]$ & $100(2)$ & Max. / min. Transmission & $0.839 / 0.99$ \\
\hline
\end{tabular}




\subsubsection{Struktur des AK Beckhaus (Universität Oldenburg)}

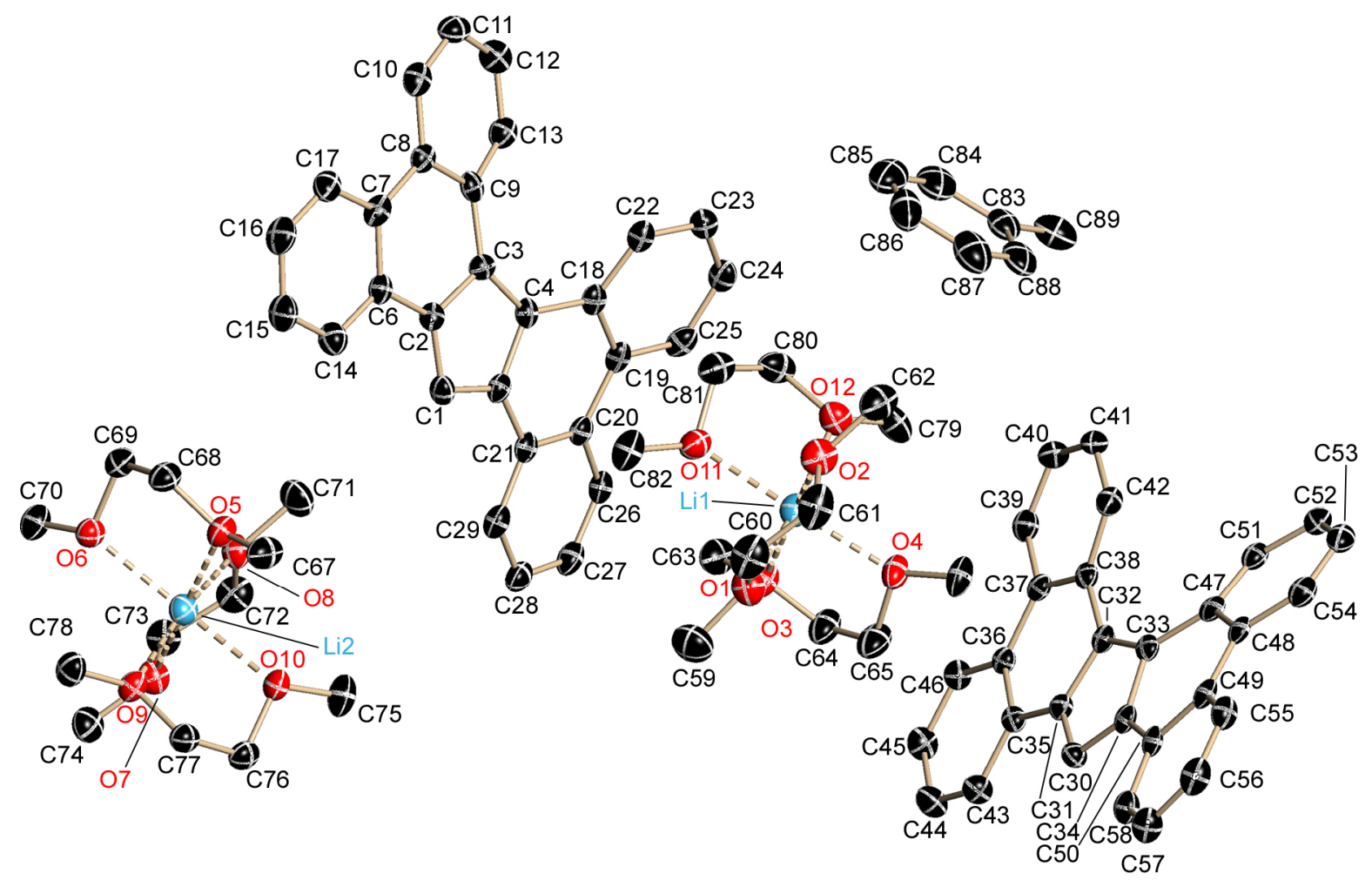

Abb. 5.111: Asymmetrische Einheit von; die anisotropen Auslenkungsparameter geben die $50 \%$ ige Aufenthaltswahrscheinlichkeit an.

Tab. 5.103: Kristallographische Daten von

\begin{tabular}{|ll|ll|}
\hline Strukturcode & & $\rho\left[\mathrm{Mgm}^{-3}\right]$ & 1.206 \\
Summenformel & $\mathrm{C}_{44.50} \mathrm{H}_{51} \mathrm{LiO}_{6}$ & $\mu\left[\mathrm{mm}^{-1}\right]$ & 0.078 \\
Molekulare Masse $[\mathrm{g} / \mathrm{mol}]$ & 688.79 & $F(000)$ & 2952 \\
Kristallgröße $[\mathrm{mm}]$ & $0.13 \times 0.13 \times 0.11$ & $\theta$-Bereich [ $\left.{ }^{\circ}\right]$ & $1.58-23.26$ \\
Raumgruppe & $P 2 / n$ & Gesammelte Reflexe & 150680 \\
$a[\mathrm{pm}]$ & $2074.8(4)$ & Unabhängige Reflexe & 10904 \\
$b[\mathrm{pm}]$ & $1542.2(3)$ & Restraints & 0 \\
$c[\mathrm{pm}]$ & $2565.8(5)$ & Parameter & 942 \\
$\left.\alpha{ }^{\circ}\right]$ & 90 & $R 1[/>2 \sigma(I)]$ & 0.0562 \\
$\beta\left[^{\circ}\right]$ & $112.477(3)$ & $w R 2[$ alle Daten $]$ & 0.1343 \\
$\gamma\left[^{\circ}\right]$ & 90 & GooF & 1.041 \\
$\mathrm{~V}\left[\mathrm{~nm}^{3}\right]$ & $7.59(1)$ & Restdichte: & \\
$Z$ & 8 & Max. $/$ min. $\left[10^{-6} \mathrm{epm}^{-3}\right]$ & $0.240 /-0.241$ \\
Temperatur $[\mathrm{K}]^{3}$ & $100(2)$ & Max. / min. Transmission & $0.99 / 0.8364$ \\
\hline
\end{tabular}




\section{Abkürzungsverzeichnis}

\begin{tabular}{|c|c|}
\hline AAV & Allgemeine Arbeitsvorschrift \\
\hline An & Anthracen \\
\hline $\mathrm{Bu}$ & Buthyl \\
\hline CyLi & Cyclopropyllithium \\
\hline DCM & Dichlormethan \\
\hline DEE & Diethylether \\
\hline DIOPAn & Diisopropylphosphoranylanthracenen \\
\hline DME & Dimethoxyethan \\
\hline DMF & Dimethylformamid \\
\hline DMSO & Dimethylsulfoxid \\
\hline HFAC & Hexafluroacetylacetonatanionen \\
\hline HOMO & highest occupied molecule orbital \\
\hline$i \operatorname{Pr}$ & iso-Propyl \\
\hline i. Vak. & im Vakuum \\
\hline LDA & Lithiumdiisopropylamid \\
\hline LUMO & lowest unoccupied molecule orbital \\
\hline Lsg. & Lösung \\
\hline Me & Methyl \\
\hline NBS & N-Bromsucinimid \\
\hline$n B u L i$ & $n$-Buthyllithium \\
\hline NMR & nuclear magnetic resonance \\
\hline PET & Photoinduzierter Elektronentransfer \\
\hline $\mathrm{Ph}$ & Phenyl \\
\hline PhLi & Phenyllithium \\
\hline PMDETA & $N, N, N ', N$ ', N"-Pentamethyldiethylentetraamin \\
\hline ppm & parts per million \\
\hline Py & 2-Pyridyl \\
\hline RT & Raumtemperatur \\
\hline sBuLi & sec-Buthyllithium \\
\hline tBuLi & tert-Buthyllithium \\
\hline THF & Tetrahydrofuran \\
\hline TMEDA & $\mathrm{N}, \mathrm{N}, \mathrm{N}^{\prime}, \mathrm{N}^{\prime}-$ Tetramethylethylendiamin \\
\hline TrMEDA & N,N,N'-Trimethylethylendiamin \\
\hline
\end{tabular}




\section{Verzeichnis der nummerierten Verbindungen}

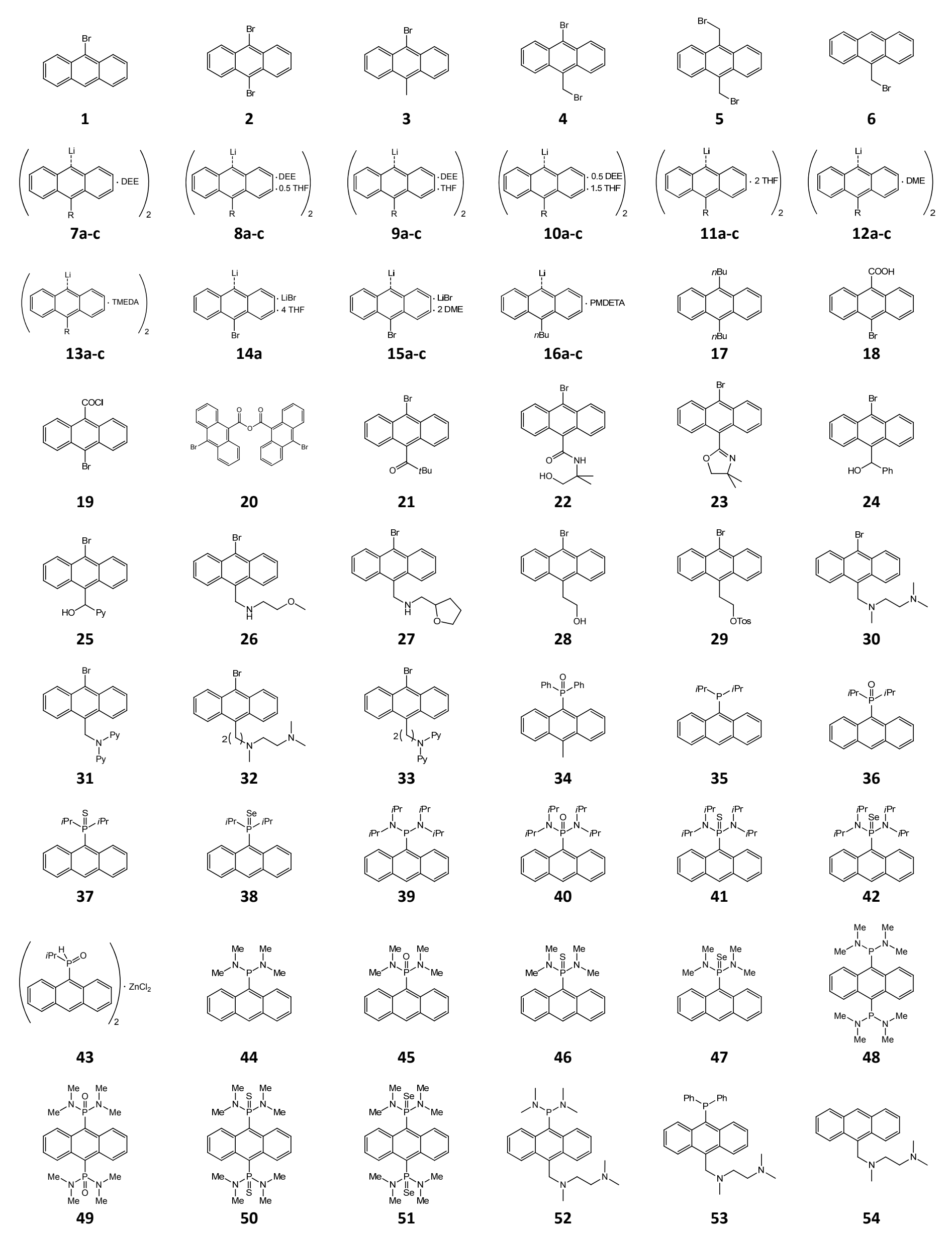




\section{Literaturverzeichnis}

[1] G. Plinus d. Ä., Naturkunde / C. Plinius Secundus d. Ä. Hrsg. und übers. von Roderich König, Roderich König, Darmstadt, 1997.

[2] B. Schäfer, Naturstoffe der chemischen Industrie, Elsevier GmbH, Spektrum Akademischer Verlag, Heidelberg, 2007.

[3] A. V. Bulatov, R. M. Lobkovskaya, M. L. Khidekel, A. N. Chekhlov, R. P. Shibaeva, Izv. Akad. Nauk SSSR, Otdel. Khim. Nauk 1980, 1203.

[4] C. Graebe, C. Liebermann, Ber. Dtsch. Chem. Ges. 1868, 1, 49-51.

[5] C. Graebe, C. Liebermann, Ber. Dtsch. Chem. Ges. 1869, 2, 14.

[6] C. Graebe, C. Liebermann, Ber. Dtsch. Chem. Ges. 1869, 2, 332-334.

[7] H. Caro, C. Graebe, C. Liebermann, Ber. Dtsch. Chem. Ges. 1870, 3, 359-360.

[8] S. Brockert, H. Emde, I. Rheinholz, E. Thiel, Chemie für die Zukunft, BASF AG, Ludwigshafen, 1990.

[9] W. Brita, Applica 2003, 5, 4-16.

[10] H. Du, R.-C. A. Fuh, J. Li, L. A. Corkan, J. S. Lindsey, Photochem. Photobiol. 1998, 68, 141-142.

[11] G. Wedler, Lehrbuch der Physikalischen Chemie, Wiley-VCH Verlag GmbH, Weinheim, 1997.

[12] P. W. Atkins, Physikalische Chemie, Wiley-VCH Verlag GmbH, Weinheim, 2001.

[13] L. Horner, H.-W. Flemming, Phosphorus, Sulfur Silicon Relat. Elem. 1985, 22, 161-167.

[14] A. Schmillen, R. Legler, Zahlenwerte und Funktionen aus Naturwissenschaft und Technik: Lumineszenz organischer Substanzen, Springer Verlag, Berlin, 1967.

[15] Z. Fei, N. Kocher, C. J. Mohrschladt, H. Ihmels, D. Stalke, Angew. Chem. 2003, 115, 807-811.

[16] K. Akasaka, T. Suzuki, H. Ohrui, H. Meguro, Anal. Lett. 1987, 20, 731-745.

[17] H. Schindlbauer, H. Hagen, Monatsh. Chem. 1965, 1, 285-299.

[18] R. A. Bissell, A. P. de Silva, H. Q. N. Gunaratne, P. L. M. Lynch, G. E. M. Maguire, K. R. A. S. Sandanayake, Chem. Soc. Rev. 1992, 21, 187-195.

[19] A. P. de Silva, R. A. D. D. Rupasinghe, J. Chem. Soc., Chem. Commun. 1985, 16691670.

[20] M. E. Huston, K. W. Haider, A. W. Czarnik, J. Am. Chem. Soc. 1988, 110, 4460-4462.

[21] S. K. Kim, J. H. Lee, J. Yoon, Bull. Korean Chem. Soc. 2003, 24, 1032-1034.

[22] M. Ciampolini, M. Formica, V. Fusi, A. Saint-Mauricec, M. Micheloni, N. Nardi, R. Pontellini, F. Pina, P. Romani, A. M. Sabatini, B. Valtancoli, Eur. J. Inorg. Chem. 1999, 2261-2268.

[23] F. A. Khan, K. Parasuraman, K. K. Sadhu, Chem. Commun. 2009, 2399-2401.

[24] B. Bag, P. Bharadwaj, J. Chem. Sci. 2005, 117, 145-151.

[25] K. S. Kim, E. J. Jun, S. K. Kim, H. J. Choi, J. Yoo, C.-H. Lee, M. H. Hyuna, J. Yoon, Tetrahedron Lett. 2007, 48, 2481-2484.

[26] A. T. Bens, Doktorarbeit, Deutschland (Düsseldorf), 2001.

[27] G. H. Schwab, Dissertation, Deutschland (Göttingen), 2008.

[28] D. Stern, Diplomarbeit, Deutschland (Göttingen), 2006.

[29] P. Nanjappan, A. W. Czarnik, J. Am. Chem. Soc. 1987, 109, 1826-1833.

[30] G. Greiner, I. Maier, J. Chem. Soc., Perkin Trans. 2 2002, 1005-1011.

[31] T. Gunnlaugsson, A. P. Davis, J. E. O'Brien, M. Glynn, Org. Lett. 2002, 4, 2449-2452.

[32] A. Tamayo, E. Oliveira, B. Coveló, J. Casabo, L. Escriche, C. Lodeiro, Z. Anorg. Allg. Chem. 2007, 633, 1809-1814.

[33] A. P. Prasanna de Silva, R. A. D. D. Rupasinghe, J. Chem. Soc., Chem. Commun. 1985, 1669-1670.

[34] B. Bock, K. Ziemer, C. Näther, J. Organomet. Chem. 1996, 511, 29-35.

[35] A. Krasovskiy, B. F. Straub, P. Knochel, Angew. Chem., Int. Ed. Engl. 2006, 45, 159162.

[36] B. F. Duerr, Y.-S. Chung, A. W. Czarnik, J. Org. Chem. 1988, 53, 2120-2122. 
[37] J. H. K. Yip, J. Prabhavathy, Angew. Chem., Int. Ed. Engl. 2001, 40, 2159-2162.

[38] O. Reiser, Chemie in unserer Zeit 2001, 35, 94-100.

[39] S. Höger, Chemie in unserer Zeit 2001, 35, 102-109.

[40] T. Ezuhara, K. Endo, O. Hayashida, Y. Aoyama, New J. Chem. 1998, 183-188.

[41] N. Miyaura, A. Suzuki, Chem. Rev. 1995, 95, 2457-2483.

[42] A. Suzuki, J. Organomet. Chem. 2002, 653, 83-90.

[43] R. B. D. de la Mare, J. H. Ridd, Aromatic Substitution Nitration and Halogenation, Butterworths Scientific Publications, London, 1959.

[44] E. de Barry Barnett, M. A. Matthews, Chem. Ber. 1926, 59, 1429-1438.

[45] D. E. Pearson, M. G. Frazer, V. S. Frazer, L. C. Washburn, Synthesis 1976, 9, 621-623.

[46] D. Bogdal, M. Lukasiewicz, J. Pielichowski, Green Chem. 2004, 6, 110-113.

[47] H. A. Muathen, Helv. Chim. Acta 2003, 86, 164-168.

[48] M. K. Chaudhuri, A. T. Khan, P. K. Patel, Tetrahedron Lett. 1998, 39, 8163-8166.

[49] J. H. Boyer, T. Manimaran, J. Chem. Soc., Perkin Trans. 1 1989, 1381-1385.

[50] T. Anderson, Liebigs Ann. Chem. 1862, 122, 294-308.

[51] O. Cakmak, L. Aydogan, K. Berkil, I. Gulcin, O. Buyukgungor, Beilstein J. Org. Chem. 2008, 4, no. 50.

[52] T. Hökelek, A. Tutar, O. Cakmak, Acta Crystallogr., Sect. E: Struct. Rep. Online 2002, E58, o10-o12.

[53] M. Hartmann, M. Räthe, Z. Chem. 1979, 19, 373-374.

[54] P. Mamalis, J. Green, D. J. Outred, M. J. Rix, J. Chem. Soc. 1965, 1829-1843.

[55] S. Duan, J. Turk, J. Speigle, J. Corbin, J. Masnovi, R. J. Baker, J. Org. Chem. 2000, 65, 3005-3009.

[56] S. E. Webber, J. H. Clements, Macromolecules 2004, 37, 1531-1536.

[57] J. H. Clements, S. E. Webber, J. Phys. Chem. B 1999, 103, 9366-9377.

[58] S. H. Yang, O. J. Shon, K. M. Park, S. S. Lee, H. J. Park, M. J. Kim, J. H. Lee, J. S. Kim, Bull. Korean Chem. Soc. 2002, 23, 1585-1589.

[59] A. D. Mosnaim, D. C. Nonhebel, J. Chem. Soc. C: Org. Chem. 1970, 942-946.

[60] A. Tamayo, B. Pedras, C. Lodeiro, L. Escriche, J. Casabó, J. L. Capelo, B. Covelo, R. Kivekäs, R. Sillanpää, Inorg. Chem. 2007, 46, 7818-7826.

[61] T. Lazarides, M. A. H. Alamiry, H. Adams, S. J. A. Pope, S. Faulkner, J. A. Weinstein, M. D. Ward, Dalton Trans. 2007, 1484-1491.

[62] A. Tamayo, C. Lodeiro, L. Escriche, J. Casabó, B. Covelo, P. González, Inorg. Chem. 2005, 44, 8105-8115.

[63] N. M. Shavaleev, Z. R. Bell, T. L. Easun, R. Rutkaite, L. Swansonb, M. D. Ward, Dalton Trans. 2004, 3678-3688.

[64] R. A. Bissell, E. Calle, A. P. de Silva, S. A. de Silva, H. Q. N. Gunaratne, J.-L. HabibJiwan, S. L. A. Peiris, R. A. D. D. Rupasinghe, T. K. S. D. Samarasinghe, K. R. A. S. Sandanayake, J.-P. Soumillion, J. Chem. Soc., Perkin Trans. 2 1992, 1559-1564.

[65] M. Bullpitt, W. Kitching, J. Org. Chem. 1976, 41, 760-766.

[66] I. Cade, N. J. Long, A. J. P. White, D. J. Williams, J. Organomet. Chem. 2006, 691, 13891401.

[67] F. de Montigny, G. Argouarch, C. Lapinte, Synthesis 2006, 2, 293-298.

[68] N. Krause, Metallorganische Chemie, Spektrum, Akad. Verl., Heidelberg, 1996.

[69] W. E. Bachmann, M. C. Kloetzel, J. Org. Chem. 1938, 3, 55-61.

[70] E. Müller, T. Töpel, Chem. Ber. 1939, 72, 273-290.

[71] B. M. Mikhailov, Izv. Akad. Nauk SSSR, Otdel. Khim. Nauk 1948, 4, 420-426.

[72] B. M. Mikhailov, Chem. Abstr. 1948, 43, 208g-209c.

[73] B. M. Mikhailov, N. G. Chernova, Zh. Obshch. Khim. 1959, 29, 222-228.

[74] B. M. Mikhailov, V. P. Bronovitskaya, J. Gen. Chem. USSR 1953, 23, 127-131.

[75] B. M. Mikhailov, V. P. Bronovitskaya, J. Gen. Chem. USSR 1952, 22, 195-201.

[76] B. M. Mikhailov, N. G. Chernova, Dokl. Akad. Nauk SSSR 1952, 48, 967-970.

[77] B. M. Mikhailov, N. G. Chernova, J. Gen. Chem. USSR 1951, 21, 1689-1665.

[78] A. Maercker, Angew. Chem. 1987, 99, 1002-1019; Angew. Chem., Int. Ed. Engl. 1987, 26, 972-989.

[79] P. Walker, J. Org. Chem. 1961, 26, 2994-2995.

[80] G. Rio, B. Sillion, Séances Acad. Sci. 1957, 244, 623-626. 
[81] H. Lehmkuhl, A. Shakoor, K. Mehler, C. Krüger, K. Angermund, Y.-H. Tsay, Chem. Ber. 1985, 118, 4239-4247.

[82] U. Herrmann, B. Tiimmler, G. Maass, P. K. T. Mew, F. Vogtle, Biochemistry 1984, 23, 4059-4067.

[83] Y. Matsubara, A. Kimura, Y. Yamaguchi, Z.-i. Yoshida, Org. Lett. 2008, 10, 5541-5544.

[84] R. Schröck, K. Angermaier, A. Sladek, H. Schmidbaur, J. Organomet. Chem. 1996, 509, 85-88.

[85] G. Schwab, D. Stern, D. Leusser, D. Stalke, Z. Naturforsch., B: Chem. Sci. 2007, 62, 711-716.

[86] S. Wandtke, Bachelor, Deutschland (Göttingen), 2009.

[87] A. Visscher, Bachelor, Deutschland (Göttingen), 2009.

[88] N. Finkelmeier, Diplom, Deutschland (Göttingen), 2008.

[89] G. Schwab, D. Stern, D. Stalke, J. Org. Chem. 2008, 73, 5242-5247.

[90] H. L. Lewis, T. L. Brown, J. Am. Chem. Soc. 1970, 92, 4664-4670.

[91] I. Craubner, Z. Phys. Chem. (Munich) 1966, 51, 225.

[92] T. L. Brown, Adv. Organomet. Chem. 1966, 3, 365-395.

[93] D. Margerison, J. P. Newport, Trans. Faraday Soc. 1963, 59, 2058-2063.

[94] W. McLean, P. T. Murray, T. Baer, R. C. Jarnagin, J. Chem. Phys. 1978, 69, 2715-2718.

[95] W. M. Scovell, B. Y. Kimura b, T. G. Spiro, J. Coord. Chem. 1971, 1, 107-114.

[96] L. D. McKeever, R. Waack, J. Chem. Soc. D, Chem. Commun. 1969, 750-751.

[97] M. Weiner, G. Vogl, R. West, Inorg. Chem. 1962, 1, 654-658.

[98] R. R. Fraser, T. S. Mansour, Tetrahedron Lett. 1986, 27, 331-334.

[99] S. Akiyama, J. Hooz, Tetrahedron Lett. 1973, 42, 4115-4118.

[100] G. G. Eberhardt, W. A. Butte, J. Org. Chem. 1964, 29, 2928-2932.

[101] C. Strohmann, K. Strohfeldt, D. Schildbach, J. Am. Chem. Soc. 2003, 125, 1367213673.

[102] W. Zarges, M. Marsch, K. Harms, G. Boche, Chem. Ber. 1989, 122, 2303-2309.

[103] H. Hope, P. P. Power, J. Am. Chem. Soc. 1983, 105, 5320-5324.

[104] D. Thoennes, E. Weiss, Chem. Ber. 1978, 111, 3157.

[105] U. Schümann, J. Kopf, E. Weiss, Angew. Chem. 1985, 97, 222-223; Angew. Chem., Int. Ed. Engl. 1985, 24, 215-216.

[106] R. A. Barlett, H. V. R. Dias, P. P. Power, J. Organomet. Chem. 1988, 341, 1.

[107] D. Stern, A. Visscher, S. Wandtke, D. Stalke, J. Org. Chem. 2009, in preperation.

[108] M. A. Beno, H. Hope, M. M. Olmstead, P. P. Power, J. Organomet. Chem. 1985, 4, 2117.

[109] S. P. Patterman, I. L. Karle, G. D. Stucky, J. Am. Ceram. Soc. 1970, 92, 1150.

[110] H. Ott, U. Pieper, D. Leusser, J. Henn, D. Stalke, Angew. Chem. 2009, 121, 3022; Angew. Chem., Int. Ed. Engl. 2009, 48, 2978.

[111] I. Kondolff, H. Doucet, M. Santelli, Tetrahedron 2004, 60, 3813-3818.

[112] D. Seebach, Angew. Chem. 1988, 100, 1685; Angew. Chem., Int. Ed. Engl. 1988, 27, 1624.

[113] M. L. Pratt, Mini-Rev. Org. Chem. 2004, 1, 209.

[114] D. A. Price, N. J. Simpkins, Tetrahedron Lett. 1994, 35, 6159.

[115] B. J. Bunn, N. J. Simpkins, J. Org. Chem. 1993, 58, 533.

[116] W. Glaze, R. West, J. Am. Chem. Soc. 1960, 82, 4437.

[117] D. Y. Curtin, E. W. Flynn, J. Am. Chem. Soc. 1959, 81, 4714.

[118] H. J. S. Winkler, H. Winkler, J. Am. Chem. Soc. 1966, 88, 969.

[119] R. Huisgen, W. Mack, Chem. Ber. 1960, 93, 332.

[120] H. Schmidbaur, A. Schier, U. Schubert, Chem. Ber. 1983, 116, 1938.

[121] T. Kottke, R. J. Lagow, D. Hoffmann, R. D. Thomas, Organometallics 1997, 16, 789.

[122] C. A. Ogle, B. K. Huckabee, H. C. Johnson, Organometallics 1993, 12, 1960.

[123] M. A. Nichols, P. G. Williard, J. Am. Chem. Soc. 1993, 115, 1568.

[124] M. M. Olmstead, P. P. Power, J. Am. Chem. Soc. 1990, 112, 8008.

[125] M. Geissler, J. Kopf, B. Schubert, E. Weiss, W. Neugebauer, P. von Rague Schleyer, Angew. Chem. 1987, 99, 569-570; Angew. Chem., Int. Ed. Engl. 1987, 26, 587-588.

[126] G. Schwab, D. Stern, D. Leusser, D. Stalke, Z. Naturforsch., B: Chem. Sci. 2008, 62, 711-716. 
[127] F. A. Carey, R. J. Sundberg, Organische Chemie, Wiley-VCH Verlag GmbH, Weinheim, 2004.

[128] F. A. Cotton, M. W. Extine, C. W. Rice, Inorg. Chem. 1978, 17, 176-186.

[129] E. Wolcan, G. Torchia, J. Tocho, O. E. Piro, P. Juliarena, G. Ruiz, M. R. Féliz, J. Chem. Soc., Dalton Trans. 2002, 2194-2202.

[130] S. Furukawa, S. Kitagawa, Inorg. Chem. 2004, 43, 6464-6472.

[131] R.-Q. Zou, C.-S. Liu, X.-S. Shi, X.-H. Bu, J. Ribas, Cryst. Eng. Comm. 2005, 7, 722-727.

[132] M. J. Byrnes, M. H. Chisholm, J. A. Gallucci, Y. Liu, R. Ramnauth, C. Turro, J. Am. Chem. Soc. 2005, 127, 17343-17352.

[133] C.-S. Liu, X.-S. Shi, J.-R. Li, J.-J. Wang, X.-H. Bu, Cryst. Growth Des. 2006, 6, 656-663.

[134] M. M. Amini, A. Azadmeher, H. R. Khawasi, S. W. Ng, J. Organomet. Chem. 2007, 692, 3922-3930.

[135] C.-S. Liu, J.-J. Wang, L.-F. Yan, Z. Chang, X.-H. Bu, E. C. Sanudo, J. Ribas, Inorg. Chem. 2007, 46, 6299-6310.

[136] R. Inglis, L. F. Jones, K. Mason, A. Collins, S. A. Moggach, S. Parsons, S. P. Perlepes, W. Wernsdorfer, E. K. Brechin, Chem. Eur. J. 2008, 14, 9117-9121.

[137] J.-J. Wang, C.-S. Liu, T.-L. Hu, Z. Chang, C.-Y. Li, L.-F. Yan, P.-Q. Chen, X.-H. Bu, Q. Wu, L.-J. Zhao, Z. Wang, X.-Z. Zhang, Cryst. Eng. Comm. 2008, 10, 681-692.

[138] C.-S. Liu, L.-F. Yan, Z. Chang, J.-J. Wang, Acta Crystallogr., Sect. E: Struct. Rep. Online 2008, 64, m15-m16.

[139] C.-S. Liu, L.-Q. Guo, L.-F. Yanb, J.-J. Wang, Acta Crystallogr., Sect. C: Cryst. Struct. Commun. 2008, 64, m292-m295.

[140] C.-S. Liu, P.-Q. Chen, Z. Chang, J.-J. Wang, L.-F. Yan, H.-W. Sun, X.-H. Bu, Z. Lin, Z.-M. Li, S. R. Batten, Inorg. Chem. Commun. 2008, 11, 159-163.

[141] S. M. A. Karim, R. Nomura, F. Sanda, S. Seki, M. Watanabe, T. Masuda, Macromolecules 2003, 36, 4786-4789.

[142] R. Nomura, S. M. A. Karim, H. Kajii, R. Hidayat, K. Yoshino, T. Masuda, Macromolecules 2000, 33, 4313-4315.

[143] M. A. J. Bayliss, R. B. Homer, J. Chromatogr. 1988, 445, 393-402.

[144] A. J. Blake, R. S. Grimditch, M. Schröder, Acta Crystallogr., Sect. C: Cryst. Struct. Commun. 1995, C51, 1472-1474.

[145] S. H. Kang, H. Ma, M.-S. Kang, K.-S. Kim, A. K.-Y. Jen, M. H. Zareie, M. Sarikaya, Angew. Chem. 2004, 116, 1538-1542; Angew. Chem., Int. Ed. Engl. 2004, 43, 15121516.

[146] T. G. Gant, A. I. Meyers, Tetrahedron 1994, 50, 2297-2360.

[147] B. W. Gung, X. Xue, H. J. Reich, J. Org. Chem. 2005, 70, 3641-3644.

[148] I. Ravikumar, B. N. Ahamed, P. Ghosh, Tetrahedron 2007, 63, 12940-12947.

[149] A. P. de Silva, H. Q. N. Gunaratne, C. P. McCoy, Nature (London) 1993, 364, 42-44.

[150] A. Tamayo, L. Escriche, J. Casabó, B. Covelo, C. Lodeiro, Eur. J. Inorg. Chem. 2006, 15, 2997-3004.

[151] E. U. Akkaya, M. E. Huston, A. W. Czarnik, J. Am. Chem. Soc. 1990, 112, 3590-3593.

[152] H. Mao, J. B. Thorne, J. S. Pharr, R. E. Gawley, Can. J. Chem. 2006, 84, 1273-1279.

[153] J. Wesemann, P. G. Jones, D. Schomburg, L. Heuer, R. Schmutzler, Chem. Ber. 1992, $125,2187-2197$.

[154] A. J. Deeming, C. M. Martin, Chem. Commun. 1996, 53-54.

[155] W.-Y. Wong, F.-L. Ting, Y. Guo, Z. Lin, J. Cluster Sci. 2005, 16, 185-199.

[156] D. M. P. Mingos, T. E. Müller, J. Organomet. Chem. 1995, 500, 251-259.

[157] M. Osawa, M. Hoshino, T. Wada, F. Hayashi, S. Osanai, J. Phys. Chem. A 2009, 113, 10895-10902.

[158] T. Sasamori, A. Tsurusaki, N. Nagahora, K. Matsuda, Y. Kanemitsu, Y. Watanabe, Y. Furukawa, N. Tokitoh, Chem. Lett. 2006, 35, 1382-1383.

[159] F. Yang, P. E. Fanwick, C. P. Kubiak, Inorg. Chem. 2002, 41, 4805-4809.

[160] F. Yang, P. E. Fanwick, C. P. Kubiak, Organometallics 1999, 18, 4222-4225.

[161] W. Schlenk, A. Thal, Ber. Dtsch. Chem. Ges. 1913, 46, 2840-2854.

[162] T. Kottke, D. Stalke, J. Appl. Crystallogr. 1993, 26, 615-619.

[163] D. Stalke, Chem. Soc. Rev. 1998, 27, 171-178.

[164] Bruker AXS, APEX2 v2009.3-0, Madison, 2009.

[165] Bruker AXS, Saint v7.46a, Madison, 2009. 
[166] G. M. Sheldrick, SADABS 2008/2, Göttingen, 2008.

[167] G. M. Sheldrick, TWINABS 2008/1, Göttingen, 2008.

[168] G.M. Sheldrick, XS in SHELXTL v2008/2, Madison, 2008.

[169] G. M. Sheldrick, Acta Crystallogr., Sect. A. 2008, 64, 112-122.

[170] G. M. Sheldrick, XL in SHELXTL v2008/2, Madison, 2008.

[171] G. M. Sheldrick, XP in SHELXTL v2008/2, Madison, 2008.

[172] P. Müller, R. Herbst-Irmer, A. L. Spek, T. R. Schneider, M. R. Sawaya, Crystal strukture refinement; Crystallographer's Guide to SHELXL, Oxford University Press, Oxford, 2006.

[173] P. Emsley, K. Cowtan, Acta Crystallogr., Sect. D 2004, D60, 2126-2132.

[174] P. Emsley, B. Lohkamp, W. G. Scott, K. Cowtan, Acta Crystallogr., Sect. D. 2010, D66, in press.

[175] A. Lorbach, H.-W. Lerner, M. Bolte, Acta Crystallogr., Sect. C 2007, C63, m174.

[176] D. Stern, M. Granitzka, T. Schulz, D. Stalke, Z. Naturforsch., B: Chem. Sci 2010, 65b, 719-724.

[177] Bruker AXS, XSHELL v4.01, Madison, 2001. 


\section{Publikationen}

2010

D. Stern, N. Finkelmeier, J. Henn, K. Meindel, D. Stalke, Angew. Chem. 2010, accepted.

From the Lithium-2-anilide-2-fluoro-1,3-diaza-2-sila-cyclopentene- $\mathrm{GaCl}_{3}$ Adduct to 1,4,6-Triaza-5-gallium-7-sila-cyclo-3-heptene - Experimental and Quantumchemical Results. A. Fischer, D. Stern, A. Torn, S. Abraham, D. Stalke, U. Klingebiel, Z. Anorg. Allg. Chem. 2010, in press.

Polymorphism of Dibromo-tetrakis(tetrahydrofuran- $k \mathrm{O}$ )magnesium(II). D. Stern, M. Granitzka, T. Schulz, D. Stalke, Z. Naturforsch., B 2010, 65b, 719.

High Yield Access to Silylene $\mathrm{RSiCl}\left(\mathrm{R}=\mathrm{PhC}(\mathrm{NtBu})_{2}\right)$ and Its Reactivity toward Alkyne: Synthesis of Stable Disilacyclobutene. S. Sen, H. Roesky, D. Stern, J. Henn, D. Stalke, J. Am. Chem. Soc. 2010, 132, 1123.

Reactivity Studies of a $\mathrm{Ge}^{\prime}-\mathrm{Ge}^{\prime}$ Compound with and without Cleavage of the Ge-Ge Bond. Sakya S. Sen, D. Kratzert, D. Stern, H. W. Roesky, D. Stalke, Inorg. Chem. 2010, 49, 5786.

Synthesis and Structural Characterization of Heterobimetallic Bismuth Complexes with Main Group and Transition Metals. B. Nekoueishahraki, A. Jana, H. W. Roesky, L. Mishra, D. Stern, D. Stalke, Organometallics 2009, 28, 5733.

Synthesis and Structural Characterization of Aluminum Iminophosphonamide Complexes. B. Nekoueishahraki, H. W. Roesky, G. Schwab, D. Stern, D. Stalke Inorg. Chem. 2009, 48, 9174.

A comparison of a microfocus $X$-ray source and a conventional sealed tube for crystal structure determination. T. Schulz, K. Meindl, D. Leusser, D. Stern, J. Graf, C. Michaelsen, M. Ruf, G. M. Sheldrick, D. Stalke, J. Appl. Crystallogr. 2009, 42, 885 .

6-Benzyl-3,4-dimethoxy-10-methylpyrido[2',1':2,3]imidazo[4,5-c]isoquinolin5(6H)-one. K. Meindl, D. Stern, F. Mert-Balcib, U. Beifuss, Acta Crystallogr., Sec. E. 2009, E65, 02464-02465.

Solvent-Separated and Contact lon Pairs of Parent Lithium Trimethyl Zincate. S. Merkel, D. Stern, J. Henn, D. Stalke, Angew. Chem., Int. Ed. 2009, 48, 6350.

Addition of Dimethylaminobismuth to Aldehydes, Ketones, Alkenes, and Alkynes. B. Nekoueishahraki, S. P. Sarish, H. W. Roesky, D. Stern, C. Schulzke, D. Stalke, Angew. Chem., Int. Ed. 2009, 48, 4517. 
2008 Template and pH-Mediated Synthesis of Tetrahedral Indium Complexes $\left[\mathrm{Cs} \subset\left\{\ln _{4}(\mathrm{~L})_{4}\right\}\right]^{+}$and $\left[\ln _{4}\left(\mathrm{H}^{N} \mathrm{~L}\right)_{4}\right]^{4+}$ : Breaking the Symmetry of N-Centered $C_{3}(\mathrm{~L})^{3-}$ To Give Neutral [In4(L)4]. R. W. Saalfrank, H. Maid, A. Scheurer, F. W. Heinemann, R. Puchta, W. Bauer, D. Stern, D. Stalke, Angew. Chem., Int. Ed. 2008, 47, 8941.

Structural and Variable-Temperature NMR Studies of 9Diisopropylphosphanylanthracenes and 9,10-Bis(diisopropylphosphanyl)anthracenes and Their Oxidation Products. G. Schwab, D. Stern, D. Stalke, J. Org. Chem. 2008, 73, 5242.

2007 Syntheses and Structures of 9-Bromo-10-diphenylphosphanylanthracene and its Oxidation Products. G. Schwab, D. Stern, D. Stalke, Z. Naturforsch., B 2007, $62,711$.

\section{Teilnahme an Konferenzen}

2009 17. Jahrestagung der DGK (Hannover) - 4 Posterbeiträge

DFG 1178 Jahrestreffen (Kloster Banz) - 1 Posterbeitrag

2008 32. NMR-Benutzertagung (Karlsruhe)

Chemie Dozententagung (Göttingen)

5th European charge density meeting in Kombination mit dem DFG 1178 Jahrestreffen (Gravedona) - 1 Posterbeitrag

2007 31. NMR-Benutzertagung (Karlsruhe)

Bruker AXS Anwendertreffen (Göttingen)

1. Chemie-Forum (Göttingen) - 1 Vortrag

DFG Jahrestagung (Ulm) - 1 Posterbeitrag

2006 4th European charge density meeting (Brandenburg) 


\section{Lebenslauf}

\section{Persönliche Daten}

Name: Daniel Stern

Geburtdatum: $\quad 26.10 .1980$

Geburtsort: Hameln

Familienstand: ledig

\section{Ausbildung}

seit Januar 2007

Dissertation im Arbeitskreis von Prof. Dr. D. Stalke an der GeorgAugust-Universität in Göttingen.

Oktober 2001 -

Studium der Chemie an der Georg-August-Universität in Göttingen.

November 2006

September $2000-$

Zivildienst im Seniorenzentrum Eterna, Bad Gandersheim

August 2001

Juli 2000

Abschluss des Roswitha-Gymnasiums mit der allgemeinen Hochschulreife.

Juli 1997 -

Besuch des Roswitha-Gymnasiums in Bad Gandersheim.

Juni 2000

Juni 1997

Abschluss der Realschule Bad Gandersheim mit dem Erweiterter Sekundarschulabschluss I.

Juli $1993-$

Besuch der Realschule in Bad Gandersheim.

Juni 1997

Juli 1991 -

Besuch der Orientierungsstufe in Bad Gandersheim.

Juni 1993

Juli 1989 -

Besuch der Grundschule in Bad Gandersheim.

Juni 1991

Juli 1987 -

Juni 1989

Besuch der Grundschule in Seesen.

Daniel Stern 